RENATO RIBEIRO FRANCO

GEAR CONTACT FATIGUE FAILURE EFFECTS EVALUATION UNDER PROCESS UNCERTAINTY INFLUENCE 
RENATO RIBEIRO FRANCO

\section{GEAR CONTACT FATIGUE FAILURE EFFECTS EVALUATION UNDER PROCESS UNCERTAINTY INFLUENCE}

Thesis submitted to Escola Politécnica da Universidade de São Paulo as a requisite of Doctor Science Degree.

São Paulo

2019 
RENATO RIBEIRO FRANCO

\section{GEAR CONTACT FATIGUE FAILURE EFFECTS EVALUATION UNDER PROCESS UNCERTAINTY INFLUENCE}

Thesis submitted to Escola Politécnica da Universidade de São Paulo as a requisite of Doctor Science Degree.

Mechanical Engineering Program

Supervisor: Gilberto Francisco Martha de Souza 
Este exemplar foi revisado e corrigido em relação a versão original, sob responsabilidade única do autor e com a anuência de seu orientador.

São Paulo, de de

Assinatura do autor:

Assinatura do orientador:

Catalogação-na-publicação

Franco, Renato Ribeiro

GEAR CONTACT FATIGUE FAILURE EFFECTS EVALUATION UNDER PROCESS UNCERTAINTY INFLUENCE/ R. R. Franco -- São Paulo, 2019. 237 p. edição revisada.

Tese (Doutorado) - Escola Politécnica da Universidade de São Paulo. Departamento de Engenharia Mecatrônica e de Sistemas Mecânicos.

1.Confiabilidade 2.Falhas 3.Engrenagens 4.Erros de Processo I.Universidade de São Paulo. Escola Politécnica. Departamento de Engenharia Mecatrônica e de Sistemas Mecânicos II.t. 


\begin{abstract}
AKNOWLEDGE
I would like to thanks God for this experience. I'm thankful for all that I've been through since I'd started this PHD. It was very rich in human practices including classes that I had the chance of learning, people that I had the opportunity of knowing, teachers that kindly guided me through the knowledge path.

I'm thankful to professor Gilberto Francisco Martha de Souza for his supervision in this work. Once again, I learned that with a wisdom supervision, daily activities are more proficient and less hard.

I'm thankful to my wife and my little daughter for understanding my absence. I'm also thankful that more than understanding, I also had their support, it was very important for me. Thanks also to my parents, which always supported me in every steps taken in my education.

Finally, thanks to FCA and Brazilian government for the funding that made this research possible. I'm also thankful for FCA, USP and UFTPR human and lab structure support in the activities done to make this research happen. I am really proud of this work.
\end{abstract}




\section{RESUMO}

A análise de tensão é essencial na atividade de engenharia devido à sua finalidade inerente de dimensionar componentes. Nesta pesquisa, a análise de tensão também tem uma importância especial devido a intenção de reproduzir em banco de teste de engrenagens, a magnitude de tensão similar as tensões existentes em uma engrenagem de primeira marcha de um câmbio disponível no mercado.

As normas ISO 6336 e AGMA foram as diretrizes para o projeto de engrenagens, assim como a análise de tensão e a avaliação de fadiga. De acordo com essas normas, a geometria de engrenagem e as propriedades do material são fatoreschave para falhas por fadiga. Neste cenário, o objetivo desta pesquisa é avaliar os resultados experimentais da falha de fadiga de contato por engrenagem com base na metodologia ISO 6336, revelando a quantidade de área danificada após o ciclo de carregamento. Nesta pesquisa também se discute a influência da incerteza do processo de manufatura sobre tais resultados. Com base na análise de modelagem de confiabilidade, que visa apontar diferenças induzidas por erros de processo.

Os resultados experimentais apresentam treze dentes danificados para cada uma das cinco engrenagens avaliadas. Esta é uma contribuição importante desta pesquisa, uma vez que define a quantidade de dano resultante do ciclo de fadiga previsto na ISO 6336 dentro dos limites definidos nesta pesquisa, representados pela geometria da engrenagem e os parâmetros de teste, incluindo a carga, a velocidade de deslizamento, a viscosidade do óleo e a temperatura.

Outra importante contribuição desta pesquisa é a avaliação da área de dano por pitting, considerando as incertezas associadas ao processo de fabricação de engrenagens. Esse esforço de avaliar as incertezas do processo tem como objetivo indicar os procedimentos de controle de fabricação do processo que podem contribuir para reduzir a quantidade de áreas danificadas durante a vida operacional das engrenagens.

Finalmente, também foram pesquisados a influência de dois diferentes revestimentos na área danificada pela fadiga de contato e seus impactos na análise de confiabilidade. Estes testes também foram baseados na mesma metodologia utilizada para testes de fadiga de engrenagens não revestidas (ISO 6336). Os 
resultados apontam para uma nova aplicação de revestimento muito promissora para retardar a ocorrência de pitting durante a vida operacional da engrenagem.

Palavras-Chave: Análise de Tensão, Confiabilidade, Falha por fadiga. 


\begin{abstract}
Stress analysis has special importance on engineering activity due to its inherent intention of dimensioning components. Stress analysis has also a special importance on this research due to the intention of reproducing in a gear bench test, the amount of stress similar to the real stress distribution existing in a first gear present in a real gear box available in the market.

In this research, ISO 6336 and AGMA standards were the guidelines for gear design, stress analysis and fatigue evaluation. According to those standards, gear geometry and material properties are key factors for fatigue failure evaluation, allowing the definition of the amount of cycles that the gear would face till fatigue failure. In this scenario, the main objective of this research is to evaluate gear fatigue failure experimental results based on ISO 6336 methodology, revealing the amount of damaged area after fatigue cycle. The thesis also discusses the manufacturing process uncertainty influence on such results. Based on reliability modeling analysis, which aims to point out differences induced by process errors.

Thus, this research is based on analysis of five pinion gears experimental fatigue test output, according to ISO 6336 procedure aiming at defining gear fatigue damage. The experimental results show thirteen teeth damaged area for each one of the five gears. This represents an important contribution of this research once it defines the amount of damage resulted by fatigue cycle predicted at ISO 6336 within the boundaries defined on this research represented by gear geometry and test parameters including load, sliding speed, oil viscosity and temperature.

Another important contribution of this research to gear designers is the evaluation of pitting gear damage area taking into account uncertainties associated with gear manufacturing process. This effort of evaluating process uncertainties aims to indicate process manufacturing control procedures that could contribute to reduce the amount of pitting damaged area during gear operational life.

Finally, coating influence on final fatigue damaged area and its impact on reliability analysis were also researched, based on the same methodology used for fatigue testing of uncoated gears (ISO 6336). The output results point to a very promising new coating application to delay pitting occurrence during gear operational life.
\end{abstract}


Key Words: Fatigue Failure, Reliability, Stress Analysis. 


\section{Figure List}

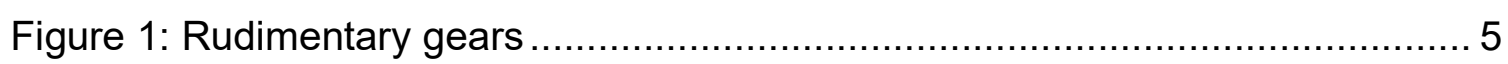

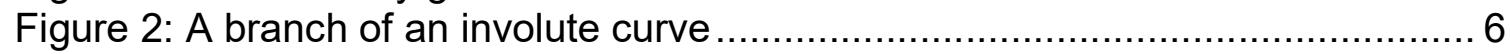

Figure 3a: Pitch point and gear ratio relations ............................................ 8

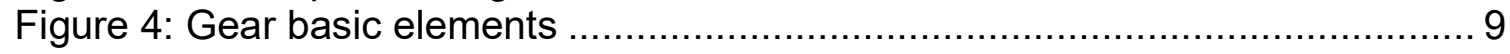

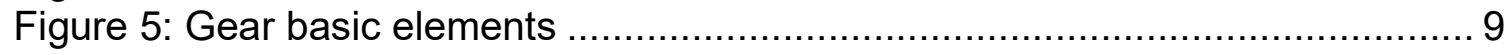

Figure 6: Gear tooth parameter ................................................................. 11

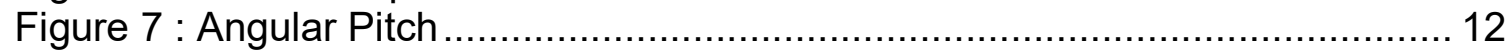

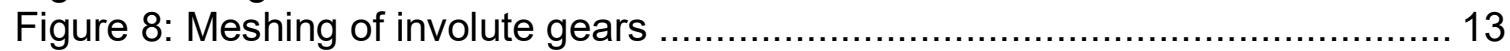

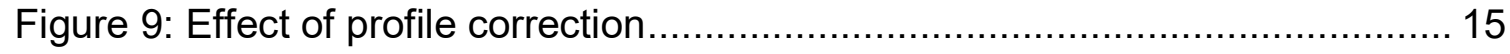

Figure 10: Involute profiles as equidistant curves............................................ 16

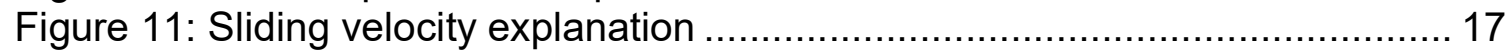

Figure 12: SEM micrograph showing Abrasion ............................................. 19

Figure 13: Severe Abrasion......................................................................... 20

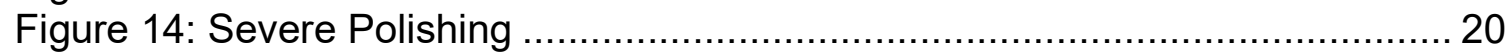

Figure 15: Fretting Corrosion.................................................................... 21

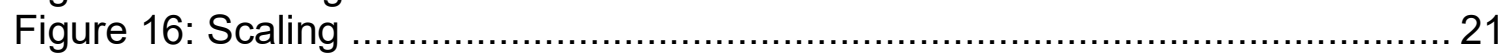

Figure 17: (a) Cavitation damage showing sandblasted appearance and (b) cavitation crater showing deep, rough clean and honeycomb appearance........... 22

Figure 18: Severe Erosion........................................................................ 23

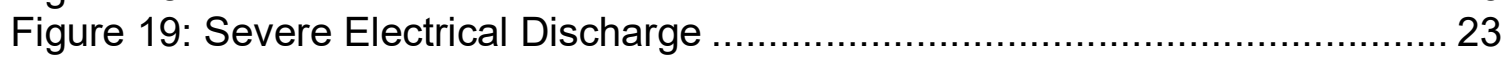

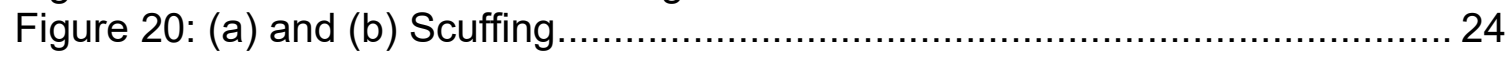

Figure 21: (a) initial pitting and (b) progressive pitting ..................................... 25

Figure 22: Nature of tooth engagement in spur gear drive .............................. 26

Figure 23: Gear meshing mechanism emphasizing Rolling (R) and Sliding (S)

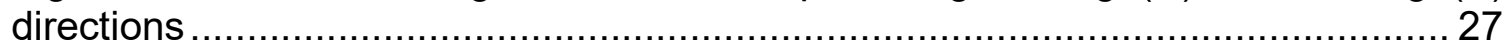

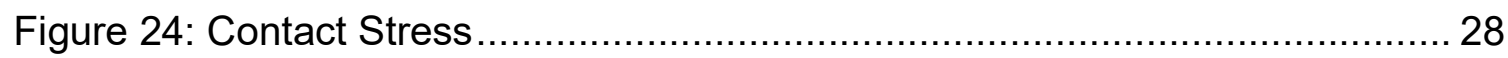

Figure 25: Stress distribution on contacting surface due to rolling, sliding and

combined effects .................................................................................. 30

Figure 26: formation of (a) micro-pits and (b) macro-pits from the grow of micro-

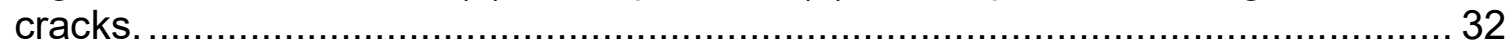

Figure 27: Subcase fatigue (Black narrows are indicating cracks) ..................... 33

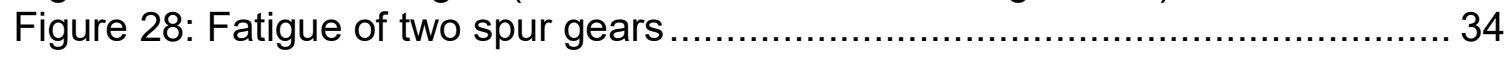

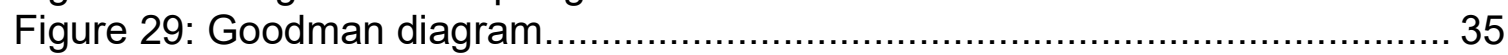

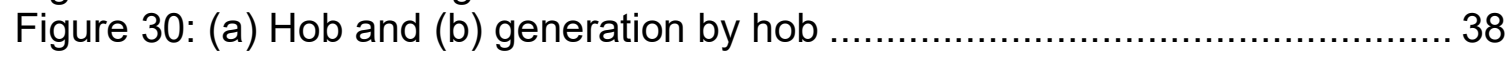

Figure 31: Hob Tool concept for process strategies ....................................... 38

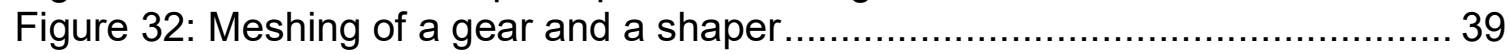

Figure 33: Shaper and generation by shaping............................................ 40

Figure 34: Tool and workpiece assignment for gear shaving ............................ 41

Figure 35: Gear grinding kinematics and contact ......................................... 42

Figure 36: Gear honing tool .................................................................... 43

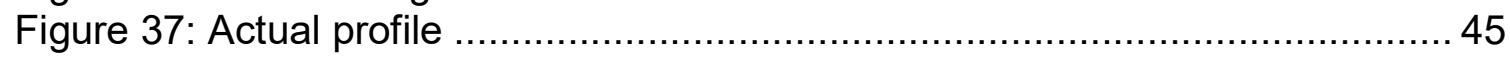

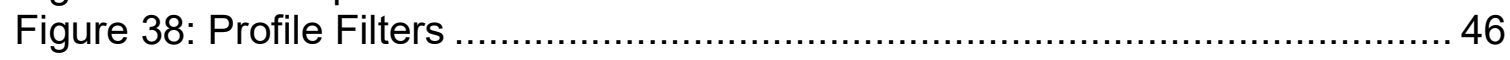

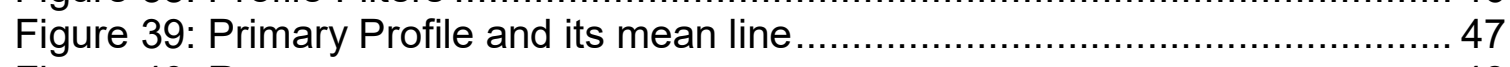

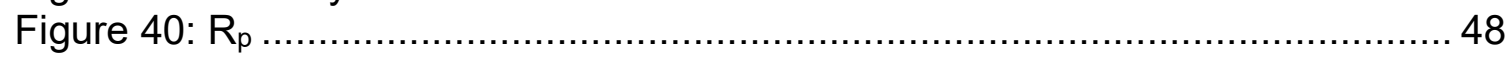




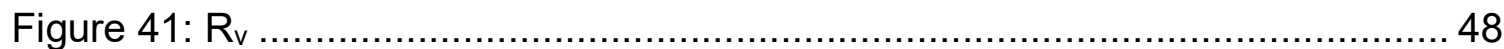

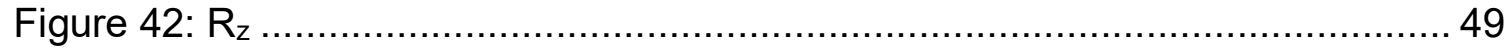

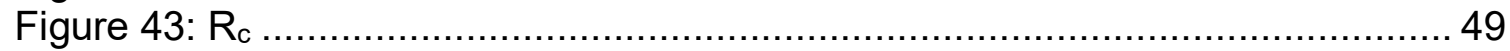

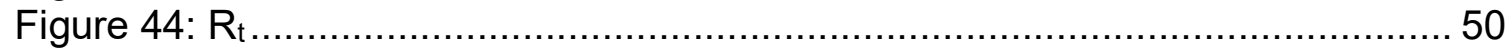

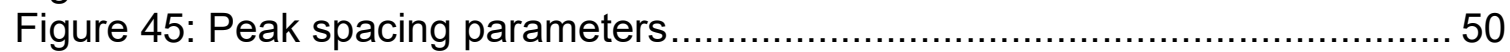

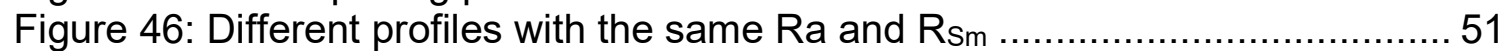

Figure 47: P N curve for surface failure on roller tests ................................ 52

Figure 48: Dynamic Viscosity Concept............................................................ 54

Figure 49: Kinematic viscosity $x$ temperature define the Index Viscosity curve..... 56

Figure 50: Geometry of conformal and counterformal contacts...........................57

Figure 51: $L_{10}$ fatigue life relative to book value as a function of specific film thickness; the data are for a series of cylindrical roller bearings .........................6 60

Figure 52: Relations between contact fatigue macropitting and micropitting and film

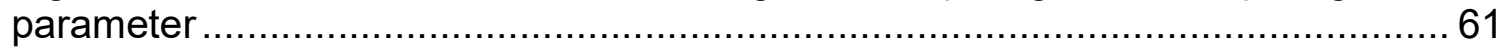

Figure 53: Normal probability density functions for selected values of $\mu$ and $\sigma^{2} \ldots 80$ Figure 54: Lognormal probability density functions with $\theta=0$ for selected values of $w^{2}$

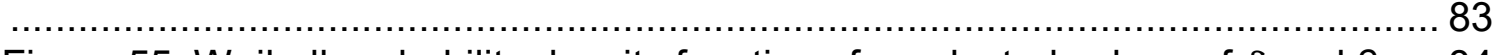

Figure 55: Weibull probability density functions for selected values of $\delta$ and $\beta \ldots . .84$

Figure 56: Methodologies Schematic Overview ............................................. 85

Figure 57: Back to Back Bench Test picture illustrating gears setting under test at

UTFPR. ......................................................................................... 88

Figure 58: Schematic view of measurement direction ...................................... 91

Figure 59: shows Wenzel WGT 350 used on gear measurement. ........................ 93

Figure 60: New gear designed final geometry highlighting in red pitch line and between black lines is seen the single contact area........................................... 98

Figure 61: Gear F01 tooth 3 cross section view image done on SEM to indicate damaged mechanism............................................................................ 101

Figure 62: Gear F01 tooth 10 cross section view done on SEM to indicate damaged mechanism........................................................................................ 102

Figure 63: Gear F01 tooth 10 pitting geometry analyzed by $\mathrm{CCl}$ revealing its width, length and depth.................................................................................. 102

Figure 64: Gear F01 tooth 10 pitting geometry analyzed by $\mathrm{CCl}$ revealing its width, length and depth.................................................................................. 103

Figure 65: Gear F01 tooth 3 damaged area after images after (a) one hour on K9 load stage, (b) more three hours on K10 load stage and (c) more extra three hours

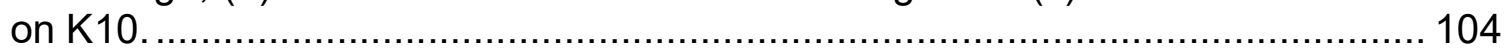

Figure 66: Damaged area identified using MatLab routine on gear F01 Tooth 3 after (a) one hour on K9 load stage, (b) more three hours on K10 load stage and (c) more

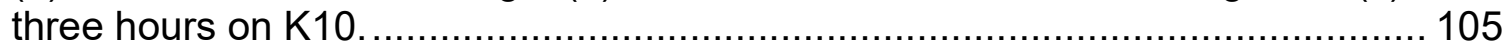

Figure 67: F01 Damaged area variation between fatigue cycle phases, considering: (i): one hour on $\mathrm{K} 9$ load stage, (ii) more three hours on K10 load stage and (iii) more three hours on K10............................................................................ 107

Figure 68: F02 Damaged area variation between fatigue cycle phases, considering: (i): one hour on K9 load stage, (ii) more three hours on K10 load stage and (iii) more three hours on K10................................................................................ 108 
Figure 69: F03 Damaged area variation between fatigue cycle phases, considering: (i): one hour on $\mathrm{K} 9$ load stage, (ii) more three hours on K10 load stage and (iii) more three hours on $\mathrm{K} 10$. 108 Figure 70: F04 Damaged area variation between fatigue cycle phases, considering: (i): one hour on $\mathrm{K} 9$ load stage, (ii) more three hours on K10 load stage and (iii) more three hours on $\mathrm{K} 10$. 109

Figure 71: F05 Damaged area variation between fatigue cycle phases, considering: (i): one hour on $\mathrm{K} 9$ load stage, (ii) more three hours on K10 load stage and (iii) more three hours on K10. 109

Figure 72: Final damaged area variation observed on gears F01 till F05 teeth after last fatigue cycle step (6h K10). 111

Figure 73: Ordinated final damaged area variation observed on gear F01 till F05 teeth after last fatigue cycle step (6h K10). 112 Figure 74: Gears F01(A) till F05 (E) final damaged area data plotting highlighting tendency behavior.

Figure 75: Weibull Probability Plot for pitting damage area for gears F01 to F05 115 Figure 76: Weibull cumulative pitting damaged area distribution for five gears... 117 Figure 77: Tooth surface with process errors in comparison with ideal geometry 118 Figure 78: Gear process errors $x$ damaged area by pitting in \%. 122 Figure 79: Gear tooth illustrating (a) F03 final geometry impacted by fpt (b) F04 final geometry impacted by fpt and fha and (c) F01 final gear geometry impacted by fpt,

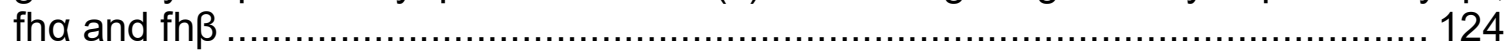

Figure 80 shows material chemical evaluation .............................................. 128

Figure 81: shows material chemical evaluation ............................................ 129

Figure 82: shows material chemical evaluation.......................................... 130

Figure 83: shows material chemical evaluation ............................................ 130

Figure 84: Gear B01 tooth 1 damaged area after images after (a) one hour on K9 load stage, (b) more three hours on K10 load stage and (c) more extra three hours on $\mathrm{K} 10$.

Figure 85: Gear D02 tooth 8 damaged area after images after (a) one hour on K9 load stage, (b) more three hours on K10 load stage and (c) more extra three hours on K10.

Figure 86: Damaged area identified using MatLab routine on gear B01 Tooth 1 after (a) one hour on K9 load stage, (b) more three hours on K10 load stage and (c) more three hours on $\mathrm{K} 10$. 133

Figure 87: Damaged area identified using MatLab routine on gear D02 Tooth 8 after (a) one hour on K9 load stage, (b) more three hours on K10 load stage and (c) more three hours on $\mathrm{K} 10$. 133

Figure 88: B01 Damaged area variation between fatigue cycle phases, considering: (i): one hour on $\mathrm{K} 9$ load stage, (ii) more three hours on K10 load stage and (iii) more three hours on $\mathrm{K} 10$. 137

Figure 89: B02 Damaged area variation between fatigue cycle phases, considering: (i): one hour on $\mathrm{K} 9$ load stage, (ii) more three hours on K10 load stage and (iii) more three hours on $\mathrm{K} 10$. 138

Figure 90: B03 Damaged area variation between fatigue cycle phases, considering: (i): one hour on $\mathrm{K} 9$ load stage, (ii) more three hours on K10 load stage and (iii) more three hours on $\mathrm{K} 10$. 138 
Figure 91: B04 Damaged area variation between fatigue cycle phases, considering: (i): one hour on $\mathrm{K} 9$ load stage, (ii) more three hours on K10 load stage and (iii) more three hours on $\mathrm{K} 10$. 139

Figure 92: B05 Damaged area variation between fatigue cycle phases, considering: (i): one hour on $\mathrm{K} 9$ load stage, (ii) more three hours on K10 load stage and (iii) more three hours on $\mathrm{K} 10$. 139

Figure 93: D01 Damaged area variation between fatigue cycle phases, considering: (i): one hour on $\mathrm{K} 9$ load stage, (ii) more three hours on K10 load stage and (iii) more three hours on $\mathrm{K} 10$. 141

Figure 94: D02 Damaged area variation between fatigue cycle phases, considering: (i): one hour on $\mathrm{K} 9$ load stage, (ii) more three hours on K10 load stage and (iii) more three hours on K10. 142

Figure 95: D03 Damaged area variation between fatigue cycle phases, considering: (i): one hour on $\mathrm{K} 9$ load stage, (ii) more three hours on K10 load stage and (iii) more three hours on $\mathrm{K} 10$. 142

Figure 96: D04 Damaged area variation between fatigue cycle phases, considering: (i): one hour on $\mathrm{K} 9$ load stage, (ii) more three hours on $\mathrm{K} 10$ load stage and (iii) more three hours on $\mathrm{K} 10$. 143

Figure 97: D05 Damaged area variation between fatigue cycle phases, considering: (i): one hour on $\mathrm{K} 9$ load stage, (ii) more three hours on K10 load stage and (iii) more three hours on K10............................................................................. 143

Figure 98: Ordinated final damaged area variation observed on gear B01 till B05 teeth after last fatigue cycle step (6h K10). .................................................. 145 Figure 99: Ordinated final damaged area variation observed on gear D01 till D05 teeth after last fatigue cycle step (6h K10). .................................................... 146 Figure 100: Gears B01 (A) till B05 (E) final damaged area data plotting highlighting tendency behavior............................................................................. 148

Figure 101: Gears D01(A) till D05 (E) final damaged area data plotting highlighting tendency behavior................................................................................... 149

Figure 102: Weibull Probability Plot for pitting damage area for gears B01 to B05 150

Figure 103: Weibull cumulative pitting damaged area distribution for gears D01 to

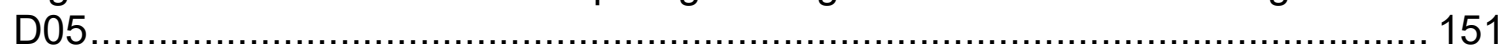

Figure 104: Weibull cumulative pitting damaged area distribution for B01 till B05

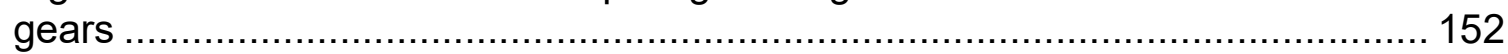

Figure 105: Weibull cumulative pitting damaged area distribution for D01 till D05

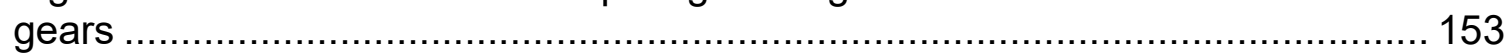

Figure 106: B Gear process errors $x$ damaged area by pitting in \%................... 157

Figure 107: D Gear process errors $x$ damaged area by pitting in $\% \ldots \ldots \ldots \ldots \ldots \ldots . . . . . . . . . .158$

Figure 108: Cr-DLC surface characterization .............................................. 159

Figure 109: DLC surface characterization ................................................ 161

Figure 110: DLC thickness evaluation on D03 tooth3 upper view ......................... 162

Figure 111: DLC thickness evaluation on D03 tooth5 not meshed flank .............. 162

Figure 112 DLC thickness evaluation on D03 tooth5 meshed flank .................... 163

Figure 113 DLC thickness evaluation on D05 tooth3 upper view ....................... 163

Figure 114 DLC thickness evaluation on D05 tooth3 not meshed flank ............... 164

Figure 115 DLC thickness evaluation on D05 tooth3 meshed flank .................... 164 


\section{Table List}

Table 1: Gears general classification (AGMA 933-B03 Basic Gear Geometry, 2003)

Table 2: Nomenclature of gear failure mode (Adapted from AGMA 1010 - E95c

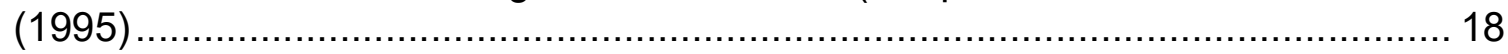

Table 3: Gear tooth quality achievable according to the manufacture method (Linke et al., 2016) ............................................................................................. 44

Table 4: Dynamic and kinematics viscosity units, ASM Handbook (1992) ............ 55

Table 5: - Lubrication regimes classification for gears according to Hutchings (1992),

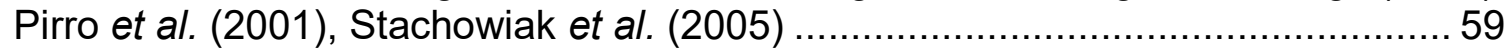

Table 6: Strain and Material properties (Linke et al., 2016) ................................. 62

Table 7: test parameters and schedule defined for gear experimental tests. ........90

Table 8: Design variables influence in stress simulation. ................................. 96

Table 9: Stress Analysis Comparisons of FCA and FZG and Designed Gear....... 97

Table 10: Gear geometry comparisons of FZG and new designed gear. .............. 98

Table 11: Chemical analysis results of Designed Gear ........................................ 99

Table 12: Roughness Measurements executed on Pinion ................................... 99

Table 13: Roughness Measurements executed on Ring ................................. 100

Table 14: Hardness, case depth and core hardness measurements executed on Designed Gear ....................................................................................... 100

Table 15: Gear F01 to F05 teeth damaged area by Pitting (in \% of total gear flank area).................................................................................................... 113

Table 16: Comparison of gear flank mean damage area by pitting and mean process

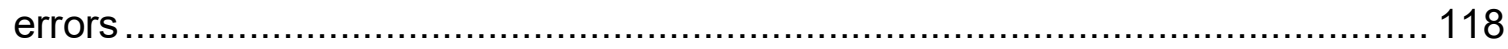

Table 17: Statistical data summary results............................................. 119

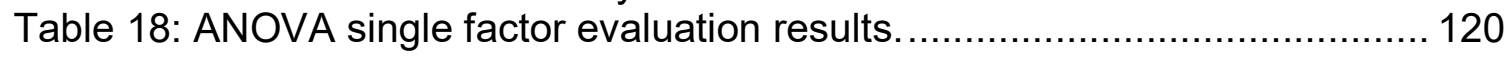

Table 19: Tukey test pairwise comparisons..................................................... 121

Table 20: Influence of $f_{h \alpha}$ and $f_{h \beta}$ process errors on contact stress.................... 124

Table 21: Roughness measurements performed on pinion cross direction, comparing as shaved and coated gears.................................................. 126

Table 22: Hardness, case depth and core hardness measurements of researched

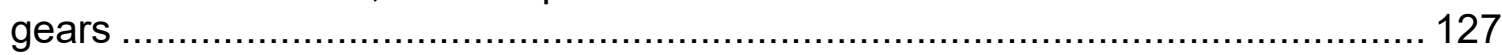

Table 23: Chemical composition revealed by EDS ........................................ 128

Table 24: Gears B01 to B05 teeth damaged area by Pitting (in \% of total gear flank area) ................................................................................................... 147

Table 25: Gears D01 to D05 teeth damaged area by Pitting (in \% of total gear flank area) ....................................................................................................... 147

Table 26: Comparison of gear flank mean damage area by pitting and mean process errors for B01 to B05 ........................................................................... 154

Table 27: Comparison of gear flank mean damage area by pitting and mean process

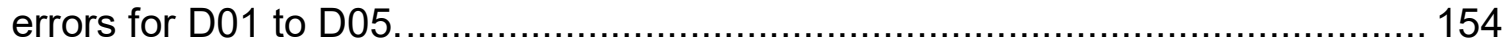

Table 28: Statistical data summary results for B01 till B05.............................. 155

Table 29: Statistical data summary results for D01 till D05.......................... 155

Table 30: ANOVA single factor evaluation results for B01 till B05...................... 155

Table 31: ANOVA single factor evaluation results for D01 till D05 ..................... 156 
Table 32: Tukey test pairwise comparisons.

156

Table 33: Tukey test pairwise comparisons

157 


\section{SYMBOLS LIST}

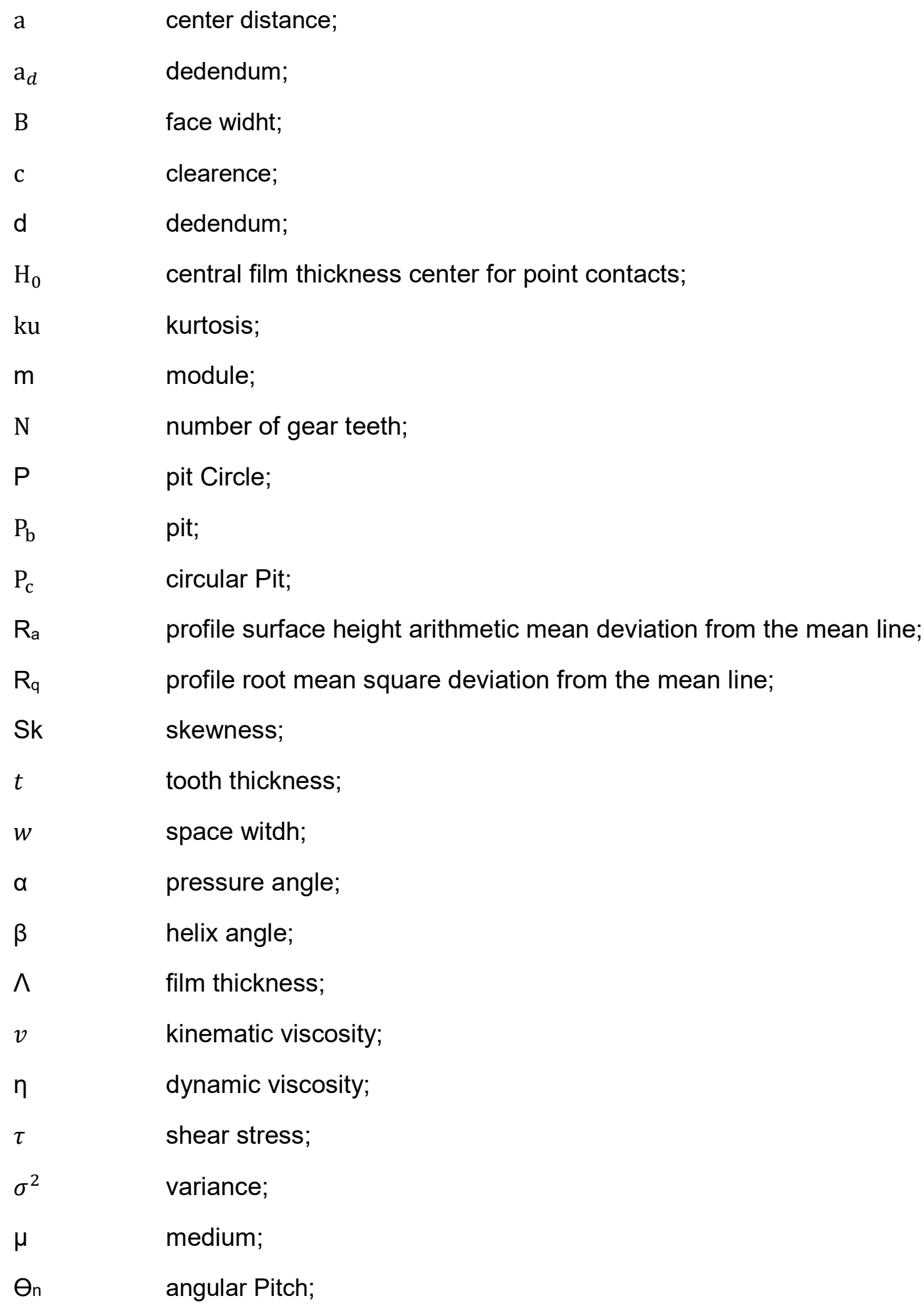

a

$a_{d}$

B

C

d

$\mathrm{H}_{0}$

$\mathrm{ku}$

m

$\mathrm{N}$

P

$\mathrm{P}_{\mathrm{b}}$

$\mathrm{P}_{\mathrm{c}}$

$\mathrm{R}_{\mathrm{a}}$

$\mathrm{R}_{\mathrm{q}}$

Sk

$t$

w

$\alpha$

$\beta$

$\wedge$

$v$

$\eta$

$\tau$

$\sigma^{2}$

$\mu$

$\theta_{\mathrm{n}}$

center distance;

dedendum;

face widht;

clearence;

dedendum;

central film thickness center for point contacts;

kurtosis;

module;

number of gear teeth;

pit Circle;

pit;

circular Pit;

profile surface height arithmetic mean deviation from the mean line;

profile root mean square deviation from the mean line;

skewness;

tooth thickness;

space witdh;

pressure angle;

helix angle;

film thickness;

kinematic viscosity;

dynamic viscosity;

shear stress;

variance;

medium;

angular Pitch; 


\section{Summary}

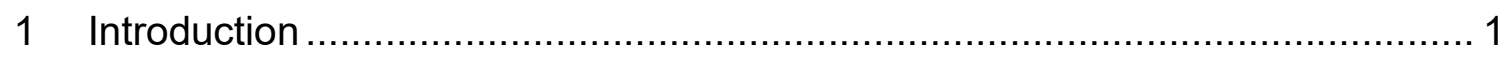

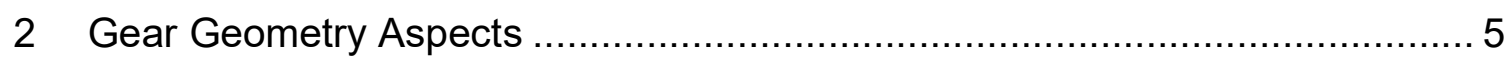

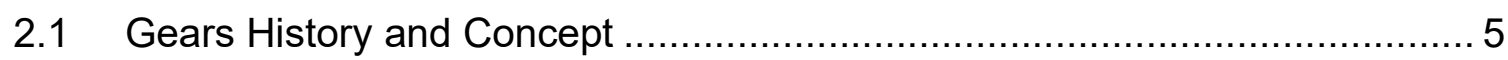

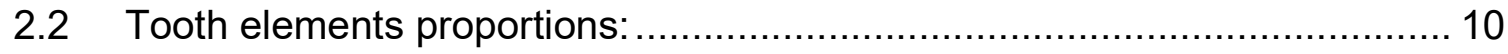

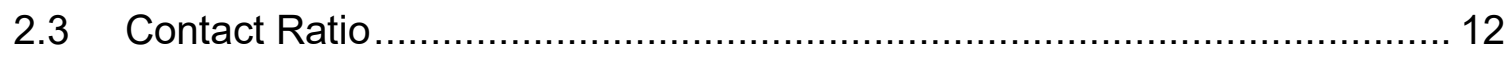

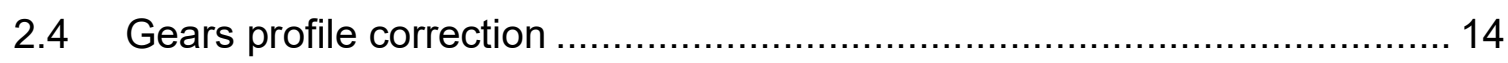

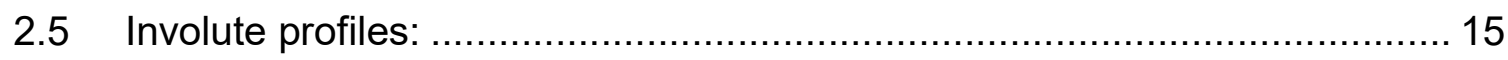

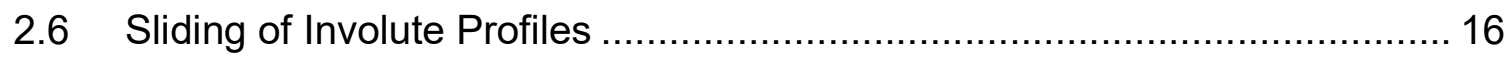

3 Gears Failure Modes and Associate Variables .......................................... 18

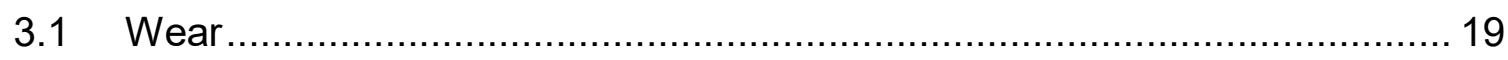

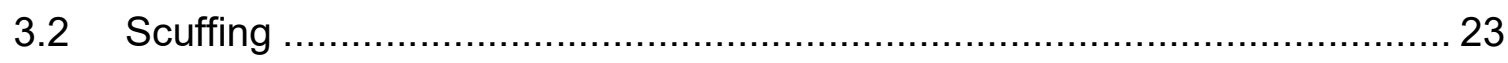

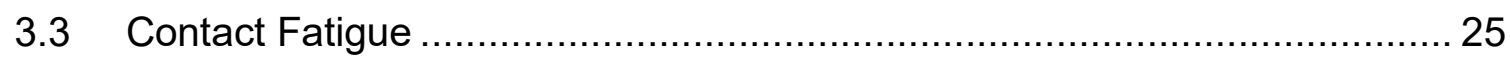

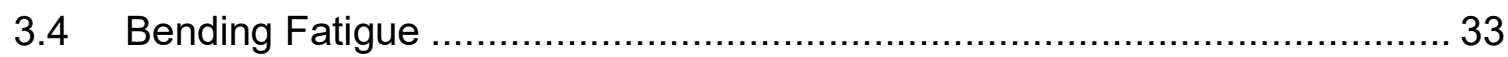

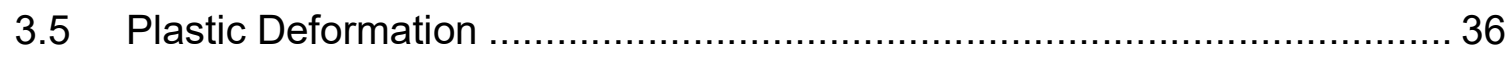

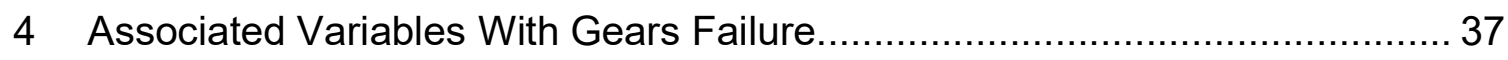

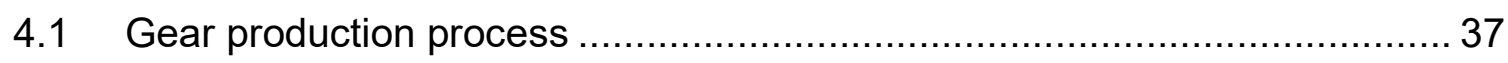

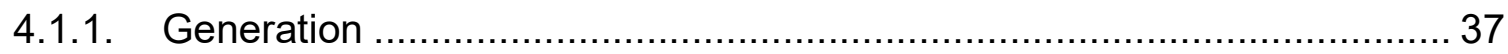

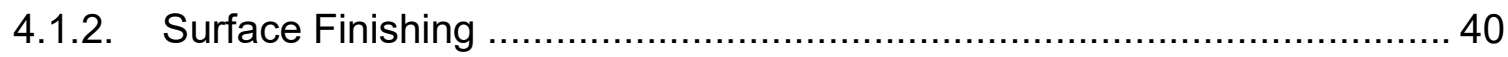

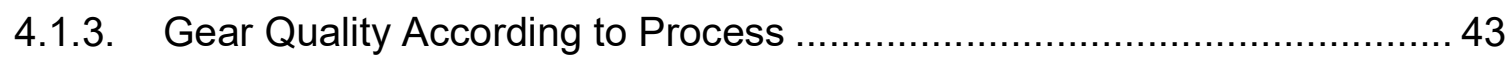

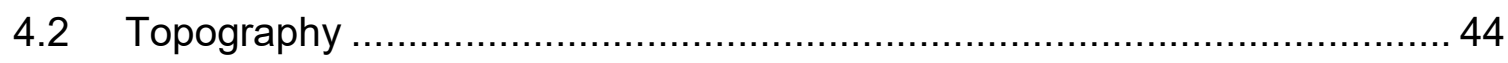

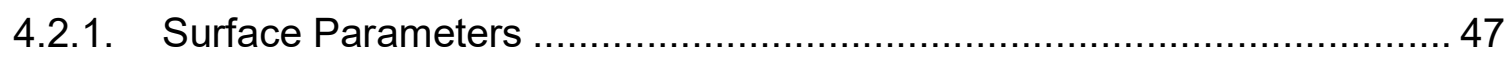

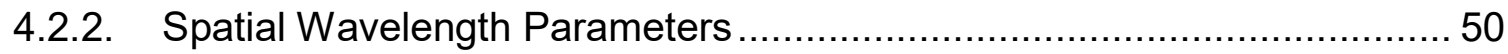

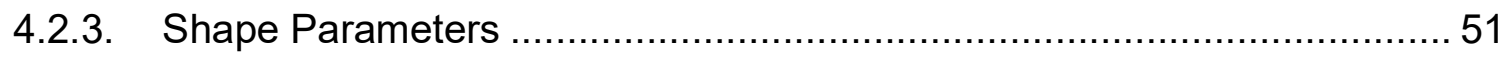

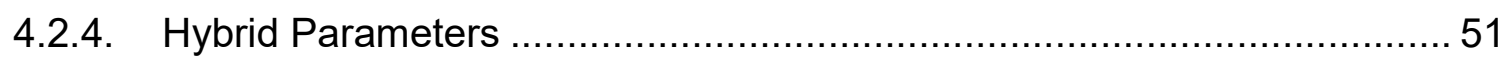

4.3 Process influence on Roughness and gears failure analysis.....................52

4.4 Lubricants and their fundamental properties ........................................... 53

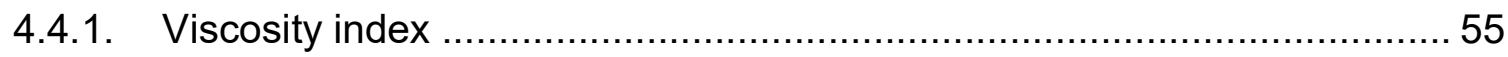

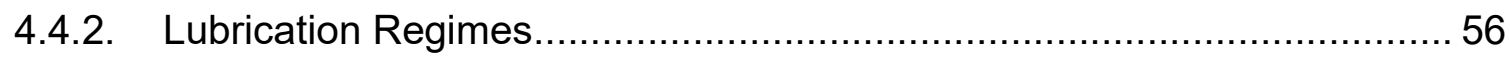

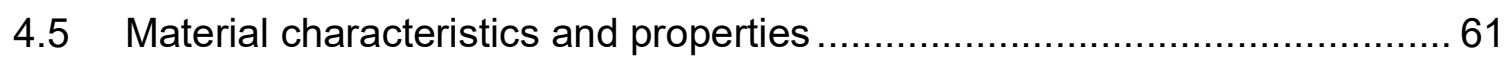

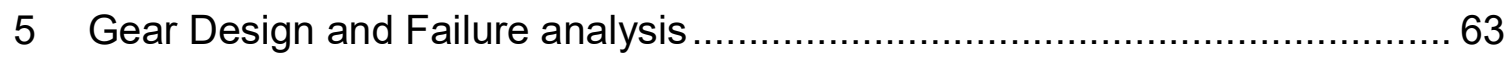

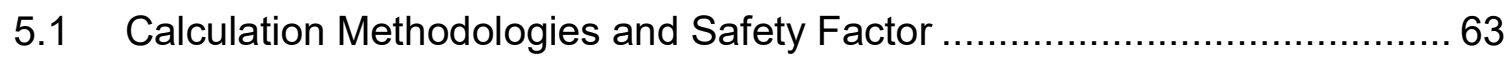




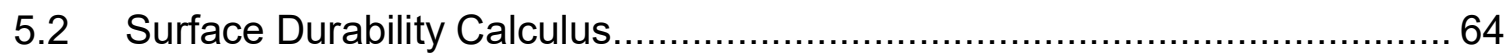

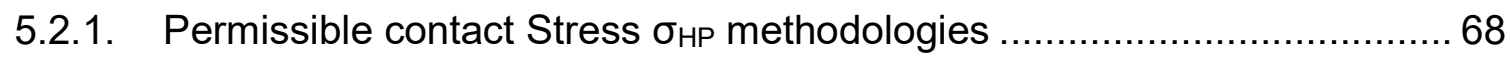

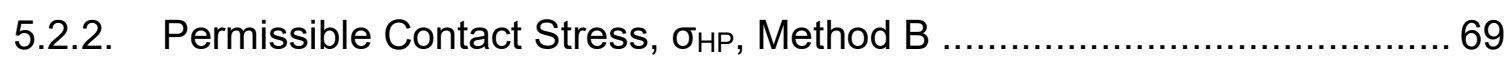

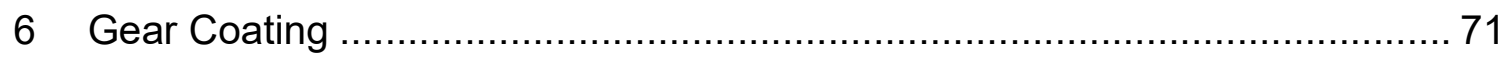

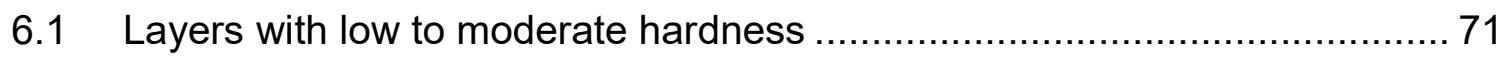

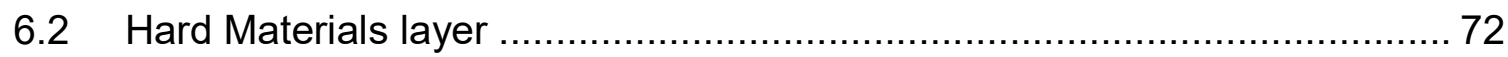

6.3 DLC and BALINIT ${ }^{\circledR} \mathrm{C}$ Coating concepts and published results ...................73

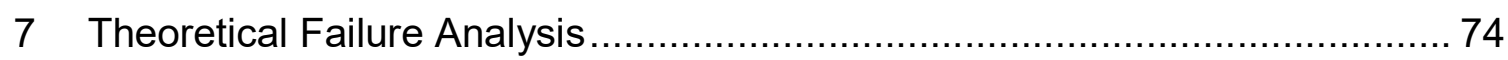

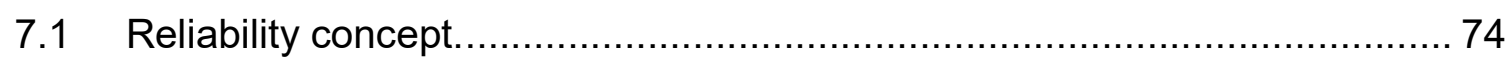

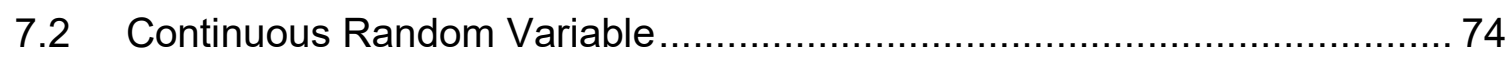

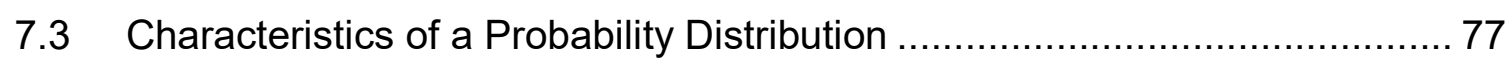

7.4 Reliability and Related Distribution .................................................... 79

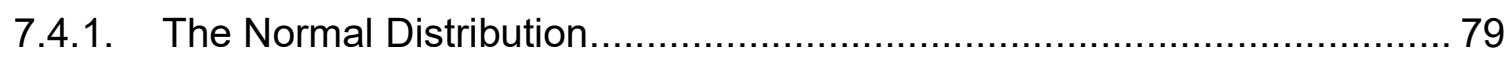

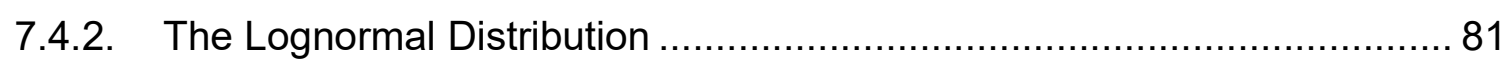

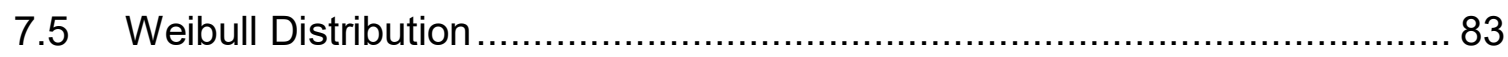

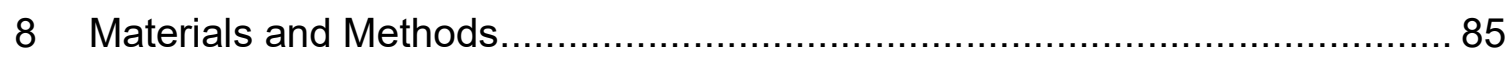

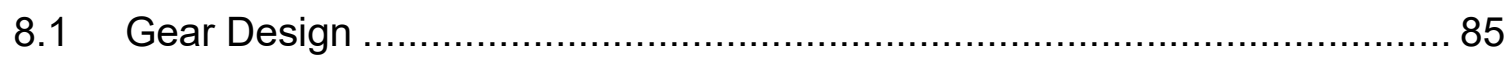

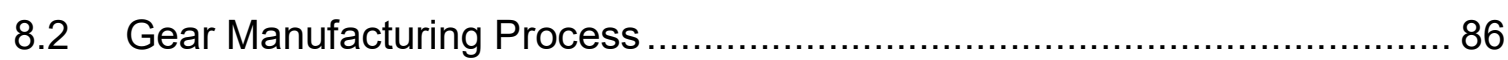

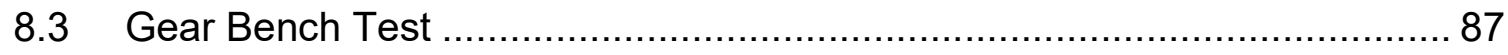

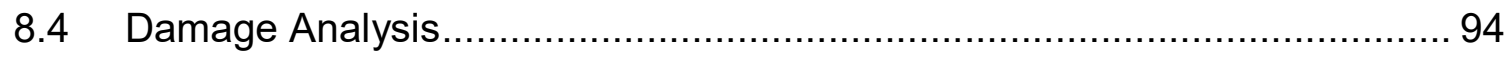

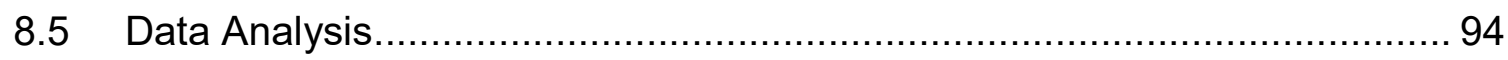

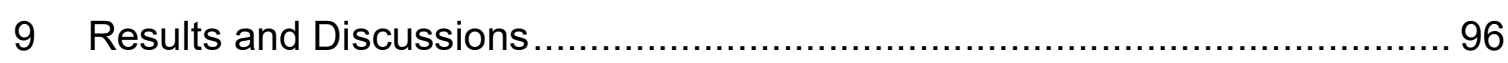

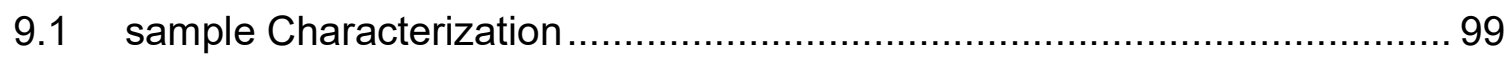

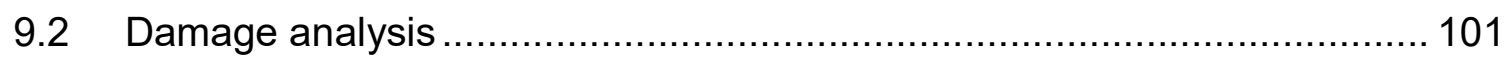

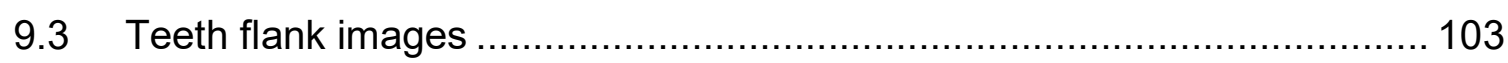

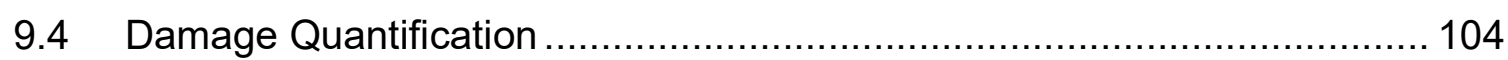

9.5 Pitting Damage area analysis and damage behavior (grow) …............... 105

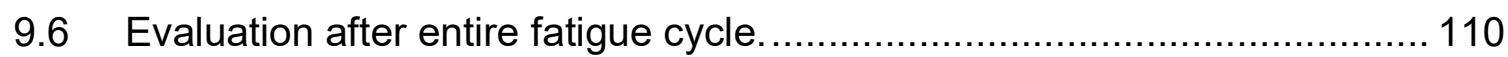

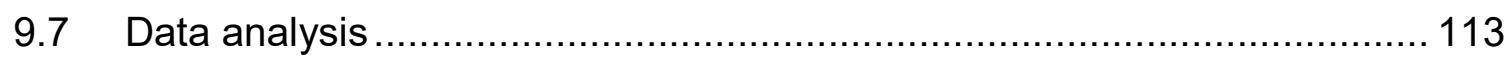

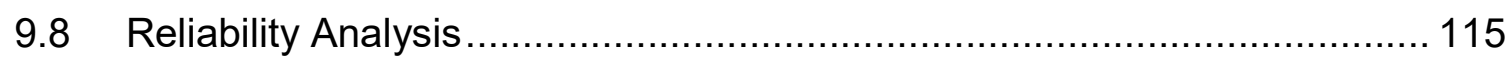

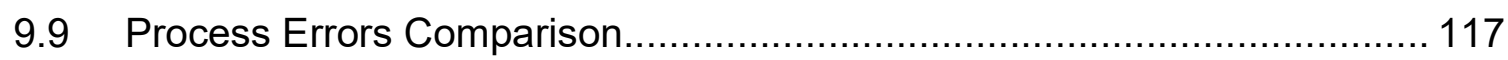

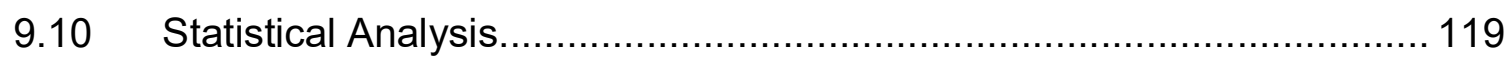

9.11 Adding coated gears w-dlc and dlc results (specific objective vii)......... 125

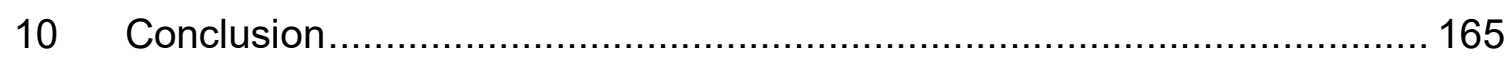




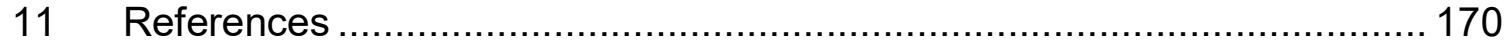

APPENDIX A - Flank surface images and MatLab routine of F01 till F05 ........... 175

APPENDIX B - Flank surface images and MatLab routine of B01 till B05 .......... 190

APPENDIX C - Flank surface images and MatLab routine of D01 till D05 .......... 205

APPENDIX D - Flank profile measurements of F01 till F05 ............................. 220

APPENDIX E - Flank profile measurements of B01 till B05 ............................. 223

APPENDIX F - Flank profile measurements of D01 till D05...........................226

APPENDIX G - Flank Helix measurements of F01 till F05 ….......................... 229

APPENDIX H - Flank Helix measurements of B01 till B05 …............................ 232

APPENDIX I - Flank Helix measurements of D01 till D05 ….............................. 235 


\section{INTRODUCTION}

Reliability is a worldwide research trend due to its inherent objective of understanding failure mechanism and revealing its occurrence possibility. Countless researches such as those presented by He et al. (2013), Karabay et al. (2018), Li et al. (2017) and Nejad et al. (2014) have been developed on this topic on different areas and industrial sectors. Many of those results have already been shared on renowned international journals and magazines or even their concepts had been added widely used by customers.

On specific literature is found that reliability studies had started when industry had identified some failures which were not understood at that time. Those failures were deeply studied and the reliability methodology found its first steps back in the 1950s, with fundamental concepts defined by a mathematician named Robert Lusser (Bradley, 2017). Later on 1960s and 1970s this methodology had been more structured and its application found a wide range of use. In more recent years, reliability has also been focus of several researches due to the potential benefit of conducting evaluations aiming to define or clarify the associated operational risks with the object under evaluation.

Reliability concept and methodology is a strategic approach for industry since it enables process or product engineers to evaluate life prediction of components or systems. Some important systems, which should be the focus of failure analysis and prediction methods, are wind turbines, bucket wheel excavators, machine tools and automotive transmissions. On all those examples, gears are present and play an important role in the systems. Gears are recognized by its relevant purpose of transmitting power on mechanical industry, but naturally, they are suitable to present several failures modes.

Some of those failure modes are object of investigation of AGMA (1995), Moorthy (2013) and Morita (2016) and many other researches which are dedicated to understand the influence of tribological aspects on failure mode development. In the present research, emphasis is dedicated on pitting evaluation. Pitting is an ordinary failure mode in gears; fundamentally, it can be defined as a type of gear tooth surface failure due to contact fatigue. This failure mode has been considered 
an important cause of engineering design failures and it is focus of several researches as those presented by Aslantas et al (2004), Chen et al. (2018) Terrin, et al. (2017); Lanzuttia, et al. (2017); Neptu, et al. (2013); Olsson (2016); Sidiqui (2013) and also is currently focus of studies on gear industry.

As pitting is a fatigue phenomenon, one important physical characteristic for gear failure modes investigation is surface characterization, which, in fact, could be more explored and this is the path this research will be guided on. One important aspect that could be highlighted here is that, even though this failure mode has been considered an important problem, there are few researches exploring manufacturing process errors influence on gear tooth fatigue published on both national and international literature.

So, the research question which is raised up is: Do process errors influence gear failures by pitting?

The main objective of this research is to evaluate gear fatigue failure results based on ISO 6336 methodology, revealing the amount of damaged area after the defined fatigue cycle. The research aims also to explore the manufacturing process uncertainty influence on such results, mainly the influence of tooth profile geometry variation.

The specific objectives of this research are to: (i) Design a gear based on FZG Type C geometry, which correlates dynamic behavior with FCA transmission 1st gear; (ii) Machine such gear and submit it to the same thermochemical treatment used on ordinary gears of automotive gearbox available in the market. The purpose of using the same process is to preserve mechanical and metallurgical characteristics such as hardness profile and microstructure aspects, (iii) Define tests parameters and cycles based on FZG tests. (iv) Perform tests, (v) Analyze tooth flank damage of each tested gear using tooth flank damaged images processed by software, (vi) Perform data analysis and results discussion. (vii) Repeat steps (iv), (v) and (vi) for DLC - Diamond Like Carbon Coated gear and W-DLC - Tungsten Diamond Like Carbon Coated gear to evaluate the influence of coating on fatigue damage. 
This research is based on the aim of evaluating a standard method of gear engineering design, based on experimental analysis to deeply understand pitting occurrence as much as to propose alternatives solution to avoid or delay its occurrence.

This study represents a rare and extensive observation of the entire engineering development seen on specific literature regarding gear design.

On the perspective of evaluating the influence of manufacturing process errors on final fatigue damage area by pitting a meticulous, accurate and appropriate evaluation procedure is required.

For this research, several evaluation steps were carefully selected to obtain trustworthy and representative experimental results to evaluate manufacturing process errors effects on gear micro-geometry. Though, to reach the micro-geometry evaluation level, several variables needed to be carefully evaluated and controlled to support an accurate output. For that reason, the following resources were used, considering: (i) the software used for virtual analysis (IsoCad), which is the same used for gear design and gear fatigue evaluation, which is based on ISO 6336 methodology; (ii) gear production tools, which were designed and produced by auto transmission industry supplier. (iii) Selection of fatigue bench test, which was redesigned and renewed, aiming at reducing vibration trough axles and housing stiffness redesign and improving temperature controls, preparing it to support the designed experiments. (iv) Selection of metrology equipment, which are key elements for this research, The machines used were a dedicated 3D gear measuring equipment to evaluate production process, recently purchased by FCA to replace an older equipment, and Roughness evaluation equipment.

This work is also relevant and important due to the aim of setting a guide of gear experimental evaluation, from design until fatigue data and Reliability analysis, which is also very rare on specific literature.

This thesis will be divided into the following chapters:

- Literature Review: it will be presented in seven items comprehending: (i) Gear Design, split in: Gear History and Concept, Gear Types and Design Concepts, Tooth Elements Proportion, Contact Ratio, Gears Profile Correction, Involute Profiles, 
Sliding of Involutes Profile. (ii) Gear Failure modes, split in: Wear, Scuffing, Contact Fatigue, Bending Fatigue, Plastic deformation. (iii) Associated Variables with Gear Failure: Gear Production Process, Surface Finishing, Gear Quality According to Process, Topography, Process Influence on Roughness and Gears Failure Analysis, Lubricants and Fundamental Properties, Material Characteristics and Properties, (iv) Gear Design and Failure Analysis: Surface Durability Calculus, (v) Gear Coating: Layers with Low and Moderate Hardness, Hard Material Layers DLC and W-DLC Coating Concepts and Published Results. (vi) Theoretical Failure Analysis: Reliability Concept, Continuous Random Variable, Characteristics of a Probability Function, Normal and Related Distribution, Weibull Distribution.

- Materials and Methods: In chapter 8 each one of the five steps Methodology will be detailed discussed. Those Steps are: Gear Design, Gear Manufacturing Process, Gear Bench Test, Damage Analysis and Experimental Data Analysis.

- Results and Discussion: In this chapter 9, results and discussions in alignment with methodology are presented.

- Conclusions: In chapter 10 conclusions are presented.

- On Appendix much of the data used to support this research are presented. 


\section{GEAR GEOMETRY ASPECTS}

\subsection{GEARS HISTORY AND CONCEPT}

It had been registered by Asian communities, since 2600 before Cristian era, the use of rudimentary components to transmit power and motion from one wheel to another. Those wheels perimeter were basically assembled with cylindrical woods arranged to drive the next wheel. Figure 1 illustrates this rudimentary system (Juvinall, 2008).

Figure 1: Rudimentary gears

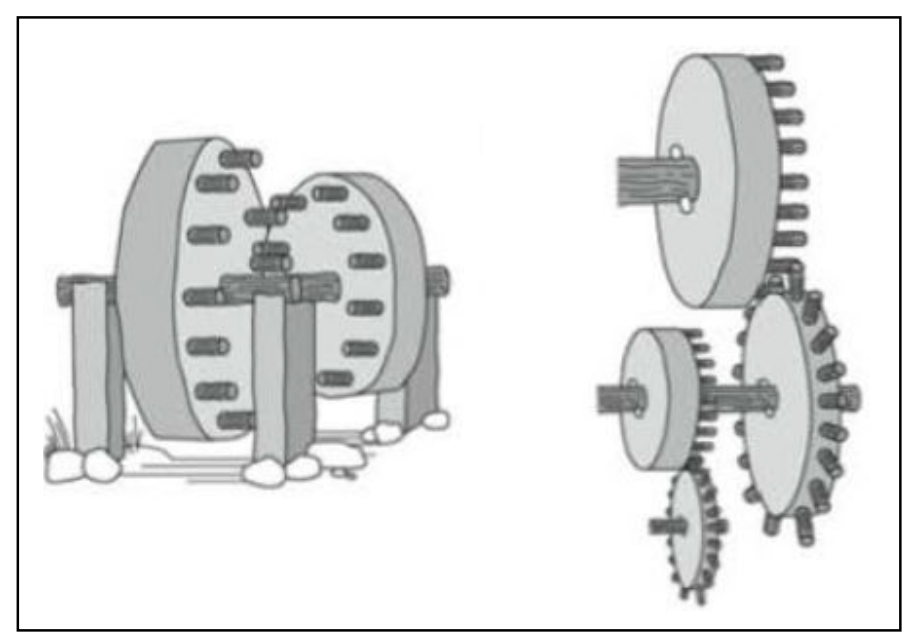

Source: Juvinall, 2008

Still before Christ, Archimedes also knew basic gears design concepts and used then in some of his discovers such as war machines. Later on, Leonardo da Vinci also knew gears concepts and applied them on many of his projects such as Fighting Vehicle and Arch Bridge (Maitra, 2001).

In contrast with early gears developments, on more recent age, much has been researched to remain its original concept of transferring power and movement, but also to add smooth and silence on teeth contact and movement. One theory that support this approach is the contact theory sustained by the involute curve. Figure 2 illustrates a branch of an involute curve. 
Figure 2: A branch of an involute curve

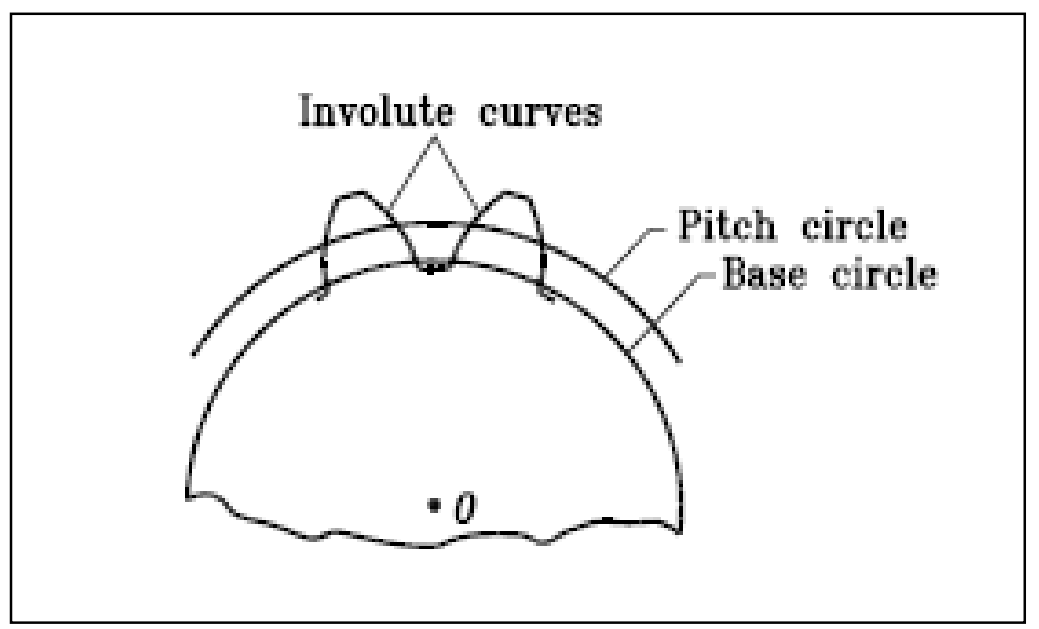

Source: Litvin et al, 2004

Involutes were originally suggested by Leonard Euler, but latter enhanced by prof. Roberts Wills as a practical shape curve as known today. The basic principle of this theory is that contact points in gears are given by involutes in touch. Those involutes are designed to sustain a relative velocity between the two surfaces in contact during their movement. In other words, the shape of the curve is designed to promote constant angular velocity ratio during the teeth contact of two rotating gears (Maitra, 2001).

Gears are not the only known technique of transmitting power and movement smoothly. It is also possible to execute this transmission with different mechanical architectures such as: i) cylinders or wheels connected by belt or chains: those architectures have an advantage of vibration absorbing capacities, but on the other hand, they usually require longer center distances. ii) cylinders or wheels in direct contact: these architectures are based on friction to provide movement on driven cylinder, the torque or power transmission limit of this application is reached when the torque in transfer overcomes the friction forces interaction, then slippage will occur. The slippage will impact the initial transmission accuracy and angular velocity will no longer be constant between both elements on contact. In fact, to deal with this slippage the teeth wheel or gear has the capability to allow slippage on meshing and preserve angular velocity constant on involute curves in contact. In the next items sliding and rolling phenomenon will be deeply discussed. 
1.1 Gears types and design concepts

According to AGMA 933-B033 (2003) gear general classification comprehends four types: (a) those transmitting power and motion between parallel shafts; (b) those for shafts with intersecting axes; (c) those where shafts rolls with cylinder and (d) those where shafts rolls with cone. Table 01 shows more details of this classification and also exhibits examples for each gear class.

Table 1: Gears general classification (AGMA 933-B03 Basic Gear Geometry, 2003)

\begin{tabular}{|l|l|l|l|}
\hline \multicolumn{1}{|c|}{ Pitch } & Relation of axes & Direction of teeth & \multicolumn{1}{c|}{ Name of gear } \\
\hline Cylinders & Parallel & $\begin{array}{l}\text { Parallel to axis } \\
\text { Oblique to axis }\end{array}$ & $\begin{array}{l}\text { Spur } \\
\text { Helical or herringbone }\end{array}$ \\
\hline Cones & Intersecting & $\begin{array}{l}\text { Intersecting axis } \\
\text { Offset from axis }\end{array}$ & $\begin{array}{l}\text { Straight-tooth bevel } \\
\text { Spiral bevel }\end{array}$ \\
\hline Plane & Rolling with cylinder & $\begin{array}{l}\text { Parallel to axis } \\
\text { Oblique to axis }\end{array}$ & $\begin{array}{l}\text { Spur rack } \\
\text { Helical rack }\end{array}$ \\
\hline Plane & Rolling with cone & $\begin{array}{l}\text { Intersecting axis } \\
\text { Offset from axis }\end{array}$ & $\begin{array}{l}\text { Straight-tooth ring gear } \\
\text { Spiral ring bevel }\end{array}$ \\
\hline
\end{tabular}

Several concepts are important to understand basic gear design, according to AGMA 933-B03 (2003). Those concepts are: (i) gear center: defined as a point of intersection of the gear axis in a plane of rotation; (ii) center line: connects two gear centers in a plane of rotation, figure 03 shows this line; (iii) The center distance is the distance between two gears centers; (iv) gear ratio: it's a relation of number of gear teeth of two different gears in contact; (v) The pitch point: located on line of centers, it splits the center distance according to gear ratio (valid for parallel axis), figure 03a also shows it; (vi) The pitch circles: Is defined by the circle which contains all pitch points of two meshing gears, those circles are tangent of each other and represents contact points where pure rolling is seen (valid for parallel axis), figure $03 \mathrm{~b}$ demonstrate it. 
Figure 3a: Pitch point and gear ratio relations

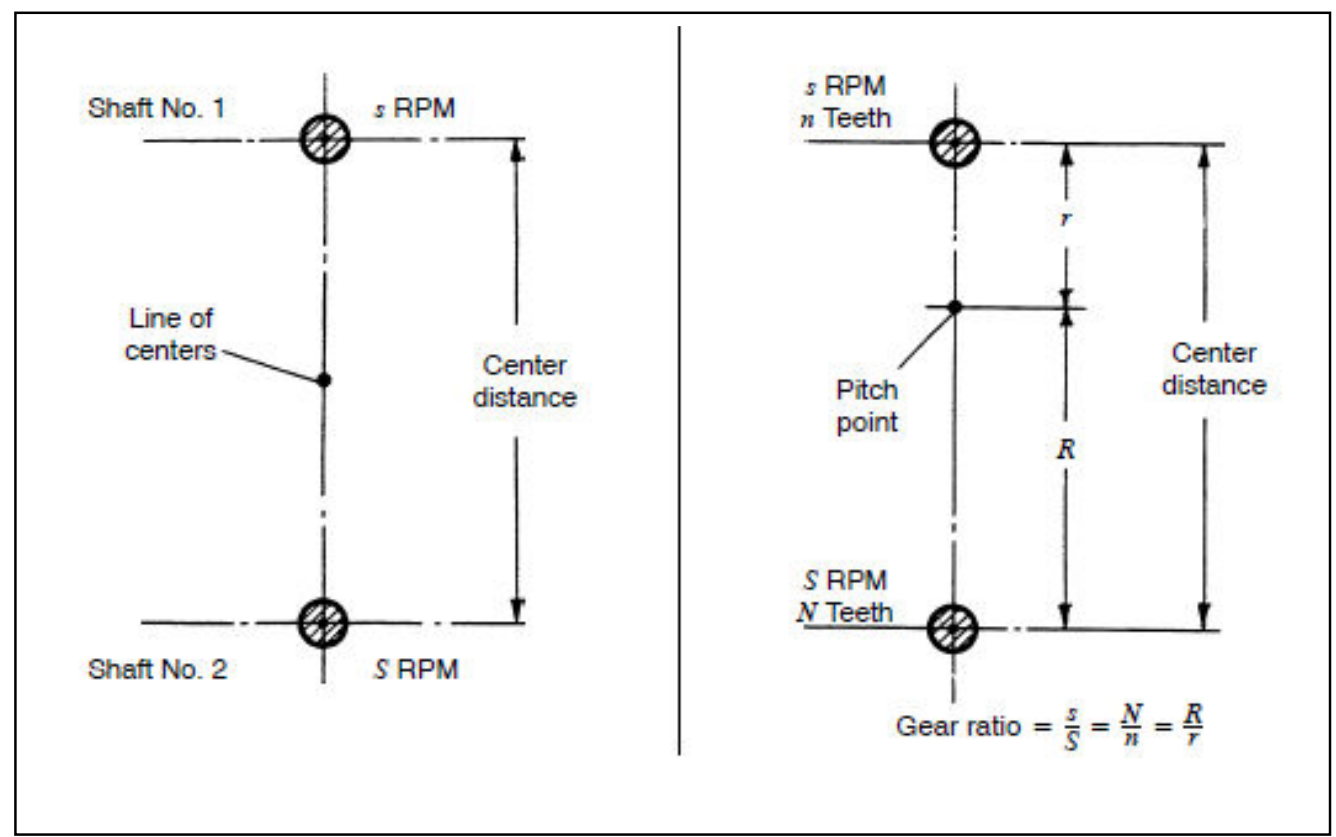

Source: AGMA, 2003

Figure 3b: Fundamental gear terms and relations

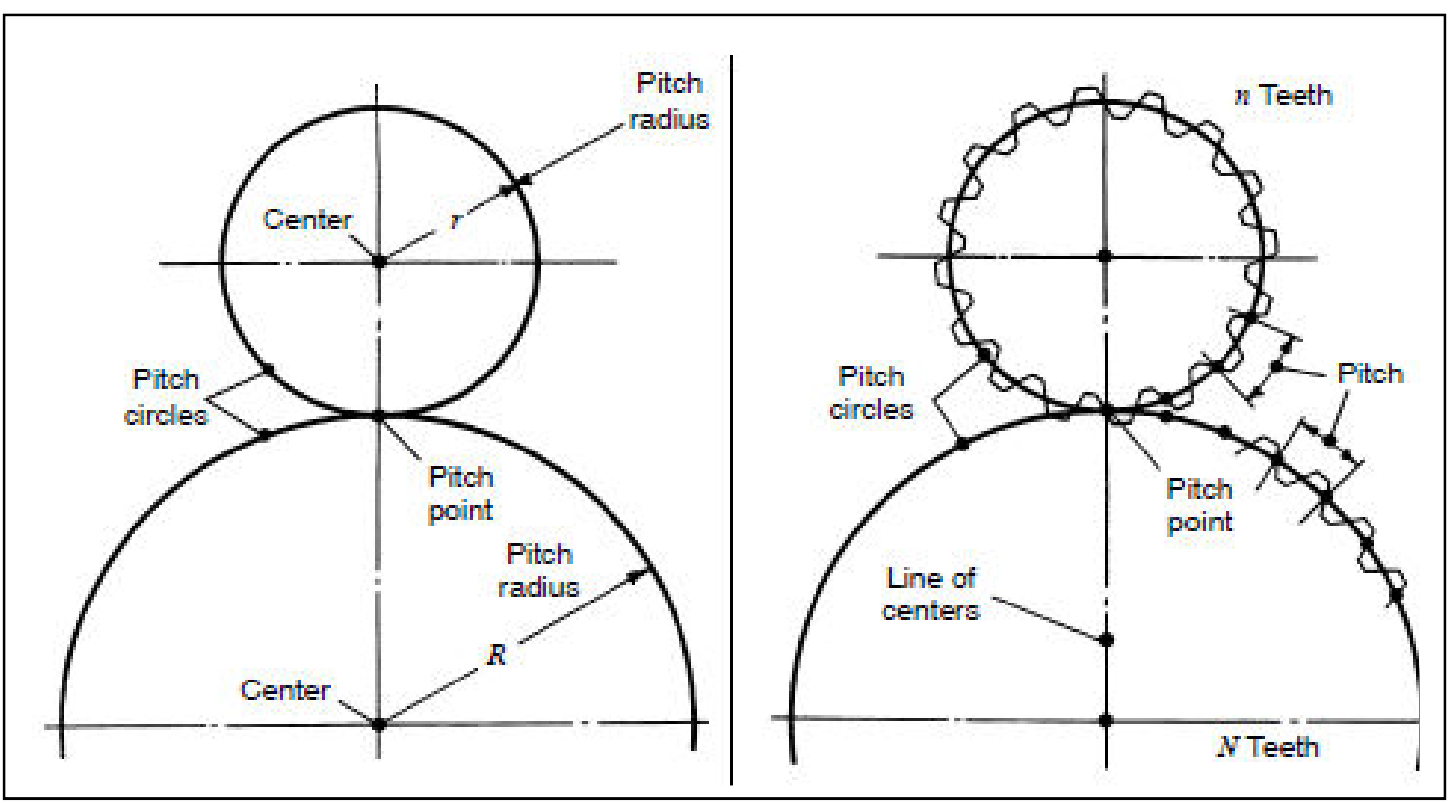

Source: AGMA, 2003 
(vii) The pitch line: This is the line in the rack-cutter that rolls with the gear pitch circle. This line location is composed of two directions: It passes through the pitch points and it is also perpendicular to the line of centers, as shown in figure 4.

Figure 4: Gear basic elements

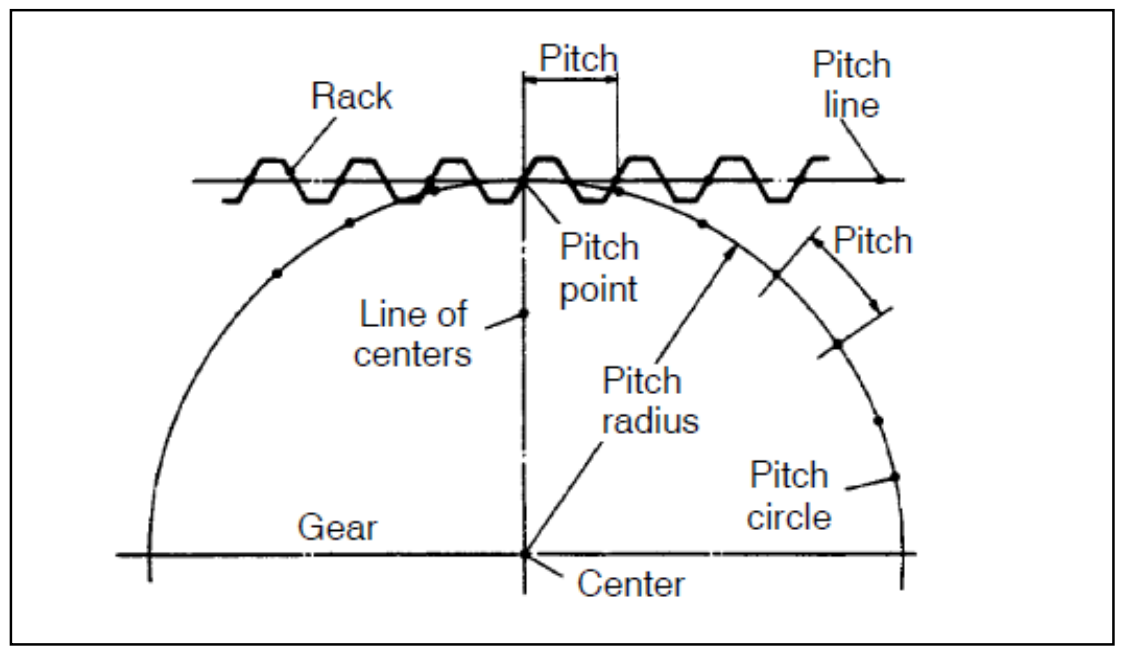

Source: AGMA, 2003

Figure 5: Gear basic elements

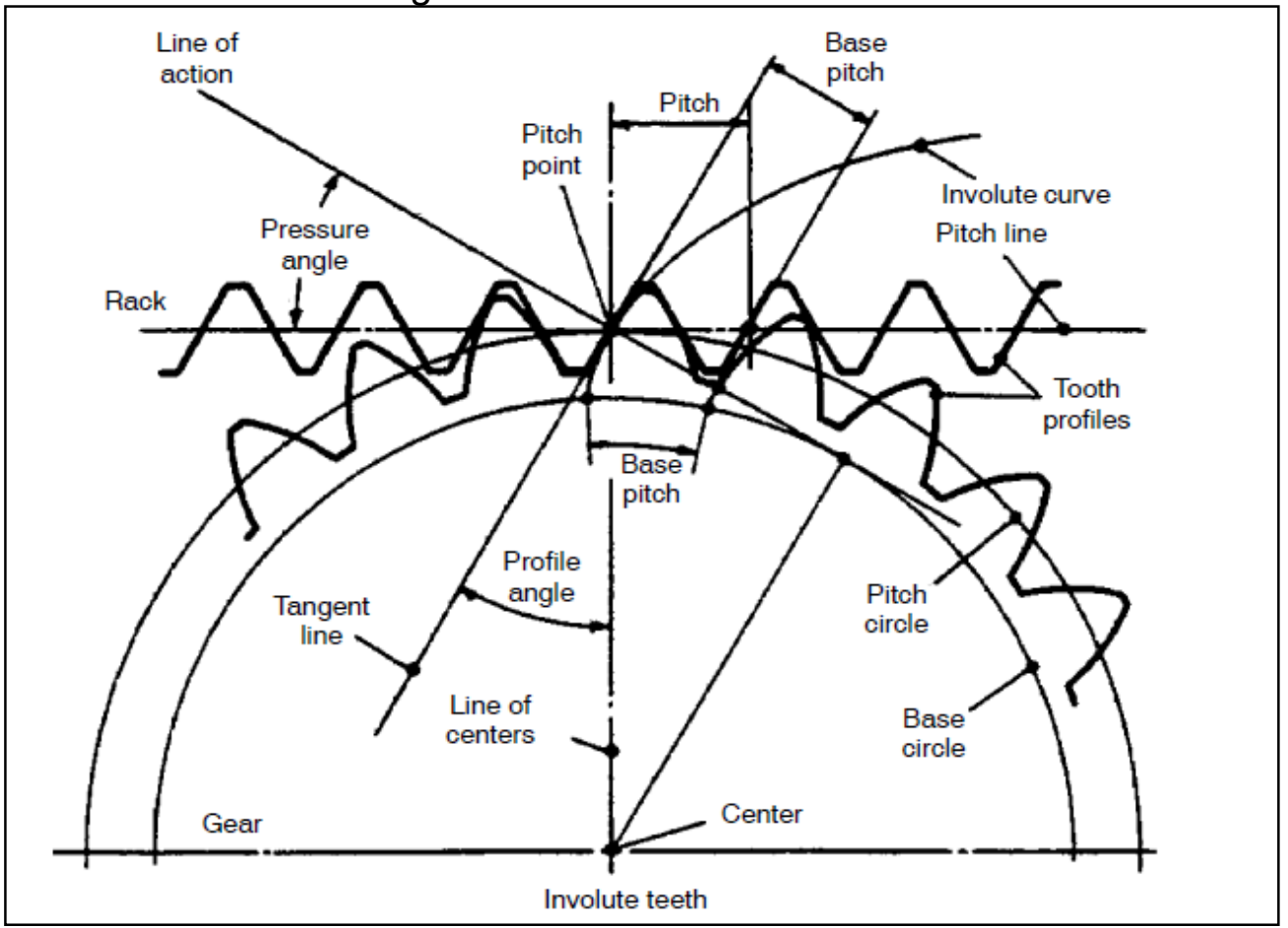

Source: AGMA, 2003 
(viii) Line of action: This is the line along tooth pressure acts and it is also the path of the point in contact. This line passes through the pitch point and is normal to tooth profile (see figure 5); (ix) The pressure angle: This angle specifies the pressure or force direction between meshing teeth, it's the angle between the pitch line and line of action (see figure 5); (x) The profile angle: This angle gives the direction of the tangent to a tooth profile, based on pitch point. This angle will be defined by tooth profile and line of centers (see figure 5); (xi) The base circle: It's tangent to line of action and it's also the circle were the involutes (or involute tooth) profile are based (see figure 5); (xii) The base pitch: In an involute framework, equivalent teeth sides are basically parallel lines regularly spaced each other. The pitch line is the perpendicular distance between such lines measured in the base circle.

\subsection{TOOTH ELEMENTS PROPORTIONS:}

The Pitch circle is the orientation for gear tooth parameters and also for elements proportions calculations (see figure 06). Pitch circle is calculated by equation 01 and represents the number of teeth per inch of gear diameter:

$$
\mathrm{P}=\frac{\mathrm{N}}{\mathrm{d}}=\frac{\pi}{P C}
$$

In which $P c$ is another important parameter to design tooth, the circular pitch. It is an arc of the pitch circle, the circular pitch or base pitch is the distance between two neighboring teeth. The circumference of pitch circle is defined on equation 02.

$$
\mathrm{L}=P c \mathrm{~N}=\pi \mathrm{d}
$$

Where $\mathrm{N}$ is the number of gear teeth. The module $\mathrm{m}$ is represented by equation 03 , Figure 06 illustrates those parameters. The module unit is in millimeters, while the unit for $P$ is $(1 /$ in. $)$. 


$$
\mathrm{m}=\frac{P c}{\pi}=\frac{\mathrm{d}}{\mathrm{N}}
$$

The addendum (a) is the radial distance between the addendum circle and the pitch circle (equation 04). The dedendum (b) is the radial distance between the pitch circle and the dedendum circle (equation 05). The clearance is important to allow top teeth movement on the meshing and also to drain the lubricant with eventual wear debris. The clearance is calculated according to equation 06 . The tooth thickness $(t)$ and the space width $(w)$ are arcs measured along the pitch circle (defined according to equation 07).

$$
\begin{gathered}
\mathrm{a}=\mathrm{m} \\
\mathrm{b}=1,25 \mathrm{~m} \\
\mathrm{c}=\mathrm{b}-\mathrm{a} \\
\mathrm{t}=\mathrm{w}=\frac{P c}{2}
\end{gathered}
$$

Figure 6: Gear tooth parameter

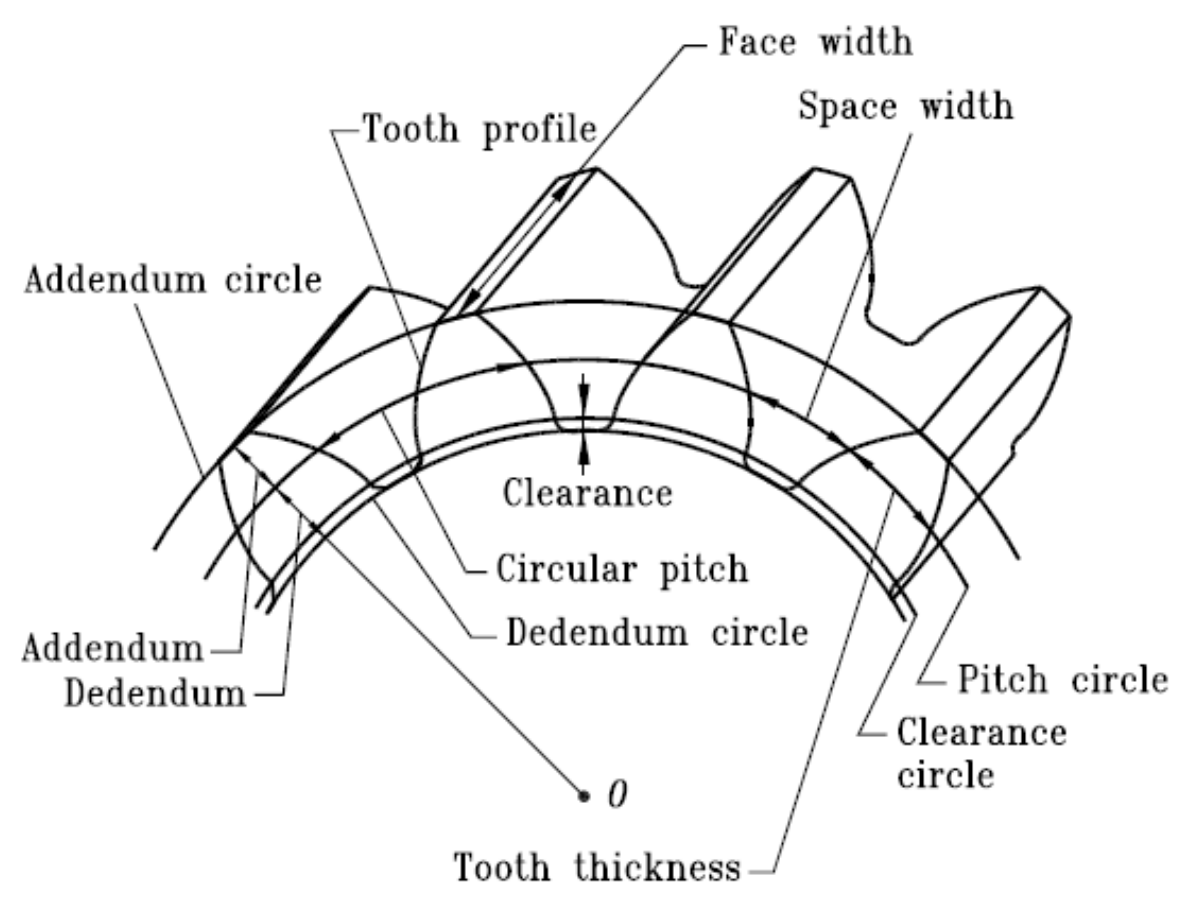

Source: Litvin et al., 2004 


\subsection{CONTACT RATIO}

The contact ratio physically means a measure of the average number of teeth in contact while tooth comes and goes out of contact with the mating gear. A contact ratio equal 1 means that a pair of tooth is in contact during the course of action. $A$ contact ratio equal 1,5 means that while one tooth from mating gears is in contact, a second tooth from the mating gears is also in mesh for $50 \%$ of the meshing period (Maitra, 2001). In other words, a contact ratio of 1.5 means that $50 \%$ of the time two teeth in mating gears are in contact. Another important aspects of contact ratio concept is related to (i) force and load distribution during power transmission and also (ii) angular pitch. Figure 7 illustrates angular Pitch $\Theta_{\mathrm{n}}$ and equation 8 defines its calculation (Litvin et al, 2004).

Figure 7 : Angular Pitch

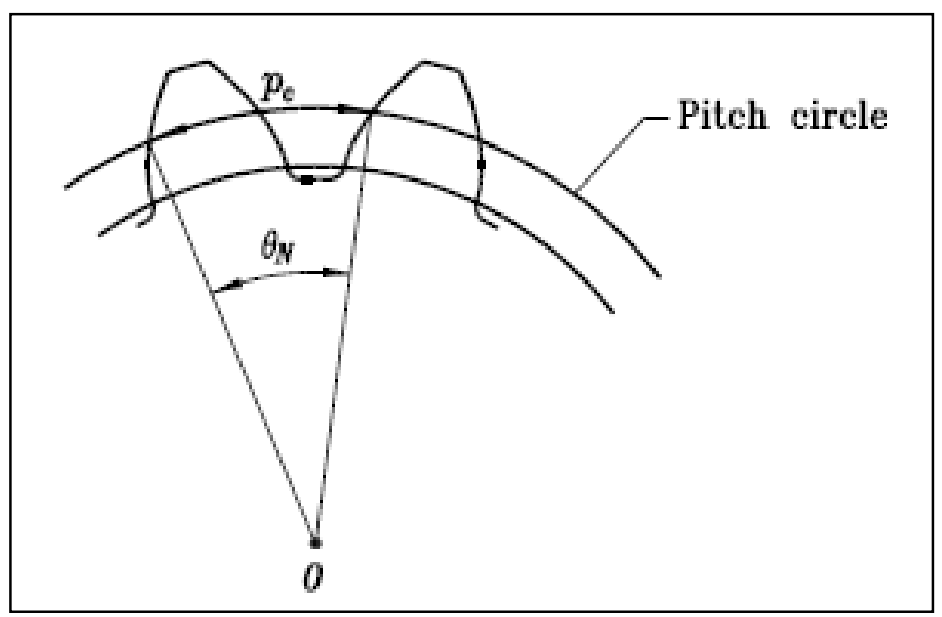

Source: Litvin et al, 2004

$$
\Theta_{n}=\frac{P_{c}}{r p_{i}}=\frac{2 \pi}{\mathrm{N}_{\mathrm{i}}}
$$

In figure 08 points $\mathrm{B} 1$ and $\mathrm{B} 2$ indicate the points of contact at the line of action in the beginning and at the end of meshing for the shown tooth profiles $\beta-\beta$ and $\gamma-$ Y. Point B1 is the intersection of the line of action and the gear addendum circle and Point B2 is the intersection of the line of action and the pinion addendum circle. There is also a third point in the line of action, Point $M$, which is the current point of tangency of the tooth profiles. Between point B1 to B2 during this profile meshing, 
consequently there's an angle $\mathrm{B}_{1} \mathrm{O}_{1} \mathrm{~B}_{2}$, which allows de calculation of contact ratio as shown on equation 9:

$$
m c=\frac{\widehat{\bar{B}_{1} B_{2}}}{\theta_{n}}
$$

Another approach of calculating the $\mathrm{mc}$ is using equation 10 , where $\mathrm{I}$ is the length where the parts have meshed in line of action. It's the contact movement lengthways in the line of action for the duration of the cycle of meshing; $\mathrm{Pb}$ is the space measured along their common normal in between neighboring tooth profiles:

$$
m c=\frac{l}{P_{b}}
$$

Figure 8: Meshing of involute gears

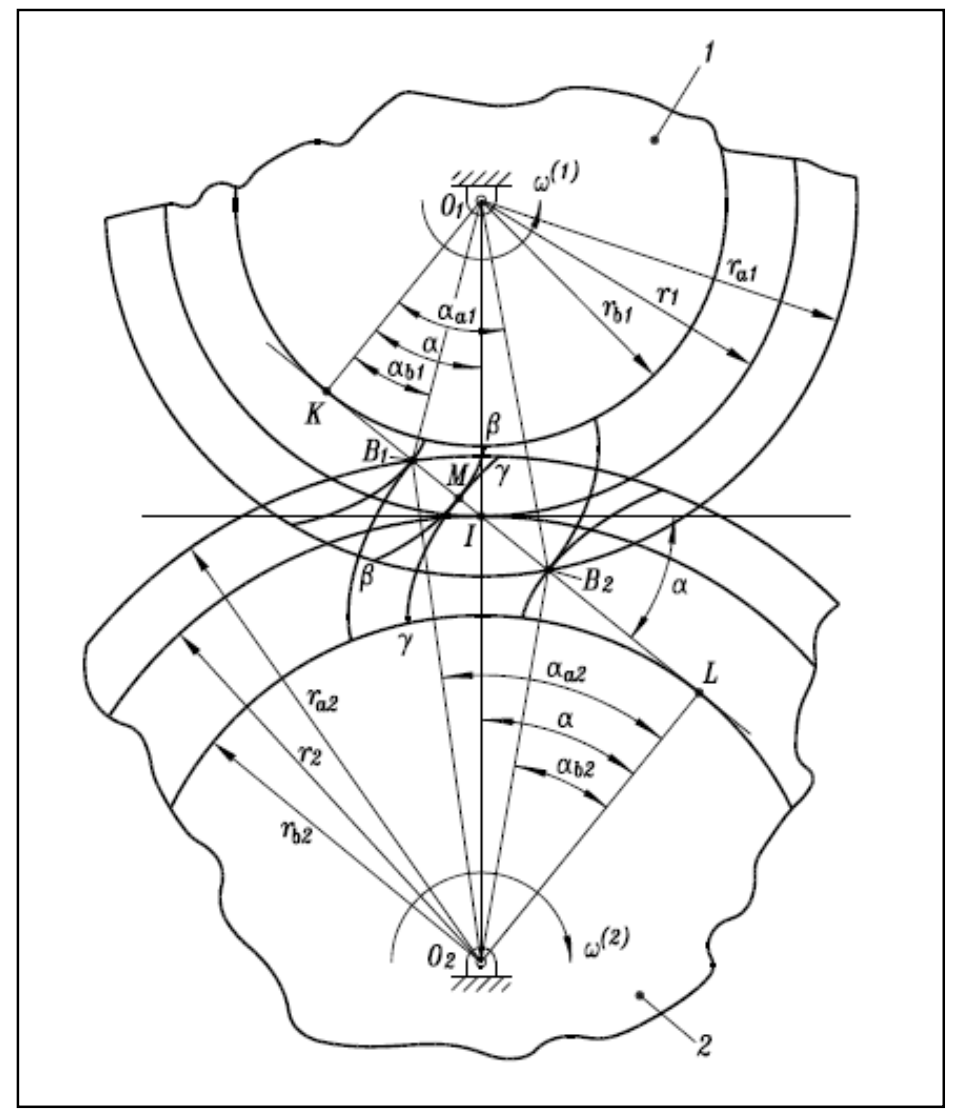

Source: Litvin et al, 2004 


\subsection{GEARS PROFILE CORRECTION}

Profile correction is increasing its popularity in modern gear design in most of the power transmission system. Initially, the main goal of this correction was to avoid undercutting or to define a center distance. Those objectives are still valid, but it uses is not limited to them. Nowadays, pinions and gears are generated with positive correction due to the following benefits: (i) Avoid undercutting; (ii) pre-define a center distance; (iii) The thickness of tooth at the root is increased, resulting in greater load carrying capacity of the teeth; (iv) Improvement of sliding and contact relations; (v) To alter the beginning of the effective profile away from the base circle. In the tooth bottom, the specific contact pressure tends to be increased due to the geometry and its fast modification. The tooth bottom is also the region were highest sliding velocity and compressive stress occurs (Maitra, 2001).

The profile modification can be either positive or negative, according to the project and its objective. In a positive correction the addendum is increased by an extent of $x m$, consequently the dedendum is decreased by the same extent. In this modification, the root-fillet becomes smaller and has an unfavorable effect on stress concentration. In figure 09 it's possible to visualize that the positive correction influence is equivalent of shifting the teeth radially out of the pitch circle. Keeping number of teeth and the modulus, this action reduces the space between tooth and increase the tooth thickness at the pitch circle.

For negative correction the addendum is decreased by an extent of $x m$, consequently the dedendum is increased by the same extent. This modification, weakens the teeth and reduce the tooth strength. However, this unfavorable effect is annulled in case of gears with a greater number of teeth (Maitra, 2001). 
Figure 9: Effect of profile correction

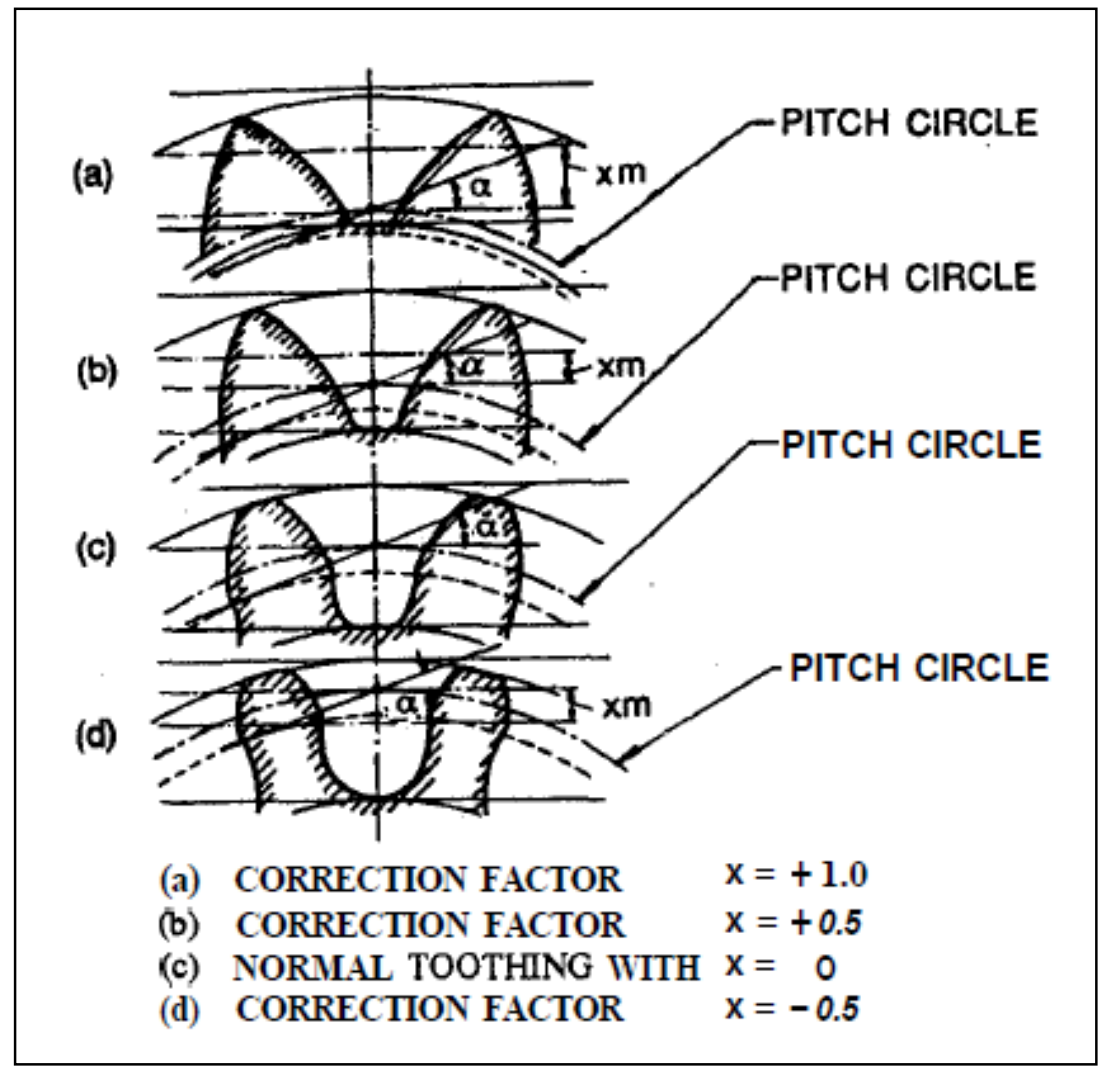

Source: Maitra, 2001

\subsection{INVOLUTE PROFILES:}

In a rack-cuter tooth profile is a parallel straight line as seen on figure 5, that's why Litvin (2004) states that one benefit of involute gearing is that the teeth profiles are equidistant curves as seen on figure 10. Still on those curves it is possible to measure the normal to the profiles between them. This distance is seen on figure 11 as $P_{b}$ and its equal to $P_{n}$ of the rack cutter, the expression 11 defines it as:

$$
P_{b}=P_{n}=P_{c} \cos \alpha_{c}
$$

But, $\mathrm{Pb}$ is also calculate as (seen on expression 12), where $\widehat{\mathrm{MN}}$ is measured along the base circle and it physically means the distance in between two involute curves. 


$$
P_{b}=\widehat{M N}
$$

Figure 10: Involute profiles as equidistant curves

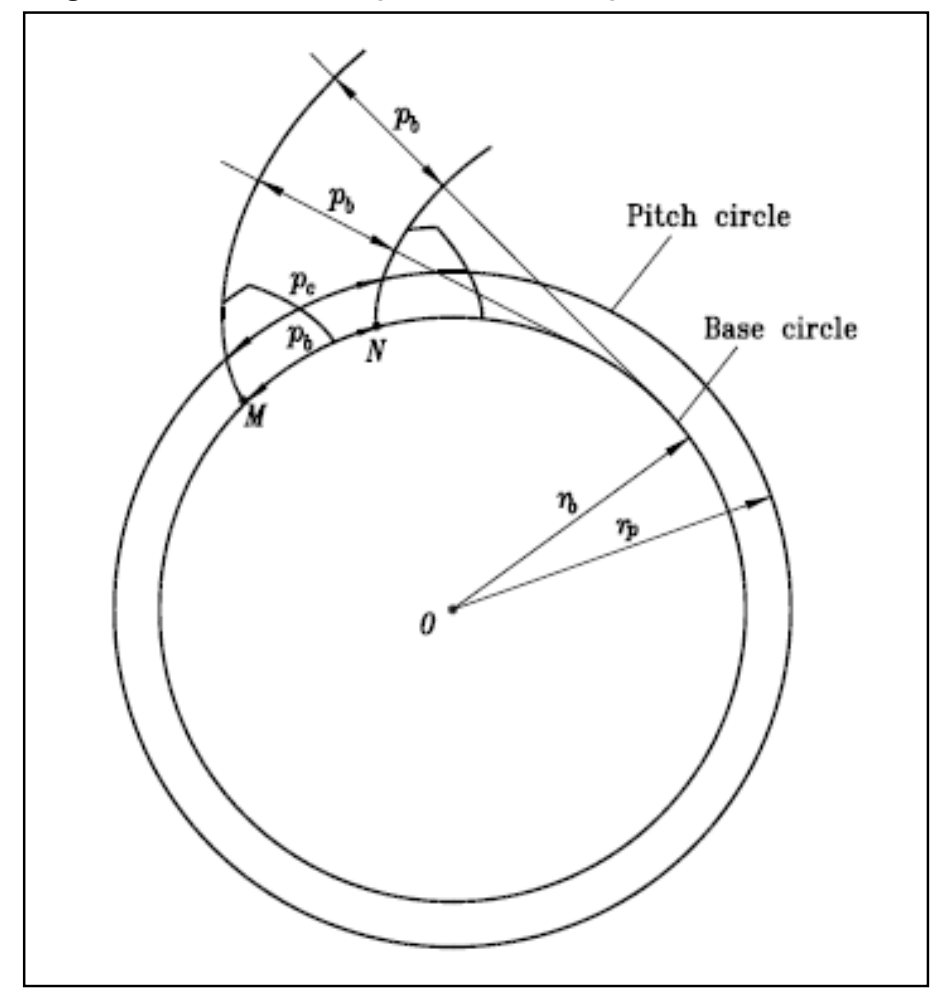

Source : Litvin et al, 2004

\subsection{SLIDING OF INVOLUTE PROFILES}

In figure 11 is seen the line of action KL. It's also possible to see that the tangent of two involutes profiles in mesh belongs to the line of action KL. Still on figure 11, the point I seen on line of action, also represents a tangent of two involutes while in meshing. Nevertheless, this point I is the specific point where pure rolling is seen (the instantaneous center of rotation). The sliding velocity at point $I$ is equal to zero. Despite of this point I, any other relative tooth profile motion which occurs alongside the line of action will show rolling and sliding. In this way, considering teeth profiles tangent at point $M$ on line of action, means that $M_{1}$ of profile $\beta \beta$ coincides 
with $M_{2}$ of profile $Y y$, than the velocity of point $M_{1}$ and $M_{2}$ is shown on equation 13 (Litvin et al, 2004).

$$
V^{(12)}=V^{(1 M)}-V^{(2 M)}=\left(\omega^{1} x \overline{O M}_{1}\right)-\left(\omega^{2} x \overline{O M}_{2}\right)
$$

Figure 11: Sliding velocity explanation

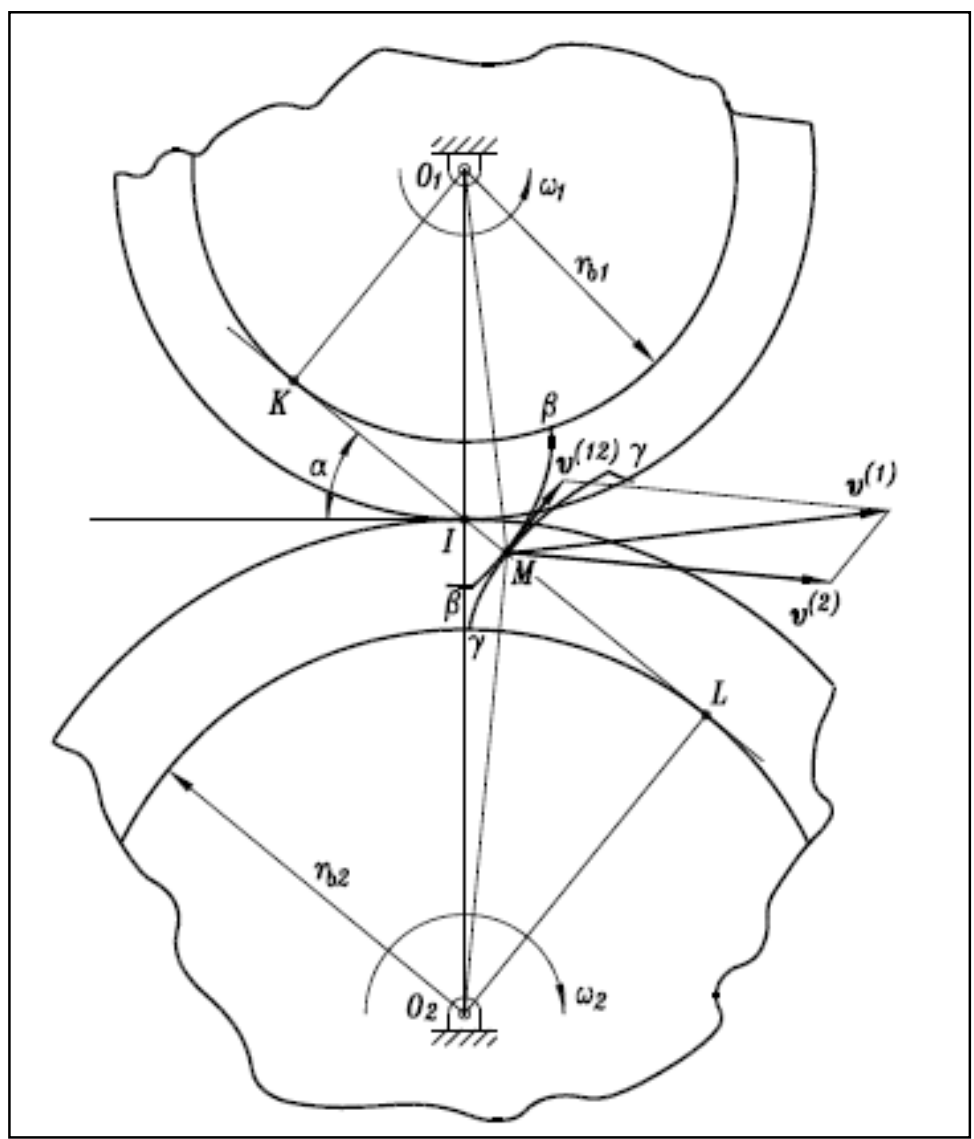

Source : Litvin et al, 2004 


\section{GEARS FAILURE MODES AND ASSOCIATE VARIABLES}

According to NBR 5462 (ABNT, 1994) failure is defined when a component has lost the capacity of performing its function. This standard also states a failure criteria concept as, a set of rules applicable on the judgment of failures types and severities for defining the acceptable degradation limits until loss of performance. Those concepts have been used for several components and systems reliability studies in Brazil. In special, on transmission components and systems this concept is also important, appropriate and aligned with international studies.

Several specific literatures such as those presented by Smith (2003), Naunheimer et al. (2011), and some standards have classified and defined gears failures and its modes, AGMA 1010-E95C (AGMA, 1995) summarizes then in five general classes as can be seen in first column of table 02 . On the second column, each class has also been subdivided into more detailed failure modes.

Table 2: Nomenclature of gear failure mode (Adapted from AGMA 1010 E95c (1995)

\begin{tabular}{|l|l|}
\hline \multicolumn{1}{|c|}{ Classes } & \multicolumn{1}{c|}{ Detailed failure mode } \\
\hline Wear & Adhesion \\
& Abrasion \\
& Polishing \\
& Fretting Corrosion \\
& Scaling \\
& Cavitation \\
& Erosion \\
& Electrical Discharge \\
\hline Scuffing & Scuffing \\
\hline Contact fatigue & Subsurface (Pitting or macropitting) \\
& Surface (micropitting) \\
\hline Bending fatigue & Subcase fatigue \\
\hline Plastic deformation $\quad$ and & Low cycle fatigue \\
Fracture & Plastic cycle fatigue \\
& Brittle fracture \\
& Ductile fracture \\
& Mixed mode fracture \\
& Tooth shear \\
& Fracture after plastic deformation \\
\hline
\end{tabular}




\subsection{WEAR}

According to Hutchings (1992), Wear occurs when two solid surfaces slides against each other, its consequence is an amount of material removed from sliding bodies. AGMA 1010-E95c (AGMA, 1995) has the same definition, but as seen on table 2 the Wear class is divided in (i) Adhesion, (ii) Abrasion, (iii) Polishing, (iv) Fretting Corrosion, (v) Scaling, (vi) Cavitation, (vii) Erosion and (viii) Electrical Discharge.

Adhesion is known as material transfer from one tooth to the other while meshing. This phenomenon is due to micro welding and subsequently material displacement of one tooth adhered to another. It can be classified as: (i.1) Mild: it refers to local micro imperfection. It's usually there, but difficult to be detected. (i.2) Moderate: Remove the machining marks, and if it's limited to this aspect, can be consider acceptable. (i.3) Severe: It's better classified as scuffing.

Abrasion is a result of hard particles contact on gear tooth. It can be caused by tooth protuberance sliding against other tooth surface or by free hard particles rolling and sliding on tooth surface. On both mechanism, the final result is material removal or displacement. Figure 12 shows a SEM micrograph abrasion and figure 13 shows mild and moderate abrasion.

Figure 12: SEM micrograph showing Abrasion

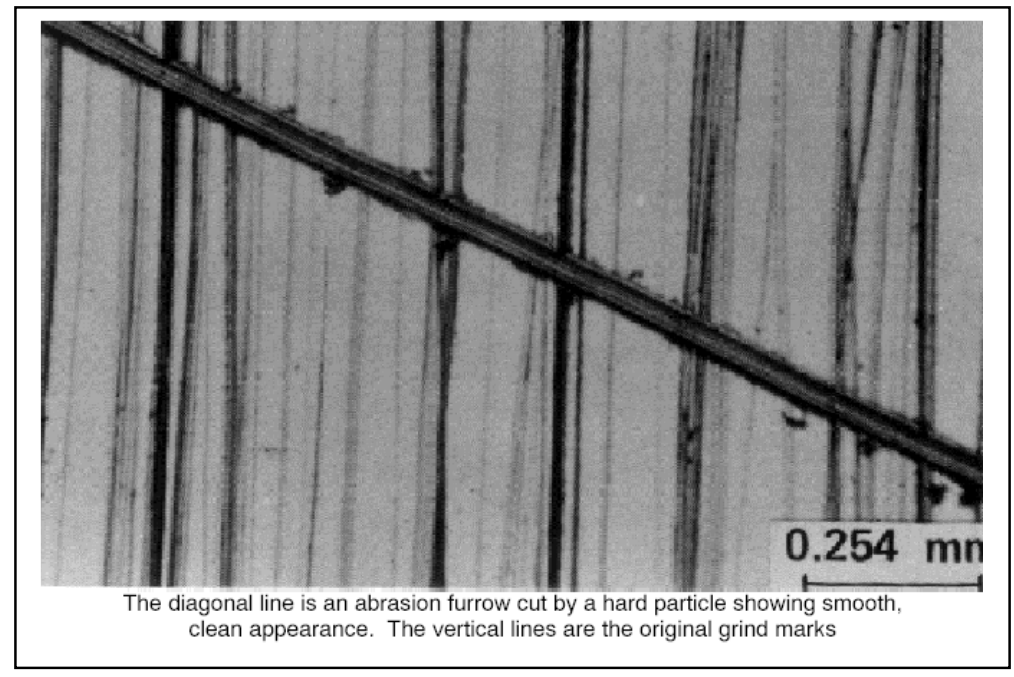

Source: ANSI-AGMA 1010 E95c (AGMA, 1995) 
Figure 13: Severe Abrasion

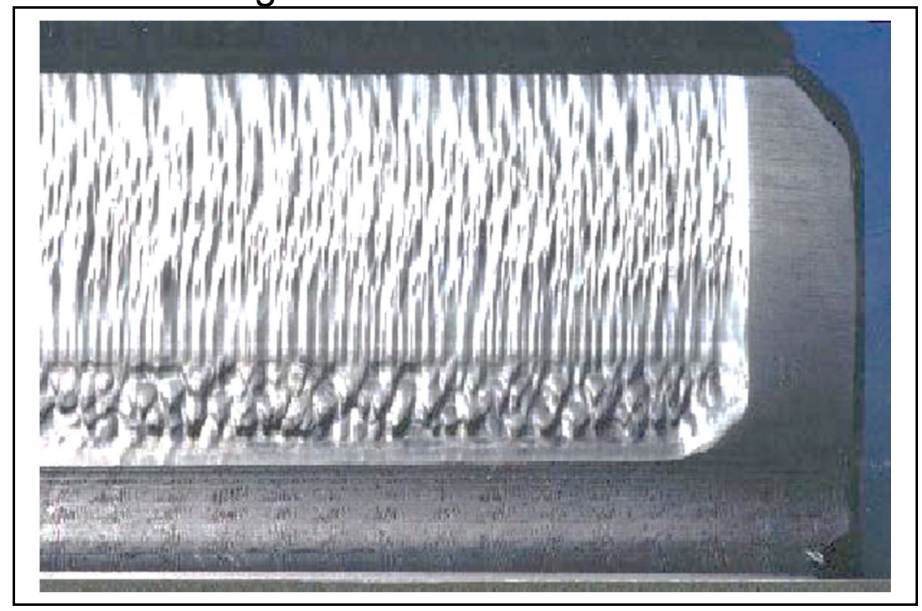

Source: ANSI-AGMA 1010 E95c (AGMA, 1995)

Polishing is a fine scale abrasion that induces a mirror like finish in a smooth or wavy surface. A SEM analysis will show fine scratches oriented in the direction of sliding. Figure 14 shows a Severe Polishing.

Figure 14: Severe Polishing

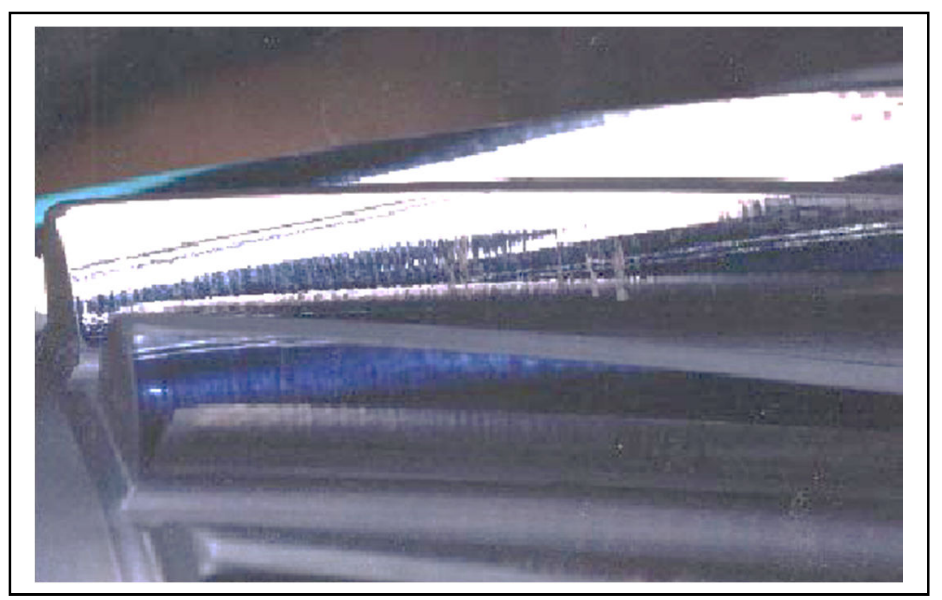

Source: ANSI-AGMA 1010 E95c (AGMA, 1995)

Fretting Corrosion is typical of surfaces under compression and with a very reduced vibration mode. Under these conditions the lubricant is completely expelled from the contact zone between the flank of the teeth, allowing adhesion between the asperities. The relative movement causes the breaking of this adhesion with the consequent generation of particles of iron oxides which are hard and abrasive. The presence of these oxides forms a barrier that prevents the lubricant to reach the 
contact region allowing again the adhesion of asperities and making the phenomenon cyclical. Figure 15 illustrates a fretting corrosion.

Figure 15: Fretting Corrosion

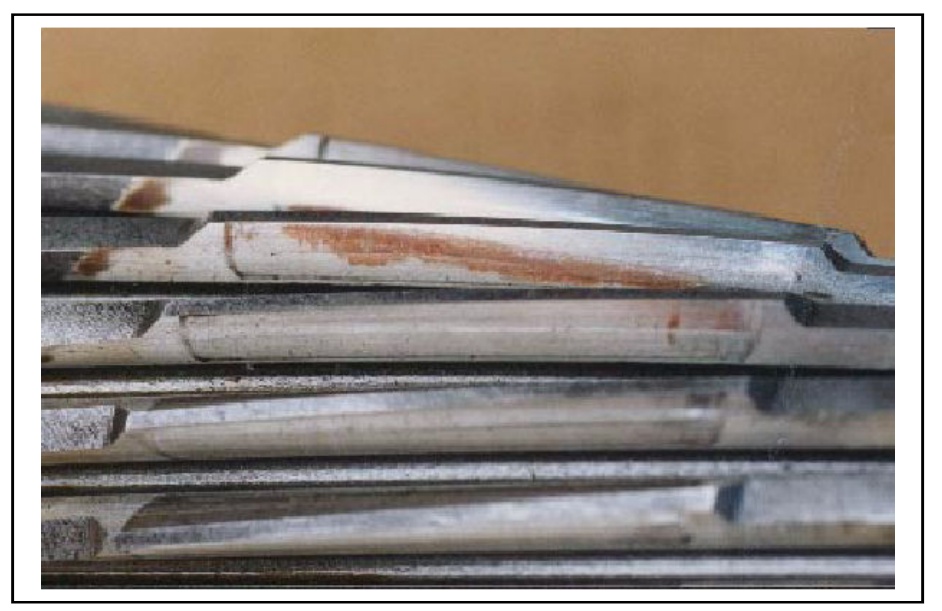

Source: ANSI-AGMA 1010 E95c (AGMA, 1995)

Scaling happens when the heat treatment process induces raised and irregular areas under the flank of the tooth. In operation these areas will undergo pressure and material removal can happen quickly. The surface will have a metallic sheen similar to a scale, hence the origin of the name of the wear. Figure 16 shows scaling.

Figure 16: Scaling

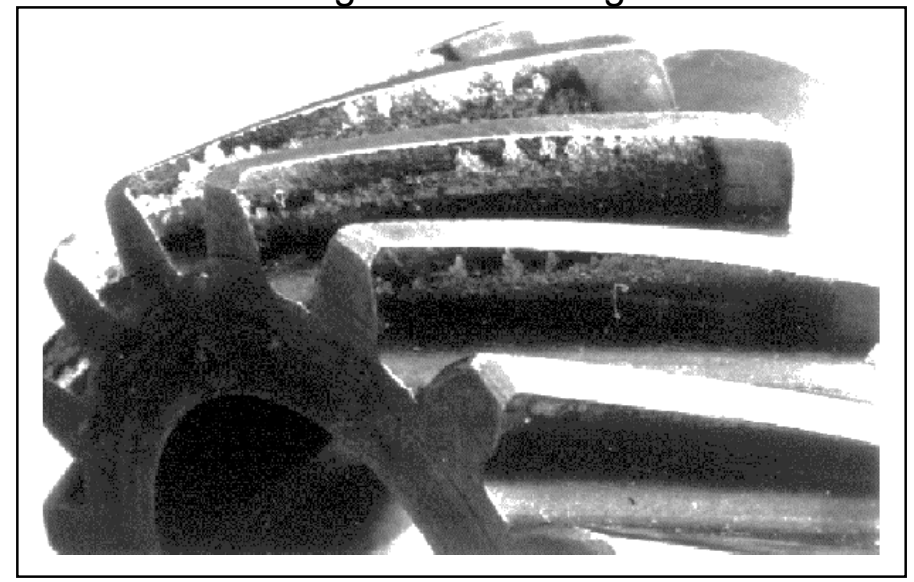

Source: ANSI-AGMA 1010 E95c (AGMA, 1995) 
Cavitation consists of the nucleation and implosion of lubricating fluid bubbles generating wear of the surface flank under an erosion and abrasion process. Figure 17 (a) and (b) show cavitation damage in gears.

Figure 17: (a) Cavitation damage showing sandblasted appearance and (b) cavitation crater showing deep, rough clean and honeycomb appearance.

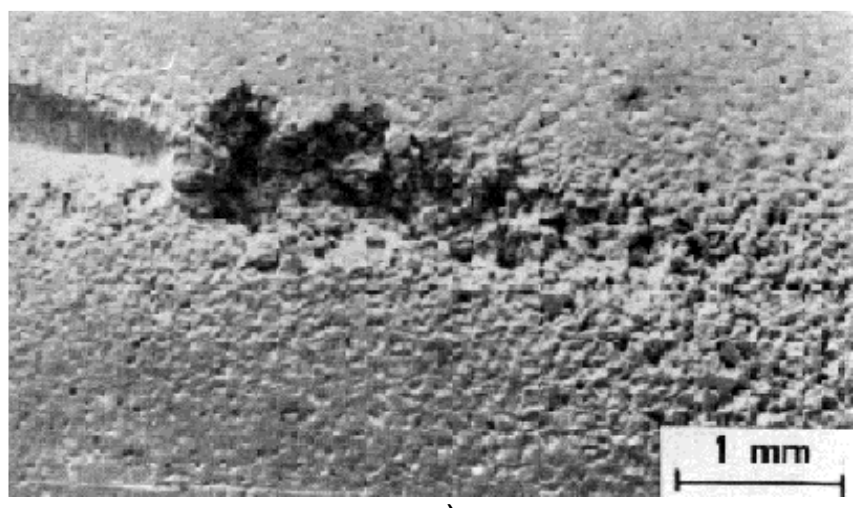

a)

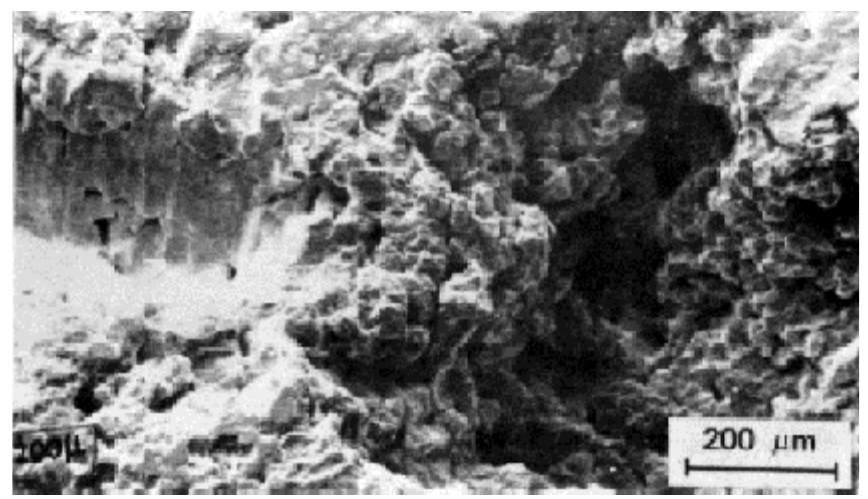

b)

Source: ANSI-AGMA 1010 E95c (AGMA, 1995)

Erosion in gears is caused by lubricating fluid passage at high speed on the tooth flank surface. Erosion is favorable to occur in gears subject to high speeds. Figure 18 shows gears which suffered severe erosion.

Electrical Discharge conducted through meshing gears are capable of traversing the lubricant film and producing temperatures high enough to cause a local melting of the tooth flank material. The damage takes the form of microscopic craters and the re-casting of the material promotes changes in the metallurgical characteristics of 
the material in the contour region of the crater. Figure 19 shows electrical discharge failure on gear.

Figure 18: Severe Erosion

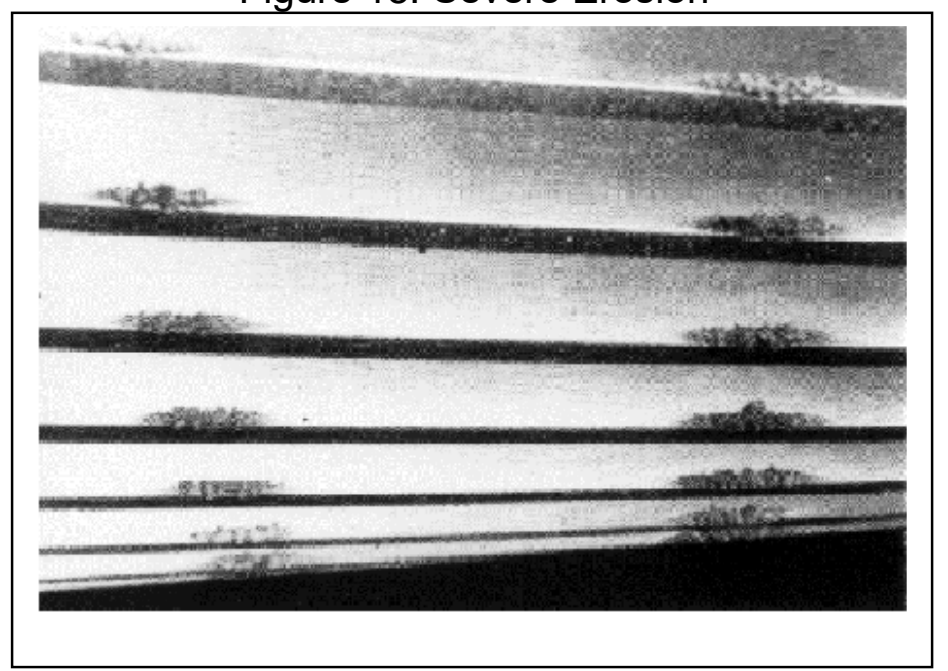

Source: ANSI-AGMA 1010 E95c (AGMA, 1995)

Figure 19: Severe Electrical Discharge

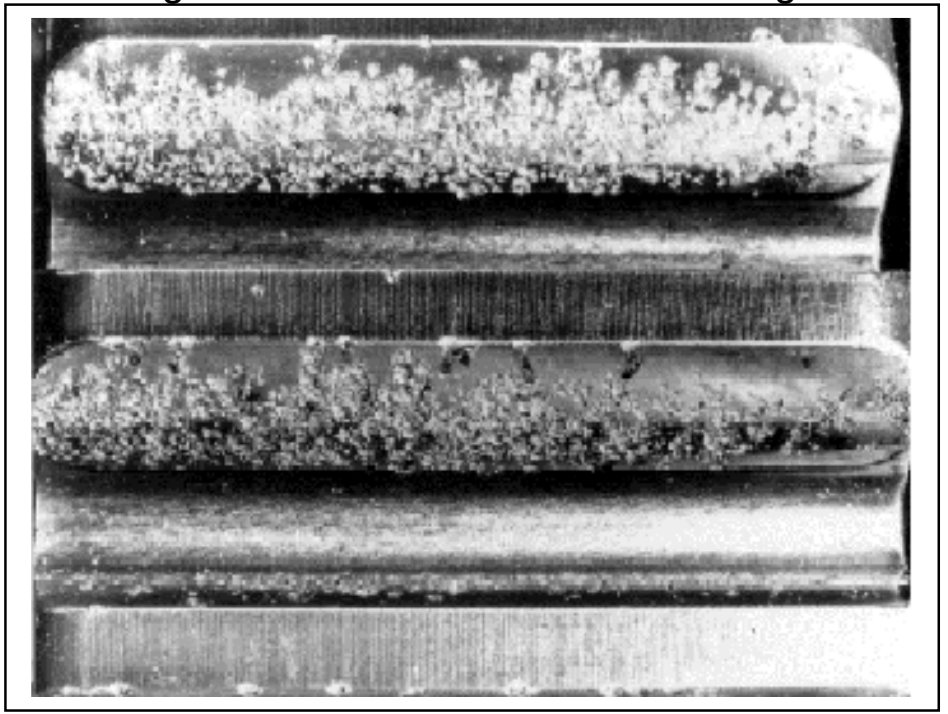

Source: ANSI-AGMA 1010 E95c (AGMA, 1995)

\subsection{SCUFFING}

Scuffing is defined in AGMA 1010-E95 (AGMA, 1995) is a serious type of wear, which can instantly damage the surfaces of teeth that are in relative motion. 
In fact, a single overheat can lead to a catastrophic failure. This type of damage usually happens in areas of high pressure contact and high speed relative slip (high temperature), close to the head and to the tooth root. A very high pressure favors the rupturing of the lubricating film, which is precisely the layer that prevents direct contact between the metals. Right after film breakdown, the surfaces will get in contact and an adhesion due to a micro-welding will be seen, the adhesion will induce material transfer from one tooth flank to another. The phenomenon can continues with the tear off a small portion of material from one of the two surfaces. Figure 20 illustrates a Scuffing damage in gears. Sometimes this failure class is wrongly called scoring. Naunheimer et al. (2011) sustain that a higher viscosity oil, with the capacity of remaining the lubricant film under operational stress application and temperature, can prevent scuffing. These authors also say that E.P (Extreme Pressure) additives can also help in this prevention.
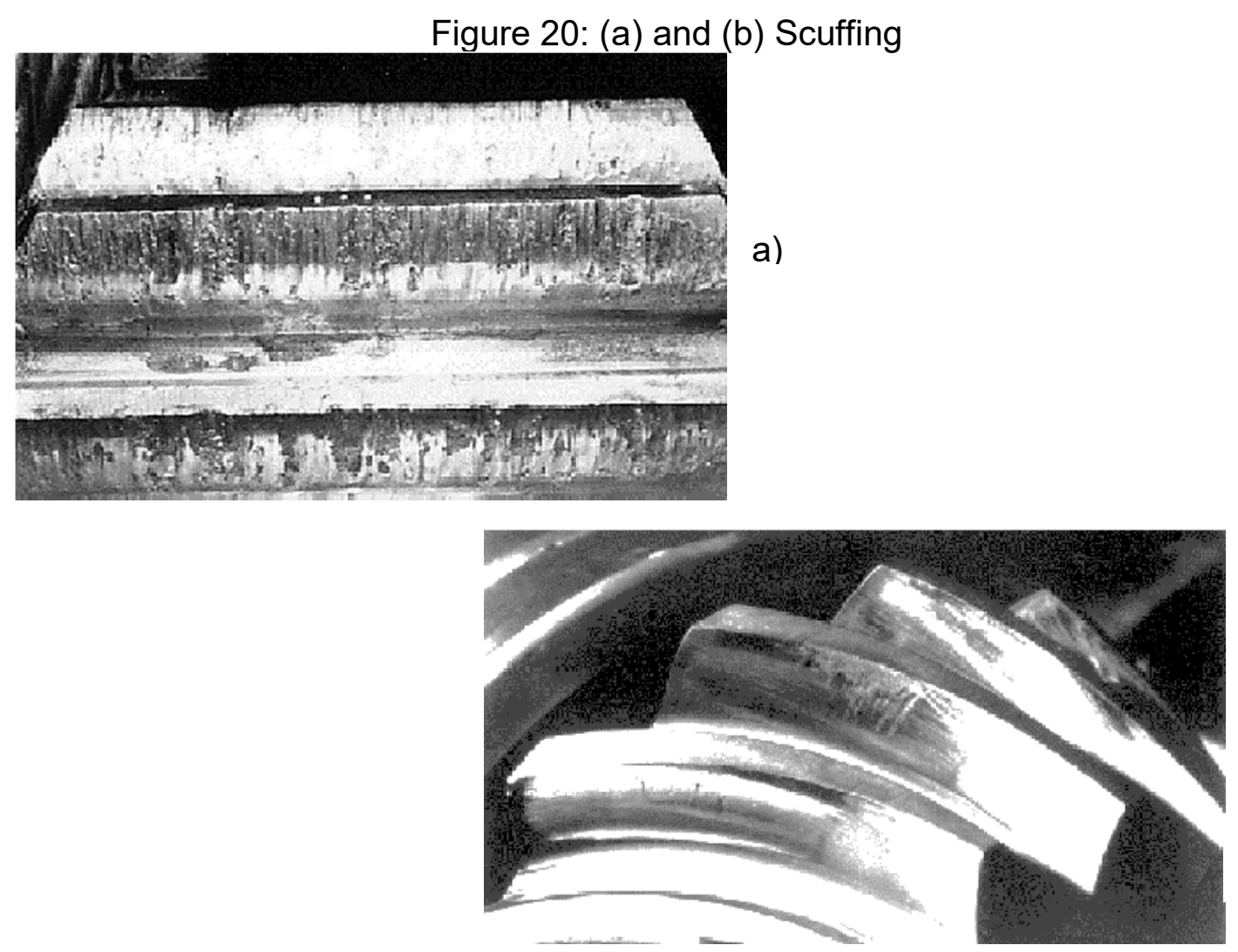

b)

Source: ANSI-AGMA 1010 E95c (AGMA, 1995) 


\subsection{CONTACT FATIGUE}

Pitting is an ordinary failure mode in gears. Fundamentally, it is a type of gear tooth subsurface failure due to fatigue. The repeated contact forces can cause fatigue cracks and the consequent detachment of fragments of material from the surface of the teeth. It looks like craters distributed alongside teeth width. Figure 21 illustrates (a) an initial pitting and (b) a progressive pitting. Its occurrence is directly correlated with adopted design criteria. Standards ISO 6336-2 (ISO, 1996) and 6336-5 (ISO, 1996) show that pitting is highly related to surface hardness, material, design and loading cycles to which gears are submitted.

Figure 21: (a) initial pitting and (b) progressive pitting

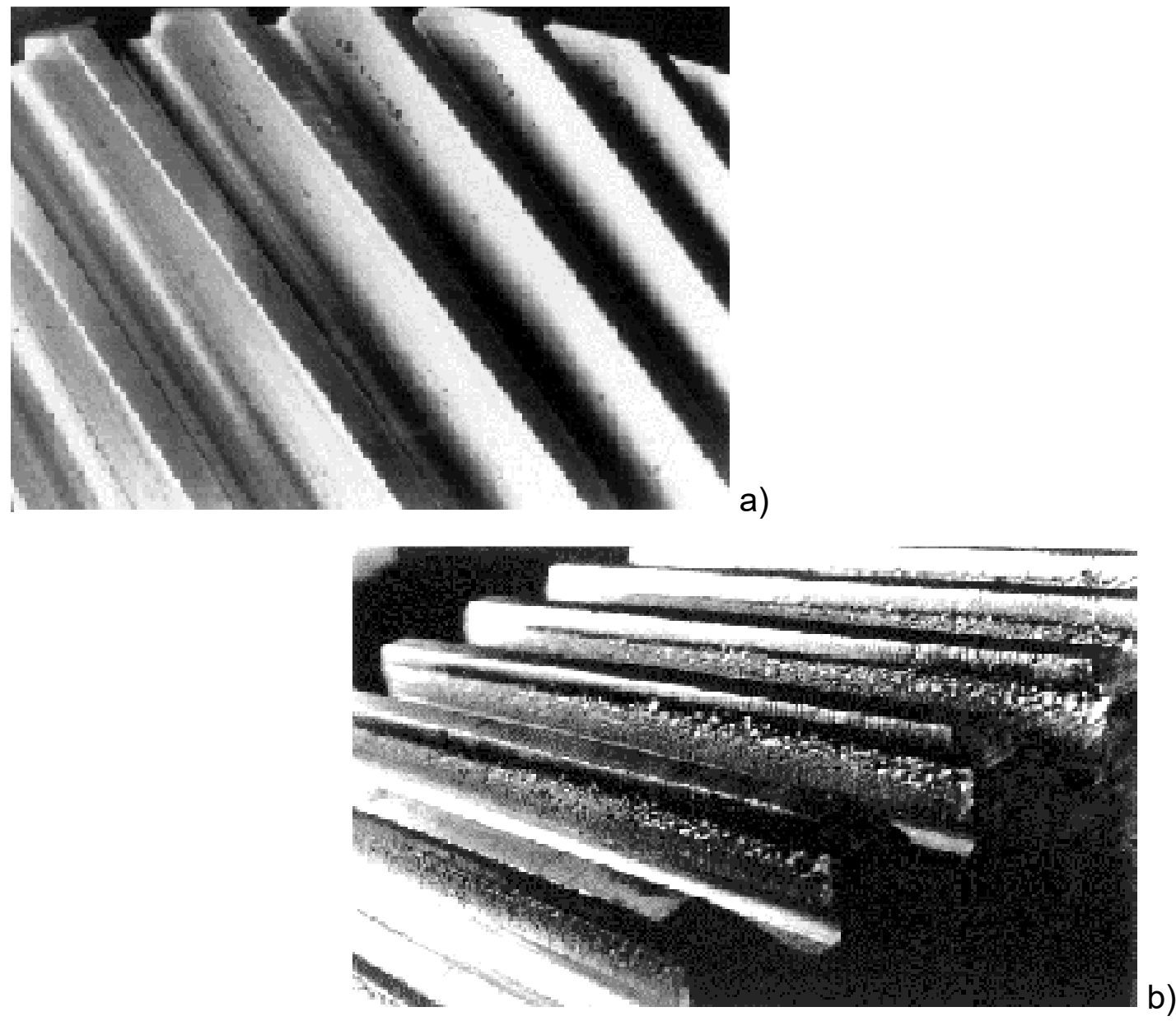

Source: ANSI-AGMA 1010 E95c (AGMA, 1995) 
Pitting ${ }^{1}$ occurrence has been perceived mainly on pitch line surroundings. This fact can be sustained by the analysis that pitch line supports maximum dynamic loads due to reduced contact area. Figure 22 shows a schematic view of tooth engagement in spur gear drive. As can be seen, on pitch line, generally a single tooth contact is observed. Another important point to consider when analyzing pitting occurrence on pitch line surroundings is the sliding velocity direction changing and the frictional forces reaction as seen on figure 23

Figure 22: Nature of tooth engagement in spur gear drive

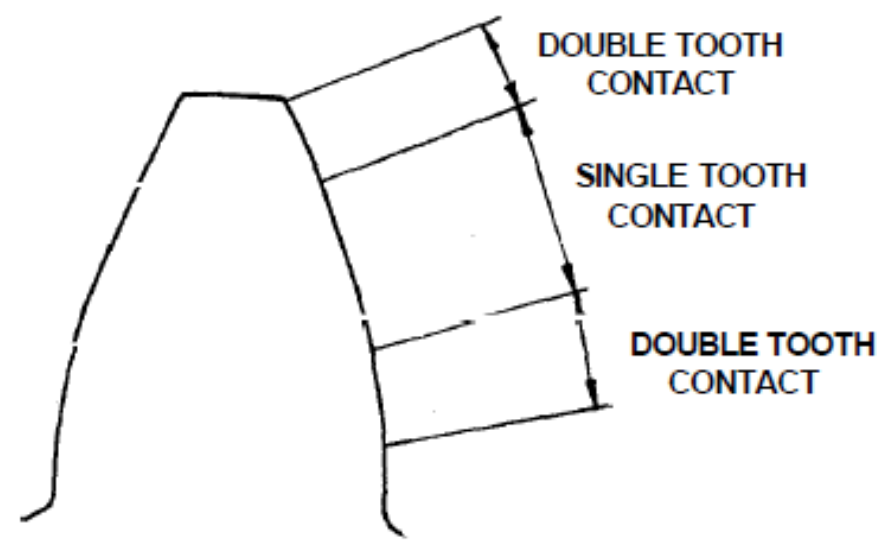

Source: Maitra, 2001.

\footnotetext{
1 Ding and Rieger (2003) define spalling as a contact fatigue failure effect. The authors also defined the associated mechanism as a subsurface crack nucleated and propagate on material, consequently a material detachment is seen. This definition is similar to pitting definition used in this thesis.
} 
Figure 23: Gear meshing mechanism emphasizing Rolling (R) and Sliding (S) directions

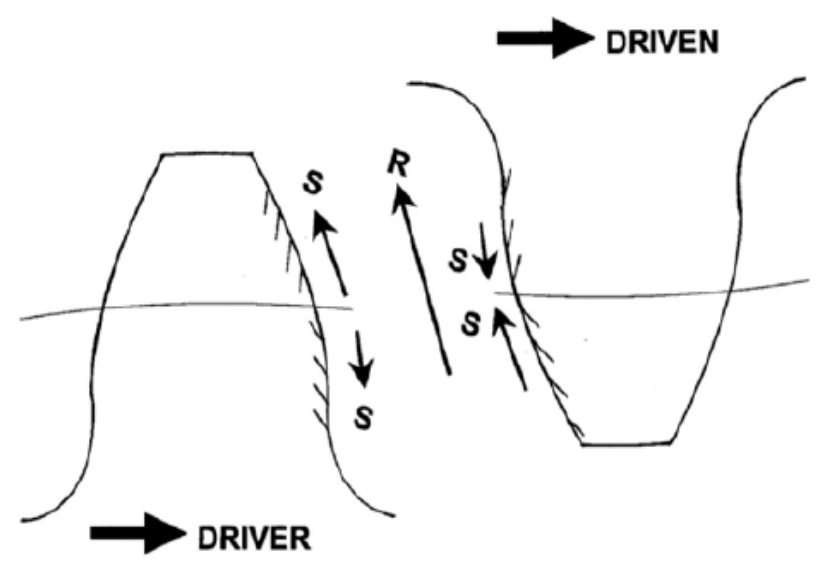

Source: Errichello, 2012.

When meshing gears have the same material, the pitting is expected to occur first on the pinion teeth, because it performs a higher number of revolutions, consequently, it performs a higher number of relative teeth contacts per gear. (GLODEZ et al, 1997).

Glodez et al (1997) and (1999) suggest that pitting is initiated at machining marks on material surface or at inclusions under surface in the area of maximum contact stress. Nevertheless Maitra (2001) emphasizes that pitting is basically a contact fatigue failure mode. Several variables have influence on this failure mode such as contact pressure, sliding velocity, lubricant viscosity and other factors like frictional forces have also effect on tooth surface pits phenomenon. However, a more complete model which take in account all those parameters above mentioned are less usual and despite of the significant effort done on gear design research, contact stress calculations are mainly based on hertzian equation studied by a German physicist, Heinrich Hertz.

Heinrich Hertz investigated the contact between two curved surfaces. He established an expression of the surface stress generated on this contact, which is knew as Hertz stress or hertzian stress. The Hertz equations, hence, are used to 
calculate the contact stresses which prevail in case of tooth surfaces of two mating spur gears. Although, it has been considered an approximation, the gears contact under loading can be taken as a realistic approximation of two cylinders with the same radius of curvature on contact point. Figure 24 illustrate this analysis, where it can be seen on (a) two cylinders in contact under compression and (b) gear teeth in matching at the pitch point, the area of contact under load is a narrow rectangle of width $B$ and length $L$. The stress distribution form is elliptical through the width. The maximum stress value is given by equation 14 :

$$
P_{c(\max )}=\frac{4 F}{\pi B L}
$$

Where $B$ is calculate by equation 15 ,

$$
B=\sqrt{\frac{8 F}{\pi L} X \frac{\left(1-v_{1}^{2}\right)}{E_{1}}+\frac{\left(1-v_{2}^{2}\right)}{E_{2}} /\left[\left(\frac{1}{D_{1}}\right)+\left(\frac{1}{D_{2}}\right)\right]}
$$

Figure 24: Contact Stress

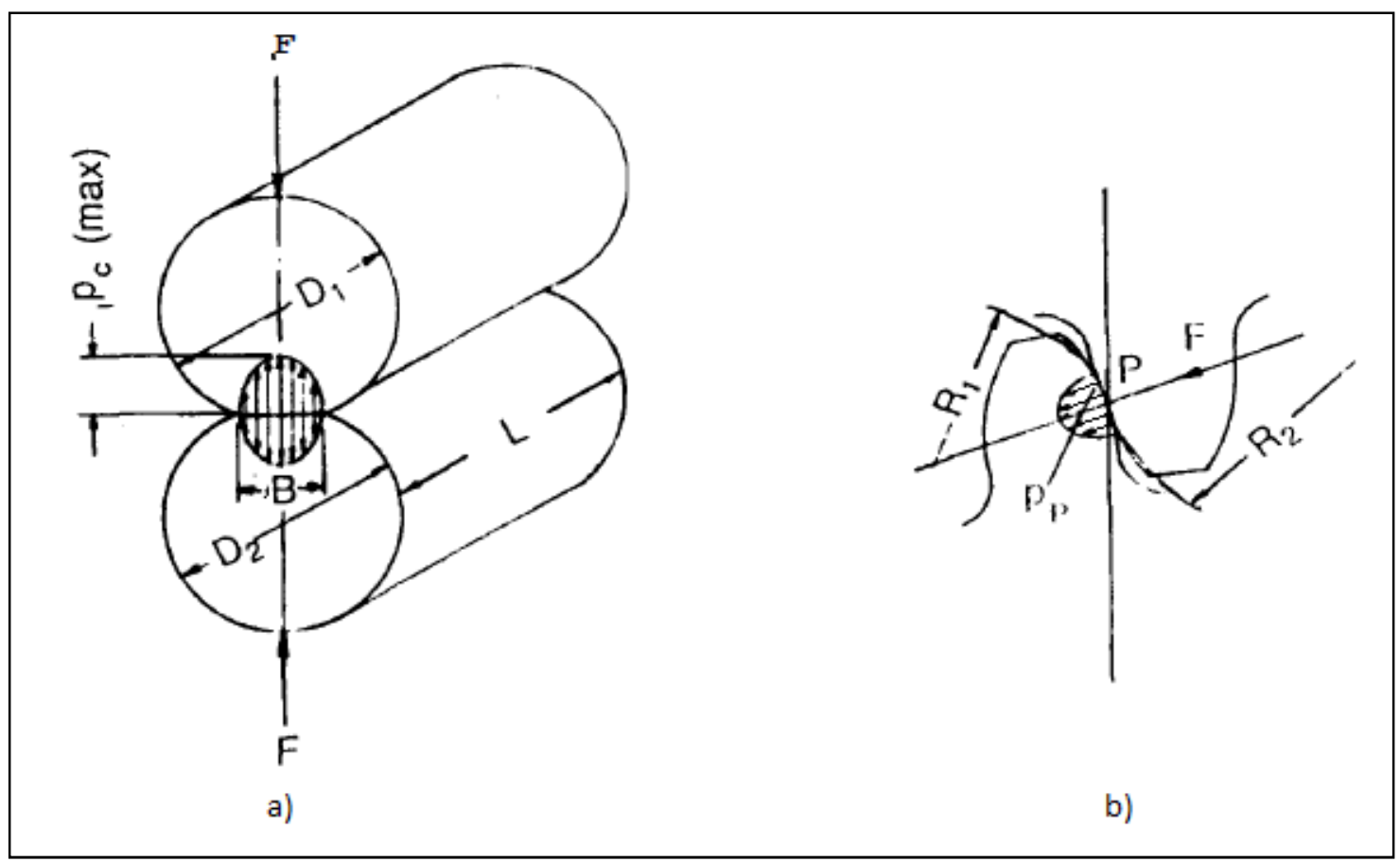

Source: Maitra, 2001 
Here, $\mathrm{F}$ is the applied force, $\mathrm{v}_{1}$, and $\mathrm{v}_{2}$, are the Poisson's ratios of the two materials of cylinders with diameters $D_{1}$ and $D_{2}$, and $E_{1}$ and $E_{2}$ are the respective elasticity modulus. Relating equations 14 and 15, replacing diameters by respective radius and assuming 0.3 to Poisson's ratio, a simplified equation (equation 16) is presented.

$$
P_{c(\max )}=\sqrt{0,35 \frac{F \frac{1}{R_{1}}+\frac{1}{R_{2}}}{L \frac{1}{E_{1}}+\frac{1}{E_{2}}}}
$$

The assumption of two cylinders contact be similar to the teeth contact on pitch line incomes reasonably accurate results in these region, since, on meshing gears, the contact region is theoretically a line. Each individual surface in the contact point has its own curvature according to the designed tooth profile dimension, as well as the immediate contact position on the line of action during sliding and rolling that occurs on the course of action.

Nevertheless curvature radius change continuously on involute curves and in the base circle area, the angular variation is even more significant. In the base circle surrounding the radius tends to zero and subsequently the stress tends to infinity. In this way, Hertz equation does not yield accurate stress results in this area, also, the contact in this area should be avoided due to high stress unfavorable effects. Contact stress equation has also another prerogatives which bounds its application. It is valid only when elastic limit is not exceeded and that the contact band is subjected to only compressive stress.

Considering the bounds of hertzian equation application, it's a good approximation of the real stress distribution which in fact occurs on gears contact. Bartz (1973) stated that the combination of rolling and sliding between the two contact surfaces induces a stress, which distribution is shown on Figure 25. In this case, fatigue crack will initiate if the subsurface shear stress is over material endurance. The crack will propagate parallel to surface, a second propagation direction may lead cracks to surface releasing upside. Eventually, oil penetrate on 
nucleated fatigue crack increasing surroundings stress and induces its propagation due to hydraulic wedge action.

Many variables define the gear tooth capacity to resist a surface fatigue failure caused by contact pressure, but surface durability improvement is a good choice when capacity to resist to this failure is under discussion. To design and to estimate a gear life time, the contact stress is calculated and gears are designed to maintain its surface stress lower than surface durability, which is directly related to gear material. (Maitra, 2001).

Figure 25: Stress distribution on contacting surface due to rolling, sliding and combined effects

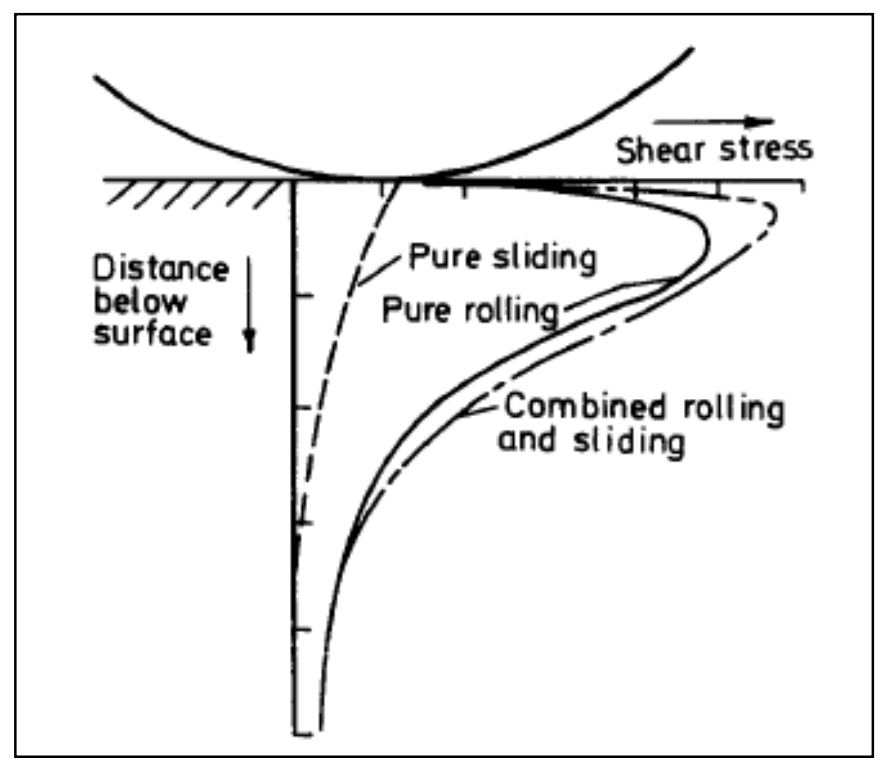

Source: Bartz, 1973

In modern gear design, pitting estimation is usually based on empirical results shared on international standard as DIN, ISO and AGMA (GLODEZ et al., 1997). In fact, ISO 6336-2 (ISO, 1996) defines different methodologies for defining loading calculation and 6336-5 (ISO, 1996) exhibits experimental life cycle results based on material capacity. 
Micropitting is defined by ASM (1992) as microscale contact fatigue. It is caused by relative metal surfaces contact during rolling and sliding resulting in micro roughness and micro craters scale. As a final result it can also end in polished surface. Moorthy e Shaw (2013) have defined micropits as small craters formed on the surface of the tooth, often in the negative slip region, below the primitive circle.

It produces a gradual damage which is a complex result of surface topography and its lubricant interaction. This remains valid even under severe contact loading, because a thin lubricant film between contacting surfaces still exists, that's EHD (elastrohydrodynamic). An early cycling damage is due to plastic deformation, which induces flattening asperities tips as the surface-finishing topography is been packed down. Right after, remains only smooth waves and scratches at the bottom of deeper furrows (ASM, 1992).

Moorthy e Shaw (2013) also have stated that progressive micro-pitting is the primary mode of contact fatigue in gears. It is associated with formation and propagation of micro cracks contrary to a sliding orientation on tooth flank surface. As much as the micro-cracks propagates, it tends to propagate parallel to the contact surface at a depth equivalent to asperity scale shear stresses, It disconnects material above it forming a micro-pit as shown on figure 26a. The progress of micro-pitting damage alters a micro-geometry of the tooth profile which is, in fact, losing material. As the material is displaced, it alters the profile contact area. Those modifications will also alters tension distribution on the gear teeth under contact fatigue. As this area is already damage, under rolling stress phenomenon, the micro-cracks at the bottom can induce a nucleation and propagation of other cracks resulting in a macro pit as seen on figure $26 \mathrm{~b}$. 
Figure 26: formation of (a) micro-pits and (b) macro-pits from the grow of microcracks.

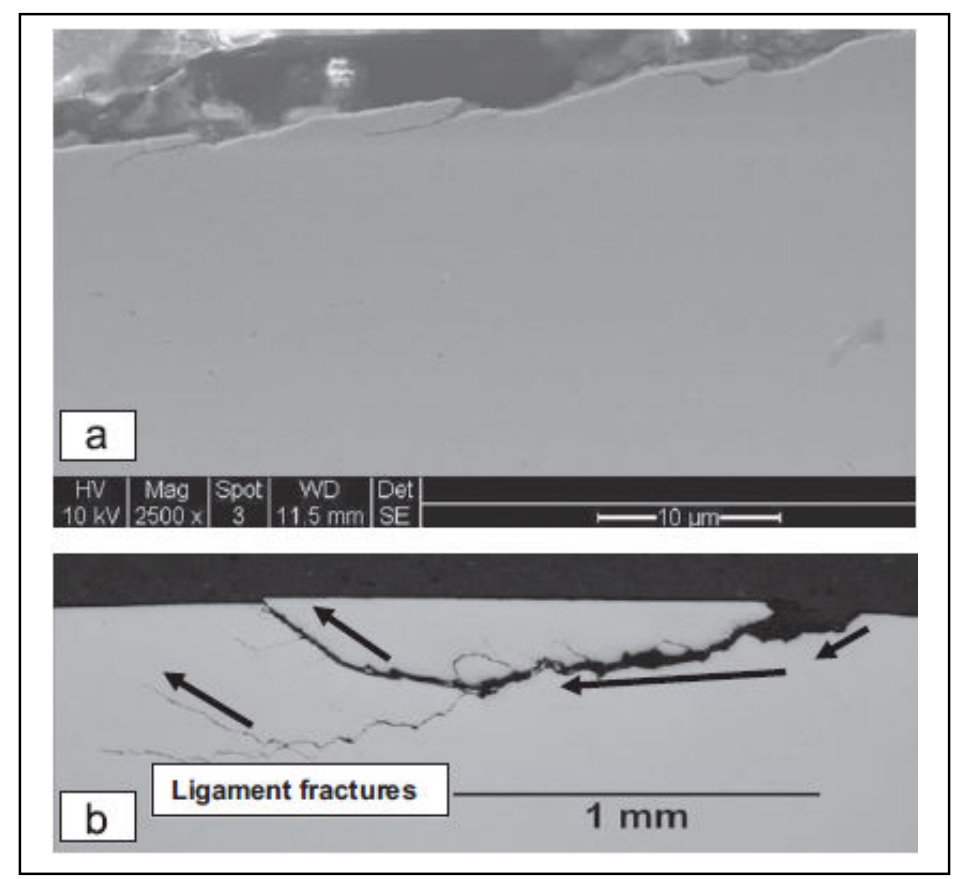

Source: Moorthy e Shaw, 2013

Two variables are directly related to micropitting occurrence: (i) EHD film thickness to roughness and (ii) surface microgeometry. In this manner, if the EHD film is thick enough to prevent high contact stress interaction with asperity interactions, micropitting will be avoided. On the other hand, film thickness will offer a prevention means for general micropitting, but localized microppiting is still possible to occur due to surface aspects for instance, nicks and dents, which in fact, reduce film thickness. Those surface characteristics are related to asperities height and sharpness.

Subcase fatigue is also a contact fatigue mechanism. Although the crack depth are much deeper than macro pitting, it is also influenced by material and by alternating hertzian shear stress. This kind of failure is traditionally caused by a too thin hardened case such as nitrited gear tooth or insufficient case hardness. The critical point is the existence of a gradient of decreasing hardness (shear strength) from the case to the core. Once nucleated, cracks will tends to propagate parallel to the case hardened area till a second crack formation that tend to propagate to material 
surface, resulting in macropitting. Figure 27 shows a micrograph of a roller specimen and gear tooth both submitted to fatigue test. Those specimen indicate a subcase fatigue cracks which nucleated and propagated during test (ASM, 1992).

Figure 27: Subcase fatigue (Black narrows are indicating cracks)

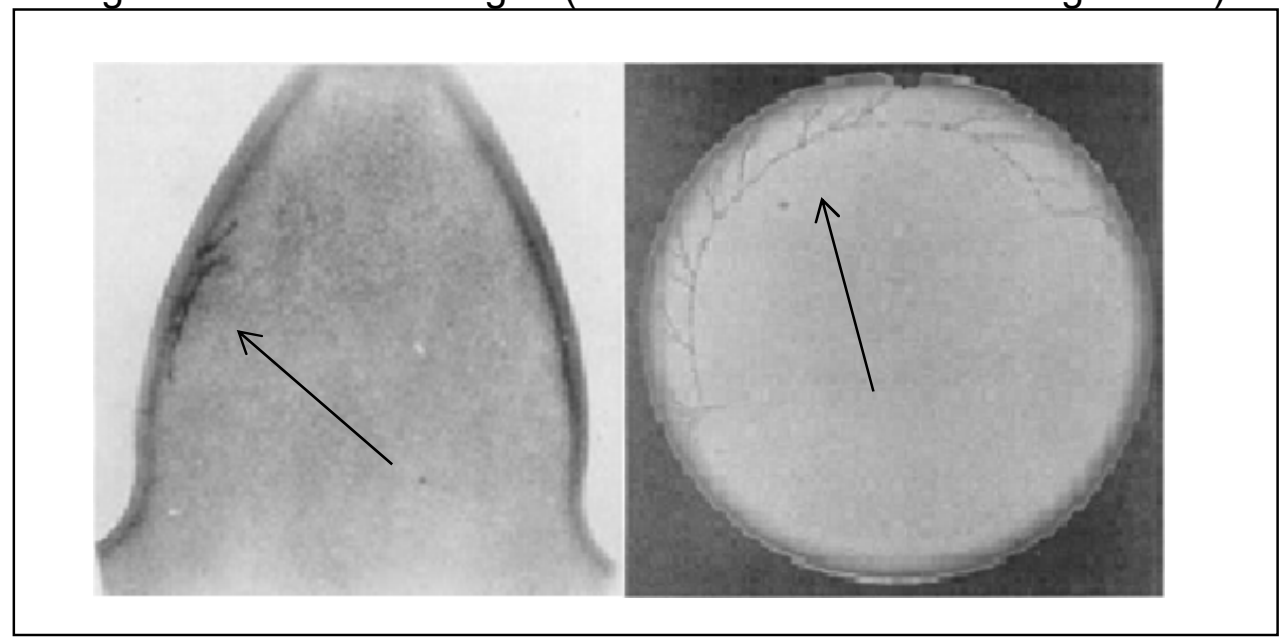

Source: ASM, 1992

\subsection{BENDING FATIGUE}

Bending fatigue fracture is a result of repetitive stress action exceeding or close to the fatigue strength limit of the material. A tiny crack is nucleated on higher tension area under cyclic loading. The crack can also begin with an impurity or tool mark inclusion. If the cyclic loading continues, the crack will propagate till the residual section of the tooth no longer supports the load. At that moment, the tooth will break abruptly. The fractured surface consists of two different zones: the surface fractured by fatigue and the surface fractured abruptly (residual surface). The surface fractured by fatigue is characterized by a series of contour lines to a focal point, reminiscent of "beach marks". It is flat, matte and velvety. In the case where the point of origin of the crack is subsurface, the focal point (eye) will appear with a strong brightness. The surface fractured by fatigue and the surface fractured abruptly can be seen on figure 28. (ANSI-AGMA 1010 E95c) 
Figure 28: Fatigue of two spur gears

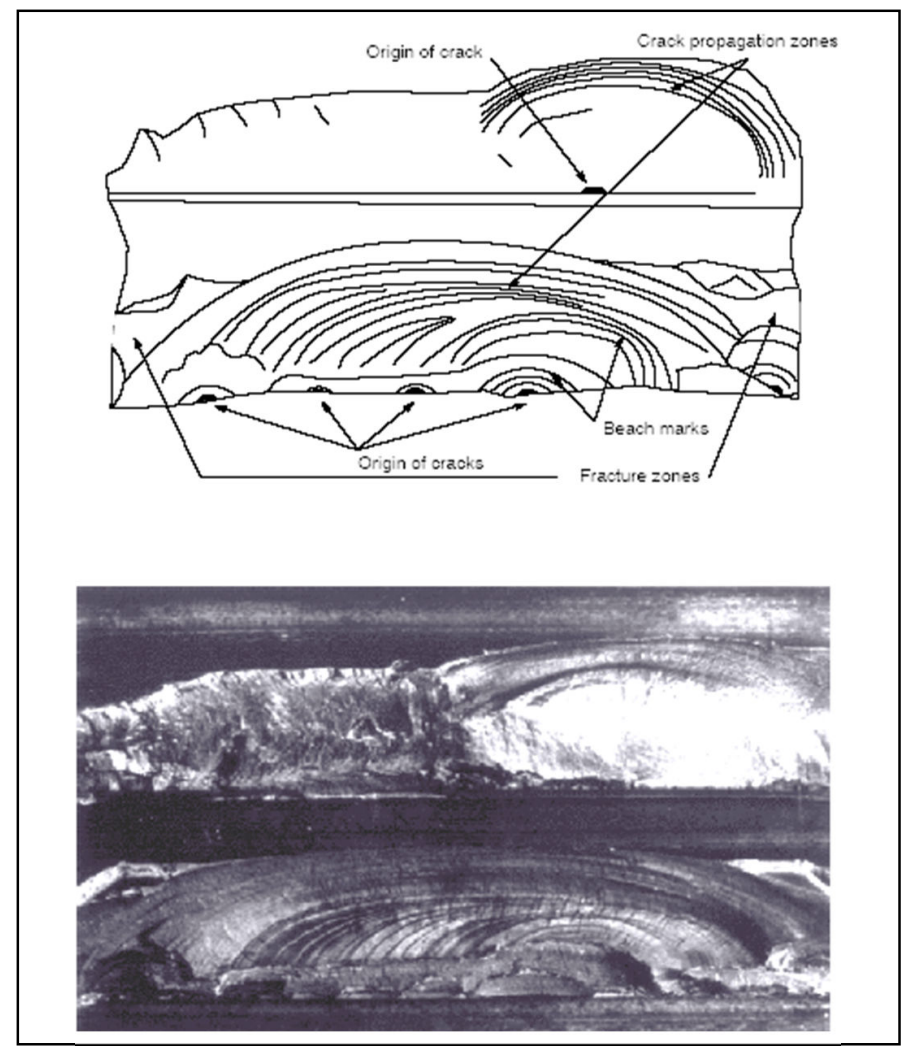

Source: ANSI-AGMA 1010 E95c

Low cycle fatigue is defined as the fatigue in which macroscopic plastic deformations occur in each cycle, and wherein the number of cycles before breakage is low (1000 to 10000). The cracks nucleation occur both inside the tooth and on its surface. In low cycle fatigue the nucleation step is shorter than propagation (ASM, 1992).

On the other hand, a high cycle fatigue is defined as the cyclic fatigue in which efforts are maintained below the yield stress of the material and wherein the number of cycles before breakage is higher than 10.000. It is the most common mode of bending fatigue. The cracks usually reveal themselves in the tooth root, however, they may arise on the side (abnormal load distribution due to pitting) or on the ends of the band (load poorly distributed or bevels poorly executed). In high cycle fatigue the nucleation phase is longer than propagation. Figure 29 illustrates a diagram made by Goodman, which exhibits the alternating stress limits reduction according 
to mean stress increase under high cycle fatigue area. Alternating stress $\sigma_{a}$ can be calculated using equation 17 and the mean stress $\sigma_{\mathrm{m}}$ can be calculated using equation 18.

Figure 29: Goodman diagram

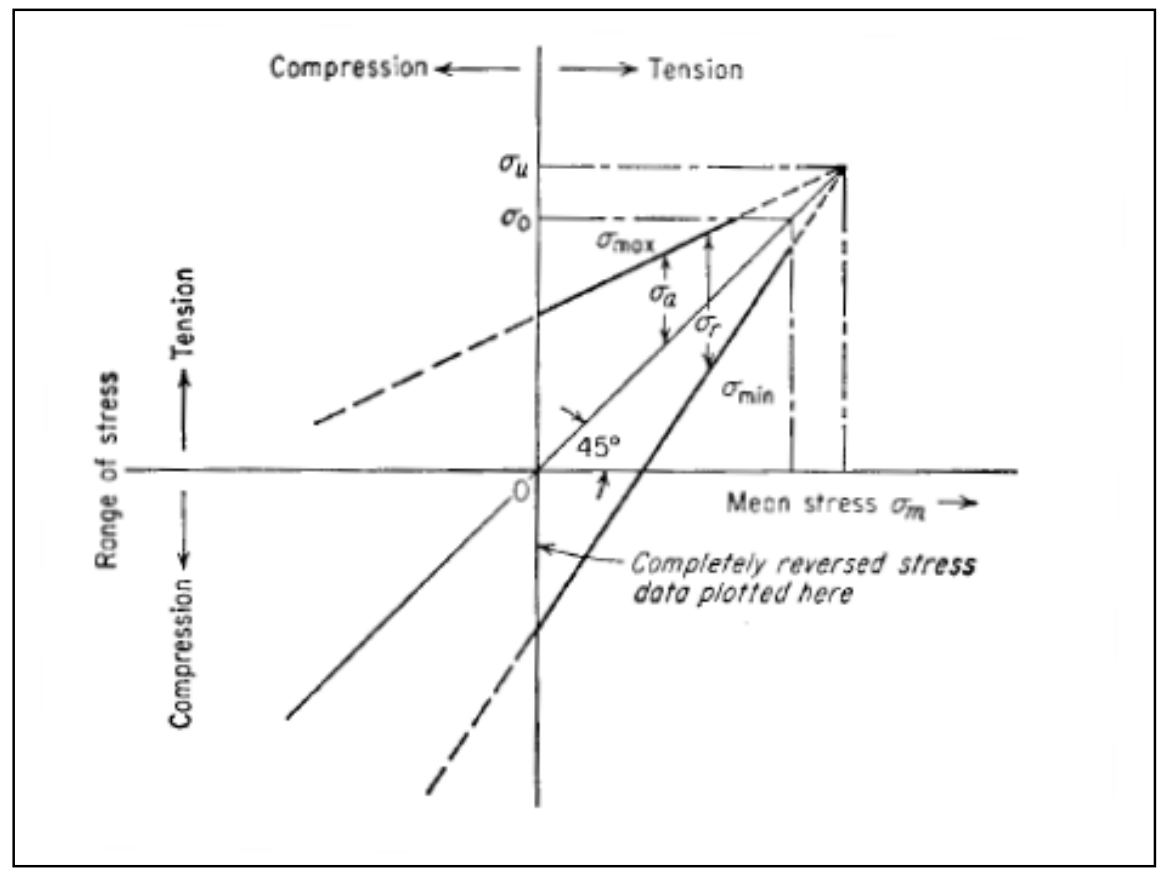

Source: Dieter, 1988

According to Goodman diagram, to avoid a failure due to fatigue, the allowable alternating stress $\left(\sigma_{a}\right)$ tends to reduce when $\sigma_{m}$ nominal value increases, as can be seen on Figure 29. The alternating stress is allowable till $\sigma_{0}$ which represents the material yield stress. If $\sigma_{m}$ (shown) increases and became greater than $\sigma_{0}$ a failure due to Yielding will occur. $\sigma_{\mathrm{u}}$, represents the material ultimate strength (Dieter, 1988).

$$
\begin{gathered}
\sigma_{a}=\frac{\sigma_{r}}{2}=\frac{\sigma_{\max }-\sigma_{\min }}{2} \\
\sigma_{m}=\frac{\sigma_{\max }+\sigma_{\min }}{2}
\end{gathered}
$$


Regardless of this classification of low or high cycle fatigue, which is related to load and cycle, its outcome in gears analysis is often a broken tooth. Traditionally a tooth break results in several teeth destroyed in a meshing gears, which usually ends a transmission service life. Therefore, in gear design, a safety factor choice ought to be larger than a chosen safety factor for delay pitting occurrence (ISO, 6336-2, 1996).

\subsection{PLASTIC DEFORMATION}

When a tooth is overloaded it can fail by plastic deformation. A permanent deformation that rises when the yield strength of the material is exceeded on the contact. If this tooth fractures this can be classified as:

(i) Brittle fracture: the tooth before breaking presents reduced plastic deformation, (ii) Ductile fracture: the tooth before breaking presents plastic deformation, (iii) Mixed fracture mode: the tooth presents brittle and ductile zone in the fractured region, (iv) Tooth shear: When the break occurs by shear stress, the fracture surface appears as a machined surface. It is a typical failure due to high overload on a single tooth, (v) Fracture after plastic deformation: This type of fracture starts with large plastic deformation. Typically all the teeth are damaged, since the material cannot stand the load applied: (v.i) when the load exceeds the resistance of the material (cold flow followed by fracture); (v.ii) when the material is weakened by heating (hot flow followed by fracture). 


\section{ASSOCIATED VARIABLES WITH GEARS FAILURE}

\subsection{GEAR PRODUCTION PROCESS}

According to Mazzo (2013), there are two gears teeth manufacturing processes: (a) with debris displacement, including: Forming, performed with Milling and Brochure process; and Generation, which is performed with Milling process. (b) without debris displacement, including process such as Melting, Stamping, Molding with Rolling Mills, Sintering, Plastic Injection of Resins and Forgings. Hereunder, the discussion will be focused on generation processes, due to its worldwide application on industry.

\subsubsection{Generation}

The Milling principle is based on meshing tool and blank, analogous to pinion and gear meshing. But on cutting, the aim is to originate final or very similar to final tooth shape starting from a base casted disc (blank) and removing material revealing teeth geometries. Traditionally, two tools can be chosen for using on generation; (i) Hob and (ii) Shaper. The tool will be chosen due to parts geometry and quality requirements and volume of production. (KLOCKE et al., 2016)

Milling can be used for both roughing and finishing, but If required by design, there is also available a finishing process. (KLOCKE et al., 2016)

Hob is a continuous gear generating method and it's the most common method used to cut spur and helical gear tooth. Figure 30 shows a schematic view of this tool and process. This method is relatively flexible to teeth design, but there is limiting aspect on indentations geometry. The teeth need to be sufficient wide in proportion to height to roll the hob in and out without interferences (MAZZO,2013). 
Figure 30: (a) Hob and (b) generation by hob

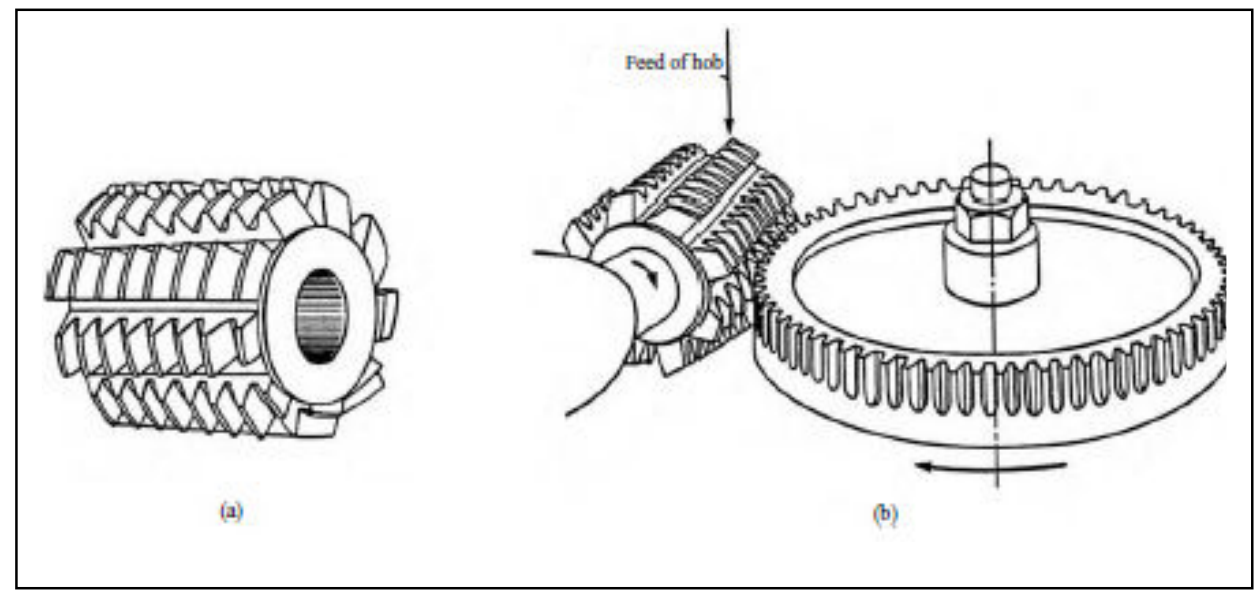

Source: Litvin et al, 2004

Klocke et al.(2016) state that a high accuracy in teeth geometry and in involute profile are obtained by this process, it is also possible to make a surface finishing using hob. Figure 31 shows a tool configuration to allow the Hob usage for gear teeth roughing and finishing with just one tool with two different areas.

Figure 31: Hob Tool concept for process strategies

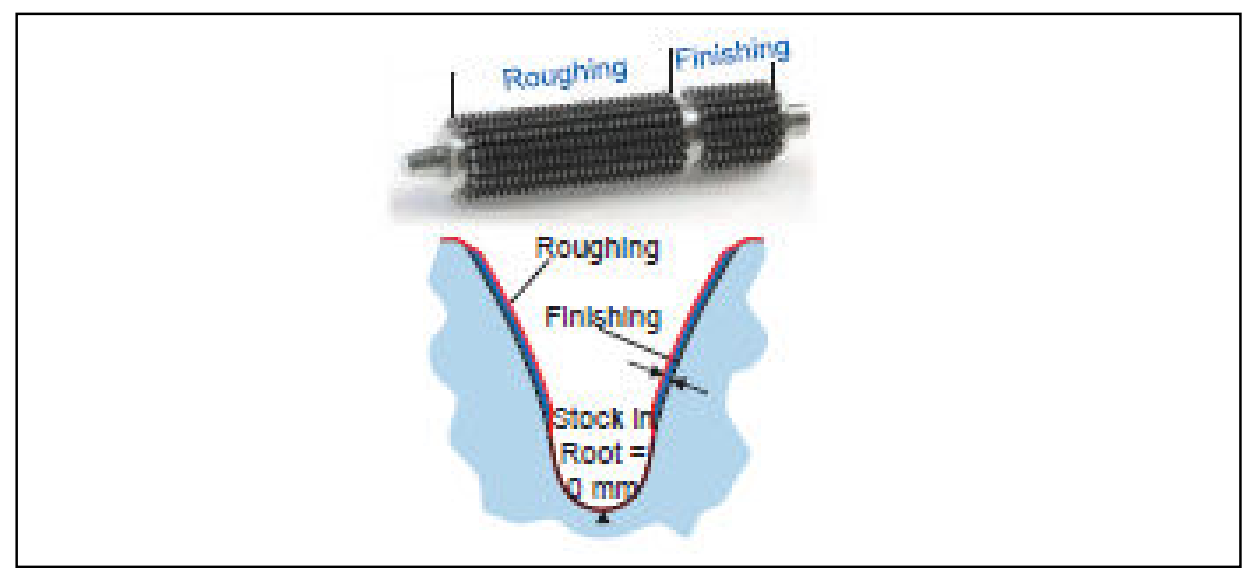

Source: Klocke et al., 2016

Despite of this possibility, surface finishing with hobbing is not as good as the ones obtained by shaping and shaving. Hob leaves a succession of slight radial tooth 
marks on gear teeth flank surface. Those marks thickness are influenced by the rate at which the hob moves axially across the face of the gear. So, according to gear design and application, it might be required to work on flank surface to adequate the final profile by a finishing operation (Endoy, 1990).

Shaping works under the gear rolling in mesh principle, which is similar of two meshing gears. This process will be done by a shaper and a gear (Mazzo, 2013) as shown in figure 32. Using this process it is possible to outcomes both external and internal spur gears, helical gears and spline. Besides the conventional blanks, with this process it is also possible to cut flanges and blanks with narrow recesses for cutter clearances. Some gears are only feasibly generated by shaping, such as: cluster gears and shoulder gear (Litvin et al., 2004).

Figure 32: Meshing of a gear and a shaper

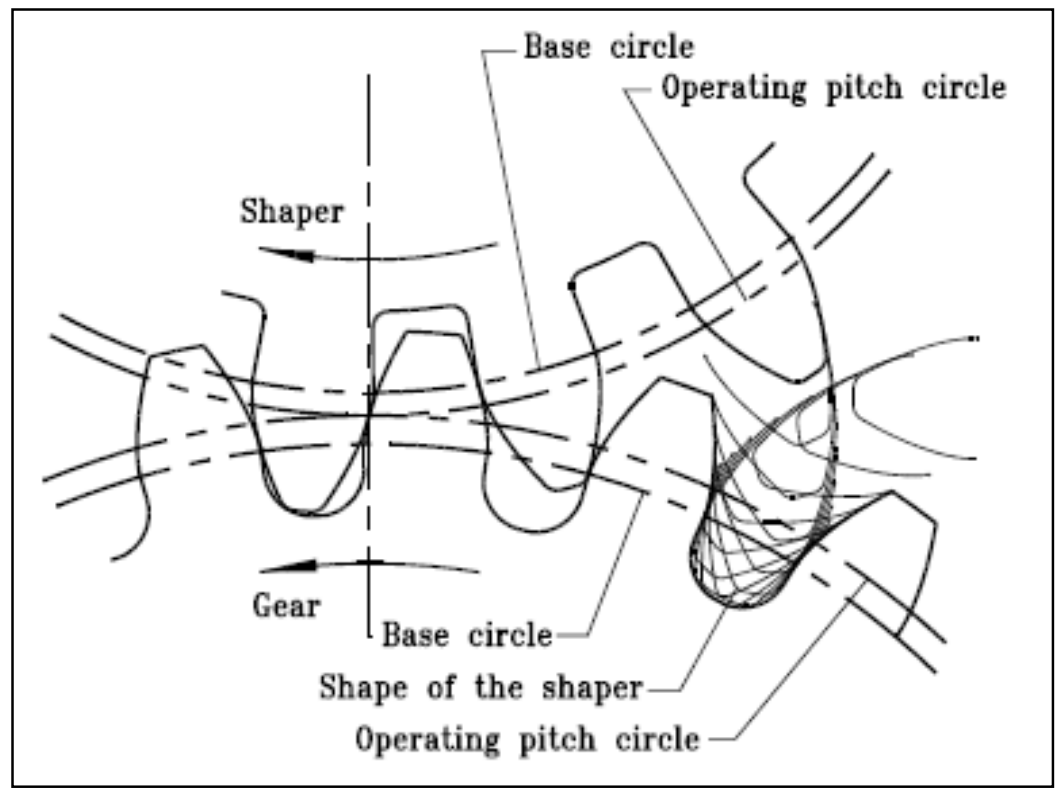

Source: Litvin et al., 2004

The working principle is based on a rotating blank (around its axis), while the cutter stands around the face of the work blank. Figure 33 shows a shaper and a generation by shaping process. The generating feed is defined by working rotation, while the cutting speed is a function of cutter stroke length and stroking level. Ever 
since, a relief action is provided for the return stroke due to shaper one direction cut. This process is also indicated when gear design requests special surface finishing, with a reduced roughness and high accuracy. This outcome combination is feasible because generating speed is not a function of stroking speed. If stroking speed is increased, a larger number of flats will tough generate the profile and the outcome is a special finish (Endoy, 1990).

Figure 33: Shaper and generation by shaping

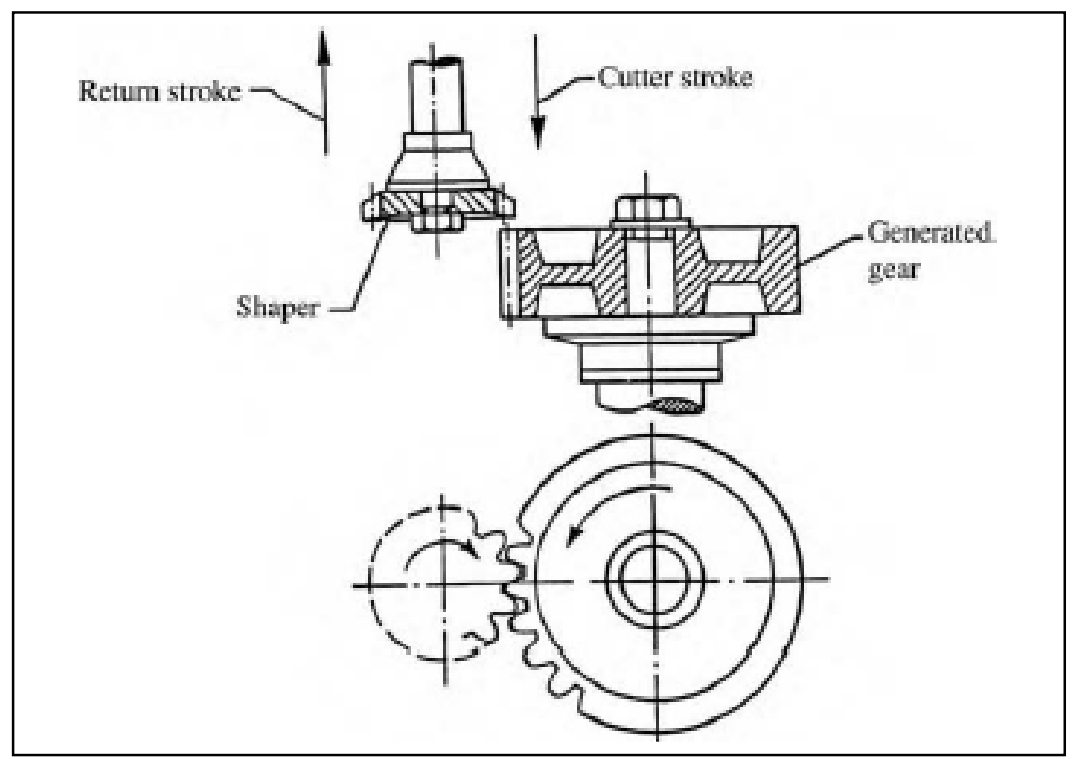

Source: Litvin et al, 2004

\subsubsection{Surface Finishing}

Shaving is a gear flank surface finishing process, applicable to internal and external gears. This process application is restricted by workpieces size and quality specifications. Since it is processed prior to the heat treatment it is not applicable to high accuracy gears which are usually obtained by grinding or honing.

Its operation is analogous of two meshing gears, in which elements are rotating tight together in meshing with one gear crossing the tooth face of the other. The shaving cutter is essentially a helical gear with helix angle different than that of 
the gear to be shaved, the cutting edges are made of teeth face unevenness. Figure 34 illustrates a shaving cutter.

The cutter is the driven and the work gear rotates without obstruction among centers. The angular difference between the helix angle of cutter and gear defines the angle which both axes from work gear and cutter are traversed while shaving. Infeed is done by approximating cutter and gear centers, thought the work gear tooth thickness is changed by approaching its center upwards getting closer to the cutter. This movement increases pressure among cutter and work gear, the cycle is therefore completed by two finishing strokes without vertical feed (Endoy, 1990).

Figure 34: Tool and workpiece assignment for gear shaving

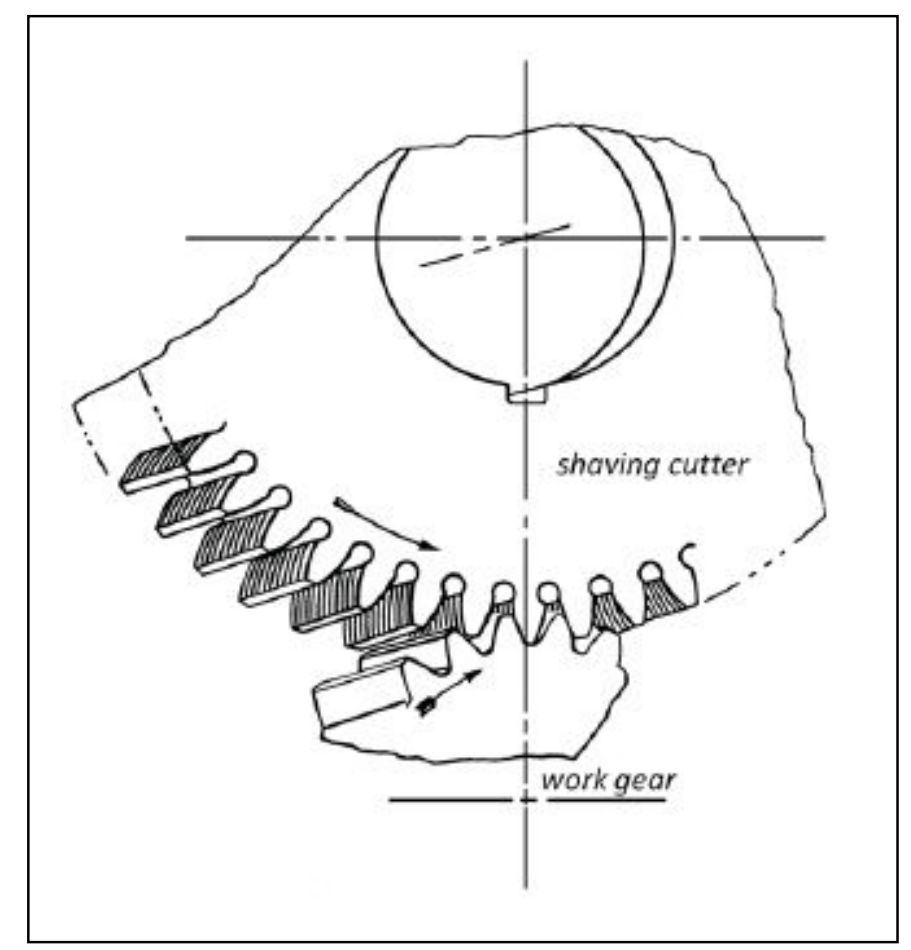

Source:.Linke et al, 2016

Grinding represents one of those possible hard finishing process, which can improve, load carrying capacity and noise performance (Klocke et al., 2016). Reishawer developed a method using a worm shaped grinding wheel which is knew as the most productive ones. Worn wheel grinding outcome is a high quality gear 
with accurate profile, tolerance index and smooth surface finish. Worm wheel principle is similar to hobbing, but a larger grinding wheel diameter is used instead and the process is based on several passes taken through the gear surface. Figure 35 illustrates a gear grinding tool (Klocke et al., 2016).

Grinding takes place during wheel stroke up and down and infeed is a result of centers approximation. Basically, right after each wheel pass, the tool (working gear) is moved slighted closer to wheel. This process has similar gear geometry restrictions, as much as hobbing process does. Geometry restriction becomes more severe due to grinding wheel diameters, which are much larger than hob diameters. As hob, it's also not suitable for shoulders gears. Changing grinding worm profile allows performing tip and root relief, reducing workpiece and grinding wheel distance results in gear teeth longitudinal modifications and a crowned teeth is an outcome of center distances modifications on work side (Endoy, 1990).

Figure 35: Gear grinding kinematics and contact

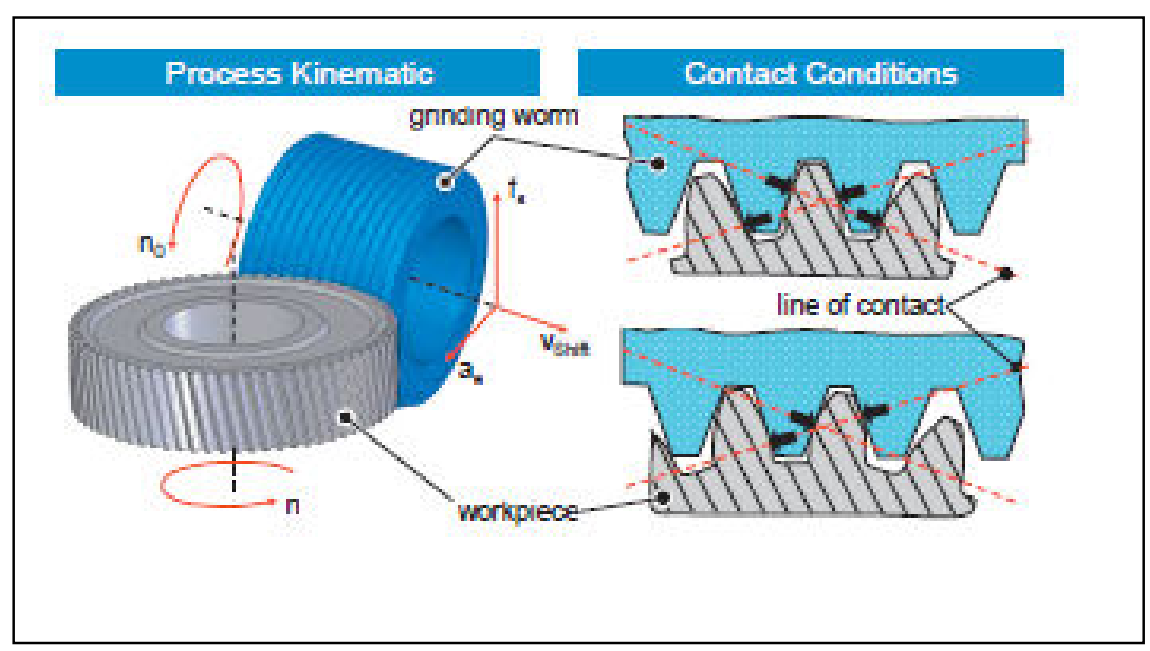

Source: Klocke et al., 2016

Gear Honing, according to Endoy (1990), is spur and helical gears surface finishing process that takes place after heat treatment. It is indeed called a hard finishing process, which is capable of removing grooves and marks to obtain expressive improvements in involute profile and helix angle. 
Gear honer working principle has similarities with shaving process. The tool is an abrasive material helical gear which rotates with workpiece crossed axes, in a controlled meshing liaison. The honing tool crosses gear surface, changes rotation direction and then perform a return journey crossing back gear surface. It's a stepping forward and back mechanism, changing rotation direction at each step (stroke), while the workpiece rotates without restrictions despite of the centers. The cutting is sustained increasing pressure among workpiece and horning gear. Figure 36 illustrates a honing tool design.

Remarkable honing outcome advantages are generate noise quality improvement and a wear life prolongation of shaved and hardened gears. The noise improvement is due to groove and marks removal and the wear life prolongation regards to surface finishing and load carrying capacity increased due to larger surface contact area in meshing gears.

Figure 36: Gear honing tool

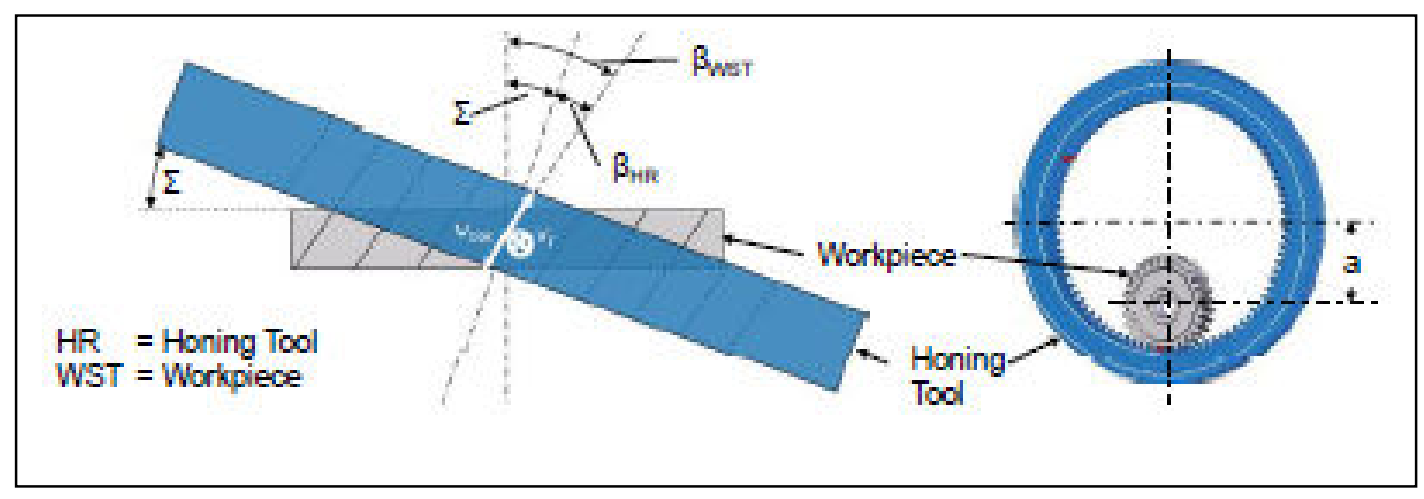

Source: Klocke et al., 2014

\subsubsection{Gear Quality According to Process}

Gear quality criteria is detailed by ISO 1328 (ISO, 1995). The standard purposes are to specify an accuracy classification system to gear flanks, to define suitable descriptions for gear tooth accuracy as much as its evaluation system. The gear quality classification system contains 12 grades of accuracy, ranging from grade 1 , which is given to the best accuracy, till 12 , given to the largest admissible deviations. 
Linke et al. (2016) correlated gear quality criteria achievable according to the manufacture method. On table 03 is seen such correlation, which is based on ISO 1328 (DIN 3962 to 3964). As observed in the table 03 each manufacturing method has an inherent capacity to induce a range of quality to gear. On this table, the dark blue sections mean accuracy ordinarily feasible on production scale level of several manufacturers involved on Linke et al. (2016) research. For superior requirements, such as test or master gears, which means small scale or even prototype, the light blue area represents increased qualities that are also possible to obtain by gear manufacture (Linke et al, 2016).

Table 3: Gear tooth quality achievable according to the manufacture method (Linke et al., 2016)

\begin{tabular}{|r|l|l|l|l|l|l|l|l|l|l|l|l|}
\hline Pressed, stamped & & & & & & & & & & & & \\
\hline Hobbed, shaped & & & & & & & & & & & & \\
\hline Shaved & & & & & & & & & & & & \\
\hline Ground & & & & & & & & & & & & \\
\hline $\begin{array}{l}\text { Quality in accordance with ISO 1328 } \\
\text { (DIN 3962 to DIN 3964) }\end{array}$ & 1 & 2 & 3 & 4 & 5 & 6 & 7 & 8 & 9 & 10 & 11 & 12 \\
decreasing quality $\rightarrow$ \\
\hline
\end{tabular}

According to Linke et. al. (2016), four functional groups are defined in DIN 3961 (G, L, T, N) aiming to simplify the tolerance specifications, where $(G)$ is the uniformity of the transmission of motion, $(L)$ is the quiet running and dynamic load capacity, $(\mathrm{T})$ is the static load capacity (load distribution) and $(\mathrm{N})$ means no indication of the function. The functional group $(L)$ is suitable to investigate pitch, transverse profile and flank deviation.

\subsection{TOPOGRAPHY}

Real surfaces presents particularities that are consequence of employed manufacturing processes, whether it be a turning, milling, grinding or any other machining process. The outcome surface, which is a result of those machining process looks like as a set of irregularities, tending to form a characteristic pattern or texture throughout on its length (Thomas, 1999). 
To evaluate surface purposes some important and useful definition are required as much as a geometric approach. In surface metrology, measuring are performed on deviations from an ideal form. In this manner, an ideal reference line is demanded and that's the "M" system principle (NBR ISO 4287, 2002).

The reference line is the midline, defined as the line located in the middle part of the roughness profile, with the same shape of the geometric profile, arranged parallel to the profile general direction, within the length limits of measurement basis and also splits equally areas bellow and above it.

The geometric approach is required to define an actual profile, which is a resulting from the intersection of the workpiece surface and a plane normal to that surface, figure 37 illustrates it.

Figure 37: Actual profile

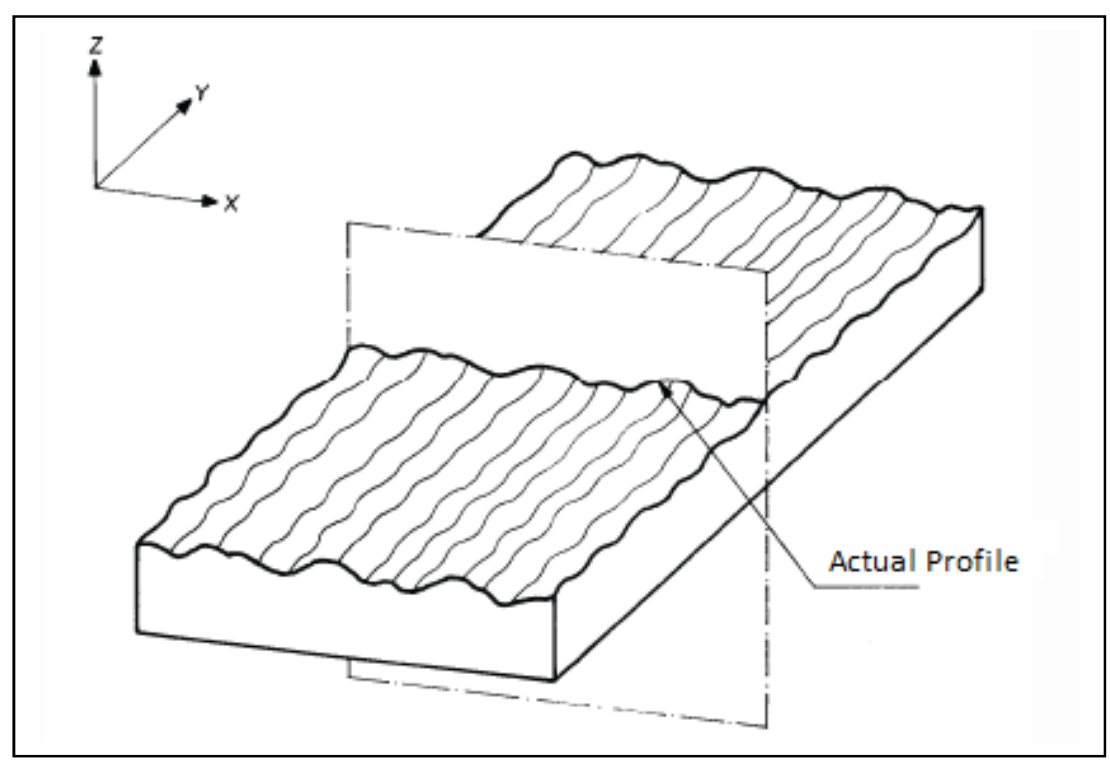

Source: NBR ISO 4287, 2002

The measured profile is the outcome shape of probe scanning the actual profile. The probe mechanically filters this shape due to its tip radius and the skid system. Stepping forward, it is important to apply a first filter (see figure 38) according to NBR ISO 4287 and ISO 16610 required for the primary profile reveal. 
Figure 38: Profile Filters

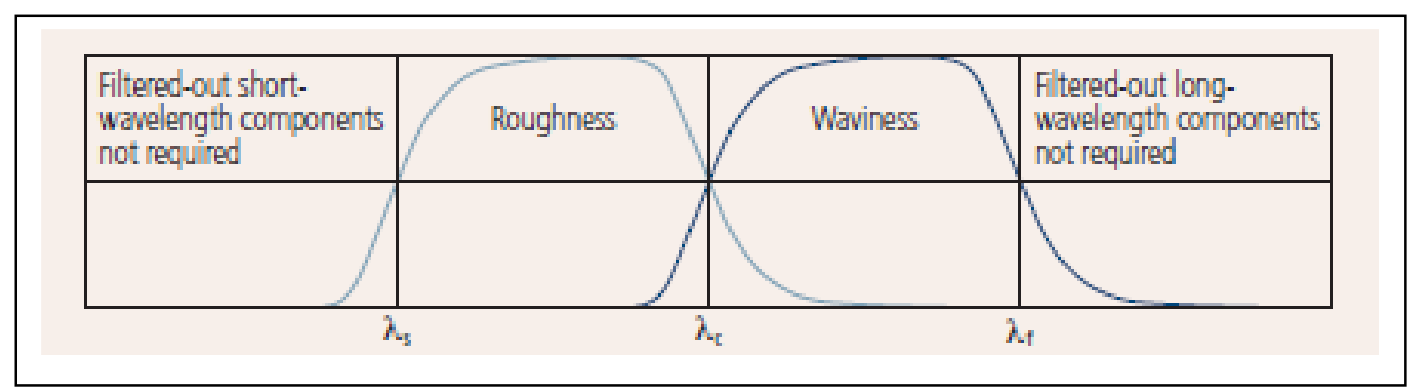

Source: NBR ISO 4287, 2002

The primary profile (P-profile) is the electronic low-pass filtering shape outcome of the measured profile with a cut-off wavelength $\lambda_{s}$. This procedure eliminates the shortest wavelength components that are considered irrelevant to a roughness measurement. Primary profile parameters are nominated $P$ and calculated within the sampling lengths. At this step the analysis can take two distinct components: (i) the roughness and (ii) the waviness.

The roughness is formed by grooves or marks left by the agent which attacked the surface in the machining process and is overlaid on the waviness profile and the roughness profile (R-profile) is the electronic high-pass filtering outcome shape of the primary profile with a cut-off wavelength $\lambda_{c}$. This procedure eliminates the longer wavelength components (NBR ISO 4287, 2002).

The waviness is the set of repeated irregularities in waves of a length much larger than their amplitude and that can occur due to deficiencies in the machining machine movements, deformation in the heat treatment, residual stresses, etc.

The waviness profile (W-profile) is the electronic low-pass filtering outcome shape of the primary profile with the cut-off wavelength $\lambda_{c}$ followed by high-pass filtering with the cut-off wavelength $\lambda_{\mathrm{f}}$ (NBR ISO 4287, 2002).

In Figure 39 in seen the total length of the surface profile recorded. (NBR ISO 4287, 2002). 
Figure 39: Primary Profile and its mean line

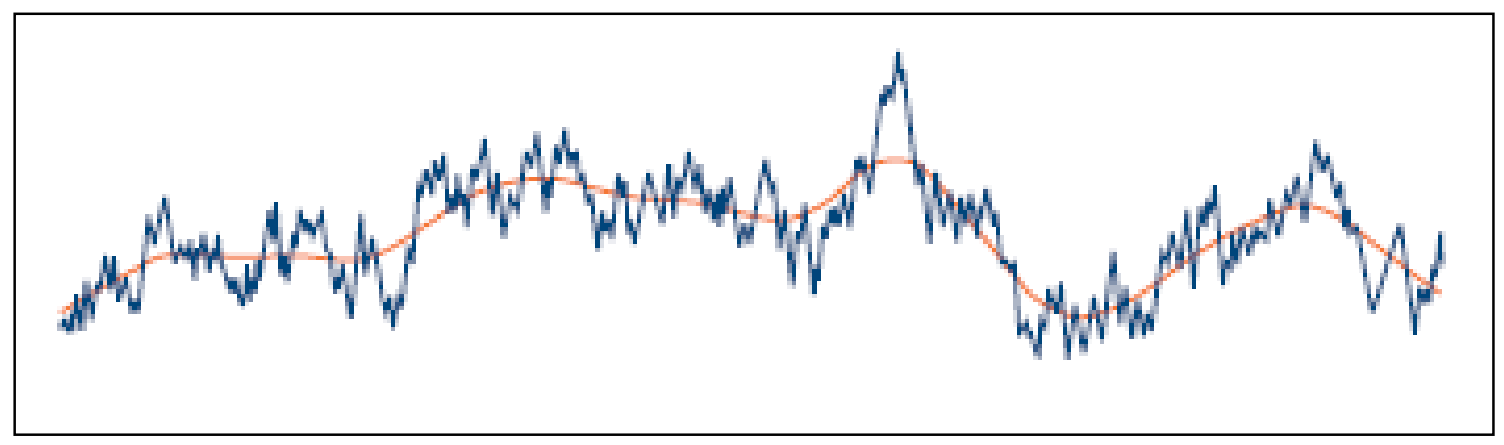

Source: adapted from NBR ISO 4287, 2002

\subsubsection{Surface Parameters}

According to Czanderna (2002), an extensive variety of surface parameters have been established to describe surfaces roughness, but they can be classified into four categories: height parameters, shape parameters, wavelength parameters, and a combinations known as hybrid parameters.

a. Height Parameters. The most commonly used are the roughness average $\mathrm{Ra}$ and the rms roughness also called $\mathrm{Rq}$. $\mathrm{Ra}$ is known as the profile surface height arithmetic mean deviation from the mean line and can be calculated according to expression 19.

$$
R a=\frac{1}{L} \int_{0}^{L}|Z(x)| d x
$$

$\mathrm{Rq}$ is defined as the profile root mean square deviation from the mean line and can be calculated as seen on expression 20 .

$$
R q=\sqrt{\frac{1}{L} \int_{0}^{L} Z^{2}(x) d x}
$$


Where:

$Z_{p}$ is the height of the surface above the mean line at a distance $x$ from the origin and $L$ is the profile evaluation length.

In addition to those two averaging height parameters, a variety of other height parameters have been defined for various applications, including several for characterizing peak-to-valley height as (NBR ISO 4287, 2002):

$R_{p}$ is the highest peak of the $Z_{p}$ profile peaks at sampling length, more details can be seen on figure 40 .

Figure 40: $R_{p}$

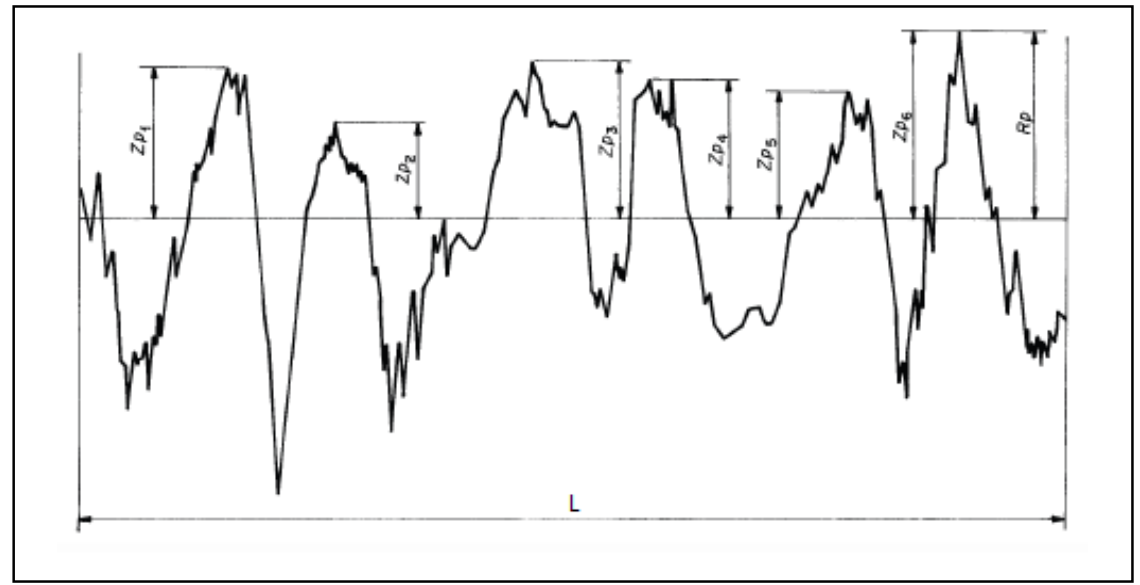

Source: NBR ISO 4287, 2002

$R_{v}$ is the Greater depth of profile valley at sampling length, more details can be seen on figure 41.

Figure 41: $R_{V}$

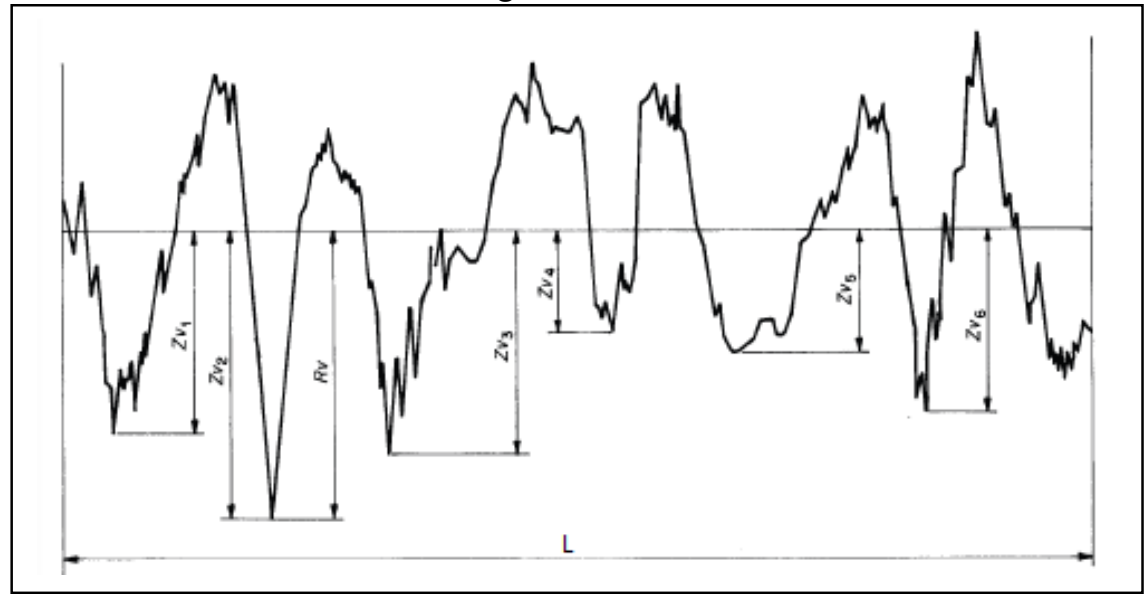

Source: NBR ISO 4287, 2002 
$R_{z}$ is the sum of the maximum height of the peaks of the $Z_{p}$ profile and the largest of the depths of the valleys of the profile $Z_{v}$, at the sampling length. $R_{Z}$ is calculated as $R_{p}+R_{v}$, and more details can be seen in figure 42 .

Figure 42: $R_{z}$

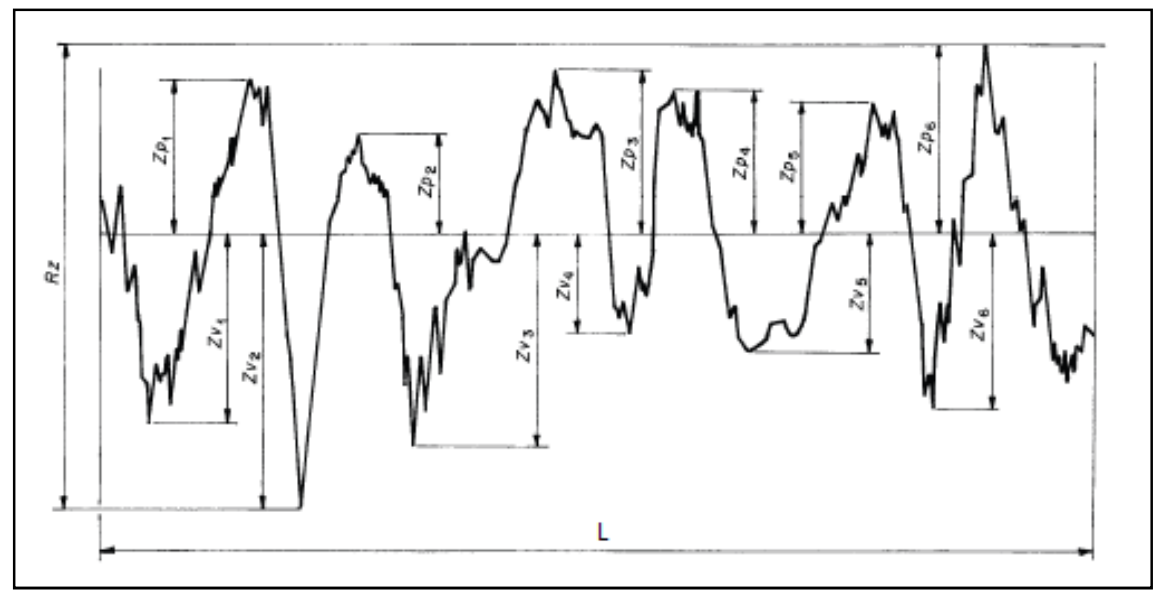

Source: NBR ISO 4287, 2002

$R_{c}$ is the mean value of the heights of the profile elements $Z_{t}$ at the sampling length, More details can be seen on figure 43.

Figure 43: $R_{c}$

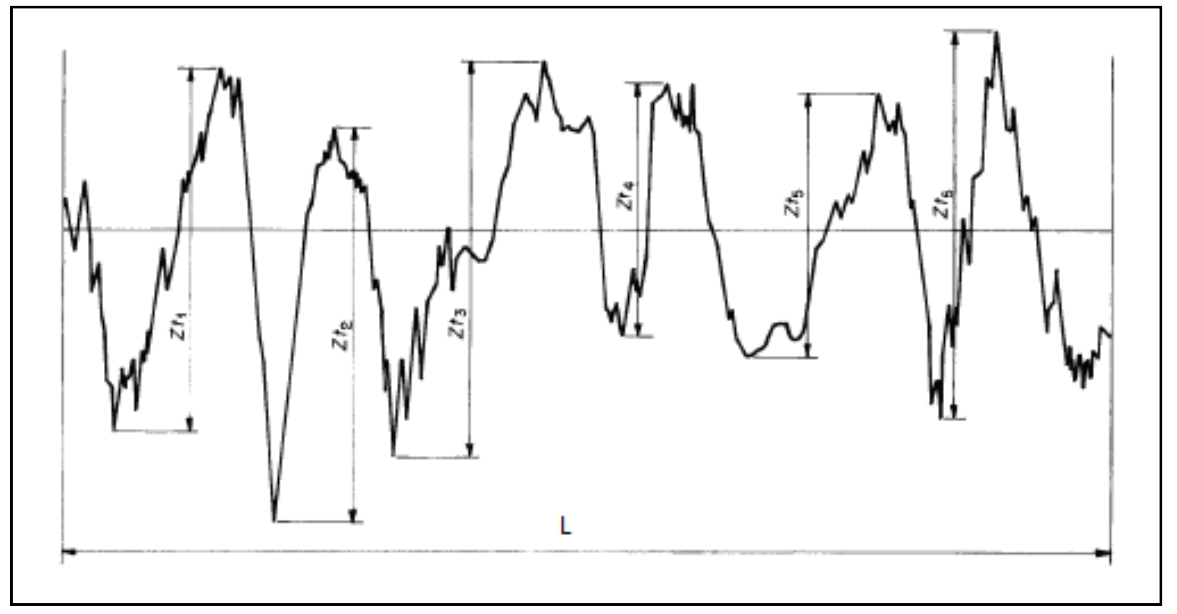

Source: NBR ISO 4287, 2002

$R_{t}$ is the sum of the highest peak of the $Z_{p}$ profile and the largest depths of the valleys of the $Z_{v}$ profile in the evaluation length, details can be seen in figure 44 . 
Figure 44: $R_{t}$

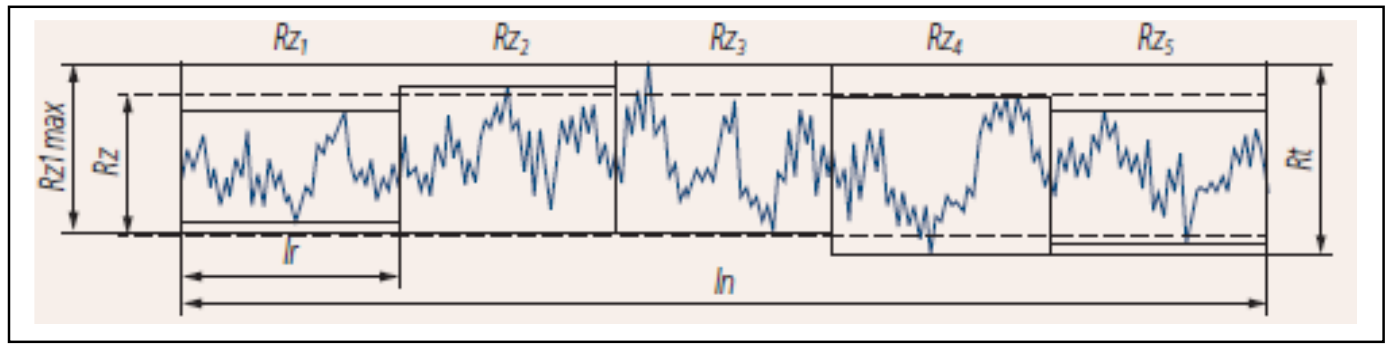

Source: adapted from NBR ISO 4287, 2002

\subsubsection{Spatial Wavelength Parameters}

Spatial Wavelength Parameters are useful to define the distance between peaks and valleys of the profile under analysis.

In NBR 4287 (ABNT, 2002) a classic wavelength parameter is the mean width of profile elements $R_{S m}$, it has been calculated for a surface profile as the average distance between consecutive negative slopes crossing profile with the mean line, and can be calculated as shown by equation 21 . The peak spacing parameters are shown on figure 45 .

$$
R S m=\frac{1}{m} \sum_{i=1}^{m} X s_{i}
$$

Figure 45: Peak spacing parameters

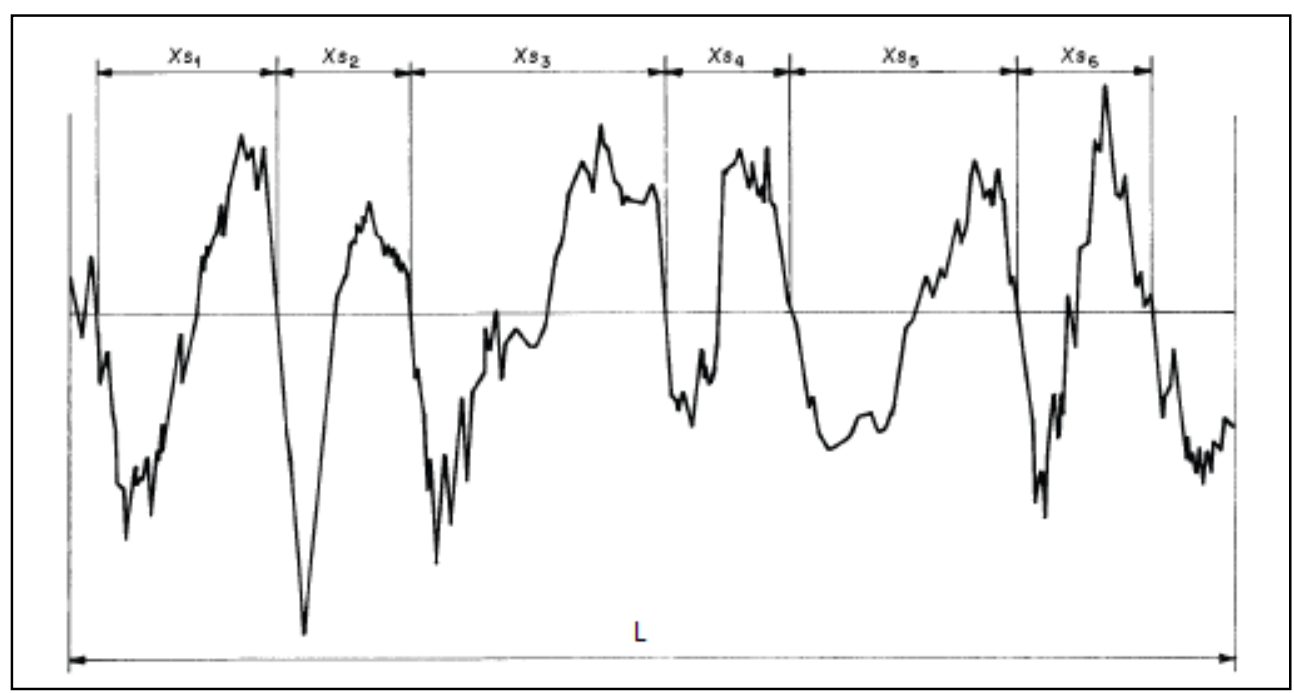

Source: NBR ISO 4287, 2002 


\subsubsection{Shape Parameters}

The periodic profiles shown in figure 46 have the same $\mathrm{Ra}$ and spatial wavelength, but different shapes. Therefore, they might perform unequally for diverse applications. It is also seen in figure 46 a dashed line for each profile, which represent the mean line. Shape parameters are useful to quantify the practical differences between surfaces. In this perspective, the skewness $\left(R_{s k}\right)$ is a measure of the symmetry of the profile about the mean line. Back on figure 46 , the skewness is positive for profile (a) and negative for profile (b). The other two profiles are symmetrical about the mean line and have zero skewness. The skewness $\left(R_{s k}\right)$ can be calculated as shown by equation 22 :

$$
R s k=\frac{1}{R q^{3}}\left[\sqrt{\frac{1}{L r} \int_{0}^{L r} Z^{3}(x) d x}\right]
$$

Figure 46: Different profiles with the same $\mathrm{Ra}$ and $\mathrm{R}_{\mathrm{Sm}}$

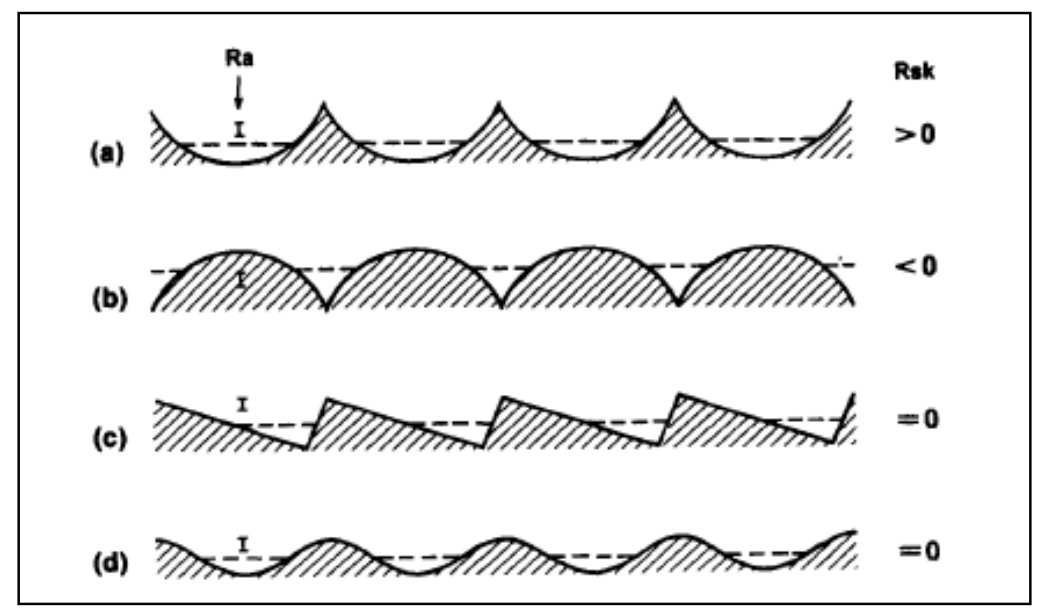

Source: Czanderna, 2002

\subsubsection{Hybrid Parameters}

A quantitative concept combination of height deviation and lateral displacement are slopes and curvature. Hybrid parameters are used in tribology as much as in elastic contact and thermal conductance (Czanderna, 2002). 


\subsection{PROCESS INFLUENCE ON ROUGHNESS AND GEARS FAILURE ANALYSIS.}

Linke et al. (2016) have correlated process and surface roughness and have indicated that on ground gears an allowable $R a$ is ranging from 0,3 to $0,8 \mu \mathrm{m}$ and $R_{z}$ ranging from 4 to $6,3 \mu \mathrm{m}$. They've also considered that grinding steps can take overall influence on tooth flank surface if they lead to expressive marks which influence local stress. Another important consideration done was related to milled gears roughness acceptable limits, in which $R_{a}$ upper limit is $1,6 \mu m$ and $R_{z}$ upper limit is set on $16 \mu \mathrm{m}$.

The influence of roughness on fatigue life evaluation of a roller has been studied by Nakanishi et al. (1987). They had tested two different groups of final roughness, $R_{\max }=2 \mu \mathrm{m}$ and $R_{\max }=10 \mu \mathrm{m}$. So, in figure 47 is seen the evaluation done by the authors of the roller load capacity under fatigue test based on two mentioned groups roughness. The authors concluded that durability is influenced by initial surface roughness.

Figure 47: $\mathrm{P} N$ curve for surface failure on roller tests ${ }^{2}$

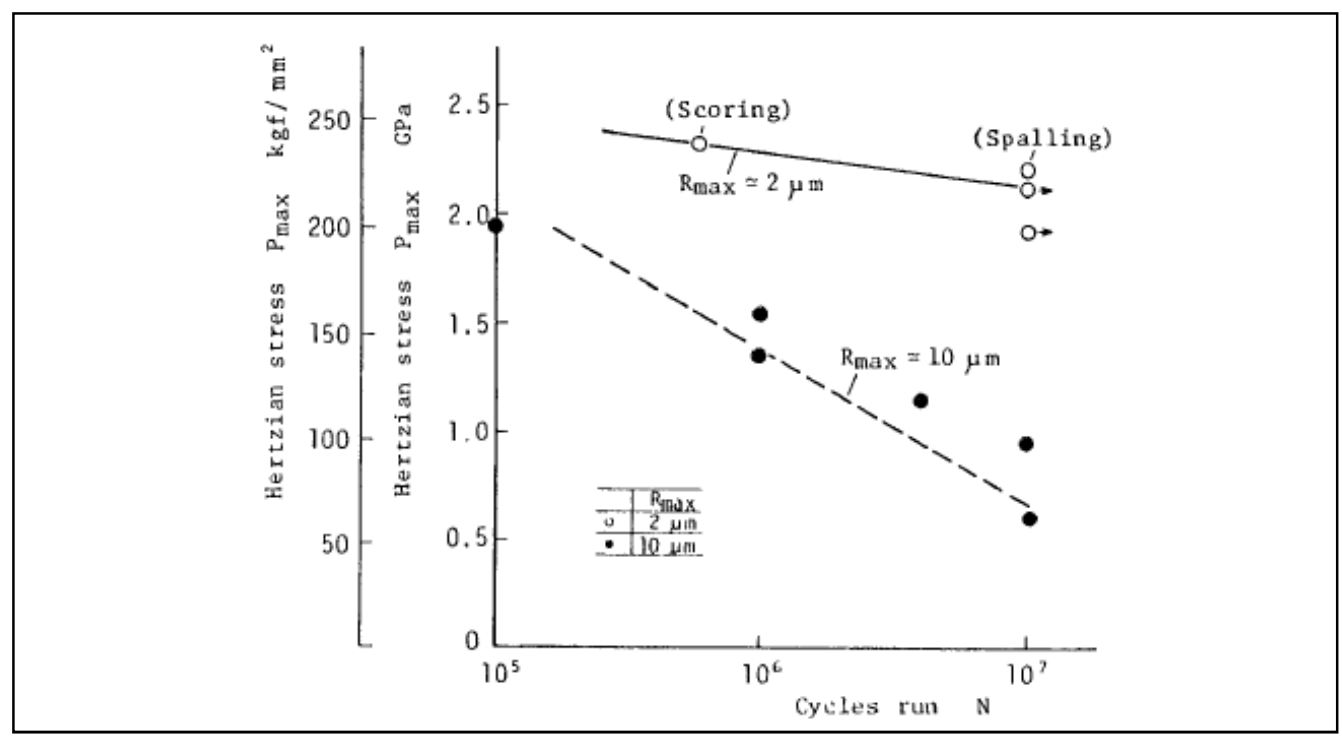

Source: Nakanishi et al, 1987

2 ASTM 5182 (2008) defines Scoring as a "severe form of wear characterized by the formation of extensive grooves and scratches in the direction of the sliding". 
The Nakanishi et al. (1987) research obtained an important understanding of roughness influence on rollers fatigue cycle. Those results highlight that roughness must be controlled in any component life evaluation, otherwise it can add an uncontrolled influence on final evaluations.

In this perspective, gears production and life evaluation ought to take into consideration roughness influence on failures. That's the reason of a meticulous roughness control in gear manufacturing process on this research.

\subsection{LUBRICANTS AND THEIR FUNDAMENTAL PROPERTIES}

An extensive range of material in different phases such as gases, liquids or solids stages have been used for lubricating purpose. The use of lubricants are indicated when it is intended to reduce frictional force between surfaces. The lubricant working principle is to introduce an extra material layer between sliding surfaces. This extra material layer has a property of a lower shear strength than the previous surfaces in contact (Hutchings, 1992).

One example of this working principle is illustrated in figure 48 , containing a stationary surface, a moving surface and a lubricant in between. The oil in contact with sliding surface will keep the same velocity $U$ as the surface, on the other border, oil in contact with a stationary surface will not move, it will have zero speed. In the middle of it, the oil film might be seen as an amount of many layers, each one drawn by the layer above it at a portion of velocity $U$ that is relative to its distance above the stationary plate. In figure 48 , a force $F$ ought to be present in the moving surface to overcome the friction among fluid layers. This friction is a result of a very important feature of a lubricate oil, called viscosity. (Pirro et al., 2001).

ASM (1992) has defined viscosity as the measurement of a fluid withstands to a flow and according to Hutchings (1992), viscosity offers a quantity of a fluid resistance to a shearing stream. Both definitions are very constructive to phenomenon understanding.

Back in figure 48, the force $F$ is proportional to fluid viscosity. This variable can be measured by defining the force required to overcome fluid friction in a film of 
known dimensions. Viscosity determined in this way is called dynamic or absolute viscosity ( $\eta$ ) and it is a function of the internal friction of a fluid.

Figure 48: Dynamic Viscosity Concept

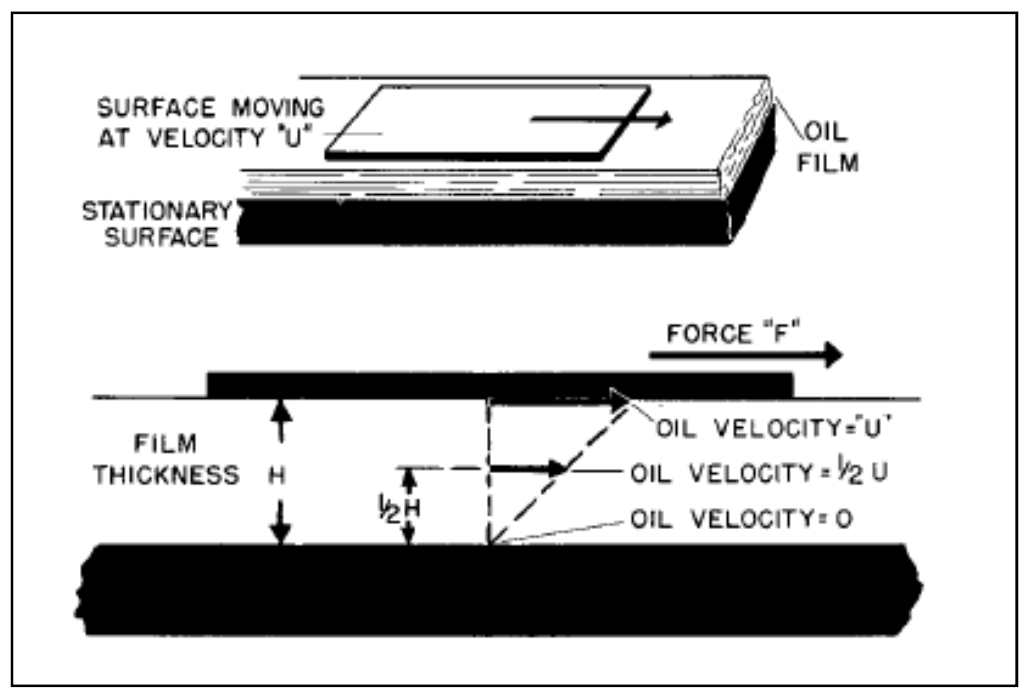

Source: Pirro et al, 2001

An expression can be defined with discussed variables: Velocity $U$ is increasing as the distance $y$ from the fixed surface is also increasing and Viscosity offers a quantity of a fluid resistance to a shearing stream. It is possible to correlate those concepts and define shear stress $(\tau)$ acting on those planes as can be seen on expression 23:

$$
\sigma_{x y}=\tau=\eta \frac{d u}{d y}
$$

The reference units used are in poise $(P)$ or centipoise $(C p ; 1 C p=0,01 P)$, or in SI units in pascal-seconds (Pa.s; $1 \mathrm{~Pa} \cdot \mathrm{s}=10 \mathrm{P})$.

Another important viscosity characterization is knew as kinematic viscosities (v) and its unit is shown in table 04 . It has been used widely due to its relation with fluid density. Basically it is the dynamic viscosity divided by its density both measured at the same temperature and in consistent units. The reference units are the stokes 
(St) or centistokes (cSt; $1 \mathrm{cSt}=0.01 \mathrm{St}$ ), or in SI units, square millimeters per second $\left(\mathrm{mm}^{2} / \mathrm{s} ; 1 \mathrm{~mm}^{2} / \mathrm{s}=1 \mathrm{cSt}\right)$. (Pirro et al., 2001).

Table 4: Dynamic and kinematics viscosity units, ASM Handbook (1992)

\begin{tabular}{|c|c|c|c|c|}
\hline Viscosity & Dimension & C.G.S & S.I & Comparison \\
\hline $\begin{array}{c}n \\
\text { dynamic }\end{array}$ & $M L^{-1} T^{-1}$ & Poise & Pascal Segundo & $1 c P_{0}=1 \mathrm{mPa} . \mathrm{s}$ \\
\hline$v$ & $P_{0}=\frac{g}{\mathrm{~cm}} \cdot \mathrm{s}$ & Pa.s $=\frac{\mathrm{Kg}}{\mathrm{m}} \cdot \mathrm{s}$ & \\
\hline $\begin{array}{c}\text { kinematics } \\
\text { kines }\end{array}$ & & Stokes $=\mathrm{cm}^{2} / \mathrm{s}$ & $\mathrm{m}^{2} / \mathrm{s}$ & $1 \mathrm{cSt}=1 \mathrm{~mm}^{2} / \mathrm{s}$ \\
\hline
\end{tabular}

Viscosity is a key aspect when choosing oil for designing a lubricate operation. A special attention ought to be given on this subject due to temperature influenced characteristics. In fact, mineral and synthetic oils viscosity decreases when the temperature increases. In this way, It is also important to take into consideration surfaces or elements in contact operational temperatures. In addition, for an appropriate lubricant selection, viscosity needs to be high enough to allow a suitable lubrication film formation, but limited to an upper value otherwise excessive oil friction losses will be verified (Hutchings, 1992).

\subsubsection{Viscosity index}

The viscosity index is a method which relates a numerical value to the temperature influence on oil property changing. This comparison is performed based on relative changes of two randomly designated sorts of oil that vary widely in this characteristic. When the temperature has low impact on viscosity change, its recognized as a High VI. On the other hand, when temperature has high influence on viscosity change, it is recognized as Low VI. Figure 49 shows the line $0 \mathrm{VI}$, related to a naphthenic oil, which viscosity is highly influenced by temperature. It's also possible to see a line $100 \mathrm{VI}$, related to a paraffinic oil, which viscosity has low temperature influence. Arbitrarily assigned 100 to the first and 100 to the second, respectively. The viscosity index (VI) of an intermediate oil, between 0 and 100 . 
Figure 49: Kinematic viscosity $x$ temperature define the Index Viscosity curve

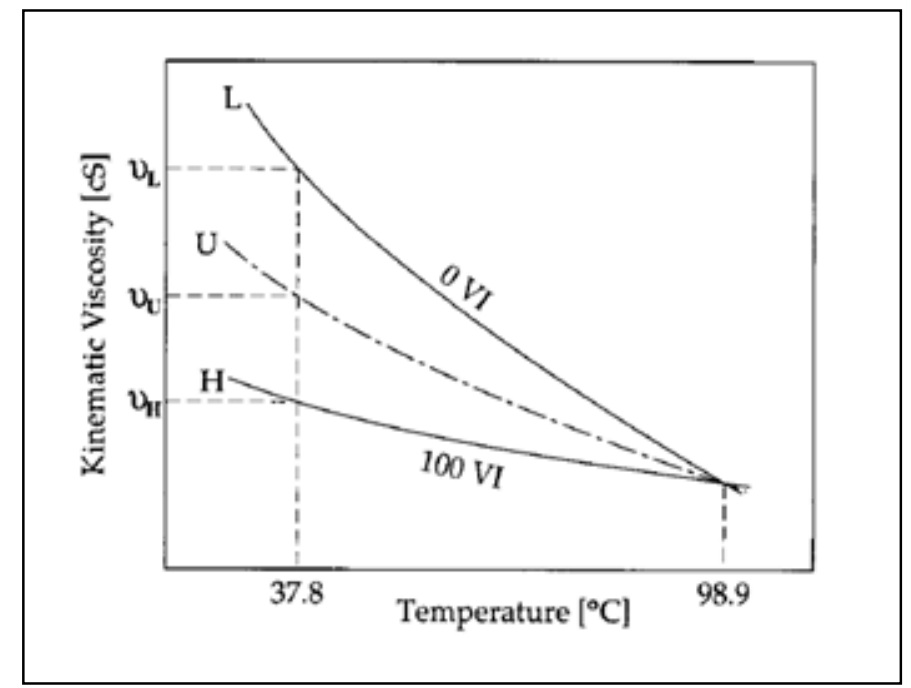

Source: STACHOWIAK et al. ,2013

\subsubsection{Lubrication Regimes}

Lubrication may undertake a variety of forms according to lubricant properties, contact geometry and loading contact and pressure. Two geometries calls attention for lubricated surfaces discussions, conformal and counterformal, as illustrated in figure 50 .

On conformal surfaces sliding elements are separated by an expressive film thickness hydrodynamically formed by surface velocities or by an externally pressurized lubricant. Basically conformal geometry is found on sliding journal and trust bearing, as much as on seals (ASTM, 1992).

On counterformal geometries surface contact areas are smaller than on conformal, this reduced contact is also recognized as Hertzian conjunction. In these geometries, lubricants will face a very high pressure and very thin film is formed, generally, similar to surfaces roughness height. Under high pressure elastic deformations of bearings surface will also takes place affecting lubricants performance as much as some lubricants properties as pressure-viscosity and temperature-viscosity coefficient have strong influence on sliding surface contacts 
temperature and friction. In this scenario, elastohydrodynamic theories has been used to define lubricant film thickness, pressure and distribution (ASTM, 1992).

Figure 50: Geometry of conformal and counterformal contacts

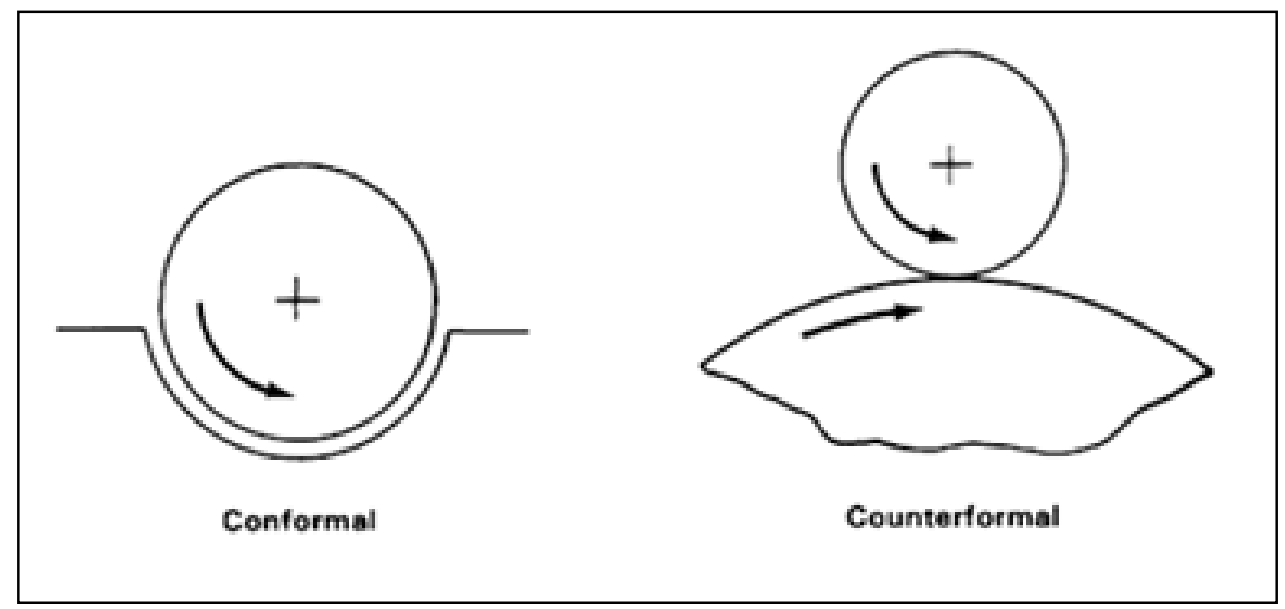

Source: ASTM, 1992)

Lubrication regime have been discussed by Hutchings (1992), Pirro et al (2001), Stachowiak et al (2005), which are: (i) The Hydrodynamic, ii) The Elastohydrodynamic, (iii) Boundary lubrication.

The Hydrodynamic is a case of conformal geometries, where both dimensions are so matched that barely exists a small gap, separating both surfaces. The narrow space among sliding surfaces area are filled with lubricant thick fluid or grease film, which is enough to ensure a full separation between asperities. As those surface are in relative motion this induces a forces on fluid which are directly related to fluid viscosity. Hence those forces will induce hydrodynamic pressure, which is responsible for supporting normal load.

The Elastohydrodynamic lubrication is a case of counterformal contact, which is based on a contact point or line. Due to this geometric approach, pressure in the contact region tends to be usually much higher than those encountered in hydrodynamic lubrication. Examples of this type of lubrication can be observed on gear teeth contacts or between a ball and inner track in a ball bearing. Both examples are based on contacts concentrated in small areas and local pressure among steel components can range up to GPa. In these settings, lubricant viscosity dependence 
on pressure shows an important function. In high pressure conditions lubricant viscosity rise up and also tends to increase film thickness beyond estimated by hydrodynamic theory.

Boundary lubrication is a case where contact between asperities will occur, due to hydrodynamics forces being unable to maintain even a EHD thin film among sliding surfaces. The low hydrodynamics forces are due to low sliding speeds or very high contact pressure. On this scenario, high friction and wear rates will take place. This aggressive scenario to sliding parts will remain if boundaries lubricants are not added. Boundary lubricants works principle is to arrange surface molecular films which has the capability of inducing repulsive forces between them. This feature provides the capability of carrying much of the load avoiding or limiting asperities contact or adhesion.

Classification methodology is based on specific film thickness $\Lambda$. The specific film thickness is an important variable associated with lubricating film ability to prevent or minimize wear or scuffing. This parameter is related to the RMS roughness of the surface tested and can be calculated as seen on equation 24 .

$$
\Lambda=\frac{H_{0}}{\sqrt{R_{q A}^{2}+R_{q B}^{2}}}
$$

Where:

$\mathrm{H}_{0}$ is the center lubricant film thickness for point contacts, $\mathrm{R}^{2}{ }_{\mathrm{qA}}$ and $\mathrm{R}^{2}{ }_{\mathrm{qB}}$ are the combined RMS surface roughness of contact surfaces $A$ and $B$.

Table 05, thus, summarizes the gear lubrication regime classification according to Hutchings (1992), Pirro et al. (2001), Stachowiak et al. (2005).

Despite of differences in classification names and deviating $\Lambda$ values for mixed lubrication limits, concepts are aligned and according to Hutchings (1992), 
Pirro et al. (2001), Stachowiak et al. (2005) and all authors also consider specific film $\Lambda$ as an important parameter for damage analysis.

Table 5: - Lubrication regimes classification for gears according to Hutchings (1992), Pirro et al. (2001), Stachowiak et al. (2005)

\begin{tabular}{|c|c|c|}
\hline $\begin{array}{c}\Lambda \text { Values film } \\
\text { classification }\end{array}$ & Regime & Observation \\
\hline$\Lambda>2$ & $\begin{array}{c}\text { Full film or } \\
\text { Full EHD }\end{array}$ & $\begin{array}{r}\text { Contact surfaces completely separate } \\
\text { by lubricant film. }\end{array}$ \\
\hline $0.7<\Lambda<2$ & $\begin{array}{c}\text { Mixed film or } \\
\text { Partial EHD }\end{array}$ & $\begin{array}{r}\text { Contact surfaces partially separated by } \\
\text { lubricant film, occurring at some points } \\
\end{array}$ \\
\hline$\Lambda<0.7$ & Boundary & Films breaks down and surfaces \\
& film & $\begin{array}{r}\text { contact occurs, predominating contact } \\
\text { metal vs. metal. }\end{array}$ \\
\hline
\end{tabular}

According to Pirro et al. (2005) fatigue life is also correlated to specific film. On figure 51 is shown an effect of $\Lambda$ on the life of a series of cylindrical roller bearing. On the right area in the graph with $\Lambda$ ranging up from 3 or 4 is seen a full EHD region and relative $L_{10}$ fatigue analyzed is the highest seen on graph. Progressively decreasing $\Lambda$, film is getting thinner and asperities starting penetrating the film and fatigue life is also decreasing. This area is represented in graph as the partial EHD.

Decreasing even more film thickness $\Lambda$ to extremes values lower than 1 , asperities will get in contact constantly. Fatigue life is fastly reduced if effective additives with antiwear are not added on oil. This discussed area is represented as boundary lubrication in the graph. These ranges for $\Lambda$ are considered to be applicable to rolling element bearings according to Pirro et al. (2005) but can also guide analysis for gear failure modes. 
Figure 51: $L_{10}$ fatigue life relative to book value as a function of specific film thickness; the data are for a series of cylindrical roller bearings

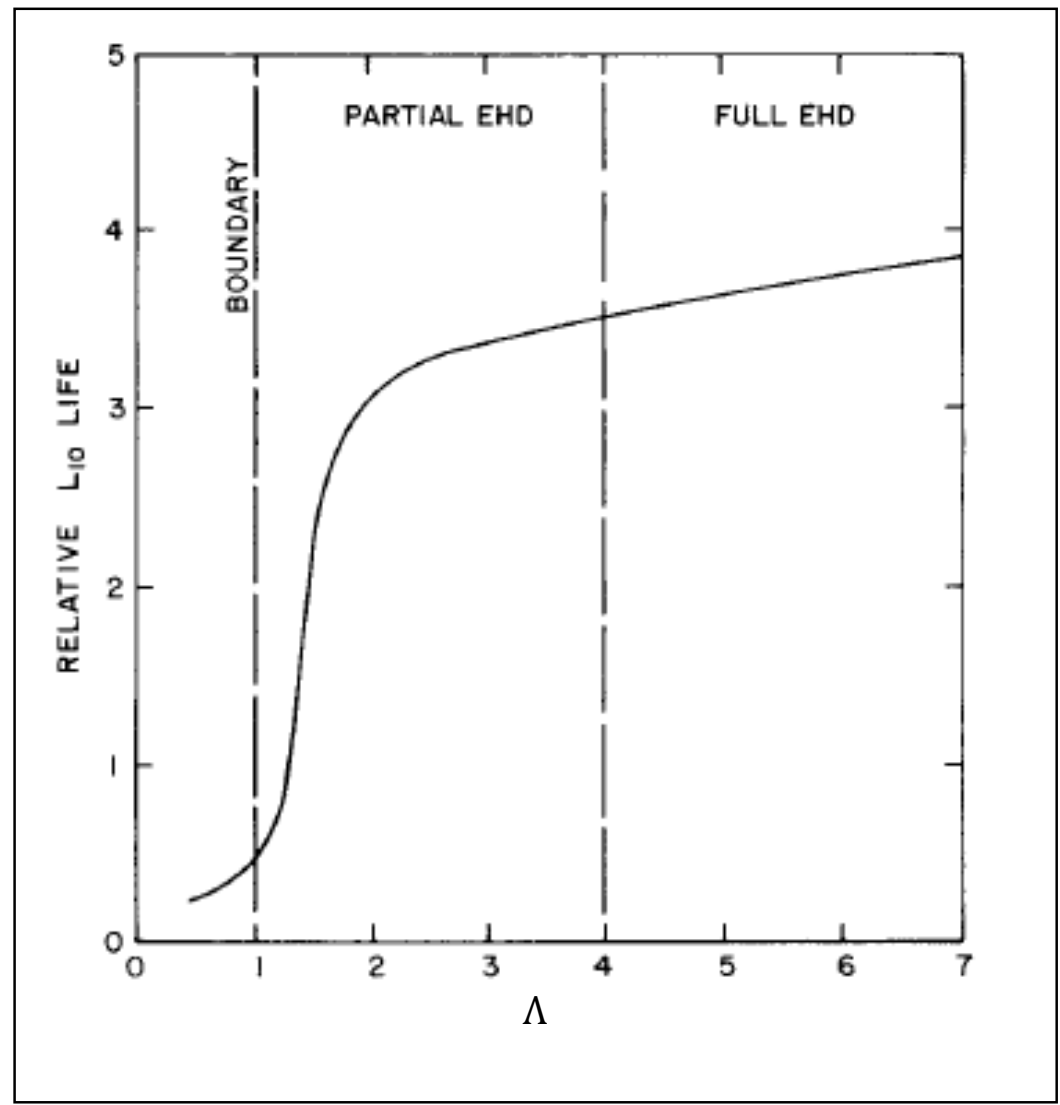

Source: Pirro et al., 2001

ASM (1996) has also analyzed the film thickness influence on gear failure modes as can be seen on figure 52 . When boundary lubrication regime is in action and pitting failure is fairly short in occurrence. As a matter of fact, macropitting might not be the first failure mode. Failures such micropitting, adhesion, or scuffing tends to happen in advance. As much $\Lambda$ growths, macropitting life expectancy is increased, and micropitting, adhesion (or scuffing) have reduced the possibility of occurrence. For higher value of $\Lambda$, macropitting may still occur, but the contact fatigue life is relatively long.

In gear fatigue evaluation it is important to observe that several variables influence the occurrence of giving failure mode. In special, teeth meshing describes a non conformal contact, which induces a high contact pressure, that associated with 
oil viscosity, temperature, sliding speed, material roughness and hardness will define the value of $\Lambda$, as seen on equation 24 .

Figure 52: Relations between contact fatigue macropitting and micropitting and film parameter

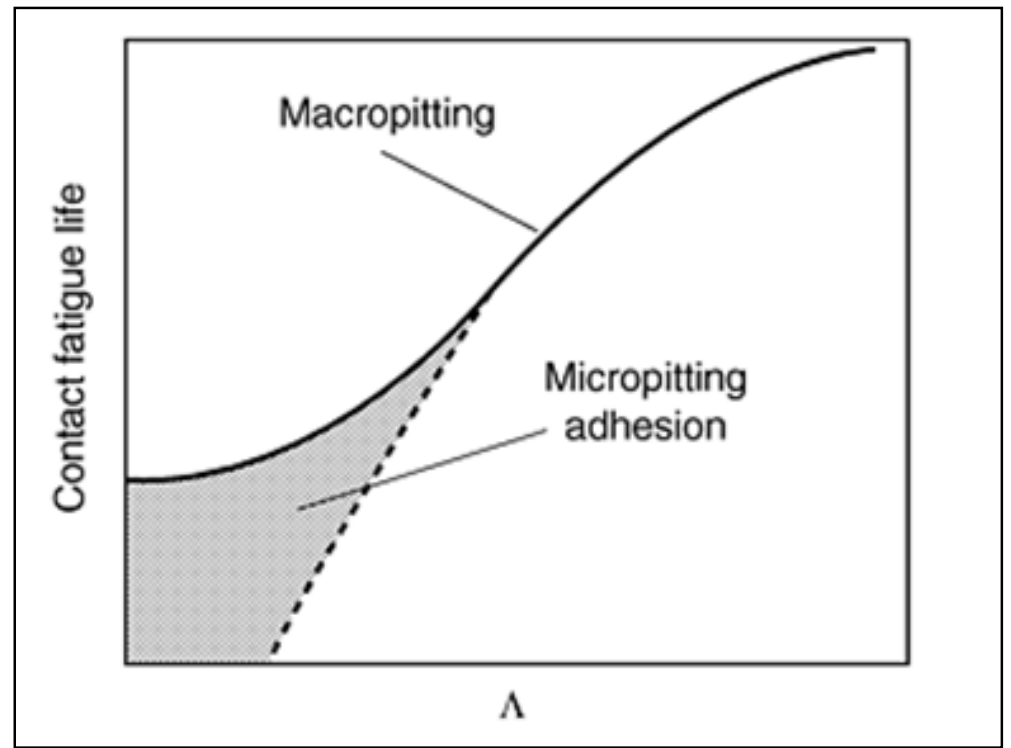

Source: ASM,1996

\subsection{MATERIAL CHARACTERISTICS AND PROPERTIES}

High capacity gears drive demands an extensive knowledge on strain effects on gears and also materials that matches with those requirements and even how to enhance material properties through bulk heat treatment and surface-layer hardening (heat treatment, coating, and mechanical hardening).

The demand in the final state or, in other words, strength values struggled are inherently connected with the selected material and its treatment and hardening. The strain complexity on gear teeth is identified on surface, even though, its case and core also ought to be a topic for special requirements. Table 06 correlates strain effects and material properties required to avoid the occurrence of specific failure modes (Linke et al., 2016). 
Table 6: Strain and Material properties (Linke et al., 2016)

\begin{tabular}{|c|c|c|}
\hline \multicolumn{2}{|c|}{ Effect of the strain } & Requirements \\
\hline \multicolumn{2}{|c|}{$\begin{array}{l}\text { Fatigue } \\
\text { Fatigue (fatigue fracture), } \\
\text { contact fatigue/surface layer fatigue (pitting, } \\
\text { micropitting) }\end{array}$} & $\begin{array}{l}\text { High endurance limit with the highest possible ductility in the } \\
\text { case region in particular, high structural homogeneity (ferrite- } \\
\text { free), continuous drop in state of hardness/strength from the sur- } \\
\text { face to the core, residual compression stresses in the surface layer }\end{array}$ \\
\hline \multicolumn{2}{|c|}{ Plastic deformation (up to overload fracture) } & High static strength with high ductility, structural homogeneity \\
\hline \multirow{3}{*}{ Wear } & Abrasion (wearing away) & $\begin{array}{l}\text { High hardness and sufficient ductility; structure with hard phases } \\
\text { (e.g., carbide, nitride) in a basic solidifying material }\end{array}$ \\
\hline & Adhesion (scuffing) & $\begin{array}{l}\text { Dissimilar case with low adhesive binding strength (low } \\
\text { predisposition to scuffing); i.e., avoidance of "purely" metal- } \\
\text { metal pairing, good running-in characteristics (contact pattem) }\end{array}$ \\
\hline & $\begin{array}{l}\text { Tribo-oxidation (wearing } \\
\text { away) }\end{array}$ & Reaction-resistant protective layer; e.g., oxidation layer \\
\hline
\end{tabular}

According to Linke et al. (2016), the gear design objective is to assure that the load capacity required for the tooth flank, tooth root and the entire cross section must be guaranteed by the material (with or without treatment suitable to the strain). In fact, the material property must be enough to exceed required strength with a grade of probability flank: $\sigma_{\mathrm{HP}}>\sigma_{\mathrm{H}}$; The Standards ISO $6336-1$ till 3 and 5 (ISO, 1996) are basically a guide procedure for these principles. 


\section{GEAR DESIGN AND FAILURE ANALYSIS}

\subsection{CALCULATION METHODOLOGIES AND SAFETY FACTOR}

ISO 6336 - 1 till 3 and 5 (ISO, 1996) establish a calculation methodology for loading capacity of spur and helical gears aiming at modeling pitting resistance and bending strength capacity. This standard requires that detail drawing or similar form are available, even though it's known that on primary designing stages all information are not available. Those missing values shall be approximated or empirical values shall be used. For some circumstances it's also appropriate to use constant factors to approximate values, this strategy also requires to assure that safety margin is adequately increased. Latter on development phases, a more accurate calculation can be provided when manufacturing phases are approaching. The correct use of this methodology requires knowledge on concerns, such as: (i) number of load repetition and allowable stress of material, (ii) failure effects, (iii) suitable safety factor, which are more detailed as follows:

(i) Number of load repetition revolutions and material capability is deeply related to application and available material selected to support this cycles. There are very important aspects on gear design.

(ii) Gear should have been object of study by an engineer to evaluate damage impact on gear and its acceptable limit. This previous study is absolutely required to study time to failure and damage limit on gears.

(iii) The least and also very important variable is related to how close will be the calculated limit and real loads. A suitable failure probability and a safety factor shall be wisely selected to run into required reliability, as much as testing conditions are controlled and gear final use are straightly similar to it, the safety factor shall be lower and more economical 
manufacturing procedures may be permissible. When safety factors are established on load, this needs to be clearly declared, and safety factor can be calculated as specific calculated load capacity divided by the specific operating load transmitted.

On ISO 6336-1 (ISO, 1996) it has also been taken into considerations influence factors applied on gear loading capacity. Those factors are result of empirical and analytical analysis and they've been grouped into factors:

(i) Those defined by gear geometry or which have been established by agreement,

(ii) Those that account for more than a few influences and which are considered as independent of each other, but, which may however influence each other into a degree that no numerical value can be assigned. These include the factors $\mathrm{K}_{\mathrm{A}}, \mathrm{K}_{\mathrm{v}}, \mathrm{K}_{\mathrm{H \alpha}}, \mathrm{K}_{\mathrm{H} \beta}$, or $\mathrm{K}_{\mathrm{Fa}}$ and the factors influencing allowable stress, which will be discussed in the sequence of the test.

\subsection{SURFACE DURABILITY CALCULUS}

Surface durability or also known as surface load capacity calculus has its foundation on contact stress $\sigma_{H}$ at the pitch point or at the inner point of single pair tooth contact. It is traditionally taken into consideration the highest one $\sigma_{H}$ for defining gear capacity. $\sigma_{H}$ shall be smaller than $\sigma_{H P}$. Three groups can be found for $\sigma_{H}$ calculation, (i) Spur gears, (ii) Helical gearing with overlap ratio $\geq 1$, (iii) Helical gearing with overlap ratio < 1 (ISO 6336-2, 1996). 
(i) Spur gears

a. spur pinion: $\sigma_{H}$ is calculated at the inner point of single pair tooth contact. If, under some conditions, $\sigma_{H}$ at the pitch point is greater, naturally it will became the determinant stress.

b. Spur wheel: $\sigma_{H}$ is calculated at the pitch point. In exceptional conditions, mainly in the case of small transmission ratios, $\sigma_{H}$ is bigger at the inner point of single pair tooth contact of the wheel and is therefore the determinant stress. On the other hand, for internal teeth, $\sigma_{H}$ is calculated at the pitch point.

(ii) Helical gearing with overlap ratio $\epsilon \beta \geq 1$

$\sigma_{H}$ is calculated at the pitch point for pinion as much as for wheel.

(iii) Helical gearing with overlap ratio $\epsilon \beta<1$

$\sigma_{H}$ is calculated by linear interpolation among two limiting values, i.e. $\sigma_{H}$ for spur gears and $\sigma_{\mathrm{H}}$,

Pinion contact stress calculus:

According to ISO 6336-2 (1996), equation 25 defines the contact stress $\sigma_{H}$ for the pinion.

$$
\sigma_{H}=Z_{B} \sigma_{H 0} \sqrt{K_{A} K_{V} K_{H \beta} K_{H \alpha}} \leq \sigma_{H P}
$$

Where:

$\sigma_{H P}$ It is the permissible contact stress. It represents the contact stress limit values rather based on materials tests using meshing gears as tests pieces. As much as gears tested and test schedules are similar to gear service and operational conditions more reliable are data achieved on testing. 
$\sigma_{\mathrm{HO}}$ is the nominal contact stress at pitch point. It is the induced stress in perfect gearing (without transmission error) by application of static nominal torque. Equation 26 defines the nominal contact stress $\sigma_{\text {HO }}$ at the pitch point.

$Z_{B} \quad$ is the single pair tooth contact factor. $Z_{B}$ transforms contact stress at the pitch point into contact stress at the inner point of single pair tooth contact on the pinion.

$\mathrm{K}_{\mathrm{A}}$ is the application factor. This factor adjusts the nominal load $\mathrm{Ft}$ in order to compensate for incremental gear loads from external sources, taking into account load increments due to externally influenced variations of input and output torque.

$\mathrm{K}_{\mathrm{V}} \quad$ is the dynamic factor. This factor takes into account the effects of gear tooth accuracy grade on load, taking into account load increments due to internal dynamic effects.

$\mathrm{K}_{\mathrm{H} \beta}$ is the face load factor for contact stress. Taking into account effects of nonuniform load distribution over the face width on the surface stress. The extent of this uneven distribution is influenced by (i) gear tooth manufacturing accuracy (lead, profile and space), (ii) alignment of the mating gears rotating axes, (iii) elastic deformations of gear units elements: shafts, bearings, housing, (iv) bearing clearances, (v) hertzian contact and bending deformation at the tooth surface, (vi) thermal deformations due to operating temperatures, (vii) centrifugal deflections due to operating speed, (viii) helix modifications including tooth crowning and relief, (ix) running effects and (x) additional shaft loads, (xi) gear geometry.

$\mathrm{K}_{\mathrm{Ha}}$ is the transverse load factor for contact stress. Taking into account effects of non-uniform load distribution of transverse direction. The extent of this uneven distribution is influenced by (i) deflections under load, (ii) profile modifications, (iii) tooth manufacturing accuracy, (iv) running-in effects. 


$$
\sigma_{H 0}=Z_{H} Z_{E} Z_{\epsilon} Z_{\beta} \sqrt{\frac{F_{t}}{d_{1} b} * \frac{u+1}{u}}
$$

Where:

$\mathrm{Z}_{\mathrm{H}} \quad$ is the zone factor. Taking into account influences of flank curvature on Hertzian contact stress at the pitch point. It's also important because this factor alters tangential force at the reference cylinder to normal force at the pitch cylinder.

$Z_{E} \quad$ is the elastic factor. Taking into account influences of material properties elasticity module $E_{1}, E_{2}$ and Poisson's ratio $v_{1}$ and $v_{2}$ on the contact stress.

$Z_{\epsilon} \quad$ is contact ratio factor. Taking into account the influence of the transverse contact and overlap ratios on the surface load capacity of cylindrical gears. In other words, effective length of the lines of contact.

$Z_{\beta} \quad$ is helix angle factor. Taking into account influences of the helix angle on surface load capacity, such as the variation of the load along the lines of contact.

$F_{t} \quad$ is defined as nominal tangential load per mesh, $F_{t}$ is derived from the nominal torque or power transmitted by gear pair and it's calculated in the transverse plane at the reference cylinder.

b is the face width, the smallest value are found at the root circles of pinion or wheel in matching, disregarding any intended crosswise chamfers or toothend rounding.

$\mathrm{d}_{1} \quad$ is the reference pinion diameter.

$u \quad$ is the gear ratio $=Z_{2} / Z_{1}$. For external gears $u$ is positive, for internal it's negative. 
According to ISO 6226-2 (ISO, 1996), equation 27 defines the contact stress $\sigma_{H}$ of the wheel.

$$
\sigma_{H}=Z_{D} \sigma_{H 0} \sqrt{K_{A} K_{V} K_{H \beta} K_{H \alpha}} \leq \sigma_{H P}
$$

$Z_{D} \quad$ is the wheel single pair tooth contact factor. $Z_{D}$ converts contact stress at the pitch point into contact stress at the inner point of single pair tooth contact on the wheel.

\subsubsection{Permissible contact Stress $\sigma_{H P}$ methodologies}

According to ISO 6336-2 (ISO, 1996), five distinct methodologies can be used to define permissible contact stress, those are known as:

(i) Method A: the permissible contact stress $\sigma_{\mathrm{HP}}$ is calculated using equation 27.

(ii) Method B: the damage curves had been written off as allowable stress numbers values $\sigma_{\text {Hlim }}$ and the limited life factors $Z_{N T}$ had been defined for several traditional gears materials and heat treatments based on gear standards tests results.

Such tested values are useful to adapt the dimensions and service conditions of the desired gear pair. This will be possible by using the influence factors for lubricant, $Z_{L}$, pitch line velocity $Z_{V}$, flank surface roughness, $Z_{R}$, work hardening, $Z_{W}$, and size, $Z_{x}$. 
(iii) Method C and D: the difference of method B is that the influence factors $Z_{L}, Z_{V}, Z_{R}, Z_{W}$, and $Z_{x}$ are determined using simplified procedures.

(iv) Method $\mathrm{B}_{\mathrm{R}}$ : the material characteristic values are defined based on rolling tribology tests such as pin on disk or disk in loaded contact. The sliding parameter should be set to characterize the in service slide and roll conditions of the tooth flanks in the pitting areas risk.

Since method B is the base methodology for gear design within FCA and also IsoCad the base methodology used to perform Contact Stress Calculation, this methodology will be discussed in detail.

\subsubsection{Permissible Contact Stress, $\sigma_{\mathrm{HP}}$, Method B}

Equation 28 is useful to calculate the Permissible Contact Stress:

$$
\sigma_{H P}=\frac{\sigma_{H L i m} Z_{N T}}{S_{H M i n}} * Z_{L} Z_{V} Z_{R} Z_{W} Z_{X}=\frac{\sigma_{H G}}{S_{H M i n}}
$$

$\sigma_{H} \operatorname{Lim}$ is the allowable contact stress number for contact.

$Z_{N T} \quad$ is the life factor. It accounts for higher contact stress that might be tolerable for a limited number of cycles as compared with allowable stress (in this case, fatigue stress limit).

$\sigma_{H G} \quad$ is the pitting stress limit $\left(=\sigma_{H}{ }^{*} S_{H M i n}\right)$.

$\mathrm{S}_{\mathrm{H} \text { Min }}$ is the minimum required safety factor for surface durability.

$Z_{L} \quad$ is the lubricant factor. It accounts for the influence of lubricant film between the tooth flank on surface durability.

$Z_{R}$ is the roughness factor, taking into account the influence of surface roughness. 
$Z_{V} \quad$ is the velocity factor, taking into account the influence of pitch line velocity.

$Z_{w}$ is the work hardening factor, taking into account the surface durability influence of meshing a hardened material.

$Z_{x} \quad$ is the size factor for contact stress, taking into account tooth size influence on the permissible contact stress. Stress level at which fatigue occurs decrease with an increase of component size. 


\section{GEAR COATING}

Coating is a technical trend to extend gear lifetime. It is always performed in a finished processed gear after the execution of heat treatment suitable for the strain cycle planned for the component. Basically, two types of coating are appropriate for gears (Linke et al., 2016):

(i) layers with low to medium hardness, which are produced by galvanized or chemical coating at temperatures lower than $100^{\circ} \mathrm{C}$;

(ii) layers of very high hardness, which are mainly produced by PVD or PACVD treatment (with temperature-time curves, that might act as heat treatment as well).

\subsection{LAYERS WITH LOW TO MODERATE HARDNESS}

The aiming of this type of coating is to avoid scuffing whereas the run-in. It is also suitable for temporarily corrosion protection. On regards of loading capacity, it's has no influence on final outcome capability. Cooper plating takes place in acidic electrolytes with controlled temperature ranging from $40^{\circ} \mathrm{C}$ to $60^{\circ} \mathrm{C}$. The produced layers thickness measures from 4 to $10 \mu \mathrm{m}$ (Linke et al., 2016).

Other coating techniques intends to improve hot scuffing loading capacity or delay its appearance as phosphating. It's is done in solution of zinc or manganese phosphate in a controlled temperature ranging from $30^{\circ} \mathrm{C}$ to $95^{\circ} \mathrm{C}$. Resins have been developed as layers for decreasing friction and increasing running-in characteristics and corrosion resistance, which are inserted in the $\mathrm{M}_{0} \mathrm{~S}_{2}$, graphite or PTFE. These anti-friction coatings are very useful to avoid direct contact of the tooth flanks. Following prior fine blasting and phosphating, the coating can be processed by immersion or spraying (Linke et al., 2016). 


\subsection{HARD MATERIALS LAYER}

The aiming in this type of coating is to provide a higher resistance to micropitting and pitting, as much as increase scuffing load capacity (through friction reduction). The layers are skinny, in general narrower than $10 \mu \mathrm{m}$ and its hardiness ranges from 1000 to $3000 \mathrm{HV}$.

The steel surface layer must have a high hardness to be coated. This is necessary to guarantee support for the hard material layer. This hard material layer and base material association needs to be carefully chosen.

The choice of the coating method is influenced by the tempering resistance of the basic material and it can be produced in following methods:

(i) Chemical vapor deposition (CVD) is a technique based on reactive gases that in contact with the base material reacts forming adherent layers. This process is executed in high temperatures ranging from $700^{\circ} \mathrm{C}$ to $1050^{\circ} \mathrm{C}$, that's why it is not suitable for case-hardened or tempering gear.

(ii) Plasma-assisted chemical vapor deposition (PACVD) is a technique similar to plasma nitriding, in which components are linked as a cathode and the recipient in the anode. The reactions required for the coating are provide in gaseous form and must be swirled around, the residual gas is pumped off. This process is executed in a temperature range of $150^{\circ} \mathrm{C}$ to $700^{\circ} \mathrm{C}$.

(iii) Physical vapor deposition (PVD) is a technique based on high energy applied on a solid coating material which is evaporated and subsequent precipitation on the component. In order to obtain a uniform coating thickness, the parts must rotate at a constant speed during the process. This process is executed in a temperature ranging from $200^{\circ} \mathrm{C}$ to $500^{\circ} \mathrm{C}$

According to Xiao et al. (2014) and Morita et al. (2016), PVD hard coatings such as, TiN, WC/C and DLC have been research focus for gears. Coated surfaces with those elements present characteristics such as: low friction coefficients, good 
wear resistance and heavy carrying capacities and can thus reduce flash temperature and extend lubricants life.

\subsection{DLC AND BALINIT ${ }^{\circledR}$ C COATING CONCEPTS AND PUBLISHED RESULTS}

DLC is a pure, metal-free, amorphous carbon coating that merely comprehend carbon and hydrogen. The final coating is very hard and compact with high residual stress, which in fact inhibits a thicker layer formation of DLC (Morita et al.. ,2016).

BALINIT ${ }^{\circledR} \mathrm{C}$ is a commercial name for W-DLC supplied by Oerlikon Balzers. It uses Physical Vapor Deposition (PVD) process at temperatures below $200^{\circ} \mathrm{C}$. WDLC coating involves a $\mathrm{Cr}$ adhesion layer adjacent to the steel substrate, followed by an intermediate transition region consisting of alternating lamellae of $\mathrm{Cr}$ and WC and an outermost W-containing hydrocarbon (W-C:H) layer (Moorthy e Shaw, 2012).

The predictable beneficial of applying coating process below $<200^{\circ} \mathrm{C}$ is that this coating process technology will not affect the base material properties such as microstructure and hardness reached after carburizing and tempering heattreatment. If those properties are kept, this process are appropriate for ordinary gear steels with low tempering temperatures $\left(<200^{\circ} \mathrm{C}\right)$ (Moorthy e Shaw, 2012).

In fact, the benefits of coating technologies has been shared on international magazines, for example, Moorthy et. al (2012) demonstrated that WC- containing DLC coating (W-DLC) of gears provides satisfactory wear resistance even at high loads (1.4 GP), the tests were performed on FZG gear-scuffing test. Moorthy e Shaw (2012) have also stated on this same paper that W-DLC enhanced contact fatigue performance on FZG micropitting test.

Xiao et al. (2014) have stated that WC/C and DLC coatings benefits appeared to increase with decrease in lubricating performance. Therefore, WC/C and DLC coating are suitable for high-speed and heavy duty gear transmission. 


\section{THEORETICAL FAILURE ANALYSIS}

\subsection{RELIABILITY CONCEPT.}

According to Lewis (1994), reliability is the probability that a system will not fail under some specified settings. Hence, the primary focus on reliability is on failures and their prevention. It's also important a good overview of the differences in time expectation and main reason for failures. Usually the failure can be classified as: (i) variability due to manufacturing processes is likely to failures occurrences early in product life. These failure are known as early or infant mortality failures. (ii) The variability triggered by the functional environment is likely to random failure occurrences, as its occurrence is independent of the product's age. (iii) Finally, product life consumption is likely to failures occurrence after longer operational journeys, and it's known as aging or wear failures.

Reliability data are based on components or system failures observation. In general those failures are observed during life tests, with a significant number of items tested till a noteworthy number of failures occur. Nevertheless, life tests regularly require expressive resources to perform defined experiments. Since destructive tests and several specimens are required to support an adequate statistics approach. Traditionally, an expressive amount of money is required to obtain expressive results. Time is also an important resource to take into consideration as long as it will be required to achieve failure mode. This resource demand can be reduced if acceleration methods are applicable or if the component or system is already in use field data will be appreciated. After data collection, the subsequent analysis are performed by sampling techniques that support the probability estimation considering component or system level (Lewis, 1994).

\subsection{CONTINUOUS RANDOM VARIABLE}

According to Montgomery et al. (2003), a random variable is a function that attributes a real number to each outcome in the sample space of a random trial. The 
properties of a continuous random variable are defined in probabilities aspects. Such as, $\mathrm{P}\{\mathbf{X}<\mathbf{X}\}$ define the probability that $\mathbf{X}$ has a value less than $\mathrm{X}$. Likewise, $\mathrm{P}\{\mathrm{a}<\mathbf{X}$ $<b\}$ is the probability that $\mathbf{X}$ has a value among $a$ and $b$.

Frequently, these two probabilities describes a random variable: (i) the probability that $\mathbf{X}$ has a value less than or equal to $x$, known as the cumulative distribution function, or just CDF , defined on equation 29, and (ii) the probability that $X$ is among $x$ and $x+\Delta x$ as $\Delta x$ turns out to be infinitesimally and is defined by equation 30 . Where $f(x)$ is the probability density function, or just PDF.

$$
\begin{gathered}
F(x)=P\{x \leq x\} \\
f(x) \Delta x=P\{x \leq x \leq x+\Delta x\}
\end{gathered}
$$

It's also possible to relate those equations, in fact, if $\mathbf{X}$ vary from any values $-\infty \leq$ $\mathbf{X} \leq+\infty$, Consequently, CDF is just the integral of the PDF over all $\mathbf{X} \leq x$, as seen on equation 31.

$$
F(x)=\int_{-\infty}^{x} f\left(x^{\prime}\right) d x^{\prime}
$$

$f(\mathrm{x})$ and $\mathrm{F}(\mathrm{x})$ are standardized as :

$$
\begin{gathered}
F(x)=\int_{-\infty}^{x} f\left(x^{\prime}\right) d x^{\prime} \\
\int_{a}^{b} f(x) d x=P\{a \leq x \leq b\}
\end{gathered}
$$




$$
\begin{gathered}
P=\{-\infty \leq X \leq \infty\}=1 \\
\int_{-\infty}^{+\infty} f(x) d x=1
\end{gathered}
$$

Then, setting $\mathbf{X}=\infty$ in Equation 31, the corresponding condition on the CDF is

$$
F(\infty)=1
$$

Complementary cumulative distribution function or CCDF, is defined as seen on equation 38 with a tilde defining it is a complementary distribution. Since $\mathbf{X}>\boldsymbol{x}$ is the same as $\mathbf{X}$ not $\leq X$.

$$
\begin{gathered}
\tilde{F}=P\{\boldsymbol{x}>x\} \\
\tilde{F}=\int_{x}^{\infty} f\left(x^{\prime}\right) d x^{\prime}=1-\int_{-\infty}^{x} f\left(x^{\prime}\right) d x^{\prime} \\
\tilde{F}=1-F(x)
\end{gathered}
$$




\subsection{CHARACTERISTICS OF A PROBABILITY DISTRIBUTION}

Knowing all details of the probability density function of a random variable is frequently rare. For such situation, knowing integral properties such as mean and variance helps the analysis. (i) The mean or expectation value of $\mathbf{X}$ is defined by equation 40 and (ii) the variance, which is a quantity of the dispersion of values about the mean is given by equation 41 :

$$
\begin{gathered}
\mu=\int_{-\infty}^{+\infty} f(x) d x=1 \\
\sigma^{2}=\int_{-\infty}^{+\infty}(x-\mu)^{2} f(x) d x
\end{gathered}
$$

Any function, $\mathrm{g}(\mathrm{x})$, that is to be an average of the values of a random variable is defined by equation 42 and the amount $E\{g(x)\}$ is stated to as the projected value of $g(x)$ (Lewis, 1994).

$$
E\{g(x)\} \equiv \int_{-\infty}^{+\infty} g(x) f(x) d x
$$

If an infinitely large number of values of $\mathbf{X}$ are sampled from $f(\mathbf{x})$ and calculated $\mathrm{g}(\mathbf{x})$ for each one of them, the average of these values would be $E\{g\}$. Singularly, the $n^{\text {th }}$ moment of $f(x)$ is defined to be as shown on equation 43 .

$$
E\left\{x^{n}\right\}=\int_{-\infty}^{+\infty} x^{n} f(x) d x
$$


Based on this, $E\left\{x^{0}\right\}=1$, and the mean is just the first moment, as shown on equation 44:

$$
\mu=E(x)
$$

Likewise, the variance can be expressed as the first and second moments.

$$
\begin{gathered}
\sigma^{2}=E\left\{(x-\mu)^{2}\right\}=E\left\{x^{2}-2 x \mu+\mu^{2}\right\} \\
\sigma^{2}=E\left\{x^{2}\right\}-2 E\{x\} \mu+\mu^{2} \\
\sigma^{2}=E\left\{x^{2}\right\}-E\{x\}^{2}
\end{gathered}
$$

Two additional properties can characterize the PDF of a random variable, (i) the skewness which is defined by equation 48 , represents a measure of the asymmetry of a PDF around the mean and (ii) the kurtosis defined by equation 49 . The kurtosis, is a measure of the spread of $f(x)$ about the mean (Lewis, 1994).

$$
\begin{aligned}
& s k=\frac{1}{\sigma^{3}} \int_{-\infty}^{+\infty}(x-\mu)^{3} f(x) d x \\
& k u=\frac{1}{\sigma^{4}} \int_{-\infty}^{+\infty}(x-\mu)^{4} f(x) d x
\end{aligned}
$$




\subsection{RELIABILITY AND RELATED DISTRIBUTION}

Reliability analysis are broadly based on continuous random variables for the account of survival times, system loads and capacities, repair rates, and a diversity of another occurrences. Additionally, a considerable amount of standardized probability distributions are engaged on modeling behavior of studied variables (Montgomery, 2003).

\subsubsection{The Normal Distribution}

According to Lewis (1994), this distribution represents the most widely used in statistics studies, frequently mentioned as the Gaussian distribution. If a random variable $\mathbf{X}$ might be expressed as a sum of the random variables, $\mathbf{x}_{i}, \mathrm{i}: 1,2, \ldots, \mathrm{N}$ where no one of them is dominant, then $\mathbf{X}$ can be described as a normal distribution, even though the $\mathbf{X}_{\mathbf{i}}$; are described by non-normal distributions that may not even be the same for different values of i. Considering the following function of the random variable $\mathbf{X}$,

$$
f(x)=\frac{1}{\sqrt{2 \pi b}} e^{\left[-\frac{1}{2}\left(\frac{x-a}{b}\right)^{2}\right]}, \quad-\infty \leq x \leq \infty
$$

This function completely attends the conditions for a probability density function. (i) $f(x) \geq 0$ for all $x$. (ii) Solving the integral, shows that the condition on the PDF given by Eq. 51 is met.

$$
\int_{-\infty}^{+\infty} \frac{1}{\sqrt{2 \pi b}} e^{\left[-\frac{1}{2}\left(\frac{x-a}{b}\right)^{2}\right]}=1
$$

A unique feature of the normal distribution is that the mean and variance appear explicitly as the two parameters a and b. As shown respectively on equation 52 and 53, as much as illustrated on figure 53 (Lewis, 1994): 


$$
\begin{gathered}
\mu=\int_{-\infty}^{+\infty} d x \frac{x}{\sqrt{2 \pi b}} e^{\left[-\frac{1}{2}\left(\frac{x-a}{b}\right)^{2}\right]}=a \\
\sigma^{2}=\int_{-\infty}^{+\infty} d x \frac{(x-\mu)^{2}}{\sqrt{2 \pi b}} e^{\left[-\frac{1}{2}\left(\frac{x-a}{b}\right)^{2}\right]}=b^{2}
\end{gathered}
$$

Therefore, the normal PDF can be written directly in terms of the mean and variance as shown on equation 54 .

$$
f(x)=\frac{1}{\sqrt{2 \pi b}} e^{\left[-\frac{1}{2}\left(\frac{x-\mu}{\sigma}\right)^{2}\right]}, \quad-\infty \leq x \leq \infty
$$

Likewise, the CDF equivalent is shown on equation 55.

$$
F(x)=\int_{-\infty}^{x} \frac{1}{\sqrt{2 \pi b}} e^{\left[-\frac{1}{2}\left(\frac{x^{\prime}-\mu}{\sigma}\right)^{2}\right]} d x^{\prime}
$$

Figure 53: Normal probability density functions for selected values of $\mu$ and $\sigma^{2}$

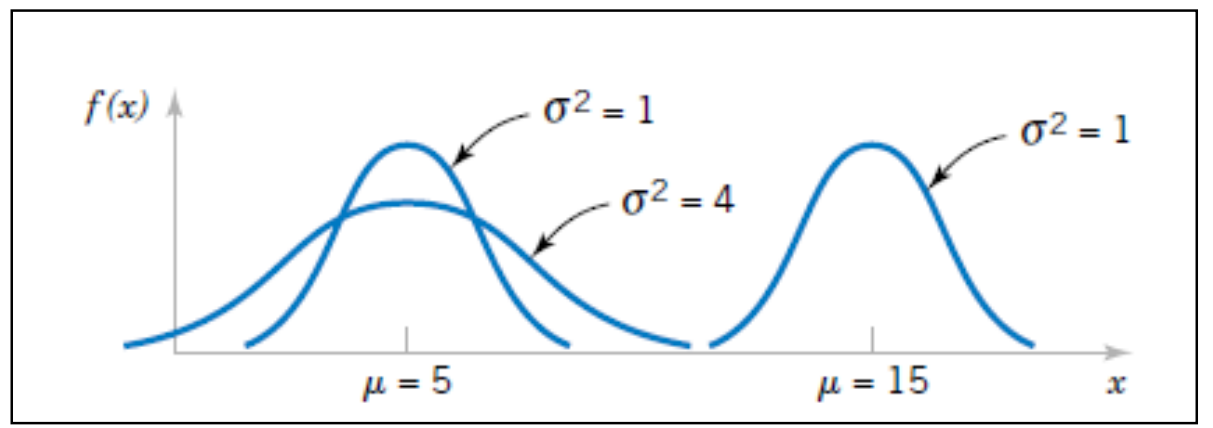

Source: Montgomery, 2003 


\subsubsection{The Lognormal Distribution}

According to Lewis (1994) Lognormal distributions are usually applied in reliability engineering to describe failure produced by fatigue, uncertainties in failure rates, and a range of other phenomenon. It has the property that if variables $x$ and $y$ have lognormal distributions, the product random variable $z=x y$ is also lognormally distributed. This distribution represents a second more often seen condition, it involves a random variable $\mathbf{Y}$ that is a product of the random variables $\mathbf{Y}_{\mathbf{i}}$. For example:

$$
\ln y=\ln y_{1}+\ln y_{2}+\cdots+\ln y_{N}
$$

The equivalence to the normal distribution is easily seen. If none terms on the righthand side has a dominant effect, then In y should be distributed normally. Thus, equation 61 defines. In other words, $x$ is distributed normally and $y$ is distributed lognormally. The first step is write the normal distribution for $x$, then the lognormal distribution for $\mathrm{y}$ is achieved. In which, $\mu_{\mathrm{x}}$ represents the mean value of $\mathbf{X}$, and $\sigma_{x}^{2}$, represents the variance of the distribution in $\mathbf{X}$.

$$
\begin{gathered}
x=\ln y \\
f_{x}(x)=\frac{1}{\sqrt{2 \pi} \sigma_{x}} e^{\left[-\frac{1}{2 \sigma_{x}{ }^{2}}\left(x-\mu_{x}\right)^{2}\right]}
\end{gathered}
$$

Assuming that $x$ be the natural logarithm of the variable $y$, aiming to calculate the PDF in $y$, thus aiming to eliminate $X$, it's defined $x=\ln y$. Equations 63 to 65 show those steps.

$$
f(y)=f_{x}(x)\left|\frac{d y}{d x}\right|
$$




$$
\begin{gathered}
\frac{d y}{d x}=\frac{d}{d y} \ln y=\frac{1}{y} \\
\mu_{x}=\ln y_{0} ; \quad \sigma_{x}=w \\
f(y)=\frac{1}{\sqrt{2 \pi} w_{y}} e^{\left[-\frac{1}{2 w^{2}}\left(\ln \frac{y}{y_{0}}\right)^{2}\right]}
\end{gathered}
$$

Integrating over $y$ with a lower limit of $y=0$ the resultant CDF is found. The outcome might be expressed in terms of the standardized normal integral as seen on equation 71. The CDF and PDF for the lognormal distribution are plotted as function of $y$, as an example shown on figure 54 (Lewis, 1994).

$$
F_{y}(y)=\Phi\left[\frac{1}{w} \ln \frac{y}{y_{0}}\right]
$$

In this distribution $\Phi$ and $w^{2}$ are the parameters, however attention is required to interpret that these are the mean and variance of the normal random variable $\mathrm{W}$. The mean of lognormal distribution may be obtained by equation 64 and the variance by equation 65 (Montgomery, 2003).

$$
\begin{gathered}
\mu_{y}=y_{0} e^{\left(\frac{w^{2}}{2}\right)} \\
\sigma^{2}=y^{2} e^{\left(w^{2}\right)\left[e^{w^{2}-1}\right]}
\end{gathered}
$$


Figure 54: Lognormal probability density functions with $\theta=0$ for selected values of $w^{2}$

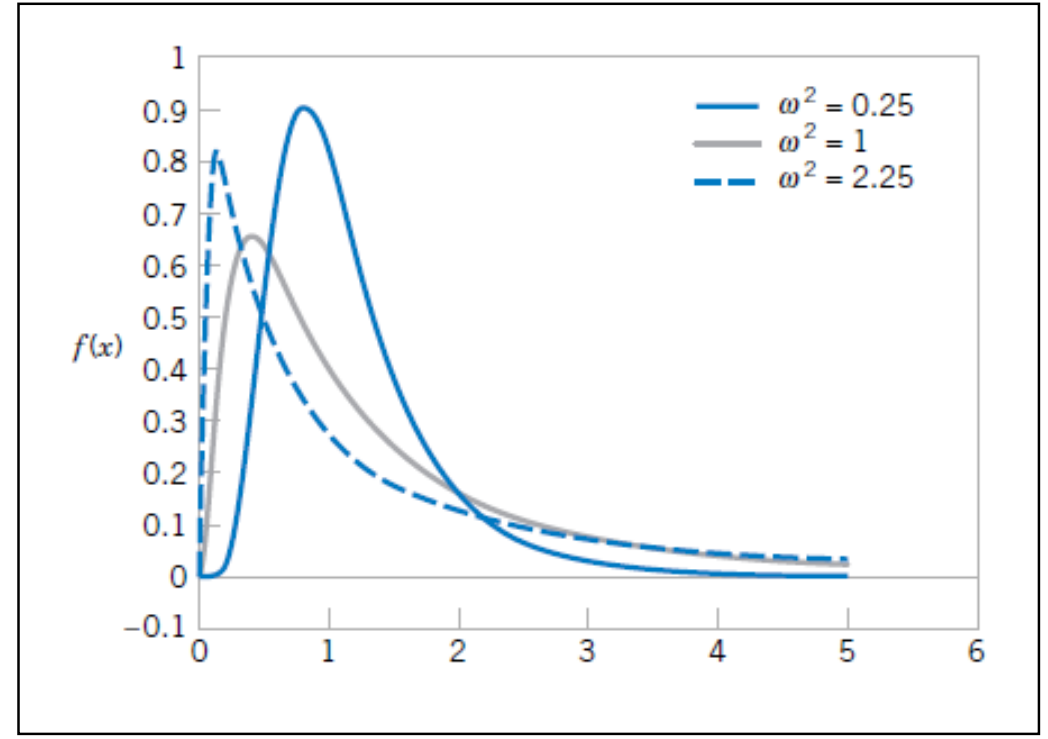

Source: Montgomery, 2003

\subsection{WEIBULL DISTRIBUTION}

The Weibull distribution is usually applied on modeling time to failure of several physical systems. The distribution parameters offer an appropriate deal of flexibility to model systems in which: (i) the number of failures increases with time, as bearing wear, (ii) decreases with time as semiconductors, (iii) or remains constant with time, as failures triggered by external shocks to the system. (Montgomery, 2003).

According to Montgomery (2003), the cumulative distribution function, CDF, is calculate by equation 66 . Where $\delta$ is the scale and $\beta$ is the shape parameter. The derivate may perform as indicated on equation 67 to obtain the PDF.

$$
\begin{gathered}
F(x)=1-e^{\left[-\left(\frac{x}{\delta}\right)^{\beta}\right]}, \quad 0 \leq x \leq \infty \\
f(x)=\frac{\beta}{\delta}\left(\frac{x}{\delta}\right)^{\beta-1} e^{\left[-\left(\frac{x}{\delta}\right)^{\beta}\right]}, \quad 0 \leq x \leq \infty
\end{gathered}
$$

The mean and the variance of the distribution are obtained from equations 68 and 69 respectively: 


$$
\begin{gathered}
\mu=\delta \Gamma\left(1+\frac{1}{\beta}\right) \\
\sigma^{2}=\delta^{2} \Gamma\left(1+\frac{2}{\beta}\right)-\delta^{2}\left[\Gamma\left(1+\frac{1}{\beta}\right)\right]^{2}
\end{gathered}
$$

The complete gama function $\Gamma(v)$ is defined by the integral seen on equation 70 .

$$
\Gamma(v)=\int_{0}^{\infty} \zeta^{v-1} e^{-\zeta} d \zeta
$$

Figure 55: Weibull probability density functions for selected values of $\delta$ and $\beta$

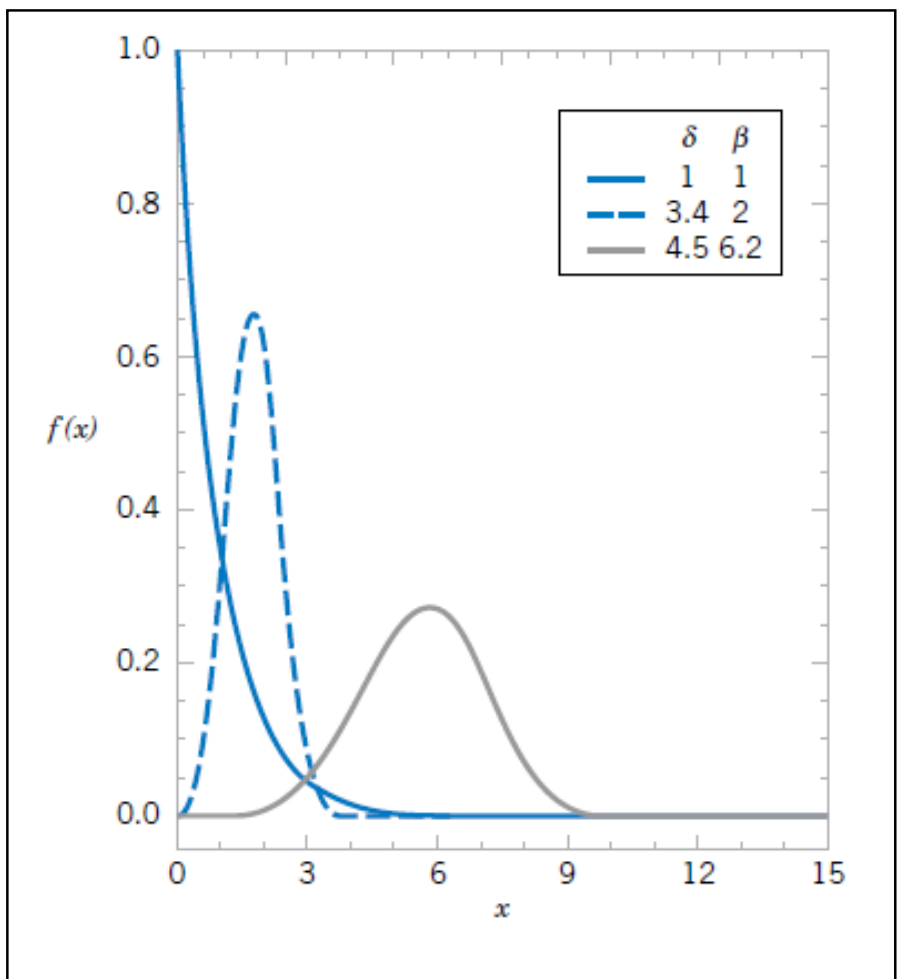

Source: Montgomery, 2003 


\section{MATERIALS AND METHODS}

The proposed methodology used to guide the experiments aiming to answer the main objective of this research is based on five steps, each step comprehends a group of activities as show on Figure 56.

Figure 56: Methodologies Schematic Overview

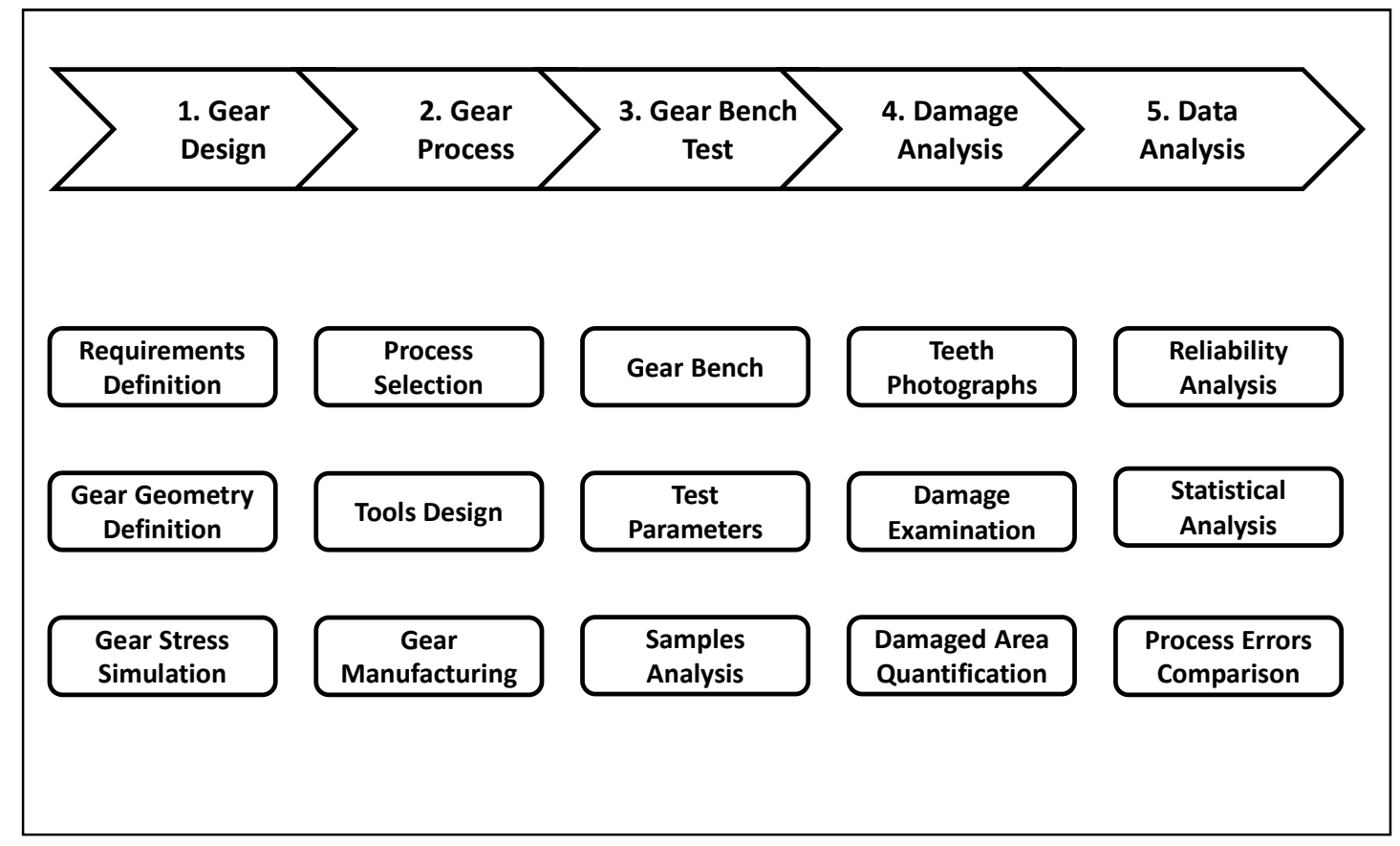

Source: Elaborated by the author

\subsection{GEAR DESIGN}

The gear design involves some important activities which correlates: (i) requirements with (ii) geometry and base material definition and (iii) stress simulation. This below listed activities interact with each other for a modern gear design. 
i) Gear Requirements defines application characteristics in which the element will be submitted to. In this research the requirements definitions amis to design a test gear with similar dynamic characteristics of FCA $1^{\text {st }}$ gear. These dynamic characteristics involves Contact Stress, Bending Stress and Sliding Speed.

ii) Gear Geometry Definition will be based on FZG gear pitting type $C$ to be used as a sample for Pitting evaluation as much as maintain bench test assembly characteristics. Material definition requirement is to keep the same used on FCA transmission $1^{\text {st }}$ gear, to maintain material base properties.

iii) Gear Stress Simulation is crucial for gear design. This research is supported by IsoCad software, which was internally developed at FCA. The software methodology is based on ISO 6336 1-3,5 (1996) standard Method B procedures for stress analysis, considering contact and bending stress simulation. All gears under evaluation will be submitted to stress simulation. Such analysis will be helpful to explore different gear geometries for the gear test set under development, as much as to evaluate variables influence on Stress. Stress simulation will also be performed on FZG gear pitting type C (FZG, 1992) and on FCA $1^{\text {st }}$ gear to allow comparisons between those samples.

\subsection{GEAR MANUFACTURING PROCESS}

It involves some important activities which correlates (i) process selection, (ii) tools manufacturing and (iii) gear manufacturing. Those listed activities below interact with each other to obtain a final gear surface geometry and outcome quality.

i) Process Selection in the present study will be kept the same process used on FCA $1^{\text {st }}$ gear. This requirement induced the choice of $\mathrm{Hob}$ and Shaving which are capable of delivering the designed surface roughness and set quality. 
ii) Tool Design was done by traditional gear machining tools suppliers. Those selected suppliers have relevant experience on Hob and Shaving design as much as on those tools manufacturing.

iii) Gear manufacturing was done by an auto industry prototype supplier, which manufacturing process is equal to ordinary industry gears process. The selected manufacturing supplier needs to be capable of delivering the designed surface roughness and set quality.

\subsection{GEAR BENCH TEST}

Gear Bench test involves some important activities such as (i) Bench Rig selection, (ii) Test parameters definition for this research and (iii) Samples Analysis. Those below listed activities have interactions for experimental gear bench design and test definition.

i) Bench Rig:

The Bench Rig defined for this research is a modified Back to Back test rig due to the capability of controlling test parameters and schedule defined for this research. The test rig can be seen on figure 57 and it's located at UTFPR Laboratory. On this equipment the desired torque can be easily adjusted according to loads previously defined by FZG standards (ASTM, 2008). The mechanisms that allow such changes are the dead weights and a lever applying the loads defined for the test. The applied load is hold by a clutch mechanism and security fasteners which guarantee that the load will not change and induce a variability on test. The housing shown on Figure 57(b) allocates the gears under test. Two parallel axles accommodate those gears, guarantee their $91.5 \mathrm{~mm}$ of center distance and, naturally, connect them the power source. The power induced by the electric motor will be transmitted through the ring to the pinion to generate the cycles defined for the test. A frequency inverter was used to control rotation, avoiding cycles variability. Before each test run, aiming to avoid variability, the rotation was adjusted by a laser tachometer, the bearings and sealings are inspected to guarantee that no leakage was occurring. 
In the gear housing shown on figure 57 (b) an oil heater was added in order to elevate the oil temperature to $90^{\circ} \mathrm{C}$ before test. It was also designed and added a cooler to preserve the temperature at $90^{\circ} \mathrm{C}$ during tests reducing oil viscosity variation and its effects on test results.

Figure 57: Back to Back Bench Test picture illustrating gears setting under test at UTFPR.

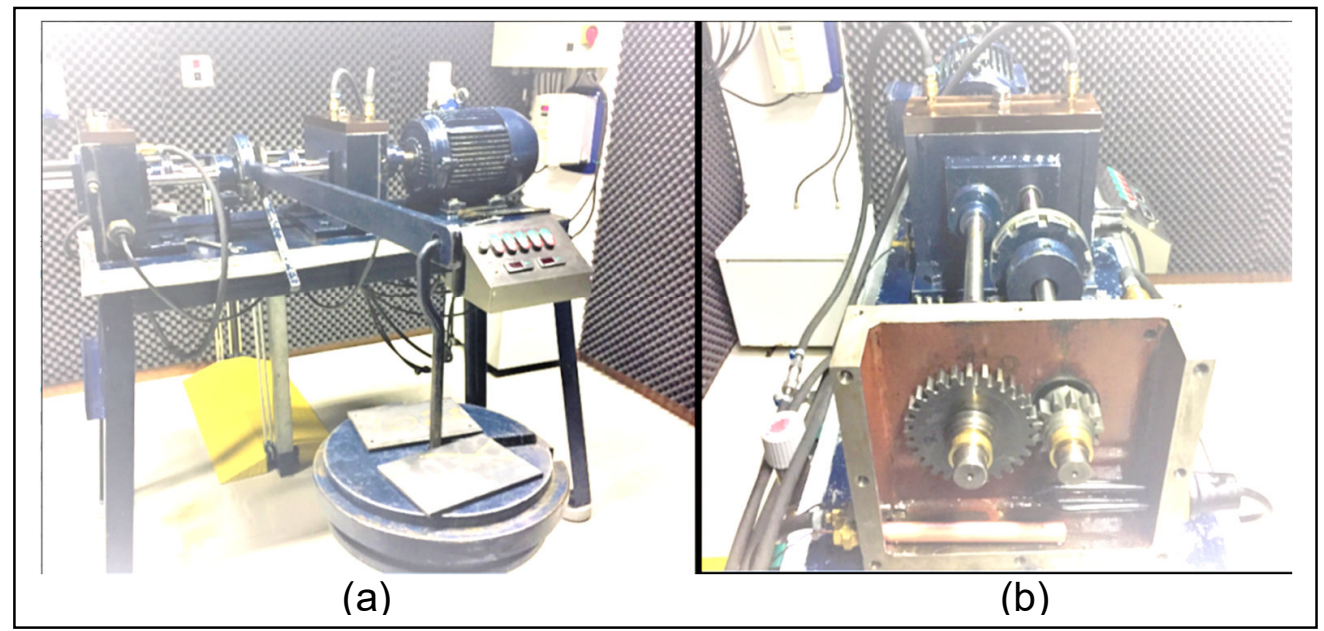

Source: Elaborated by the author

ii) Test Parameters:

ii.a) Oil Parameters, for this research the use of the same applied on FCA gear box is essential. The decision of using the same oil used on FCA gearbox oil, which is ZC 601FF (SAE 75W) and also known as low friction oil, is supported by the aim of eliminating oil properties influence on this research and allow a comparison with the FCA gear box fatigue failure. This oil viscosity and viscosity index have already been characterized in recent investigations and will be presented in this research. The defined oil temperature needs to cover the worst durability bench test conditions for FCA gear box which already considers vehicle usage temperature. 
ii.b) Test schedule definition could be based on traditional FZG methodology such as defined on ASTM D5182 (2008) to evaluate scuffing or also based on published papers such as those presented by Aslantas et al. (2004), Höhn et al. (1999), Muraro et al. (2012) and Moorthy e Shaw (2012), aiming to evaluate pitting. Nevertheless, on this research a test procedure was designed based on ISO 6336 procedures for fatigue evaluation and also to control magnitude of contact stress.

ISO 6336-5 (1996) procedure defines material capability based on experimental data, which is an important input for gear durability analysis. Therefore, for the selected material and for the chosen heat treatment, the referred standard indicated that $1.5 \mathrm{GPa}$ contact stress magnitude allows $10^{9}$ cycles till contact fatigue occurrence with limited pitting and considering life factor equal to 1 (ISO 6336 parts 1,2,3 and 5, 1996).

This stress level and cycles are traditionally used on fatigue analysis for gears, but it is still lower than the real contact stress acting on gear tooth at gear box. Therefore, three main reasons could be raised up for increasing it: (a) Set contact stress magnitude as close as possible to real values calculated for first gear at the gearbox; (b) Increased contact stress allows the use of ISO 6336-2 (1996) with a life factor 1.6 (c) A higher stress magnitude allows a reduced number of cycles until fatigue failure, which means a reduced time to failure occurrences. The reduction of time to failure facilitates the control of test parameters such sliding speed and load during the test avoiding their variability, (iv) As a consequence of reduced time to failure, the time to execute the experiment is reduced avoiding the deterioration of machine test performance during tests.

Back on ASTM D5182 (2008), K10 load stage induces the contact stress required to gear test as will be seen on details on next topic. This load stage induces $372.6 \mathrm{Nm}$ torque, which value was used as input on stress simulation of the new gear under design. The obtained stress magnitude of $2.4 \mathrm{GPa}$ is exactly the stress value applying life the factor 1.6 defined on ISO 6336-2 (1996) for life testing with limited failure by pitting. Finally, the stress 
magnitude is also close to the value defined for current gear box design. Therefore, the contact stress required is feasible using load stage K10 of FZG tests reports.

The tests have been executed in steps to allow intermediate test cycle results records. So, the first step K9 at $1750 \mathrm{rpm}$ for one hour was planned to be smaller to register initial damage appearance and right after that analysis, the gears returned to bench test. On the next step, gear tooth faced a load stage increase (to K10 load) and run 3 extra hours on test bench. Once again, the gear set was disassembled and gear teeth flanks image registered. On the last stage, the load remained at $\mathrm{K} 10$, and the gears run more 3 hours on test bench. After that the gear set was disassembled for the last time and had teeth flank images registered again. The planned and realized test schedule induces the following duty cycles: (i) K9 on $1750 \mathrm{rpm}$ run for $1.05 \times 10^{5}$ cycles and two steps of $\mathrm{K} 10$ load performed by $3.15 \times 10^{5}$ cycles. The test parameters are shown on Table 7.

Table 7: test parameters and schedule defined for gear experimental tests.

\begin{tabular}{|l|c|c|c|}
\hline Test Parameters & $1^{\text {st }}$ Step (K9) & $2^{\text {nd }}$ Step (K10) & $3^{\text {rd }}$ Step (K10) \\
\hline Oil Temperature $\left({ }^{\circ} \mathrm{c}\right)$ & 90 & 90 & 90 \\
\hline Testing timing (hours) & 1 & 3 & 3 \\
\hline Speed (rpm) & 1,750 & 1,750 & 1,750 \\
\hline Contact stress (MPa) & 2,185 & 2,427 & 2,427 \\
\hline Number of cycles & $1.15 \times 10^{5}$ & $3.05 \times 10^{5}$ & $3.05 \times 10^{5}$ \\
\hline
\end{tabular}

iii) Sample Analysis is splited in before and after Test aiming to collect relevant information for durability and reliability analysis.

iii.a) Samples Analysis Before Test for Material and Geometry Characterization.

- Base Material Chemical Analysis to confirm gear material conformity according to specifications. This analysis was done in FCA Material laboratory using optical emission spectrometer according to ISO 14707 (2015). 
- Tooth Hardness, case depth and core hardness values were performed on FCA Material laboratory using equipment and methodology according to ISO 6508-1 (2016).

- Gear Flank Surface Roughness Measurement was executed at FCA Metrology laboratory using Roughness Gauge MAHR PRK and according to ISO 1302 (2002) and 4287 (2002).

Ring and pinion roughness measurements were also executed on designed gears following the cross and longitudinal direction. The cross direction represents the contact path, which starts from the root, following to the tip of the flank as represented on figure 58. The equipment used on the measurement was a Roughness Gauge MAHR PRK.

Figure 58: Schematic view of measurement direction

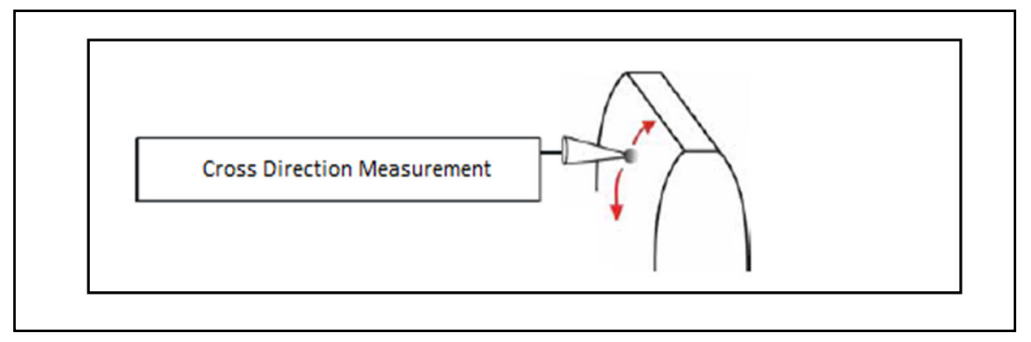

Source: Elaborated by the author

- Gear Tooth Profile Graph was measured at FCA Metrology Laboratory using Wenzel Gear Tech.

Another important steps in sample characterization are gear parameters measurements evaluation previous to tests. This evaluation was done at FCA Gear Metrology Laboratory using Wenzel Gear Tech equipment. This is a very expensive, dedicated and reliable equipment, which is currently used for gears process control. In a current process evaluation, there are several check points between process steps. But in special, right after heat treatment, some samples are daily randomly taken to check gears sizing to evaluate process errors. 
It is important to highlight that all gears used for this research after manufactured and heat treated were inspected with this same equipment to evaluate macro geometry sizing, as much as to measure process deviations or process errors. Such process errors measurements are key to the objective of this work. To perform a good inspection, pinion teeth were previously numbered during production by a stamping process to easily identify samples. As its measurements is very useful, it was taken before and after tests using Wenzel WGT 350, which measurement is taken by contact and the values read were compared to the $3 \mathrm{D}$ model upload in the software.

The Wenzel WGT 350 is a high accuracy gear inspection center. All axes are made from natural dark granite, guaranteeing excellent thermal behavior and air bearings are used on all axes to ensure smooth running and high accuracy performance. The WGT has a fully counterbalanced tailstock allowing support of pinion gears, tools and shafts. Figure 59 shows a picture of the errors measurement machine. 
Figure 59: shows Wenzel WGT 350 used on gear measurement.

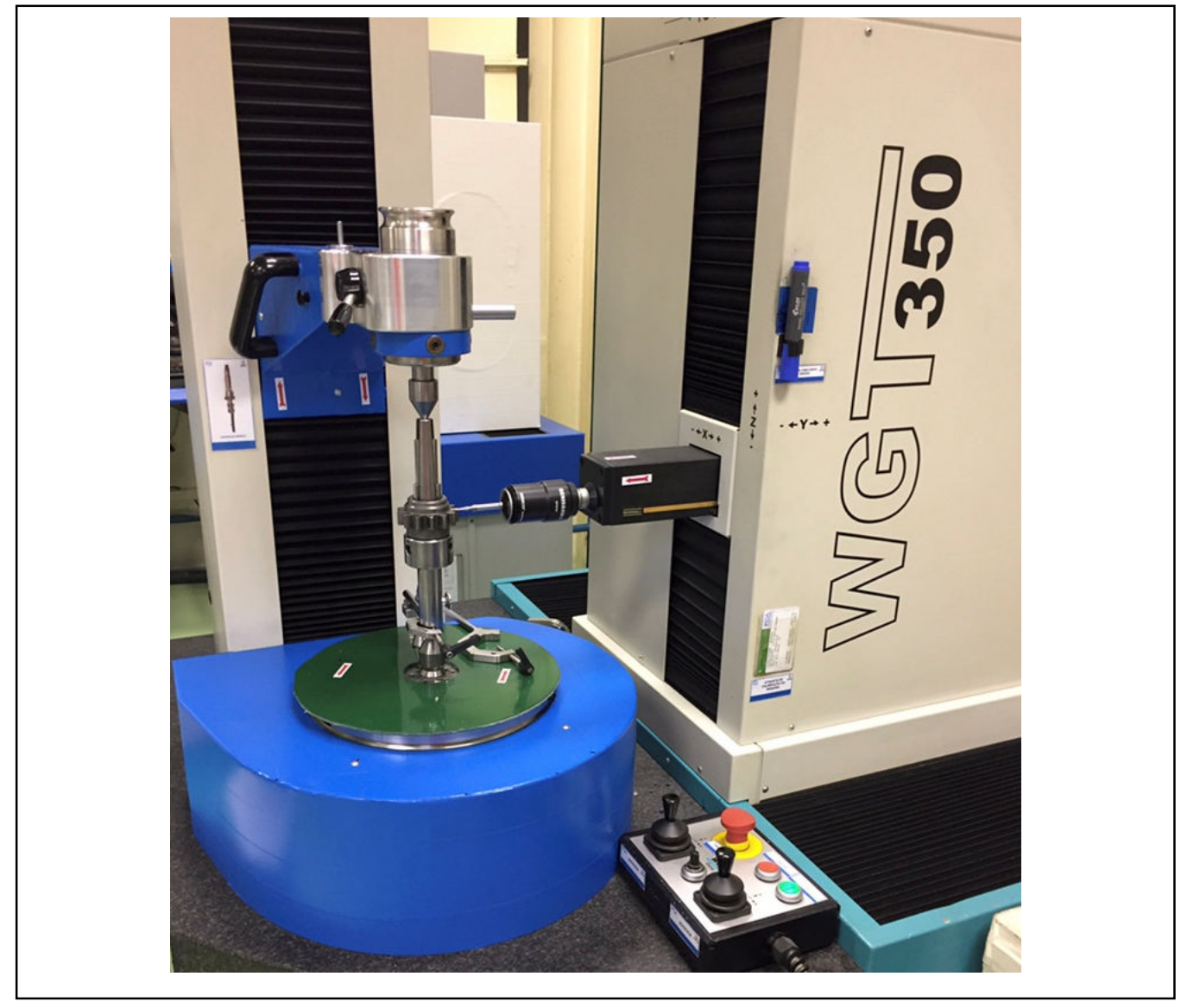

Source: Elaborated by the author

- Measure coating thickness on SEM at USP.

- Coating chemical composition was analyzed by EDS performed at USP.

iii.b) After Test.

- Gear Tooth Profile Graph was measured at FCA Metrology Laboratory using Wenzel Gear Tech.

- Teeth Hardness, case depth and core hardness values was also measured after test.

- Check final flank characteristics after test using SEM at USP. 


\subsection{DAMAGE ANALYSIS}

Involves some important activities which correlates (i) Teeth Photographs (ii) Damaged Analysis, (iii) Damaged Area Quantification. Those below listed activities interact with each other for a gear design:

i) Teeth Photographs were taken of each one of thirteen pinion teeth, right after each test schedule step in order to catalogue each tooth damage. Such imagens will be catalogued and organized to record the tooth flank damage characteristics.

ii) Damage Analysis were executed using each pinion tooth images recorded previously to identify failure mode and amount of damage area presented in each tooth flank. To perform this analysis failure definition and stress analysis knowledge were required to understand the failure modes. On this research, just pinion flank damage was evaluated. Since it was the object of study and also due to the random dynamic contact characteristic seen on of Pinion with 13 teeth and Ring with 28 teeth.

iii) Damaged Area Quantification were executed using each tooth images previously taken, each image was processed by MatLab routine in order to quantify damaged areas. This routine was developed and validated by UTFPR researchers, which have used this routine to investigate gear damage analysis and quantification.

\subsection{DATA ANALYSIS}

Involves some important activities which correlates (i) Reliability Analysis, (ii) Statistical Analysis, (iii) Process Errors Comparison. Those listed activities below interact with each other for a gear test final data analysis.

i) Reliability Analysis will support failures behavior investigation and the correspondent failure probability distribution definition. 
ii) Statistical Analysis defines a methodology path to compare final damage results, allowing a statistical comparison based on its parameters. The purpose of such comparison is to investigate if they're considered equal or not in a giving significance level.

iii) Process Errors Comparison aims to define a correlation between process errors and gear final damage after statistical comparison. Here in, the investigation aims at answering the main objective of this research. Once again, pinion flank process errors were evaluated. Since it was the object of study and also due to the random dynamic contact characteristic seen on of Pinion with 13 teeth and Ring with 28 teeth. 


\section{RESULTS AND DISCUSSIONS}

In this section, all results achieved using the methodology previously explained are presented and discussed. The results presentation sequence follows the methodology steps, beginning with gear design aiming at defining parameters variation influence on stress magnitude, as seen on Table 08.

Table 8: Design variables influence in stress simulation.

\begin{tabular}{|l|c|c|c|c|c|c|}
\hline Pressure angle () & 17 & 17 & 17 & 17 & 17 & 17 \\
\hline Addendum correction factor (mm) & 1.70 & 2.20 & 1.20 & 1.70 & 1.70 & 1.70 \\
\hline Contact widht (mm) & 14.5 & 14.5 & 14.5 & 12 & 9.5 & 14.5 \\
\hline Trochoid Radius (mm) & 2.67 & 2.67 & 2.67 & 2.67 & 2.67 & 0.9 \\
\hline Torque (Nm) & 415 & 415 & 415 & 415 & 415 & 415 \\
\hline Pinion Bending Stress (MPa) & 531.1 & 530.8 & 545.3 & 531.1 & 607.0 & 758.3 \\
\hline Ring Bending Stress (MPa) & 738.0 & 803.0 & 683.4 & 738.0 & 821.1 & 896.5 \\
\hline Contact Pressure (MPa) & 2729.6 & 2821.4 & 3142.9 & 2978.1 & 3372.7 & 2729.6 \\
\hline Pressure angle (o) & 20 & 20 & 20 & 20 & 20 & 20 \\
\hline Addendum correction factor (mm) & 1.70 & 2.20 & 1.20 & 1.70 & 1.70 & 1.70 \\
\hline Contact widht (mm) & 14.5 & 14,5 & 14,5 & 12 & 9,5 & 14.5 \\
\hline Trochoid Radius (mm) & 2.67 & 2.67 & 2.67 & 2.67 & 2.67 & 0.9 \\
\hline Torque (Nm) & 415 & 415 & 415 & 415 & 415 & 415 \\
\hline Pinion Bending Stress (MPa) & 578.8 & 573.3 & 590.6 & 578.8 & 647,6 & 757.0 \\
\hline Ring Bending Stress (MPa) & 720.9 & 775.1 & 675.7 & 720.9 & 797.2 & 823.4 \\
\hline Contact Pressure (MPa) & 2562.1 & 2490.0 & 2635.0 & 2816.6 & 3165.8 & 2562.1 \\
\hline Pressure angle (o) & 23 & 23 & 23 & 23 & 23 & 23 \\
\hline Addendum correction factor (mm) & 1.70 & 2.20 & 1.20 & 1.70 & 1.70 & 1.70 \\
\hline Contact widht (mm) & 14.5 & 14.5 & 14.5 & 12 & 9.5 & 14.5 \\
\hline Trochoid Radius (mm) & 2.67 & 2.67 & 2.67 & 2.67 & 2.67 & 0.9 \\
\hline Torque (Nm) & 415 & 415 & 415 & 415 & 415 & 415 \\
\hline Pinion Bending Stress (MPa) & 617.3 & 611.8 & 627.6 & 617.3 & 677.9 & 743.9 \\
\hline Ring Bending Stress (MPa) & 707.3 & 751.9 & 670.3 & 707.3 & 776.1 & 764.0 \\
\hline Contact Pressure (MPa) & 2427.5 & 2365.9 & 2503.4 & 2668.6 & 2999.5 & 2427.5 \\
\hline
\end{tabular}


Gear design simulation was developed according to the following variables: pressure angle, addendum value, contact width and trochoid radius. Their influence on contact pressure and also on bending stress were analyzed. This investigation was the first attempt to explore variables influence on contact stress.

On table 9 is presented the stress analysis comparison of the gear designed for the present analysis, the FZG gear type C (FZG, 1992) and the FCA 1st gear available in the market.

Table 9: Stress Analysis Comparisons of FCA and FZG and Designed Gear.

\begin{tabular}{|l|c|c|c|}
\hline \multicolumn{4}{|c|}{ Analysis by IsoCad } \\
\hline \multicolumn{1}{|c|}{ Gears Comparisons } & $\begin{array}{c}\text { New Designed } \\
\text { Gear }\end{array}$ & $\begin{array}{c}\text { FZG } \\
\text { Type C }\end{array}$ & 1st Gear \\
\hline Pinion Bending Stress (MPa) & 532.5 & 482.8 & 575.5 \\
\hline Ring Bending Stress (MPa) & 668.8 & 472.1 & 726.7 \\
\hline Contact Pressure (MPa) & $2,427.5$ & $2,030.1$ & $2,681.5$ \\
\hline Sliding speed @ 3000 rpm (m/s) & 5.5 & 4.8 & 2.4 \\
\hline Sliding speed @ 1750 rpm (m/s) & 3.2 & - & - \\
\hline
\end{tabular}

The results shown on table 8 and 9 were the input for the new gear design definition and the design final choice was to keep pressure angle $20^{\circ}$ and addendum $4.5 \mathrm{~mm}$, reaching a contact stress close to the magnitude acting on the refered1st gear. It was also important to keep this angle because it is the same pressure angle of FZG gear Type C. Contact width was also analyzed and set in $14.5 \mathrm{~mm}$, because smaller contact induced contact stress magnitude according to the requirement. Right after contact stress requirement evaluation and geometry definition, Trochoid radius was analyzed and defined in maximum, because this geometry induces bending stress of low magnitude. Hence, on figure 60 is shown the new gear designed geometry, highlighting in red the pitch diameter and in black lines the limit surface area within a single contact is observed. On table 10 are summarized gear design results. 
Figure 60: New gear designed final geometry highlighting in red pitch line and between black lines is seen the single contact area

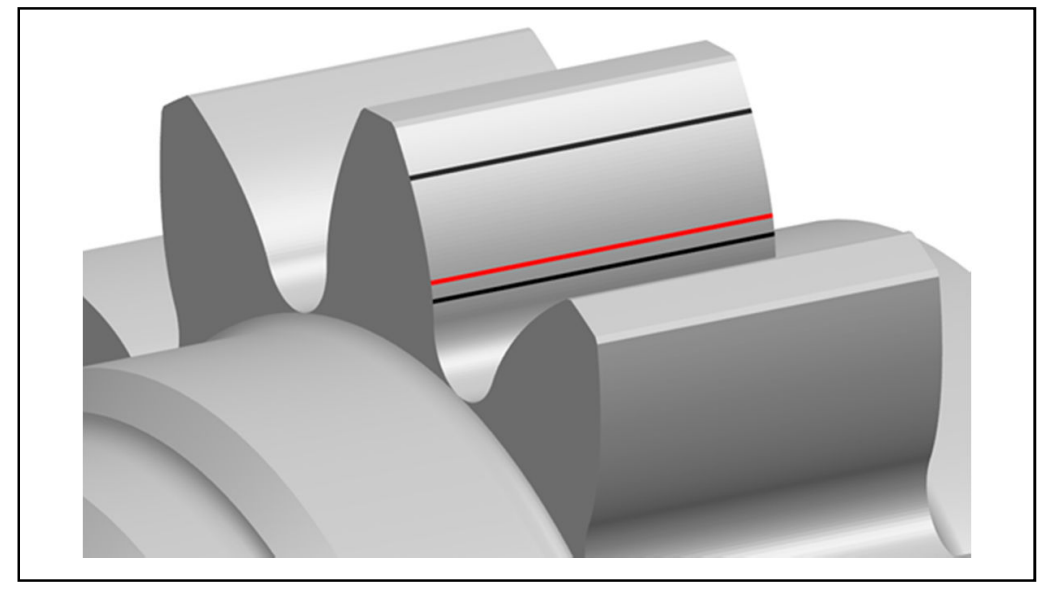

Source: Elaborated by the author

Table 10: Gear geometry comparisons of FZG and new designed gear.

\begin{tabular}{|l|c|c|c|}
\hline \multicolumn{1}{|c|}{ Geometry } & Symbol & $\begin{array}{c}\text { New Designed } \\
\text { Gear }\end{array}$ & FZG Type C \\
\hline Center distance $(\mathrm{mm})$ & $\mathrm{a}$ & 91.5 & 91.5 \\
\hline \multirow{2}{*}{ Number of teeth } & $\mathrm{Z}_{1}$ & 13 & 16 \\
\cline { 2 - 4 } & $\mathrm{Z}_{2}$ & 28 & 24 \\
\hline Module $(\mathrm{mm})$ & $\mathrm{m}$ & 4.5 & 4.5 \\
\hline Pressure angle $\left(^{\circ}\right)$ & $\alpha$ & 20 & 20 \\
\hline Helix angle $\left(^{\circ}\right)$ & $\beta$ & 0 & 0 \\
\hline Addendum $(\mathrm{mm})$ & $\mathrm{a}_{\mathrm{d}}$ & 5.8 & 5.32 \\
\hline Face width $(\mathrm{mm})$ & $\mathrm{B}$ & 16 & 14 \\
\hline \multirow{2}{*}{ Profile shift factor } & $\mathrm{X}_{1}$ & 0.378 & 0.1817 \\
\cline { 2 - 4 } & $\mathrm{X}_{2}$ & -0.539 & 0.1715 \\
\hline \multirow{2}{*}{ Pitch Diameter $(\mathrm{mm})$} & $\mathrm{dw}_{1}$ & 58.5 & 73.2 \\
\cline { 2 - 4 } & $\mathrm{dw}_{2}$ & 126.0 & 109.8 \\
\hline \multirow{2}{*}{ Tip Diameter $(\mathrm{mm})$} & $\mathrm{da}_{1}$ & 70.1 & 82.5 \\
\cline { 2 - 4 } & $\mathrm{da}_{2}$ & 128.5 & 118.4 \\
\hline
\end{tabular}

Here in, the discussion will be initially based on shaved gears and afterward coated gears will be added on the discussion. The purpose of this approach is to detail the discussion of as shaved gears process errors influence on fatigue degradation and on a next step add to the discussion coated gears and evaluate the influence of coating on the fatigue life. 


\subsection{SAMPLE CHARACTERIZATION}

Thus, two gear pairs samples (F06 and F07) were analyzed to check base material chemical composition and to evaluate surface roughness. On table 11 is shown the chemical composition analysis result for on gear samples F06 and F07. The analysis done by optical emission spectrometer shows that the material chemical composition matches with SAE 4320 specification.

Table 11: Chemical analysis results of Designed Gear

\begin{tabular}{|l|c|c|c|c|c|c|c|}
\hline \multicolumn{1}{|c|}{ Element } & $\mathbf{C}$ & $\mathbf{M n}$ & $\mathbf{N i}$ & $\mathbf{C r}$ & Mo & S & $\mathbf{P}$ \\
\hline SAE 4320 Chemical & $\mathbf{0 . 1 7}$ & $\mathbf{0 . 4 5}$ & $\mathbf{1 . 6 5}$ & $\mathbf{0 . 4 0}$ & $\mathbf{0 . 2 0}$ & Max & Max \\
Composition spec. & $\mathbf{0 . 2 2}$ & $\mathbf{0 . 6 5}$ & $\mathbf{2 . 0 0}$ & $\mathbf{0 . 6 0}$ & $\mathbf{0 . 3 0}$ & $\mathbf{0 . 0 4}$ & $\mathbf{0 . 0 3 5}$ \\
\hline Gear Pinion F06 & 0.20 & 0.61 & 1.78 & 0.53 & 0.28 & 0.03 & 0.01 \\
\hline Gear Pinion F07 & 0.19 & 0.61 & 1.79 & 0.53 & 0.28 & 0.03 & 0.01 \\
\hline Gear Ring F06 & 0.20 & 0.56 & 1.65 & 0.45 & 0.22 & 0.02 & 0.01 \\
\hline Gear Ring F07 & 0.19 & 0.61 & 1.65 & 0.46 & 0.22 & 0.01 & 0.007 \\
\hline
\end{tabular}

Therefore, on Table 12 and 13 are shown Roughness Measurements performed on shaved gear (Samples F06 e F07) for the pinion and ring respectively. The longitudinal direction is the measurement done starting from right till left. It aims to identify the roughness alongside teeth width. Measurement Length: Cut off Cross Direction: $0,08 \mathrm{~mm}$ cut off Longitudinal Direction: $0,8 \mathrm{~mm}$

Table 12: Roughness Measurements executed on Pinion

\begin{tabular}{|c|c|c|c|c|c|}
\hline \multirow{2}{*}{\multicolumn{2}{|c|}{ Parameters }} & \multicolumn{4}{|c|}{ Measured Values } \\
\hline & & \multicolumn{2}{|c|}{ Cross Direction (Profile) } & \multicolumn{2}{|c|}{ Longitudinal Direction (Lead) } \\
\hline & & F06 & F07 & F06 & F07 \\
\hline \multirow{3}{*}{$\begin{array}{c}\mathrm{Ra} \\
(\mu \mathrm{m})\end{array}$} & 1 & 0.79 & 0.75 & 0.34 & 0.53 \\
\hline & 3 & 0.69 & 0.77 & 0.38 & 0.47 \\
\hline & 5 & 0.73 & 0.78 & 0.36 & 0.48 \\
\hline \multirow{3}{*}{$\begin{array}{c}\mathrm{Rz} \\
(\mu \mathrm{m})\end{array}$} & 1 & 2.78 & 2.05 & 2.23 & 3.06 \\
\hline & 3 & 2.56 & 3.06 & 2.43 & 2.81 \\
\hline & 5 & 2.59 & 2.98 & 2.16 & 2.93 \\
\hline \multirow{3}{*}{$\underset{(\mu \mathrm{m})}{\mathrm{Rt}}$} & 1 & 3.12 & 2.24 & 2.45 & 3.65 \\
\hline & 3 & 3.05 & 3.19 & 3.05 & 3.13 \\
\hline & 5 & 2.61 & 3.05 & 2.92 & 3.74 \\
\hline
\end{tabular}


Table 13: Roughness Measurements executed on Ring

\begin{tabular}{|c|c|c|c|c|c|}
\hline \multirow{3}{*}{\multicolumn{2}{|c|}{ Parameters }} & \multicolumn{4}{|c|}{ Measured Values } \\
\hline & & \multicolumn{2}{|c|}{ Cross Direction (Profile) } & \multicolumn{2}{|c|}{ Longitudinal Direction (Lead) } \\
\hline & & F06 & F07 & F06 & F07 \\
\hline \multirow{3}{*}{$\begin{array}{c}\text { Ra } \\
(\mu \mathrm{m})\end{array}$} & 1 & 0.76 & 0.74 & 0.38 & 0.43 \\
\hline & 3 & 0.73 & 0.76 & 0.31 & 0.40 \\
\hline & 5 & 0.76 & 0.78 & 0.37 & 0.34 \\
\hline \multirow{3}{*}{$\begin{array}{c}\mathrm{Rz} \\
(\mu \mathrm{m})\end{array}$} & 1 & 2.82 & 2.22 & 2.15 & 2.85 \\
\hline & 3 & 3.29 & 4.18 & 1.68 & 2.39 \\
\hline & 5 & 1.76 & 2.10 & 2.10 & 1.99 \\
\hline \multirow{3}{*}{$\begin{array}{c}\mathbf{R t} \\
(\mu \mathrm{m})\end{array}$} & 1 & 3.50 & 2.68 & 2.81 & 4.51 \\
\hline & 3 & 4.80 & 4.81 & 2.20 & 2.83 \\
\hline & 5 & 2.05 & 2.21 & 2.31 & 2.84 \\
\hline
\end{tabular}

Roughness are also in accordance with the gear specification of having 0.8 $\mu \mathrm{m}$ of Ra Limit.

Proceeding with samples characterization, on table 14 is shown hardness measurements executed on new gear for samples number F06 and F07. As seen, the results are within specified limits and the gears are considered approved to perform the fatigue test. Those results are very important for fatigue evaluation, once according to ISO 6336 procedures (1996) the surface hardness defines the allowable stress contact ( $\sigma_{\text {HLim }}$ ).

Table 14: Hardness, case depth and core hardness measurements executed on Designed Gear

\begin{tabular}{|c|c|c|c|c|c|}
\hline \multirow{2}{*}{ Parameters } & \multirow{2}{*}{ Specified } & \multicolumn{4}{|c|}{ Identified } \\
\cline { 3 - 6 } & & \multicolumn{2}{|c|}{ Pinion } & \multicolumn{2}{c|}{ Ring } \\
\cline { 3 - 6 } & $58: 63$ & 62 & 62 & 61 & 62 \\
\hline Hardness (HRc) & 0.65 & 0.63 & 0.69 & 0.7 \\
\hline Case depth (mm) & $0.5: 0.7$ & 40 & 41 & 41 & 42 \\
\hline $\begin{array}{c}\text { Core Hardness } \\
\text { (HRc) }\end{array}$ & $\geq 34$ & 407 & \multicolumn{2}{c|}{ SAE 4320 } \\
\hline Material & SAE 4320 & \multicolumn{2}{|c|}{ SAE 4320 } \\
\hline
\end{tabular}




\subsection{Damage analysis}

The first step on damage analysis was the identification of failure mechanism to be able to classify the damage. So, for classification purpose, after test, F01 gear was evaluated using SEM (Scanning Electronic Microscope). Teeth 3 and 10 cross sections were submitted to that evaluation. The cross section were taken on pitch diameter, on longitude direction.

Figures 61 and 62 cross section views indicate a surficial and sub surficial crack induced by contact fatigue for teeth 3 and 10 . This crack seemed to be nucleated, and propagated and reached the surface and the material is subsequently detached. So, the cyclical stress applied during the test was relevant to induce fatigue crack as shown on both cross section.

In figure 61 surficial cracks induced by contact fatigue are detected. The upper arrow points to a crack nucleated and propagated, the lower arrow also points to a crack nucleated and propagated, but this one has reached surface and the material is likely to be displaced. The crack seen on this figure is on pitch diameter.

Figure 61: Gear F01 tooth 3 cross section view image done on SEM to indicate damaged mechanism.

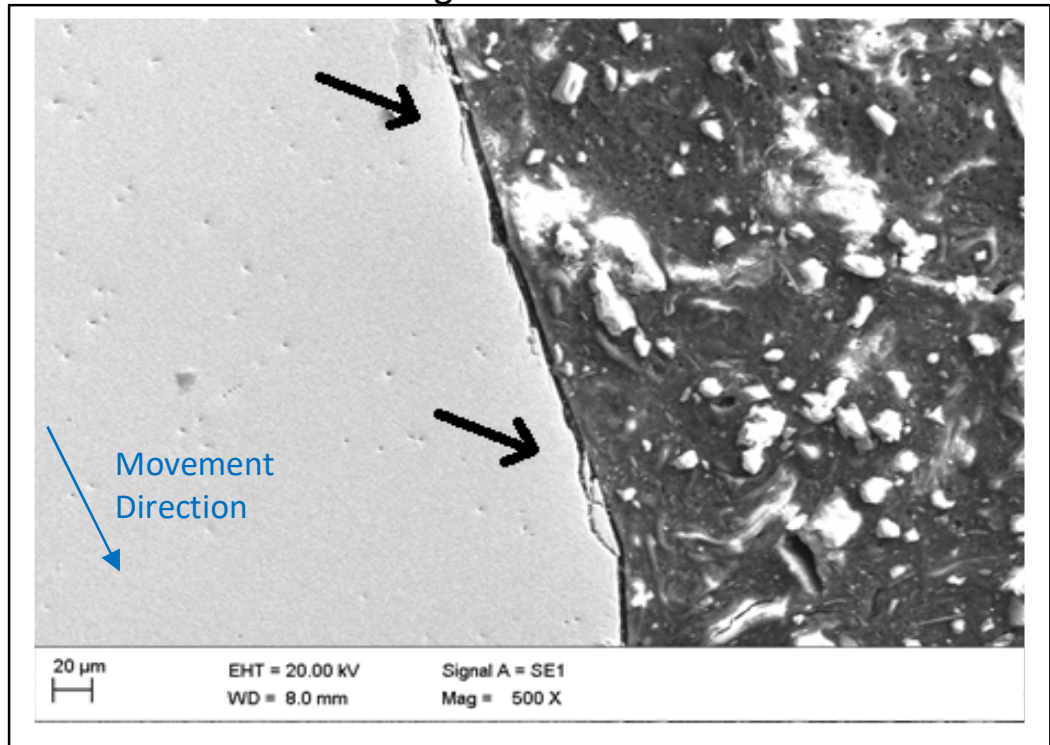

Source: Franco et. al., 2018 
Figure 62: Gear F01 tooth 10 cross section view done on SEM to indicate damaged mechanism.

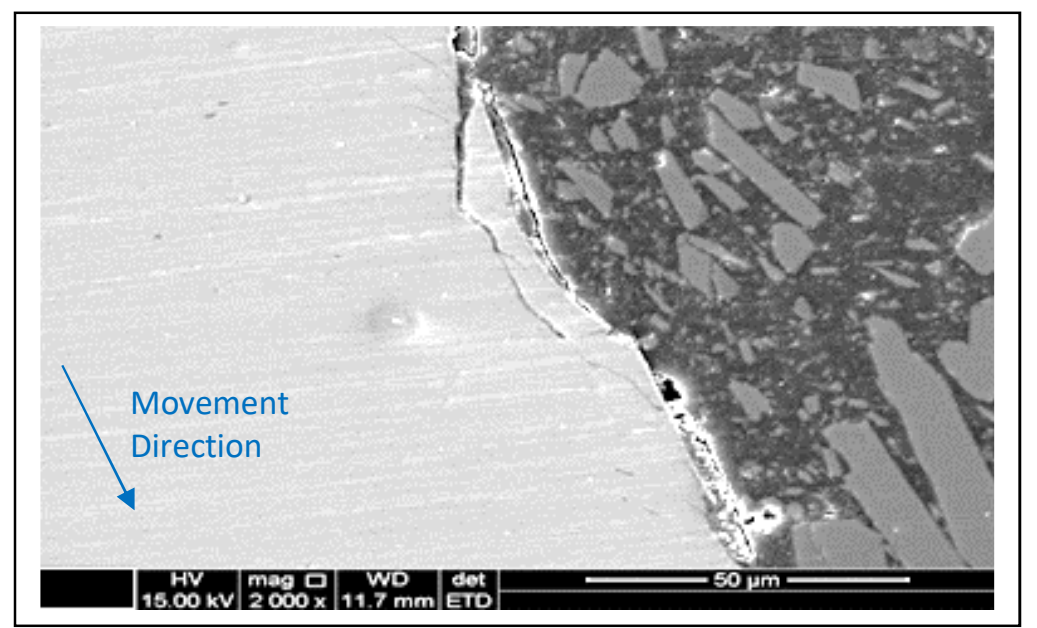

Source: Elaborated by the author

Still on F01 tooth 10, another pitting was analyzed on SEM and its image is seen on figure 62. On this figure is seen an image taken right on pitch diameter and the sliding movement highlighted with the blue narrow. On figure 63 the pit profile was taken by non-contact 3D Optical Profiler ( $C C l$ HD) in which is seen a pitting geometry emphasizing its width, length and depth.

Figure 63: Gear F01 tooth 10 pitting geometry analyzed by $\mathrm{CCl}$ revealing its width, length and depth

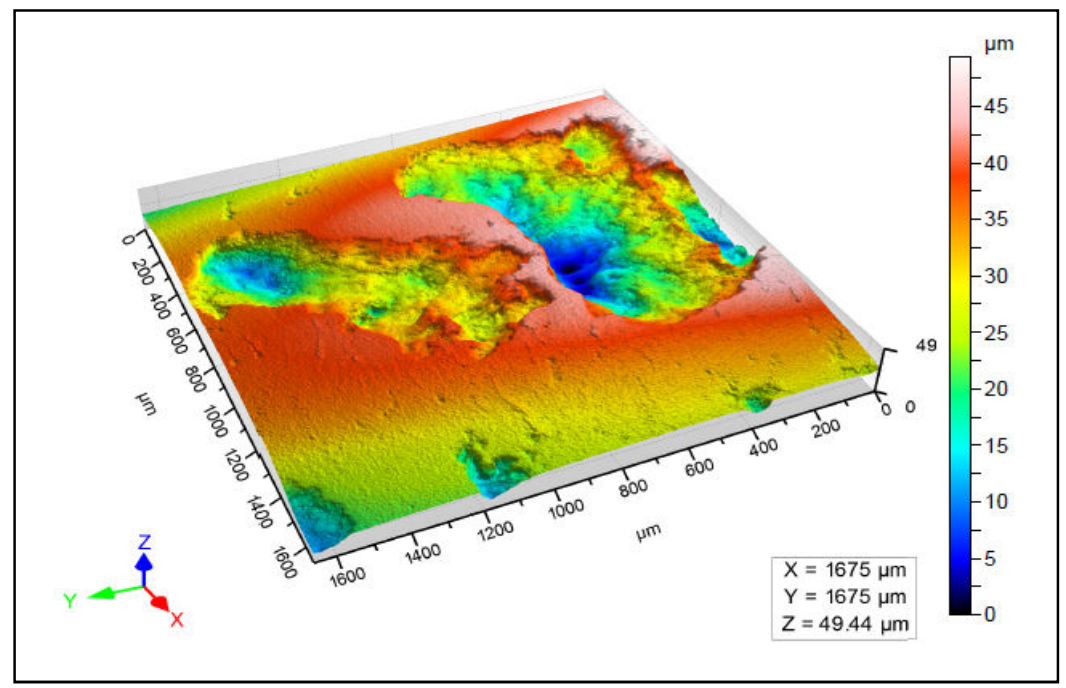

Source: Elaborated by the author 
Figure 64 is seen a zoon of picture 63. This pitch was observed on the pitch diameter the cross section was also taken on longitudinal direction and the sliding direction was seen on the figure 63 axle $\mathrm{x}$ direction.

Figure 64: Gear F01 tooth 10 pitting geometry analyzed by $\mathrm{CCl}$ revealing its width, length and depth.

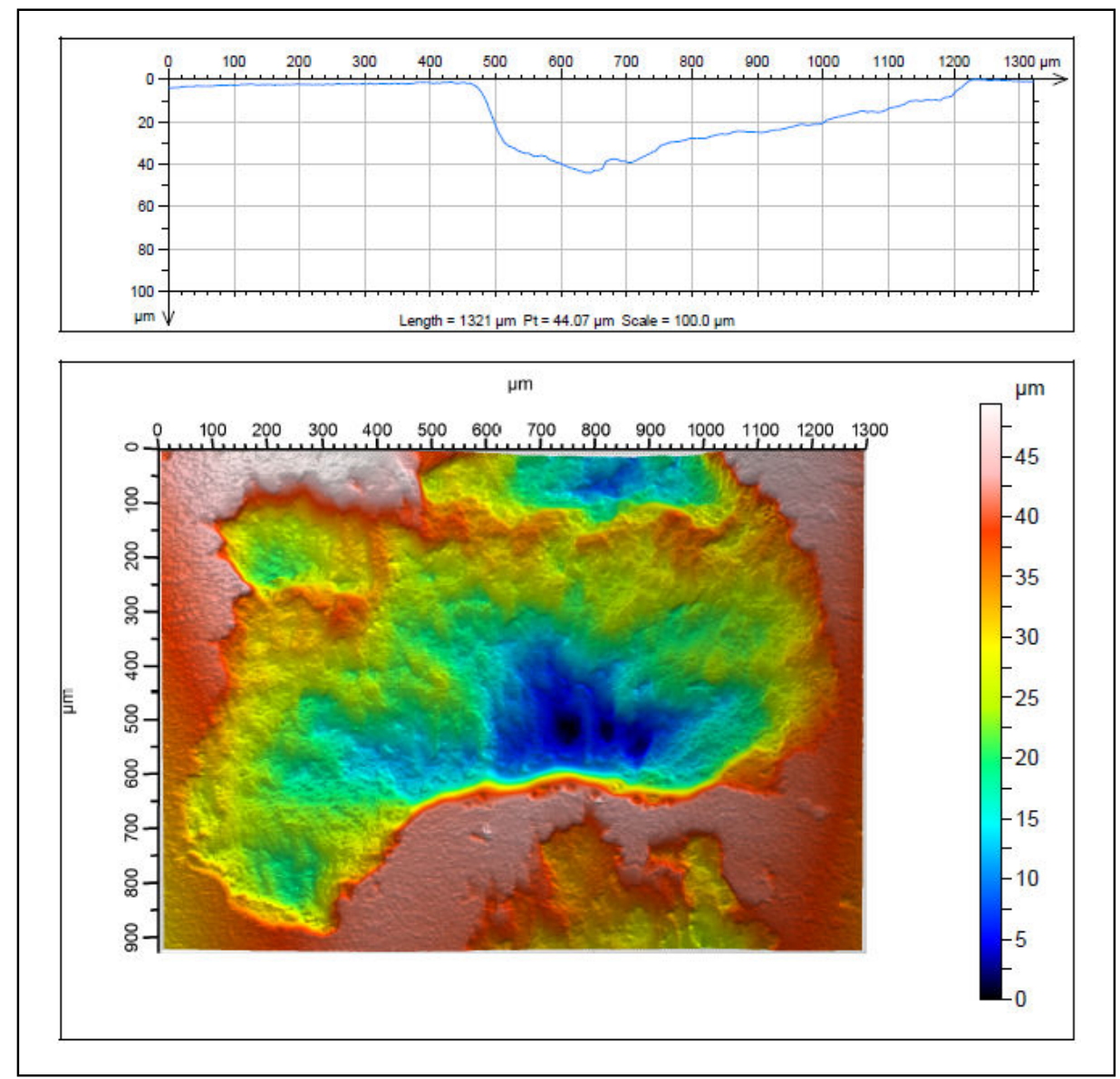

Source: Elaborated by the author

\subsection{TEETH FLANK IMAGES}

Images were taken after each fatigue cycle step defined for this research. The purpose was to register the load cycles effect on tooth flank and also to allow a damage growth evaluation. Those images were also important to use as input in a MatLab routine to quantify damage area of each pinion gear tooth. On figure 65 is 
shown a tooth flank (a) after K9 run $1 \mathrm{~h}$, (b) after $\mathrm{K} 10$ run $3 \mathrm{~h}$ and also (c) K10 run for extra $3 h$.

Figure 65: Gear F01 tooth 3 damaged area after images after (a) one hour on K9 load stage, (b) more three hours on K10 load stage and (c) more extra three hours on $\mathrm{K} 10$.

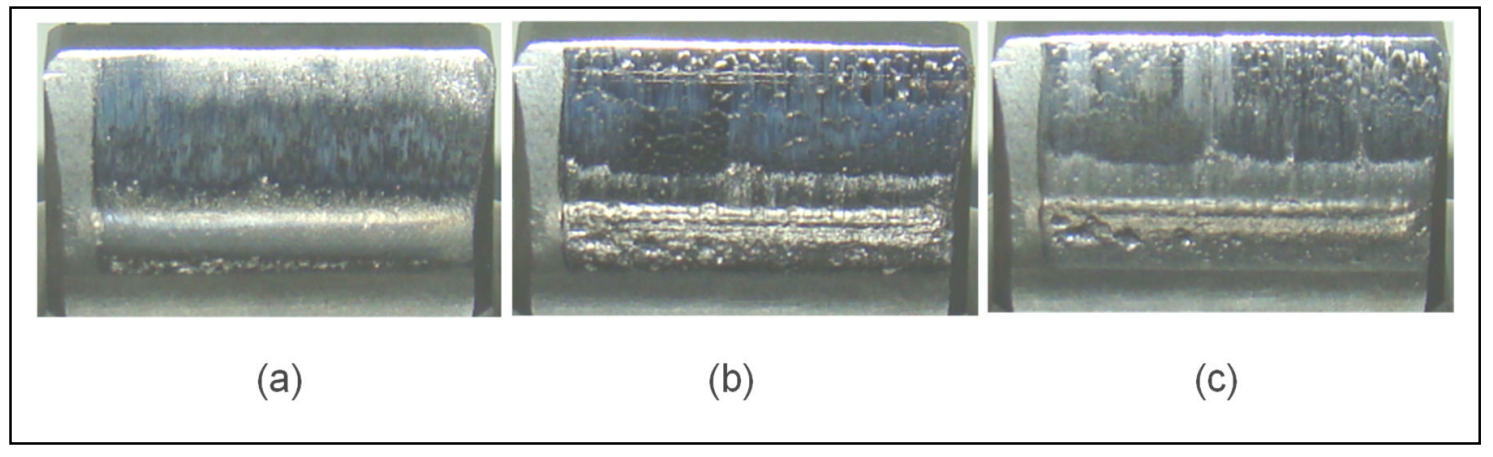

Source: Elaborated by the author

Still on figure 65 are seen two distinct areas: (i) starting from the left, one narrow area without contact. This area has been a result of a design decision of reducing the effective contact to increase contact stress; (ii) another extensive area in which contact has happened and progressive damage can be seen from (a), (b) and (c). Those increased damaged flank area seen on figure 65 are a result of the load cycles applied on Back to Back bench test under test procedure defined for this research. In appendix $A$ are shown all pictures taken at each fatigue step cycle.

\subsection{DAMAGE QUANTIFICATION}

Once this research is focused on fatigue damaged caused by pitting mechanism. this type of failure will be considered on further discussion. On figure 66, it's shown the fatigue mechanism output under study. On figure 66 the result of image processing by MatLab routine is indicated, which emphasizes the desired damaged area quantification by applying a contrast between areas on the digitalized flank surface. It considers the white area, affected by pitting, and the black area, which defines the remaining flank surface which was not affected by Pitting. On figure 66 is shown a tooth flank processed by MatLab quantification routine applied after (a) 
$\mathrm{K} 9$ run for 1 hour, (b) K10 run for 3 hours and also (c) K10 run for more 3 hours. In appendix $A$ are shown all tooth images processed by MatLab routine.

Figure 66: Damaged area identified using MatLab routine on gear F01 Tooth 3 after (a) one hour on K9 load stage, (b) more three hours on K10 load stage and (c) more three hours on K10.

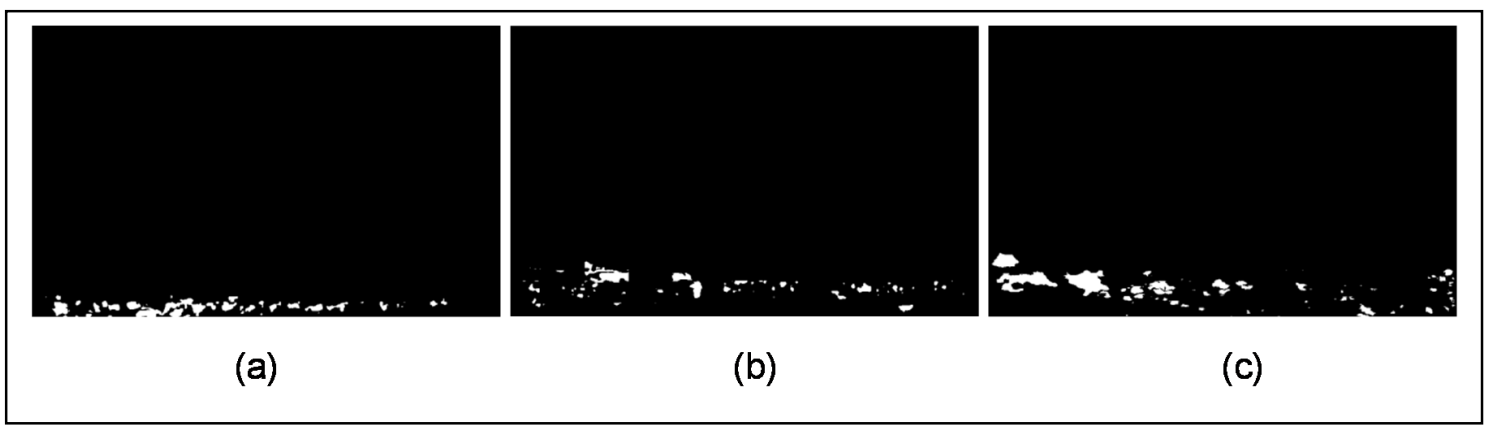

Source: Elaborated by the author

\subsection{PITTING DAMAGE AREA ANALYSIS AND DAMAGE BEHAVIOR (GROW)}

On figure 67 till figure 71 are seen damaged area evaluation at each step of the defined fatigue cycles. Each graph contains data related to one gear, starting with F01 till F05. Such graphs purpose is to allow the observation of each tooth Damaged Area (axle $Y$ ) at each step of fatigue cycle (axle $X)$. As three steps of fatigue cycle were planned, the graph shows first step (1hK09), second step (3hK10) and third step (6hK10). Thus, a tendency line was added to easily visualization of the data projection.

Based on figures 68, 69 and 70, gears F02, F03 and F04 teeth reveal a tendency of having more damaged area on the first step evaluation (1hK9). However when the second and third evaluation steps of fatigue test data are observed, the tendency is a damaged area reduction. This data behavior represents an unexpected phenomenon. To complete the evaluation, another data has a complementary and opposite observation. In this perspective, of completing the evaluation, it is important to observe once more the teeth images and the MatLab routine output. The flank images allows the observation of a growing tendency of each pitch damaged area. 
Accordingly, it's reasonable to put together two evaluations to comprehend the phenomenon: (i) a reduction of total damaged area by pitting alongside test steps evolution, (ii) On the other hand, it's also reasonable to perceive that each pitting tend to became bigger as much as the test gets closer to total designed fatigue. The pitting sizing comparisons are seen on figure 67 which makes evident the isolated fatigue mechanism revealing the pitting sizes.

Such observed behavior seems like that this phenomenon could be associate to: (i) stress, which was increased after test first step; (ii) cycle effects, which is continuously accumulated over test; (iii) dynamic effects, which are influenced by initial geometry and accumulated damage over test, and (iv) material characteristics, which influence crack nucleation and propagation. Thus, those area reduction phenomenon observed could be also associated and credit to all this highlighted variables.

The reduced area phenomenon could also be associated to: (i) deformation which is suitable to happen on this high load magnitude and consequently high compressive contact stress magnitude. This material deformation tends to induce material flow which covers pitting, as also seen on Moorthy e Shaw (2013); (ii) wear effects on the surface were seen and material layers were removed of the surface. As a result, the low depths pitting tend to be removed, reducing damaged areas. On this perspective, those deepest pitting, remained on surface, under loading cycle effects tends to be the increased size pitting observed.

The raised up question is: What are the reasons for the deepest pitting? The hypothesis to answer this question is that those pitting are outcome of increased local stress level, which could be due to: (i) local contact area reduction which tends to increase stress effect locally. This hypothesis matches with the central investigation of this thesis, which efforts are to evaluate process errors influence on final fatigue results or (ii) material heterogeneity which tends to modify the material property or stress behavior locally influencing crack nucleation and propagation, which is likely to be a random phenomenon influenced by material quality.

On figure 67 gear F01 teeth data reveal a tendency of having more damaged area on the first step evaluation (1hK9), reduce damaged area on second step, but then 
increased damaged area on the third step. The only different step of F02, F03 and F04 is the last fatigue step output, in which is seen an increased damage area. This output is going to be evaluated further based on proposed methodology following the central investigation proposed by this thesis.

Finally, on figure 71, gear F05 teeth reveal a tendency of having less damaged area on the first step evaluation (1hK9), increased damaged area on second step, then reduced damaged area to similar levels of initial damaged area in step three. Here, the only difference of gear F02, F03 and F04 is the first step which has less damage in the flank.

Here, one possible explanation is that the process variability influenced earlier the pitting outcome, revealing pitting earlier. Consequently, instead of seeing individual pitting increasing area on last fatigue step, it's anticipated and seen on fatigue second step.

Figure 67: F01 Damaged area variation between fatigue cycle phases, considering: (i): one hour on $\mathrm{K} 9$ load stage, (ii) more three hours on K10 load stage and (iii) more three hours on $\mathrm{K} 10$.

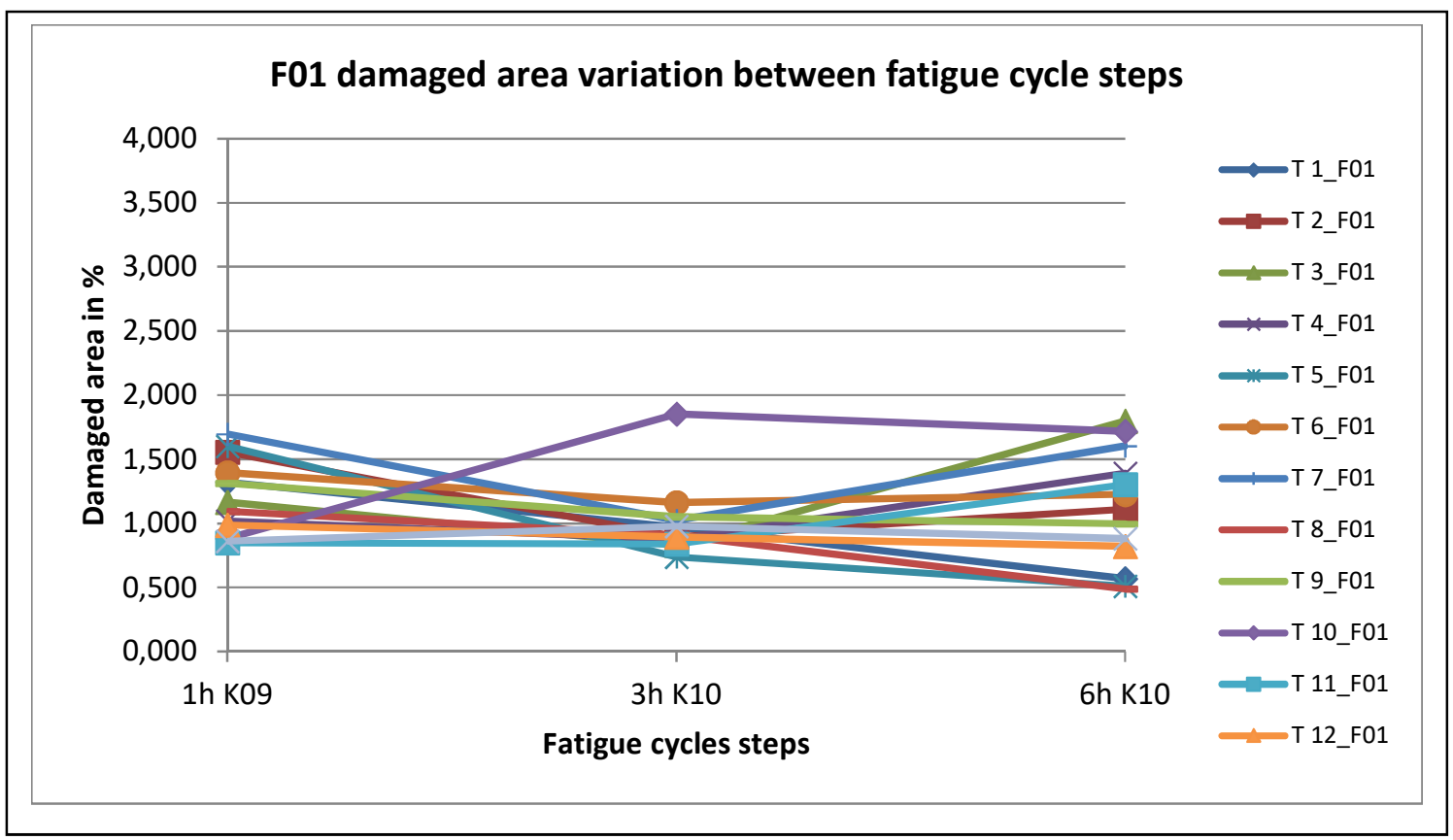

Source: Elaborated by the author 
Figure 68: F02 Damaged area variation between fatigue cycle phases, considering: (i): one hour on K9 load stage, (ii) more three hours on K10 load stage and (iii) more three hours on K10.

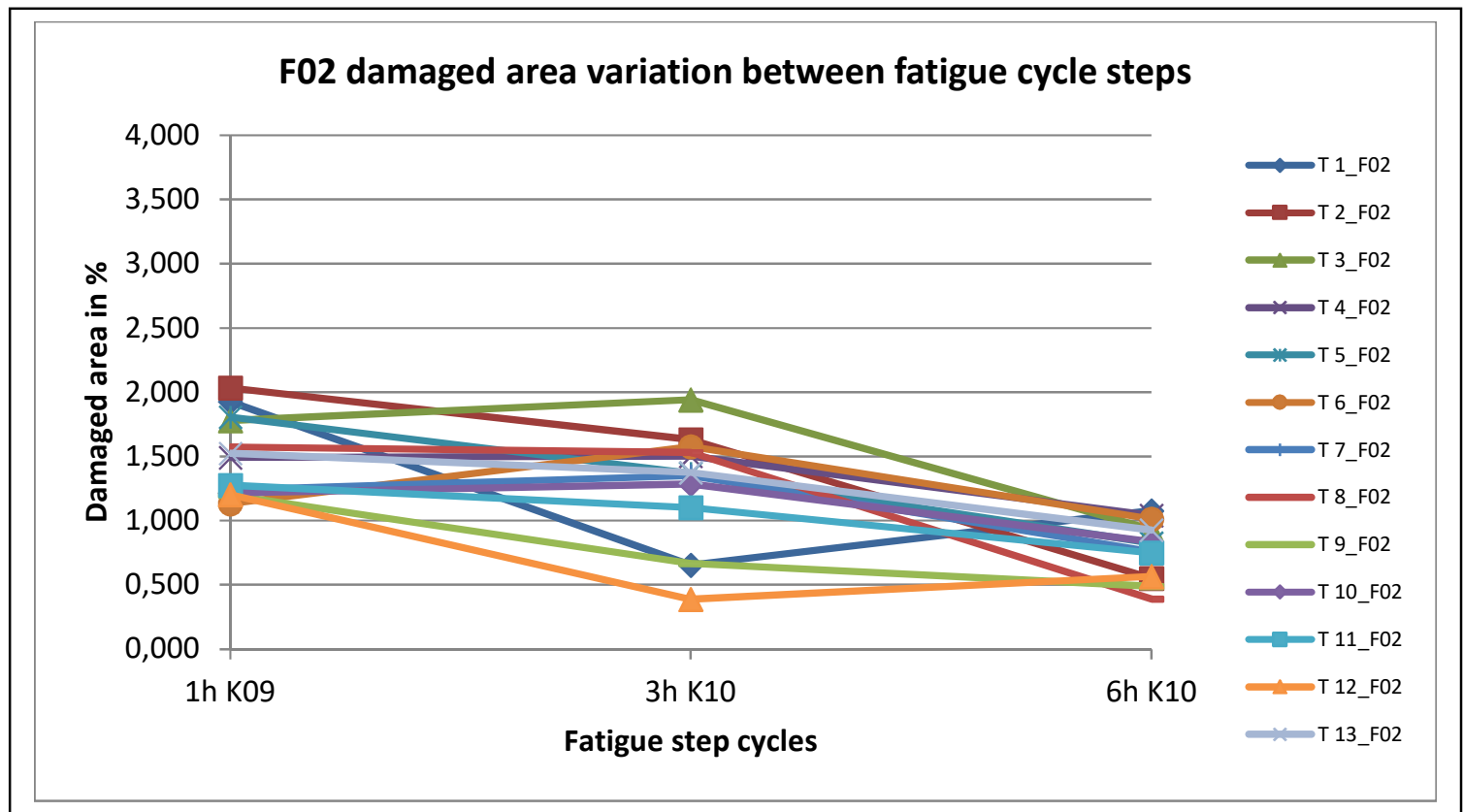

Source: Elaborated by the author

Figure 69: F03 Damaged area variation between fatigue cycle phases, considering: (i): one hour on $\mathrm{K} 9$ load stage, (ii) more three hours on K10 load stage and (iii) more three hours on K10.

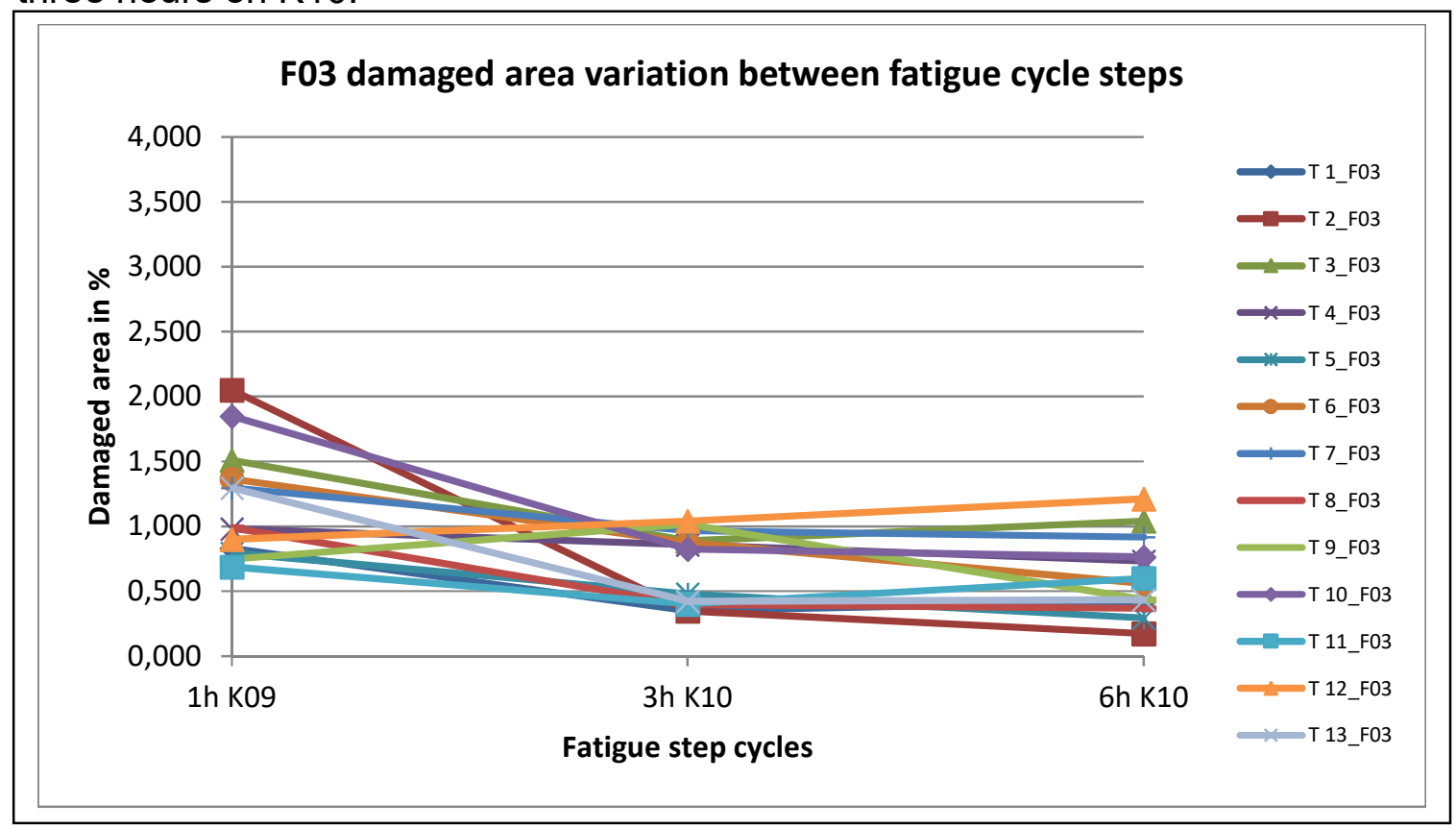

Source: Elaborated by the author 
Figure 70: F04 Damaged area variation between fatigue cycle phases, considering: (i): one hour on K9 load stage, (ii) more three hours on K10 load stage and (iii) more three hours on K10.

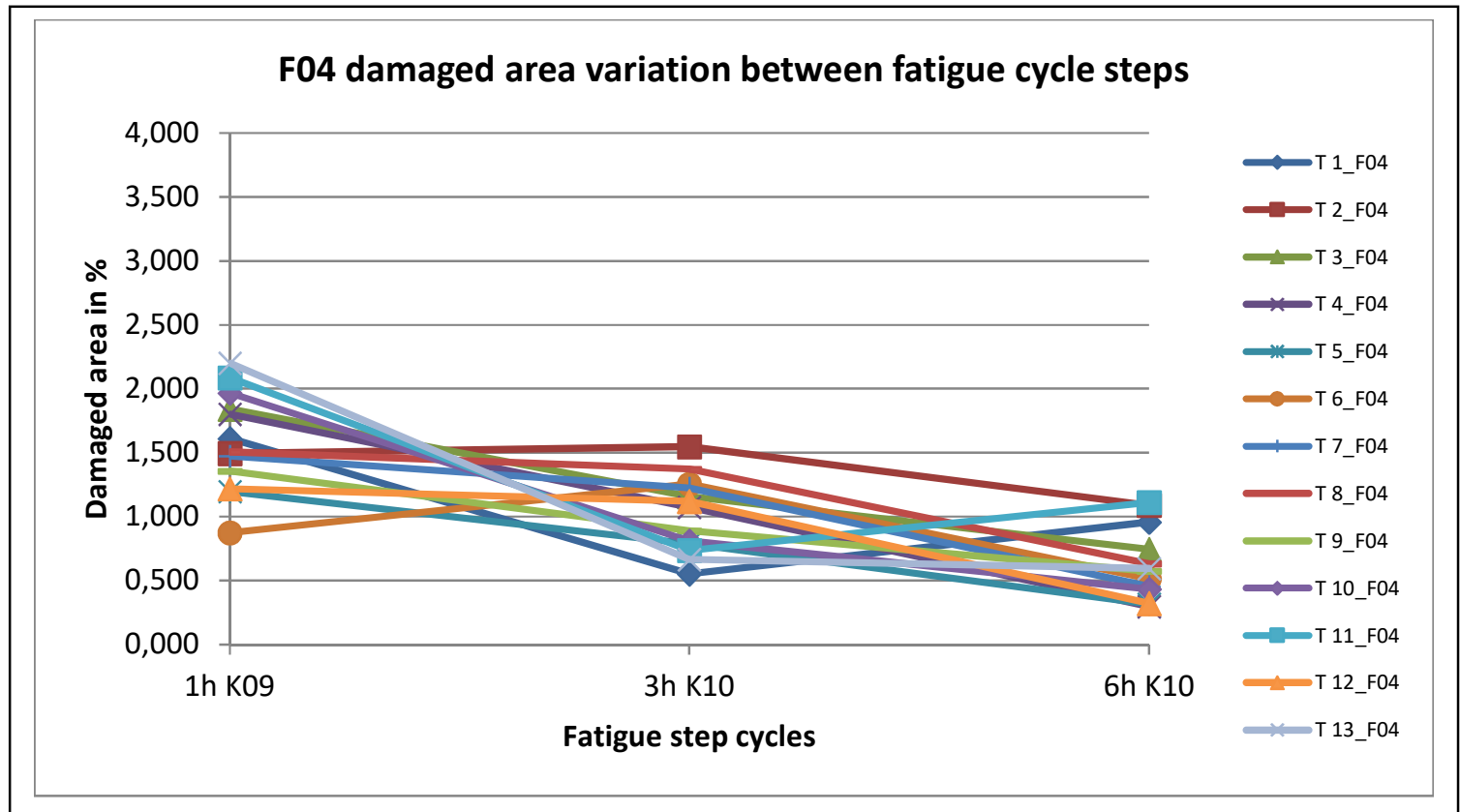

Source: Elaborated by the author

Figure 71: F05 Damaged area variation between fatigue cycle phases, considering: (i): one hour on $\mathrm{K} 9$ load stage, (ii) more three hours on K10 load stage and (iii) more three hours on K10.

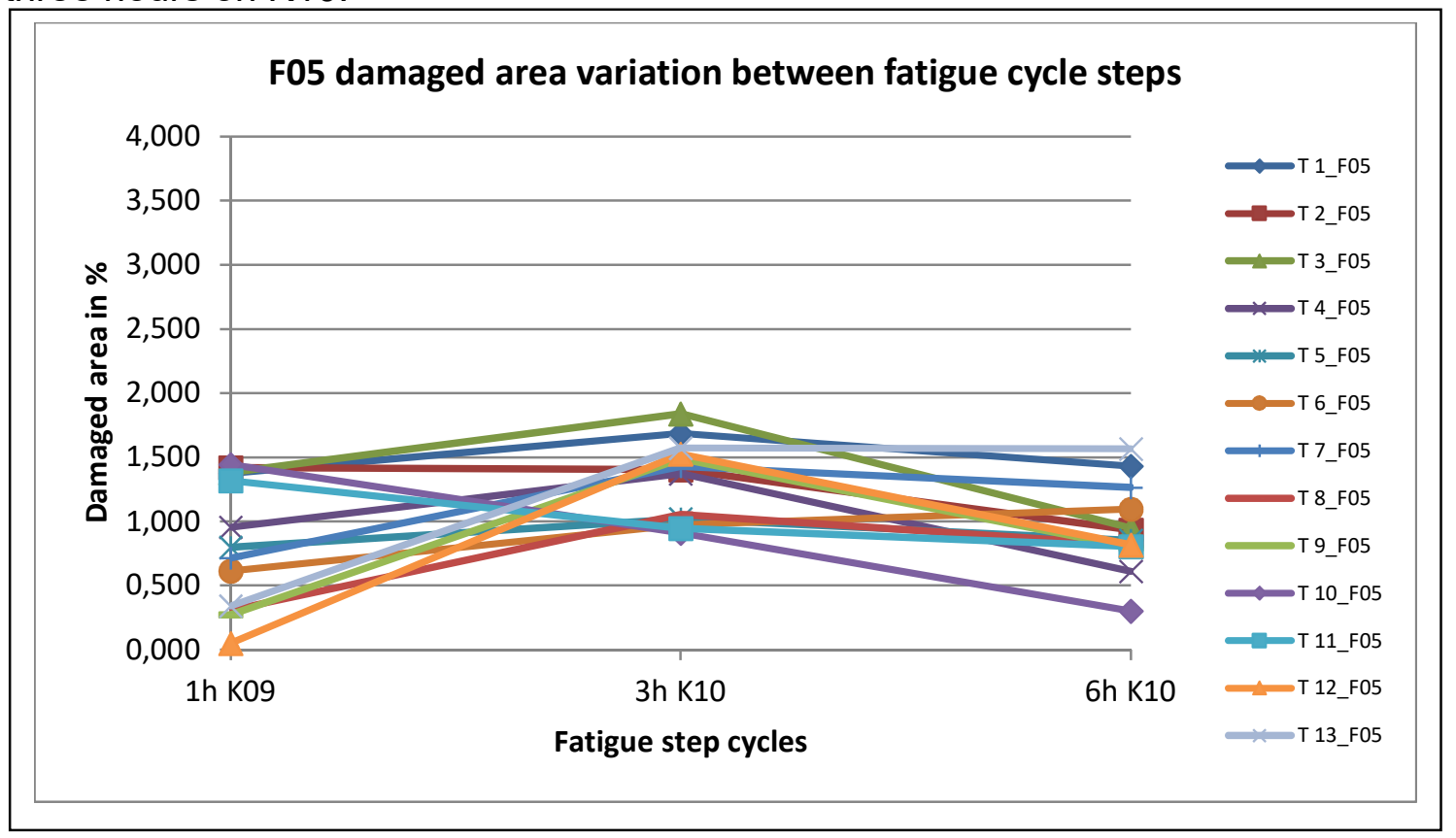

Source: Elaborated by the author 
Another important discussion is raised based on those results. Does damaged area represent a good failure criteria for pitting evaluation? This question is reasonable due to the results variability seen in this section figures. As the damaged presents variation through the initial and final test steps, this criteria could be associate to an uncertainty output.

Nevertheless, this criteria is relevant and useful, although it must be supported by other evaluations, otherwise, it could rise to a misunderstanding of the initial or partial observed phenomenon. That's why on this research, flank images were taken to have a more illustrative visualization besides profile measurements representing the geometry variation due to fatigue test were also taken to support pitting evaluation analysis.

Once again, ISO 6336 does not take other criteria into evaluation, just final damaged area. ISO 6336 also does not proceed with partial evaluation, just the final fatigue criteria. The final criteria taken considers $0.5 \%$ of flank damaged area by pitting at the end of defined duty cycle. In the next section, the final fatigue result will be the focus.

\subsection{EVALUATION AFTER ENTIRE FATIGUE CYCLE.}

Previous assessment had focused in pitting evaluation alongside fatigue cycle progression, which has shown its relevance to the phenomenon development. Here in, the focus is set to the final fatigue evaluation to support the main objective accomplishment. The evaluation done matches with the first steps in a nonparametric analysis, in which data started to be treated without an equation describing the phenomenon. Thus, on figure 72 is shown 65 bar representing each tooth of each gear tested from F01 till F05. The total damage area varies from $0.17 \%$ to $1.80 \%$ of tooth flank area. 
Figure 72: Final damaged area variation observed on gears F01 till F05 teeth after last fatigue cycle step (6h K10).

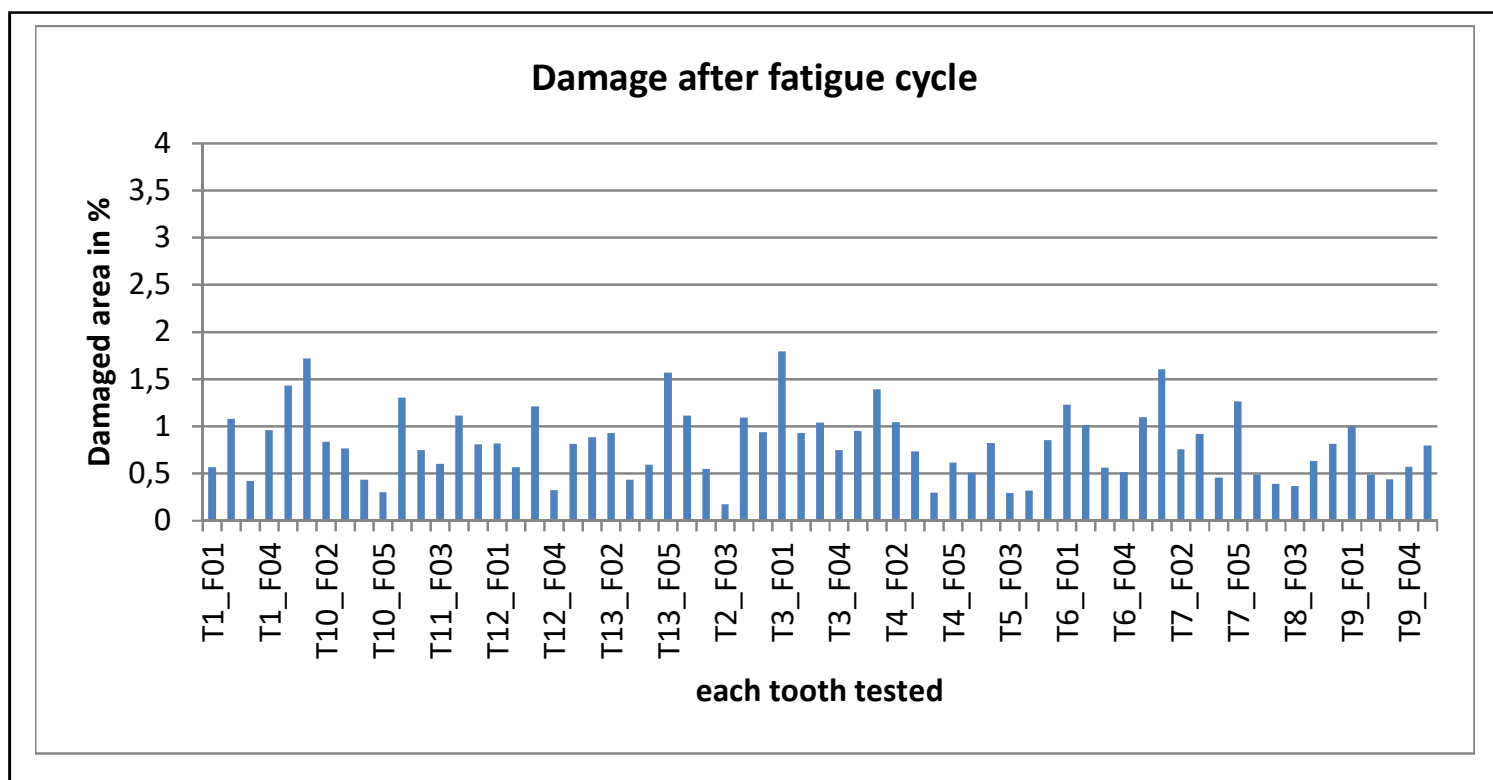

Source: Elaborated by the author

As seen on figure 73 those bars were organized by damaged area, this attempt aimed to explore the final damage behavior. To illustrate the lowest and highest damaged flank and also to observe if a non-parametric analysis would reveal a suitable function data accommodation, suggesting that might have an equation which could describe the phenomenon result. 
Figure 73: Ordinated final damaged area variation observed on gear F01 till F05 teeth after last fatigue cycle step (6h K10).

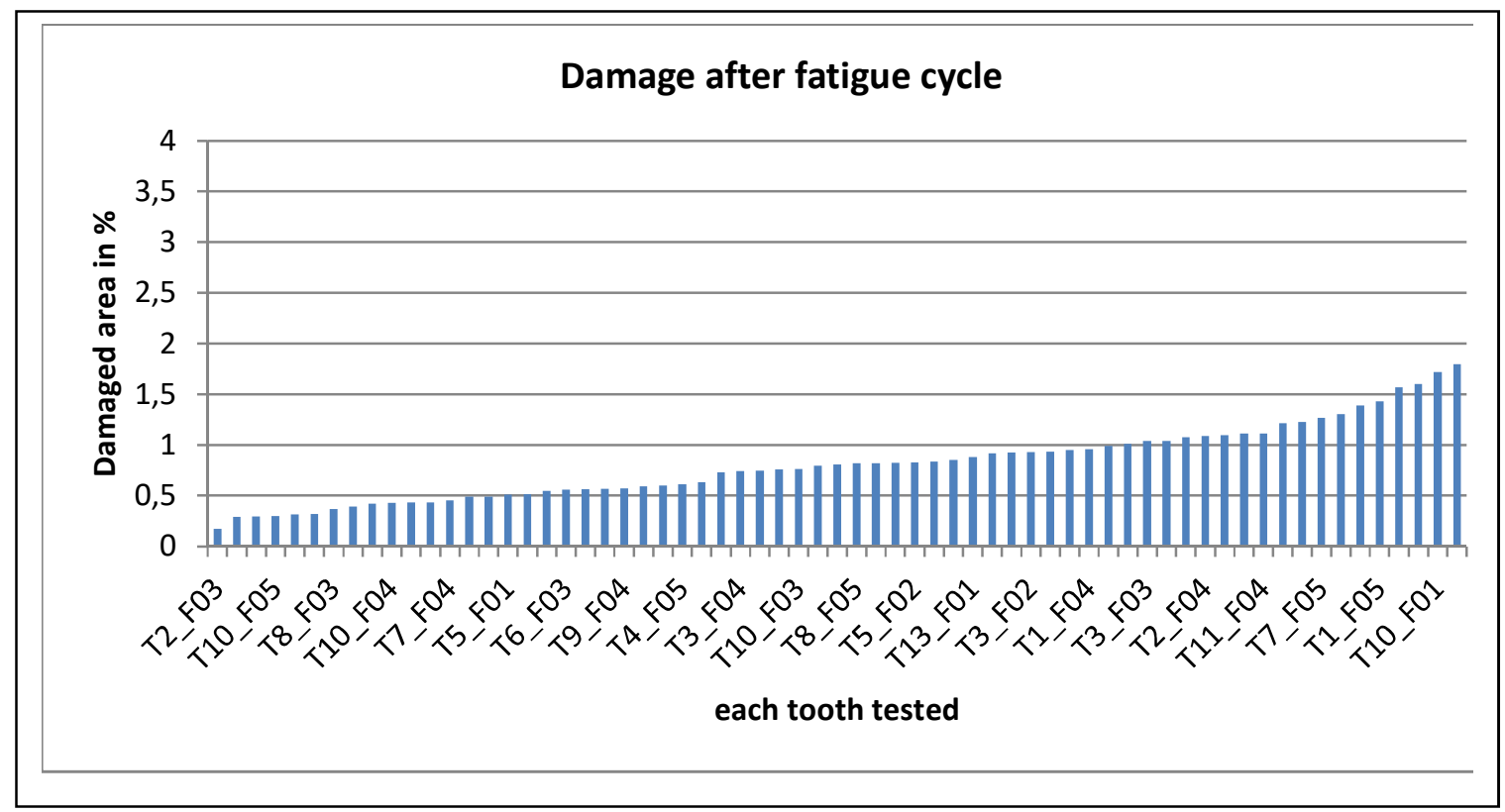

Source: Elaborated by the author

Considering that the gear design is unique for this research, material has been also analyzed and it's according to the SAE 4320 specification, all gears have been produced using the same base material, the production process was the same for all gears, the heat treatment was executed with all gears together, the final gear properties are very similar for both gears. According to ISO 6336-5 (1996), the nominal fatigue life for all gears should be the same with almost the same surface damage. Thus, the present analysis indicates that similar gear can present variation of surface fatigue damage at the end of operational life.

Taking in view that the experimental procedure is fully controlled and the test rig is fully inspected and adjusted between tests the experimental results variation could not be credited to experimental uncertainties.

In this perspective, the hypothesis raised up in the introduction of this research is going to be helpful to explain the final damaged area results shown in the present research. In special, as shown the teeth damaged area by Pitting ranged from $0.17 \%$ till $1.80 \%$. 


\subsection{DATA ANALYSIS}

The hypothesis investigation also requires data evaluation. This is a fundamental step to a more comprehensive perspective of the phenomenon under study, since what is under investigation is the failure model prediction. Here in, the discussion takes place in the first outcome of this research, that the final damaged area has variability, not predicted by the model, which is now focus of the investigation.

So, gears F01 till F05 teeth damaged area after the entire fatigue test are summarized in Table 15. Therefore, on first column is shown the tooth number and on next five columns are displayed the damage area for each gear, starting with F01 till F05.

To better evaluate the data shown on table 15, it's beneficial to observe data using a graphical perspective named box plot (Montgomery, 2012). On the graph shown on figure 74 is possible to observe in just one chart several important characteristics of the data, such as location of central tendency, variability, symmetry and in some cases, the outliers (observations seen far away from the majority of the data).

Table 15: Gear F01 to F05 teeth damaged area by Pitting (in \% of total gear flank area).

\begin{tabular}{|c|c|c|c|c|c|}
\hline Teeth & F01 & F02 & F03 & F04 & F05 \\
\hline $\mathbf{0 1}$ & 0.57 & 1.08 & 0.42 & 0.96 & 1.43 \\
\hline $\mathbf{0 2}$ & 1.11 & 0.55 & 0.17 & 1.09 & 0.94 \\
\hline $\mathbf{0 3}$ & 1.80 & 0.93 & 1.04 & 0.75 & 0.95 \\
\hline $\mathbf{0 4}$ & 1.39 & 1.04 & 0.73 & 0.30 & 0.61 \\
\hline $\mathbf{0 5}$ & 0.51 & 0.83 & 0.29 & 0.32 & 0.85 \\
\hline $\mathbf{0 6}$ & 1.23 & 1.01 & 0.56 & 0.52 & 1.10 \\
\hline $\mathbf{0 7}$ & 1.60 & 0.76 & 0.92 & 0.45 & 1.26 \\
\hline $\mathbf{0 8}$ & 0.49 & 0.39 & 0.37 & 0.63 & 0.82 \\
\hline $\mathbf{0 9}$ & 0.99 & 0.49 & 0.44 & 0.57 & 0.80 \\
\hline $\mathbf{1 0}$ & 1.72 & 0.84 & 0.76 & 0.43 & 0.30 \\
\hline $\mathbf{1 1}$ & 1.30 & 0.75 & 0.60 & 1.11 & 0.81 \\
\hline $\mathbf{1 2}$ & 0.82 & 0.57 & 1.21 & 0.32 & 0.82 \\
\hline $\mathbf{1 3}$ & 0.88 & 0.93 & 0.43 & 0.59 & 1.57 \\
\hline
\end{tabular}


Figure 74: Gears F01(A) till F05 (E) final damaged area data plotting highlighting tendency behavior.

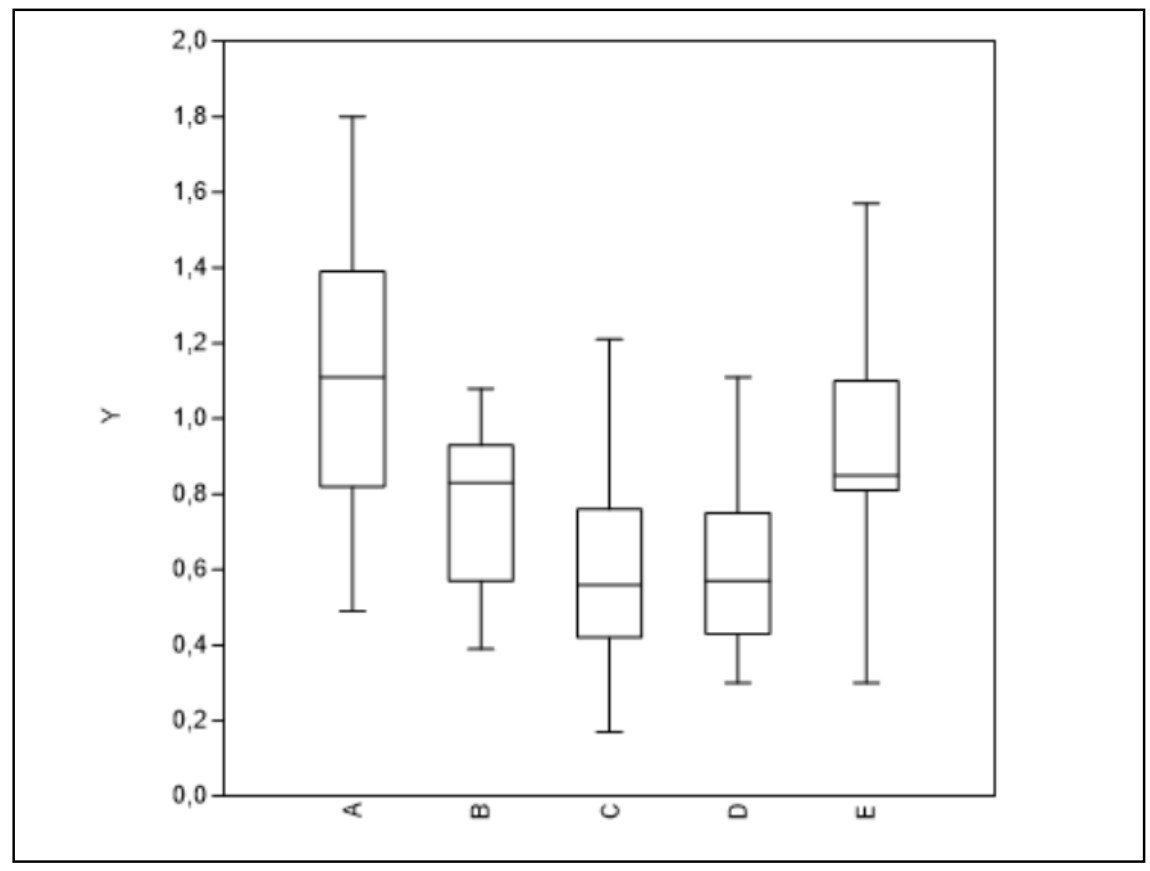

Source: Elaborated by the author

On figure 74 , thus, is seen final fatigue damage area by pitting data, starting from F01 (named as A in the graph), till F05 (named as E in the graph). In the graph is, thus, possible to compare the median value (line inside of each box) and the data symmetry (proportion of the box upper and lower to the median line).

If data set $A$ is under evaluation, it is seen that $50 \%$ of the values ranges from 0.8 till 1.4 while the median value relies on 1.1 . This represents a symmetry of the data. Data set $B$ has $50 \%$ of the values ranging from 0.6 till 0.9 . Data set $C$ has $50 \%$ of the values ranging from 0.4 till 0.7 . Data set $D$ has $50 \%$ of the values ranging from 0.4 till 0.7 and finally data set $E$ is seen that $50 \%$ of the data ranges from 0.8 till 1.1 and the median value relies on 0.85 .

It's also reasonable to say that data set $A$ minimum value is 0.5 and maximum value is 1.8 and while date set $B$ minimum value is 0.4 and maximum value is 1.1 . This complementary information allows the interpretation that data $B$ is more 
concentrated toward central tendency data. It is also possible to say that, as data set $A$ median is more prone to represent average, since data set $A$ is more symmetric. In this perspective, it is more symmetric than data set $B$.

\subsection{RELIABILITY ANALYSIS}

Initially, the cumulative distribution associated with damage area for each gear can be established. For that analysis the Weibull Distribution is selected to define cumulative distribution being a versatile distribution due to the significant influence of its shape parameters on distribution modeling. Figure 75 presents the probability plot of Weibull distribution indicating pitting damaged area distribution for each of the five gears.

Figure 75: Weibull Probability Plot for pitting damage area for gears F01 to F05

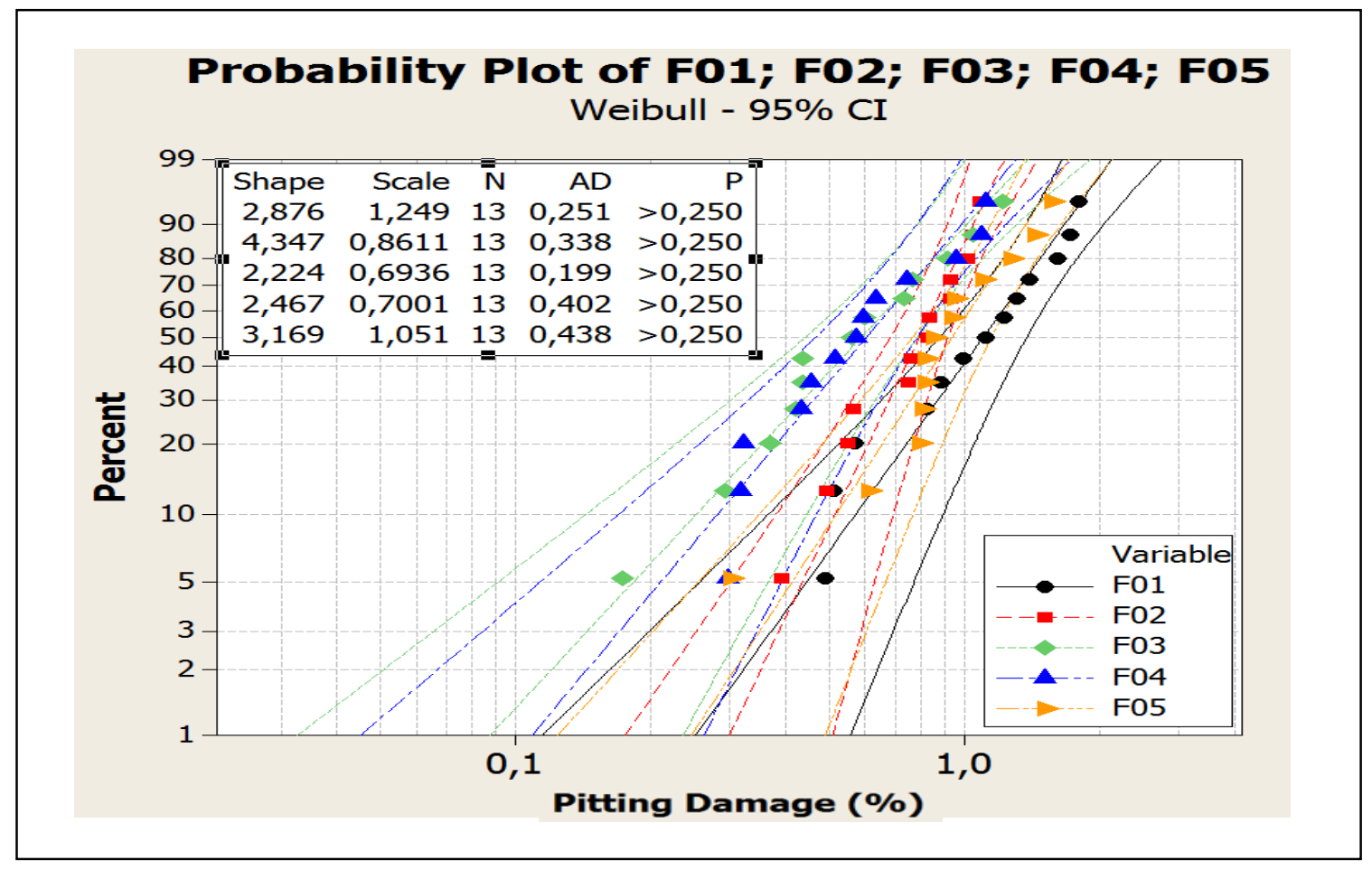

Source: Elaborated by the author 
That distribution represents a good fit for damaged area of all gears according to Anderson-Darling and P-value goodness of fit test. Gears F03 and F04 present shape parameter lower than 2.6, indicating that the distribution is positively skewed (has a right tail). Gears F01 and F05 have shape parameter between 2.6 and 3.7 indicating that the coefficient of skewness approaches zero. Finally gear F02 presents a shape parameter greater than 3.7 indicating that the distribution is negatively skewed (has a left tail). In the same figure uncertainty in the results due to the limited sample sizes are represented by confidence bounds. All experimental data are located inside the confidence bounds for each distribution.

Figure 76 presents the Weibull cumulative distribution of pitting damaged area for the five gears.

Based on Figure 76, it is possible to conclude that gears F03 and F04 have a similar pitting damaged area distribution. Gear F01 presents a spreader damaged area distribution than the other gears. Gear F02 presents the probability distribution with lowest scattering. Those results indicate that as for pitting damaged area distribution the five gears apparently present different behavior. 
Figure 76: Weibull cumulative pitting damaged area distribution for five gears.

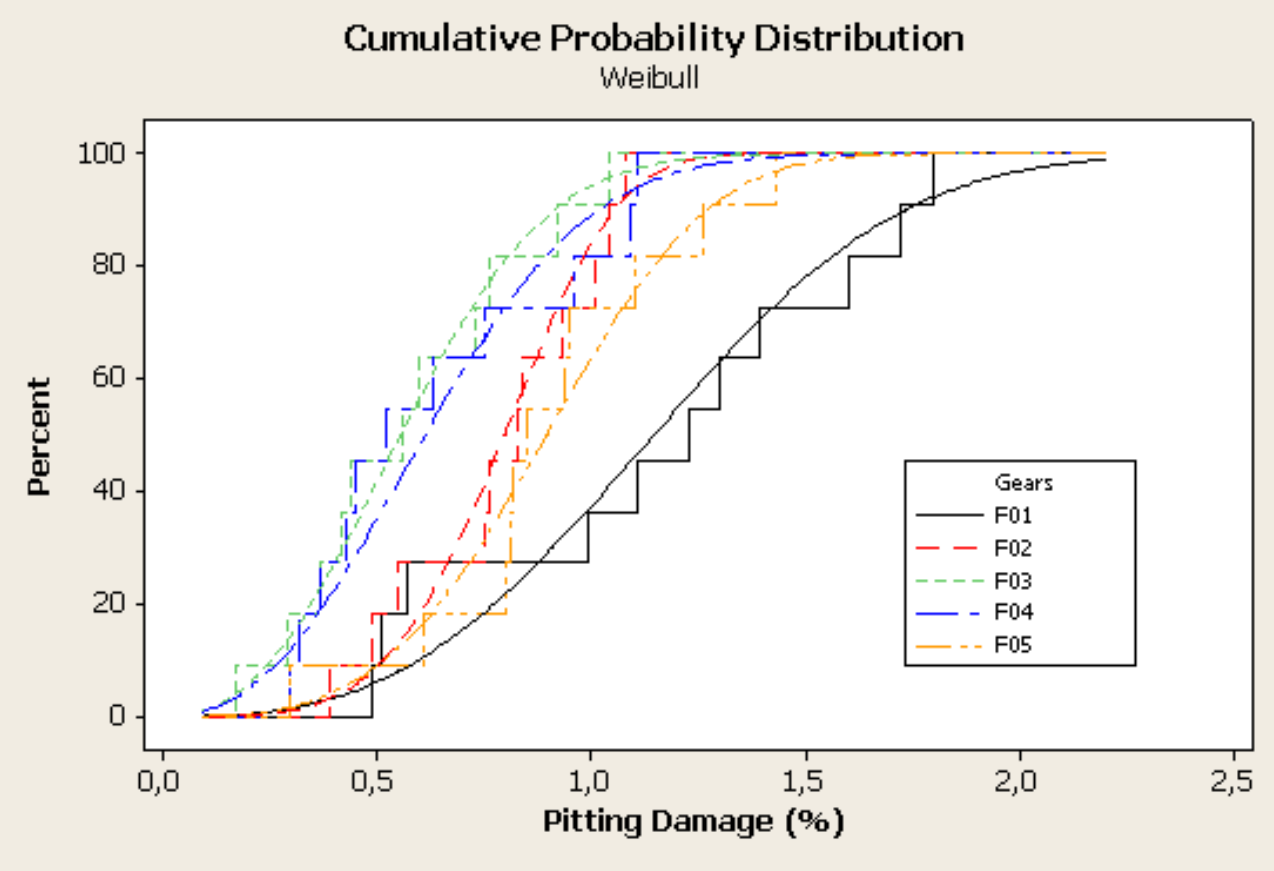

Source: Elaborated by the author

So, the research question raised up, which is unconditionally accorded to this research objective, here in is: Is there any process error influence on the data set seen in figure 76 and table 15.

\subsection{PROCESS ERRORS COMPARISON}

All gears were submitted to dimensional inspection, prior to the gear fatigue test. Hence, some important variables were measured aiming to correlate them with final tooth flank damage observed on test specimens submitted to fatigue test. Those measured variables are known as gear process errors: (i) $f_{\text {ha }}$, Profile slope deviation, (ii) $f_{f \alpha}$ Profile form deviation, (iii) $f_{h \beta}$ Helix slope deviation, (iv) $f_{f \beta}$ Helix form deviation, (v) $\mathrm{f}_{\mathrm{pt}}$ Single pitch deviation (ISO 1328-1, 1995) (ISO 1328-1, 2013). In figure 77 is shown an illustration of each one of those errors, which can be known as process errors of the designed geometry (Lin et al, 2017). 
Figure 77: Tooth surface with process errors in comparison with ideal geometry

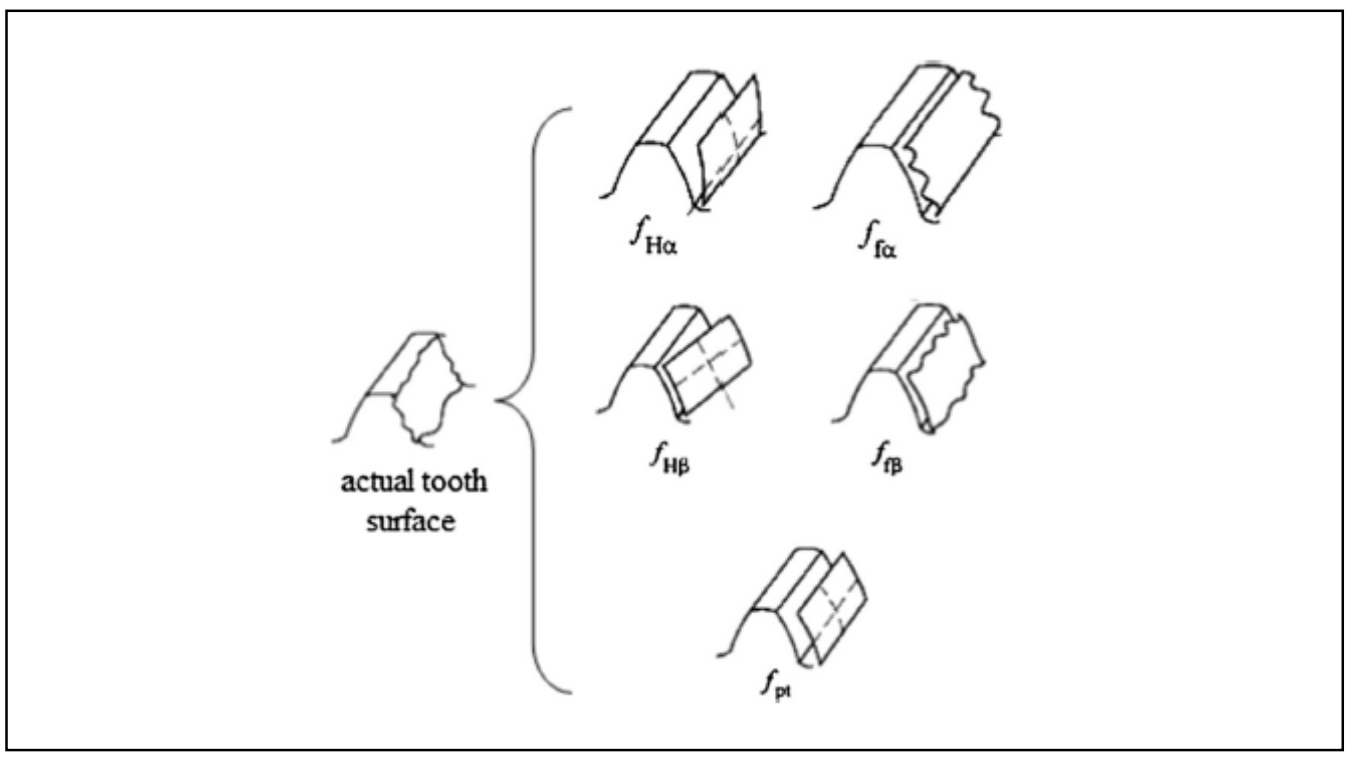

Source: Lin et al., 2017

On table 16, in first column, is indicated the mean value of final damaged area for each gear ${ }^{3}$ ordinated from the smaller to the higher damaged area by pitting. On second column is seen the sample number or gear number to which the mean final damage belongs. From the third till seventh columns are shown the mean values of the measured variables $f_{h \alpha}, f_{f \alpha}, f_{h \beta}, f_{f \beta}, f_{p t}$ of gears F01 till F05.

Table 16: Comparison of gear flank mean damage area by pitting and mean process errors

\begin{tabular}{|c|c|c|c|c|c|c|}
\hline Damaged area & Gear \# & $\mathbf{f}_{\mathbf{h} \boldsymbol{\alpha}}(\boldsymbol{\mu m})$ & $\mathbf{f}_{\mathbf{f} \boldsymbol{\alpha}}(\boldsymbol{\mu m})$ & $\mathbf{f}_{\mathbf{h} \boldsymbol{\beta}}(\boldsymbol{\mu m})$ & $\mathbf{f}_{\mathbf{f} \boldsymbol{\beta}}(\boldsymbol{\mu m})$ & $\mathbf{f}_{\mathbf{p t}}(\boldsymbol{\mu m})$ \\
\hline $\mathbf{0 . 6 1}$ & F03 & -1.28 & 13.20 & 0.95 & 11.23 & 2.50 \\
\hline $\mathbf{0 . 6 2}$ & $\mathrm{F} 04$ & -7.85 & 14.40 & 0.83 & 10.60 & 1.50 \\
\hline $\mathbf{0 . 7 8}$ & $\mathrm{F} 02$ & -2.05 & 10.93 & 0.33 & 9.53 & -0.63 \\
\hline $\mathbf{0 . 9 4}$ & F05 & -1.60 & 15.30 & -1.48 & 10.43 & 0.13 \\
\hline $\mathbf{1 . 1 1}$ & F01 & -2.10 & 10.28 & 2.98 & 10.90 & 0.50 \\
\hline
\end{tabular}

\footnotetext{
3 The ring gear process errors were not measured within FCA gear metrology laboratory, since the gear manufacturer guarantee that no error was higher than $0.8 \mu \mathrm{m}$. Despite of knowing it, the random contact seen between a 28 teeth gear and 13 teeth pinion would not provide reference to guide evaluations between gear tooth process errors and pinion tooth damage. Also, ring gear damaged area were not evaluated on this work.
} 
Those means values of $f_{h \alpha}, f_{f \alpha}, f_{h \beta}, f_{f \beta}$ and $f_{p t}$ were measured using Wenzel WGT 350 equipment, seen on figure 60 . The equipment use a contact probe which measured gear teeth and automatically calculated and display the mean values for each of the variables seen on table 16 (On Appendix $D$ and $G$ are shown details of 1,4,7,11 gear teeth were measurements done. Those teeth were selected due to its distribution over gear perimeter. Table 16 also shows that no direct correlation can be seen when all data is considered. Consequently, a statistical tool is required to support the analysis.

\subsection{STATISTICAL ANALYSIS}

Data seen on Table 15 (pag 113) are pertinent to allow a statistical analysis and to prepare input data for Analysis of Variance (ANOVA). The ANOVA is useful to analyze the differences among group means. The null hypothesis $\left(\mathrm{H}_{0}\right)$ for an ANOVA is that there is no significant difference among the groups (in the present case among the gears damaged area). The alternative hypothesis $\left(\mathrm{H}_{1}\right)$ assumes that there is at least one significant difference among the groups. The analysis considers model errors as normally and independently distributed with average zero and variance $\mathrm{S}^{2}$ (Montgomery, 2013). On table 17 is summarized some important calculations as count of repetitions on each group (one specific gear), results sum, mean value and variance.

Table 17: Statistical data summary results.

\begin{tabular}{lcccc} 
SUMMARY & & & & \\
\hline Groups & Count & Sum & Mean & Variance \\
\hline F01 & 13 & 14.42 & 1.11 & 0.20 \\
F02 & 13 & 10.15 & 0.78 & 0.05 \\
F03 & 13 & 7.96 & 0.61 & 0.09 \\
F04 & 13 & 8.04 & 0.62 & 0.08 \\
F05 & 13 & 12.24 & 0.94 & 0.11 \\
\hline
\end{tabular}

The ANOVA procedure is based on decomposition of total variation in parts which could be attributed to treatment and to residual. All calculation was performed on Microsoft Excel Data Analysis function and the output table is shown on table 18. Statistical value $F$ is calculated dividing the mean square between groups by the 
mean square within groups. So, calculated $F(5.56)$ is higher than F critical (2.53) and $p$ value is lower than adopted significance level $(\alpha=5 \%)$. This result defines that the null hypothesis of all mean values of gear damaged area between gears are equal has been rejected. Consequently, the alternative hypothesis $\left(H_{1}\right)$ is that there are differences between gears damaged area, which means the gears does have influence on mean value of final damaged area by pitting after entire fatigue cycle. This is key information for evaluation analysis, once it will guide the choice of samples to perform a geometry comparison to possibly explain final damage variations among gears. In this research, the experimental procedure is fully controlled and the test rig is fully inspected and adjusted between tests. Hence, the experimental results variation could not be credited to experimental uncertainties

Table 18: ANOVA single factor evaluation results.

\begin{tabular}{lcccccc} 
ANOVA & \multicolumn{7}{l}{ Sum of } & $\begin{array}{c}\text { Degree of } \\
\text { Source of }\end{array}$ & $\begin{array}{c}\text { Mean } \\
\text { Variation }\end{array}$ & Squares & freedom & Square & $F$ & P-value & F crit \\
\hline Between Groups & 2.39 & 4 & 0.60 & 5.56 & 0.0007 & 2.53 \\
Within Groups & 6.44 & 60 & 0.11 & & & \\
& & & & & & \\
Total & 8.83 & 64 & & & & \\
\hline
\end{tabular}

The ANOVA results indicate that the manufacturing process introduces variability in gear geometry that has significant effect on pitting damage evolution during tests. Once ANOVA is not adequate to evaluate which treatment mean value differs from other mean values, another statistical test is required. The Tukey test performs a pair to pair comparison and defines in between groups the ones which the mean value are considered unequal, considering a significance level $\alpha$.

Thus, on table 19 is shown the final output of Tukey test with the results shown above the table diagonal. Those values above the diagonal are the calculated $p$ values for the pair to pair comparison. The $p$ values which are under the adopted significance level $(\alpha)$ or smaller than 0.05 are highlighted in red. Based on this is suitable to conclude calculated p for F03 in comparison to F01 and also F04 in comparison to F01 are smaller than proposed significance level, confirming that the null hypothesis 
could be rejected, pointing the means which are considered different by the test. Those values define that gears F04 and F01, F03 and F01 mean damaged area are considered statistically different. In other words, the treatment has influence between mentioned gears. Based on those results, it is reasonable to isolate those gears results and analyze the damage area results and gear process errors of F01, F03 and F04.

Table 19: Tukey test pairwise comparisons

\begin{tabular}{|c|c|c|c|c|c|}
\hline & F01 & F02 & F03 & F04 & F05 \\
\hline F01 & - & 0.09597 & 0.002541 & 0.00304 & 0.7004 \\
\hline F02 & & - & 0.6711 & 0.7076 & 0.7219 \\
\hline F03 & & & - & 1 & 0.08611 \\
\hline F04 & & & & - & 0,09857 \\
\hline F05 & & & & & - \\
\hline
\end{tabular}

So, on figure 78 are seen mean damage area results and mean gear process errors of F04, F03 and F01, respectively. On the graph will be seen only damaged area influenced by the treatment, according to the Tukey Test. Those data are ordinated based on gear damaged area, with gear F04 mean damaged area of 0.61, gear F03 mean damaged area of 0.62 and F01 mean damaged area of 1.11. The ordination aims to allow a comparison of mean gear damaged and mean measured manufacturing process errors. 
Figure 78: Gear process errors $x$ damaged area by pitting in $\%$.

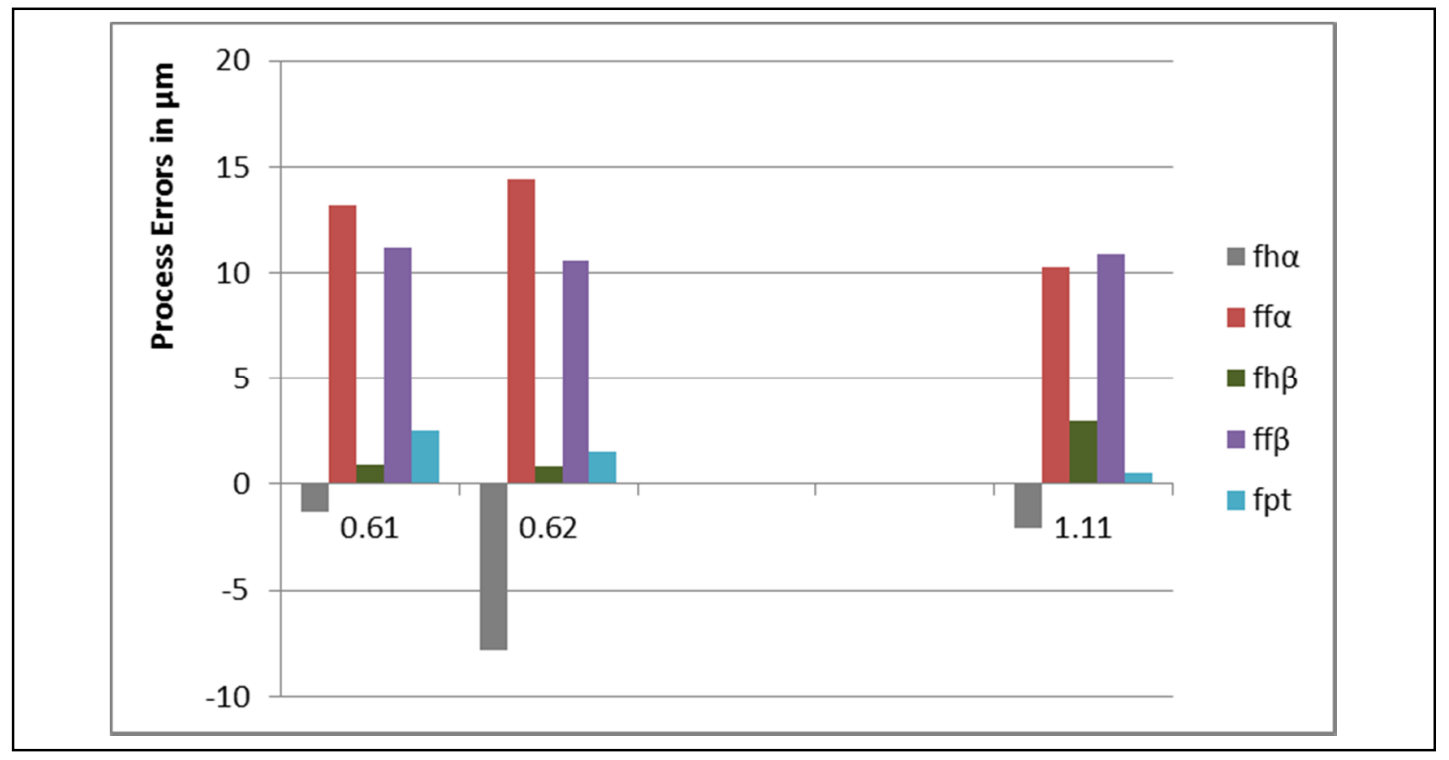

Source: Elaborated by the author

As shown on figure 78, a correlation between mean process errors measured and mean damaged is revealed. It's important to highlight that each one of those evaluated errors define a geometry adjustment of gear tooth in comparison to the designed geometry. However, some conceivable explanations of process errors influence on stress evaluation will be described individually, as seen below:

(i) Negative Profile slope deviation ( $f_{\text {ha }}$ ) as seen on gear F04 will define a new contact position on addendum. As a result, worsen contact pattern due reduced elastic deformation delimiting the real contact area. Consequently, the stress on addendum will be increased. On dedendum the contact pattern tends to improve, since elastic deformation tends to increase, enlarging the real contact area. Consequently, the contact stress will be reduced.

(ii) Positive Profile form deviation ( $f_{f a}$ ) as seen on gears F03 and F04 reveal existence of additional material on tooth profile. Under load events, this material tends to deform and increase real contact area, consequently, reducing contact stress.

(iii) Positive Helix slope deviation ( $\left.f_{h \beta}\right)$ as seen on gear F01 will induce a misalignment in pinion and ring contact plans, this tends to reduce contact line length and, consequently, initial contact stress will be increased. 
(iv) Positive Helix form deviation $\left(\mathrm{f}_{\mathrm{f} \beta}\right)$ reveal existence of additional material on tooth contact width. Under load events, this material tends to deform and increase real contact area, consequently, reducing initial contact stress. Although, based on this research results, helix form deviation variation were not significant to influence results.

(v) Positive Single pitch $\left(f_{p t}\right)$ as seen on gear F03 will increase teeth thickness by shifting the real position of pinion contact plans and, consequently, reducing the space width (space between teeth). This adjust will provide earlier contacts than previously designed, slightly reducing pinion tooth single contact area, subsequently, improving its tension distribution.

In this research the attention was done to understand the process uncertainty effects on final damage by pitting. In this perspective, on figure 79 (a), (b) and (c) is shown an illustrative and highlighted contact plans comparison modified by relevant process errors that had demonstrated influence on final damage to gears F03, F04 and F01 respectively. Such tooth geometry dispersion defined a contact plan which are different of the one previously designed to the meshing teeth as also seen on figure 79 illustrative view. In this figure, $f_{f \alpha}$ and $f_{f \beta}$ are not shown due to the intention of demonstrating a clear plan of contact.

Nevertheless, those five process errors measured (measured variables) had acted together to redefine the real tooth geometry of those evaluated samples. Such tooth geometry variation suggests that each contact phenomenon, indeed, had its particular dynamic characteristics. Thus, on figure 79 are shown meshing teeth surface plan drawing do on NX.11 after taken into consideration process errors influence. On these figure such errors are emphasized considering the same approach previously taken on the data analysis. Therefore, such drawings take into consideration the mean variation for errors to define the illustrative geometry for each tooth under evaluation.

Thus, following the Tukey test methodology and performing once more a pair to pair comparison, it's seen a final contact plan of gears (a) F03 x (c) F01 and also (b) F04 and (c) F01. 
Figure 79: Gear tooth illustrating (a) F03 final geometry impacted by fpt (b) F04 final geometry impacted by fpt and fha and (c) F01 final gear geometry impacted by fpt, fha and fh $\beta$

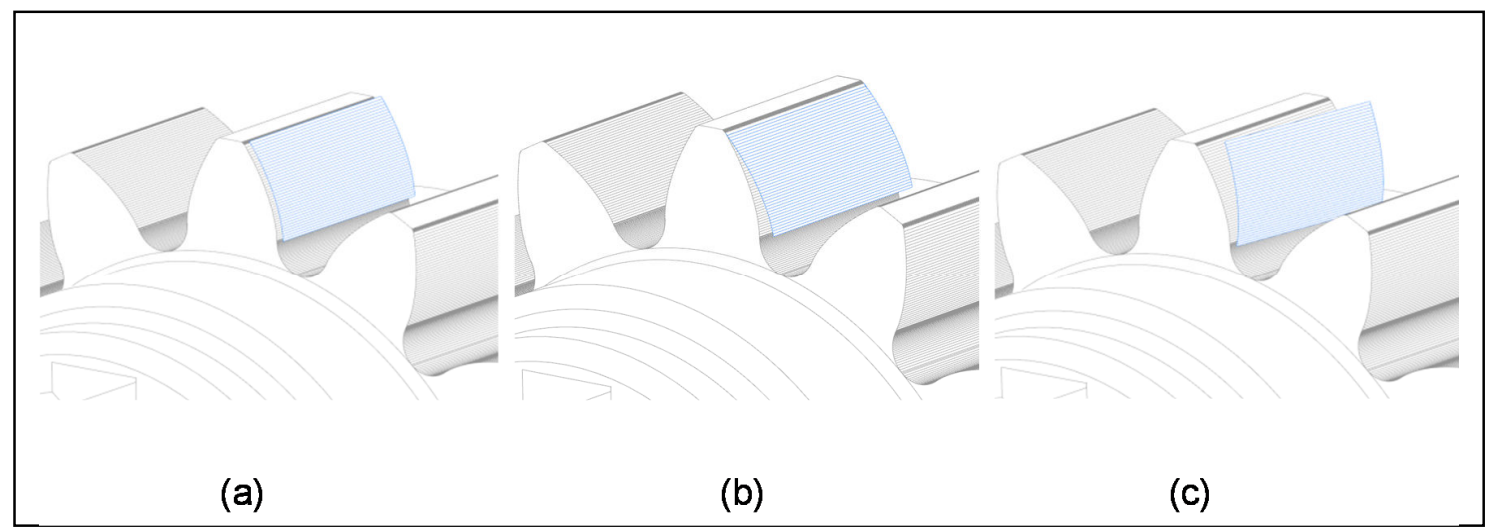

Source: Elaborated by the author

This result matches the main objective of this research, which was the evaluation of the process error influence on damaged area by pitting.

To complement the evaluation and support conclusions, once again the simulation were performed aiming to evaluate the influence done on contact stress due to process errors measured on F01, F03 and F04 gears. Due to simulation resources, this simulation was limited to evaluate $f_{h \alpha}$ and $f_{h \beta}$ influence on contact stress.

Thus, on table 20 is seen the contact stress calculated by another software which supports IsoCad simulation, the Load Pressure Distribution software. Once again, following Tukey test methodology and performing a pair to pair comparison is seen that on F01 a higher maximum contact stress is seen when compared to F03 or to F04. This results matches to previous result of mean damaged area shown on table 17 and re-written in table 20, where F01 had a higher damaged area compared to F03 or to F04.

Table 20: Influence of $f_{h \alpha}$ and $f_{h \beta}$ process errors on contact stress.

\begin{tabular}{|c|c|c|c|}
\hline & F01 & F03 & F04 \\
\hline $\begin{array}{c}\text { Damaged area } \\
\text { (\%) }\end{array}$ & 1.11 & 0.61 & 0.62 \\
\hline $\begin{array}{c}\text { Contact Stress } \\
\text { (MPa) }\end{array}$ & 3,049 & 2,461 & 2,448 \\
\hline
\end{tabular}




\subsection{ADDING COATED GEARS W-DLC AND DLC RESULTS (SPECIFIC OBJECTIVE VII).}

In this section, all results achieved using the methodology previously explained using coated gears as studied object will be presented and discussed.

\section{SAMPLE PREPARATION}

The W-DLC coating was done by Balzers using the PVD process as described on section 6.2. The PVD methodology defines that the lowest coating temperature should be $200^{\circ} \mathrm{C}$. That was the lowest coating temperature available in Balzers Brazil.

However, this temperature should influence gear properties taken after heat treatment. Some initial lab samples were coated using Brazil's chamber and in fact the temperature influenced specimen mechanical properties. Those results were not seen in this work since it was just a process setting.

Therefore, the samples were shipped to USA to be submitted to an industrial scale chamber capable of coating at $160^{\circ} \mathrm{C}$. This effort was done to guarantee that no hardness variation were seen.

The DLC coating was done by INPE (in English, Special Research Institute) using the CVD process described on section 6.2. The CVD methodology defines that the lowest coating temperature should be $90^{\circ}$, using a lab scale chamber.

\section{SAMPLE ANALYSIS}

As seen on table 21 as shaved gears (samples F06 and F07), W-DLC (Samples B06 e B07) and DLC (Samples D02 and D03) had different roughness values and based on discussed data is reasonable to highlight: 
Table 21: Roughness measurements performed on pinion cross direction, comparing as shaved and coated gears.

\begin{tabular}{|c|c|c|c|c|c|c|c|}
\hline \multirow{3}{*}{\multicolumn{2}{|c|}{ Parameters }} & \multicolumn{6}{|c|}{ Measured Values in $\mu \mathrm{m}$} \\
\hline & & \multicolumn{6}{|c|}{ Cross Direction } \\
\hline & & F06 & F07 & B07 & B08 & D02 & D03 \\
\hline \multirow{3}{*}{$\mathbf{R a}$} & 1 & 0.79 & 0.75 & 0.82 & 0.79 & 0.93 & 0.89 \\
\hline & 2 & 0.69 & 0.77 & 0.85 & 0.82 & 0.92 & 0.93 \\
\hline & 3 & 0.73 & 0.78 & 0.80 & 0.83 & 0.94 & 0.96 \\
\hline \multirow{3}{*}{$\mathbf{R z}$} & 1 & 2.78 & 2.05 & 3.21 & 3.36 & 3.14 & 2.92 \\
\hline & 2 & 2.56 & 3.06 & 3.16 & 2.60 & 2.64 & 3.37 \\
\hline & 3 & 2.59 & 2.98 & 2.62 & 2.96 & 3.19 & 3.24 \\
\hline \multirow{3}{*}{ Rt } & 1 & 3.12 & 2.24 & 4.28 & 4.53 & 3.69 & 3.49 \\
\hline & 2 & 3.05 & 3.19 & 3.51 & 2.98 & 3.47 & 4.38 \\
\hline & 3 & 2,61 & 3,05 & 3.57 & 4.39 & 3.67 & 3.74 \\
\hline
\end{tabular}

As discussed, $\mathrm{Ra}$ is known as the profile surface height arithmetic mean deviation from the mean line, this concept is helpful to evaluate the irregularities of the surface under evaluation. So, the results shown on table 22 supports that $\operatorname{Ra}(D)$ $>\operatorname{Ra}(\mathrm{B})>\operatorname{Ra}(\mathrm{F})$, suggesting that uncoated and coated surfaces have different surface roughness.

Starting from the extreme values $\operatorname{Ra}(D)$ ( $D L C)$ and $\operatorname{Ra}(F)$ (uncoated) comparison, it's possible to understand that DLC layers addition has induced a higher mean arithmetic profile surface deviation from the theoretical mean line.

An intermediate value of $\operatorname{Ra}$ for $\operatorname{Ra}(B)(W-D L C)$. The results shown that $\operatorname{Ra}(B)$ (W-DLC) is still higher than $\mathrm{Ra}(\mathrm{F})$ (uncoated), emphasizing that the same logic of inducing a higher mean arithmetic deviation from the surface deviation from mean line is still true.

As discussed, $R z$ is the sum of the maximum height of the peaks of the $Z p$ profile and the largest of the depths of the valleys of the profile $\mathrm{Zv}$, at the sampling length. So, as this parameter moderates the highest values of each samples evaluation, it tends to adjust the values, as a consequence, the results points to a very similar result of $R z$ for coated gears, but both higher than uncoated. $R z(B)=$ $R z(D)>R z(F)$. Proposing, again, that coating has increased roughness. 
As discussed, Rt is the sum of the highest peak of the $\mathrm{Zp}$ profile and the largest depths of the valleys of the Zv profile in the evaluation length (total evaluation length). The results seen on table 21 , shows that $\operatorname{Rt}(B)>\operatorname{Rt}(D)>\operatorname{Rt}(F)$. So, the result shows that W-DLC (Samples B06 e B07) has higher peaks and valleys. In other words, on the W-DLC coated surface might have been induced an asymmetry.

So, $\operatorname{Ra}(D)$ is higher than $\operatorname{Ra}(B)$ and the $\operatorname{Rt}(B)$ is higher than $\operatorname{Rt}(D)$. Based on this statement, it's coherent to assume that on W-DLC coated gears the valleys and peaks sizes pattern are more asymmetric than on DLC. Those conclusion is possible since $\operatorname{Ra}(B)$ average result is lower than $\operatorname{Ra}(D)$, but the $\operatorname{Rt}(B)$ is higher than $\operatorname{Rt}(D)$.

As seen on table 22 hardness, case depth and core hardness measurements of researched gears reveal that such properties are very similar. This results are according to expectation of not having coating process temperature influencing on material mechanical properties. Based on such results, no variation due to mechanical properties can be assumed or expected on fatigue evaluation.

Table 22: Hardness, case depth and core hardness measurements of researched gears

\begin{tabular}{|c|c|c|c|c|c|c|c|}
\hline \multirow{2}{*}{ Parameters } & \multirow{2}{*}{ Specified } & \multicolumn{6}{|c|}{ Identified } \\
\cline { 3 - 8 } & & \multicolumn{2}{|c|}{ Pinion } & \multicolumn{2}{c|}{ Pinion } & \multicolumn{2}{c|}{ Pinion } \\
\cline { 2 - 8 } & F06 & F07 & B01 & B04 & D03 & D05 \\
\hline $\begin{array}{c}\text { Hardness (HRc) } \\
\text { Case depth (mm) } \\
\begin{array}{c}\text { Core Hardness } \\
\text { (HRc) }\end{array}\end{array}$ & $58: 63$ & 62 & 62 & 59 & 61 & 61 & 60 \\
\cline { 2 - 8 } & $0.5: 0.7$ & 0.65 & 0.63 & 0.7 & 0.65 & 0.7 & 0.65 \\
\cline { 2 - 8 } Material & $\begin{array}{c}\text { SAE } \\
4320\end{array}$ & \multicolumn{2}{|c|}{ SAE 4320 } & \multicolumn{2}{c|}{ SAE 4320 } & \multicolumn{2}{c|}{ SAE 4320 } \\
\hline
\end{tabular}


Coating chemical composition

B gears and D gears tooth sample was cut to be evaluated on SEM. Each tooth was submitted to EDS evaluation analysis aiming to check chemical composition. Its results are seen on figure 80 and 82 .

Figure 80 shows material chemical evaluation

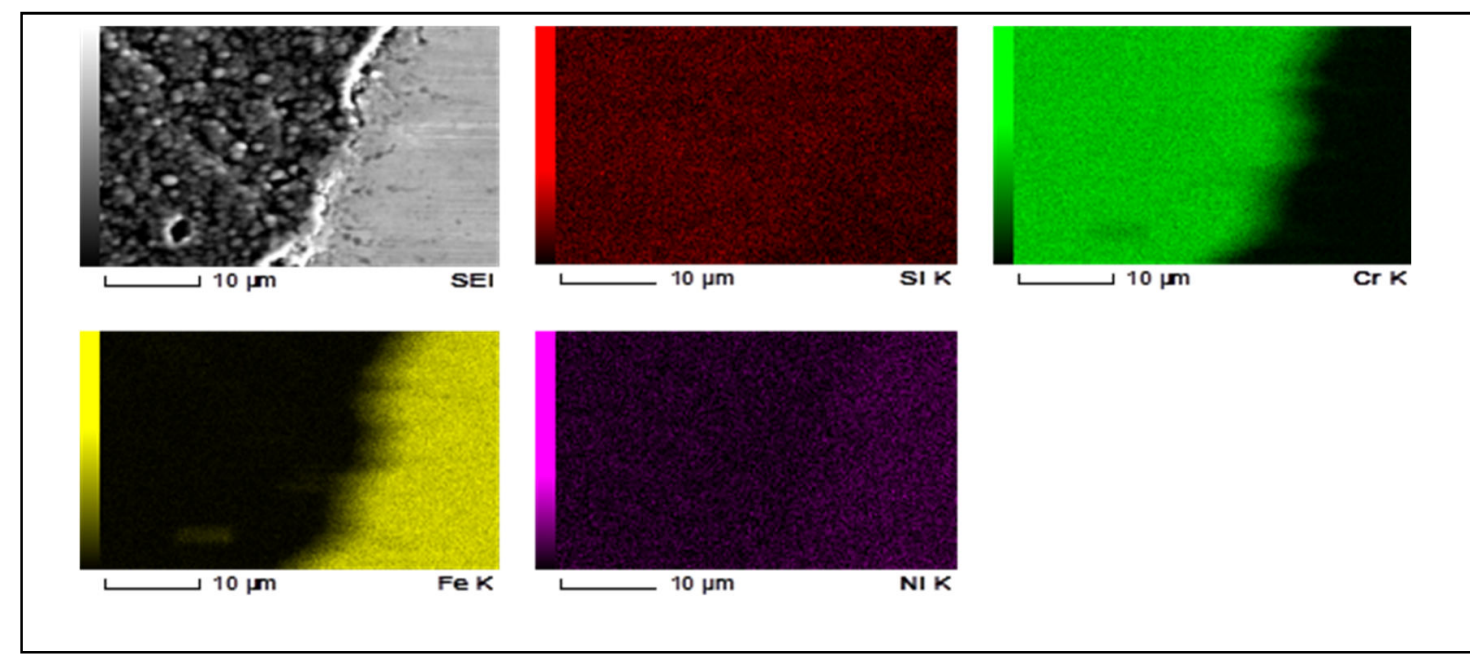

Source: Elaborated by the author

Based on chemical evaluation shown on table 23 and illustrated peaks on figure 81, the B01 till B05 gear which were coated by Balzers, in fact, were not W-DLC coating, but it was Cr-DLC coating. So, here under this new composition will be used to refer to $B$ gears samples. As defined by Balzers, Cr should just be seen on the interlayer, working as a chemical interface component to provide adherence of base material on coating initial layers.

Table 23: Chemical composition revealed by EDS

\begin{tabular}{|c|c|c|}
\hline Chemical formula & mass in \% & Atom in \% \\
\hline Si $^{*}$ & 0.15 & 0.28 \\
\hline $\mathrm{Cr}$ & 58.71 & 60.36 \\
\hline $\mathrm{Fe}$ & 40.44 & 38.71 \\
\hline $\mathrm{Ni}$ & 0.70 & 0.64 \\
\hline Total & 100.00 & 100.00 \\
\hline
\end{tabular}


Figure 81: shows material chemical evaluation

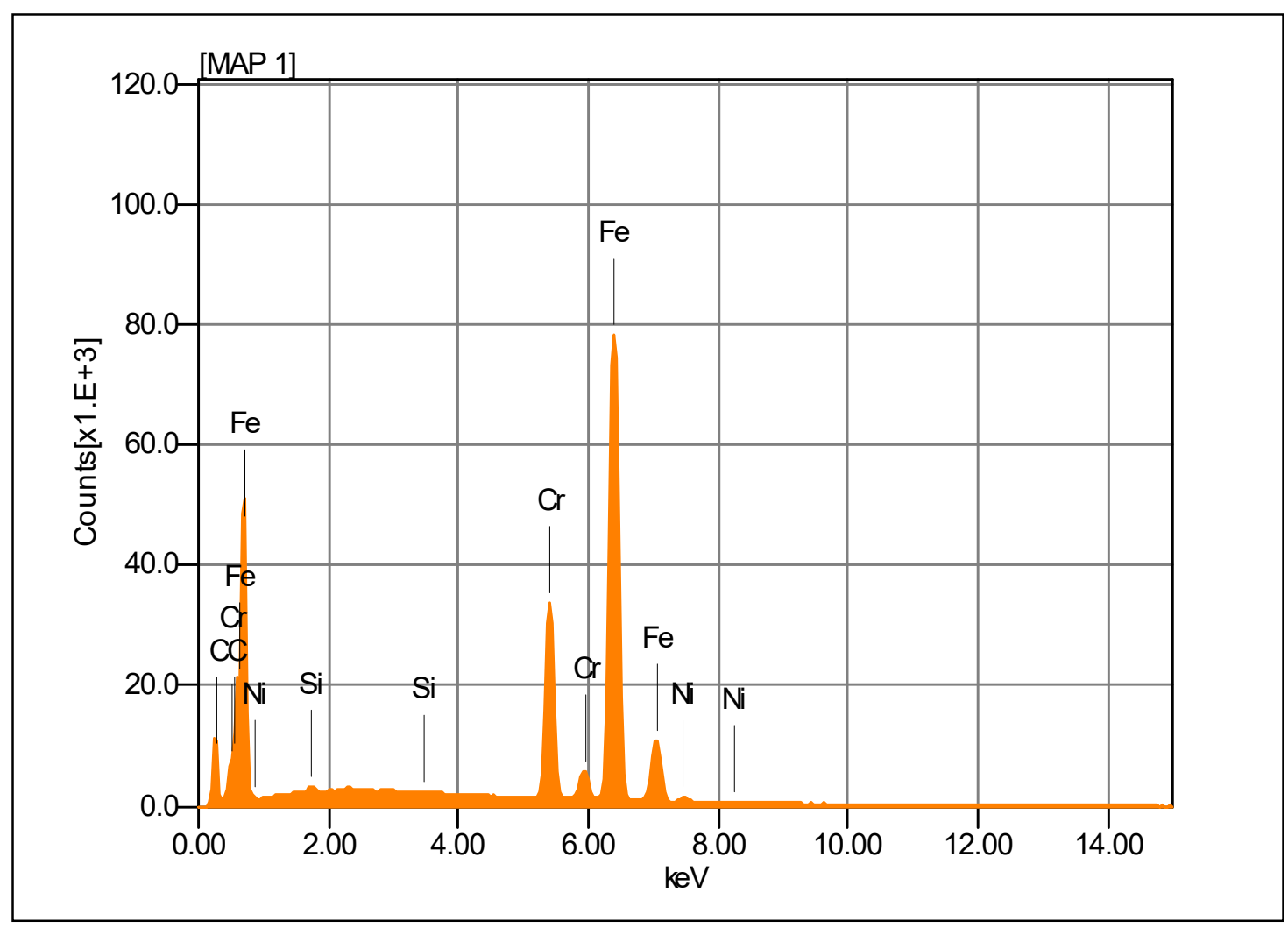

Source: Elaborated by the author

On D gears, chemical quantification won't be shown since Carbon was not excluded of the analysis. The reason why it was not excluded was to be able to better illustrate its presence. In this second coating, D gears, the interlayer was Si. The main elements are seen on figure 82, which represents part of chemical composition evaluation done by SEM - EDS. On figure 83 is seen some spikes confirming the element presence and estimated quantification. 
Figure 82: shows material chemical evaluation.

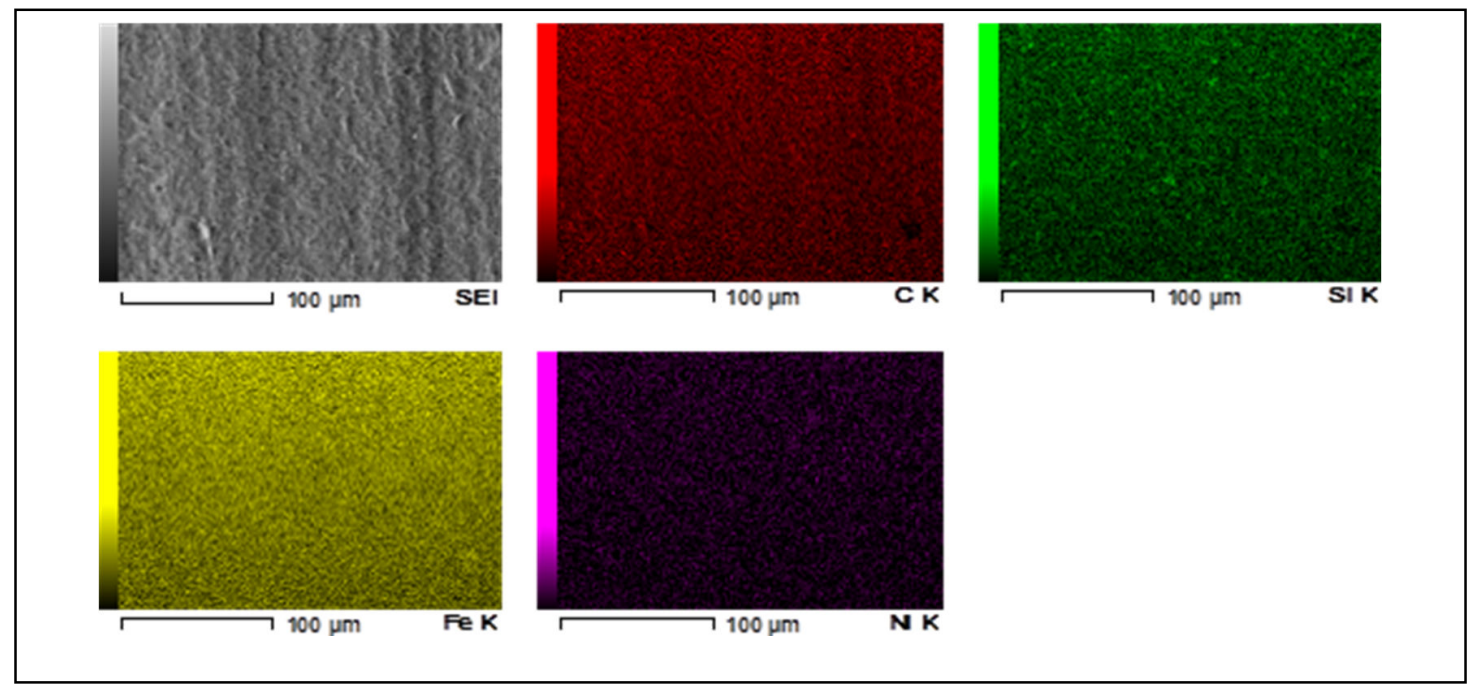

Source: Elaborated by the author

Figure 83: shows material chemical evaluation

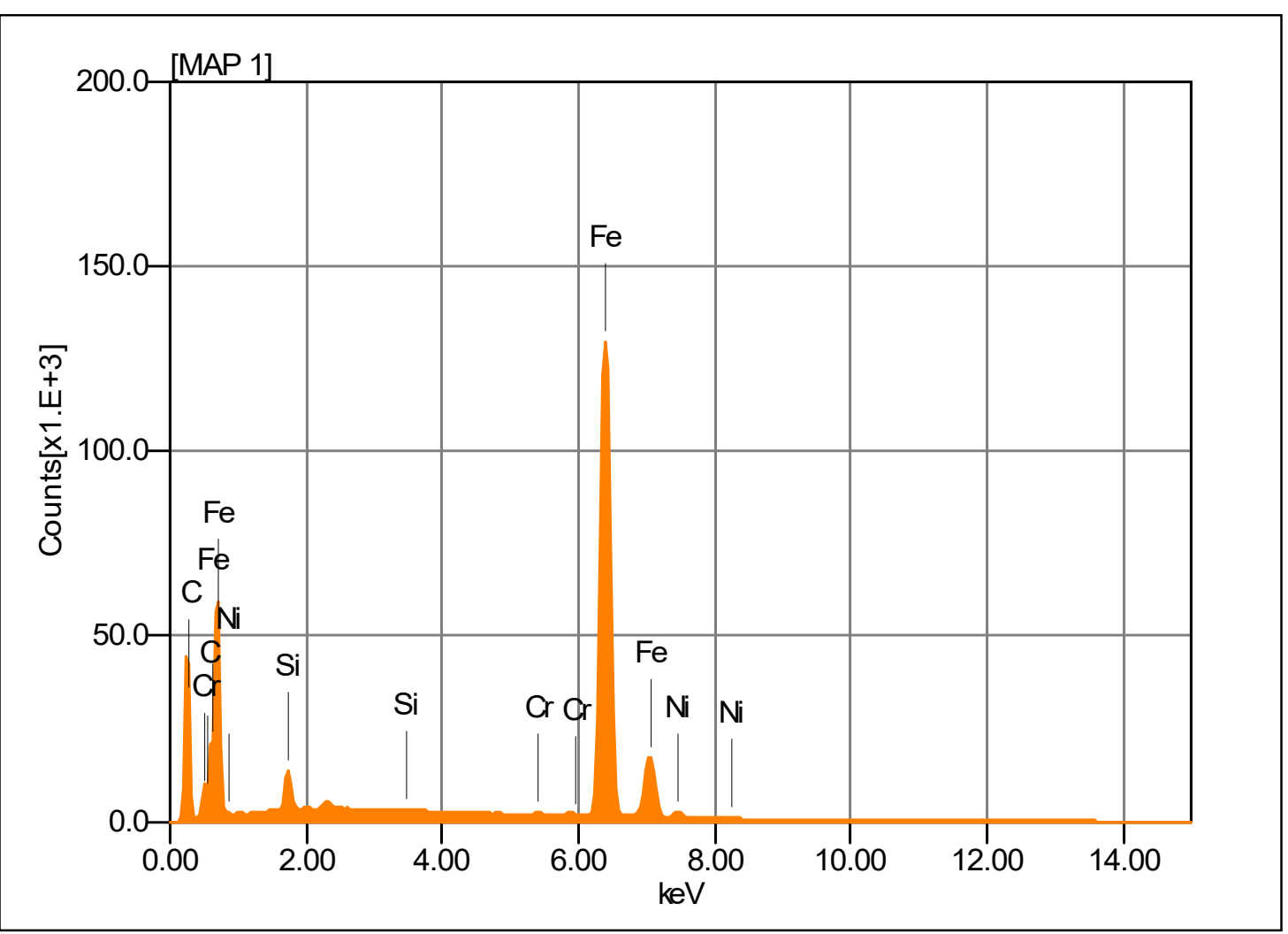

Source: Elaborated by the author 
DAMAGE ANALYSIS

Teeth flank images Photography

Once again images were taken after each fatigue cycle step defined for this research. Those images were also important to use as input in a MatLab routine to quantify damage area of each gear pinion tooth. On figure 84 is shown a gear B01 tooth 1 flank (a) after K9 run 1h, (b) after K10 run 3h and also (c) K10 run for extra $3 \mathrm{~h}$ and on figure 85 is shown a gear D01 tooth 1 flank (a) after K9 run 1h, (b) after $\mathrm{K} 10$ run $3 \mathrm{~h}$ and also (c) K10 run for extra $3 \mathrm{~h}$. In appendix A, B and C are shown all tooth images processed by MatLab routine.

Figure 84: Gear B01 tooth 1 damaged area after images after (a) one hour on K9 load stage, (b) more three hours on K10 load stage and (c) more extra three hours on $\mathrm{K} 10$.

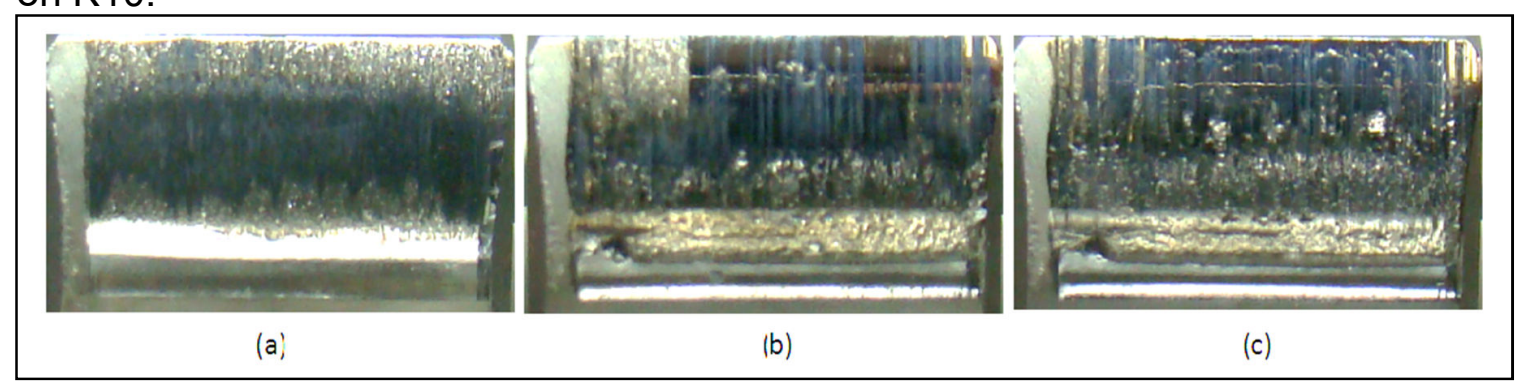

Source: Elaborated by the author

Figure 85: Gear D02 tooth 8 damaged area after images after (a) one hour on K9 load stage, (b) more three hours on K10 load stage and (c) more extra three hours on $\mathrm{K} 10$.

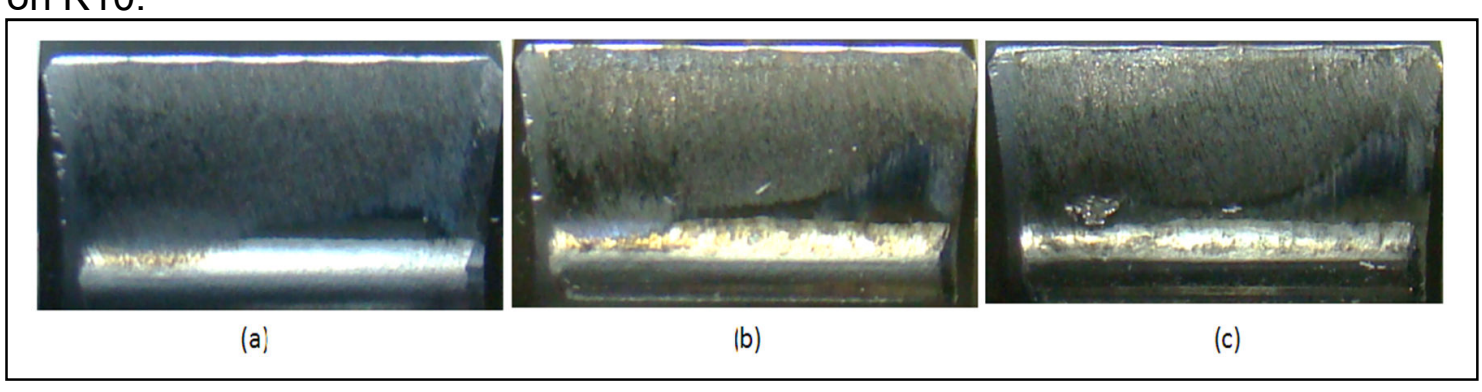

Source: Elaborated by the author 
Still on figure 84 and 85 are also seen two distinct areas as explained for the uncoated gears: (i) starting from the left, one narrow area without contact. This area has been a result of a design decision of reducing the effective contact to increase contact stress. (ii) Another extensive area in which contact has happened and progressive damage can be seen from (a), (b) and (c).

Those increased damaged flank area seen on figure 84 and 85 are result of the load cycle applied on Back to Back bench test under test procedure defined for this research.

Thus, based on figure 84 (Cr-DLC coated gear) images, is seen that after first step of fatigue test some wear is seen on flank. Then, after three hours of fatigue test, the wear seen on the gear flank is very aggressive and some pitting had appeared, but the worst damages are seen after the third step. These B01 tooth 1 damaged seem is just one representative example of all 5 gears with 13 teeth each, which were digitalized for this study, all other images can be seen on Appendix $B$.

Based on figure 85, (DLC coated gear), is seen that the gear seems like new after first fatigue cycle, it's still possible to see the shaving marks on the gear flank. After second step, although it's not seen the shaving marks, the flank is still in good appearance and no pitting seems to be present. Just after third fatigue test step some pitting are seen. Thus, it seems that this coat had influenced the gear capacity of supporting the stress cycle effects. The D02 tooth 8 damaged is just one representative example of all 5 gears with 13 teeth each, which were digitalized for this study. All other images can be seen on Appendix C.

Thus, comparing figure 65,84 and 85 is seen that DLC has the best visual results when each one of the three fatigue test steps images are compared with Cr-DLC and Uncoated gear. This visual result seen be the proposed comparison, shows that after fatigue test D02 Tooth 8 flank image is visually less damaged than F01 and B01. The F01 tooth 3 result seen on referred figure shows that it is the second best visual results and the worst result is see on Cr-DLC Gear B01 tooth 1. 
Damage Quantification

On figure 84 and 85 were seen the fatigue mechanism output under study, but on figures 86 and 87 are point out the result of image processing by MatLab routine, which emphasizes the desired damaged area quantification by applying a contrast between areas on the digitalized flank surface. It considers the white area, affected by Pitting, and the black area, which defines the remaining flank surface which was not affected by Pitting. On those figures 86 and 87 are shown a tooth flank processed by MatLab quantification routine applied after (a) K9 run for 1 hour, (b) K10 run for 3 hours and also (c) K10 run for more 3 hours. In appendix A,B and C are shown all tooth images processed by MatLab routine.

Figure 86: Damaged area identified using MatLab routine on gear B01 Tooth 1 after (a) one hour on K9 load stage, (b) more three hours on K10 load stage and (c) more three hours on K10.

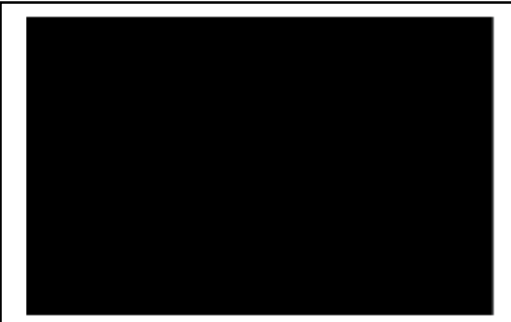

(a)

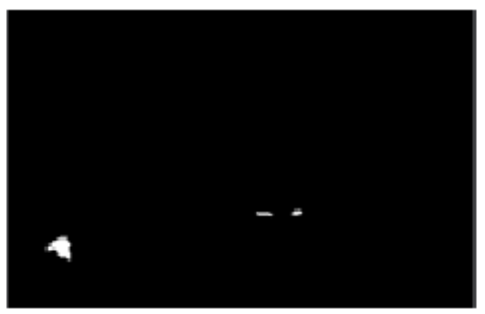

(b)

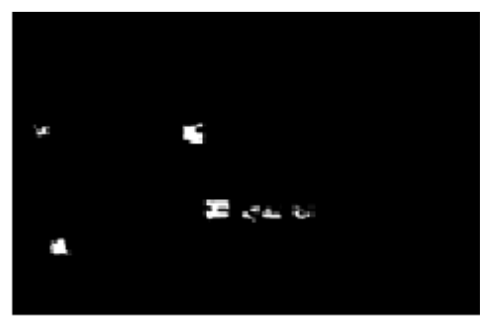

(c)

Source: Elaborated by the author

Figure 87: Damaged area identified using MatLab routine on gear D02 Tooth 8 after (a) one hour on K9 load stage, (b) more three hours on K10 load stage and (c) more three hours on K10.

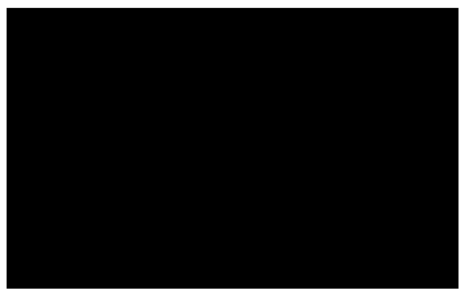

(a)

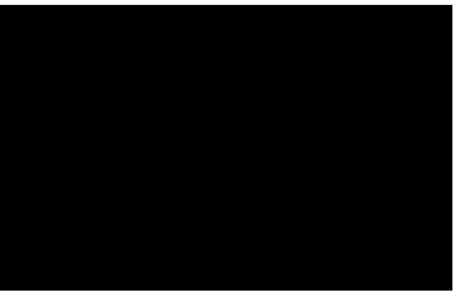

(b)

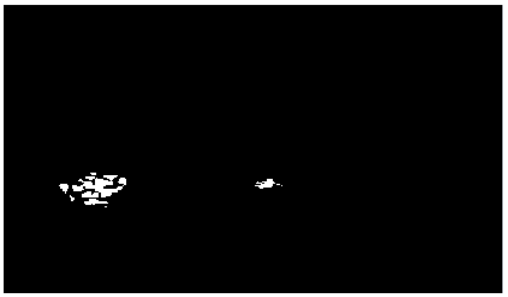

(c)

Source: Elaborated by the author 
Based on figure 86 (Cr-DLC coated gear) processed images for damaged quantification, it is seen that after first step of fatigue test no pitting is seen on flank. Then, after three hours of fatigue test some pitting is seen on the gear flank, then after the third step more pitting is seen on gear flank. The B01 tooth 1 damage analysis is just one representative example of all 5 gears with 13 teeth each, which were processed by MatLab routine on this research, all other processed images can be seen on Appendix $B$.

Based on figure 87, (DLC coated gear), it is seen that after first step of fatigue test no pitting is seen on flank. After three hours of fatigue test no pitting is seen on the gear flank. Then, just after the third step some pitting is seen on gear flank. Once again, these D02 tooth 8 damage analysis seen is just one representative example of all 5 gears with 13 teeth each, which were processed on this study. All other processed images can be seen on Appendix C.

Thus, the comparison of figure 66, 86 and 87 images supports the conclusion that DLC has the best visual results for isolated pitting damage result when each one of the three fatigue test steps images are compared with Cr-DLC and Uncoated gear. Then, the second best visual result is seen on Cr-DLC (this result will be discussed latter), followed by uncoated.

An important comment is required to explain why the damage rank established on flank images seen on figures 65,84 and 85 is different than damaged rank of MatLab output seen on figures 66,86 and 87 . On the flank images are seen different damage mechanism output, in this perspective different wear effects are seen on flank pictures. On the other hand, on MatLab routine output images are seen just an isolated damage mechanism output, which is pitting.

Once again, those images are just some examples of each fatigue step results, all flank images and also MatLab processed images are seen on Appendix A, B and C. On figure 86 and 87 it is also seen a very interesting result, as seen on those processed Mat Lab image, when pitting is seen it appeared upper then the Pitch diameter.

Now, comparing those figures with figure 66 result, which showed pitting on pitch diameter surroundings. These evaluation raised up questions: (i) Why on coated 
gears pitting appearance has been delayed when compared with uncoated? (ii) Why pitting appeared more frequently on higher position on coated gears when compared with uncoated?

Some hypothesis will also be raised up to explain those phenomenon (i) first, on coated gears friction coefficient is expected to be lower than on uncoated. According to Balzers (Balzers, 2010) those coating friction coefficient is lower than 0.2 to DLC and Cr-DLC. Despite of the reduced friction value defined by Balzers, it is an important characteristic, because on a lower friction surface, the shear stress component is also lower. The shear stress component was illustrated on figure 25. (ii) Pitting occurrence is traditionally expected to be closer to Pitch circle. This is the region of high stress level influenced by rolling and sliding. On the other hand, on coated gear, the occurrence region has been changed to higher diameters or upper position. Errichelo (2012) and Terrin et al. (2017) have seen similar phenomenon of pitch occurrence position change in relation to uncoated tested gears. On referred paper, the authors classified as PSO (Point Surface Origin), which according to their definition, this type of pitting is initiated from surface defects such as nicks, dents, grinding furrows, debris or even by previous-formed micropits.

On this perspective, the observed phenomenon of pitting changed position on coated gears matches with the phenomenon observed by Terrin et al. (2017). However, here the trigger could also be associated to detached high hardness coating fragment. Which once it is free on oil, it could flow till contact and induce a higher local contact stress level. Another possibility to stress increase could be associated to geometry modification done by the film partial removal. Which may induces surface highness variations and induce the higher localized stress. 
Pitting Damage area analysis and damage behavior (grow)

On figure 88 till figure 97 are seen damaged area evaluation at each step of the defined fatigue cycles. Each graph contains data related to one gear. So, the five initial figures have data of Cr-DLC, starting with B01 till B05 and the last five have data of DLC, starting with D01 till D05. As already exposed to uncoated gears, such graph purpose is to allow the observation of each tooth Damaged Area (axle $Y$ ) at each step of fatigue cycle (axle X). As three steps of fatigue cycle were planned, the graph shows first step (1hK09), second step (3hK10) and third step (6hk10). Thus, a tendency line was added to easier visualization of the data projection.

Hence, Cr-DLC data graph will be discussed first, which information is displayed on figures 88 till figure 92. Based on those graphs, all gears from B01 till B05 reveal a tendency of having none or low pitting damaged area on the first step evaluation (1hK9). Further on, when the second step of fatigue test data is observed, the tendency is an increased damaged area. Moving on the evaluation to the last step of fatigue test results B03, B04 and B05 total damaged area by pitting tends to reduce. While $\mathrm{B} 01$ and $\mathrm{B} 02$ final damaged area by pitting tend to increase. So, after first fatigue cycle, B01 till B05 tend to have less damaged area in comparison with shaved gears. Which is a good result to support the already mentioned explanation of reduced contact stress cycle effects due to reduced surface friction and consequently, reduced stress sliding component. But, after this first cycle gears coating shown relevant wear, in some cases scratches are seen and its base material was already exposed. After fatigue second step, accumulated fatigue increased the damaged area by pitting. The last step of fatigue test had different tendency when compared B01 and B02 with B03, B04 and B05. Consequently, the raised up question is: Why it is different? To search for an explanation was relevant to observe the data and the specimens to explain the phenomenon.

The visual inspection of B01 till B05 fatigue test images are important to understand the real phenomenon. Figure 84 observation will illustrate, but on Appendix B all gears flank images and their correspondent MatLab routine output are shown to allow the complete observation. So, on those images it is possible to have a 
complete observation of the fatigue test output on B gears flank. Thus, on Appendix $B$ gear flank pictures is also seen a severe wear effect on those gear flanks. Another important evaluation that contributes on damage evaluation is the observation of figures on appendix $\mathrm{D}$. On those figures are seen five graphs comparing profiles before and after fatigue test. The deformation seen on such graphs alters significantly the surface. This deformation tends to compress material and consequently reducing or avoid severe crack nucleation or propagation, decreasing the pitting fatigue mechanism. Based on those information, it is appropriate to observe that the reduced area damaged by pitting is associated to the amount of wear and deformation seen. Surface deformation is the predominant phenomenon explanation for the pitting damage area reduction.

Figure 88: B01 Damaged area variation between fatigue cycle phases, considering: (i): one hour on K9 load stage, (ii) more three hours on K10 load stage and (iii) more three hours on K10.

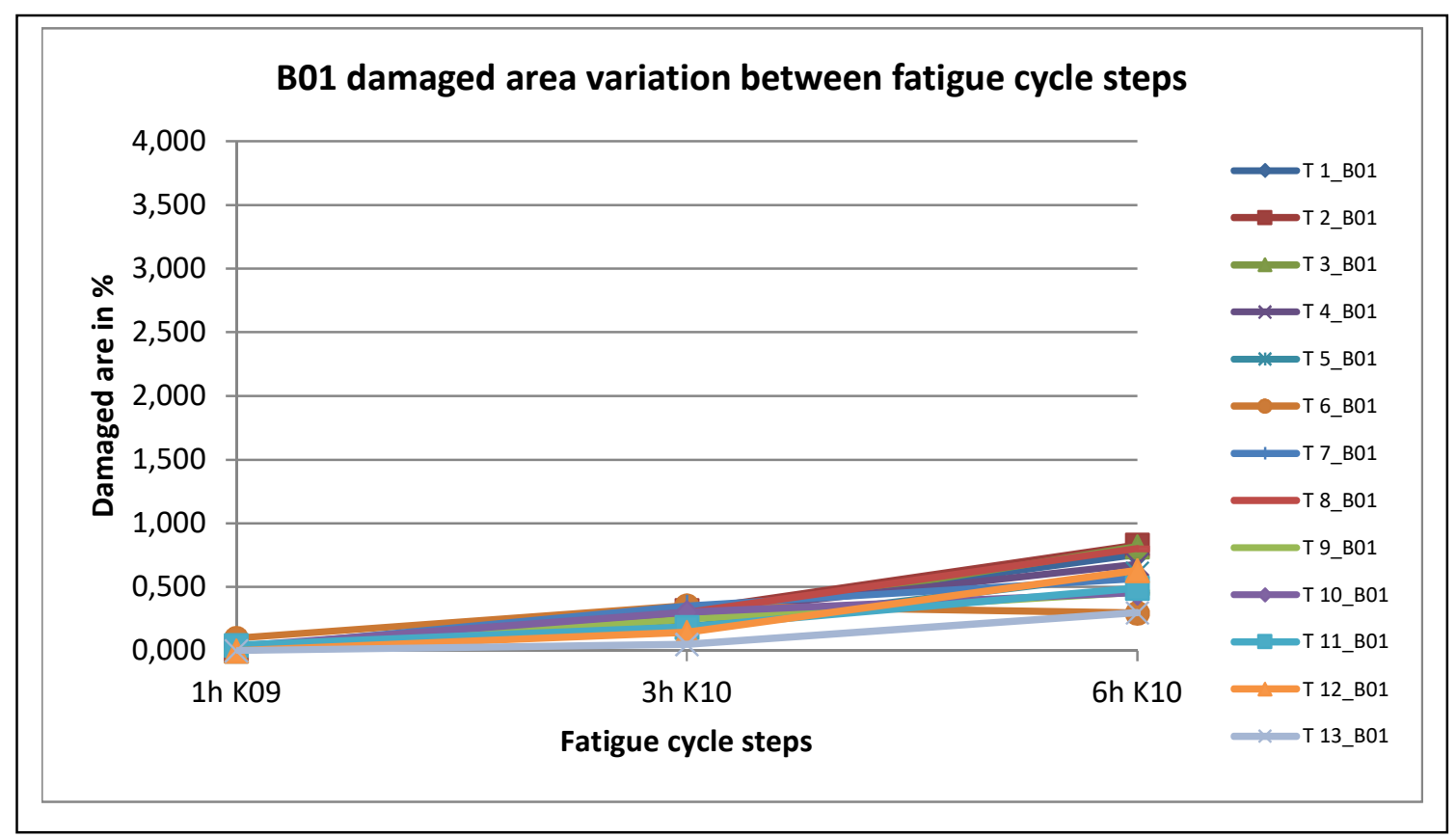

Source: Elaborated by the author 
Figure 89: B02 Damaged area variation between fatigue cycle phases, considering: (i): one hour on K9 load stage, (ii) more three hours on K10 load stage and (iii) more three hours on K10.

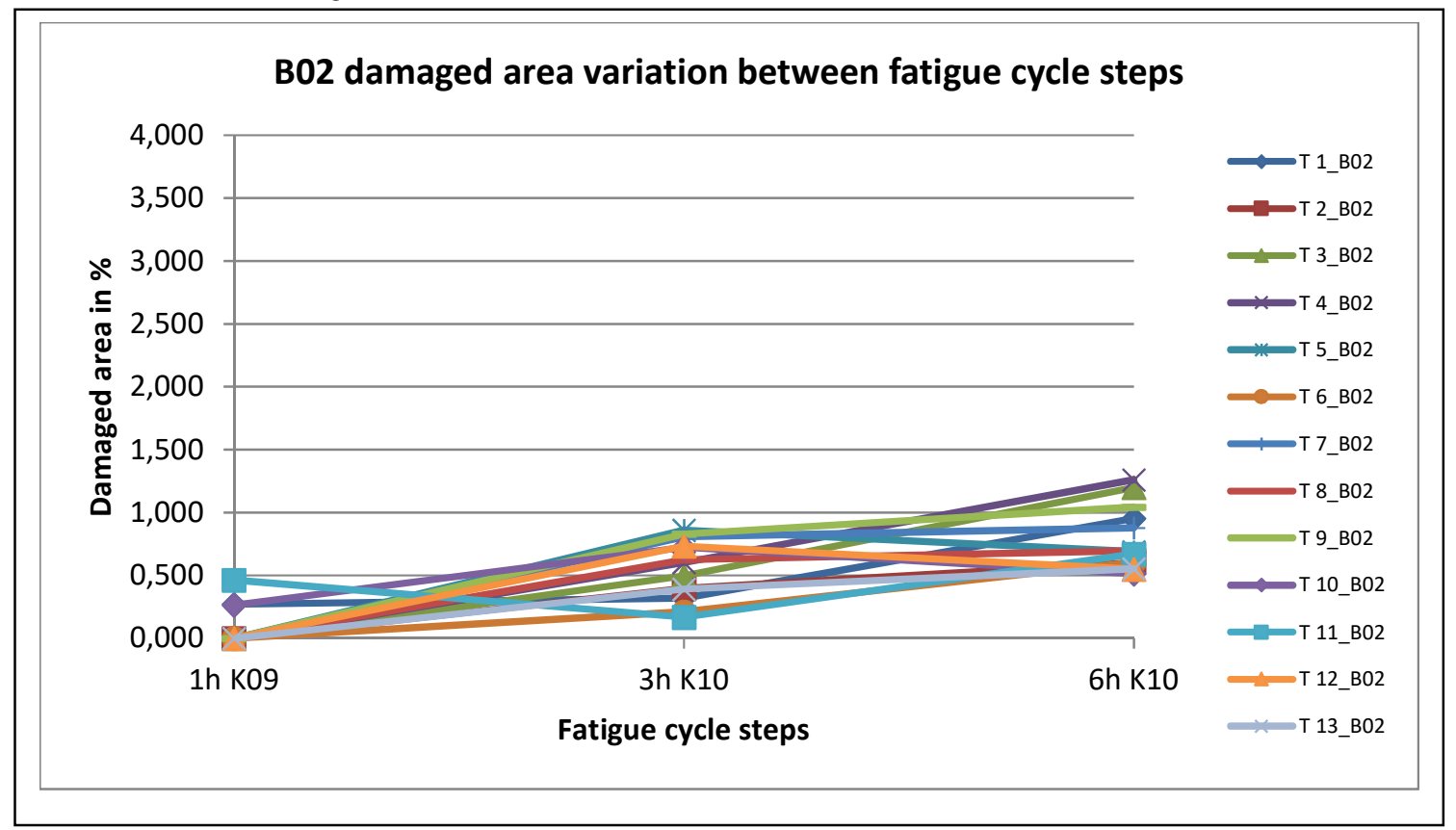

Source: Elaborated by the author

Figure 90: B03 Damaged area variation between fatigue cycle phases, considering: (i): one hour on $\mathrm{K} 9$ load stage, (ii) more three hours on $\mathrm{K} 10$ load stage and (iii) more three hours on K10.

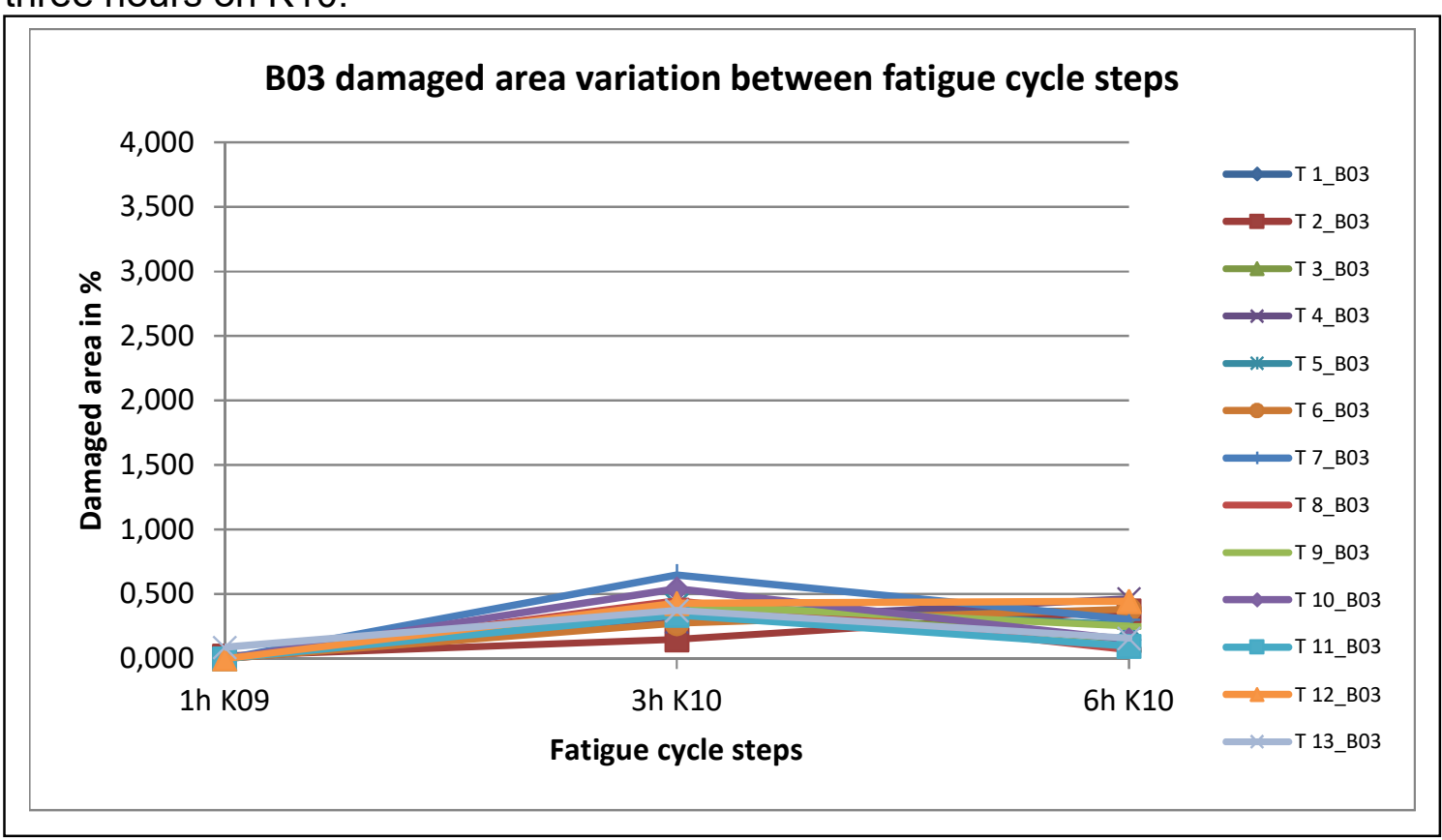

Source: Elaborated by the author 
Figure 91: B04 Damaged area variation between fatigue cycle phases, considering: (i): one hour on K9 load stage, (ii) more three hours on K10 load stage and (iii) more three hours on K10.

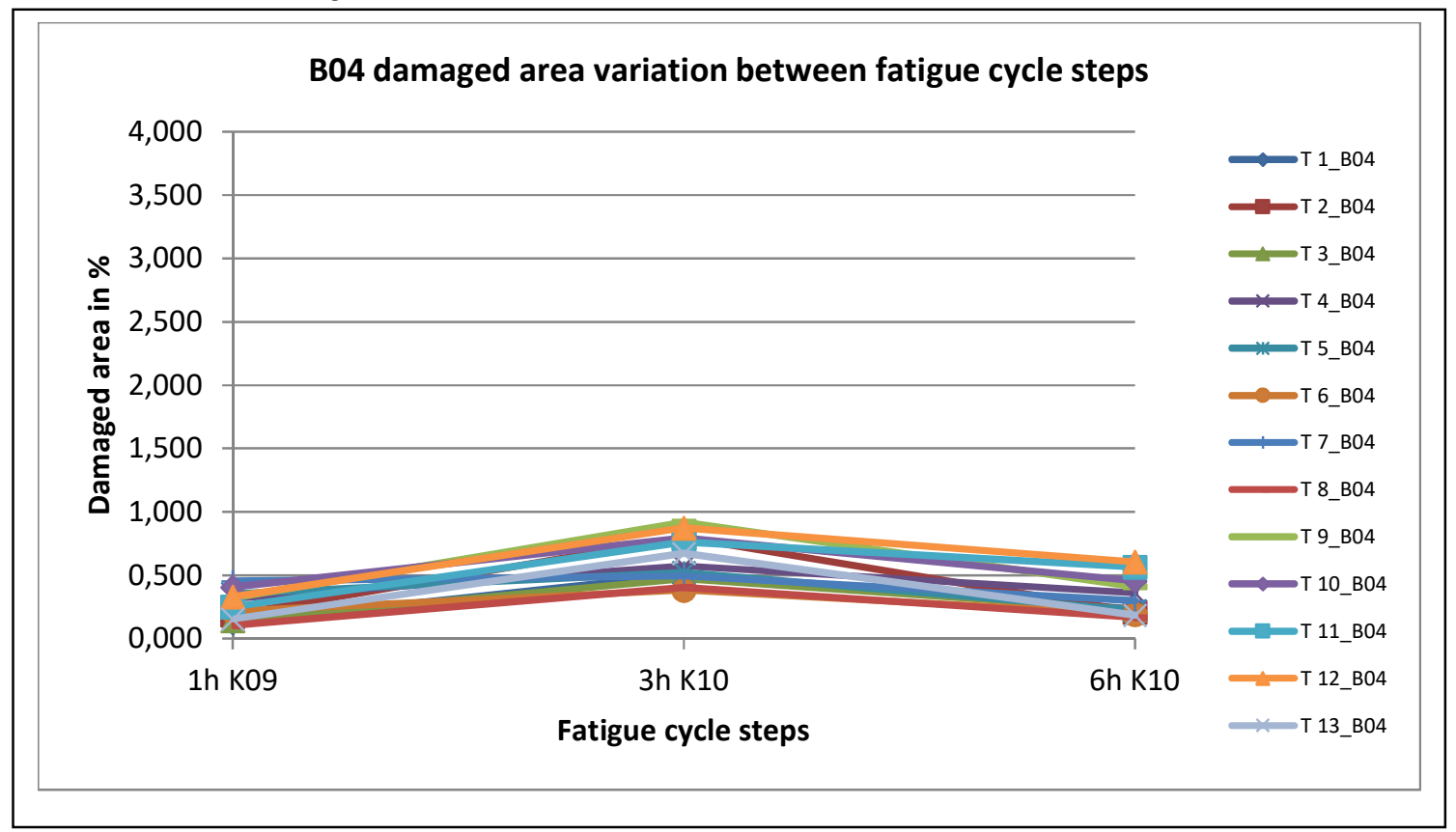

Source: Elaborated by the author

Figure 92: B05 Damaged area variation between fatigue cycle phases, considering: (i): one hour on $\mathrm{K} 9$ load stage, (ii) more three hours on K10 load stage and (iii) more three hours on K10.

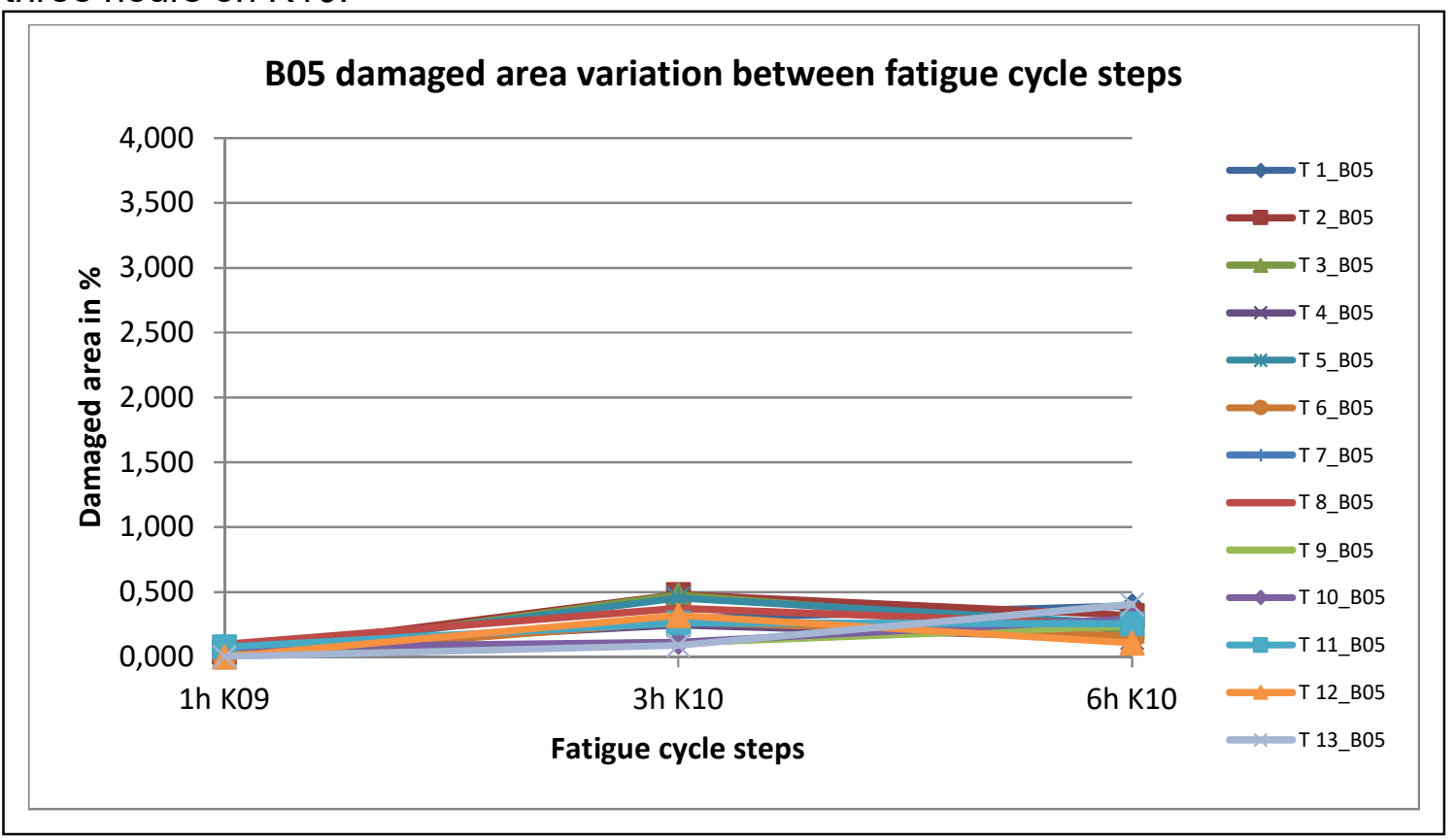

Source: Elaborated by the author 
Hereafter, DLC data graphs will be discussed. Those information are displayed on figures 93 till figure 97 . Based on those graph, all gears from D01 till D05 reveal a tendency of having none or low pitting damaged area on the first step evaluation $(1 \mathrm{hK} 9)$. Further on, when the second step of fatigue test data is observed, the tendency is an increased damaged area. Moving on the evaluation to the last step of fatigue test results, D01, D04 and D05 total damaged area by pitting tends to reduce, while D02 and D03 final damage area by pitting tends to increase.

So, after first fatigue cycle, D01 till D05 tend to have less damaged area which is a good result to support the already mentioned explanation of reduced contact stress cycle effects due to reduced surface friction and consequently, reduced stress sliding component.

After fatigue second step, once again, accumulated fatigue increased the damaged area by pitting. But, some important considerations are required. Although, the tendency of increasing is seen on all gears, total damaged area by pitting showed relevant different values. This variability is emphasized on D03. So, the raised up question here is: Has the coating been detached on D03 exposing the base material to fatigue mechanism? The visual inspection of D01 till D05 fatigue test images are important to understand this phenomenon. Thus, images seen on appendix $\mathrm{C}$ reveals that the more severe wear mechanism is seen on D03 after fatigue test second step (on those flanks the coating were detached), as much as the MatLab routine output points the increased amount of pitting.

On the other hand, on figure 85 is seen D02 tooth 8 , which coating is still there. The flank is preserved and the fatigue damaged area observed has lower values than D03 (seen on Appendix C).

The last step of fatigue test had different tendency when compared D02 and D03 with D01, D04 and D05. Consequently, the raised up question is: Why is it different? To search for an explanation was relevant to observe the data and the specimens to understand the phenomenon.

The visual inspection of D01 till D05 fatigue test images are important to understand the real phenomenon. Figure 85 observation will illustrate that even after final fatigue test, the coating was still there. But, when the pictures of Appendix C are also seen, 
especially on gears D01 and D03. It is possible to observe that the coating was removed and the base material was exposed to wear effects. Here once again is important to observe the pitch occurrence area explanation done previously. Explanation based on material surface (irregularities) and also on hard material layer detached and flowing freely on the oil, which can adhere to the surface while gears are meshing, consequently, changing local stress magnitude and influencing pitting damage area.

Also very important is the observation of Appendix $F$ figures. On those figures are seen measurements done enlightening profile (Appendix F) and helix (Appendix I) geometry before and after test. So, on referred figures are seen a low magnitude profile deformation, which tends to have a lower influence on pitting formation. Since, smaller deformation tends to keep the original surface characteristics, such peaks and valleys.

Figure 93: D01 Damaged area variation between fatigue cycle phases, considering: (i): one hour on $\mathrm{K} 9$ load stage, (ii) more three hours on K10 load stage and (iii) more three hours on K10.

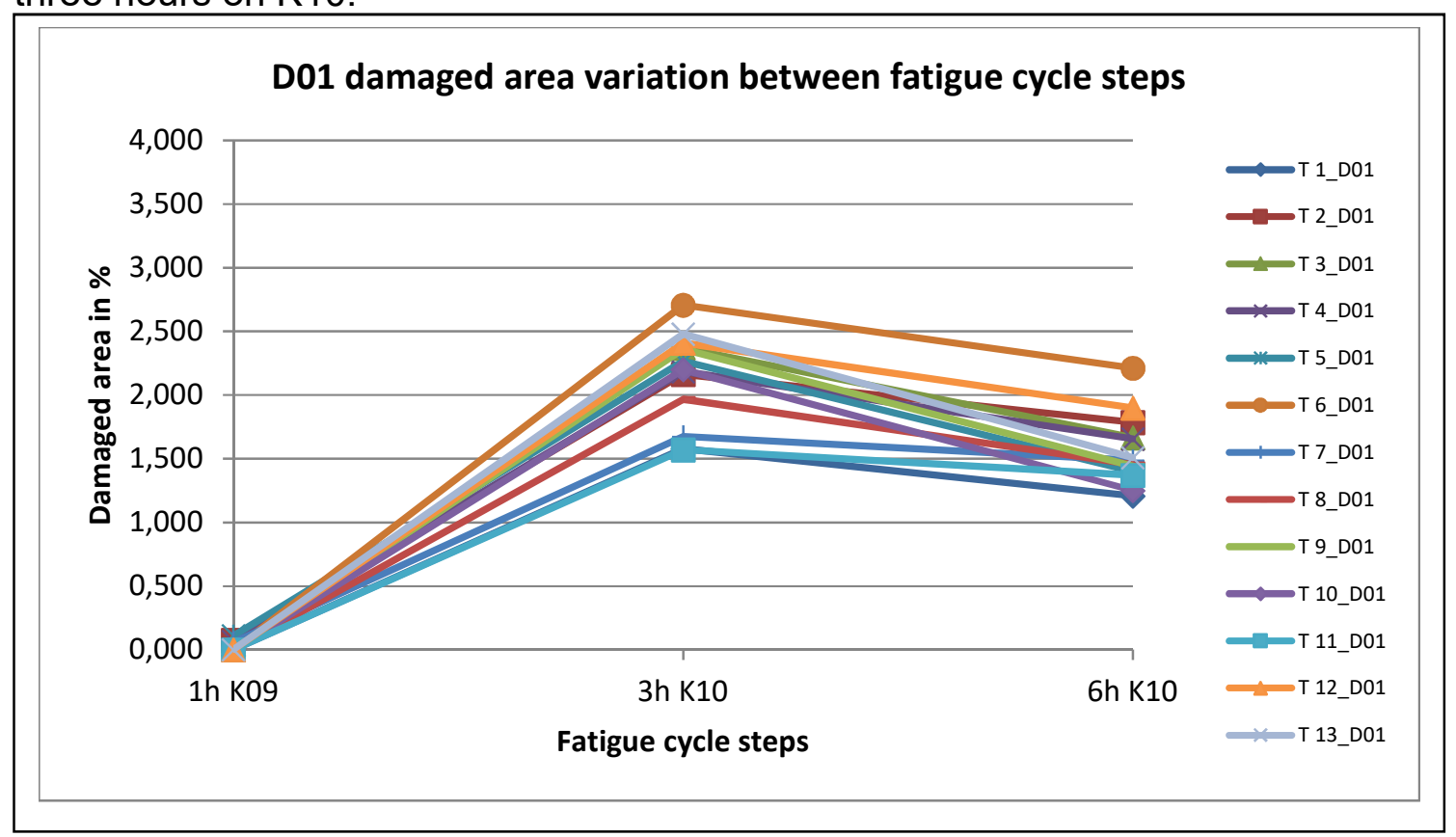

Source: Elaborated by the author 
Figure 94: D02 Damaged area variation between fatigue cycle phases, considering: (i): one hour on $\mathrm{K} 9$ load stage, (ii) more three hours on K10 load stage and (iii) more three hours on K10.

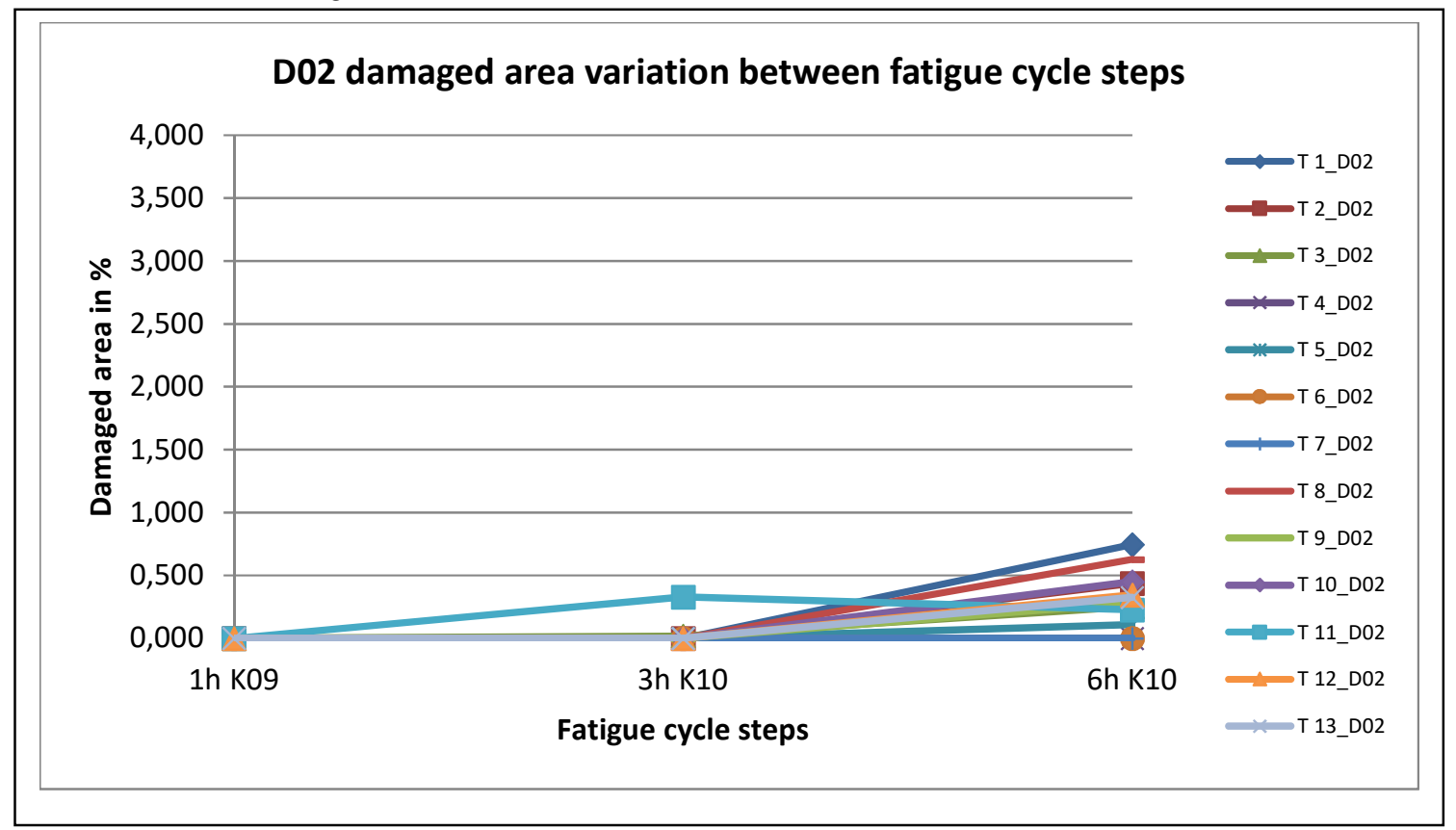

Source: Elaborated by the author

Figure 95: D03 Damaged area variation between fatigue cycle phases, considering: (i): one hour on $\mathrm{K} 9$ load stage, (ii) more three hours on K10 load stage and (iii) more three hours on K10.

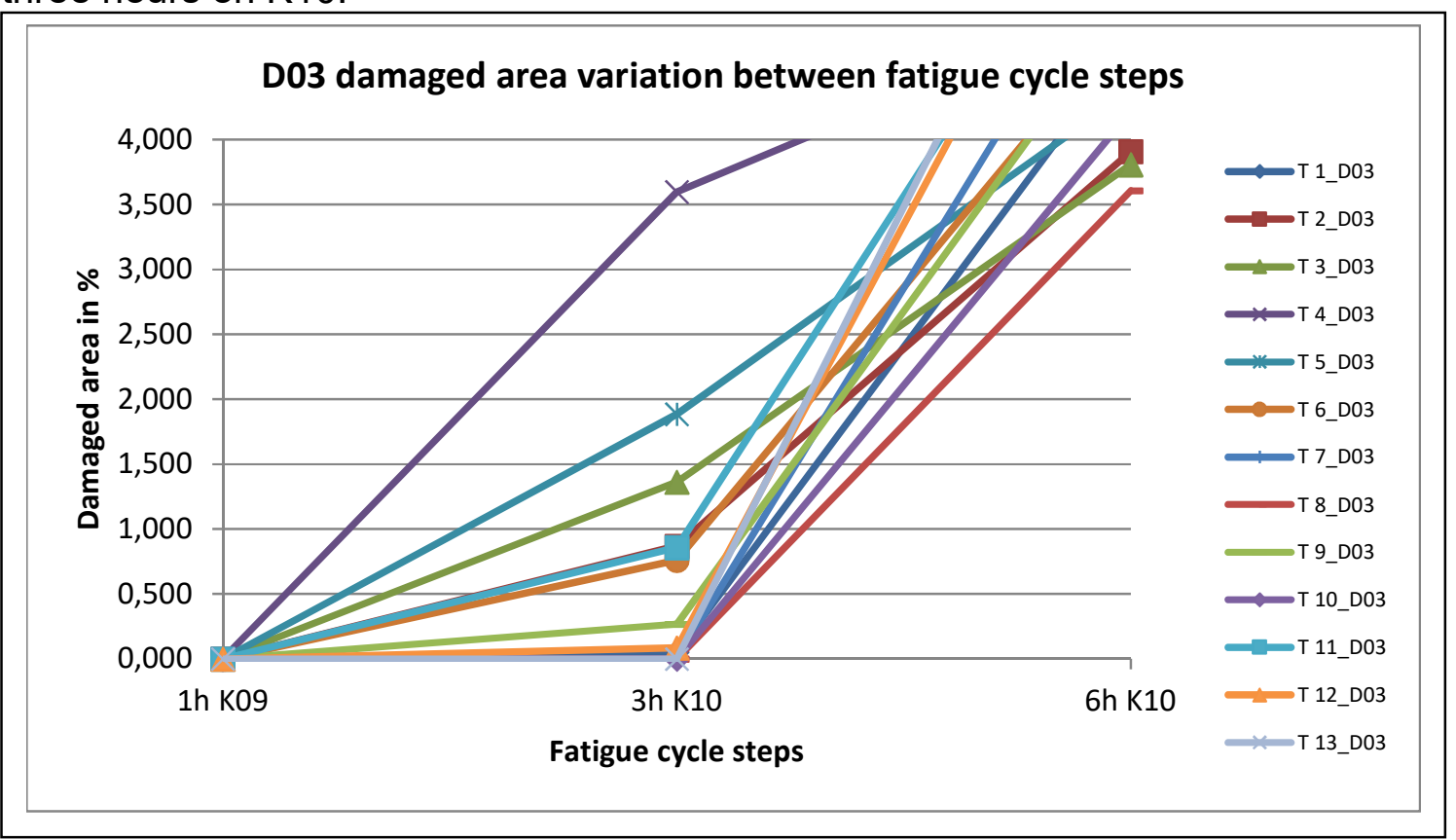

Source: Elaborated by the author 
Figure 96: D04 Damaged area variation between fatigue cycle phases, considering: (i): one hour on $\mathrm{K} 9$ load stage, (ii) more three hours on K10 load stage and (iii) more three hours on K10.

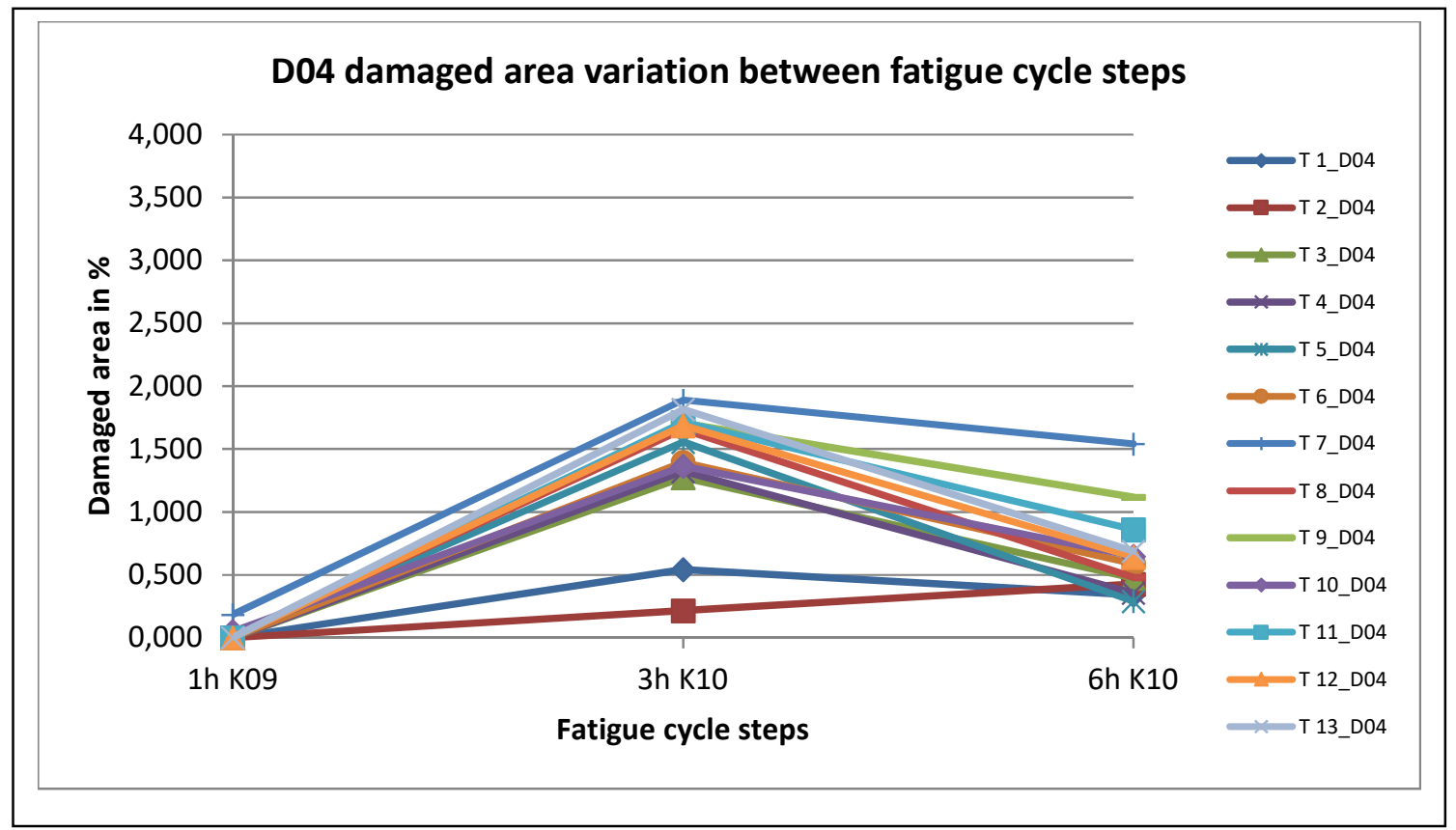

Source: Elaborated by the author

Figure 97: D05 Damaged area variation between fatigue cycle phases, considering: (i): one hour on $\mathrm{K} 9$ load stage, (ii) more three hours on K10 load stage and (iii) more three hours on K10.

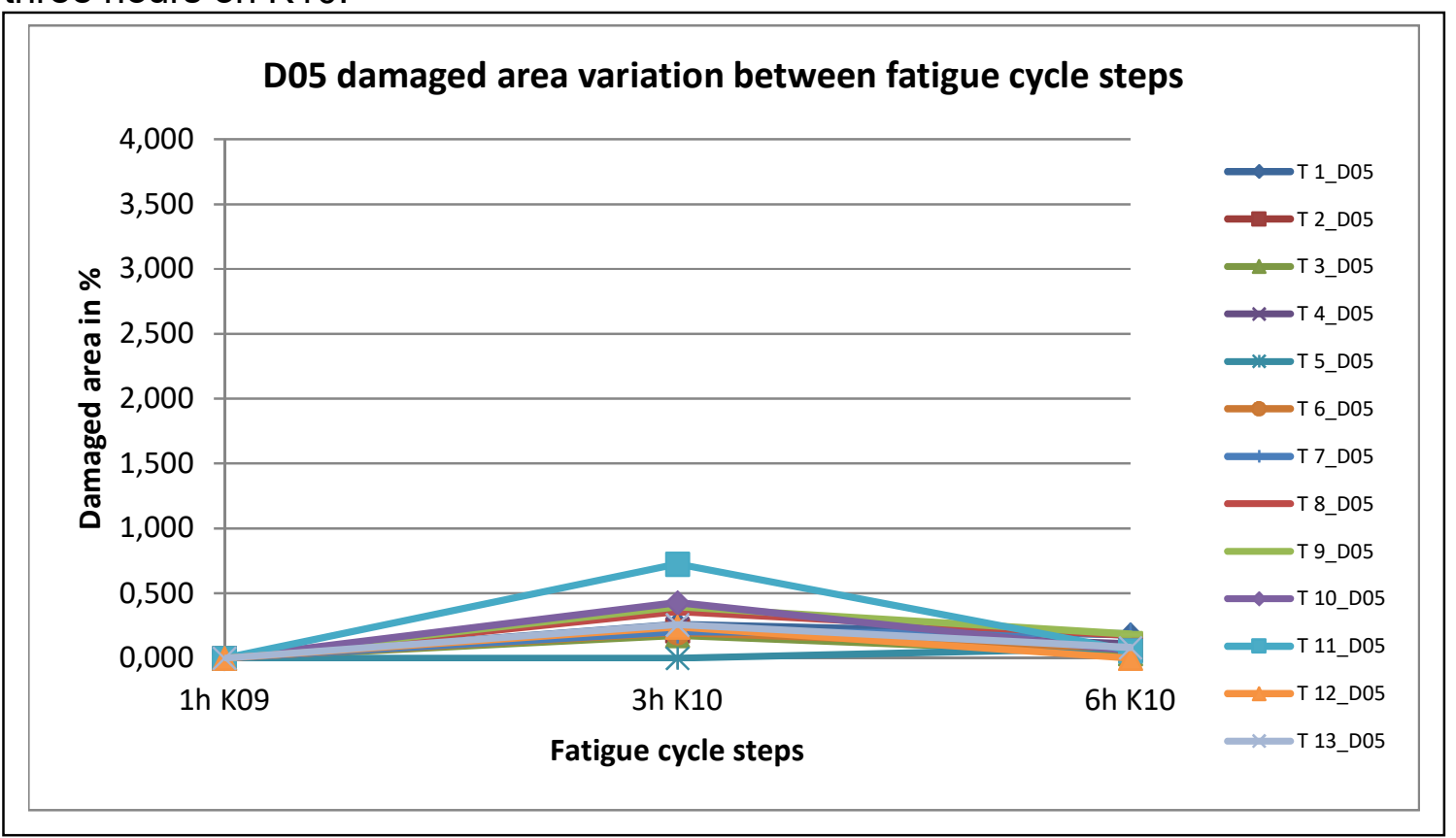

Source: Elaborated by the author 
On graph shown on figures 67 till 71 (F01 to F05 teeth), figures 88 till 92 (B01 to B05 teeth), figures 93 till 97 (D01 to D05 teeth) the scale were set to allow data comparisons. That's the reason of fixing 0.004 as the maximum value for damaged area seen on axle "damaged area".

So, based on Pitting damaged area data shown on referred graphs during fatigue step tests, DLC (D gears) has the best result after first step. After the second step, the Cr-DLC has the best values. Finally, on the last step Cr-DLC also demonstrates a low level of pitting damaged area.

The same methodology and failure criteria used for uncoated gears will be also used on next evaluation steps. The failure criteria taken considers no pitting at the end of defined duty cycle. Here in, the final fatigue result will be the focus.

EVALUATION AFTER ENTIRE FATIGUE CYCLE. OBSERVATION OF 6HK10 DATA.

Previous assessment had focused on pitting evaluation alongside fatigue cycle progression, which has shown its relevance to the phenomenon development. Here in, the focus will be set to the final fatigue evaluation to support the main objective accomplishment. Thus, on figure 98 are shown 65 bar representing each tooth of each gear tested from B01 till B05. The total damaged area varies from $0.07 \%$ to $1.27 \%$ and on figure 99 are shown 65 bar representing each tooth of each gear tested from D01 till D05. The total damaged area varies from $0.00 \%$ to $6.97 \%$. 
Figure 98: Ordinated final damaged area variation observed on gear B01 till B05 teeth after last fatigue cycle step (6h K10).

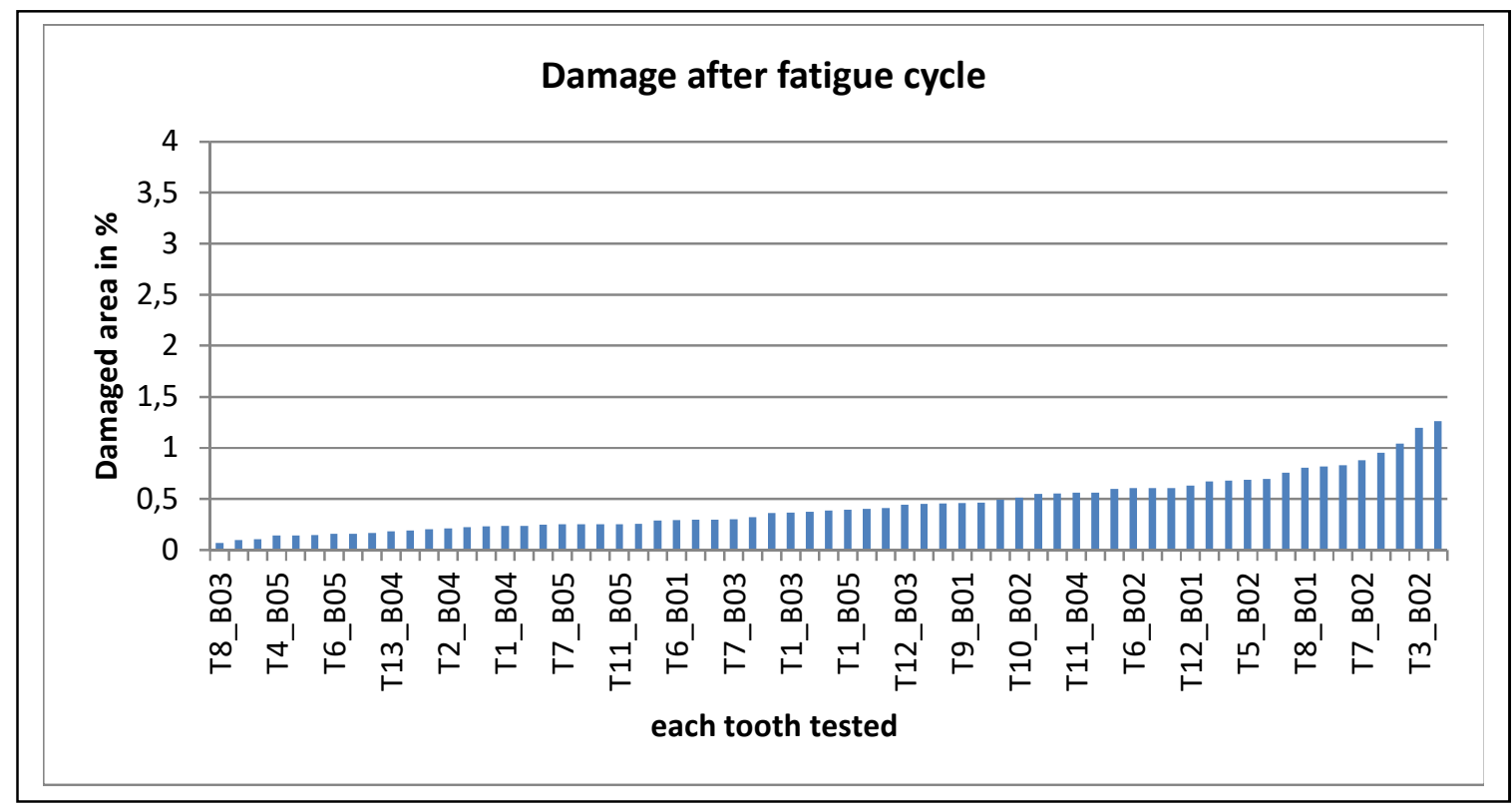

Source: Elaborated by the author

As done to uncoated gears flank damaged area by pitting analysis after fatigue cycle, here the final fatigue cycle data are seen for D01 till D05 which aim to illustrate the lowest and highest damaged flank, as much as to observe if a non-parametric analysis would reveal a suitable function data accommodation.

Once again, all considerations done for uncoated gears are valid here. The considerations were that the gear design is unique for this research, material has been also analyzed and it is according to the SAE 4320 specification, all gears have been produced using the same base material, the production process was the same for all gears, the heat treatment was executed with all gears together, the final gear properties are very similar for both gears. However, a variable was introduced here and this variable was the introduction of coating Cr-DLC or DLC. So, this surface chemical modification influenced the results and completely changed the fatigue test outcome. Thus, the present analysis indicates that coat can induce a surface fatigue damage variation at the end of operational life and, consequently, another question is raised up: Are the processer errors still influencing the final fatigue results? 
Figure 99: Ordinated final damaged area variation observed on gear D01 till D05 teeth after last fatigue cycle step (6h K10).

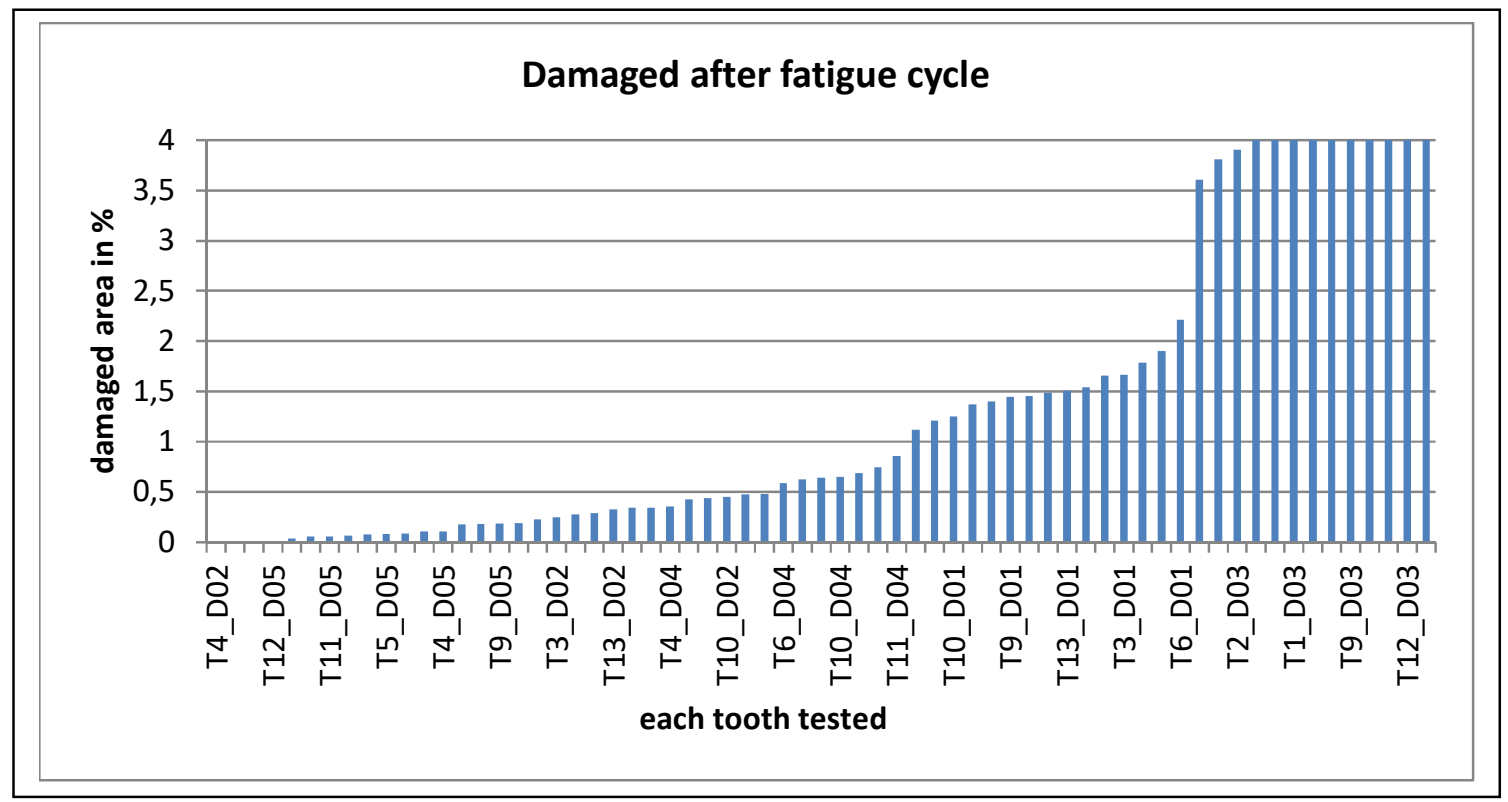

Source: Elaborated by the author

\section{DATA ANALYSIS}

Here in, the discussion takes place in the second outcome of this research, that is the evaluation of final damaged area for coated gears. Despite of not having fatigue model and failure criteria covered by the model ISO 6336, on this research the same methodology and failure criteria previously used for uncoated gears will be applied to evaluate the coated gears.

However, on coated gears, the raised up question are: (i) Does the coating addiction alter fatigue test output? (ii) Does the coating impact gears reliability? (iii) Despite of having thin chemical components layers added on base material surface, is still possible to identify process errors influencing fatigue damage?

So, gears B01 till B05 and latter D01 till D05 teeth damaged area after the entire fatigue test are summarized in Table 24 and 25. Therefore, on first column is shown the tooth number and on next five columns are displayed the damage area for each gear, starting with B01 till B05 and D01 till D05. 
Table 24: Gears B01 to B05 teeth damaged area by Pitting (in \% of total gear flank area)

\begin{tabular}{|c|c|c|c|c|c|}
\hline Teeth & B01 & B02 & B03 & B04 & B05 \\
\hline $\mathbf{0 1}$ & 0.76 & 0.95 & 0.37 & 0.24 & 0.39 \\
\hline $\mathbf{0 2}$ & 0.83 & 0.60 & 0.37 & 0.21 & 0.32 \\
\hline $\mathbf{0 3}$ & 0.82 & 1.20 & 0.15 & 0.23 & 0.20 \\
\hline $\mathbf{0 4}$ & 0.68 & 1.26 & 0.47 & 0.36 & 0.14 \\
\hline $\mathbf{0 5}$ & 0.61 & 0.69 & 0.26 & 0.22 & 0.25 \\
\hline $\mathbf{0 6}$ & 0.29 & 0.61 & 0.38 & 0.19 & 0.16 \\
\hline $\mathbf{0 7}$ & 0.57 & 0.88 & 0.30 & 0.30 & 0.25 \\
\hline $\mathbf{0 8}$ & 0.80 & 0.69 & 0.07 & 0.17 & 0.25 \\
\hline $\mathbf{0 9}$ & 0.46 & 1.04 & 0.25 & 0.41 & 0.24 \\
\hline $\mathbf{1 0}$ & 0.45 & 0.51 & 0.15 & 0.46 & 0.29 \\
\hline $\mathbf{1 1}$ & 0.49 & 0.67 & 0.10 & 0.56 & 0.26 \\
\hline $\mathbf{1 2}$ & 0.63 & 0.55 & 0.44 & 0.61 & 0.11 \\
\hline $\mathbf{1 3}$ & 0.30 & 0.55 & 0.16 & 0.19 & 0.40 \\
\hline
\end{tabular}

Table 25: Gears D01 to D05 teeth damaged area by Pitting (in \% of total gear flank area)

\begin{tabular}{|c|c|c|c|c|c|}
\hline Teeth & D01 & D02 & D03 & D04 & D05 \\
\hline $\mathbf{0 1}$ & 1.21 & 0.74 & 4.76 & 0.34 & 0.18 \\
\hline $\mathbf{0 2}$ & 1.79 & 0.44 & 3.91 & 0.43 & 0.06 \\
\hline $\mathbf{0 3}$ & 1.67 & 0.25 & 3.81 & 0.47 & 0.04 \\
\hline $\mathbf{0 4}$ & 1.66 & 0.00 & 5.07 & 0.35 & 0.11 \\
\hline $\mathbf{0 5}$ & 1.40 & 0.11 & 4.38 & 0.29 & 0.08 \\
\hline $\mathbf{0 6}$ & 2.21 & 0.00 & 4.97 & 0.59 & 0.08 \\
\hline $\mathbf{0 7}$ & 1.49 & 0.00 & 5.72 & 1.54 & 0.19 \\
\hline $\mathbf{0 8}$ & 1.46 & 0.63 & 3.61 & 0.48 & 0.18 \\
\hline $\mathbf{0 9}$ & 1.44 & 0.28 & 5.09 & 1.12 & 0.19 \\
\hline $\mathbf{1 0}$ & 1.25 & 0.45 & 4.22 & 0.65 & 0.05 \\
\hline $\mathbf{1 1}$ & 1.37 & 0.23 & 6.28 & 0.86 & 0.06 \\
\hline $\mathbf{1 2}$ & 1.90 & 0.34 & 6.61 & 0.64 & 0.00 \\
\hline $\mathbf{1 3}$ & 1.51 & 0.33 & 6.97 & 0.69 & 0.08 \\
\hline
\end{tabular}

On figure 100, thus, final fatigue damage area by pitting is presented, starting from B01 (named as A in the graph), till B05 (named as E in the graph). In the graph is, thus, possible to compare the median value (line inside each box) and the data symmetry (proportion of the box upper and lower to the median line). If data $B$ is under evaluation, it is seen that $50 \%$ of the values ranges from 0.6 till 1.0 while the 
median value relies on 0.7 . This represents an asymmetry of the data. On the other hand, for data $E$ is seen that $50 \%$ of the data ranges from 0.2 till 0.3 and the median value relies on 0.25 . Data $E$ is more symmetric than $B$. It's also reasonable to affirm that data $E$ minimum value is 0.1 and maximum value is 0.4 and while date $B$ minimum value is 0.5 and maximum value is 1.3 . This complementary information allows the interpretation that data $\mathrm{E}$ is more concentrated toward central tendency data.

Figure 100: Gears B01 (A) till B05 (E) final damaged area data plotting highlighting tendency behavior.

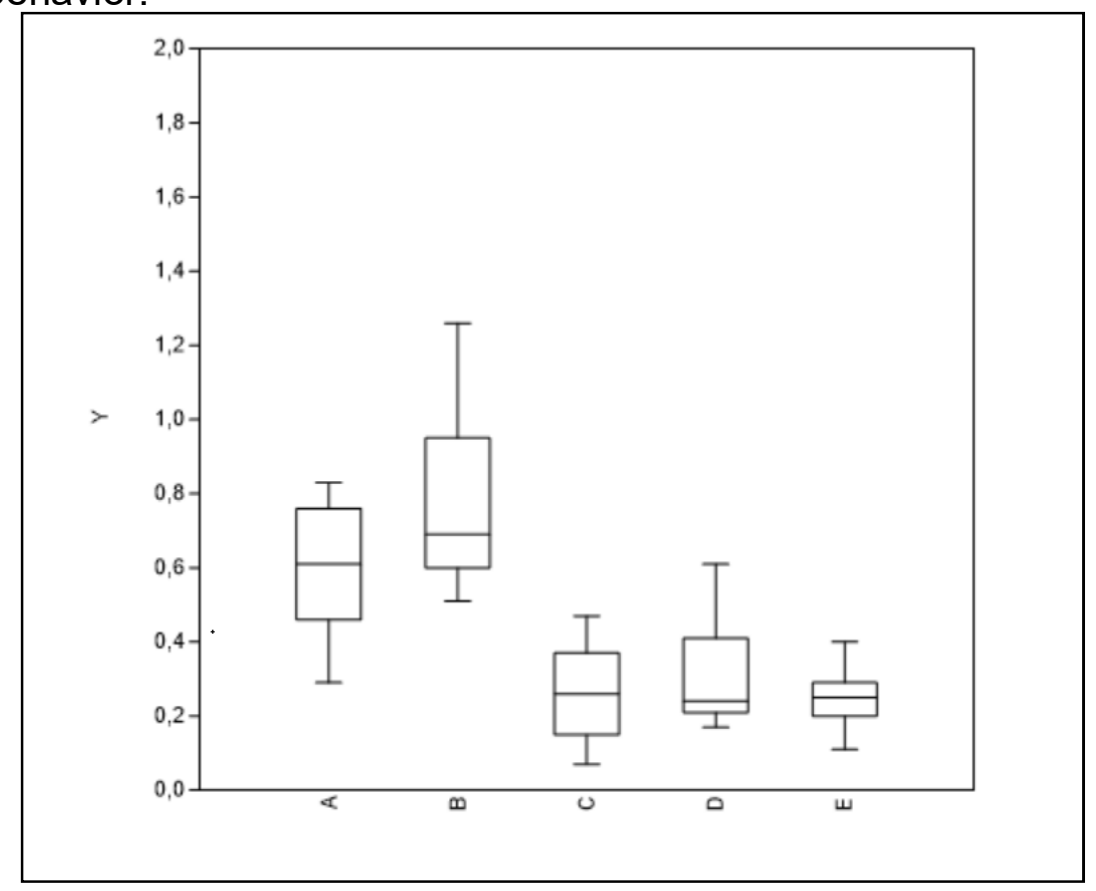

Source: Elaborated by the author

On figure 101, thus, it is also seen final fatigue damaged area by pitting data, starting from D01 (named as A in the graph), till D05 (named as $E$ in the graph). If data $A$ is under evaluation, it is seen that $50 \%$ of the values ranges from 1.3 till 1.8 while the median value relies on 1.4. This represents an asymmetry of the data. On the other hand, for data $B$ it is seen that $50 \%$ of the data ranges from 0.2 till 0.6 and the median value relies on 0.4 . On this perspective is proper to affirm hat data $B$ is more symmetric than $A$. 
It's also reasonable to analyze that data A minimum value is 1.2 and maximum value is 2.2 and while date $E$ minimum value is 0.2 and maximum value is 0.8 . This complementary information allows the interpretation that data $A$ is more concentrated toward central tendency data.

Figure 101: Gears D01(A) till D05 (E) final damaged area data plotting highlighting tendency behavior.

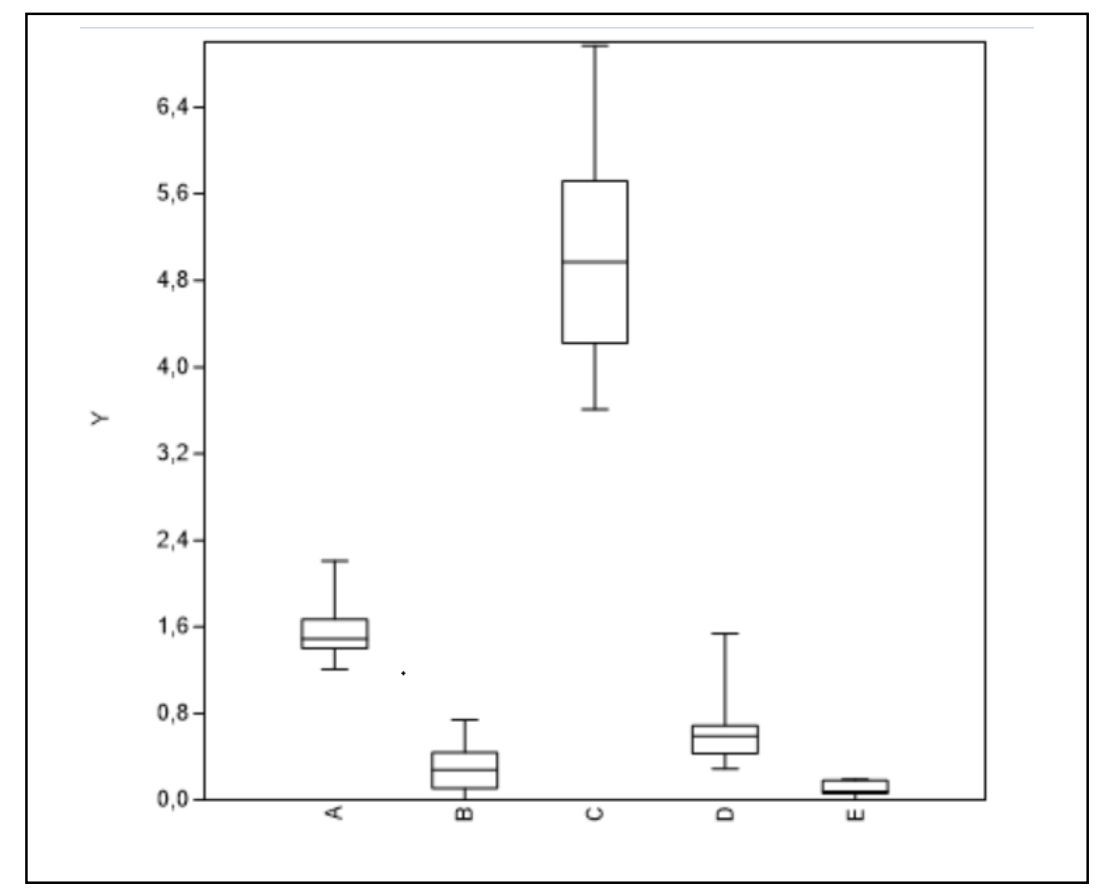

Source: Elaborated by the author

Nevertheless, comparison of figure 100 and 101reveals that B01 till B05 data median value ranges from 0.2 till 0.7 while D01 till D05 median value ranges from 0.2 till 4.8. This represents a higher variability on D gears data compared with $B$. In fact, that's the reason of having different limits values of $Y$ on $D$ graph. Because it was undesired to set the same $Y$ limits (damaged area values) based on $D$ data, otherwise the evaluation of $B$ and $F$ would not be of assistance. Then adding $F$ data on figure 74 to this comparisons, $F$ median values ranges from 0.55 till 1.1. Thus, Gears B seems to have the most stable central tendency data (median) variability, followed by $F$ gear central tendency data which has a very small difference when 
compared with B (0.05). On the last position is seen D gear central tendency data which presented a wide variability.

Reliability Analysis

Once again, the cumulative distribution associated with damaged area for each gear were established. For these analysis the Weibull Distribution was selected to define cumulative distribution being a versatile distribution due to the significant influence of its shape parameters on distribution modeling. Figure 102 presents the probability plot of Weibull distribution indicating pitting damaged area distribution for each of the B five gears and figure 103 present the same analysis but for D five gears.

Figure 102: Weibull Probability Plot for pitting damage area for gears B01 to B05

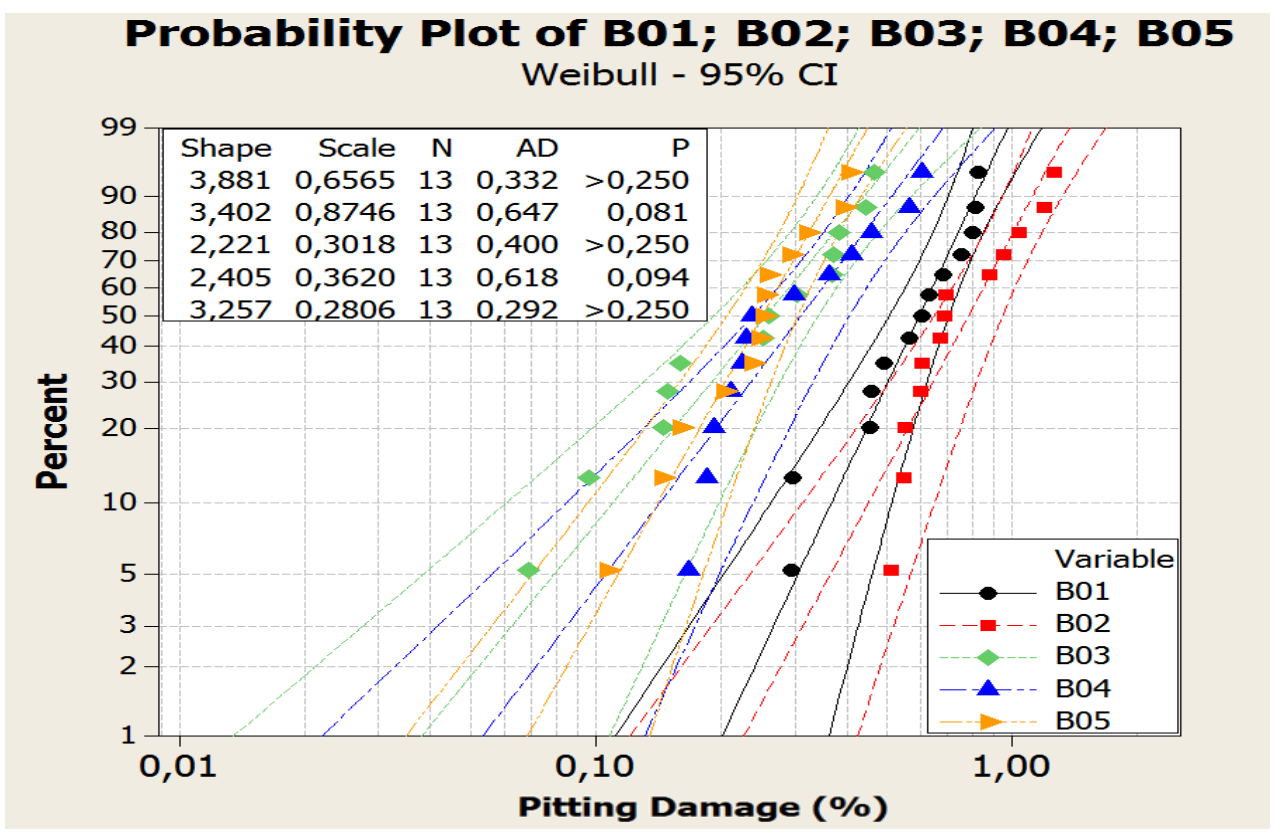

Source: Elaborated by the author

Weibull distribution represents a good fit for damaged area of all gears according to Anderson-Darling goodness of fit test. Gears B03 and B04 present shape parameter lower than 2.6, indicating that the distribution is positively skewed (has a right tail). Gears B02 and B05 have shape parameter between 2.6 and 3.7 indicating that the coefficient of skewness approaches zero. Finally gear B01 presents a shape 
parameter greater than 3.7 indicating that the distribution is negatively skewed (has a left tail). In the same figure uncertainty in the results due to the limited sample sizes are represented by confidence bounds. All experimental data are located inside the confidence bounds for each distribution.

Figure 103: Weibull cumulative pitting damaged area distribution for gears D01 to D05

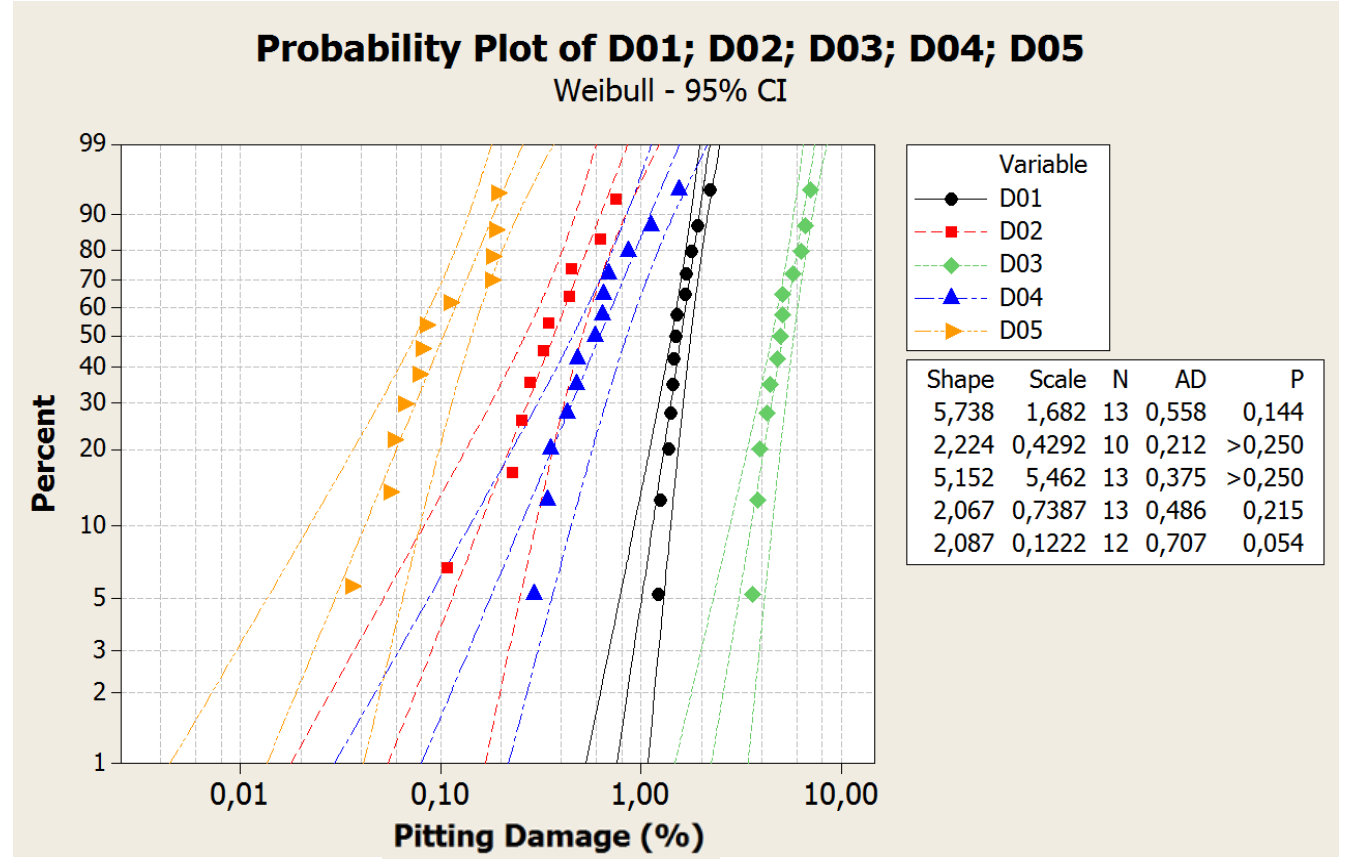

Source: Elaborated by the author

That distribution represents a good fit for damaged are of all gears according to Anderson-Darling goodness of fit test. Gears D02, D04 and D05 present shape parameter lower than 2.6, indicating that the distribution is positively skewed (has a right tail) and gears D01 and D03 present a shape parameter greater than 3.7 indicating that the distribution is negatively skewed (has a left tail). In the same figure uncertainty in the results due to the limited sample sizes are represented by confidence bounds. All experimental data are located inside the confidence bounds for each distribution.

Figure 104 presents the Weibull cumulative distribution of pitting damaged area for the B01 till B05. Based on Figure 104 it is possible to conclude that gears B03, B04 
and B05 have a similar pitting damaged area distribution. Gears B01 and B02 present a more spread damaged area distribution than the other gears. Those results indicate that as for pitting damaged area distribution the five gears apparently present different behavior.

Figure 104: Weibull cumulative pitting damaged area distribution for B01 till B05 gears

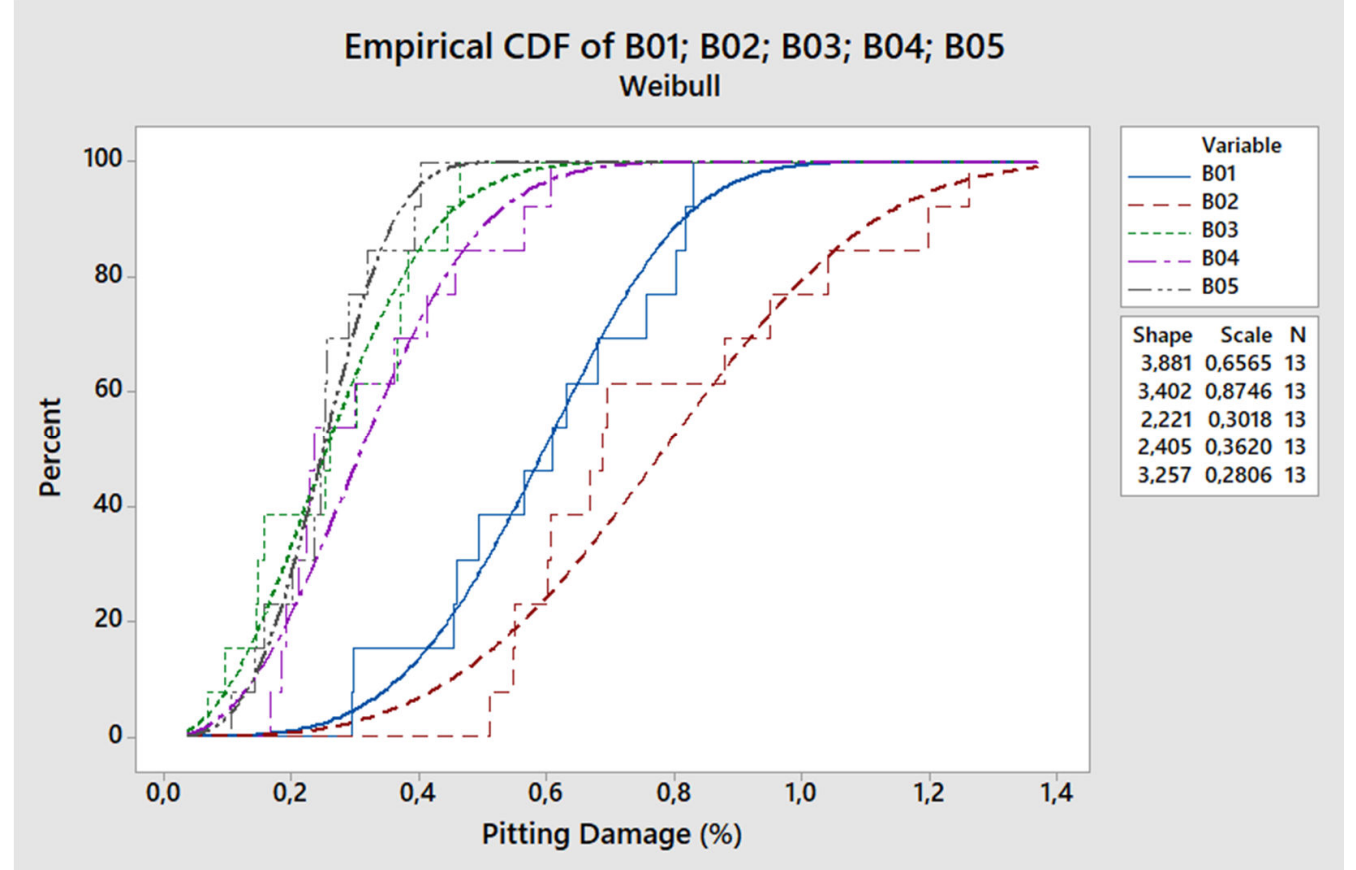

Source: Elaborated by the author

Figure 105 presents Weibull cumulative pitting damaged area distribution for all five gears. Based on Figure 105 it is possible to conclude that gears D02, D04 and D05 have a similar pitting damaged area distribution. Gear D03 presents a more spread damaged area distribution than the other gears. Although Gear D01 presents higher pitting damaged area, its probability distribution has lower scattering, as low as the D02, D04 and D05. Those results indicate that as for pitting damaged area distribution the five gears apparently present different behavior. 
Figure 105: Weibull cumulative pitting damaged area distribution for D01 till D05 gears

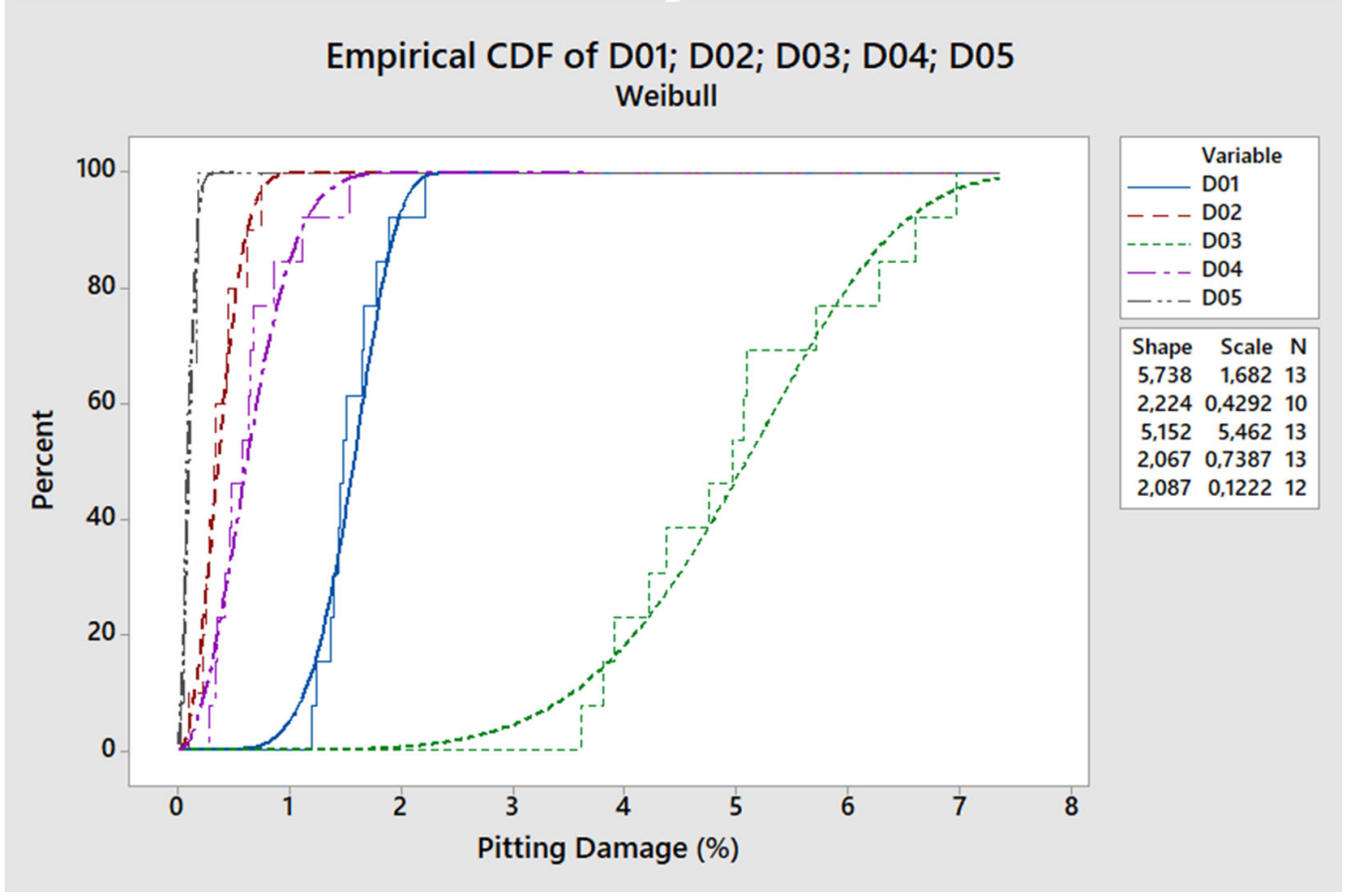

Source: Elaborated by the author

\section{Process Errors Comparison}

On table 26 , in first column, is indicated the mean value of final damaged area for each gear $B$ ordinated from the smaller to the higher damaged area by pitting. On second column is seen the sample number or gear number to which the mean final damage belongs presented. From the third till seventh columns are shown the mean values of the measured variables $f_{h \alpha}, f_{f \alpha}, f_{h \beta}, f_{f \beta}, f_{p t}$ of gears B01 till B05. On table 27 , the same variables are seen, but are seen D01 till D05 values.

Following categorically the same methodology done for gear F01 till F05 (as shaved, or in other words, without coating). Those means values of $f_{h \alpha}, f_{f \alpha}, f_{h \beta}, f_{f \beta}$ and $f_{p t}$ were measured using Wenzel WGT 350 equipment, seen on figure 60. The equipment use a contact probe which measured gear teeth and automatically calculated and display the mean values for each of the variables seen on tables 27 and 28 . 
(On Appendix E,F,H are shown details of 1,4,7,11 gear teeth were measurements done. Those teeth were selected due to its distribution over gear perimeter). Table 26 and 27 also shows that again no direct correlation can be seen when all data is considered. Consequently, once more the same statistical tools are required to support the analysis.

Table 26: Comparison of gear flank mean damage area by pitting and mean process errors for B01 to B05

\begin{tabular}{|c|c|c|c|c|c|c|}
\hline Damaged area \% & Gear \# & $\mathbf{f}_{\mathbf{h} \boldsymbol{\alpha}}(\boldsymbol{\mu m})$ & $\mathbf{f}_{\mathbf{f} \alpha}(\boldsymbol{\mu m})$ & $\mathbf{f}_{\mathbf{h} \boldsymbol{\beta}}(\boldsymbol{\mu m})$ & $\mathbf{f}_{\mathbf{f} \boldsymbol{\beta}}(\boldsymbol{\mu m})$ & $\mathbf{f}_{\mathbf{p t}}(\boldsymbol{\mu m})$ \\
\hline $\mathbf{0 , 2 5}$ & B05 & -5.83 & 13.83 & 5.20 & 12.78 & -1.50 \\
\hline $\mathbf{0 , 2 7}$ & B03 & -4.00 & 9.48 & 1.65 & 10.05 & 1.00 \\
\hline $\mathbf{0 , 3 2}$ & $\mathbf{B 0 4}$ & -5.60 & 13.95 & 2.93 & 8.45 & -1.00 \\
\hline $\mathbf{0 , 5 9}$ & $\mathbf{B 0 1}$ & 3.73 & 19.70 & 4.25 & 9.73 & 1.00 \\
\hline $\mathbf{0 , 7 8}$ & B02 & -5.60 & 9.15 & 3.53 & 8.95 & 2.75 \\
\hline
\end{tabular}

Table 27: Comparison of gear flank mean damage area by pitting and mean process errors for D01 to D05.

\begin{tabular}{|c|c|c|c|c|c|c|}
\hline Damaged area \% & Gear \# & $\mathbf{f}_{\mathbf{h} \boldsymbol{\alpha}}(\boldsymbol{\mu m})$ & $\mathbf{f}_{\mathbf{f} \alpha}(\boldsymbol{\mu m})$ & $\mathbf{f}_{\mathbf{h} \boldsymbol{\beta}}(\boldsymbol{\mu m})$ & $\mathbf{f}_{\mathbf{f} \boldsymbol{\beta}}(\boldsymbol{\mu m})$ & $\mathbf{f}_{\mathbf{p t}}(\boldsymbol{\mu m})$ \\
\hline $\mathbf{0 . 1 0}$ & D05 & -0.03 & 14.73 & 1.78 & 9.08 & -2.50 \\
\hline $\mathbf{0 . 2 9}$ & D02 & -7.58 & 9.80 & 5.80 & 11.65 & -0.25 \\
\hline $\mathbf{0 . 6 5}$ & D04 & -7.15 & 9.65 & 3.23 & 9.28 & 3.00 \\
\hline $\mathbf{1 . 5 7}$ & D01 & X & X & X & x & X \\
\hline $\mathbf{5 . 0 3}$ & D03 & -9.33 & 9.50 & 5.13 & 10.28 & 0.75 \\
\hline
\end{tabular}

Statistical Analysis

On table 28 and 29 are summarized some important calculations; such count of repetitions on each group (one specific gear), sum, mean value and variance resulted from the analysis of data. 
Table 28: Statistical data summary results for B01 till B05.

\begin{tabular}{lcccc} 
SUMMARY & \multicolumn{3}{l}{} \\
\hline Groups & Count & Sum & Mean & Variance \\
\hline B01 & 13 & 7.70 & 0.59 & 0.03 \\
B02 & 13 & 10.20 & 0.78 & 0.07 \\
B03 & 13 & 3.47 & 0.27 & 0.02 \\
B04 & 13 & 4.15 & 0.32 & 0.02 \\
B05 & 13 & 3.27 & 0.25 & 0.01 \\
\hline
\end{tabular}

Table 29: Statistical data summary results for D01 till D05.

\begin{tabular}{lcccc} 
SUMMARY & \multicolumn{3}{l}{} \\
\hline Groups & Count & Sum & Mean & Variance \\
\hline D01 & 13 & 20.35 & 1.57 & 0.08 \\
D02 & 13 & 3.79 & 0.29 & 0.06 \\
D03 & 13 & 65.37 & 5.03 & 1.18 \\
D04 & 13 & 8.45 & 0.65 & 0.12 \\
D05 & 13 & 1.29 & 0.10 & 0.00 \\
\hline
\end{tabular}

The ANOVA calculation was again performed on Microsoft Excel Data Analysis function and the output table is shown on table 30 for B01 till B05 and on table 31 for D01 till D05.

On table 30 calculated $F(24.46)$ is higher than $F$ critical $(2.53)$ and $P$ value is lower than adopted significance level (5\%). As much as on table 31 calculated $F(186.73)$ is higher than $\mathrm{F}$ critical (2.53) and $\mathrm{P}$ value is lower than adopted significance level $(5 \%)$. This defines that hypothesis of all mean values of gear damaged area between coated gears are equal has been rejected. Consequently, there are differences between treatments.

Table 30: ANOVA single factor evaluation results for B01 till B05

\begin{tabular}{|c|c|c|c|c|c|c|}
\hline $\begin{array}{l}\text { Source of } \\
\text { Variation }\end{array}$ & $\begin{array}{l}\text { Sum of } \\
\text { Squares }\end{array}$ & $\begin{array}{l}\text { Degree of } \\
\text { freedom }\end{array}$ & $\begin{array}{l}\text { Mean } \\
\text { Square }\end{array}$ & $F$ & P-value & F crit \\
\hline Between Groups & 2.89 & 4 & 0.72 & 24.46 & $4.91 \mathrm{E}-12$ & 2.53 \\
\hline Within Groups & 1.77 & 60 & 0.03 & & & \\
\hline Total & 4.66 & 64 & & & & \\
\hline
\end{tabular}


Table 31: ANOVA single factor evaluation results for D01 till D05

\begin{tabular}{|c|c|c|c|c|c|c|}
\hline $\begin{array}{l}\text { Source of } \\
\text { Variation }\end{array}$ & $\begin{array}{l}\text { Sum of } \\
\text { Squares }\end{array}$ & $\begin{array}{l}\text { Degree of } \\
\text { freedom }\end{array}$ & $\begin{array}{l}\text { Mean } \\
\text { Square }\end{array}$ & $F$ & P-value & F crit \\
\hline Between Groups & 215.72 & 4 & 53.93 & 186.73 & $3.97 E-33$ & 2.53 \\
\hline Within Groups & 17.33 & 60 & 0.29 & & & \\
\hline Total & 233.05 & 64 & & & & \\
\hline
\end{tabular}

Once again, the Tukey test is required to perform a pair to pair comparison and defines between groups the ones which the mean value are considered unequal. Thus, on table 32 and 33 are shown the final output of Tukey test with results filled above the diagonal. Those values above the diagonal are the calculated $p$ values for the pair to pair comparison. The $p$ values which are smaller than the adopted significance level $(\alpha)$ or smaller than 0,05 are highlighted in red.

So on table 32 is seen that calculated $p$ for almost all pair to pair comparisons are smaller than significance level $\alpha$, rejecting the null hypothesis for almost all comparisons. But based on the test, B03 and B04 as B03 and B05 as much as B04 and B05 are the only comparison which the null hypothesis are not rejected. In other words, the treatment has influence between B03 and B04 as B03 and B05 as much as B04 and B05.

Table 32: Tukey test pairwise comparisons

\begin{tabular}{|c|c|c|c|c|c|}
\hline & B01 & B02 & B03 & B04 & B05 \\
\hline B01 & - & 0.04323 & 0.0002152 & 0.001466 & 0.0001654 \\
\hline B02 & & - & 0.0001331 & 0.0001331 & 0.0001331 \\
\hline B03 & & & - & 0.9361 & 0.9993 \\
\hline B04 & & & & - & 0.8461 \\
\hline B05 & & & & & - \\
\hline
\end{tabular}

Therefore, as done for uncoated gears, figure 106 shows an evaluation of process errors and final damaged area by pitting. To check if process errors are still correlated with final damaged area by pitting, a consideration based on pair to pair comparisons as done on Tukey test are suitable, avoiding the comparisons which the null hypothesis were not rejected. In other words, the direct comparison of B01 
with $\mathrm{B} 02$, B01 with $\mathrm{B} 03, \mathrm{~B} 01$ with $\mathrm{B} 04$ and finally $\mathrm{B} 01$ with $\mathrm{B} 05$ which are the means that were considered statistically different, under the confidence level proposed on Tukey test, reveals that no direct correlation is established.

Figure 106: B Gear process errors $x$ damaged area by pitting in $\%$.

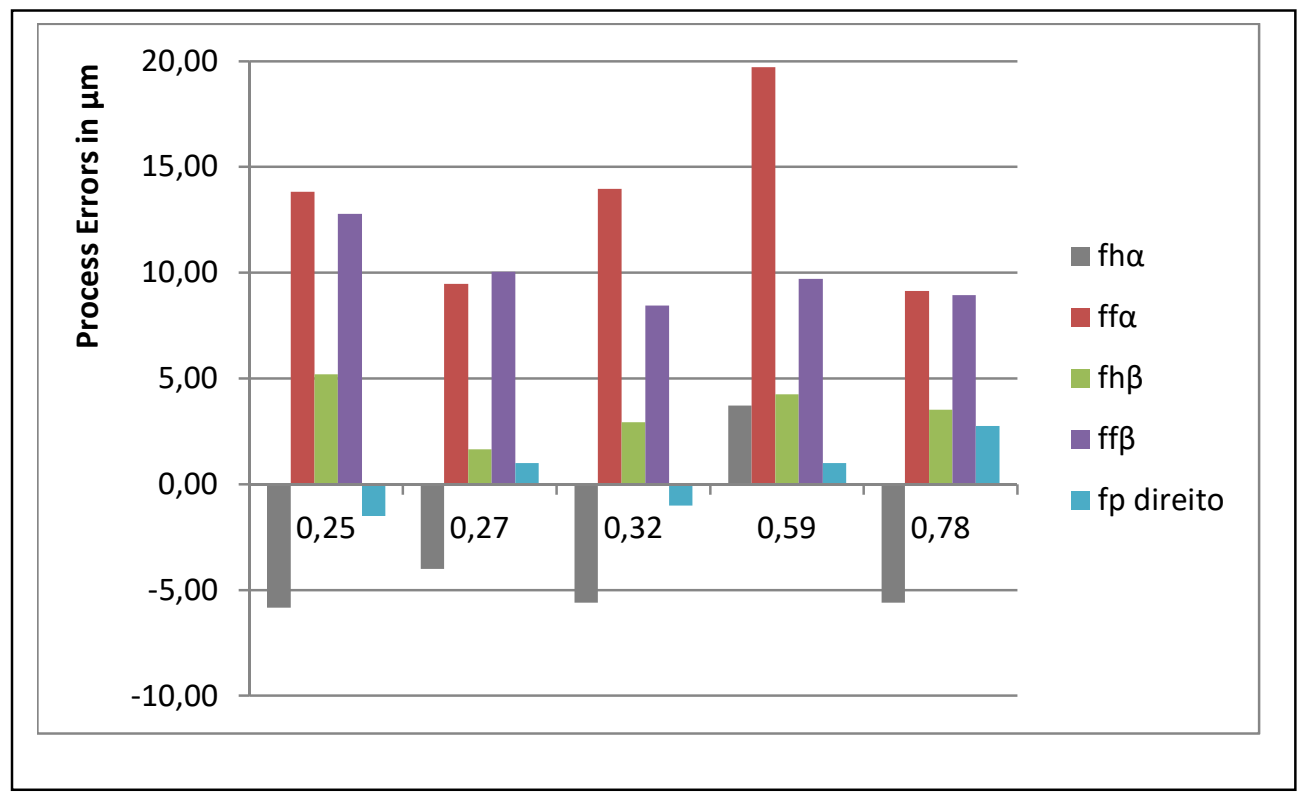

Source: Elaborated by the author

Therefore, on table 33 is seen that calculated $p$ for almost all pair to pair comparisons are smaller than significance level $\alpha$. rejecting the null hypothesis for almost all comparisons. But based on the test, D02 and D04 as D02 and D04 as much as D04 and D05 are the only comparison which the null hypothesis are not rejected. In other words, the treatment has influence between mentioned gears.

Table 33: Tukey test pairwise comparisons

\begin{tabular}{|c|c|c|c|c|c|}
\hline & D01 & D02 & D03 & D04 & D05 \\
\hline D01 & - & 0.0001336 & 0.0001331 & 0.0006284 & 0.0001331 \\
\hline D02 & & - & 0.0001331 & 0.444 & 0.8913 \\
\hline D03 & & & - & 0.0001331 & 0.0001331 \\
\hline D04 & & & & - & 0.08166 \\
\hline D05 & & & & & - \\
\hline
\end{tabular}


Once again figure 107 shows an evaluation of process errors and final damaged area by pitting. To check if process errors are still correlated with final damaged area by pitting, an reflection based on pair to pair comparisons as done on Tukey test are suitable, avoiding the comparisons which the null hypothesis were not rejected.

Figure 107: D Gear process errors $\mathrm{x}$ damaged area by pitting in $\%$.

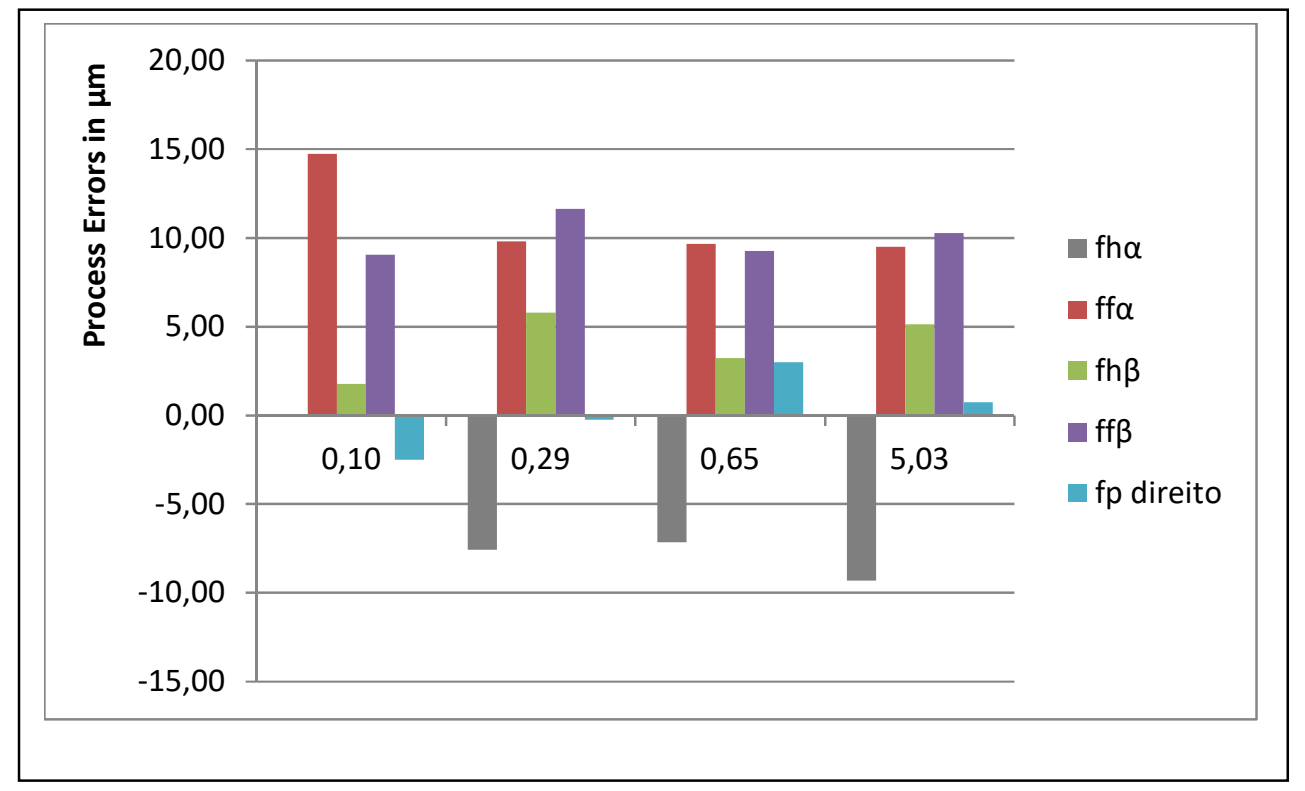

Source: Elaborated by the author

So once again, figure 107 shows an evaluation of process errors and final damaged area by pitting. To check if process errors are still correlated with final damaged area by pitting to $D$ Gears, once again the approach done on Tukey test pair to pair comparisons are appropriate, avoiding the comparisons which the null hypothesis were not rejected. In other words, the direct comparison of D01 with D02, D01 with D03, D01 with D04 and finally D01 with D05, which are the means that were considered statistically different, under the confidence level proposed on Tukey test, reveals that no direct correlation is established. In figures 106 and 107 is possible to deduct that the variables that had demonstrated influence on final fatigue damaged area by pitting on uncoated gears, are no longer highlighted as influence factors for coated gears. 
Finally, the raised up question is: If the process errors are not influencing coating, what is in fact causing the fatigue damage variation? To start to answer this question some coating surface evaluations are required.

Figure 108: Cr-DLC surface characterization

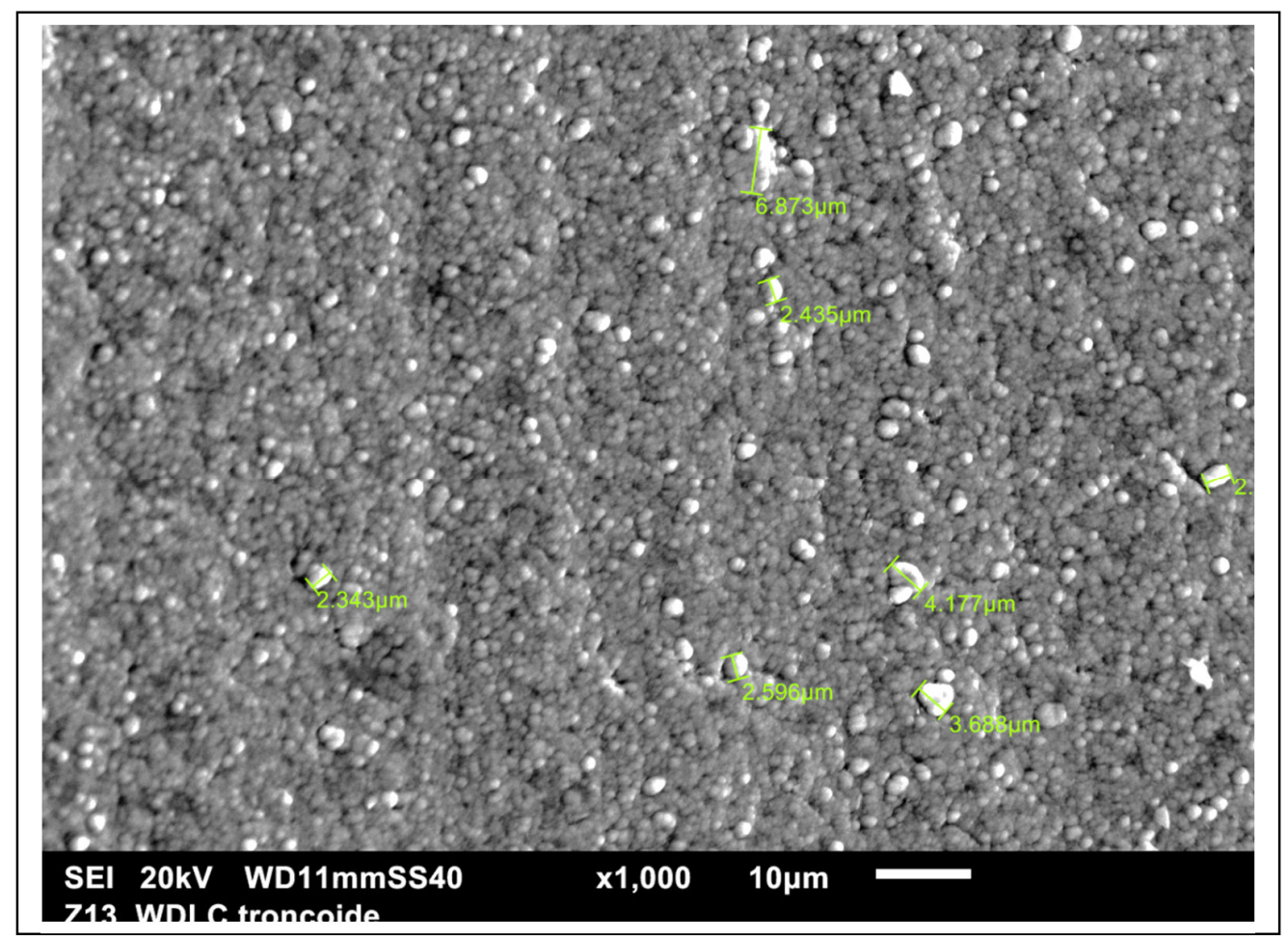

Source: Elaborated by the author

On figure 108 is shown B01 coated gear flank. This picture was taken with $\times 1000$ magnitude revealing coating surface details. As seen, the image makes visible that Cr-DLC surface is not a flat or homogeneous. The revealed surface is a heterogeneity layer which covers the base material. Some of those particles, have different morphology revealing smaller and bigger sizes particles ranging from 2.3 till $6.9 \mu \mathrm{m}$. It was not possible to characterize the particles by EDS, since the evaluation area is bigger than the particle. Similar evaluation has also been done by Bernardes et al. (2017) with lab disks evaluation. These aspects induce by itself a contact area, which could be the reason of a local stress increase, and, consequently, faster test cycles effects are seen on those gear flanks. This top view 
also helps to understand the result seen on $R_{t}$ of Cr-DLC (or previously called $\mathrm{W}$ DLC). In fact, the peaks are increased by the surface topography assumed by the particles heterogeneous disposal.

On figure 109 is shown D01 coated gear flank. This picture was taken with $x 500$ zoom revealing coating surface details. As seen, the image makes visible that DLC seemed to be more flat and homogeneous surface layer than Cr-DLC. As on the DLC coating process there is no additional of $\mathrm{W}$ or $\mathrm{Cr}$, its surface is not suitable to the different particles size formation as previously seen.

So, it's seen that DLC has a more appropriate surface to handle cyclical load, since its surface demonstrated homogeneity, which is beneficial for contact area evaluation. It is also expected to have a better lubricant regime, since DLC surface seen on SEM with 500x zoon did not shown protuberances which could influence lubricant regime or wear behavior.

Another important aspect of coating evaluation is to understand the variability seen on pitting damaged of DLC samples. The highest averaged damaged area seen was on DLC samples were D03 with 5.03 percent of damages area. On the other hand the lowest averaged damaged area seen on DLC sample was on D05 with 0.1 percent of damaged area.

This results raised up another important question: Is there any difference on coating to explain this result variability? So the hypothesis raised to answer this question will be focused on coating process. As this coating was done in a research lab, the small scale process should have induced a variability. Thus, the layers could have different thickness. So, aiming to answer the raised up question, another SEM evaluation were done. The aim was to measure the coating layer thickness of D03 and D05 and confirm the variability hypothesis.

Thus, the D03 Tooth 3 gear tooth were cross sechowed and observed on SEM. Figures from 110 till 112 is shown upper view images, not working and working flank views. Although the aim was to characterize the coating, measuring layer thickness, coating was not found in any of the observed tooth. Also, the D05 Tooth 5 was carefully prepared and also analyzed on SEM, but once again no coating were seen on SEM. Thus, on images from 113 till 115 is shown the results. The sample 
preparation were done again with another samples of each of the gear, but the same results were seen.

Figure 109: DLC surface characterization

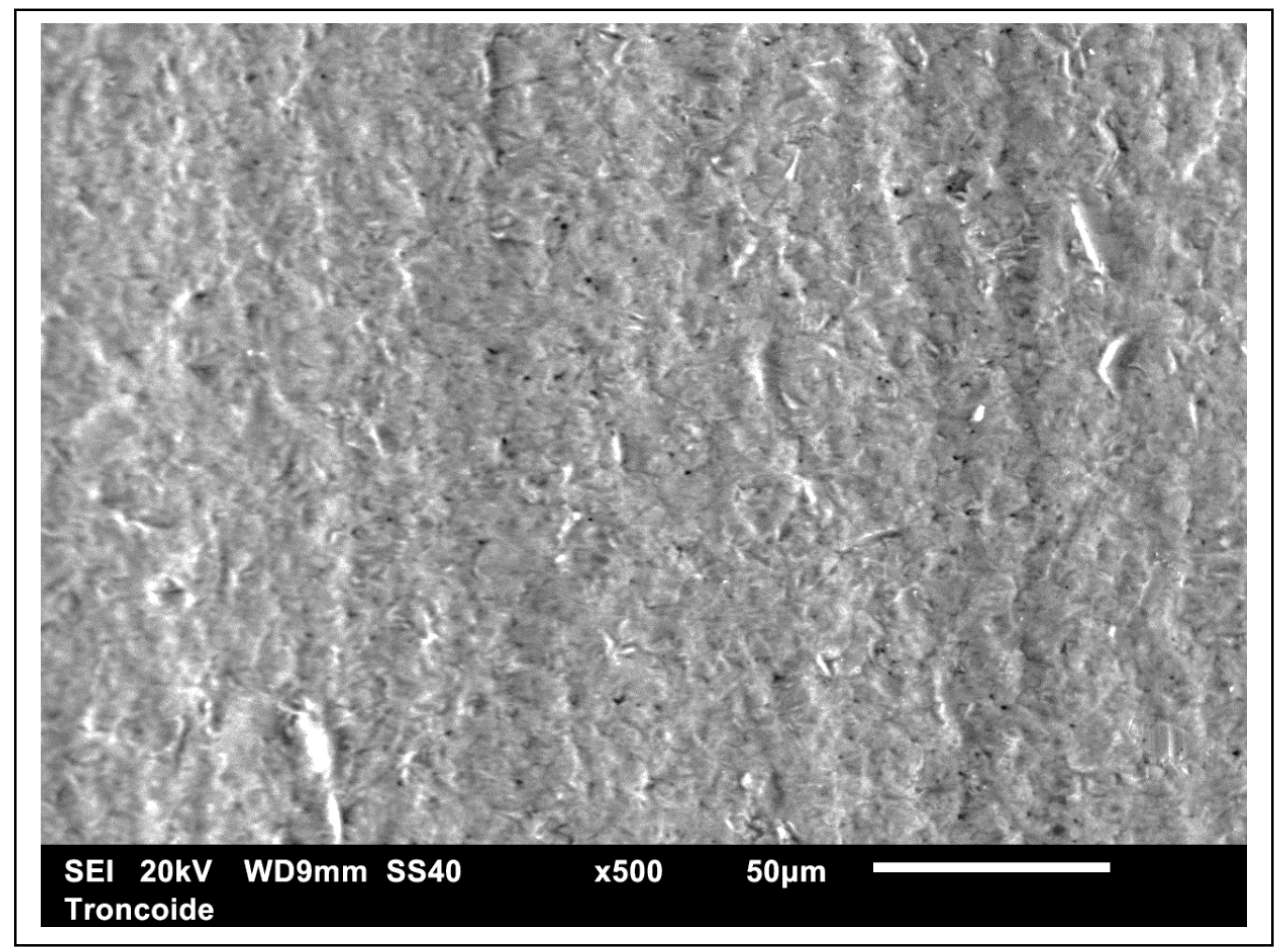

Source: Elaborated by the author 
Figure 110: DLC thickness evaluation on D03 tooth3 upper view

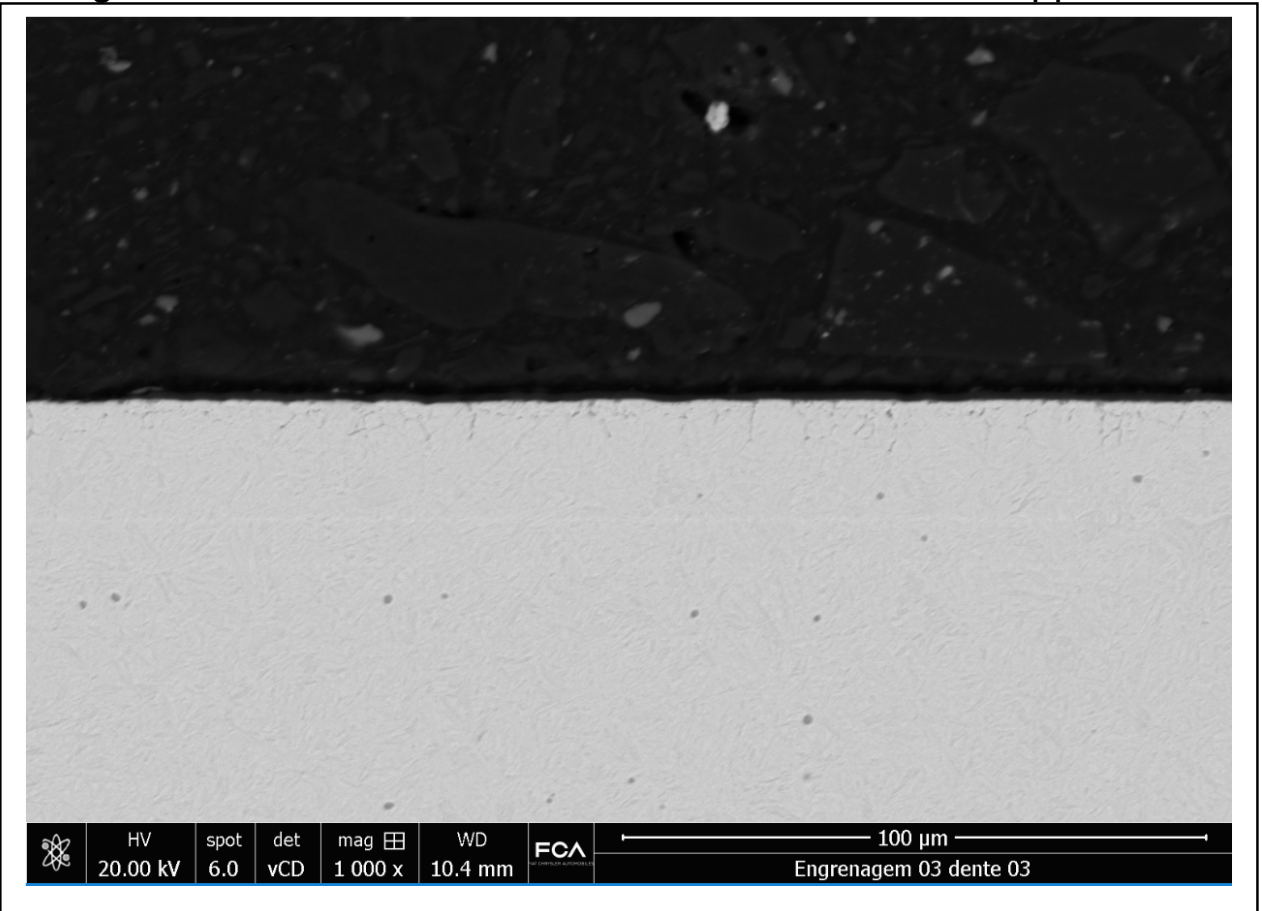

Source: Elaborated by the author

Figure 111: DLC thickness evaluation on D03 tooth5 not meshed flank

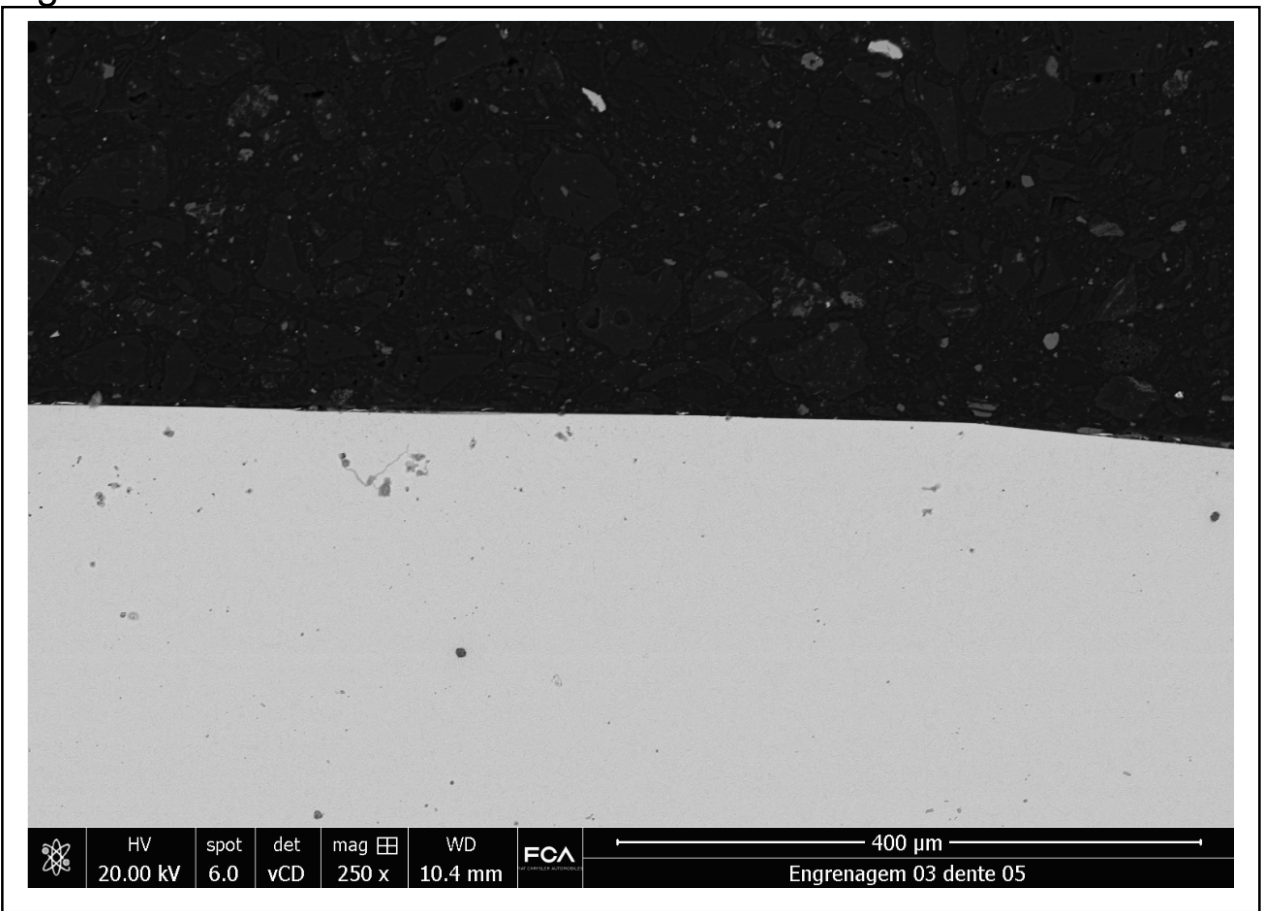

Source: Elaborated by the author 
Figure 112 DLC thickness evaluation on D03 tooth5 meshed flank

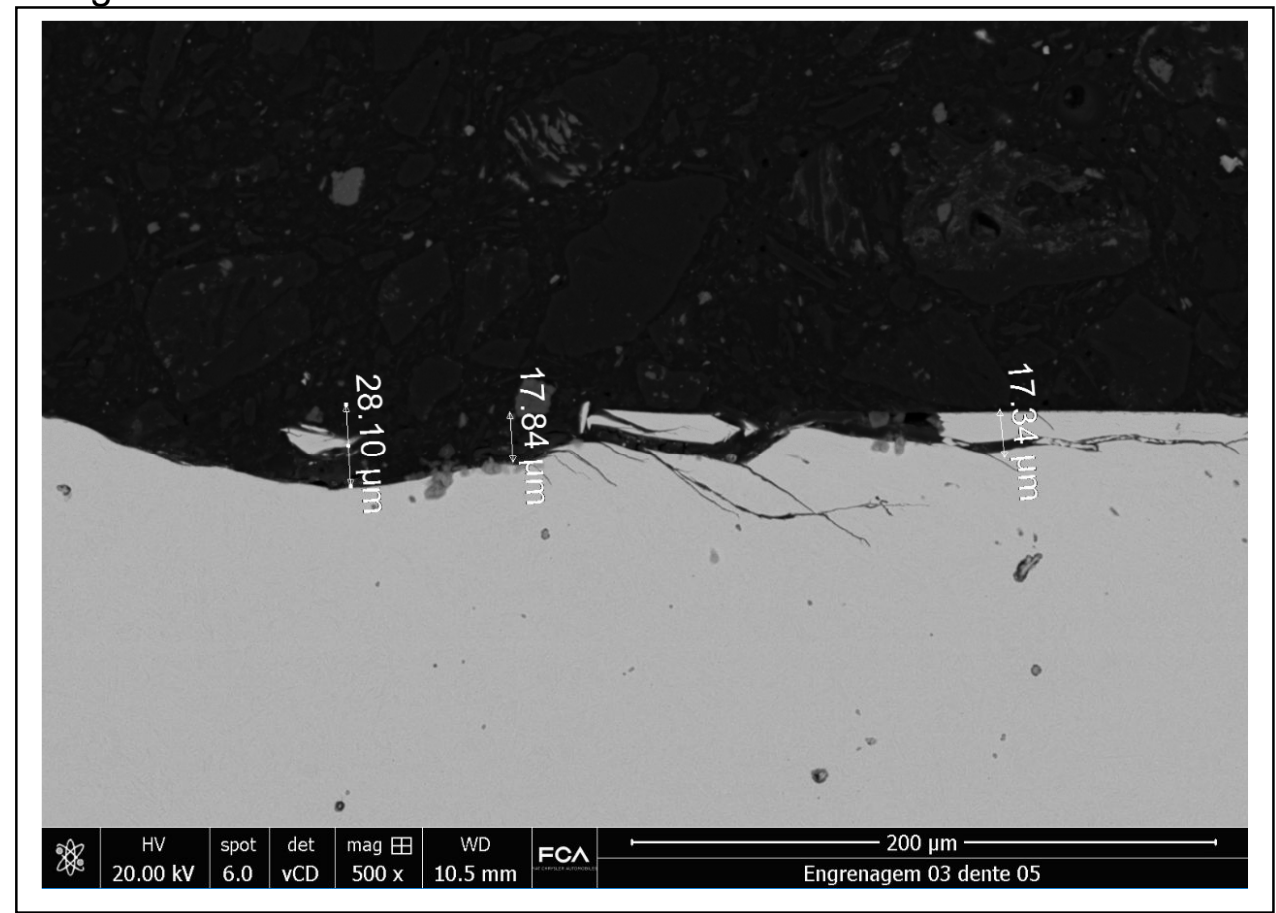

Source: Elaborated by the author

Figure 113 DLC thickness evaluation on D05 tooth3 upper view

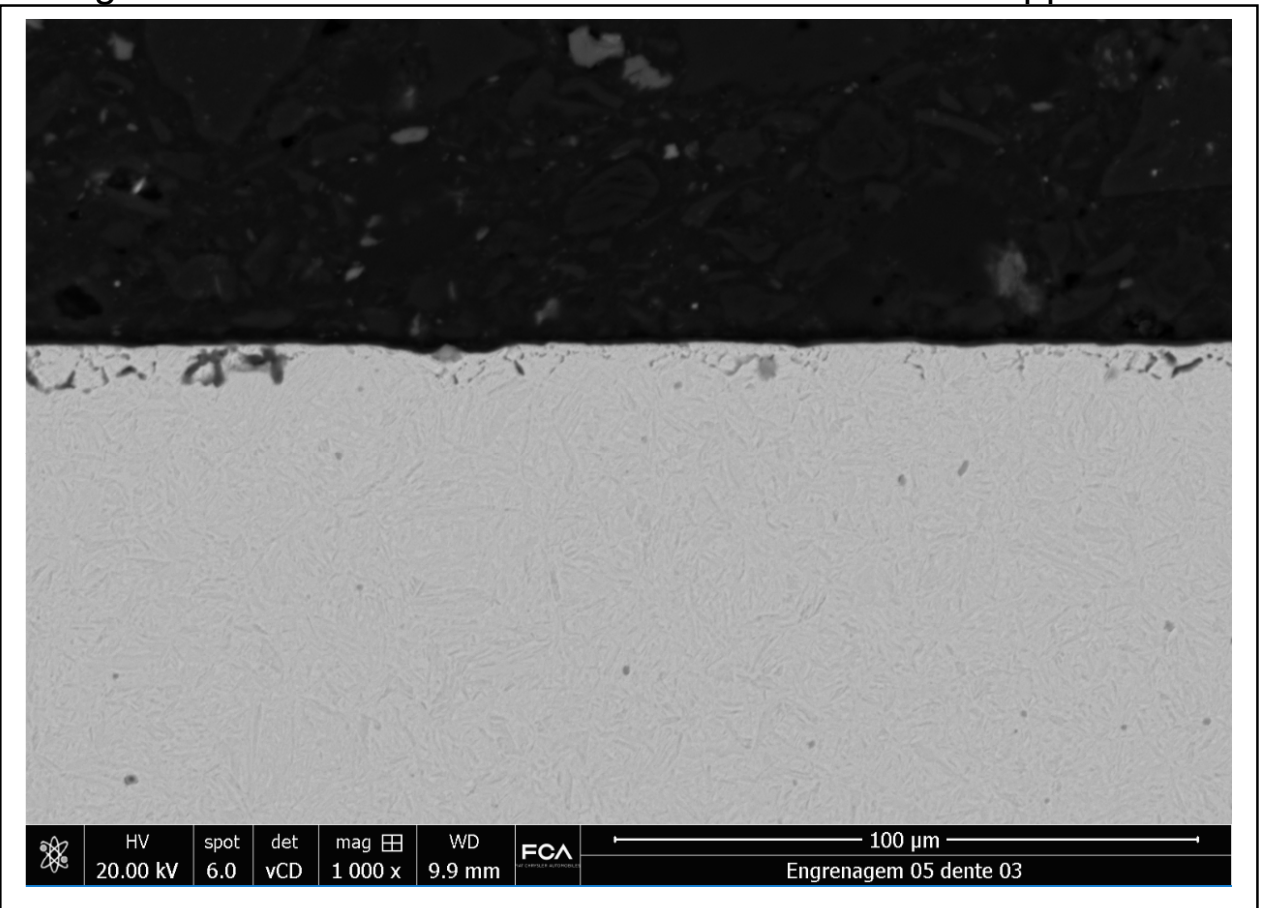

Source: Elaborated by the author 
Figure 114 DLC thickness evaluation on D05 tooth3 not meshed flank

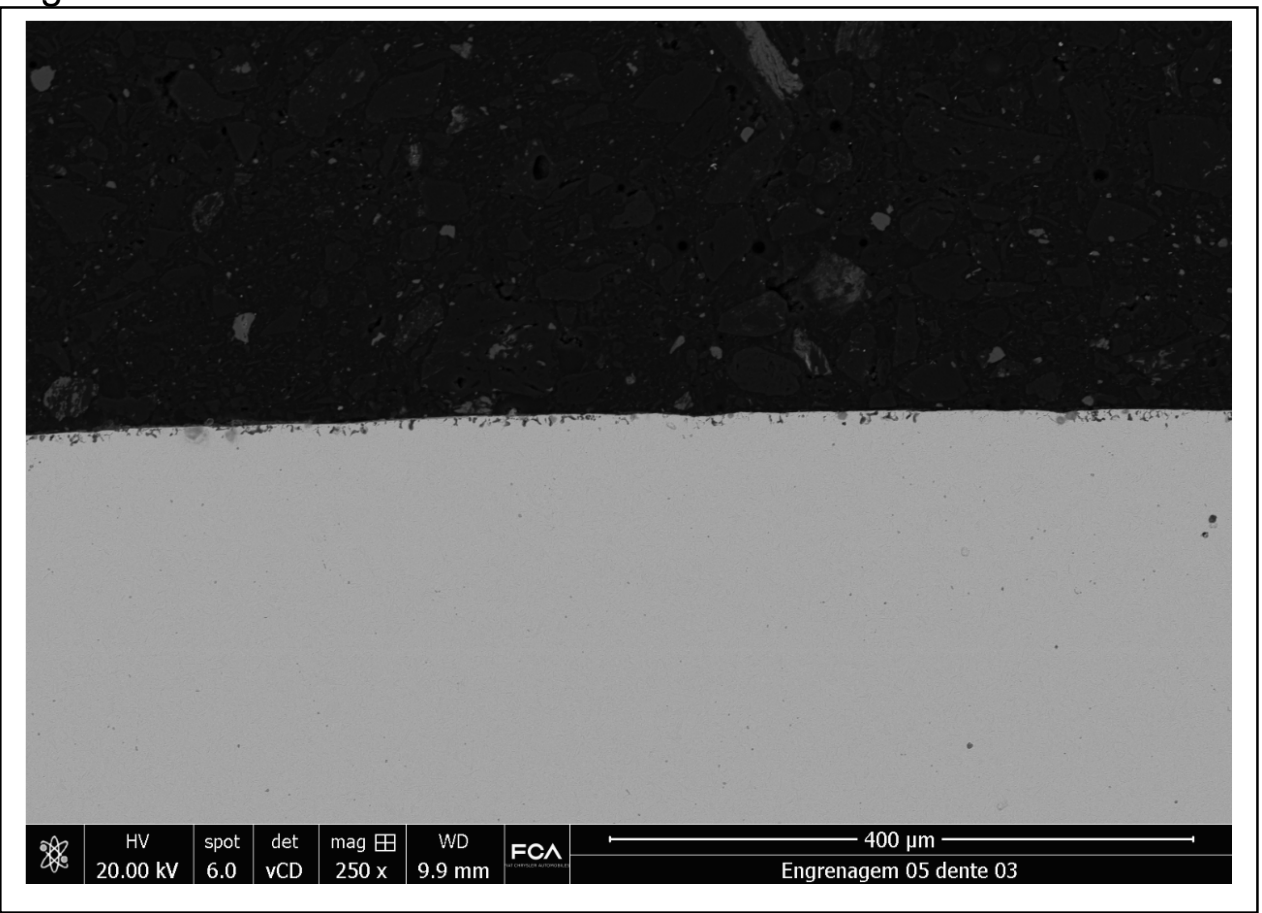

Source: Elaborated by the author

Figure 115 DLC thickness evaluation on D05 tooth3 meshed flank

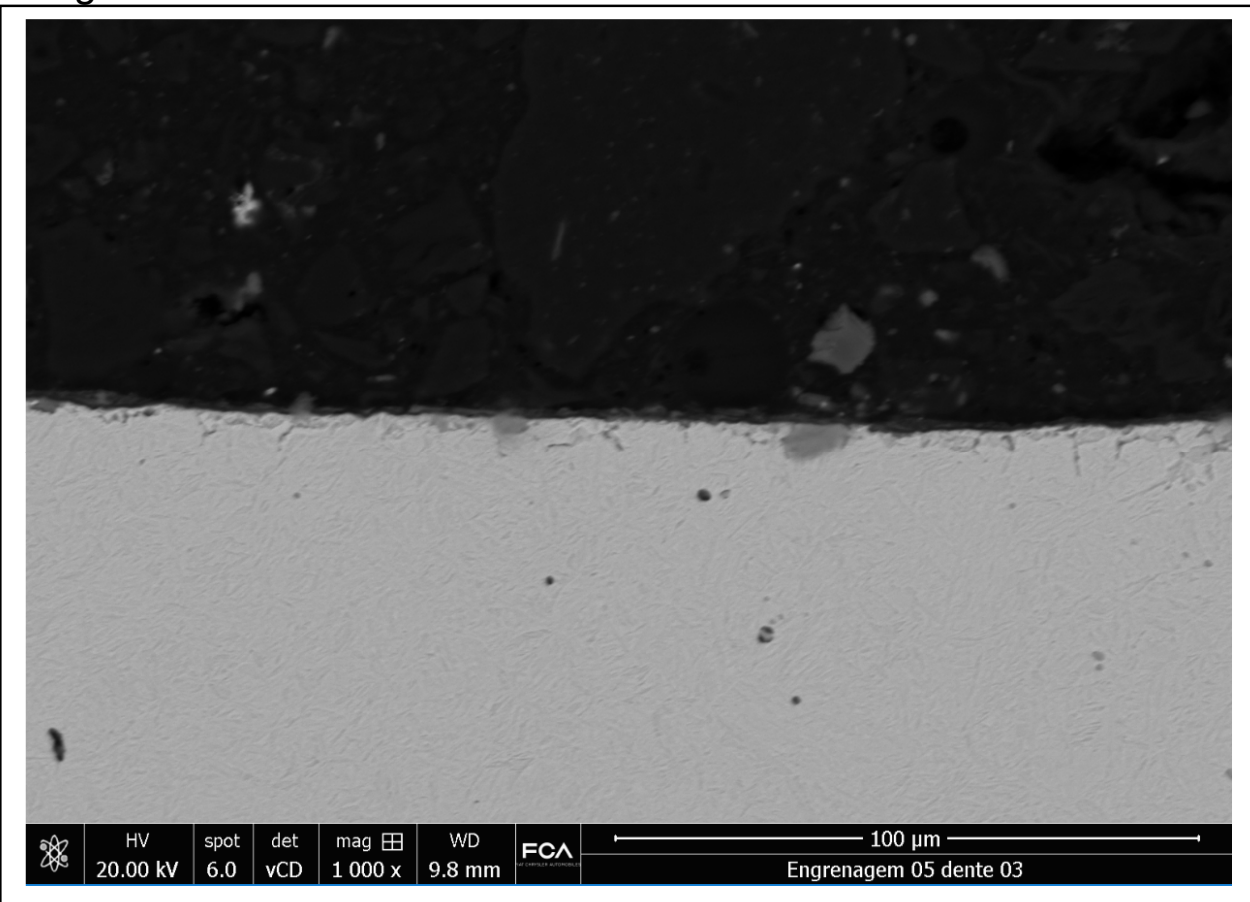

Source: Elaborated by the author 


\section{CONCLUSION}

This research presents an analysis of the pitting damaged area in spur gears based on experimental data obtained in controlled fatigue test executed in a Back to Back test machine. The gear was designed according to ISO 6336 standard.

The designed gear was more representative to replicate in a gear bench test the dynamic phenomenon which occurs on a referred first gear used in automotive transmission, based on contact stress magnitude. A contact stress magnitude of 2,427.5 $\mathrm{MPa}$ is taken when $\mathrm{k} 10$ torque category $(372.6 \mathrm{Nm}$ ) is obtained, while on FZG type C a contact stress magnitude of 2,030.1 MPa was obtained at the same torque level (K10). So, based on this data, the designed gear is representative for this and future analysis of the first gear transmission.

As a design reference standard for expert gear designers, ISO 6336 is intended to calculate the load capacity of gear. This calculation takes into consideration aspects based on drawing as much as on process. As seen on literature review, influence factors such as $\mathrm{K}_{\mathrm{A}}, \mathrm{K}_{\mathrm{v}}, \mathrm{K}_{\mathrm{H} \beta}$ and $\mathrm{K}_{\mathrm{H} \alpha}$ affects load capacity calculation and consequently adjust contact stress for the pinion. In this scenario, the referred standard takes into account the influence of process uncertainty on influence factors, in special, on $\mathrm{K}_{\mathrm{v}}, \mathrm{K}_{\mathrm{H} \beta}$ and $\mathrm{K}_{\mathrm{H \alpha}}$, to define stress increment.

Based on calculated torque magnitude, an amount of $0,5 \%$ of damaged area by pitting was expected to be observed when $6 \times 10^{5}$ loading cycles was reached for pinion. In this same standard, it's unclear the pitting starting point. In other words, it is not clearly defined the amount of cycles where pitting would begin.

However, based on this research results of Gear F01 till F05 (shaved teeth gears) pitting had been detected after the first fatigue cycle ( $\mathrm{k} 9$ for one hour). This result was not expected to be detected so early on fatigue life evaluation. This an important result due to the early unexpected pitting occurrence.

Therefore, after tests and quantifying thirteen teeth pitting damaged area of each one of the F01 and F05 pinions tested, an extensive data base was generated to support this research analysis. Such analysis points out that pitting damage area varied for each gear pinion teeth. 
Those results demonstrated that contact fatigue damaged area had been influenced by an uncertainty which impacted the final result. Thus, the contribution done here showed a widespread damage area variation after experimental fatigue test. The experimental results indicate that pitting mean damaged area varied from $0.61 \%$ to $1.11 \%$ considering five shaved gears tested

To deeply evaluate this variation statistical tools were used. Thus, a Tukey test pair to pair comparisons resulted that F01 and F03 mean damage area can be considered different as much as F01 and F04. Thus, it is possible to consider them different gears. Those gears had variations which influenced pitting damage final result. This final result alteration can be interpreted as influenced by different tooth geometry, once all other variables stated in this research had been controlled. Therefore, this geometry differences, in fact, influenced initial contact area and as a consequence, initial contact stress.

Nevertheless, this research focus had been on final damaged influenced by process uncertainty and on its impact on final tooth geometry variation. Therefore, through the experimental analysis it is possible to define that some micro geometric tooth dimensional deviations had influenced on pitting development. According to the experimental results single pitch, profile form deviation, profile and helix slope can be considered as important micro geometric deviations and could be intentionally induced by controlled manufacturing process to reduce pitting occurrence.

This research results indicate that surface damage due to pitting at end of gear operational life may vary significantly for similar gears influenced by process errors. In special, for $f_{h \beta}$ which had demonstrated a strong correlation to the amount of damaged area by pitting at the end of operational life. This contribution achieved in this research represents an important input to gear manufacturing control and gear reliability improvement path. 


\section{Coated Gears Conclusions}

Coating evaluation is note covered by the fatigue life prediction methodology used on this research, based on ISO 6336. So, this research did not compared its results directly to ISO model, but compared coated and uncoated results aiming to understand its impact on fatigue failure, as much as on reliability.

As detailed on results section and illustrated on Apendix, both coating significantly changed the experimental data output. This modification comprehends: (i) the amount of pitting damaged area, (ii) pitting occurrence position and (iii) other wear mechanism intensification and (iv) results variability.

The amount of pitting damaged area had been influenced by both coating. Statistical data evaluation is no longer suitable to establish a correlation of process errors and final fatigue damaged are by pitting. In other words, coating has covered the base material surface, removing process errors influence.

ANOVA had demonstrated DLC coated gears had introduced variability between gears D01 till D05 results. This fact allows the inference that coating introduces higher variability on results. Thus, the ANOVA results indicate that the DLC coating process introduces variability in gear flank surface after entire fatigue test.

ANOVA had also demonstrated that Cr-DLC reduced variability within each gear from B01 till B05. This means that ANOVA results indicate that the Cr-DLC coating process reduced variability within each gear flanks surface after entire fatigue test. Accordingly, it is reasonable to conclude that those variability increment seem on DLC is correlated to the coating process used to proceed with the chemical addiction on the base material. DLC has been done on Lab scale, while Cr-DLC was done on industry process, which is more stable and more capable to replicate homogeneity. Therefore, the reduced and amplified variability could be associate to the production process.

So, despite of the variation, the experimental results revealed that each coating influenced pitting appearance. Thus, DLC had demonstrated good results for avoiding or delay pitting occurrence. On the other hand, Cr-DLC fictitiously demonstrated a good result when just fatigue damaged analysis was considered. 
But when flank profile data and surface pictures taken are also into consideration, the wear phenomenon was seen. Furthermore, also based on surface characteristics seen on this coating, it's reasonable to expect that, the final coating aspects induces surface irregularities that are capable to alter local contact stress and also tribology aspects such film thickness and oil flow. As a consequence, the results seen on this research points that this coating reduces gear operational life.

The theory raised up to explain the pitting position change on coated gears may be a good phenomenon explanation. The explained PSO (Point Surface Origin), which considers that the type of pitting that is initiated from surface defects such as nicks, dents, grinding furrows, debris or even by previous-formed micropits. Consequently, this research adds extra variables, such as film detachment or even the left geometry found where the film had been removed, which characterizes a new edge formation. These research results identified that, Cr-DLC and also on some DLC samples, coating detached or its fragment tended to alter the contact area, inducing a higher localized stress.

As much as the hard layers particles are freely flowing on oil, it has a high probability of being present during gear meshing intensifying wear mechanism. In fact, Cr-DLC pictures presented on results and on Appendix B illustrated flanks surface final fatigue output with severe wear mechanism results. Thus, in gears which detachment is more frequent, wear is intensified.

Results variability was seen on both coatings. As discussed, DLC was done in a lab scale process which tends to induce a thickness layer variation and it tends to impact final result. Future Works should be based on this hypothesis to investigate this possible failure mode. Also, another suggestions to future works is to investigate the Cr-DLC hypothesis aiming to correlate the asymmetry of film particles distributed alongside different gear surface and, consequently, its impact on stress magnitude. 


\section{Future Works}

DLC results shown a potential improvement to the evaluated application. However, to be more appropriate, the hypothesis of variability needs to be confirmed and then reduced. Consequently, future works should focus in understanding significant coating process variables which influence final fatigue results. 


\section{REFERENCES}

AMERICAN SOCIETY FOR METALS HANDBOOK. Friction, lubrication and wear technology. IN: Metals Handbook, Vol. 18, p. 1879, 1992.

Fatigue and fracture. IN: Metals Handbook, Vol. 19, p. 2592 , 1992.

AMERICAN GEAR MANUFACTURERS ASSOCIATION. AGMA 2001-B88, Fundamental Rating Factors and Calculation Methods for Involute Spur and Helical Gear Teeth, AGMA Standard, 1988.

AGMA 933--B03, Basic Gear Geometry, AGMA information sheet, 2003.

AMERICAN SOCIETY FOR TESTING AND MATERIALS. ASTM D5182 - 97: Standard Test Method for Evaluating the Scuffing Load Capacity of Oils (FZG Visual Method). ASTM, 2008.

ANSI/AGMA National standard 1010-E95. Appearance of gear teeth - terminology of wear and failure, 1995.

ASLANTAS, K.; TASGETERIN, S. A Study of Spur Gear Pitting Formation and Life Prediction. Wear 257, P. 1167-1175, 2004.

ASSOCIACAO BRASILEIRA DE NORMAS TECNICAS. NBR 4287 Especificações geométricas do produto (GPS) - Rugosidade: Método do perfil - Termos definições e parâmetros de rugosidade, p.18, 2002.

NBR 5462 Confiabilidade e mantenabilidade, p.37, 1994.

BARTZ, W. J.; KRÜGER, V. K. Pitting fatigue of gears - some ideas on appearance, mechanism and lubricant influence. Tribology. Research Reports, p. 191-195, 1973.

BERNARDES, C.F., FUKUMASU, G.A.A, MACHADO, G.A.A; MACHADO I.F; Tribological Behavior of DLC and WDLC Carbon Based Coatings During Reciprocating Wear Tests. SAE International 36-0254, 2017.

BRADLEY E.; Reliability engineering: A life cycle approach. Ed. Crc Press, 2017. CZANDERNA, A., W.; MADEY, T. E. POWELL, C. J; Beam Effects, Surface Topography and Depth Profiling in Surface Analysis. Method of surface characterization Vol. 5, Kluwer Academic Publishers, 2002.

CHEN, Y.; Chen, B.; DONG, F, Yang, B. Li, S., Yu, X. Pitting Behavior of Thermally aged Z3CN20.09M Cas Stainless steel for Primary Coolant Pipe of Nuclear Power Plant. Engineering Failure Analysis 83, P. 1-8, 2018.

DIETER, G. E.; Mechanical Metallurgy, Mc Graw Hill. London, 3² Ed, 1988.

DING, Y.; Rieger, N. F.; Spalling formation mechanism for gears. Wear 254, P. 1307-1317, 2003.

DUGAS, J, P. The Process of gear shaving. Gear Technology, Michigan, January/February 1986. 
ENDOY, R. Gear Hobbing, Shaping and Shaving: A guide to cycle time estimating and process planning. Society of manufacturing engineers, Dearborn, 1990.

ERRICHELO, R. L. Morphology of Micropitting. Gear Technology November/December 2012.

FRANCO, R. F., SOUZA, G. F. M., SILVA, C. H. Experimental Uncertainty Analysis Of Gear Fatigue Life Theoretical Prediction, The 64th Annual Reliability \& Maintainability Symposium January, 2018.

FZG, "Description of the pitting test", Institute for Machine Elements - Gear Research Center, p. 08, 1992.

GLODEZ, S.; WINTER, H., STÜWE, H.P. A fracture mechanics model for the wear of gear flanks by pitting. Wear International 208, p. 177-183, 1997.

GLODEZ, S.; REN, Z. FLASKER, J. Surface Fatigue of Gear Teeth Flanks, Computer and Structures 73, p. 475-483, 1999.

HE, X., SUI, F, ZHAI, B. Liu W. Probabilistic and testing analysis for the variability of load spectrum damage in fleet. Engineering Failure Analysis 33, p. 419-429, 2013.

HOLMBERG, K.; ANDERSSON,P.; ERDEMIR, A. Global energy consumption due to friction in passenger cars. Tribology International, 2011.

HÖHN, B, R.; OSTER, P.; SCHEDL, U. Pitting Load Capacity Test on the FZG Gear Test Rig with Load-Spectra and One-Stage Investigations, Tribotest Journal 5-4 June, 1999.

HUTCHINGS,. I.M.; Tribology. Friction and wear of engineering materials. Ed. Butterworth Heinemann. Cambridge, 1992.

INTERNATIONAL STANDARD ORGANIZATION. ISO 1302 Geometrical Product Specifications (GPS) -- Indication of surface texture in technical product documentation. ISO Standard, 2002.

. ISO 1328-1 Cylindrical Gears - ISO system of accuracy Part 1: Definitions and allowable values of deviations relevant to corresponding flanks of gear teeth. ISO Standard, 1995.

ISO 1328-1 Cylindrical Gears - ISO system of flank tolerance classification - Part 1: Definitions and allowable values of deviations relevant to flanks of gear teeth. ISO Standard, 2013.

ISO 14707 Surface chemical analysis - Glow discharge optical emission spectrometry (GD-OES) - Introduction to use. ISO, 2015.

ISO 6336-1 Calculation of load capacity of spur and helical gears - Part 1 : Basic Principles, introduction and general influence factors. ISO Standard, 1996.

ISO 6336-2 Calculation of load capacity of spur and helical gears - Part 2: Calculation of surface durability (pitting). ISO Standard, 1996.

. ISO 6336-3 Calculation of load capacity of spur and helical gears - Part 3: Calculation of tooth bending strength. ISO Standard, 1996. 
ISO 6336-5 Calculation of load capacity of spur and helical gears - Part 5: Strength and quality of materials. ISO Standard, 1996.

ISO 6508-1 Metallic materials -- Rockwell hardness test -- Part 1: Test method. ISO Standard, 2016.

KARABAY, S.; ERTÜRK, A. L.; ZEREN, M.; YAMANGOLU, E. K. Failure Analysis. Engineering Failure Analysis 83, P. 47-56, 2018.

KLOCKE, F.; BRUMM, M.; REIMANN, J. Modeling of Surface Zone Influences in Generating Gear Grinding, Procedia CIRP 8, p. 21 - 26, 2013.

KLOCKE, F.; BRUMM, M.; KAMPKA, M. Process model for honing larger gears, International Gear Conference, p. 118-128, 26th-28th August 2014

KLOCKE, F.; LÖPENHAUS, C.; SARI, D. Process concepts for gear finish hobbing. 48th Conference on Manufacturing Systems, Procedia CIRP 41, p.875 880, 2016.

KUMAR, P.; HIRANI, H., AGRAWAL, A.; Fatigue failure prediction in spur gear pair using AGMA approach, Materials Today: Proceedings 4, P. 2470-2477, 2017

LANZUTTIA, A.; GAGLIARDIA, A.; RAFFAELLIA, A.; SIMONATOB, M.; FURLANETTOB, R.; MAGNANA, M.; ANDREATTAA, F.; FEDRIZZIA, L.; Failure analysis of gears, shafts and keys of centrifugal washers failed during test life, Engineering Failure Analysis 79, P. 634-641, 2017.

Li, M.; Xie, L.; Ding, L. Load Sharing Analysis and Reliability Prediction for Planetary Gear Train Helicopter. Engineering Failure Analysis 15, P. 97-113, 2017.

LEE, Y.L.; PAN, J.; HATHAWAY, R.B.; BARKEY, M.E. Fatigue Testing and Analysis: Theory and Practice. Amsterdam: Elsevier, 402p., 2005.

LEWIS, E. E. Introduction to Reliability Engineering, Ed. John Wiley and Sons, 2nd edition, Illinois, 1994.

LIN,T.; HE, Z.; Analytical method for coupled transmission error of helical gear system with machining errors, assembly errors and tooth modifications. Mechanical System and Signal Processing 91, p. 167-182, 2017.

LINKE, H.; BÖRNER J.; HEß R.; Cylindrical Gears: Calculation - Materials Manufacturing. Munich: Ed. Carl Hanser Verlag, 2016.

LITVIN L. F.; FUENTES, A. Gear geometry and applied theory, Ed. Cambridge University Press, $2^{\circ}$ Ed, 2004.

MAITRA, G., N. Handbook of Gear Design. Ed. Tata McGraw-Hill Publishing Company, $2^{\circ} \mathrm{Ed}, 2001$.

MAZZO, N. Engrenagens Cilíndricas - Da concepção à fabricação, Ed. Blucher, 2013.

MOORTHY, V.; SHAW B.A., Effect of as-ground surface and the BALINITs C and $\mathbf{N b}-\mathrm{S}$ coatings on contact fatigue damage in gears, Tribology International 51, p. 61-70, 2012. 
MOORTHY, V.; SHAW B.A., An observation on the initiation of micro-pitting damage in as-ground and coated gears during contact fatigue, Wear International 297, p 878-884, 2013.

MONTGOMERY, D. C.; RUNGER, G. C Applied Statistics and Probability for Engineers . John Wiley and Sons, 3rd edition, Illinois, 2003.

MONTGOMERY, D. C; Design and Analysis of Experiments . John Wiley And Sons, 8th Edition, Arizona State University, 2013.

MORITA, T.; INOUE, K.; DING, X.; USUI, Y.; IKENAGA, M. Effect of hybrid surface treatment composed nitriding DLC coating on friction-wear properties and fatigue strength of alloy steel. Material Science and Engineering, v. 661, p. 105114, 2016.

MURARO, M. A.; KODA, F.; REISDORFER J. U.; SILVA, C. H. The Influence of Contact Stress Distribution and Specific Film Thickness on the Wear of Spur Gears During Pitting Tests. Journal of the Brazilian Society of Mechanical Sciences and Engineering, v. 34, p. 135-144, 2012.

NAKANISHI, T.; ARIURA, Y.; UENO, T.; Load Carrying Capacity of surface hardened gears (Influence of Surface Roughness on Surface Durability) The Japanese Society of Mechanical Engineering vol. 30, n 259 p161-167, 1987.

NAUNHEIMER, H.; BERTSCHE, B.; RYBORZ, J; NOVAK, W. Automotive Transmissions. $2^{\circ}$ Ed, Springer - Verlag Berlin Heidelberg, 2011.

NEJAD, A. R.; GAO, Z.; MOAN, T. On Long-Term Fatigue Damage and Reliability Analysis of Gears Under Loads in Offshore Wind Turbine Drivetrains. International Journal of Fatigue 61, p. 116-128, 2014.

NETPU, S.; SRICHANDR, P.; Failure of a helical gear in a power plant, Engineering Failure Analysis 32, P. 81-90, 2013.

NIEMANN, G. Elementos de máquinas. Ed. Edgard Blucher, vol. 3, São Paulo, 1971.

NORTON, R. L. Projeto de máquinas: uma abordagem integrada. Ed. Bookman, $2^{\mathrm{a}}$ Ed., 2004.

OLSSON, E. OLANDER, A,, ÖBERG, M. Fatigue Of Gears In The Finite Life Regime - Experiments And Probabilistic Modelling. Engineering Failure Analysis 62, P. 276-286, 2016.

PIRRO, D., M; WESSOL, A. A. Lubrication Fundamentals, Ed. Marcel Dekker, 2nd edition, New York, 2001.

PHAM, H. Handbook of reliability engineering, Springer, London, 2003.

SIDDIQUI, N.; DEEN, K.M.; KHAN, M. Z.; Ahmad, R. Investigating The Failure Of Bevel Gears In An Aircfraft Engine, Case Studies In Engineering Failure Analysis 1, P. 24-32, 2013.

SMITH, J. D Gear noise and vibration, 2nd edition, Ed. Marcel Dekker Inc, New York, 2003. 
STACHOWIAK, G.W; BATCHELOR, A.W. Engineering Tribology, Elsevier Butterworth-Heinemann, 3rd edition, Oxford, 2005.

TERRIN, A., DENGO, C.,MENEGHETTI G. Experimental Analysis of Contact Fatigue Damage in Case Hardened Gears for Off-Highway axles. Engineering Failure Analysis 76, P. 10-26, 2017.

THOMAS, R. T. Rough Surfaces, 2nd edition, Imperial college press, London,1999. XIAO, Y.; SHI, W.; LUO, J.; LIAO Y. The tribological performance of TiN, WC/C and DLC coatings measured by the four-ball tes. Ceramics international $40, p$. 6919-6925, 2014. 
APPENDIX A - FLANK SURFACE IMAGES AND MATLAB ROUTINE OF F01 TILL F05

Figure A1 - F01 Images and quantification after each test step.

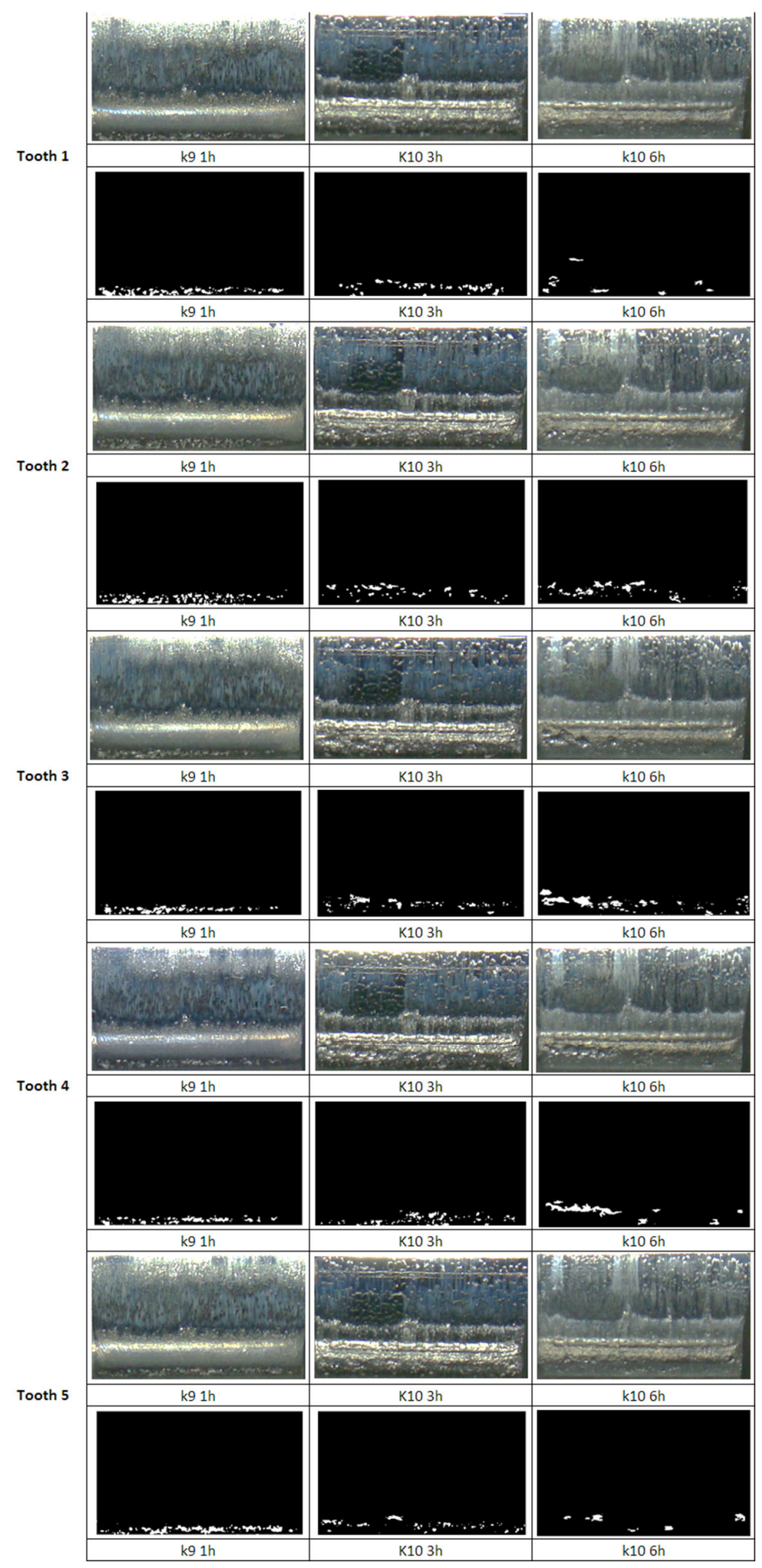


Figure A2 - F01 Images and quantification after each test step.

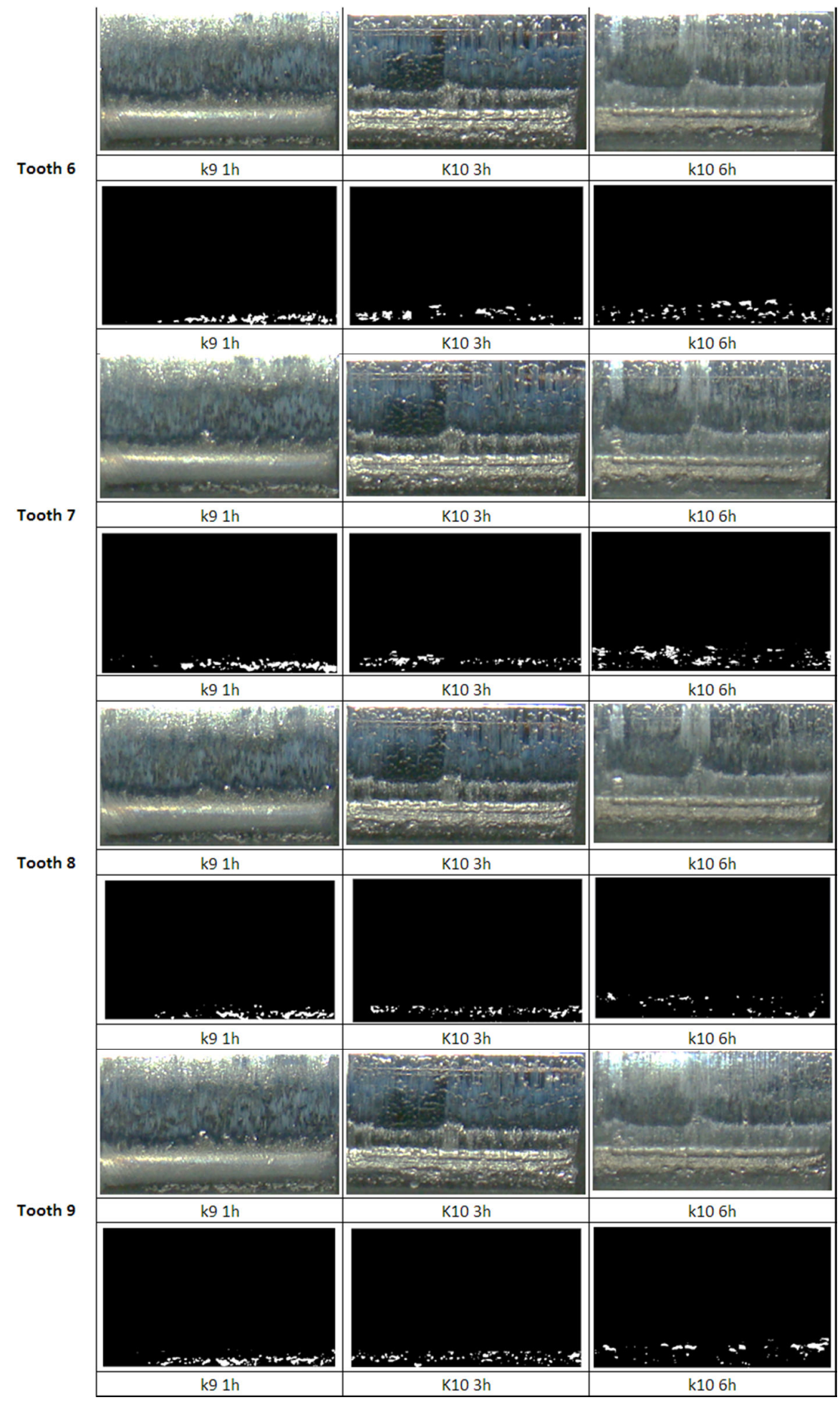


Figure A3 - F01 Images and quantification after each test step.

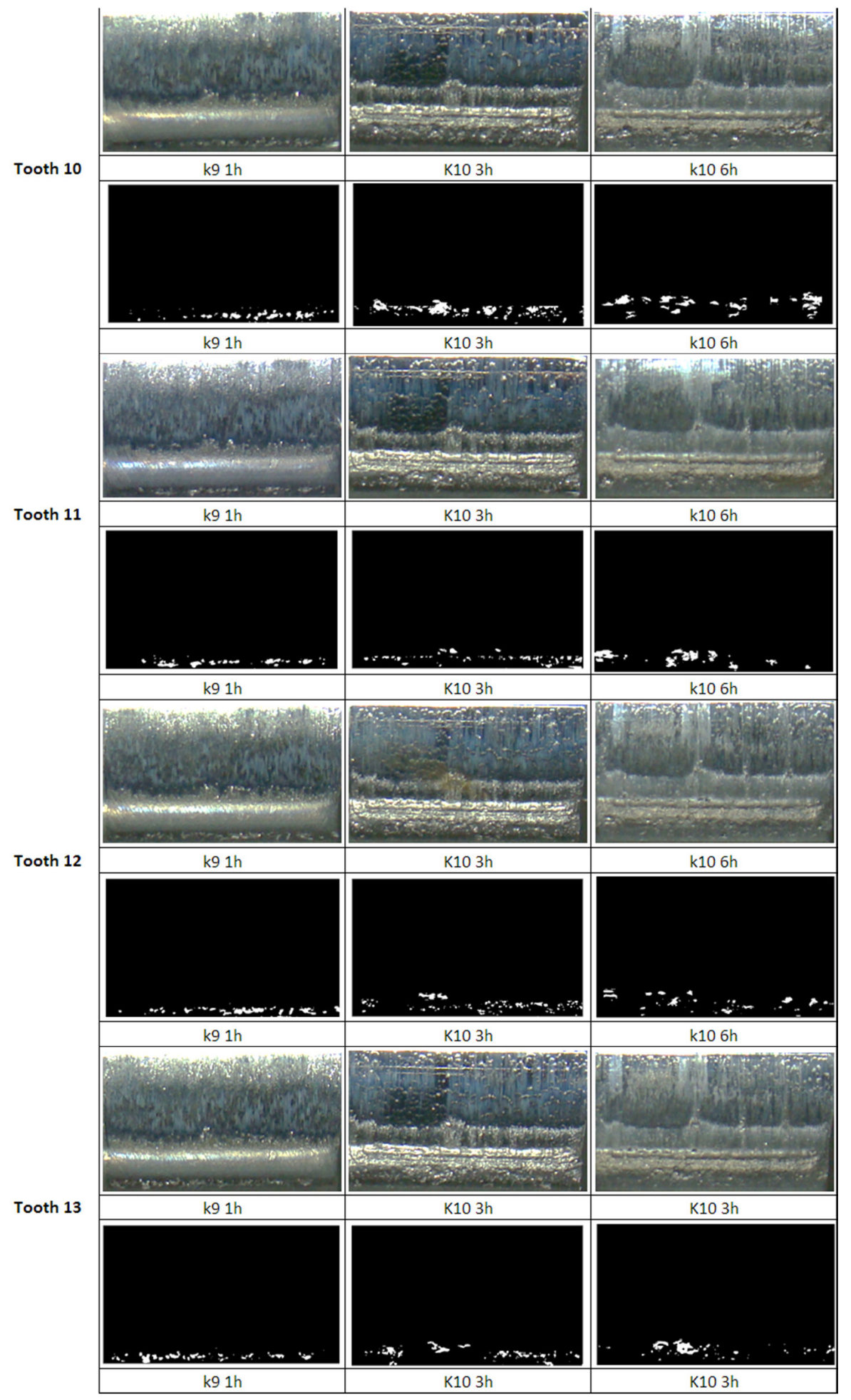


Figure A4 - F02 Images and quantification after each test step.

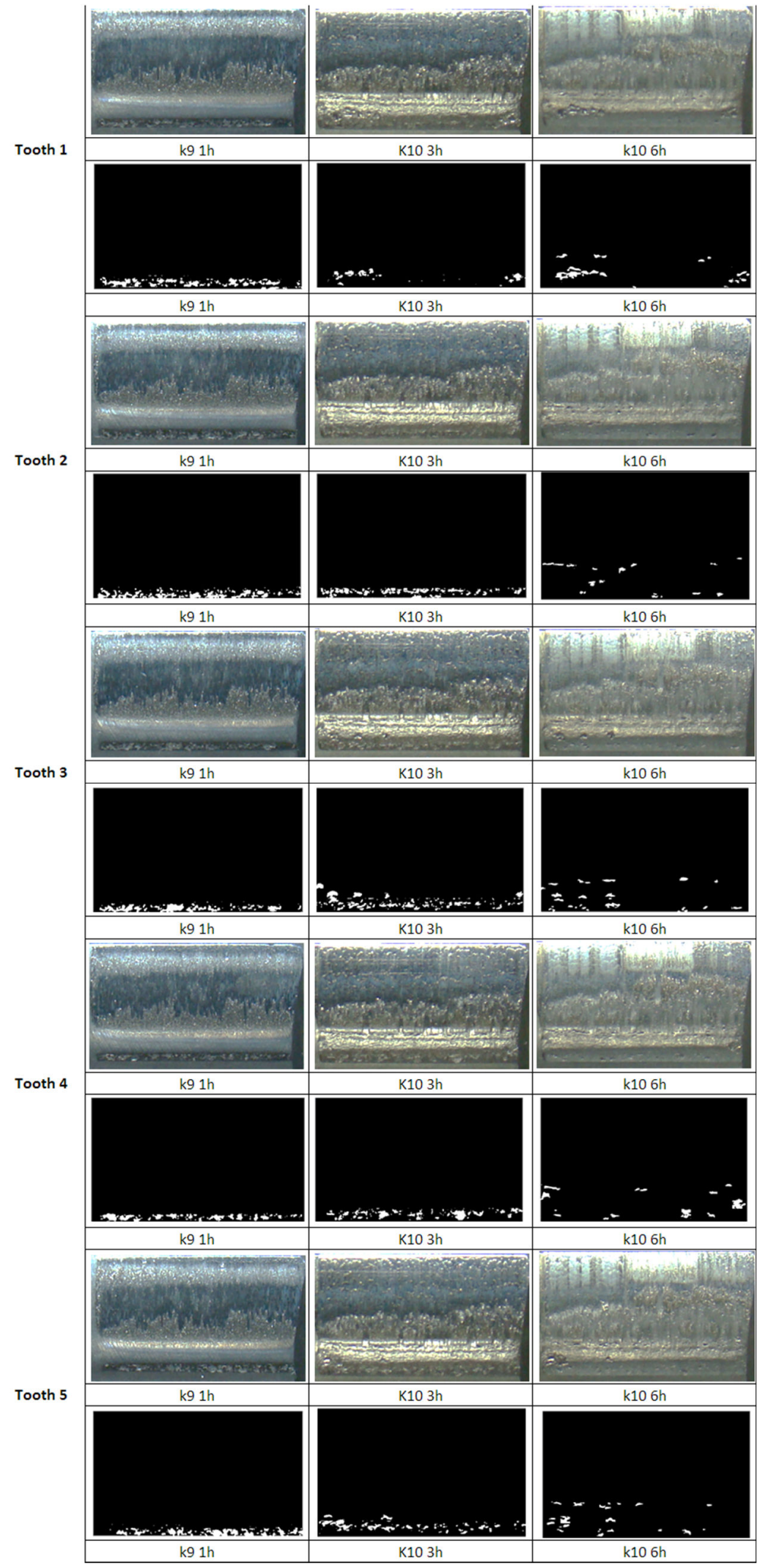


Figure A5 - F02 Images and quantification after each test step.

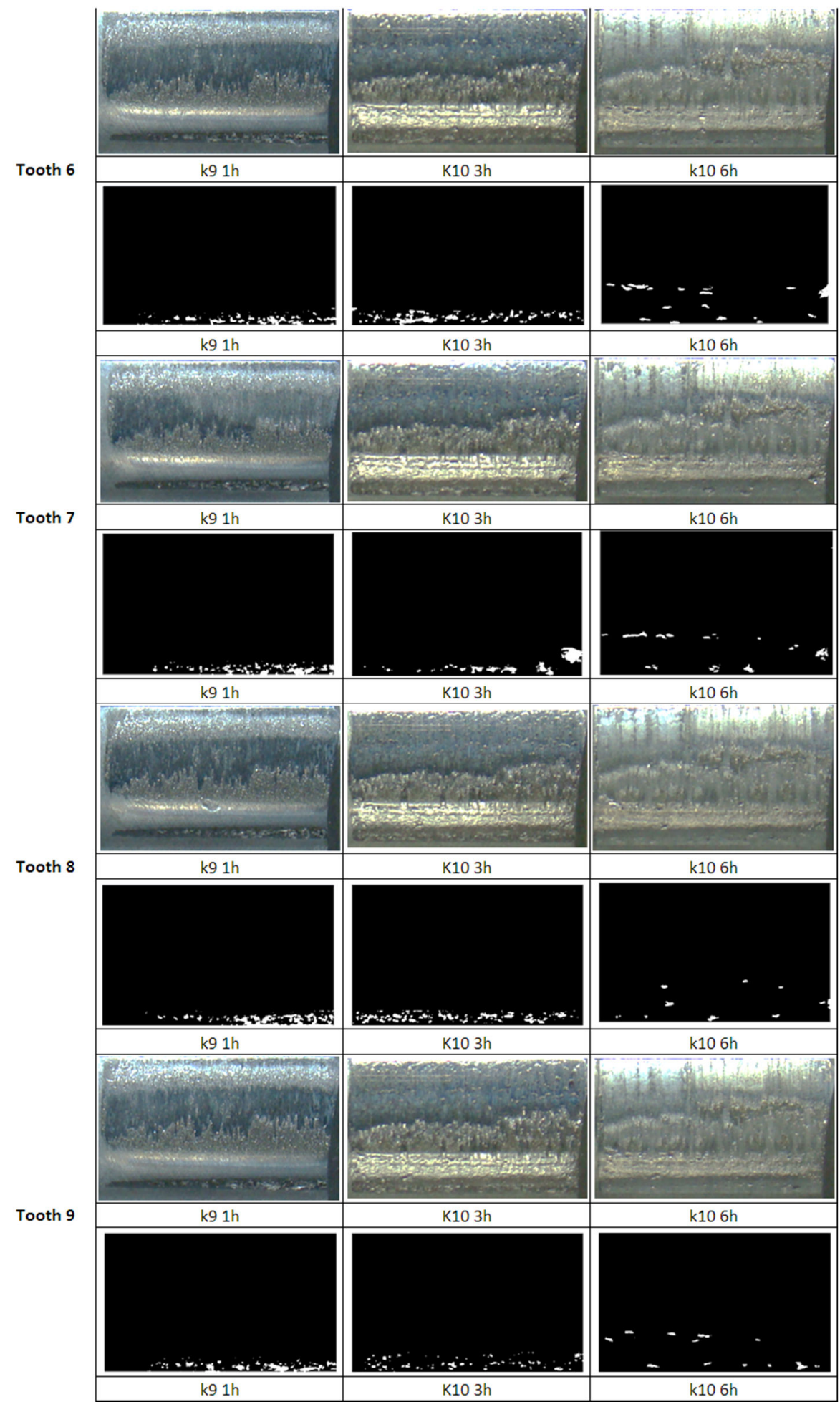


Figure A6 - F02 Images and quantification after each test step.

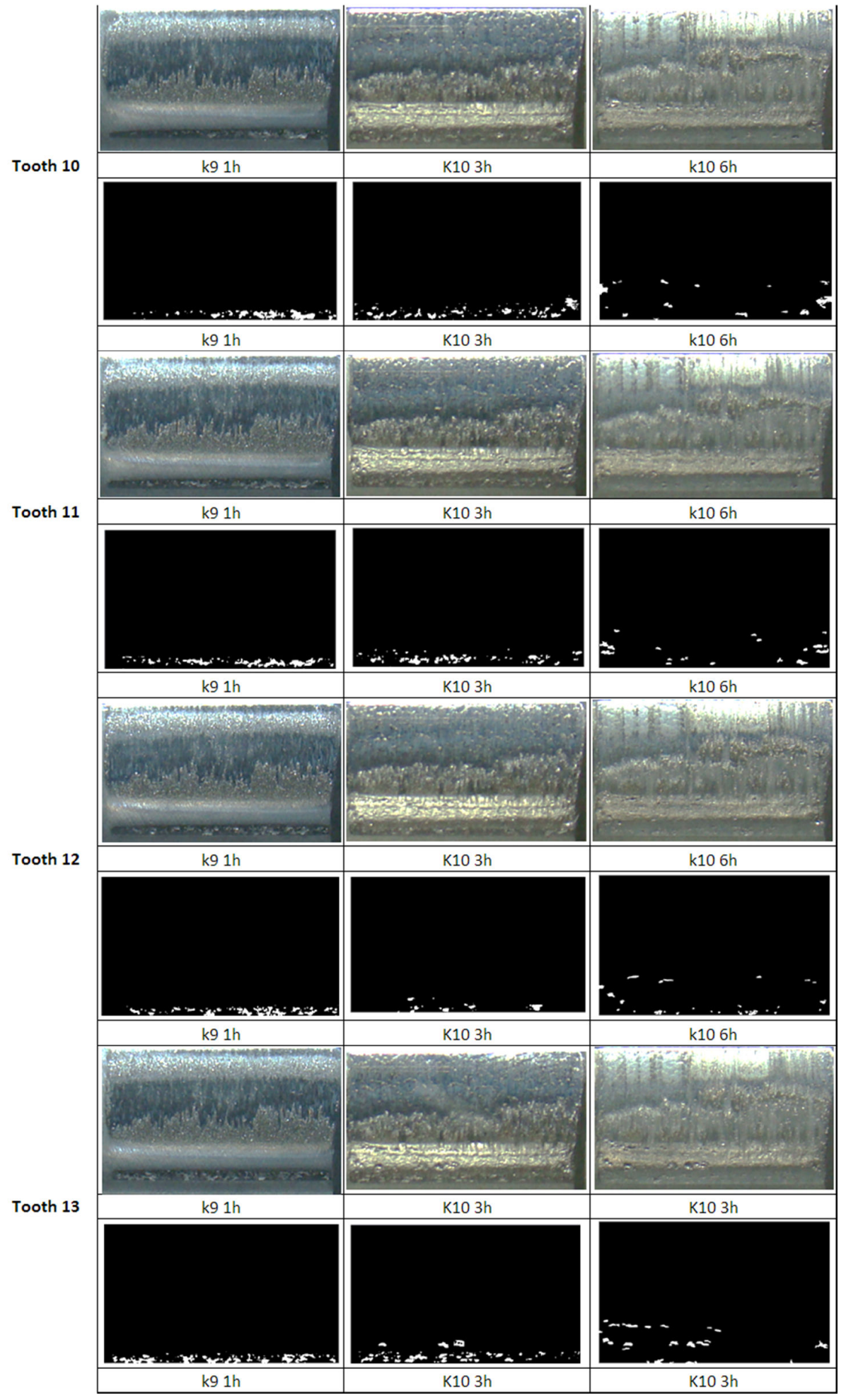


Figure A7 - F03 Images and quantification after each test step.

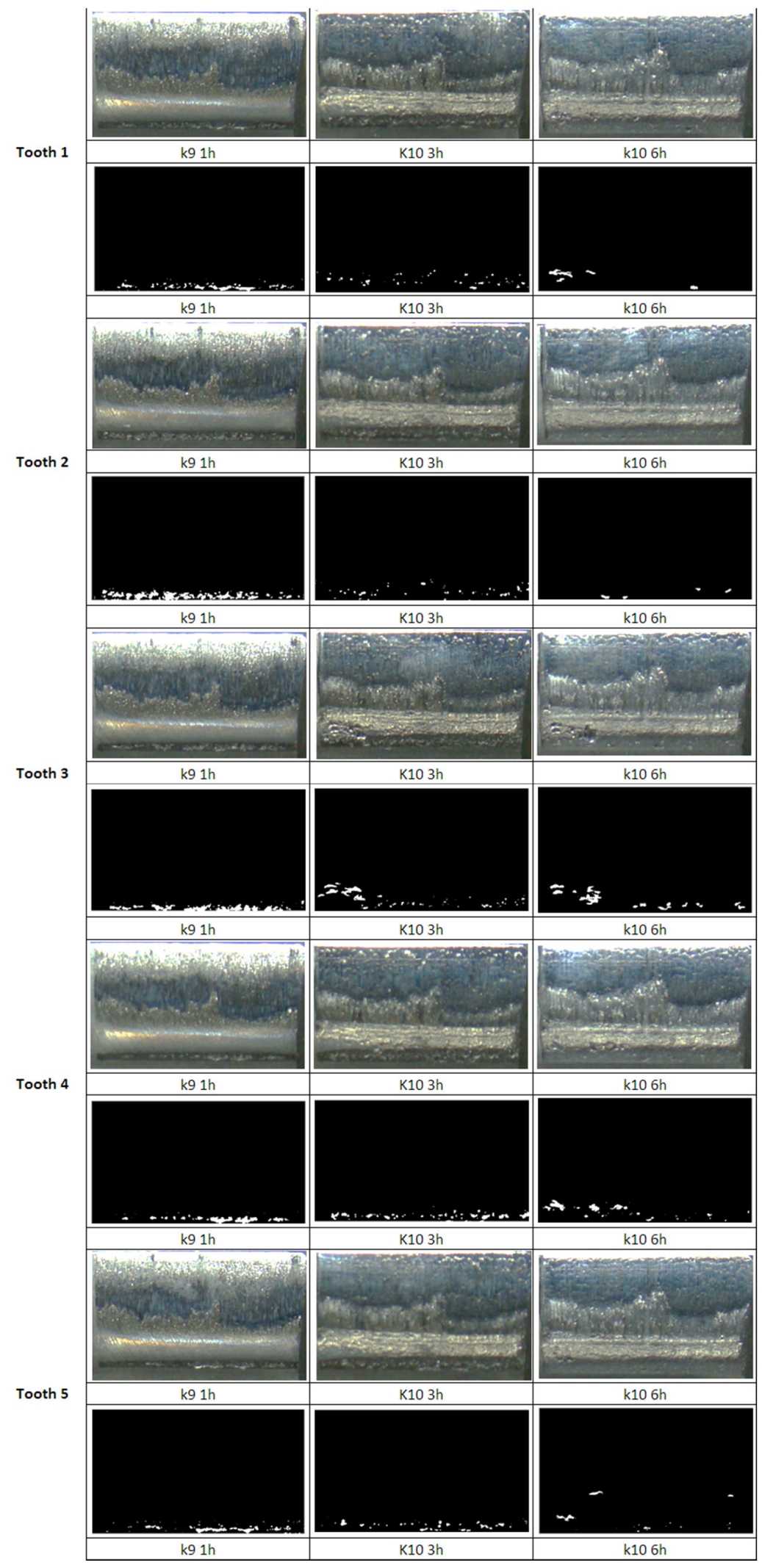


Figure A8 - F03 Images and quantification after each test step.

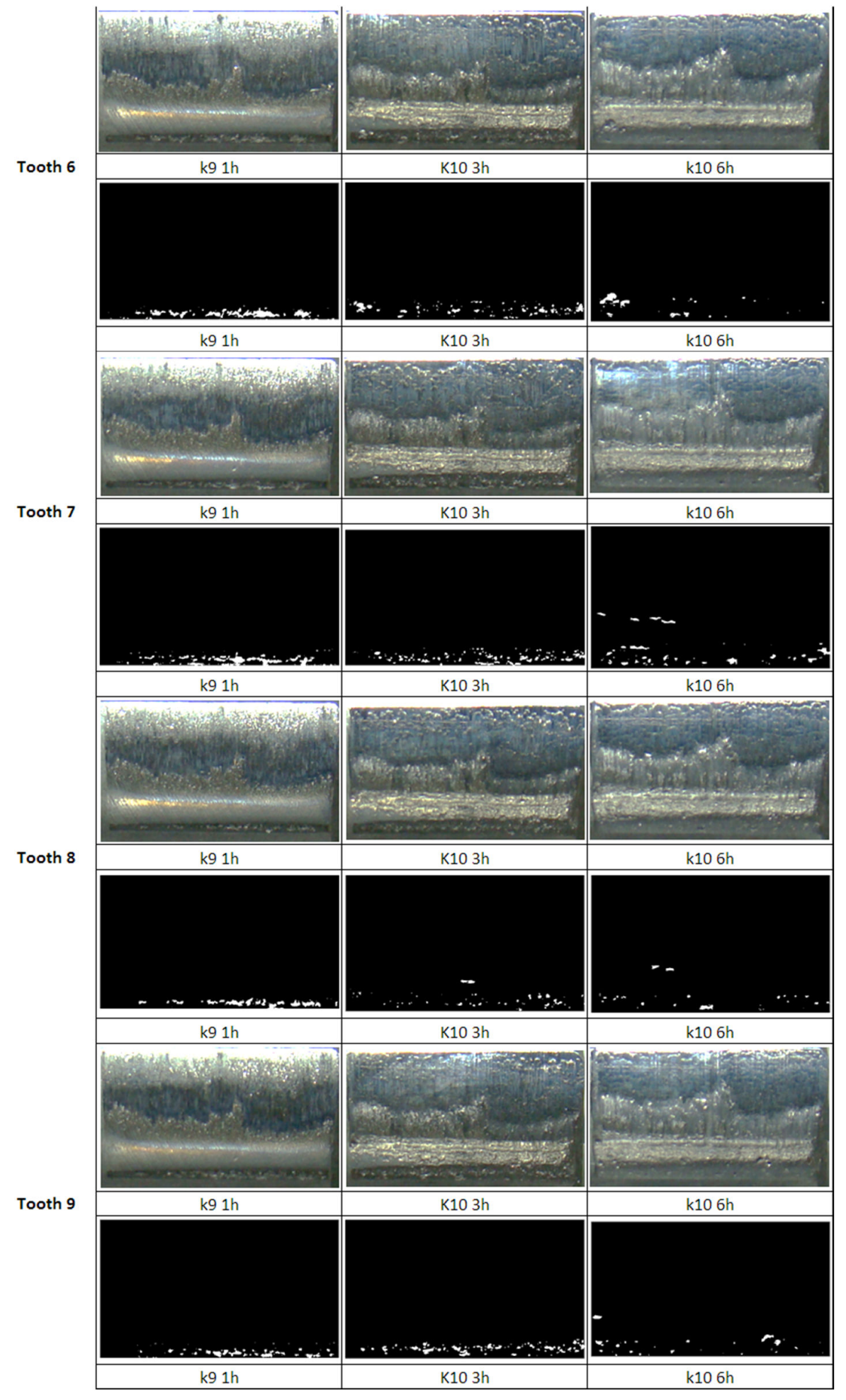


Figure A9 - F03 Images and quantification after each test step.

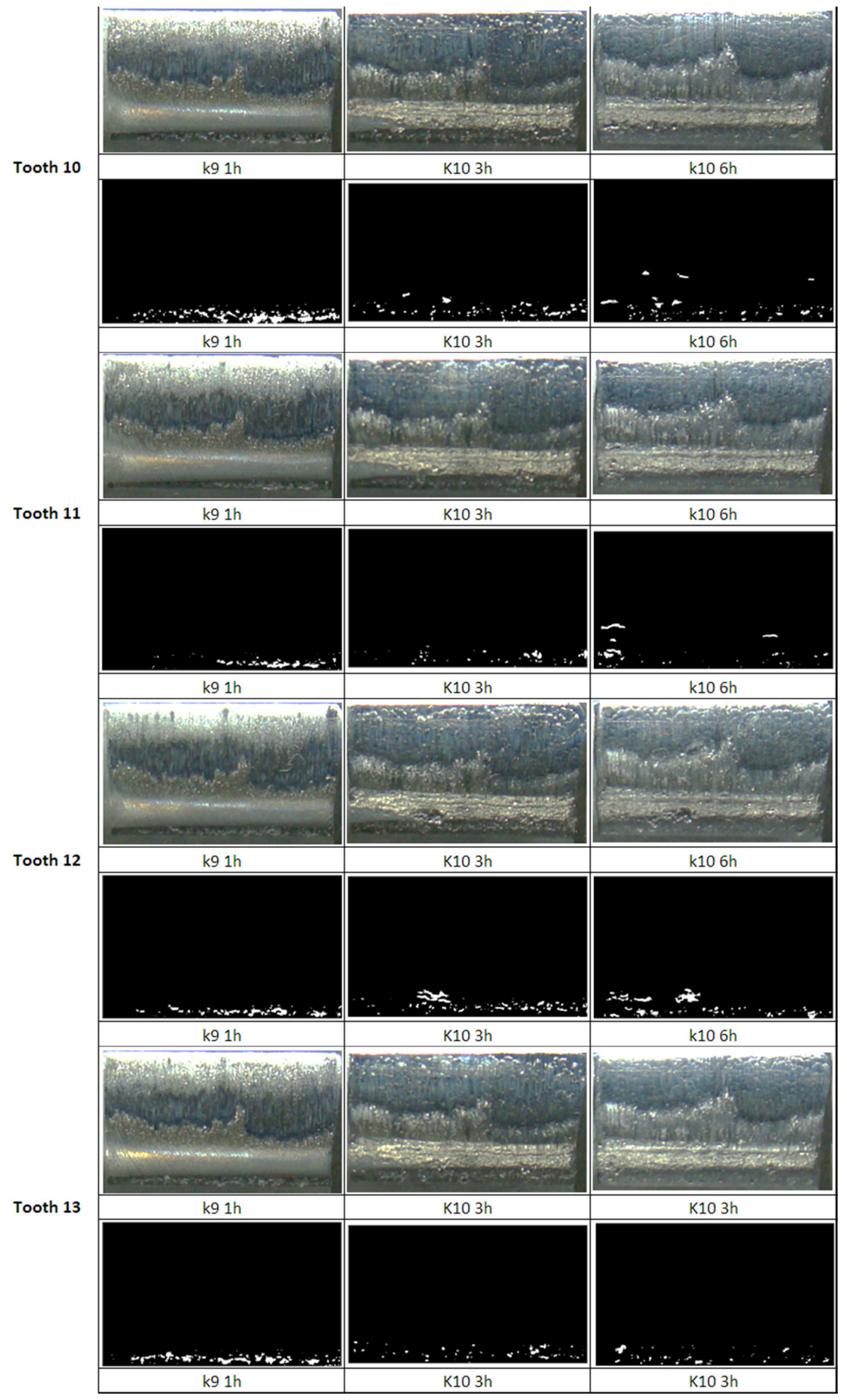


Figure A10 - F04 Images and quantification after each test step.

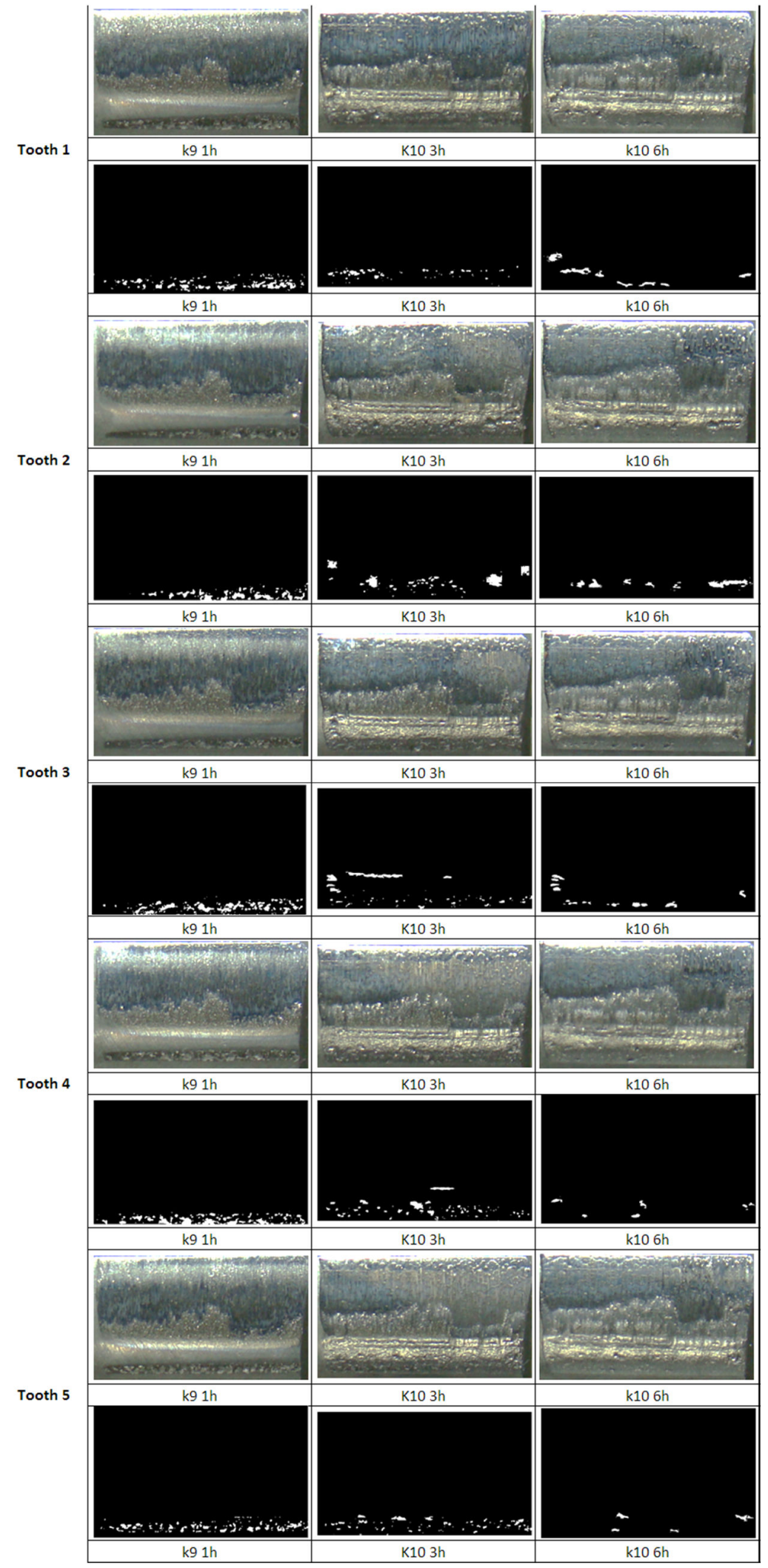


Figure A11 - F04 Images and quantification after each test step.

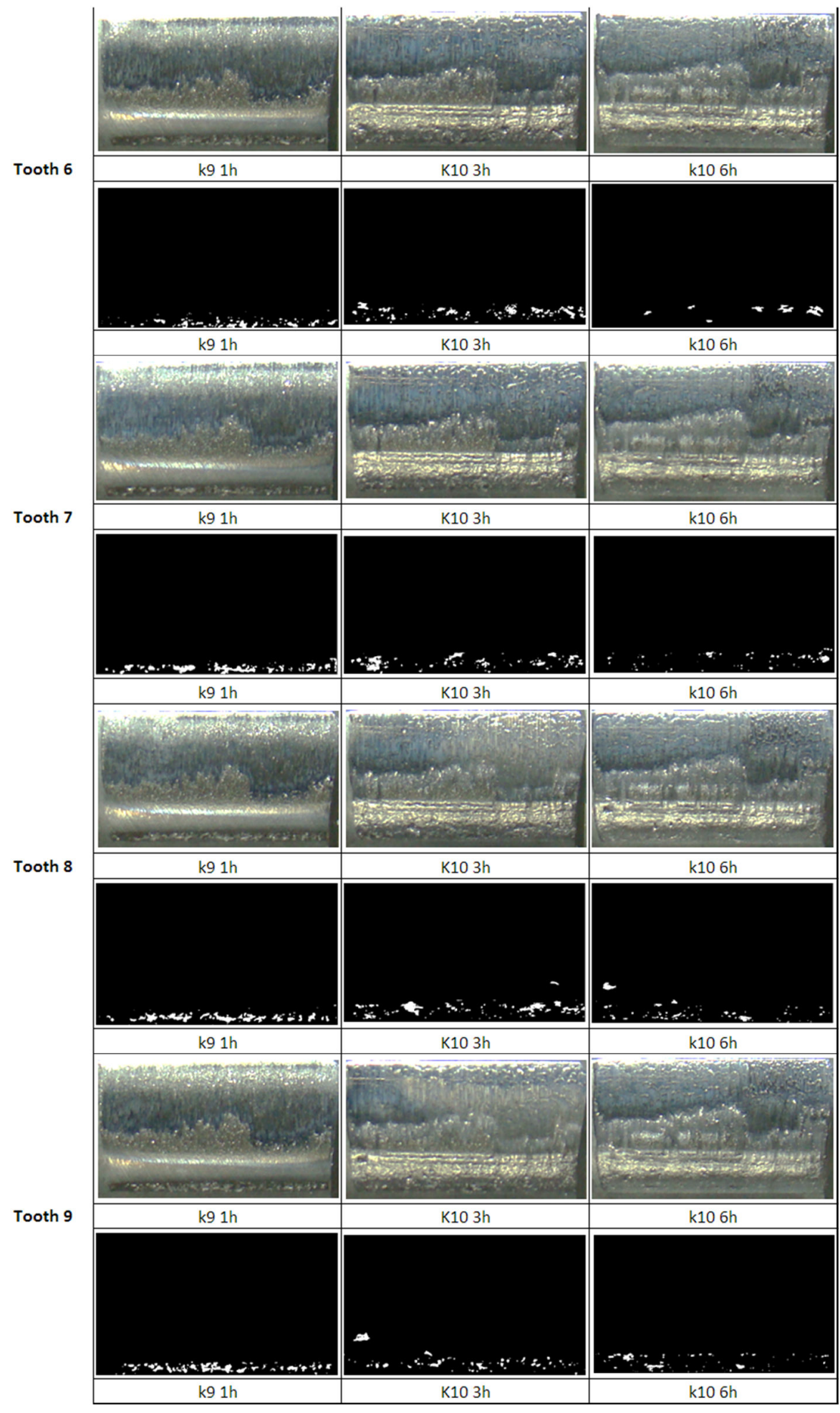


Figure A12 - F04 Images and quantification after each test step.

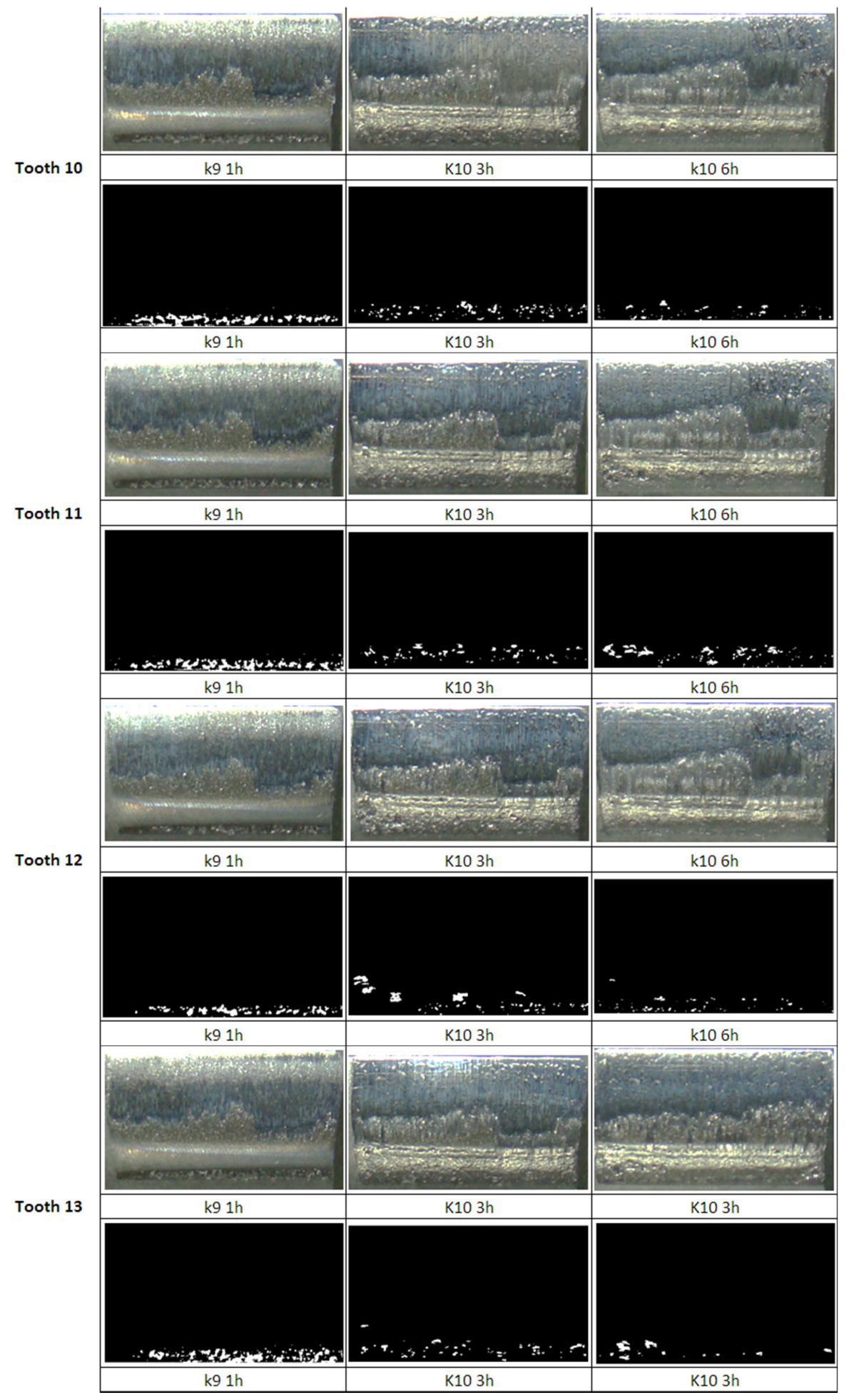


Figure A13 - F05 Images and quantification after each test step.

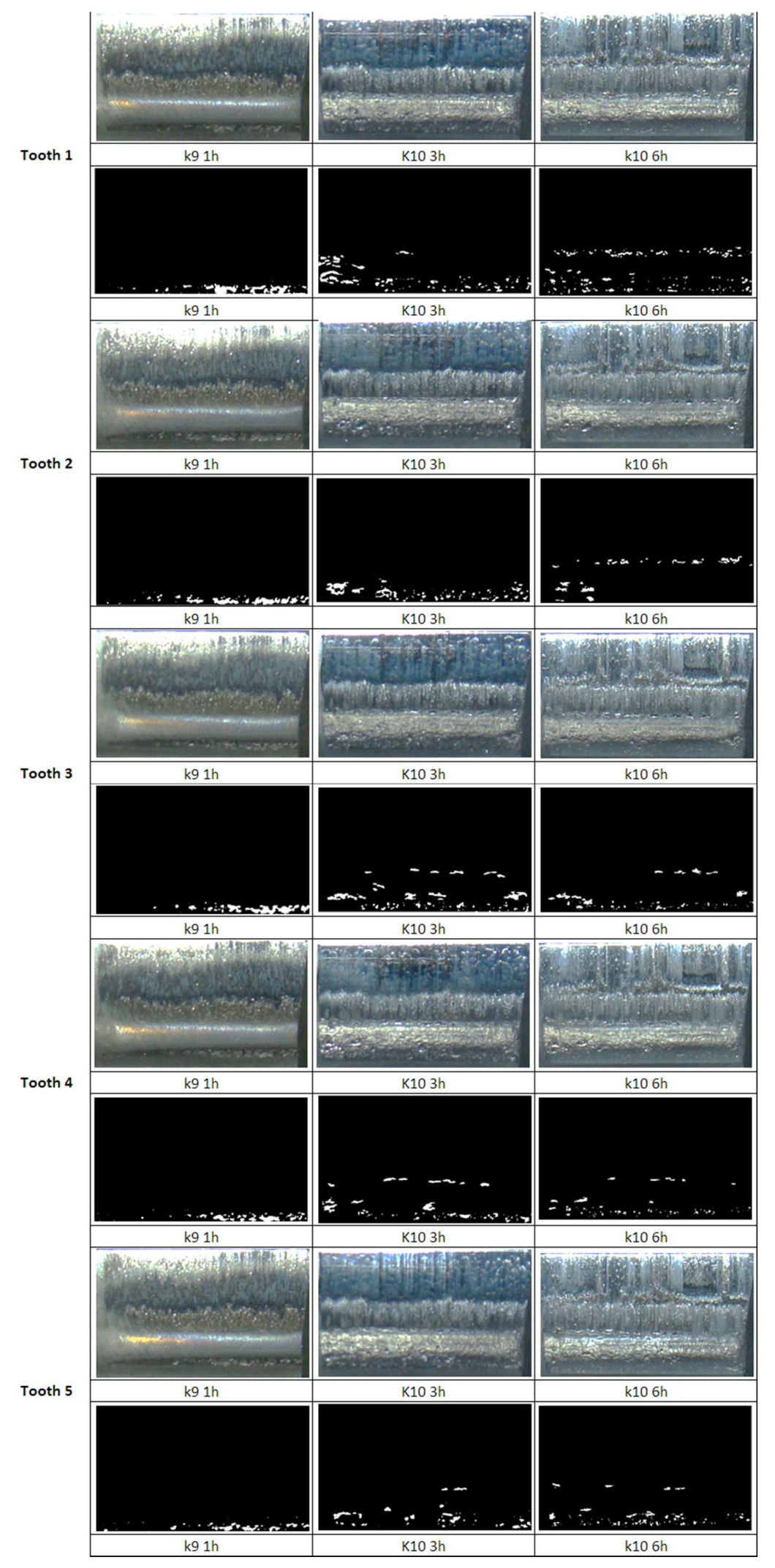


Figure A14 - F05 Images and quantification after each test step.

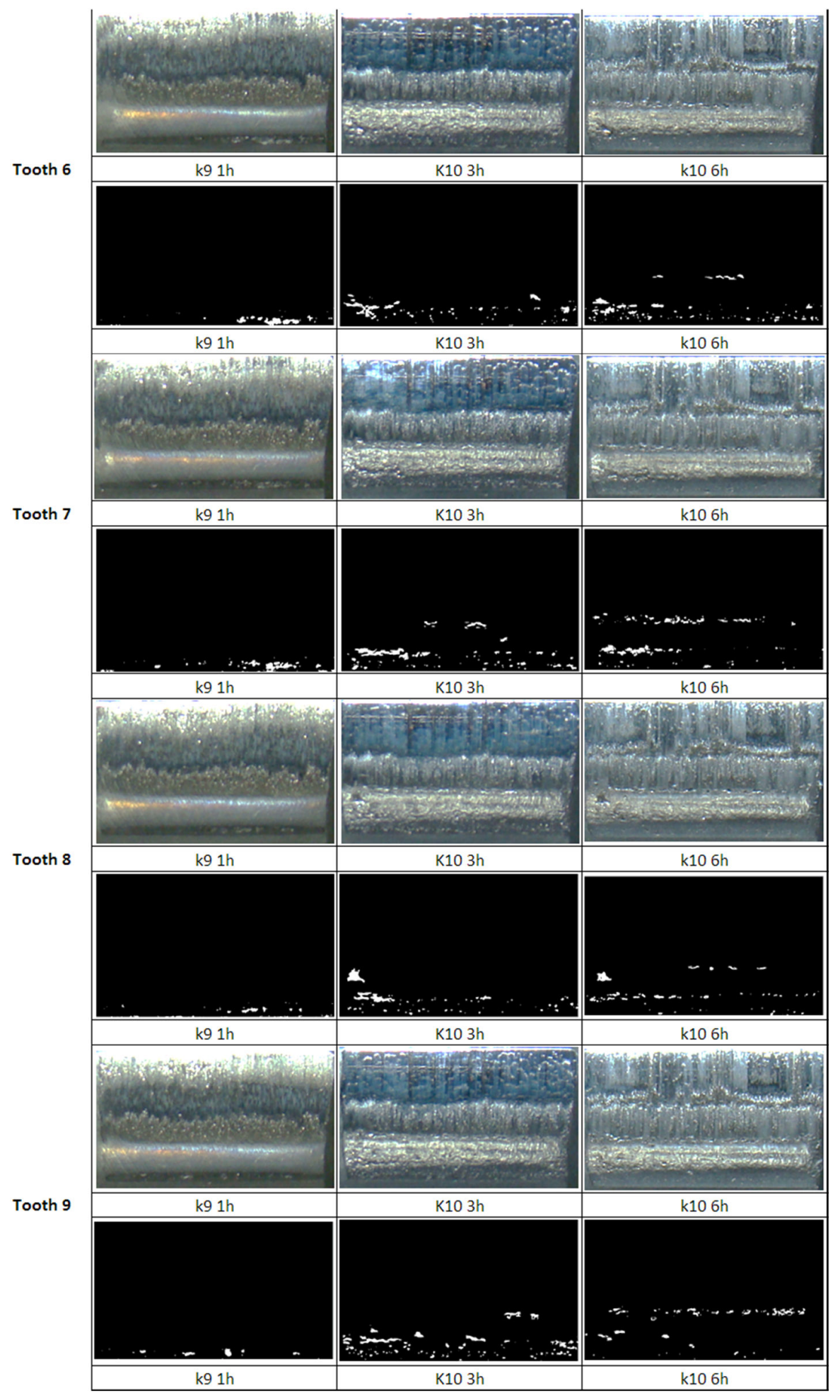


Figure A15 - F05 Images and quantification after each test step.

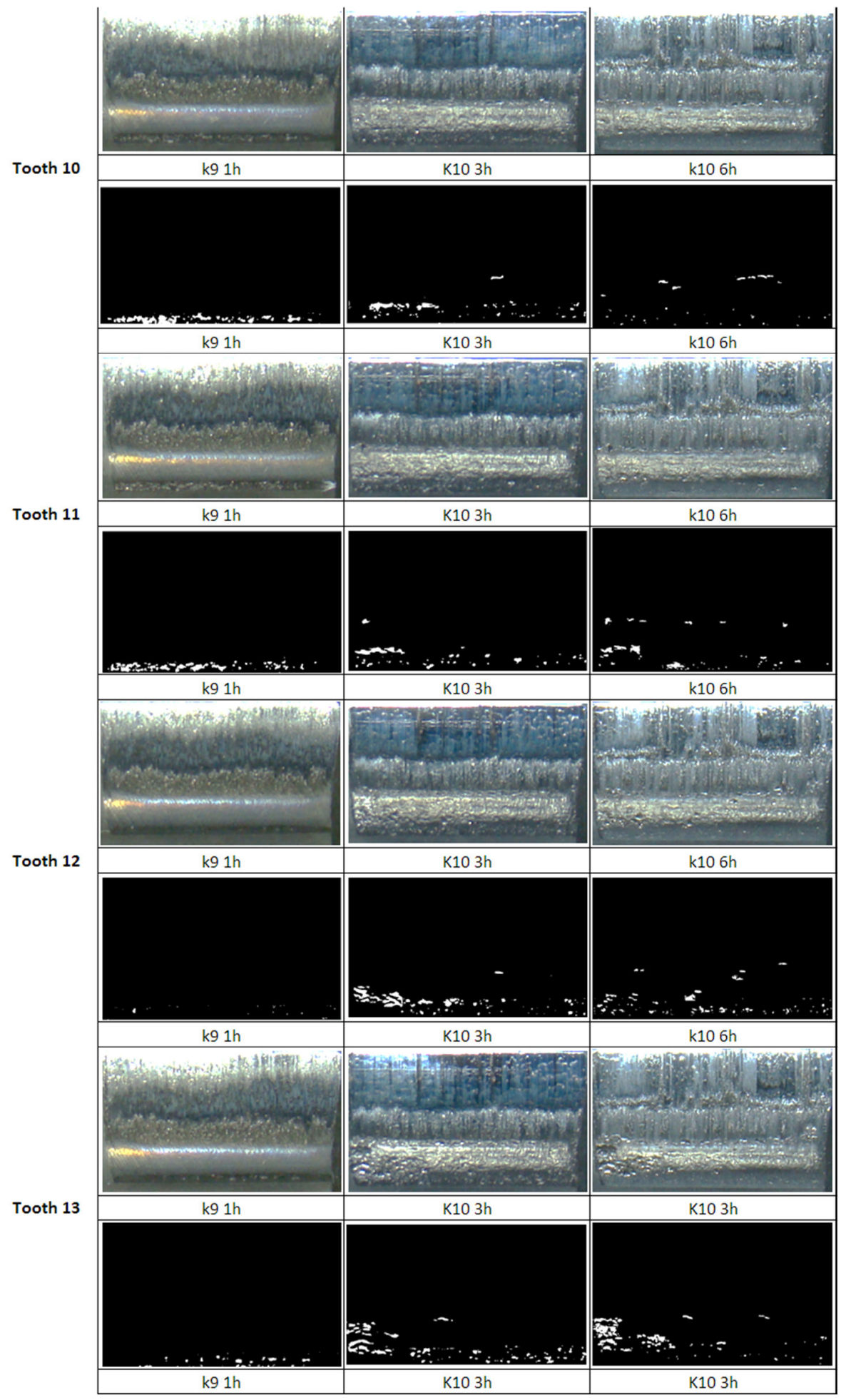


APPENDIX B - FLANK SURFACE IMAGES AND MATLAB ROUTINE OF B01 TILL B05

Figure B1 - B01 Images and quantification after each test step.

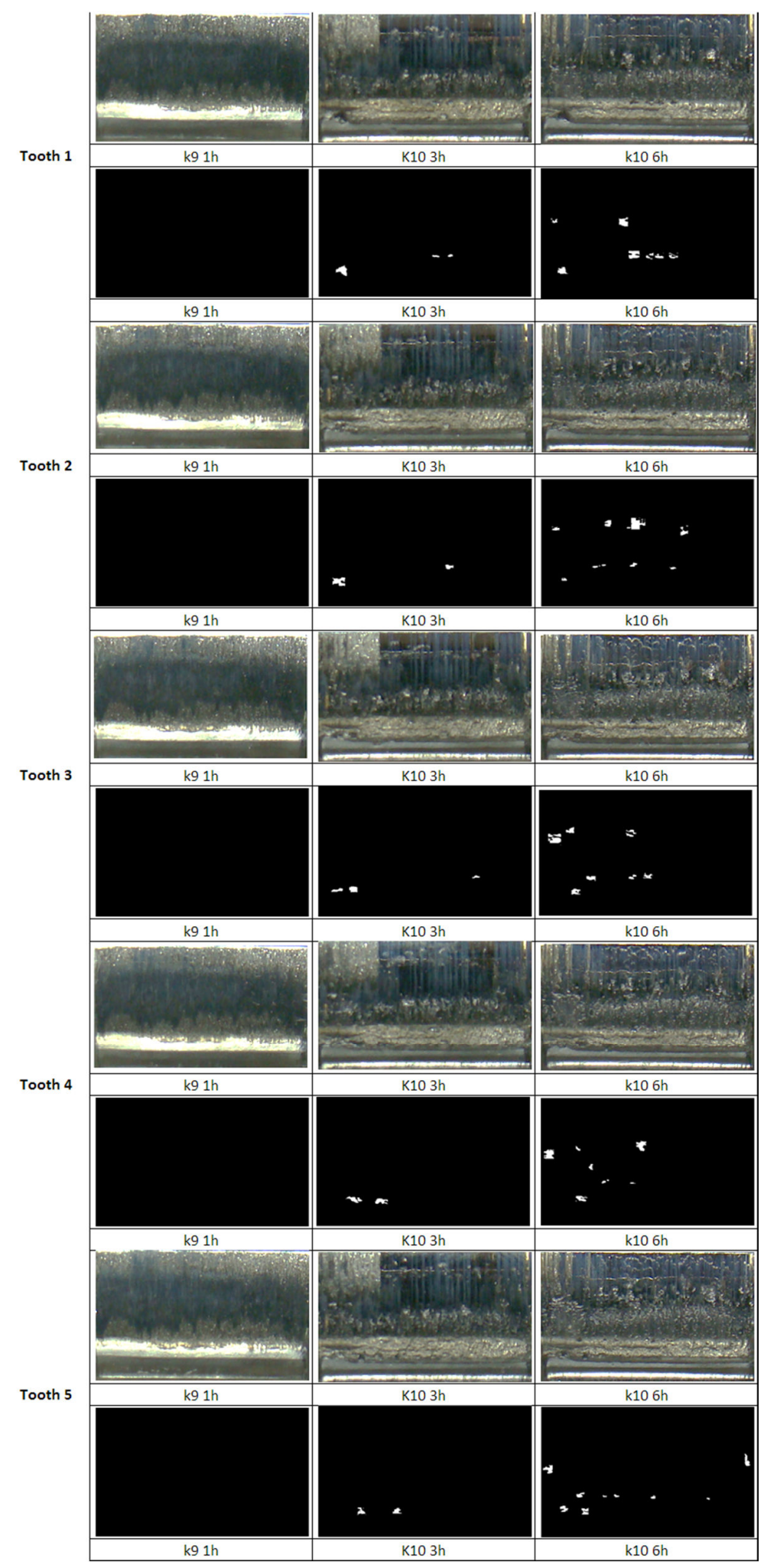


Figure B2 - B01 Images and quantification after each test step.

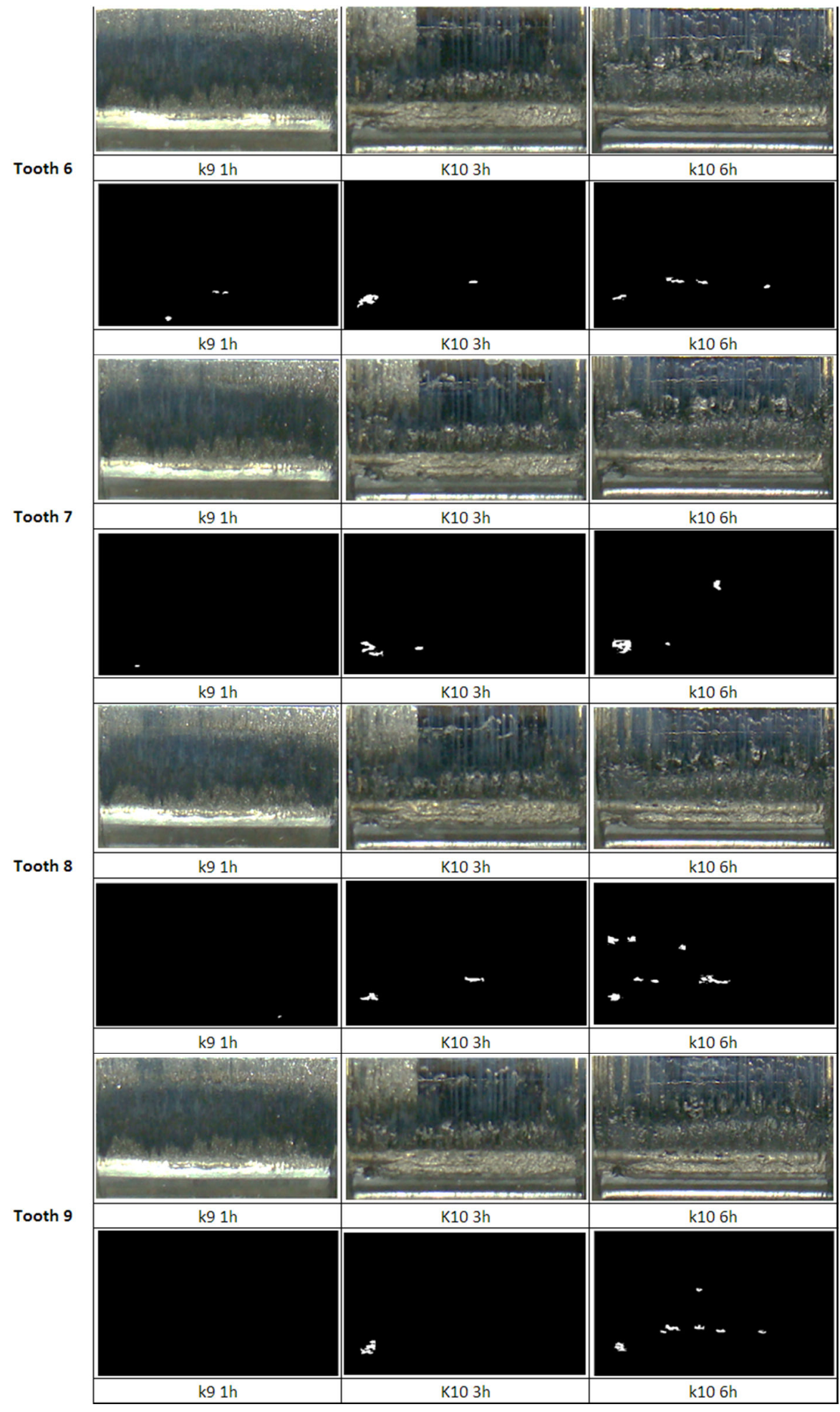


Figure B3 - B01 Images and quantification after each test step.

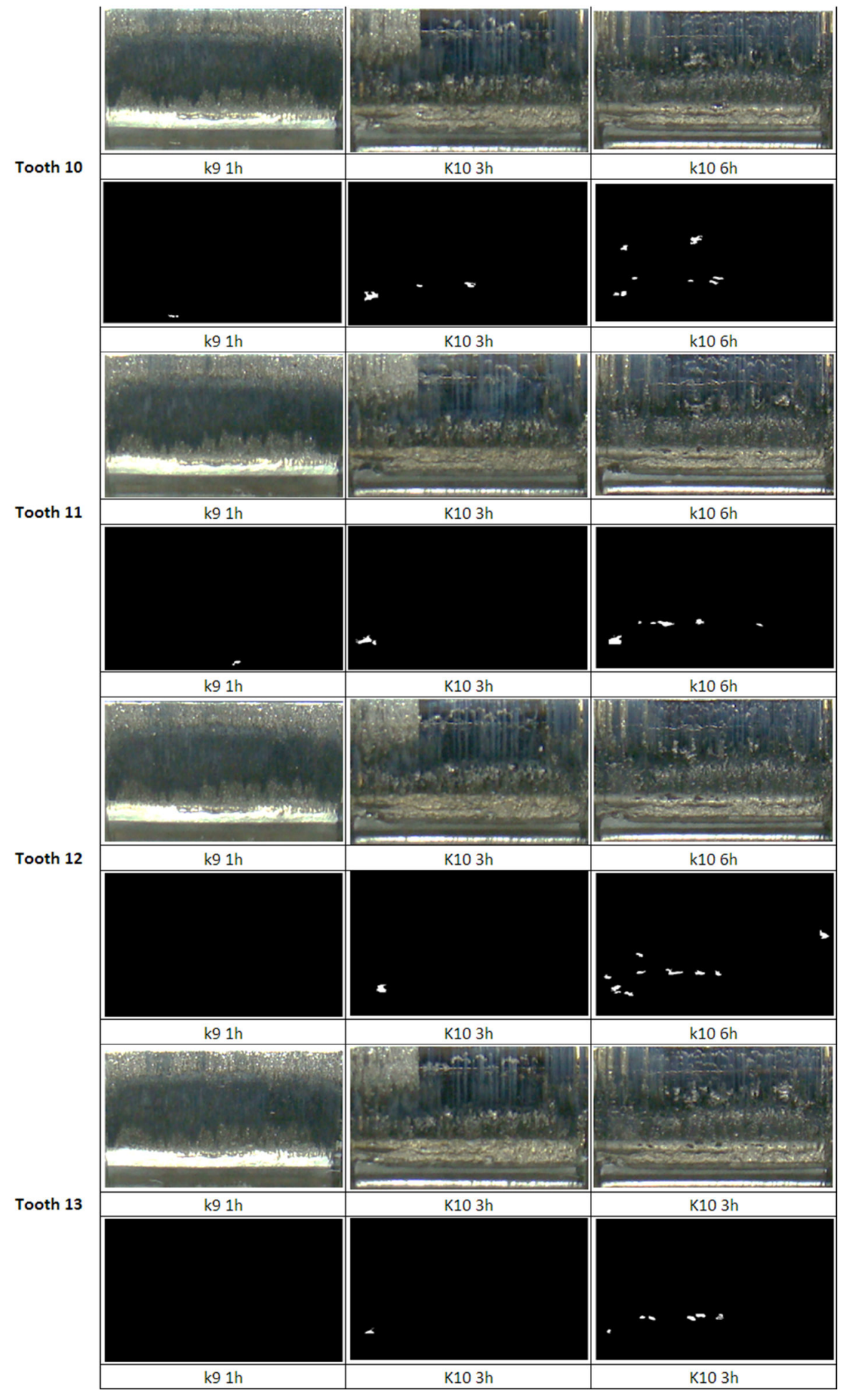


Figure B4 - B02 Images and quantification after each test step.

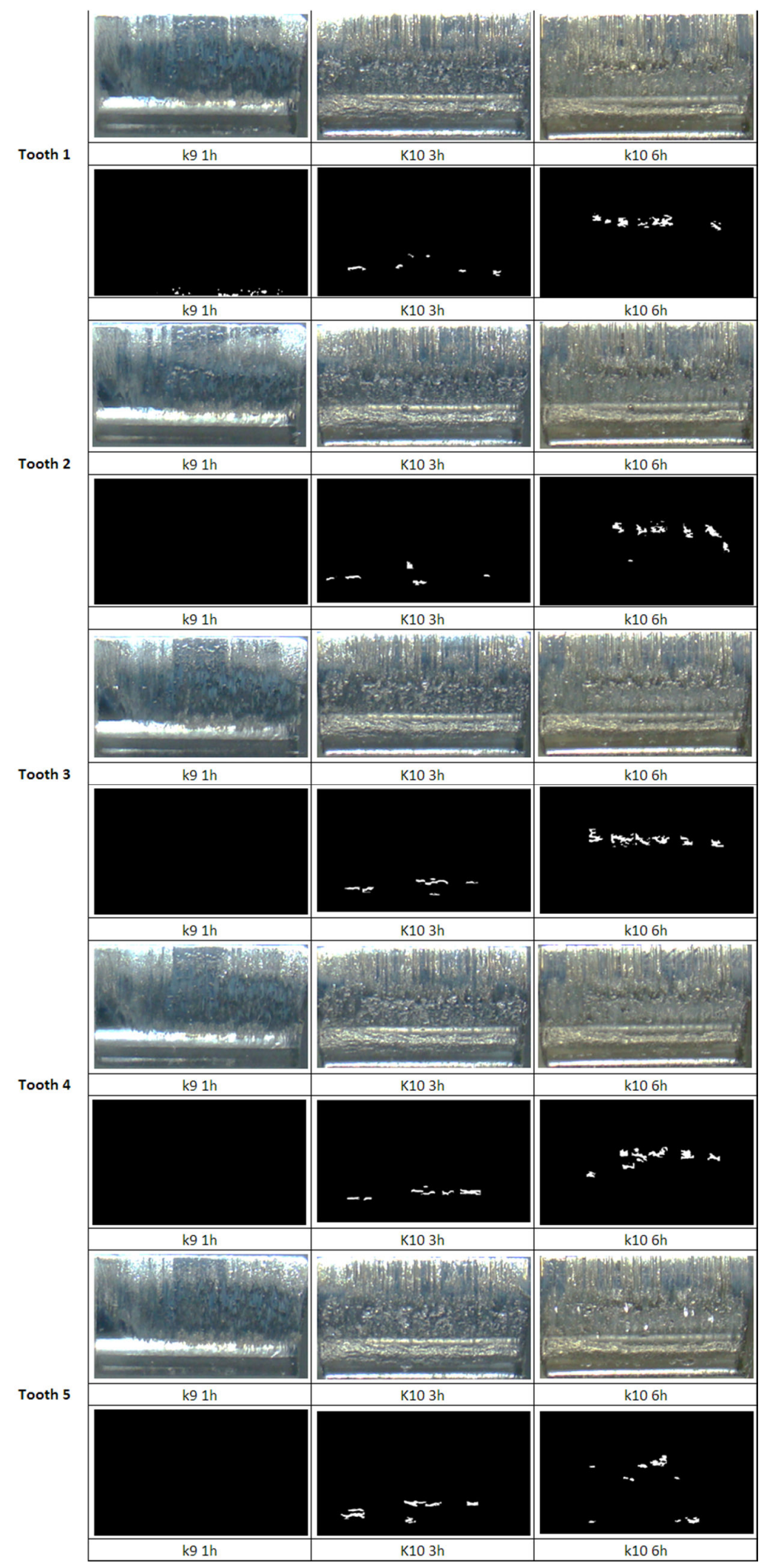


Figure B5 - B02 Images and quantification after each test step.

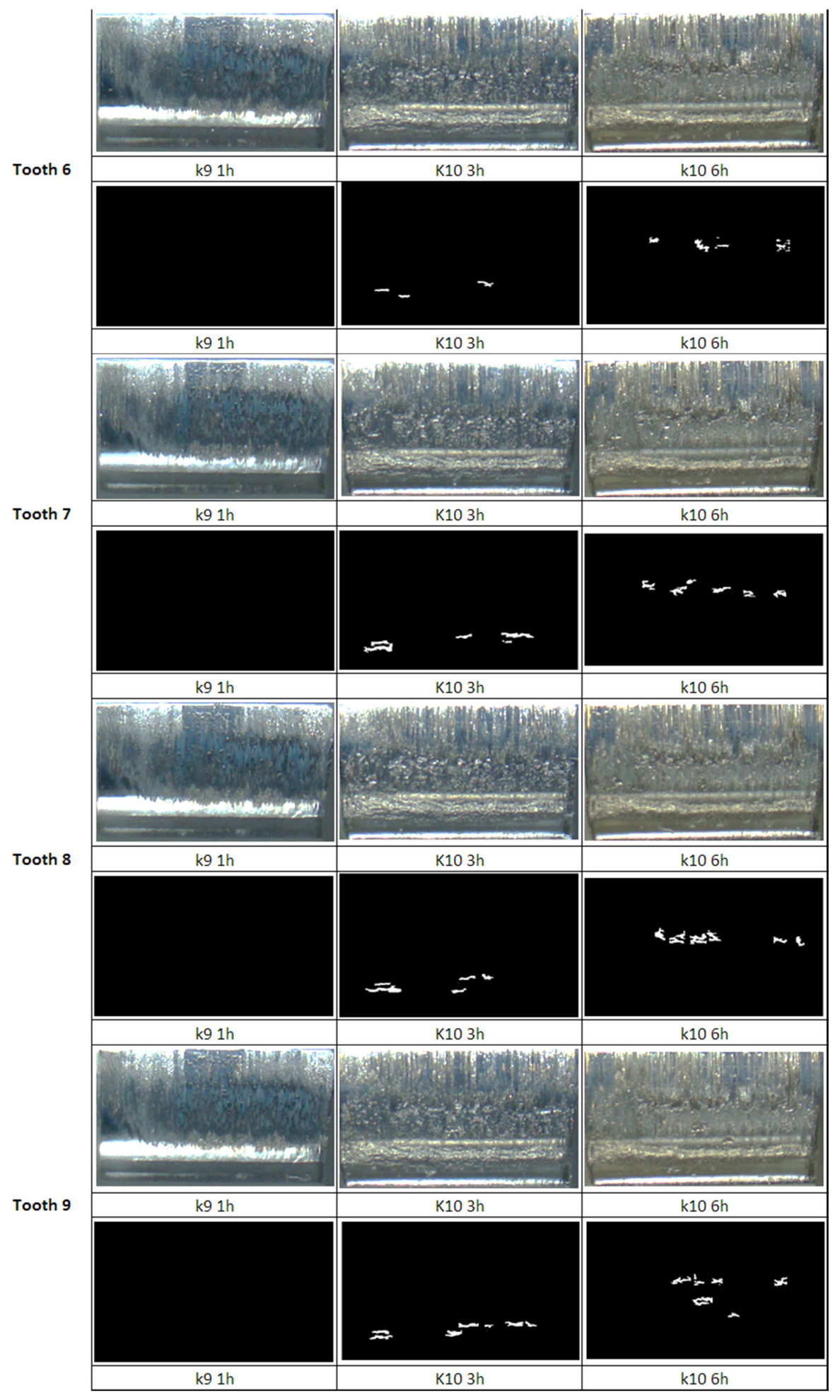


Figure B6 - B02 Images and quantification after each test step.

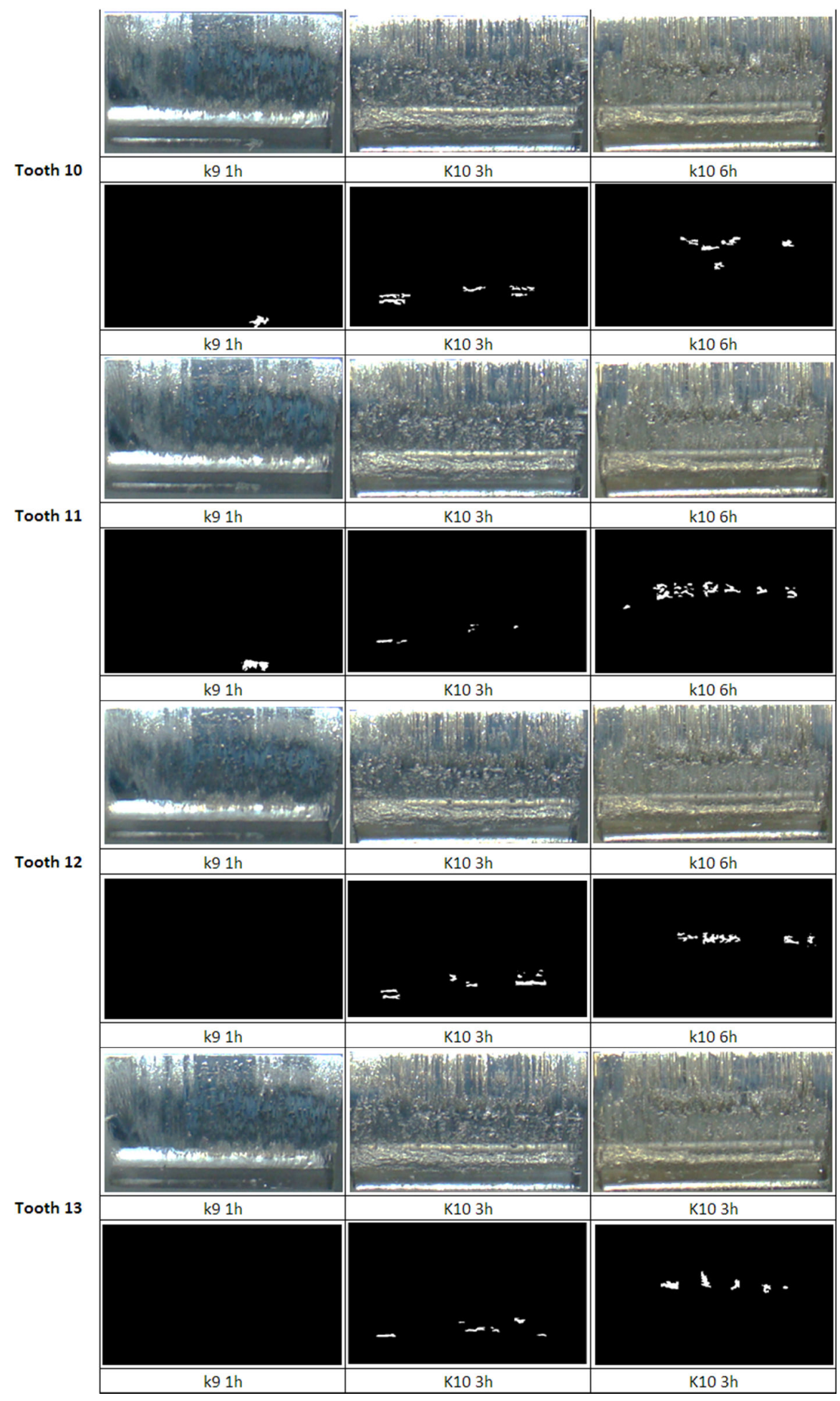


Figure B7 - B03 Images and quantification after each test step.

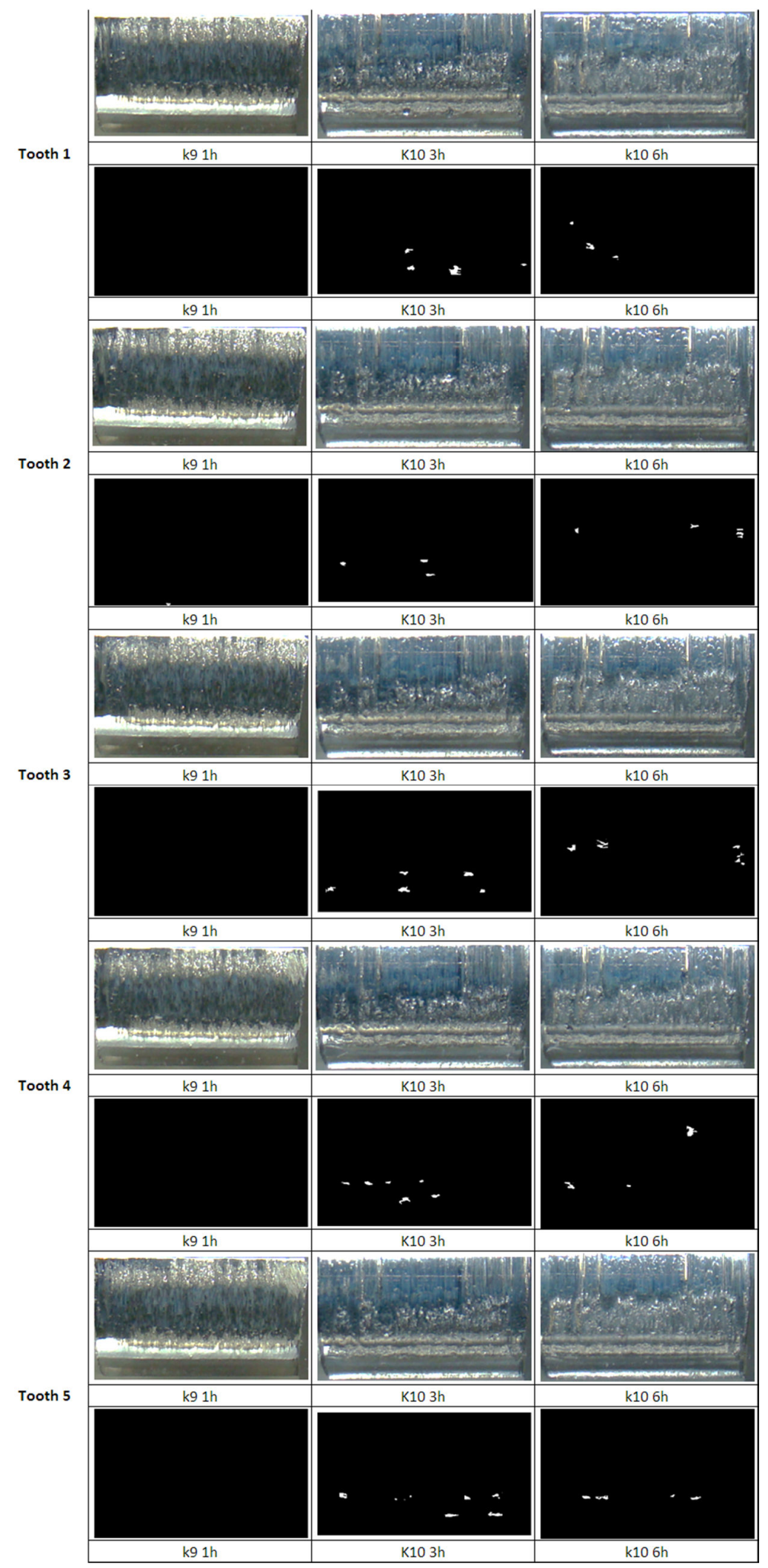


Figure B8 - B03 Images and quantification after each test step.

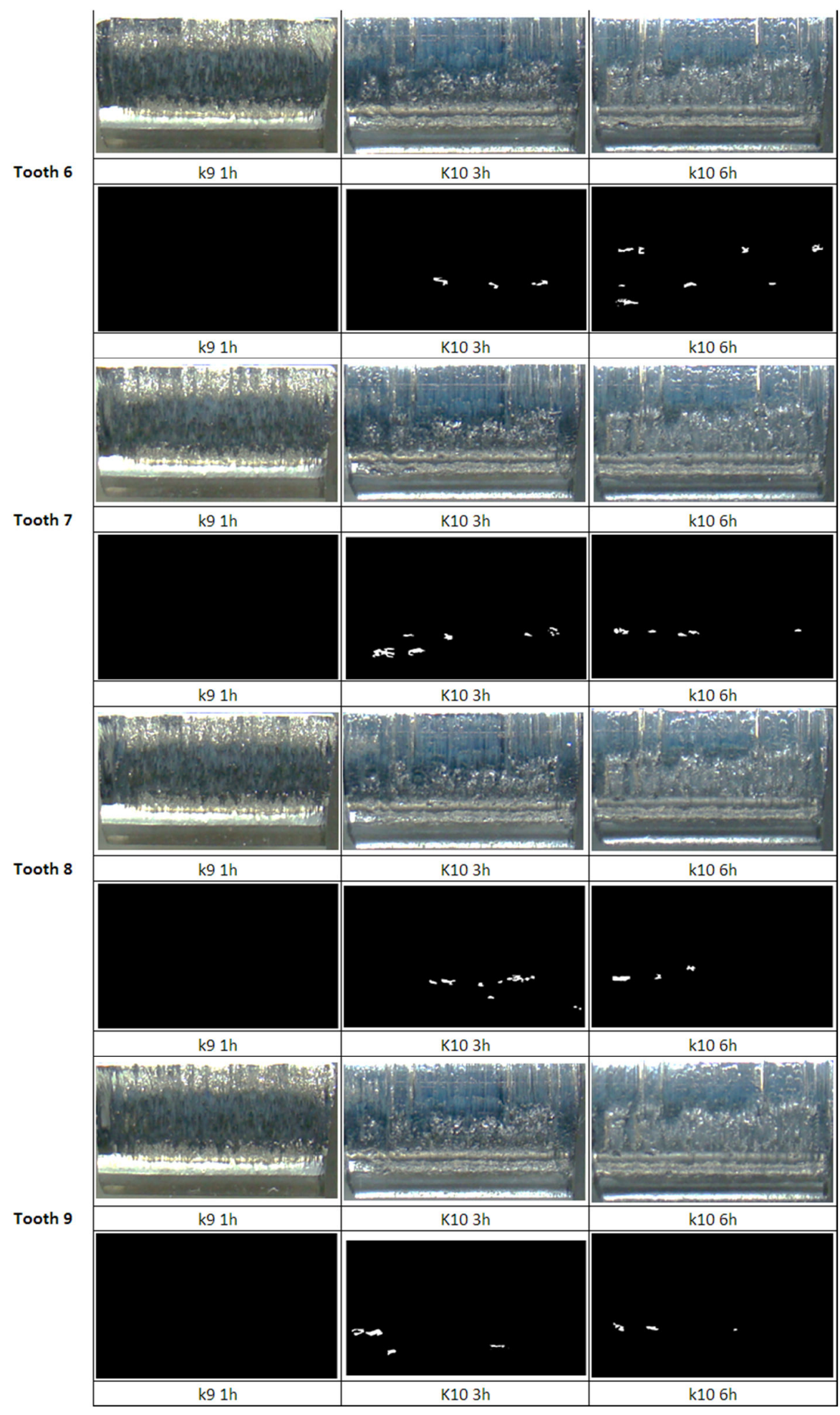


Figure B9 - B03 Images and quantification after each test step.

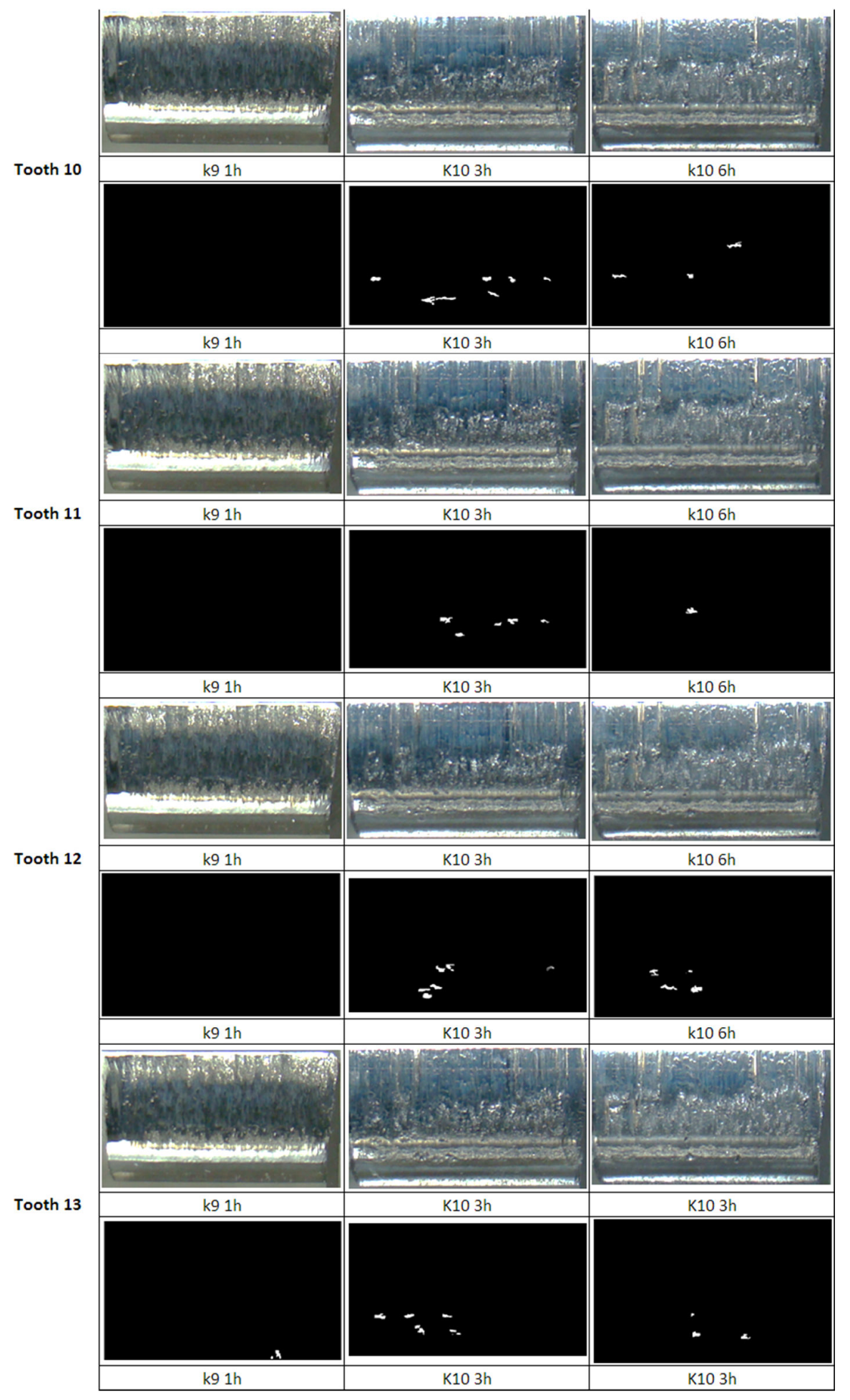


Figure B10 - B04 Images and quantification after each test step.

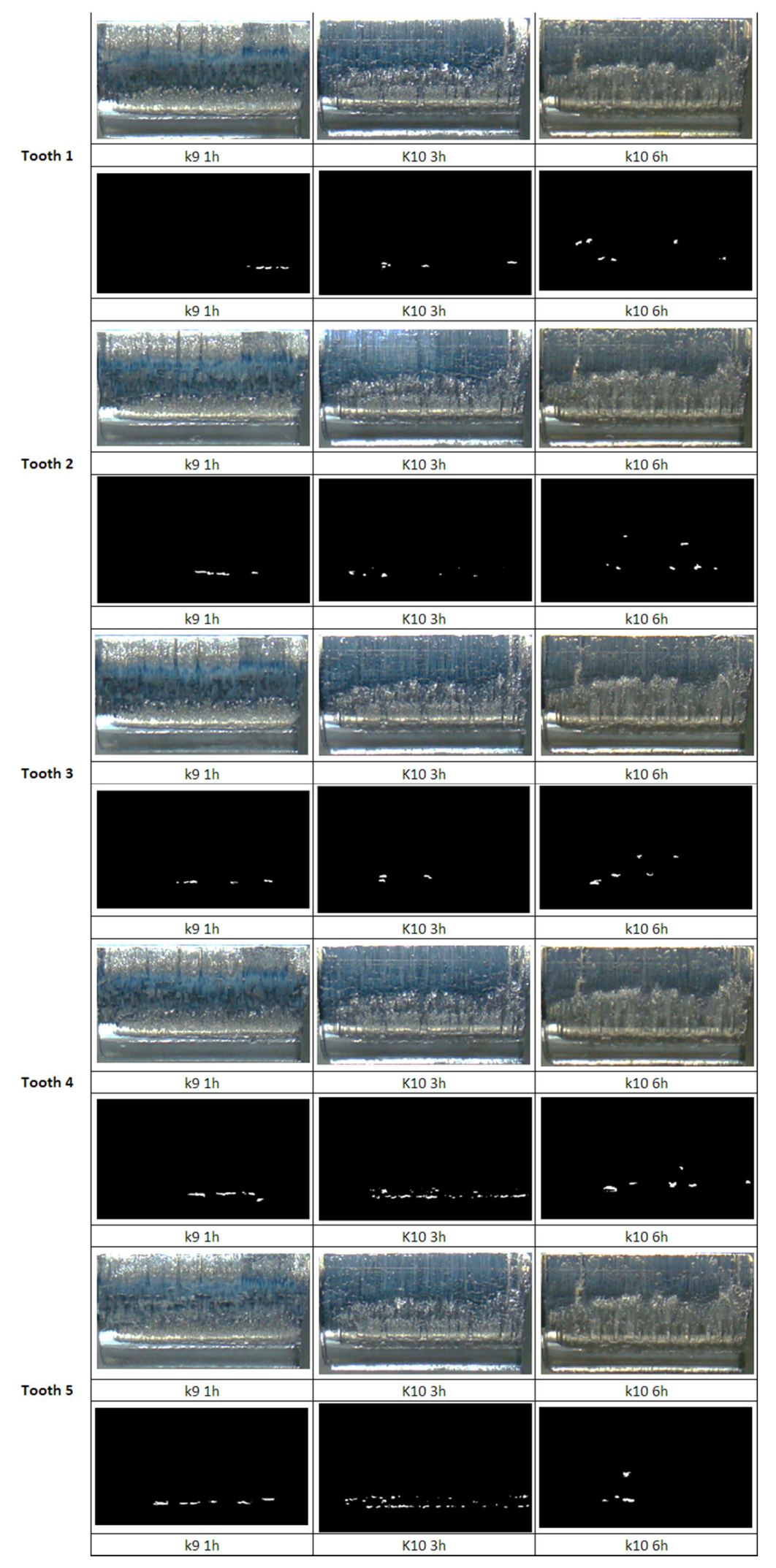


Figure B11 - B04 Images and quantification after each test step.

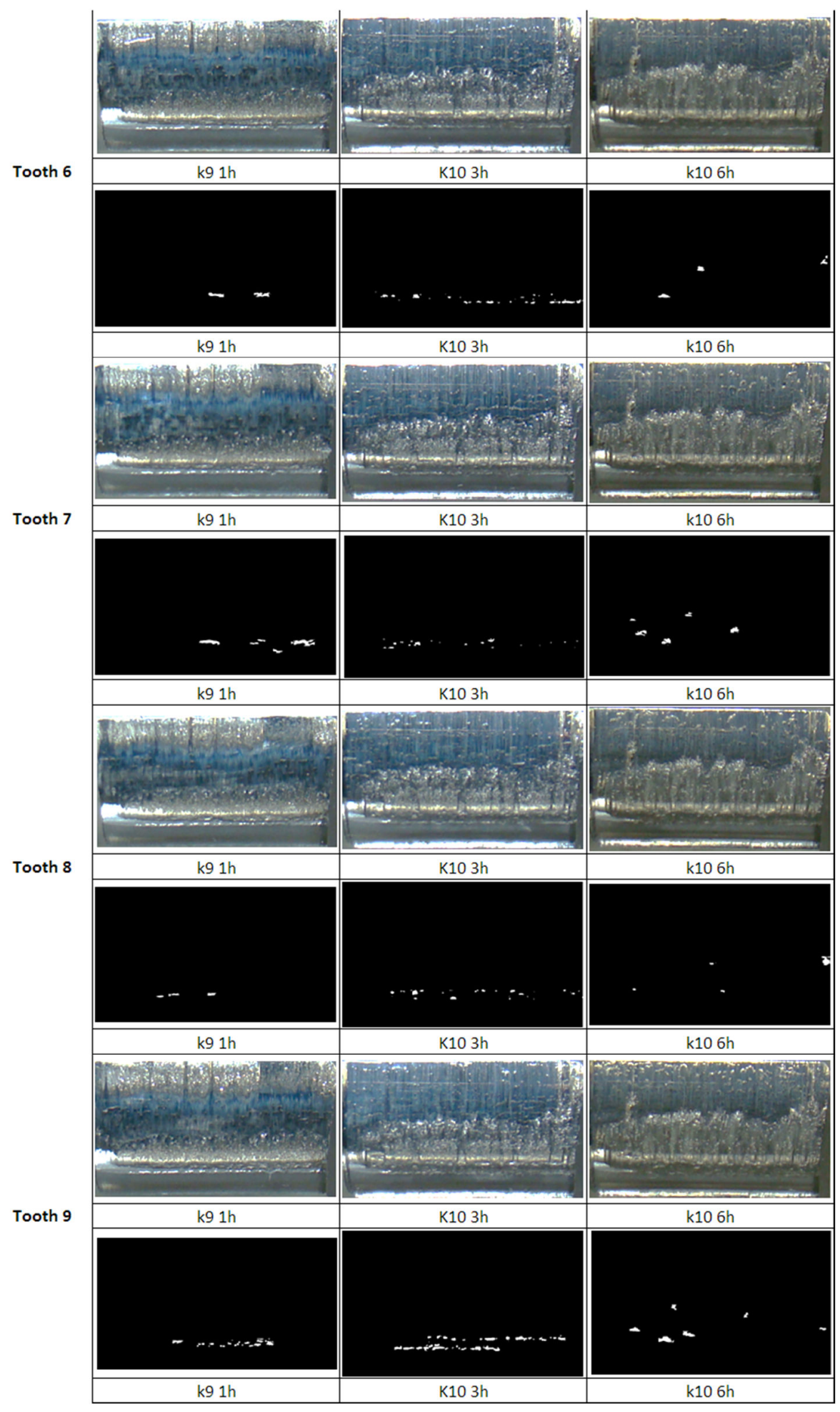


Figure B12 - B04 Images and quantification after each test step.

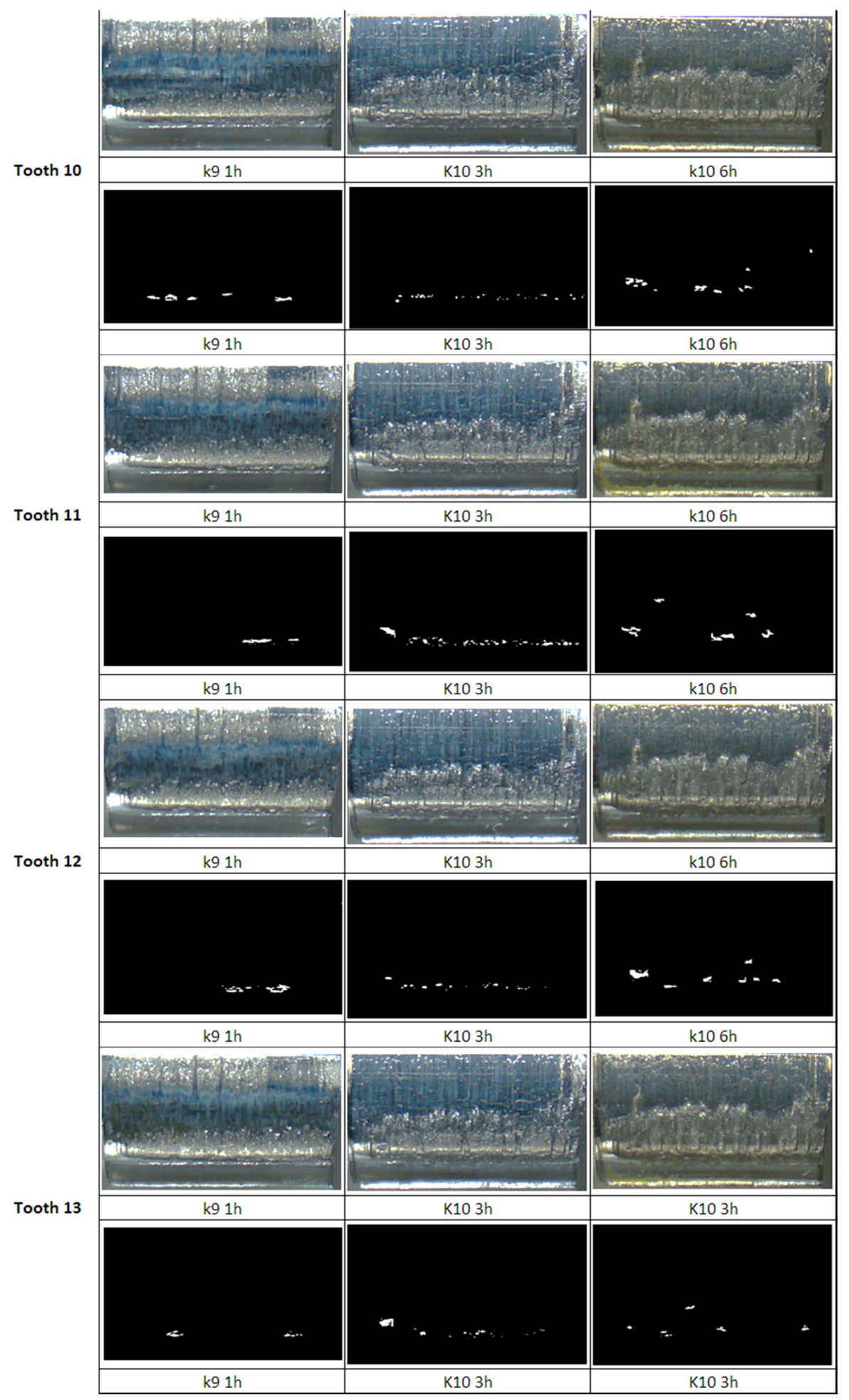


Figure B13 - B05 Images and quantification after each test step.

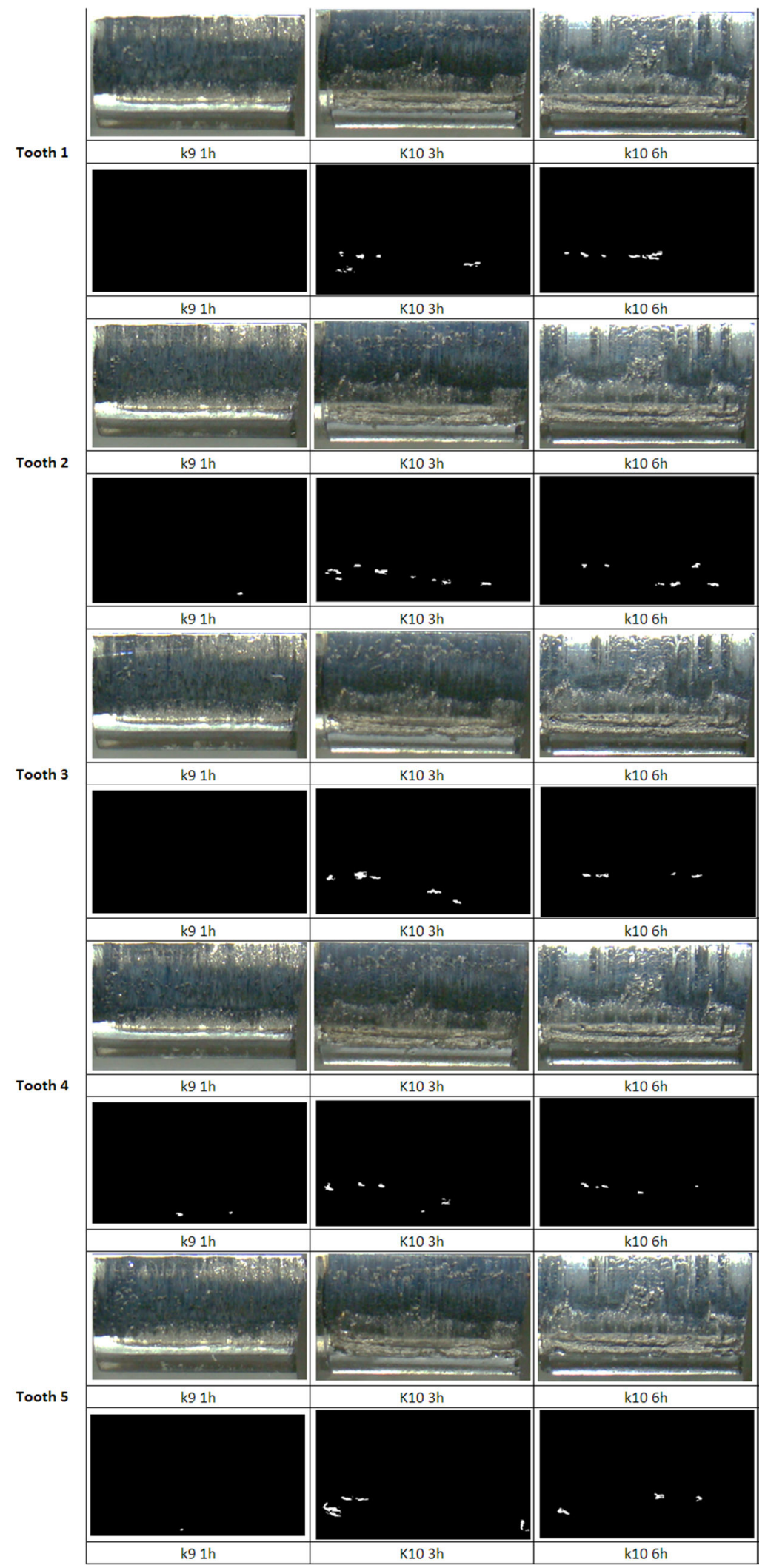


Figure B14 - B05 Images and quantification after each test step.

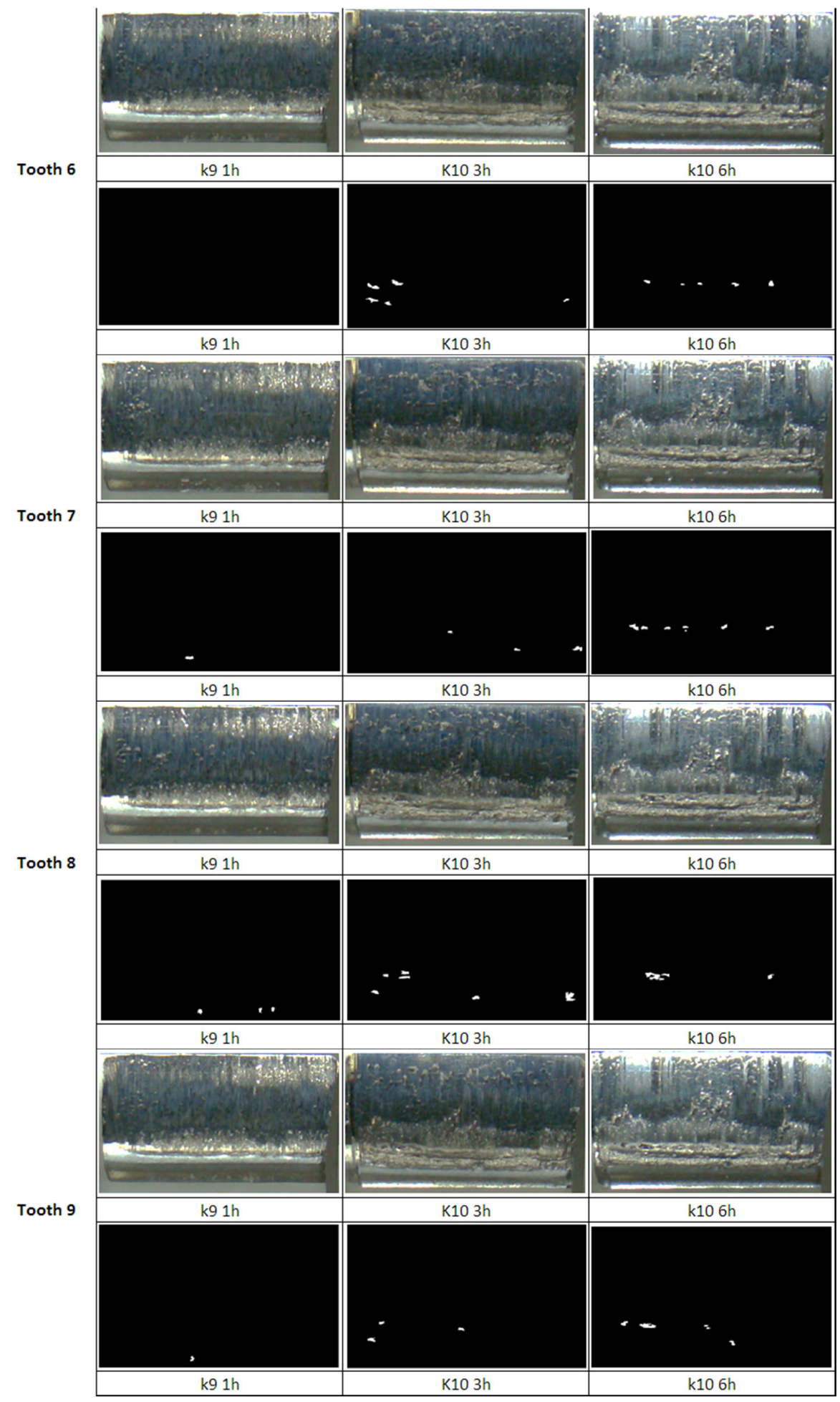


Figure B15 - B05 Images and quantification after each test step.

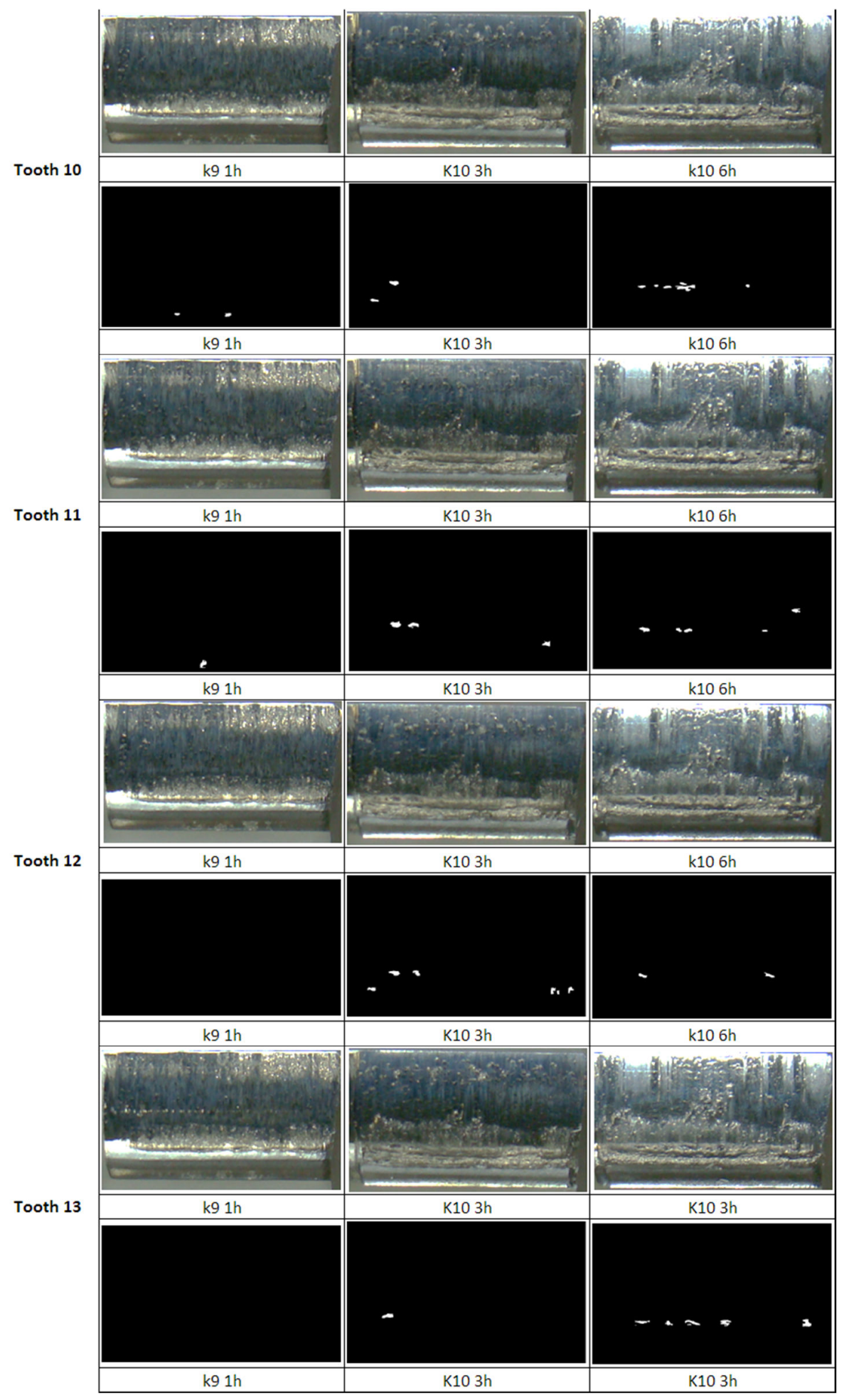


APPENDIX C - FLANK SURFACE IMAGES AND MATLAB ROUTINE OF D01 TILL D05

Figure C1 - D01 Images and quantification after each test step.

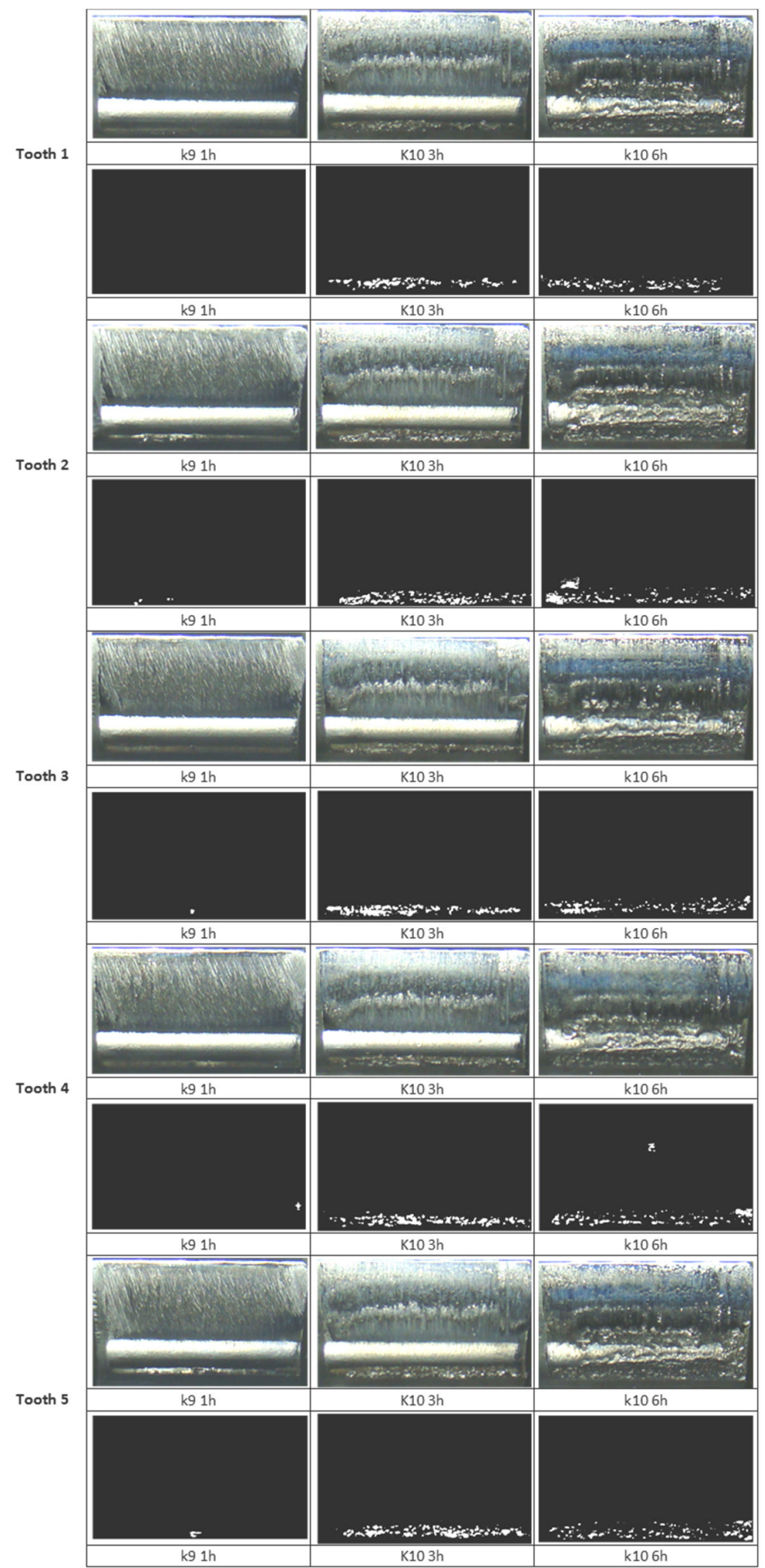


Figure C2 - D01 Images and quantification after each test step.

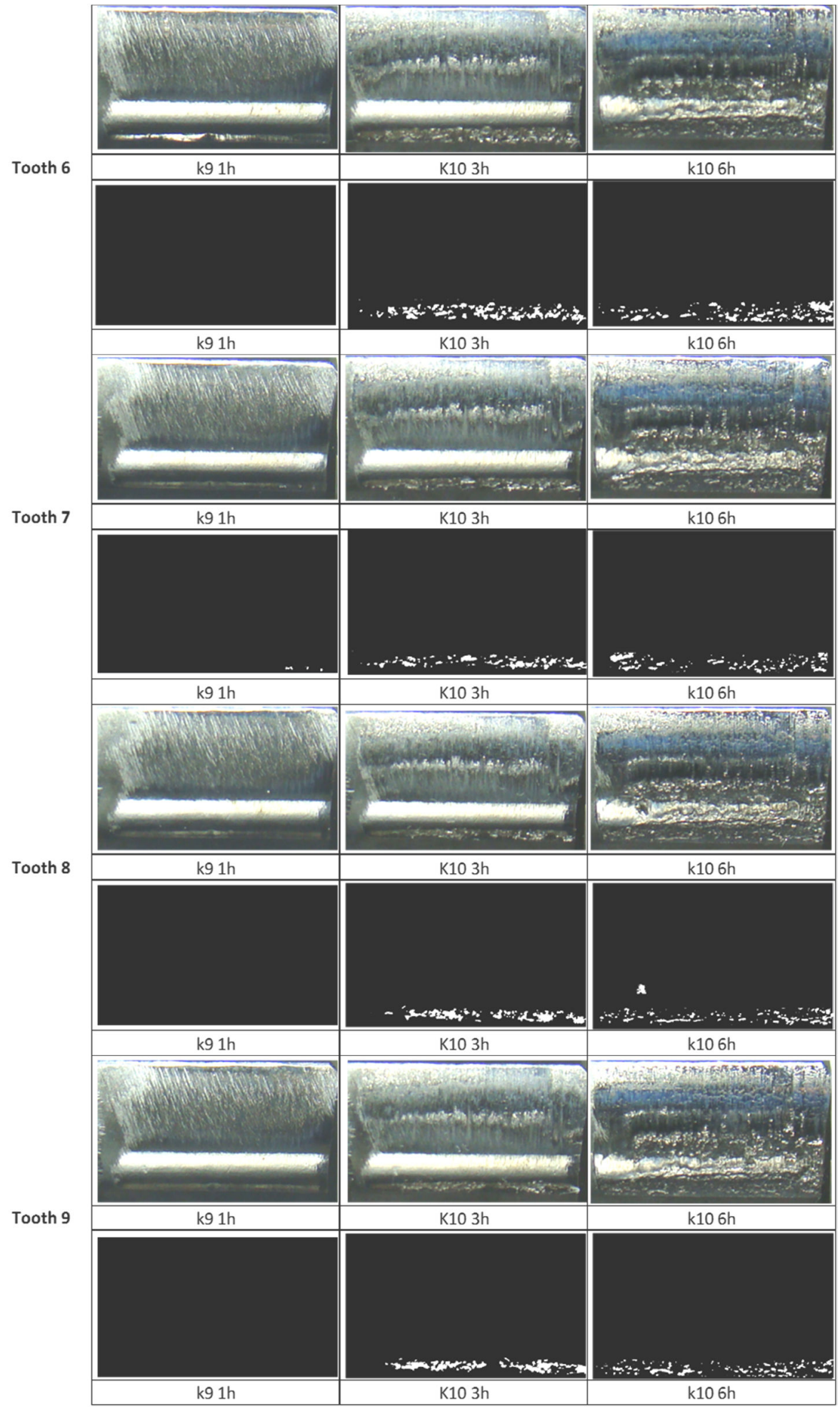


Figure C3 - D01 Images and quantification after each test step.

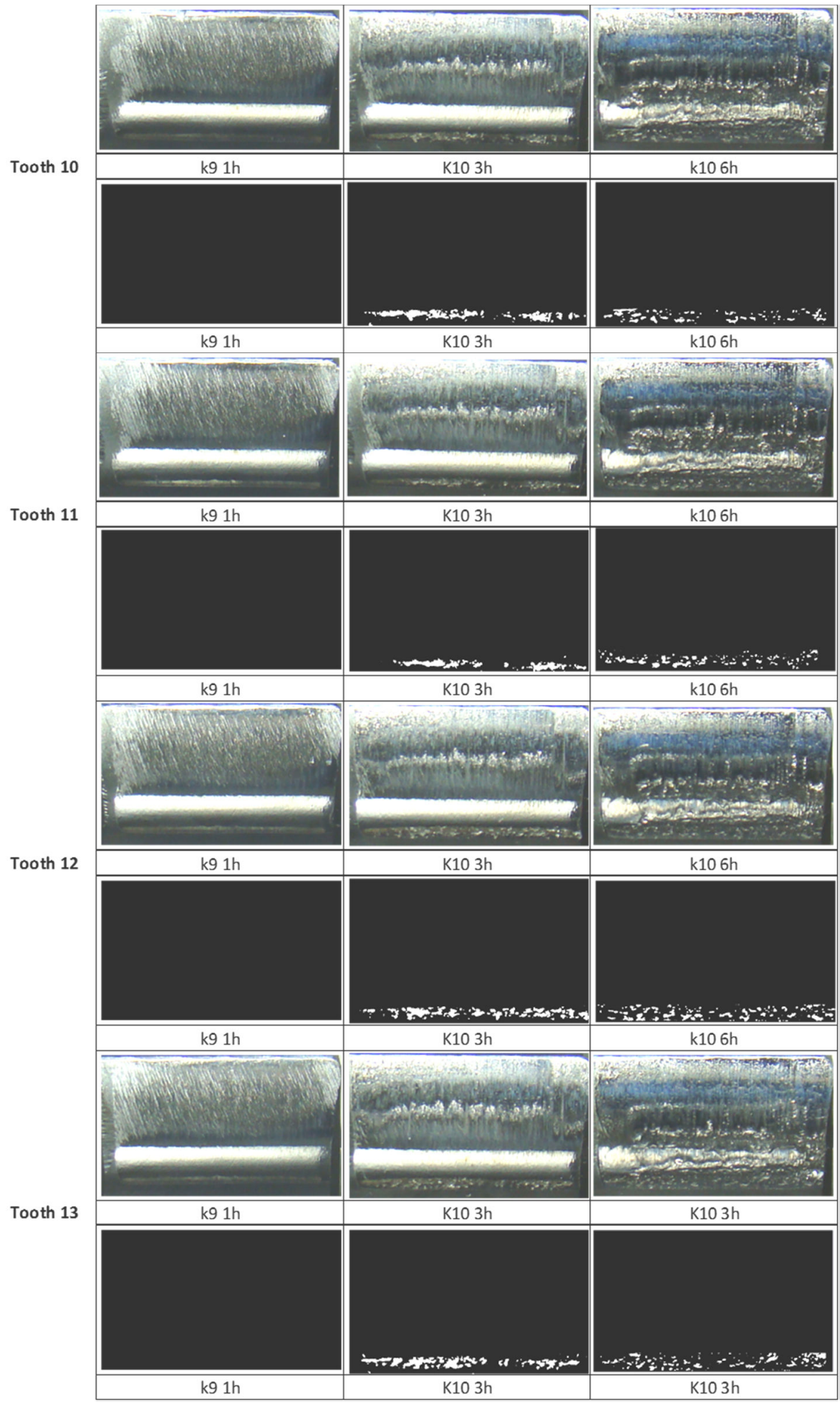


Figure C4 - D02 Images and quantification after each test step.

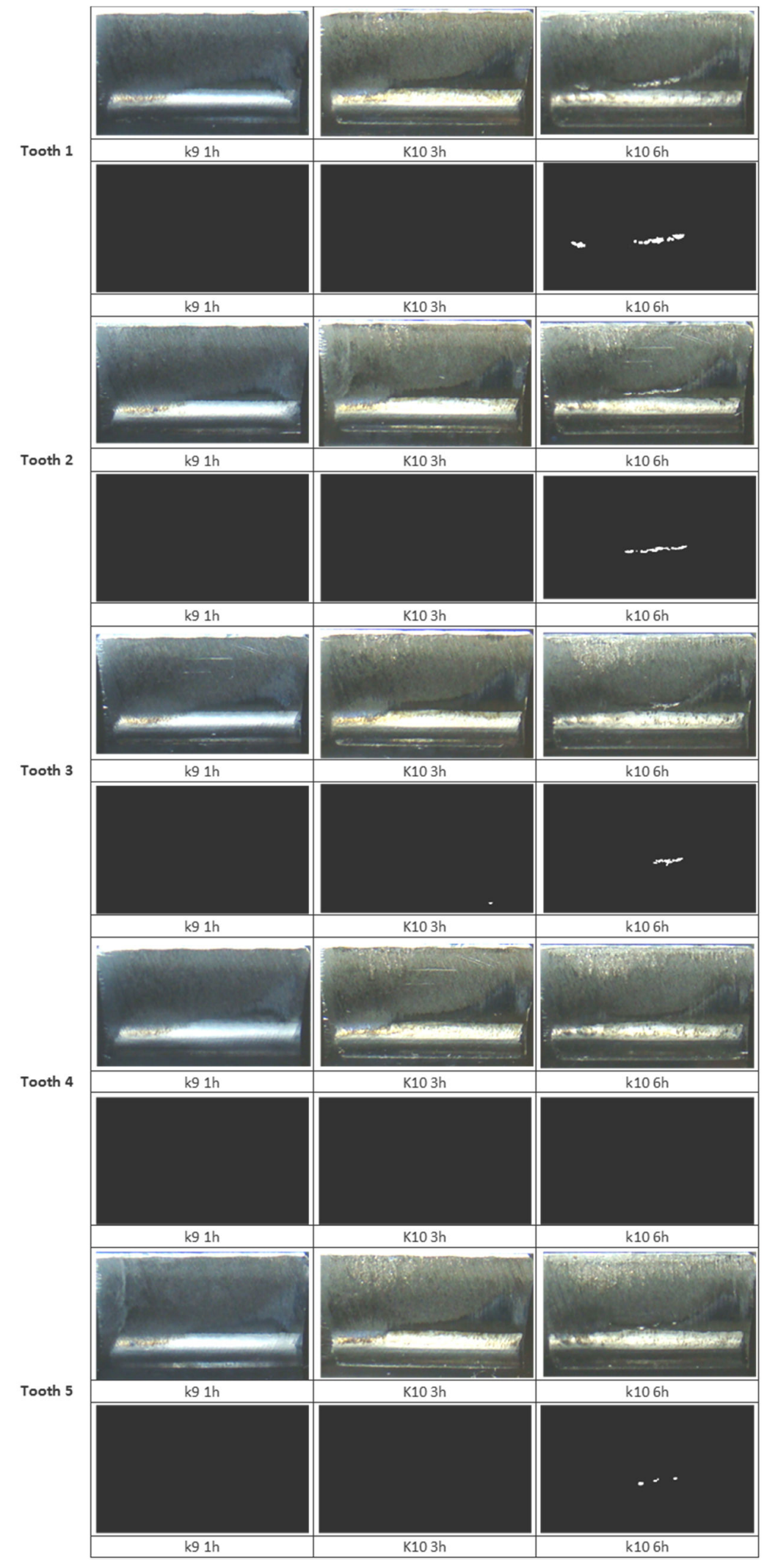


Figure C5 - D02 Images and quantification after each test step.

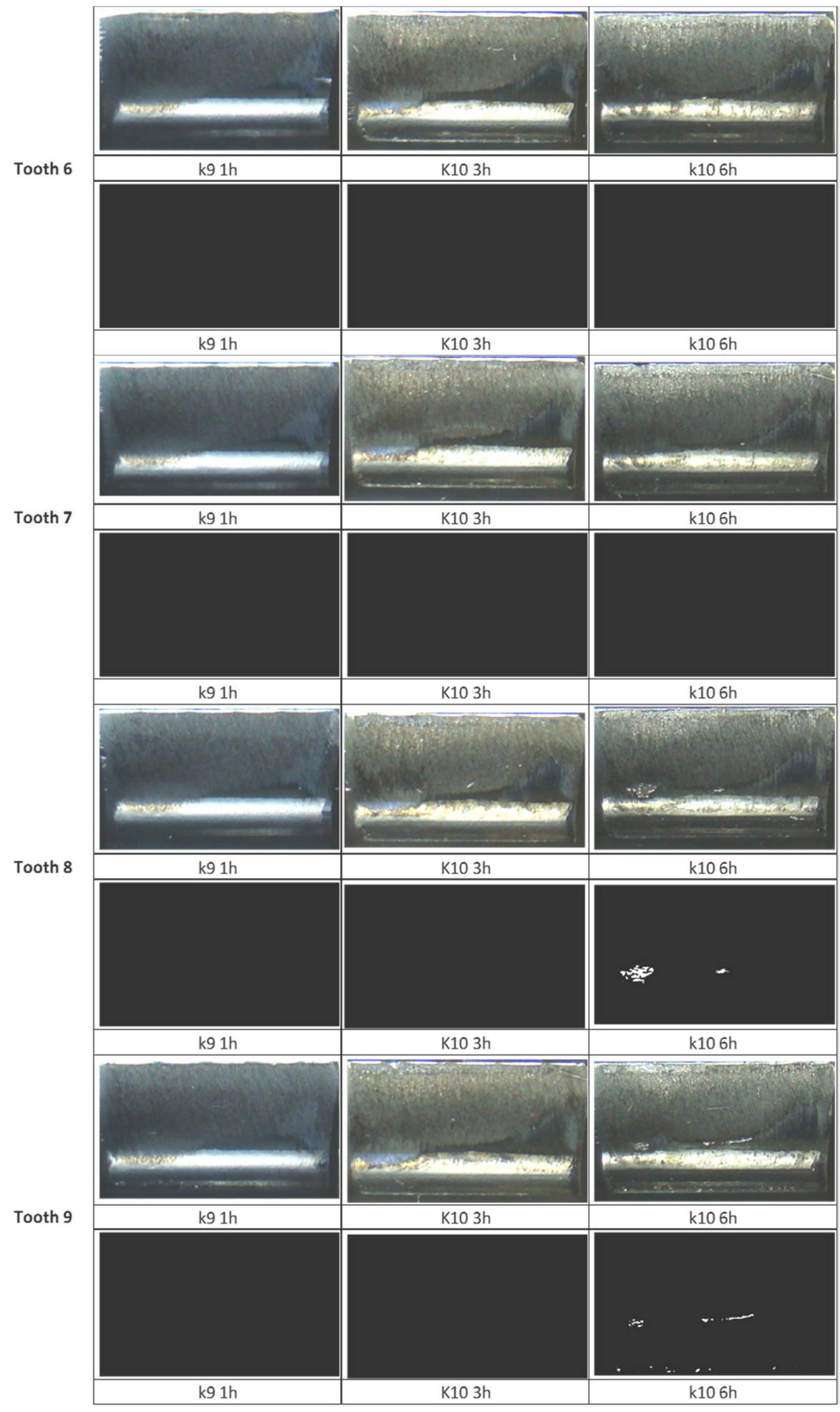


Figure C6 - D02 Images and quantification after each test step.

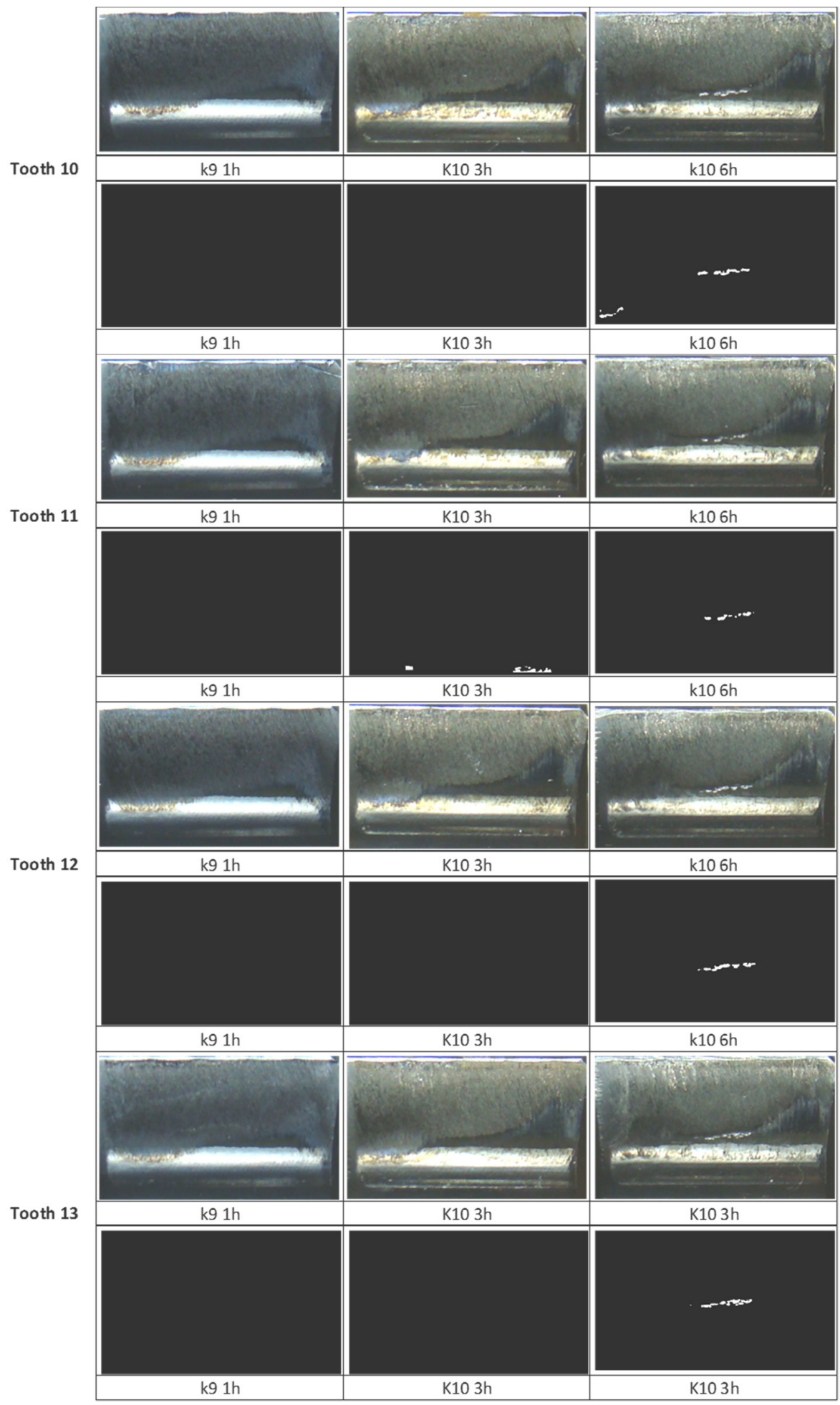


Figure C7 - D03 Images and quantification after each test step.

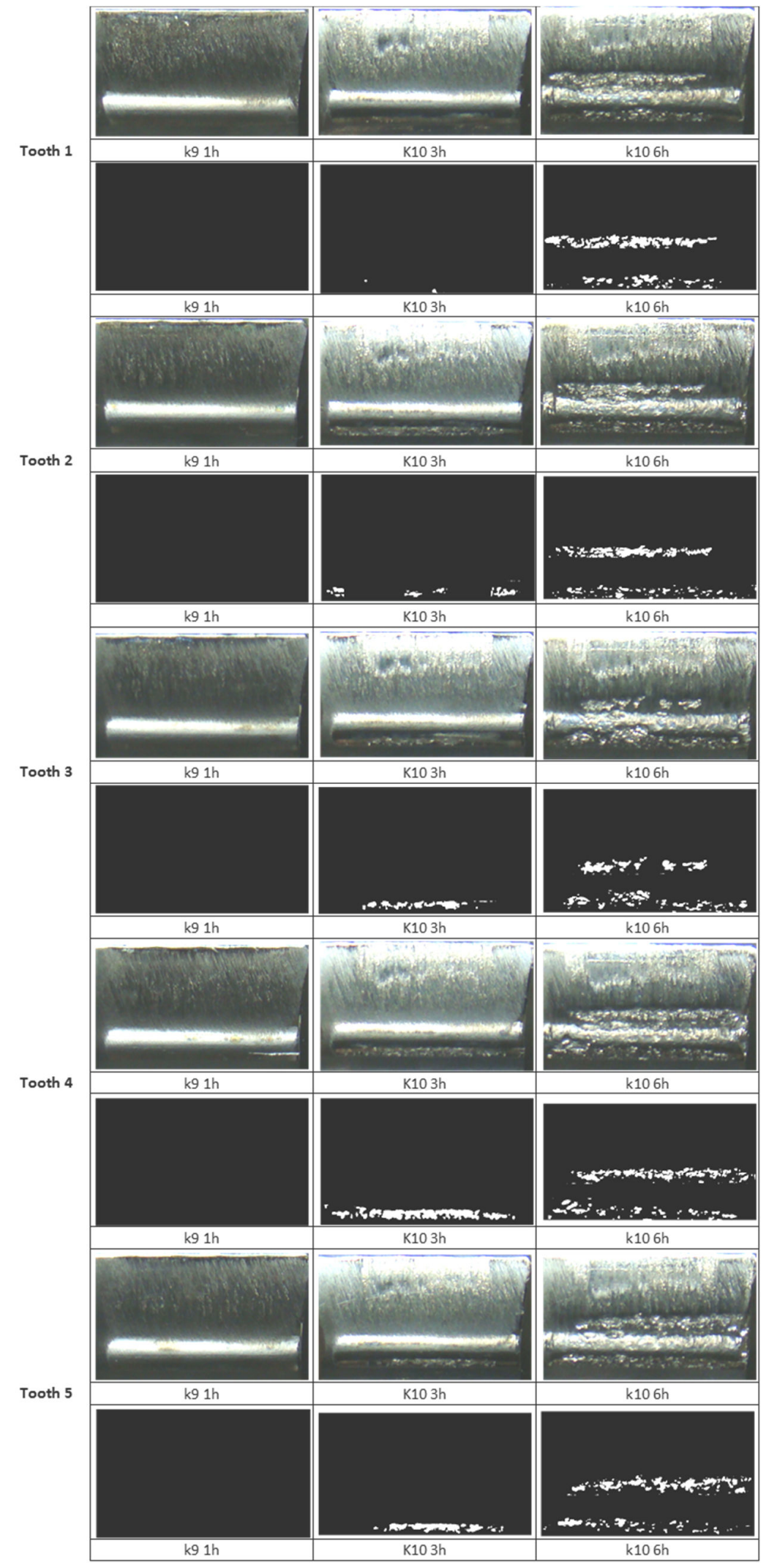


Figure C8 - D03 Images and quantification after each test step.

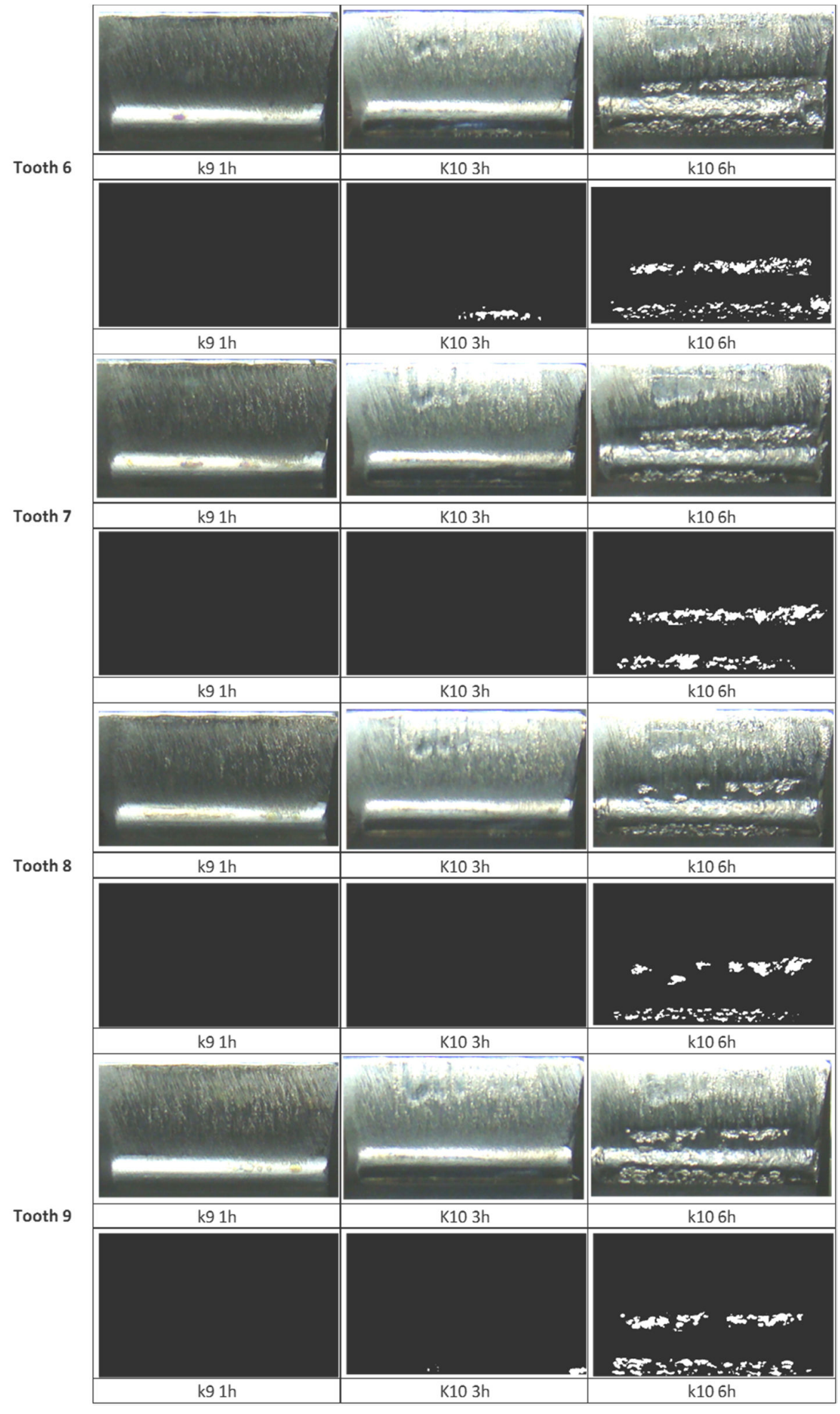


Figure C09 - D03 Images and quantification after each test step.

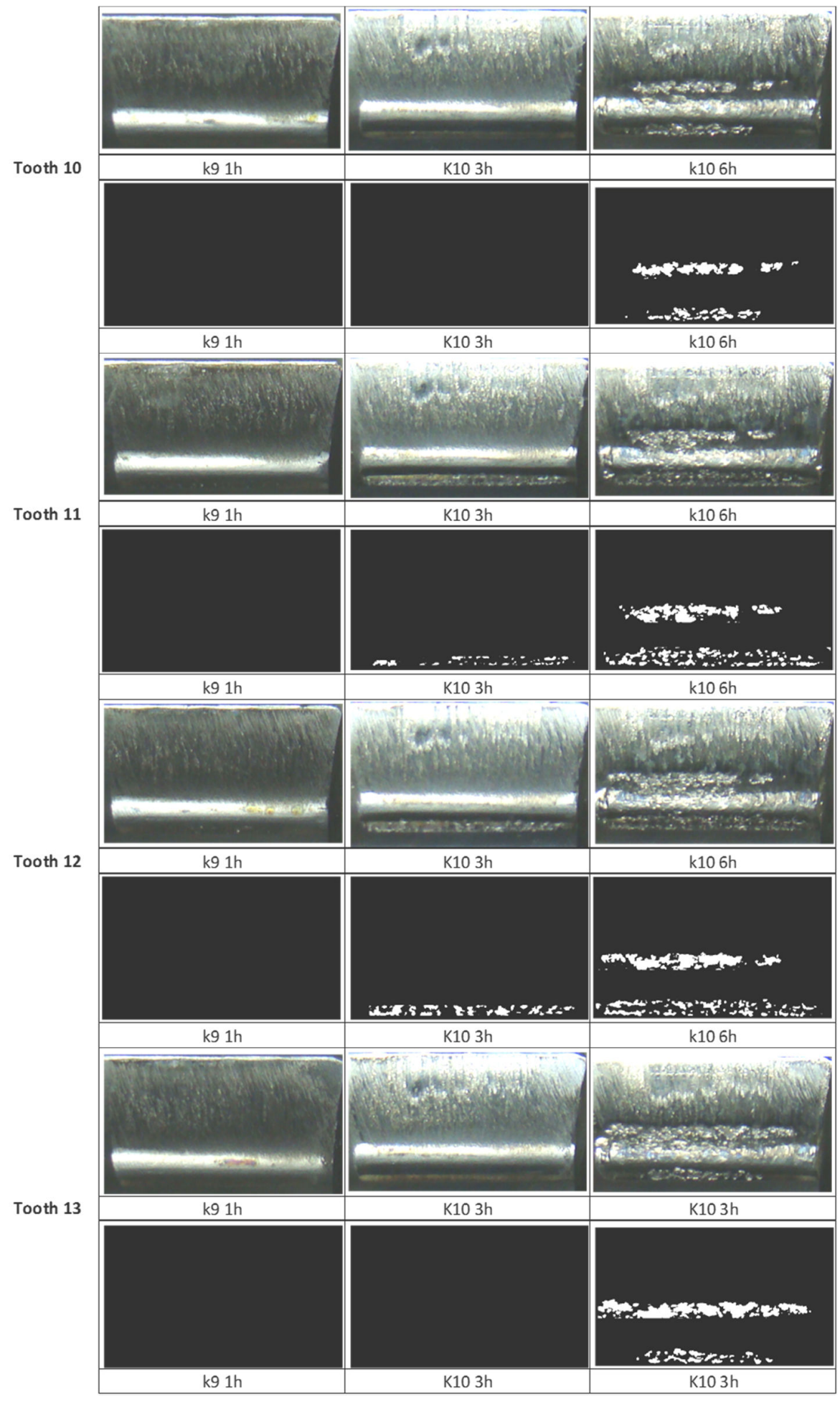


Figure C10 - D04 Images and quantification after each test step.

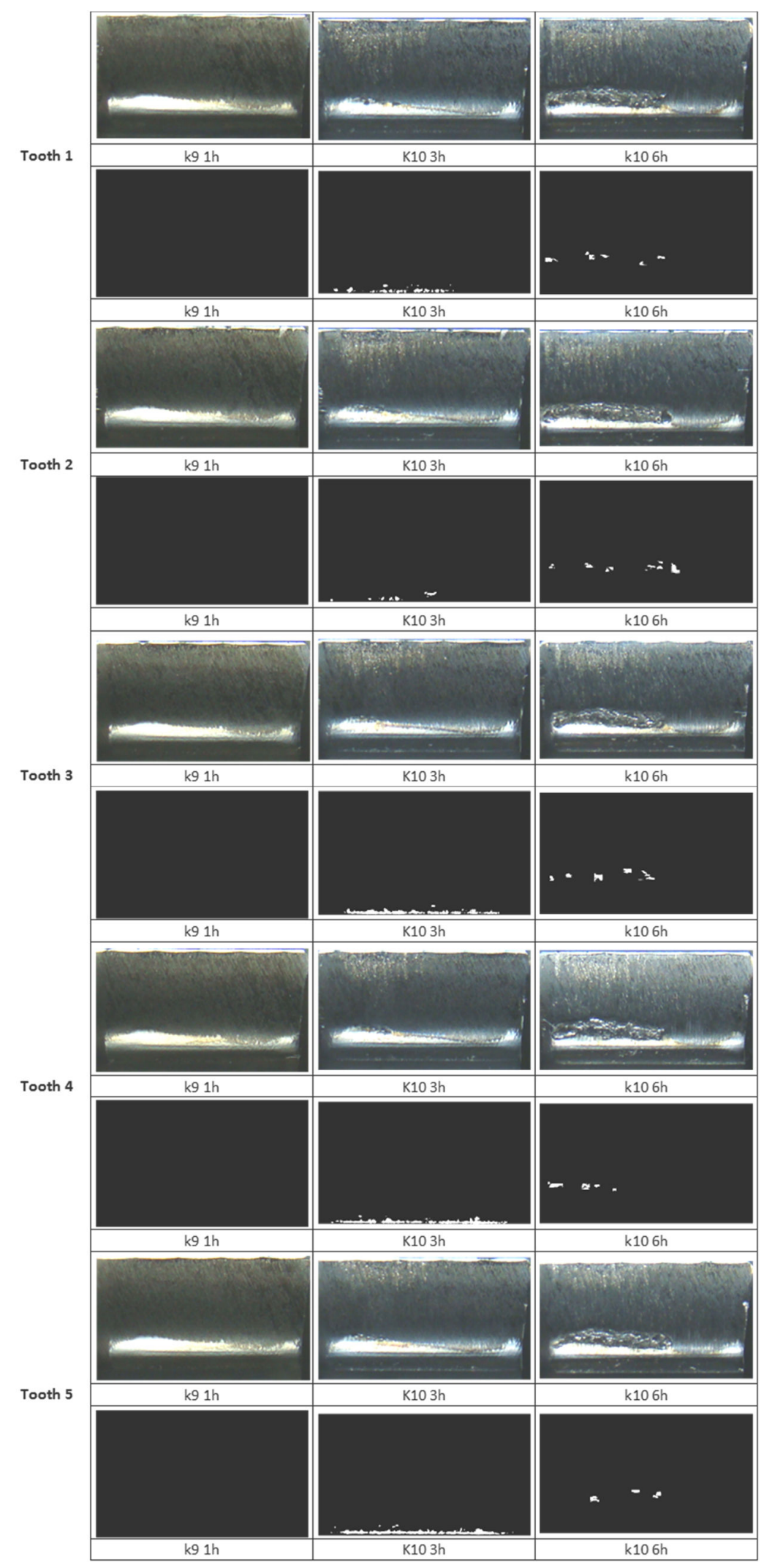


Figure C11 - D04 Images and quantification after each test step.

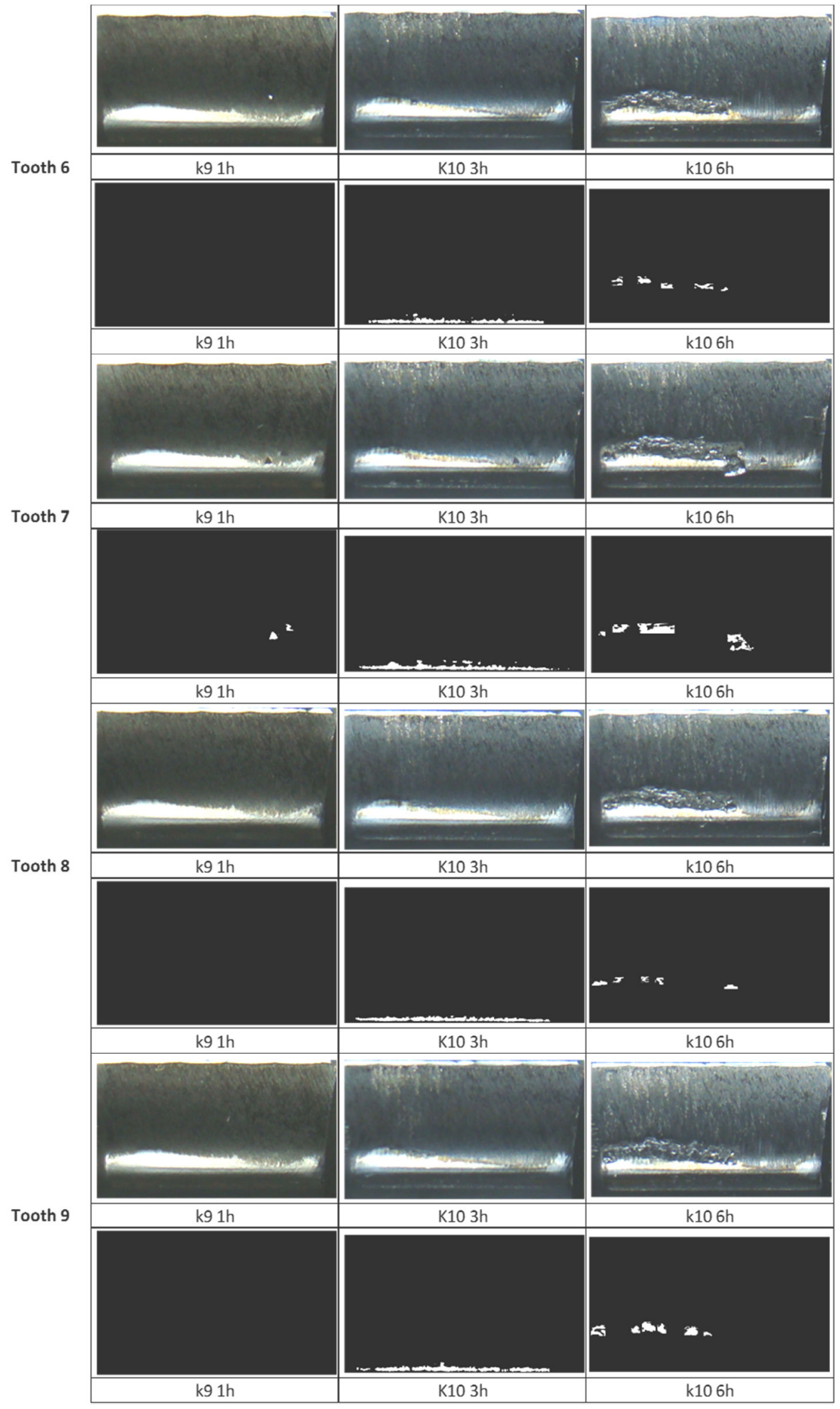


Figure C12 - D04 Images and quantification after each test step.

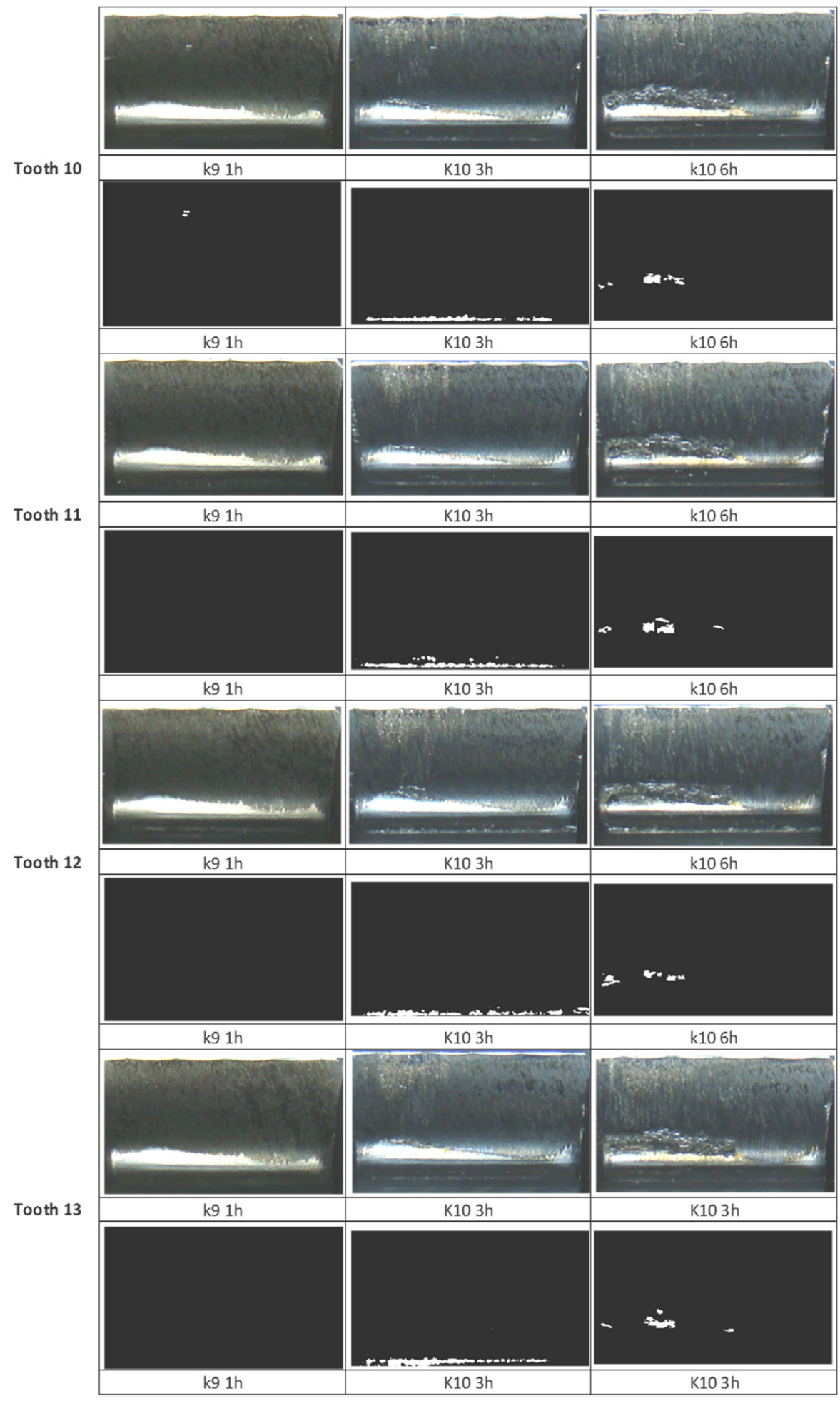


Figure C13 - D05 Images and quantification after each test step.

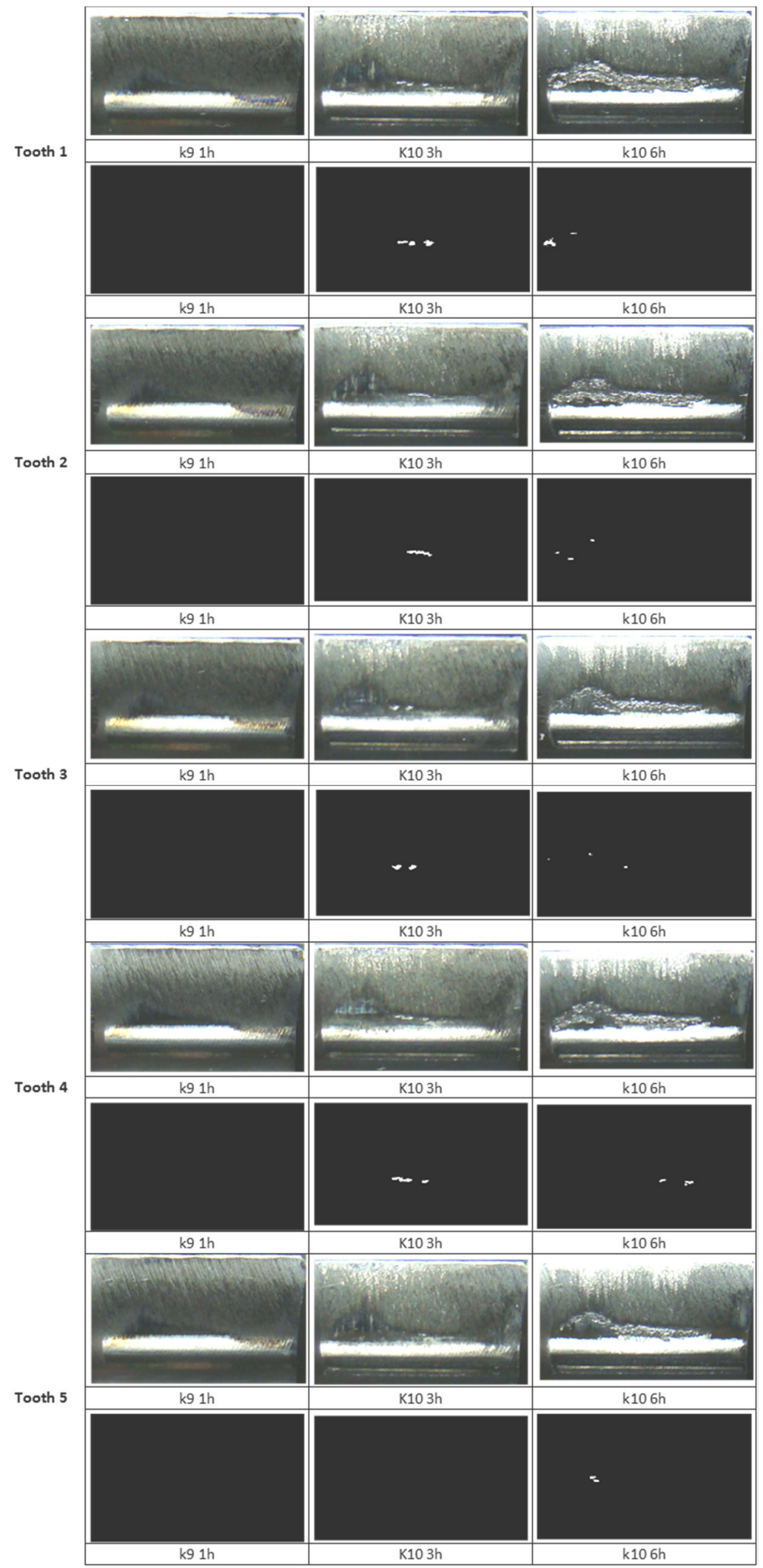


Figure C14 - D05 Images and quantification after each test step.

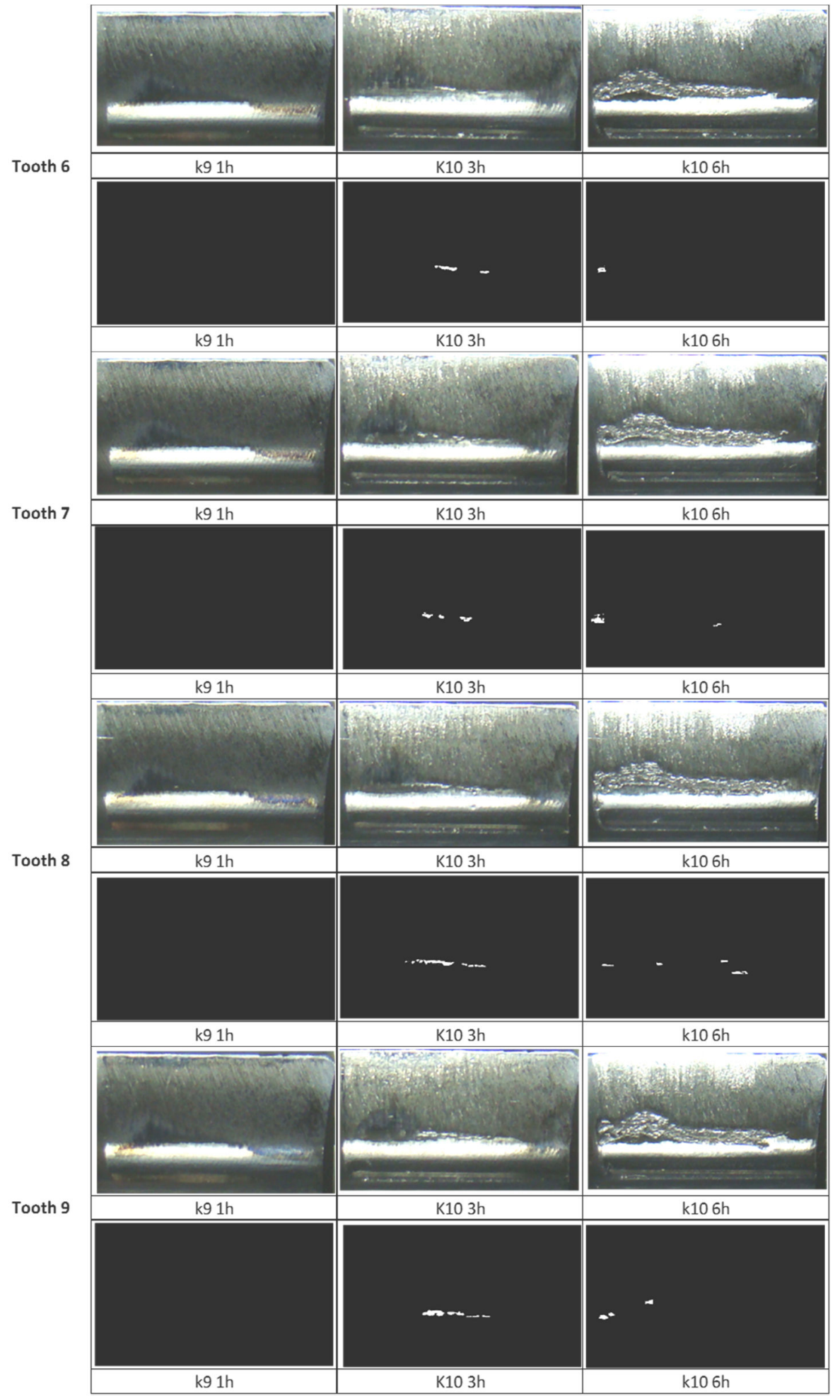


Figure C15 - D05 Images and quantification after each test step.

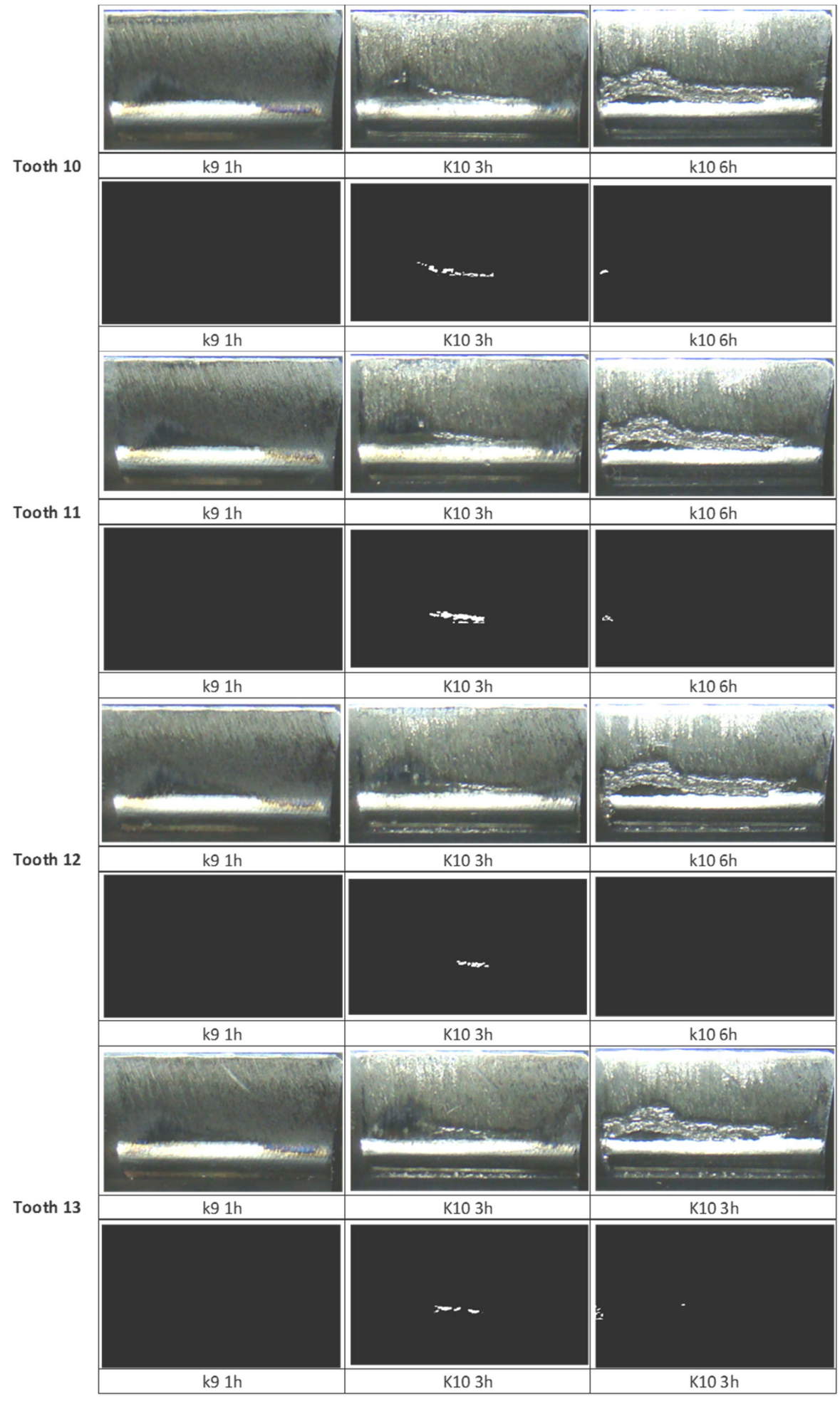




\section{APPENDIX D - FLANK PROFILE MEASUREMENTS OF F01 TILL F05}

In this section, is shown Profile plots. On each one of those figures from D1 till D5 is seen teeth 1, 4,7 and 11 measurement done on WGT350 before and after fatigue test. Here, is shown measurements collected by a MatLab routine on WGT350 graph plots, another MatLab routine were required to plot the data as seen on this section figures.

To display an organized plot, all graph will follow the referred color here in set: (i) black lines referring to data before test and (ii) blue line referring to data after test. Also important to highlight that data curves references (lower diameter measurement) were changed and set to be equal, this modification objective is to have an organized plot, clean and allowing immediate slope and form values comparisons of blue or black lines. On the other hand, a value comparison in between black line values with blue line values are no longer accurate, since its reference has been adjusted to be plotted. Even tough, it's possible to compare the pattern between before and after test of each one of those tooth.

Figure D1 - F01 Gear Profile Evaluation based on WGT 350 measurements and plotted using MatLab routine.

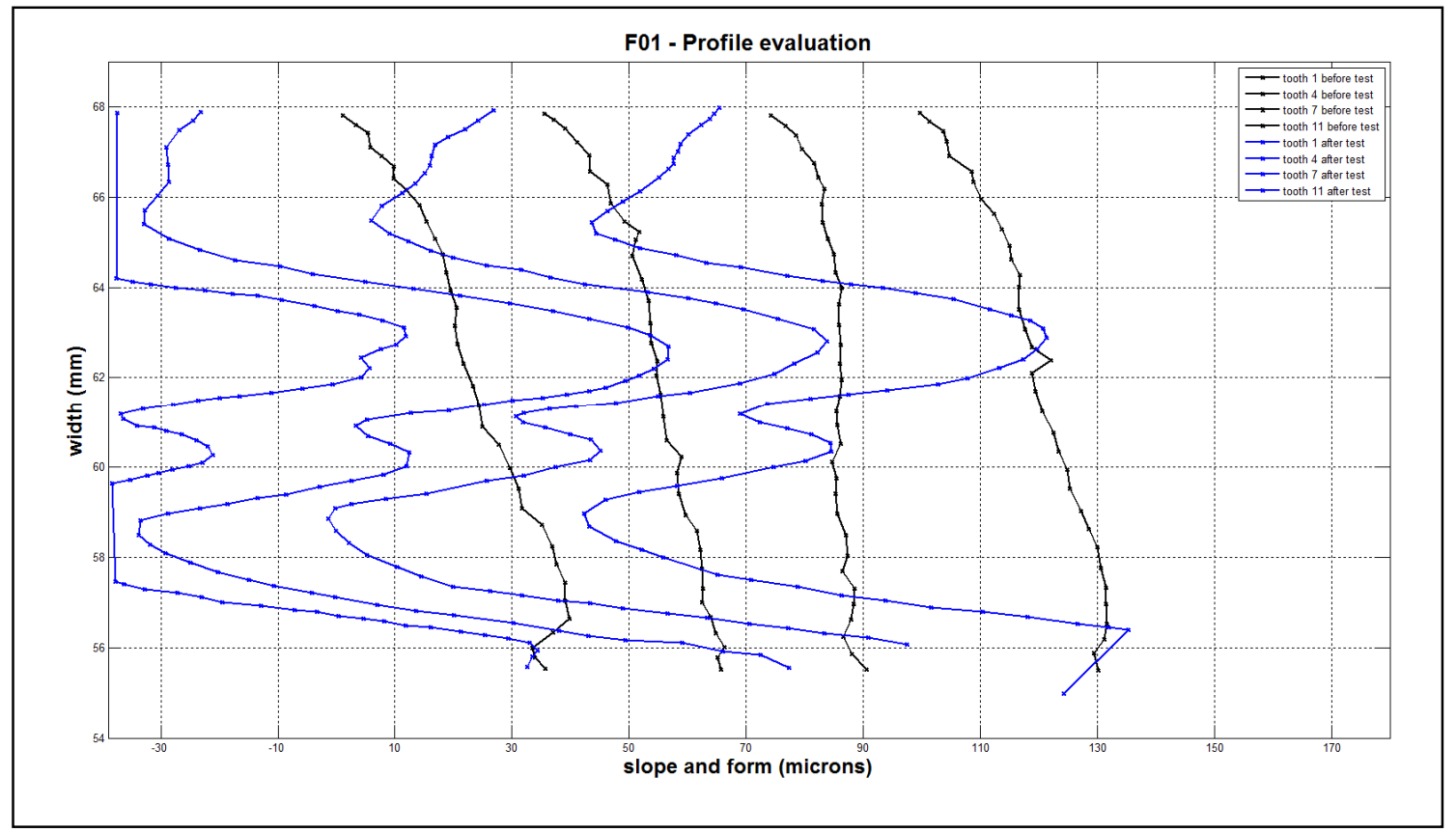


Figure D2 - F02 Gear Profile Evaluation based on WGT 350 measurements and plotted using MatLab routine.

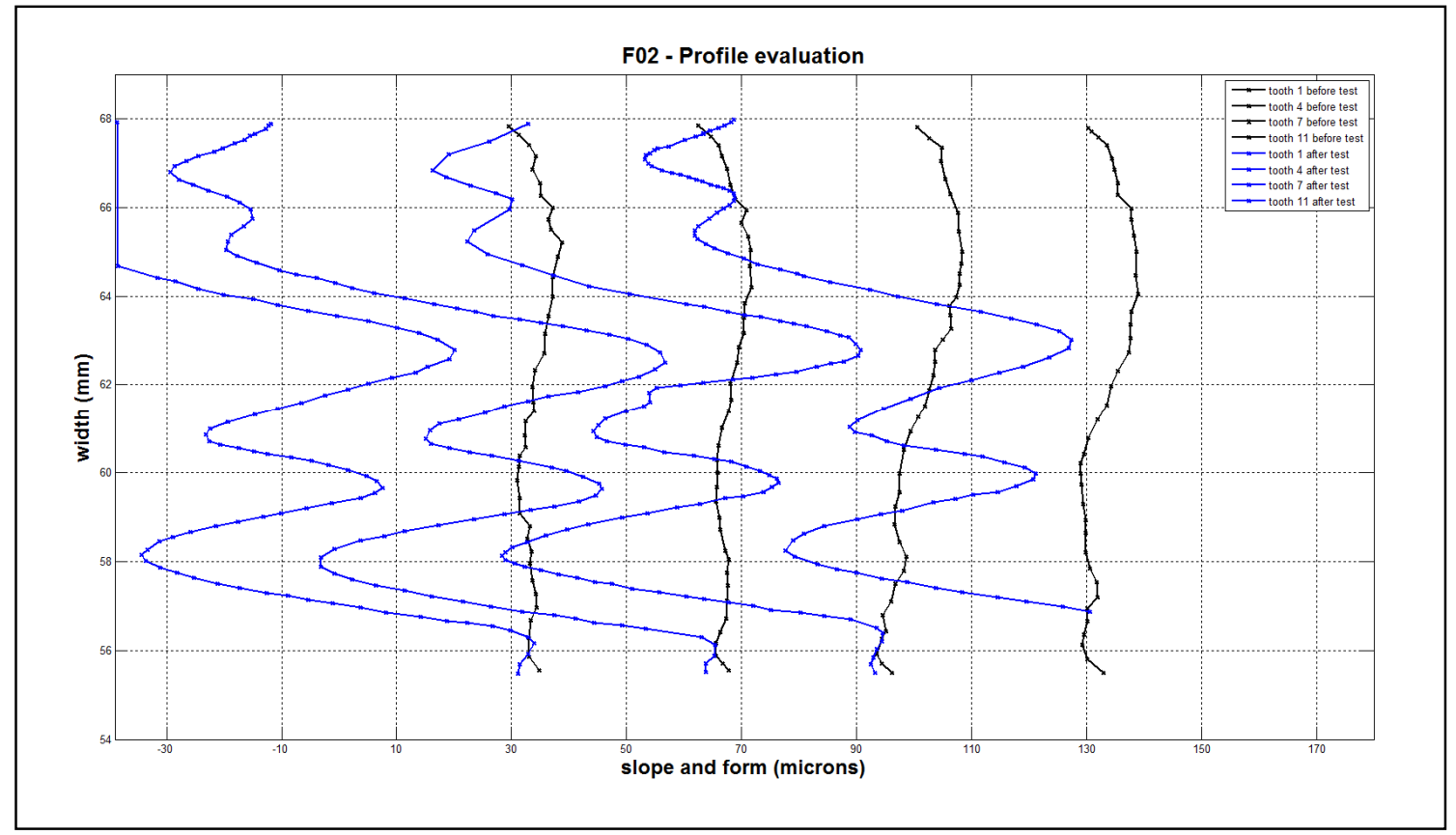

Figure D3 - F03 Gear Profile Evaluation based on WGT 350 measurements and plotted using MatLab routine.

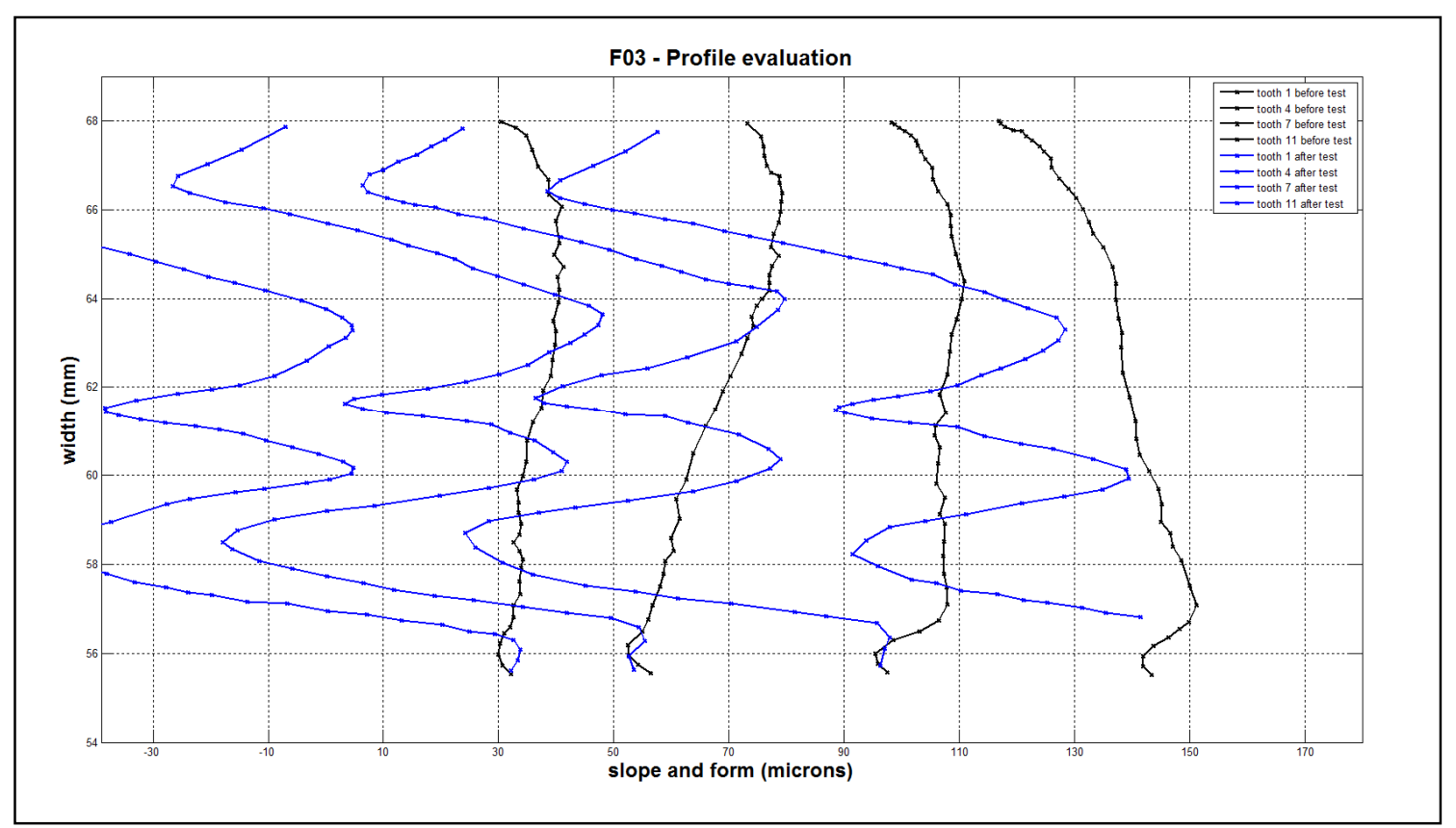


Figure D4 - F04 Gear Profile Evaluation based on WGT 350 measurements and plotted using MatLab routine.

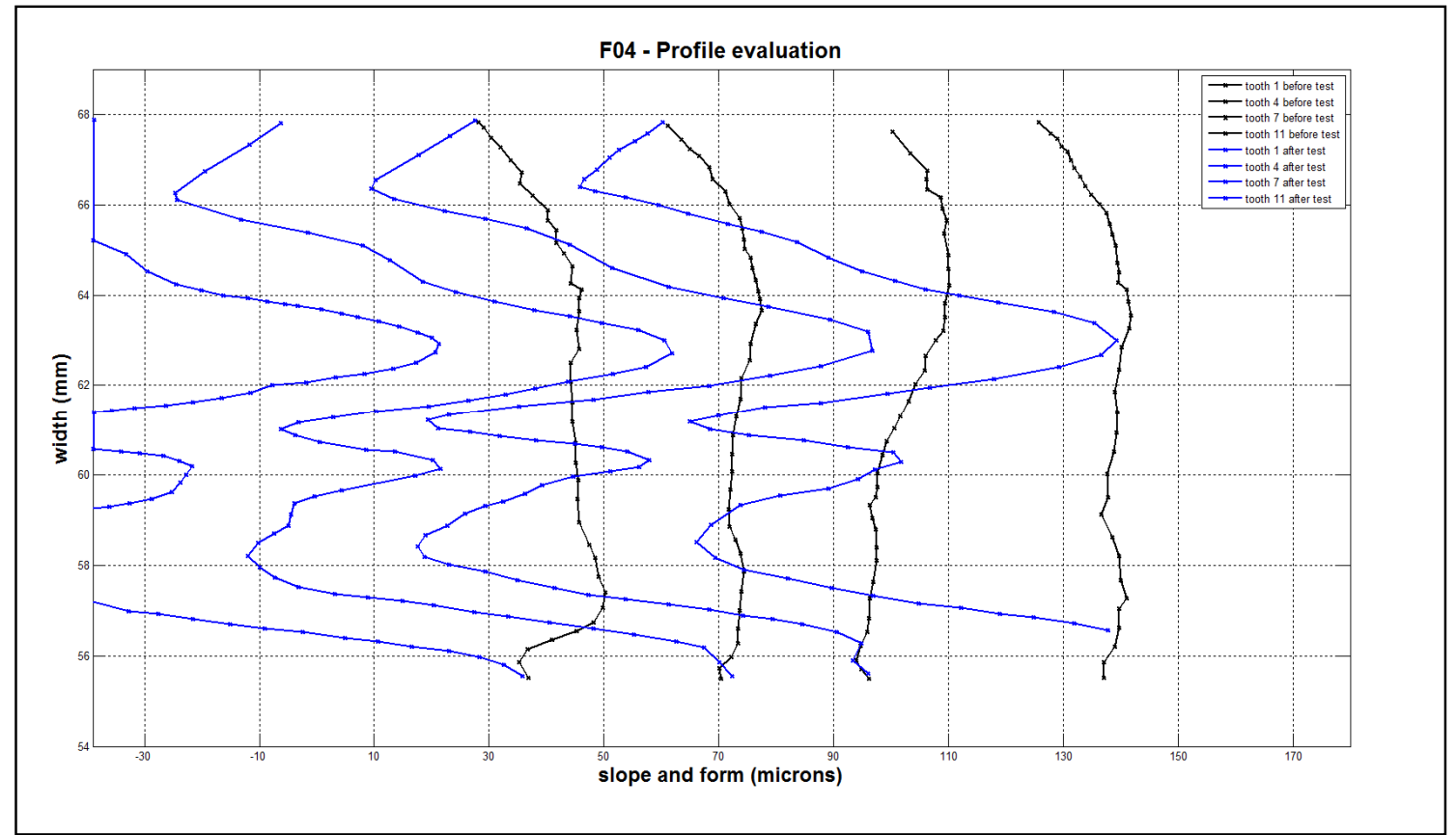

Figure D5 - F05 Gear Profile Evaluation based on WGT 350 measurements and plotted using MatLab routine

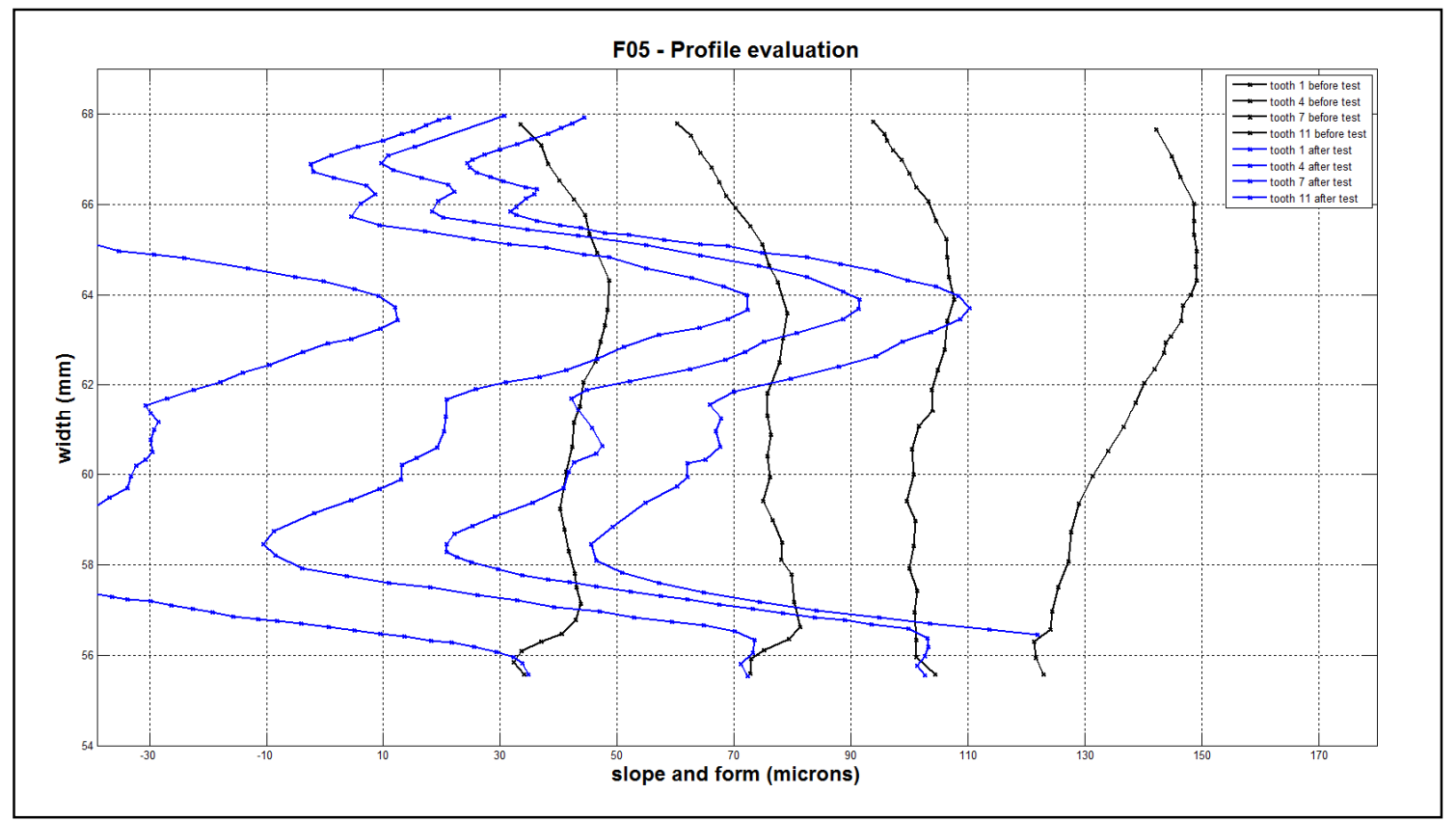




\section{APPENDIX E - FLANK PROFILE MEASUREMENTS OF B01 TILL B05}

In this section, is shown Profile plots. On each one of those figures from E1 till E5 is seen teeth 1, 4,7 and 11 measurement done on WGT350 before and after fatigue test. Here, is shown measurements collected by a MatLab routine on WGT350 graph plots, another MatLab routine were required to plot the data as seen on this section figures.

To display an organized plot, all graph will follow the referred color here in set: (i) black lines referring to data before test and (ii) blue line referring to data after test. Also important to highlight that data curves references (lower diameter measurement) were changed and set to be equal, this modification objective is to have an organized plot, clean and allowing immediate slope and form values comparisons of blue or black lines. On the other hand, a value comparison in between black line values with blue line values are no longer accurate, since its reference has been adjusted to be plotted. Even tough, it's possible to compare the pattern between before and after test of each one of those tooth.

Figure E1 - B01 Gear Profile Evaluation based on WGT 350 measurements and plotted using MatLab routine

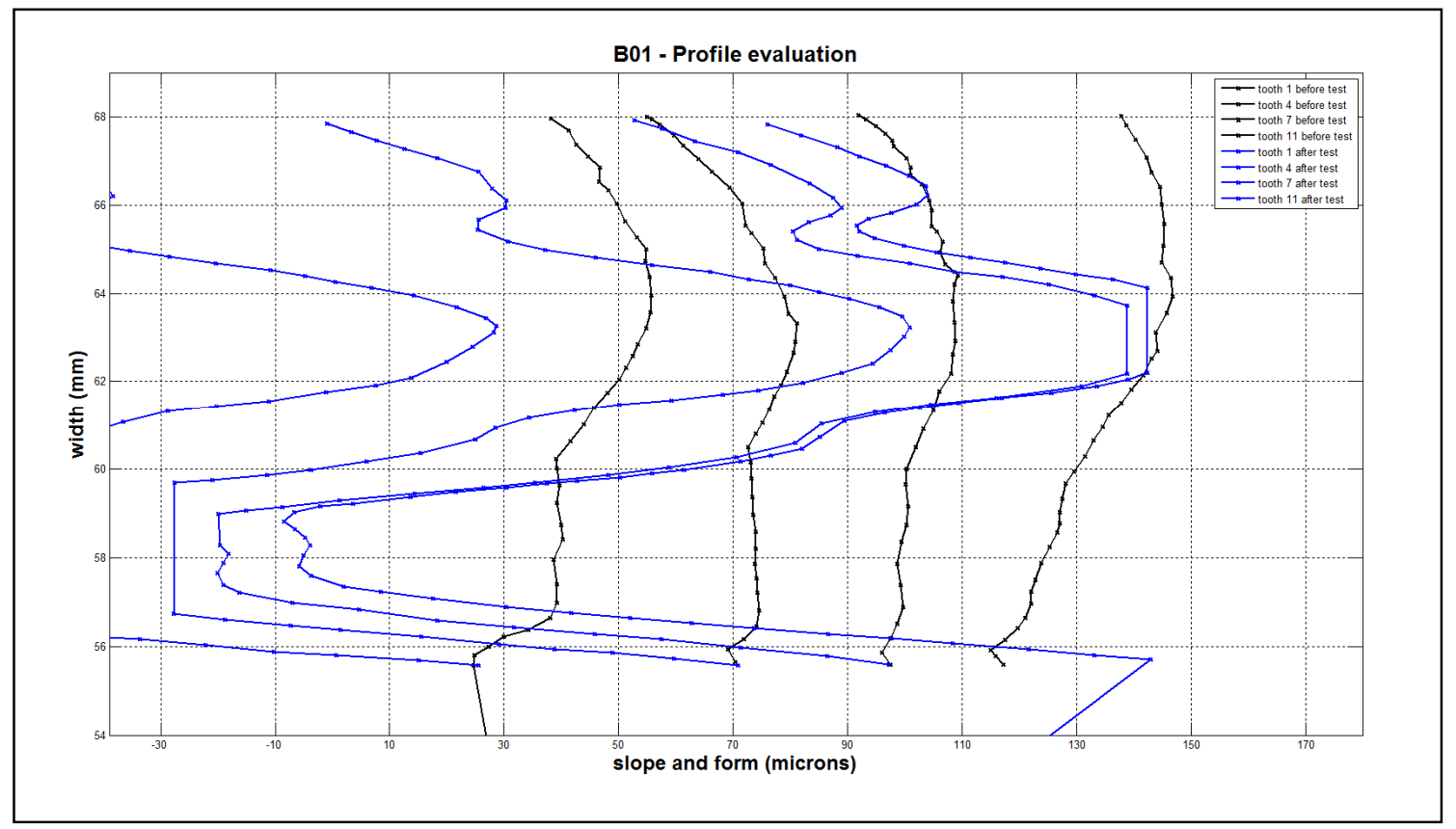


Figure E3 - B02 Gear Profile Evaluation based on WGT 350 measurements and plotted using MatLab routine.

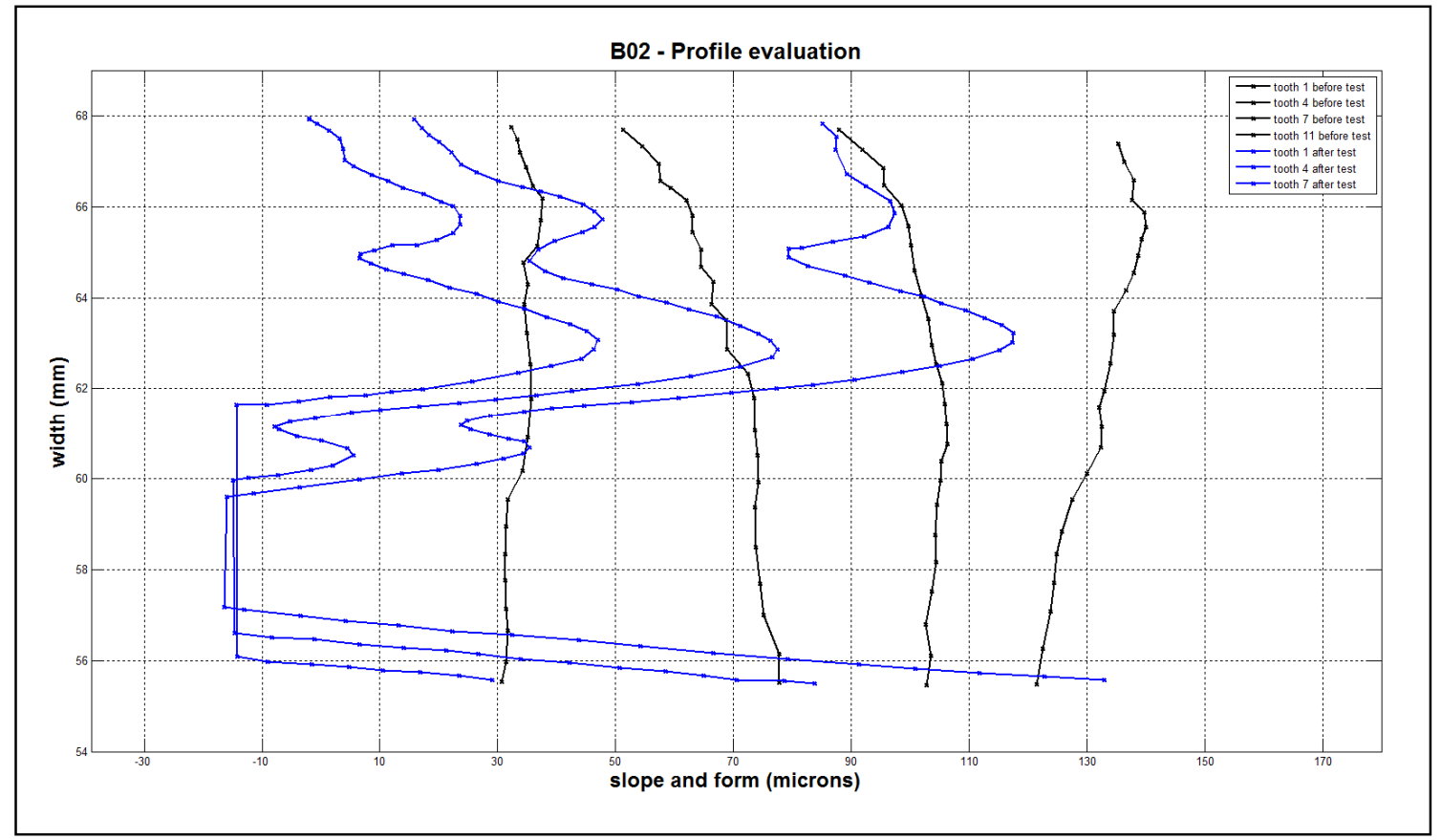

Figure E2 - B03 Gear Profile Evaluation based on WGT 350 measurements and plotted using MatLab routine.

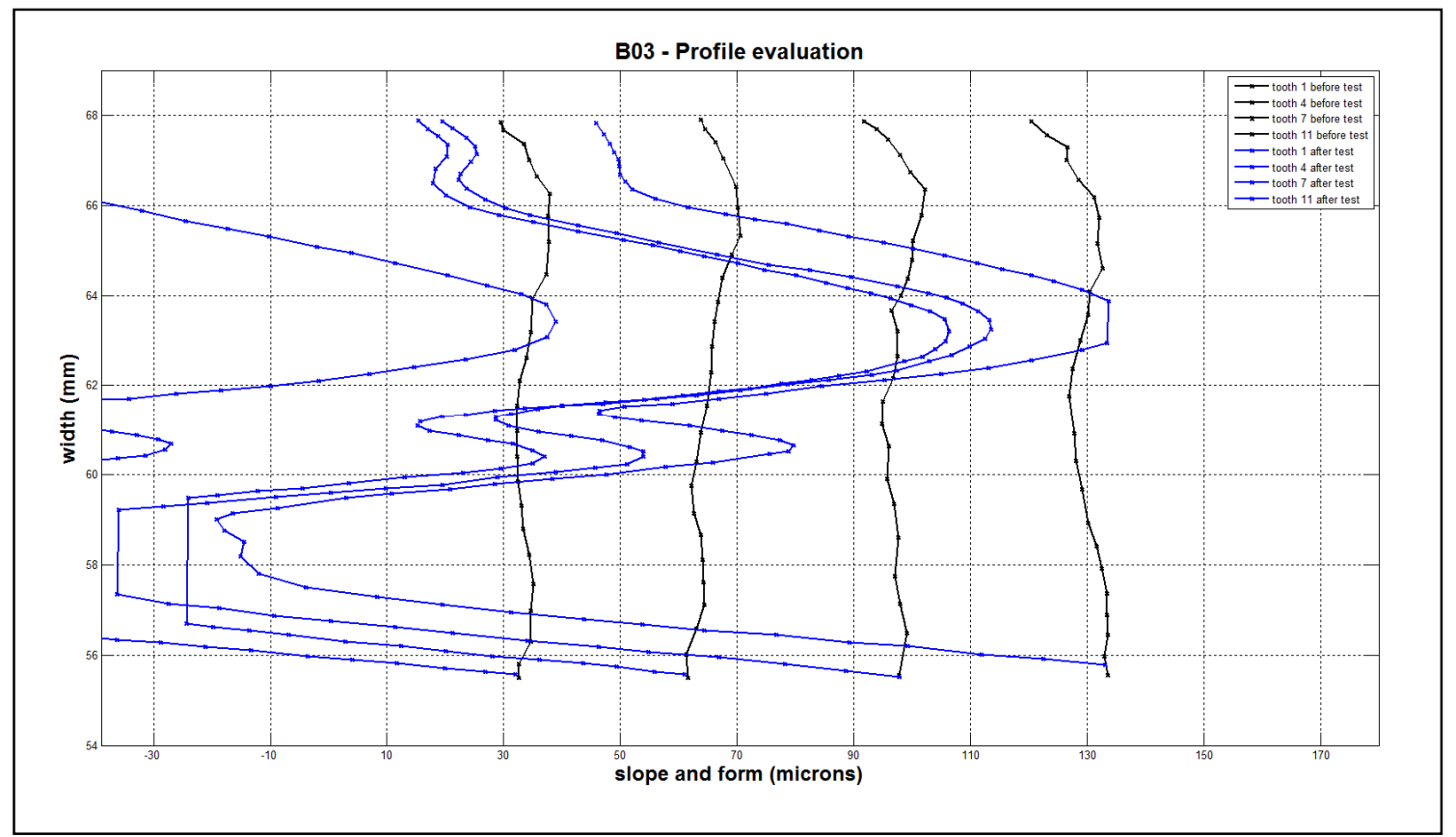


Figure E4 - B04 Gear Profile Evaluation based on WGT 350 measurements and plotted using MatLab routine.

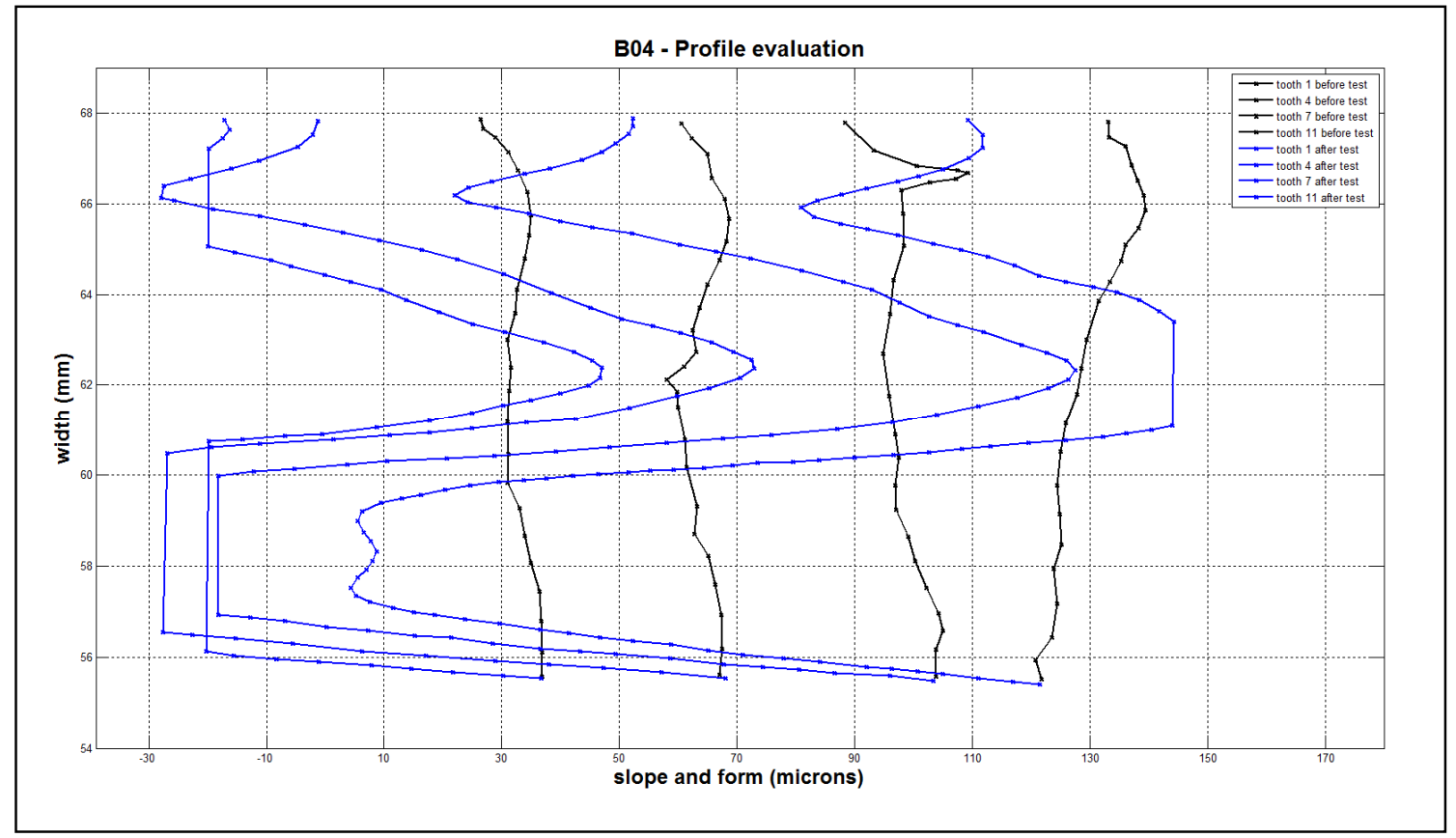

Figure E5 - B05 Gear Profile Evaluation based on WGT 350 measurements and plotted using MatLab routine.

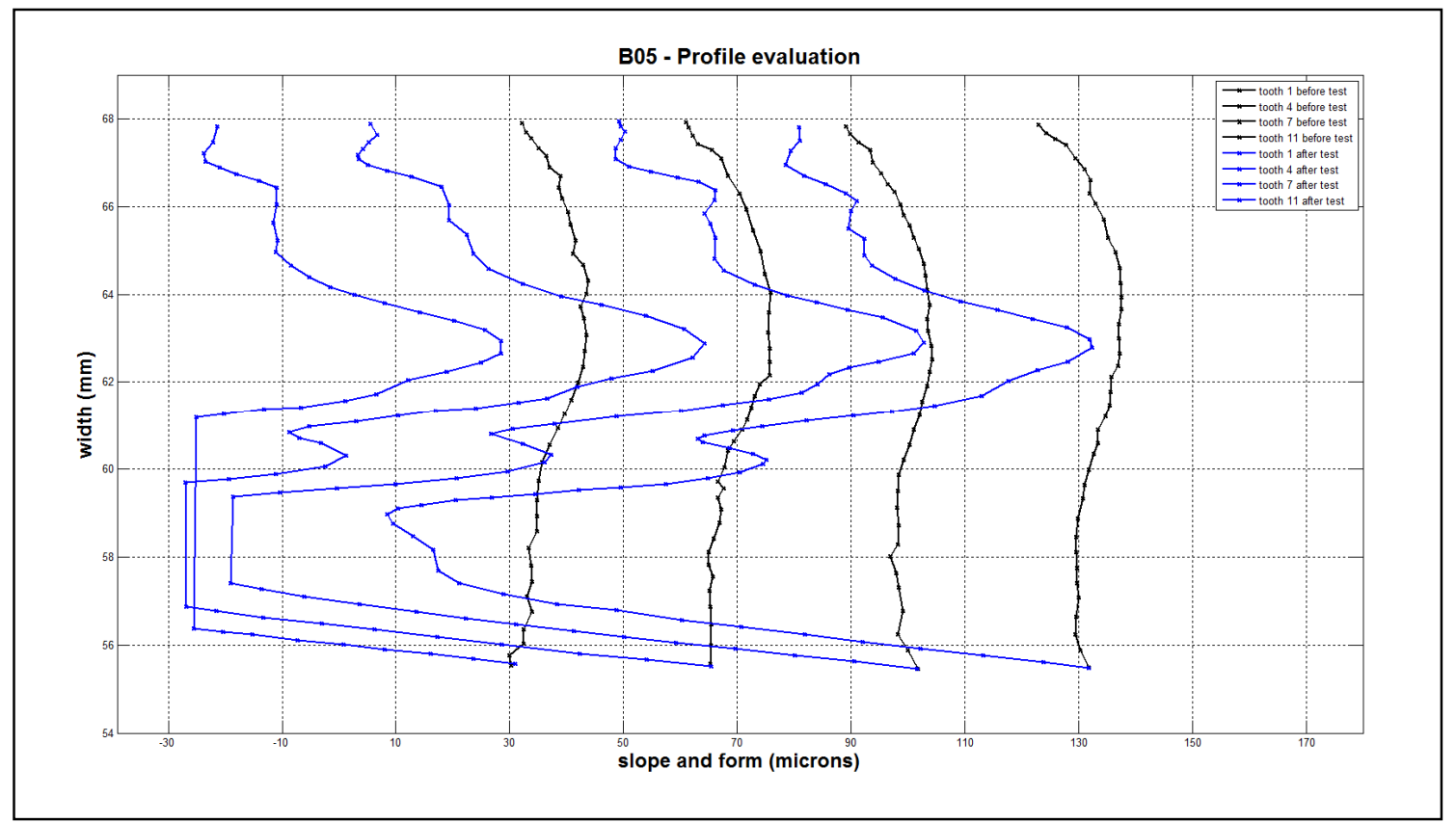




\section{APPENDIX F - FLANK PROFILE MEASUREMENTS OF D01 TILL D05}

In this section, is shown Profile plots. On each one of those figures from F1 till F5 is seen teeth 1, 4,7 and 11 measurement done on WGT350 before and after fatigue test. Here, is shown measurements collected by a MatLab routine on WGT350 graph plots, another MatLab routine were required to plot the data as seen on this section figures.

To display an organized plot, all graph will follow the referred color here in set: (i) black lines referring to data before test and (ii) blue line referring to data after test. Also important to highlight that data curves references (lower diameter measurement) were changed and set to be equal, this modification objective is to have an organized plot, clean and allowing immediate slope and form values comparisons of blue or black lines. On the other hand, a value comparison in between black line values with blue line values are no longer accurate, since its reference has been adjusted to be plotted. Even tough, it's possible to compare the pattern between before and after test of each one of those tooth.

Figure F1 - D02 Gear Profile Evaluation based on WGT 350 measurements and plotted using MatLab routine.

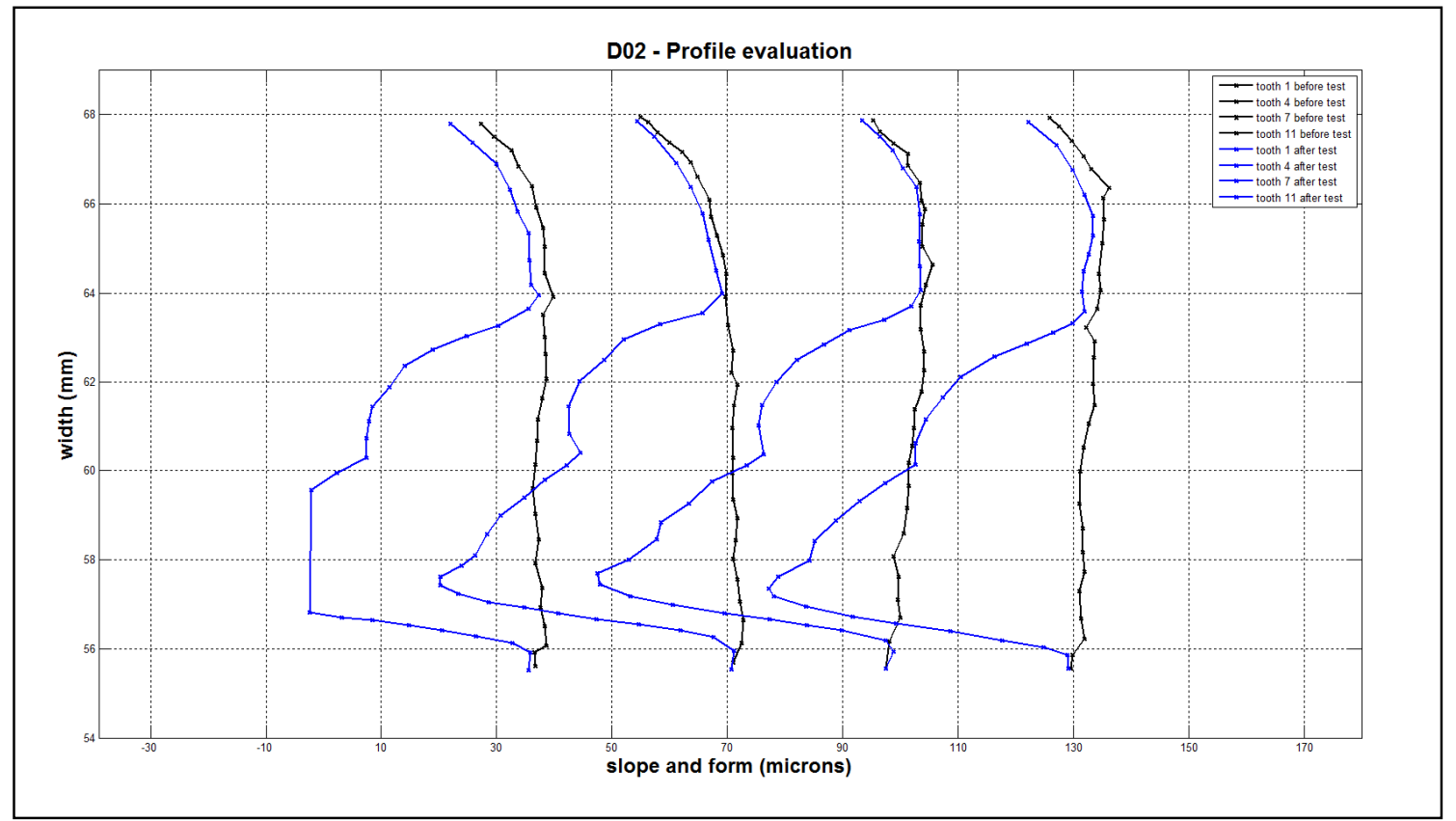


Figure F2 - D03 Gear Profile Evaluation based on WGT 350 measurements and plotted using MatLab routine.

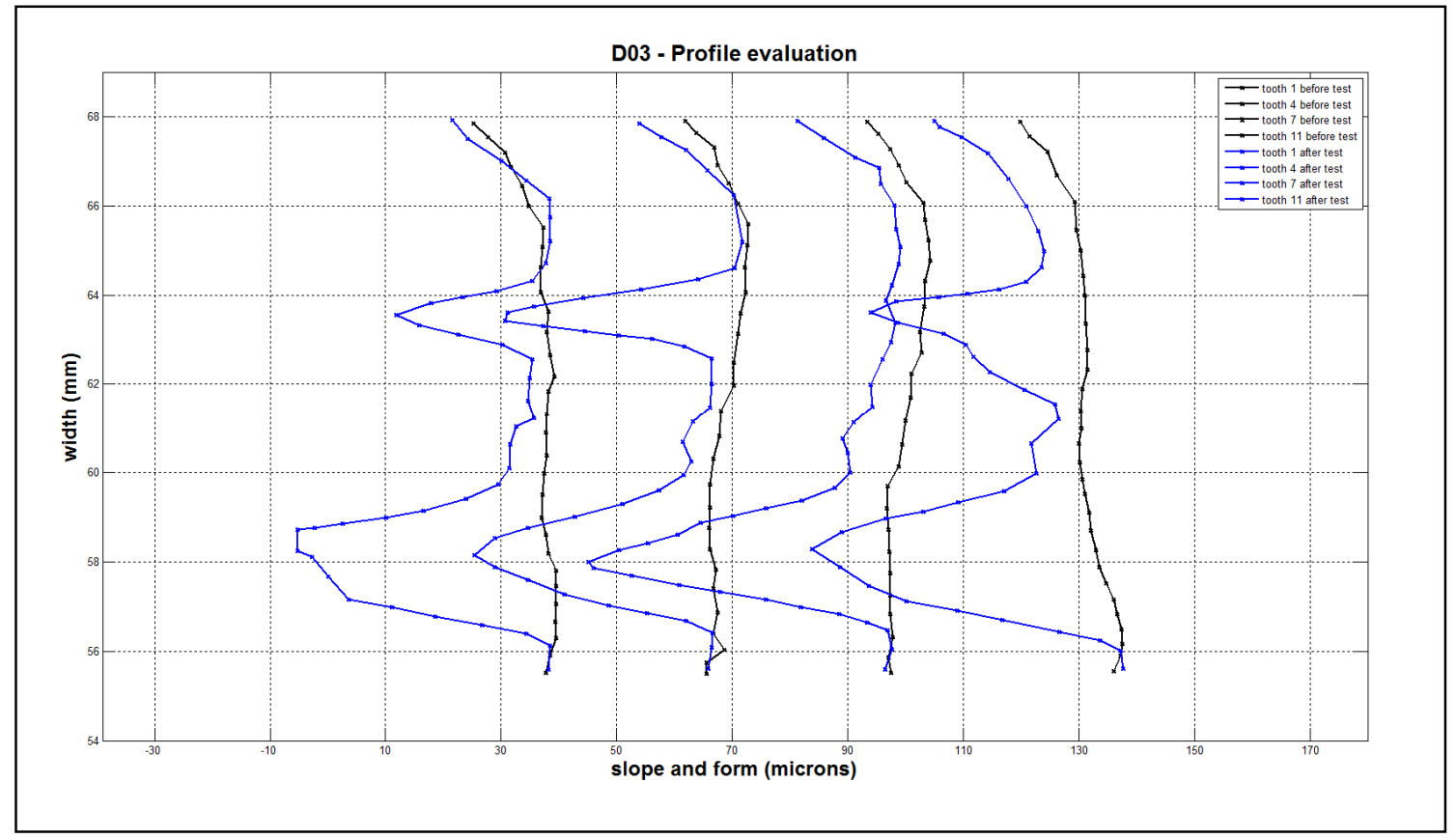

Figure F3 - D04 Gear Profile Evaluation based on WGT 350 measurements and plotted using MatLab routine.

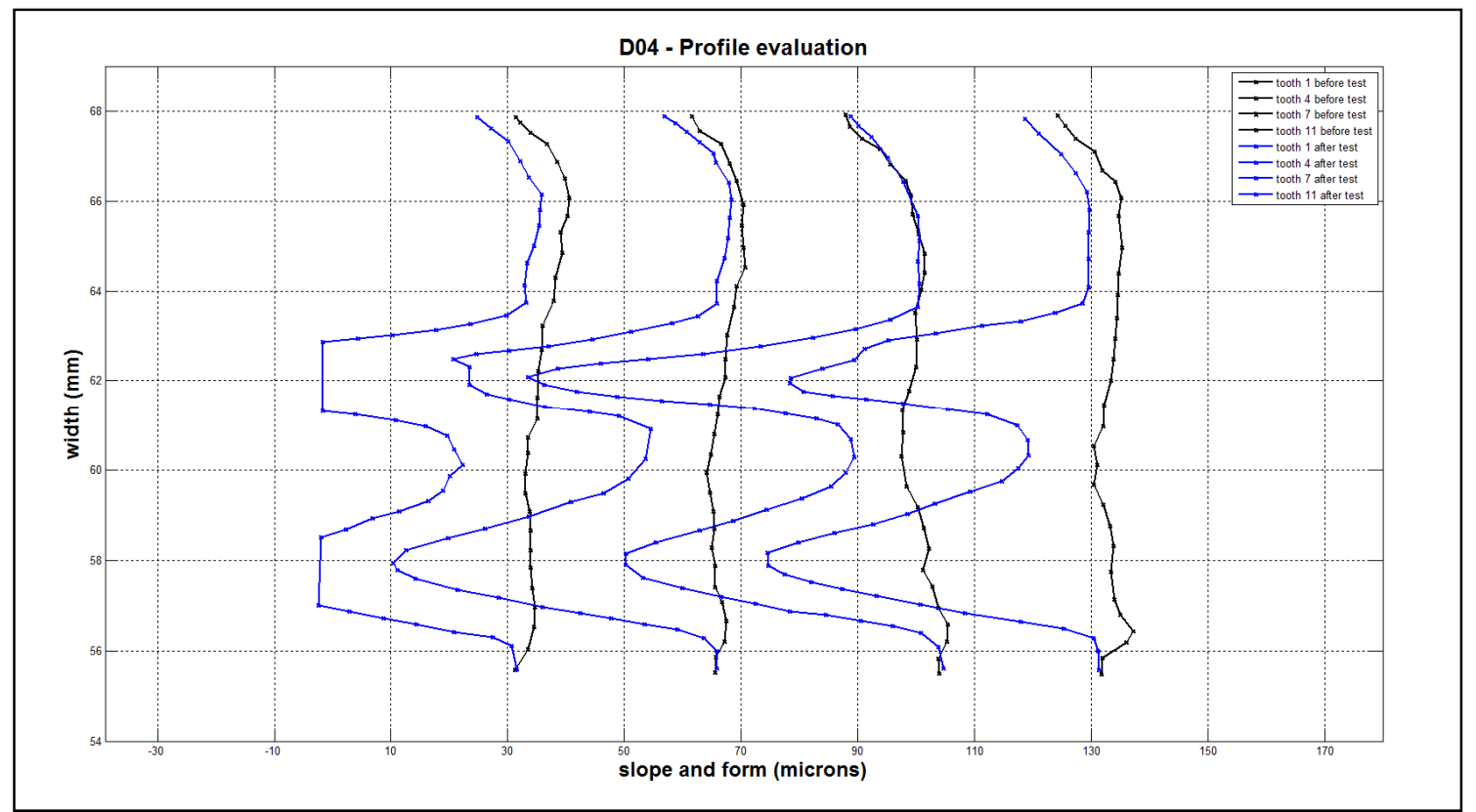


Figure F4 - D05 Gear Profile Evaluation based on WGT 350 measurements and plotted using MatLab routine.

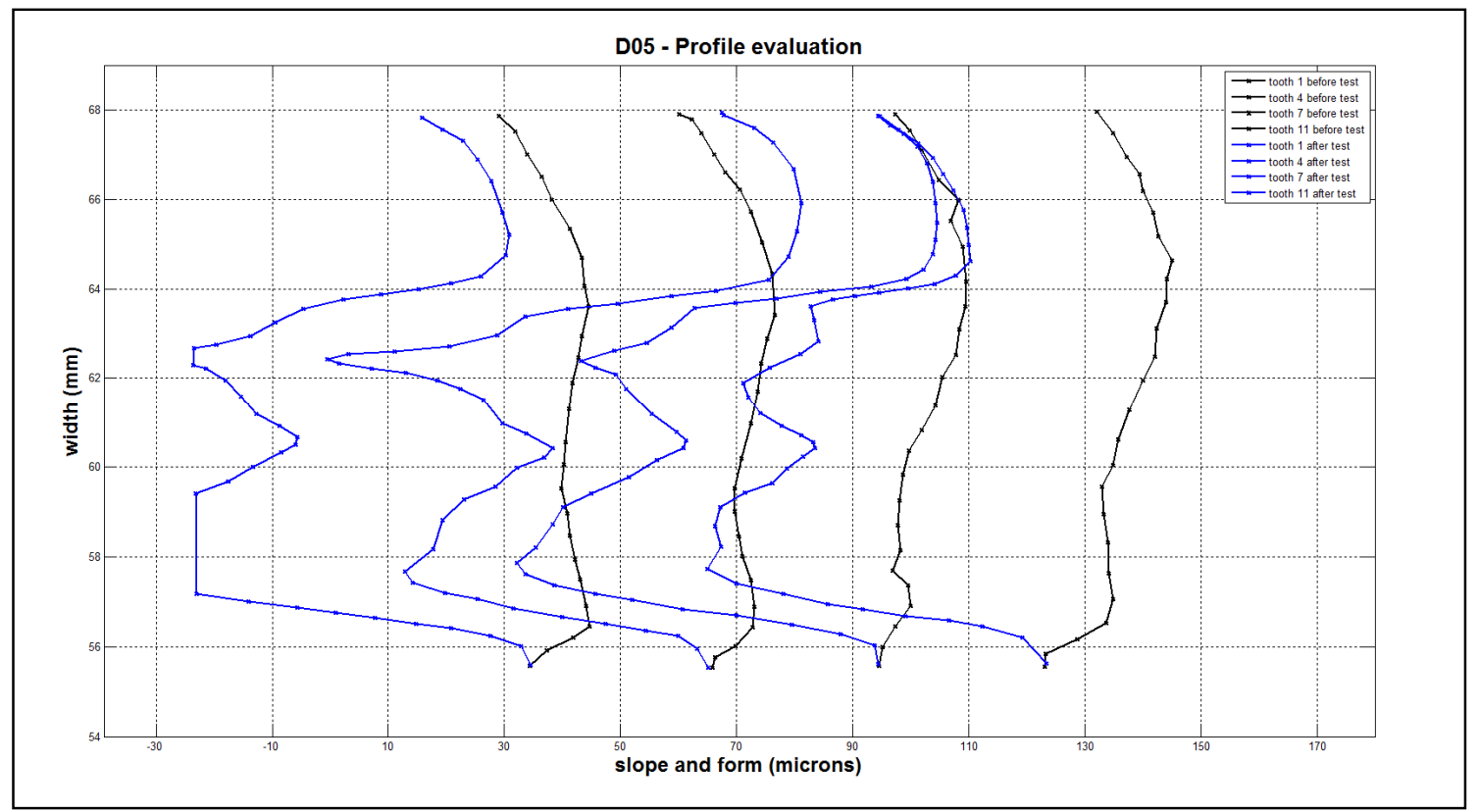




\section{APPENDIX G - FLANK HELIX MEASUREMENTS OF F01 TILL F05}

In this section, is shown Helix plots. On each one of those figures from G1 till G5 is seen teeth 1, 4,7 and 11 measurement done on WGT350 before and after fatigue test. Here, is shown measurements collected by a MatLab routine on WGT350 graph plots, another MatLab routine were required to plot the data as seen on this section figures.

To display an organized plot, all graph will follow the referred color here in set: (i) black lines referring to data before test and (ii) blue line referring to data after test. Also important to highlight that data curves references (lower width measurement) were changed and set to be equal, this modification objective is to have an organized plot, clean and allowing immediate slope and form values comparisons of blue or black lines. On the other hand, a value comparison in between black line values with blue line values are no longer accurate, since its reference has been adjusted to be plotted. Even tough, it's possible to compare the pattern between before and after test of each one of those tooth.

Figure G1 - F01 Gear Helix Evaluation based on WGT 350 measurements and plotted using MatLab routine.

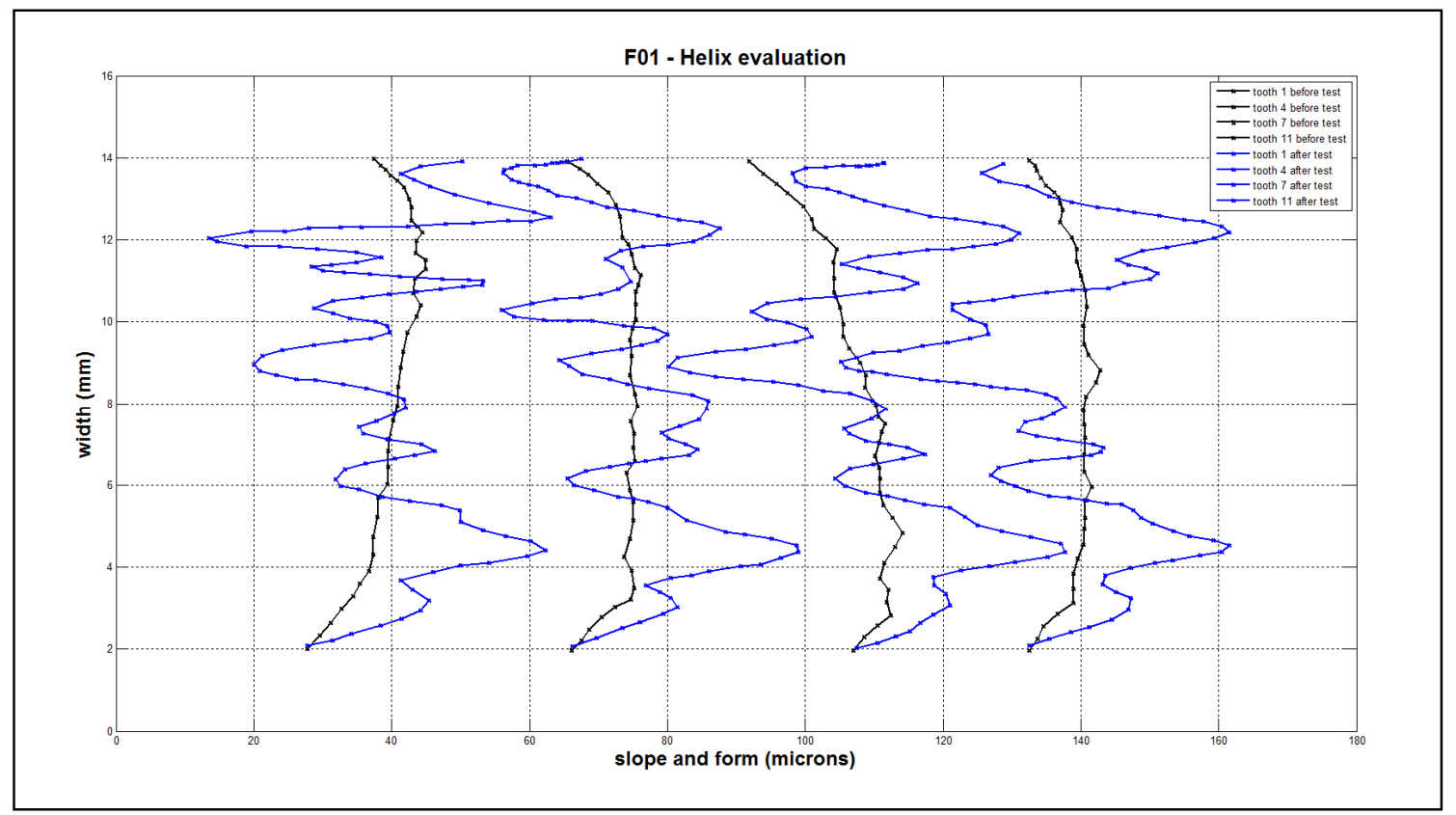


Figure G2 - F02 Gear Helix Evaluation based on WGT 350 measurements and plotted using MatLab routine.

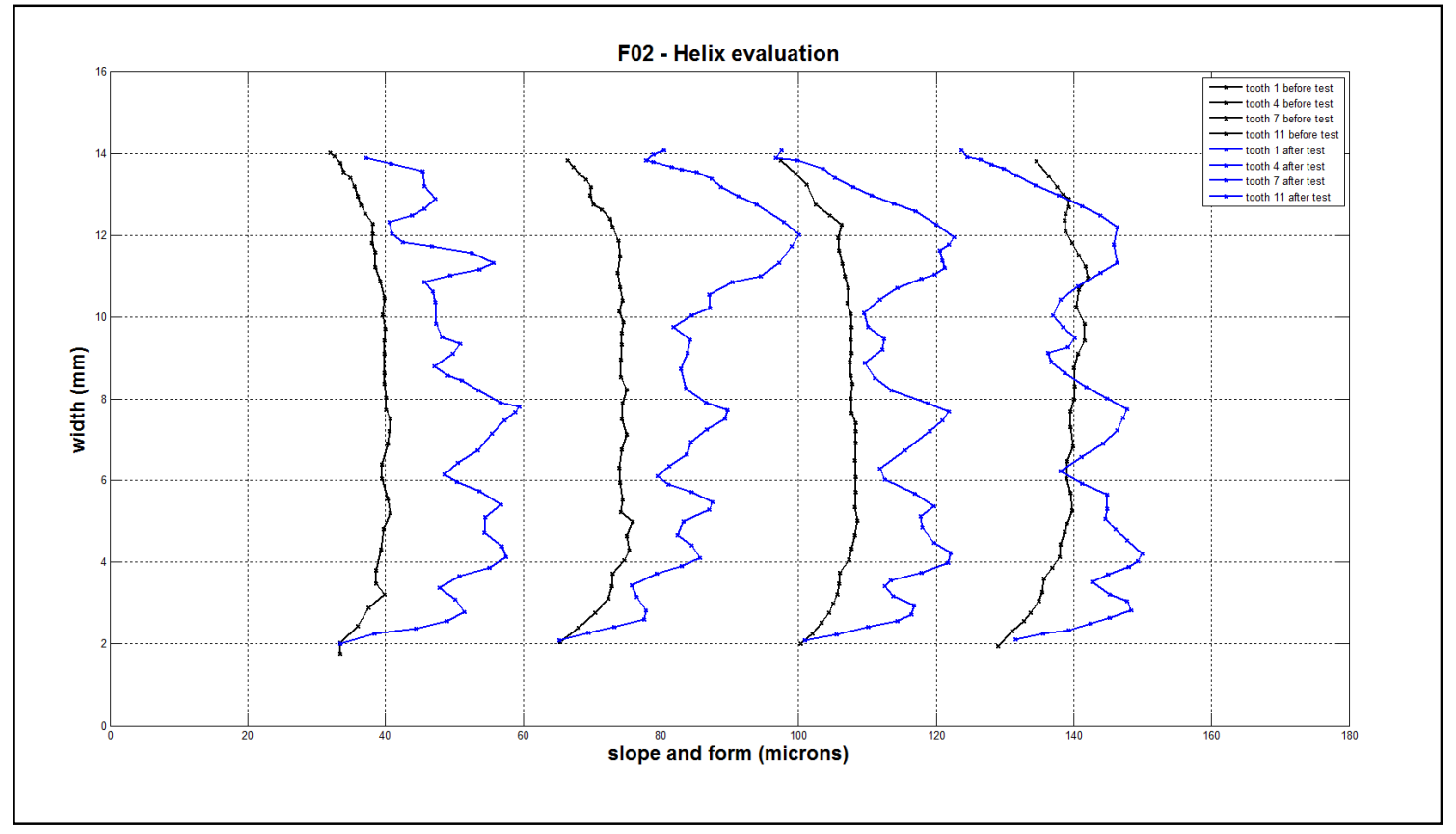

Figure G3 - F03 Gear Helix Evaluation based on WGT 350 measurements and plotted using MatLab routine.

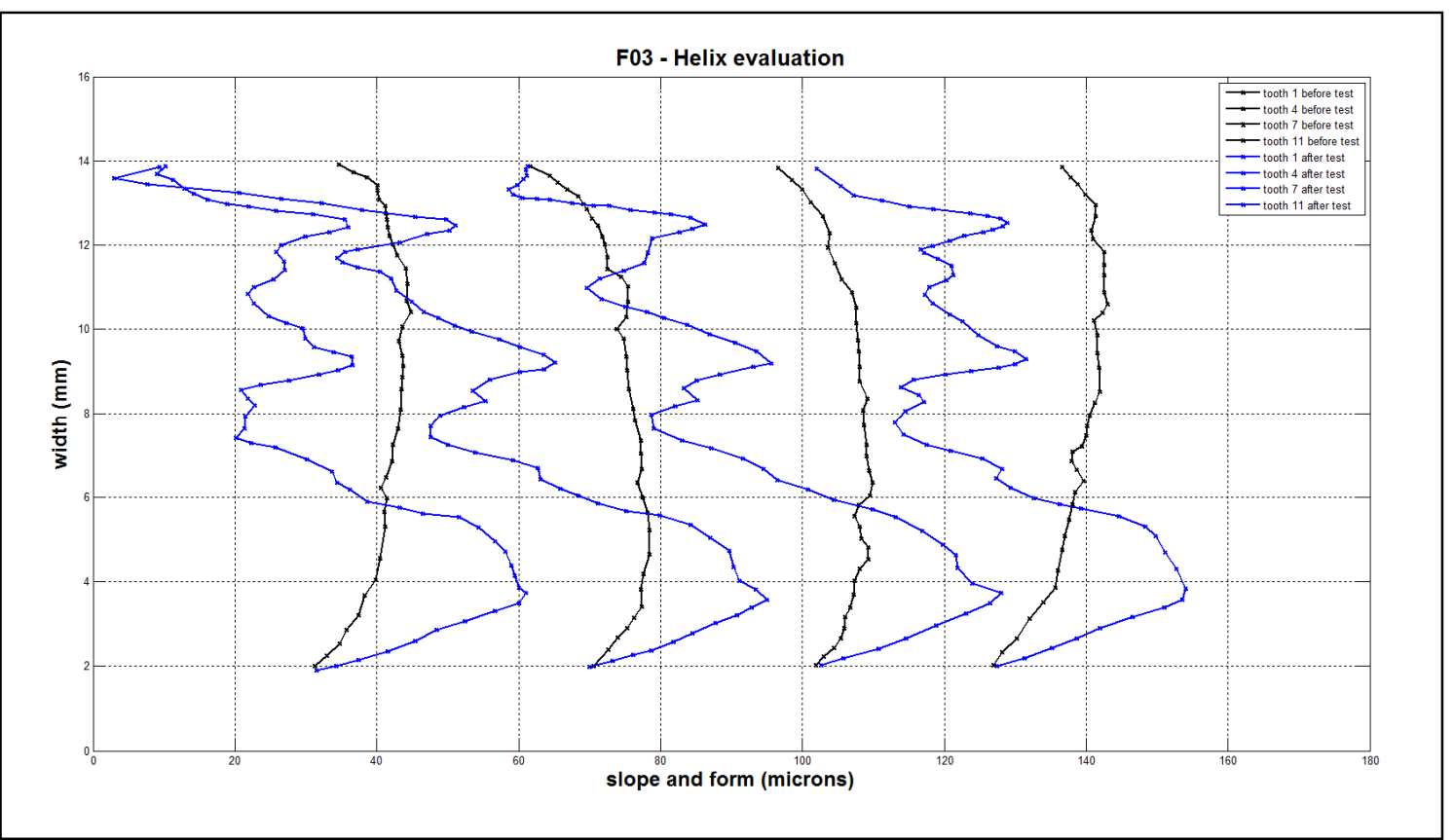


Figure G4 - F04 Gear Helix Evaluation based on WGT 350 measurements and plotted using MatLab routine.

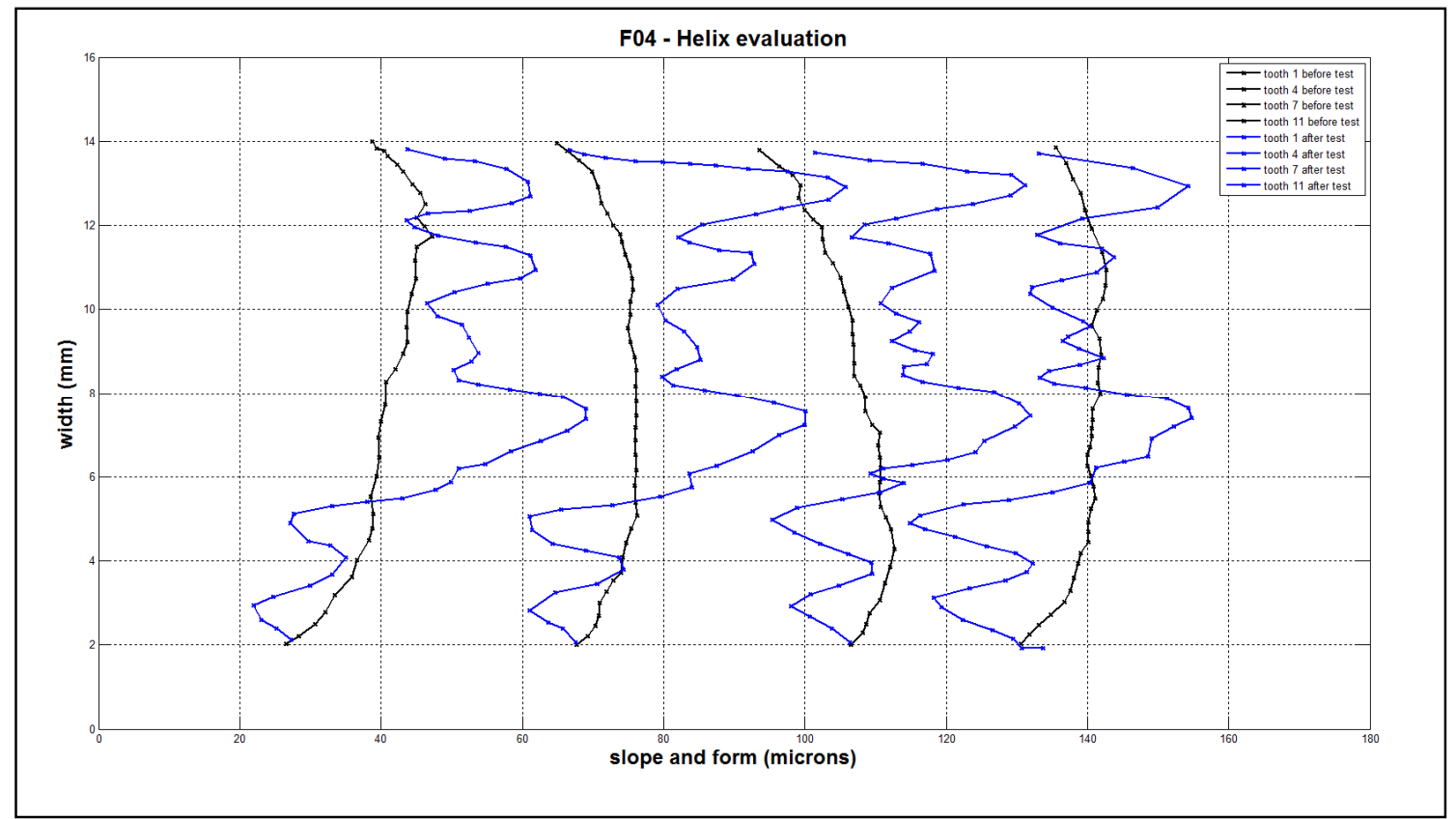

Figure G5 - F05 Gear Helix Evaluation based on WGT 350 measurements and plotted using MatLab routine.

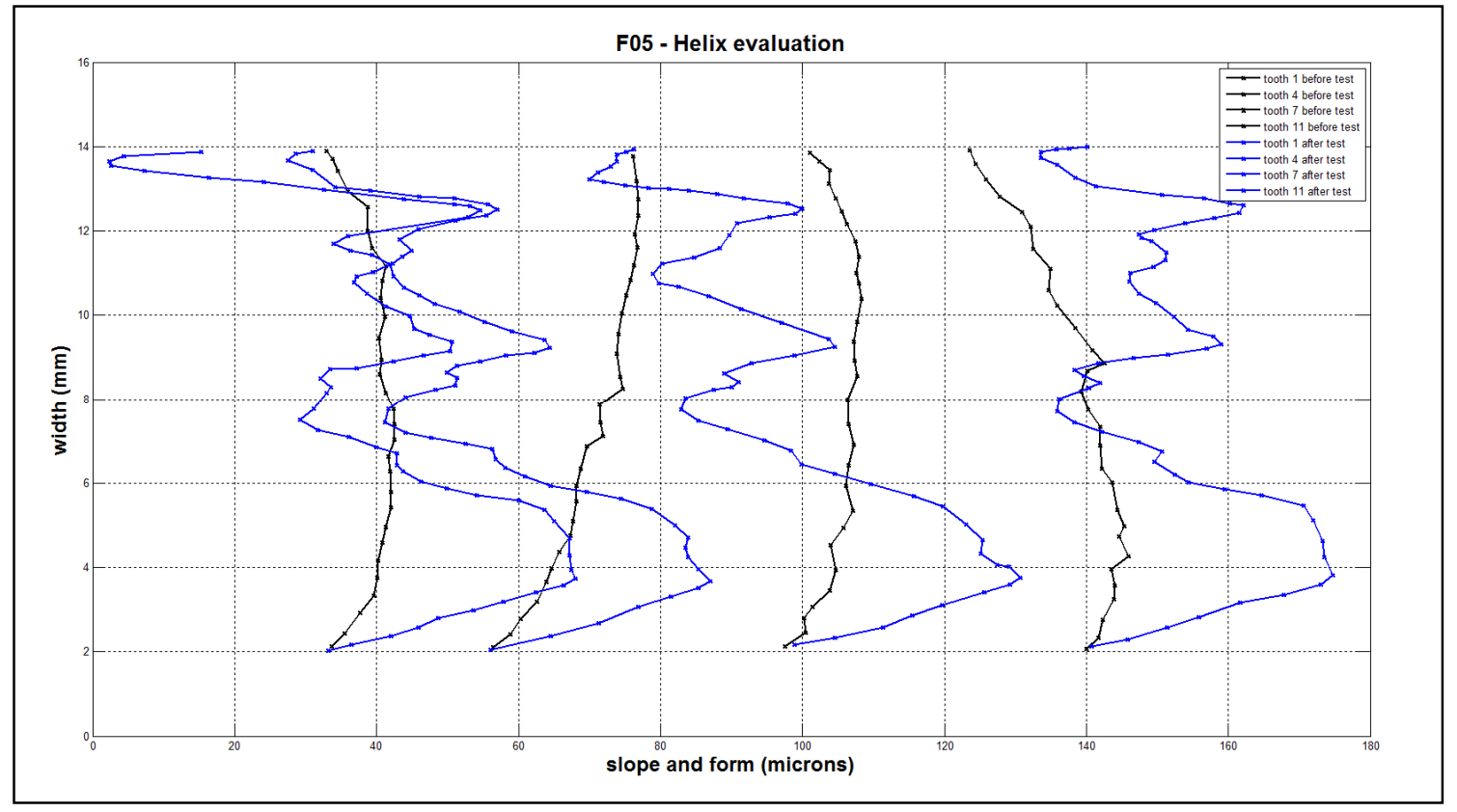




\section{APPENDIX H - FLANK HELIX MEASUREMENTS OF B01 TILL B05}

In this section, is shown Helix plots. On each one of those figures from $\mathrm{H} 1$ till $\mathrm{H} 5$ is seen teeth 1, 4,7 and 11 measurement done on WGT350 before and after fatigue test. Here, is shown measurements collected by a MatLab routine on WGT350 graph plots, another MatLab routine were required to plot the data as seen on this section figures.

To display an organized plot, all graph will follow the referred color here in set: (i) black lines referring to data before test and (ii) blue line referring to data after test. Also important to highlight that data curves references (lower width measurement) were changed and set to be equal, this modification objective is to have an organized plot, clean and allowing immediate slope and form values comparisons of blue or black lines. On the other hand, a value comparison in between black line values with blue line values are no longer accurate, since its reference has been adjusted to be plotted. Even tough, it's possible to compare the pattern between before and after test of each one of those tooth.

Figure H1 - B01 Gear Helix Evaluation based on WGT 350 measurements and plotted using MatLab routine.

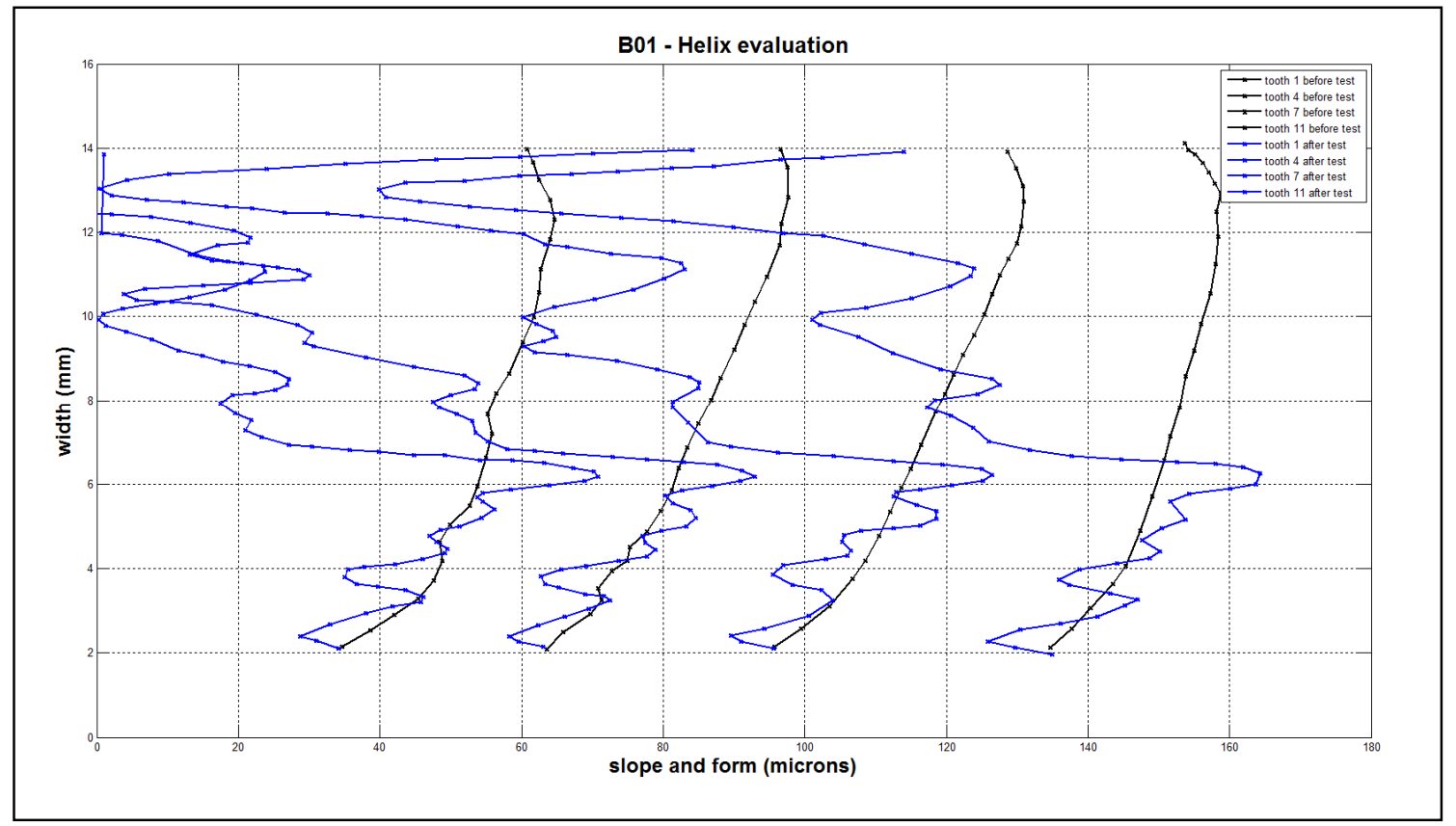


Figure H2 - B02 Gear Helix Evaluation based on WGT 350 measurements and plotted using MatLab routine.

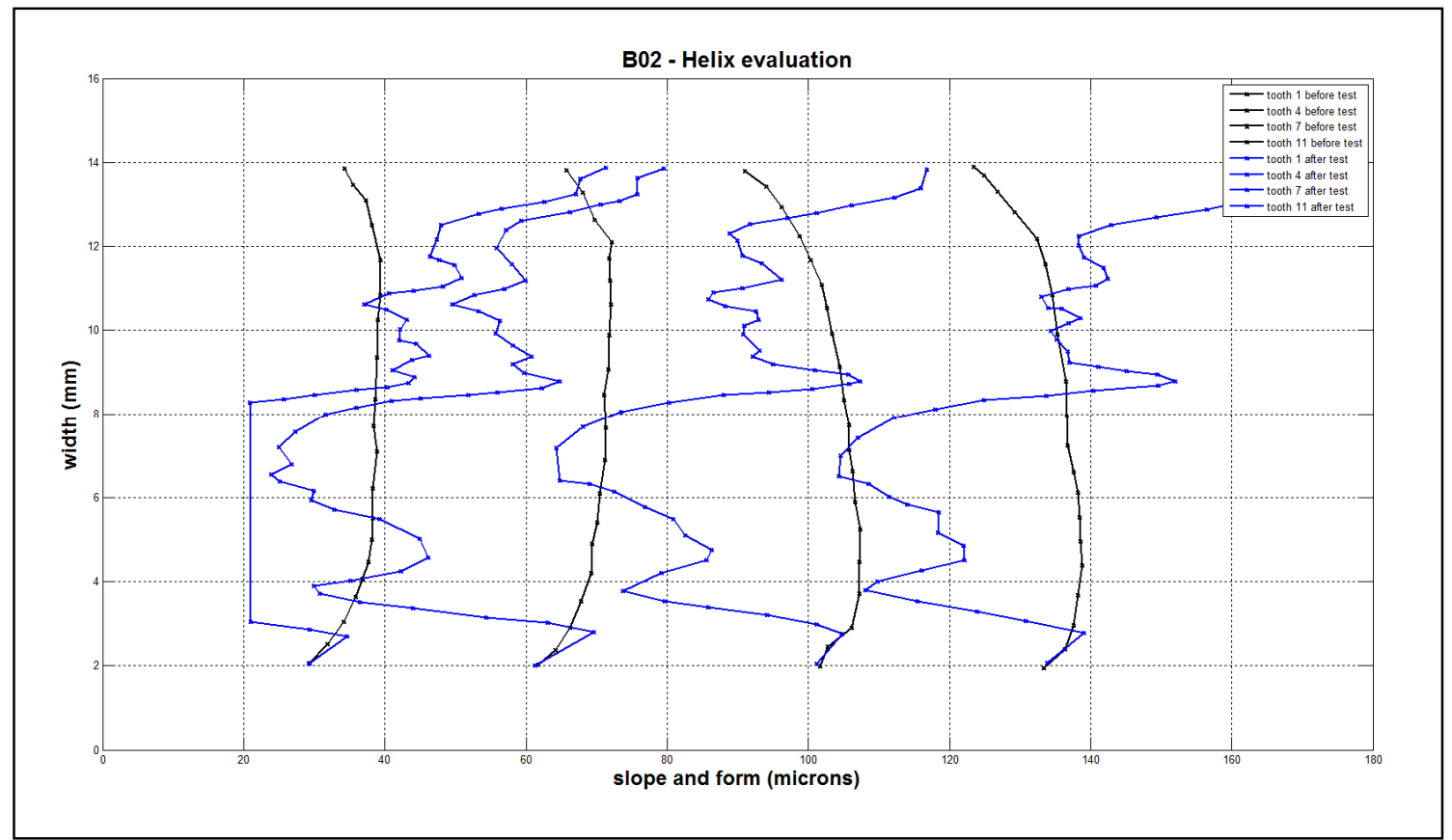

Figure H3 - B03 Gear Helix Evaluation based on WGT 350 measurements and plotted using MatLab routine

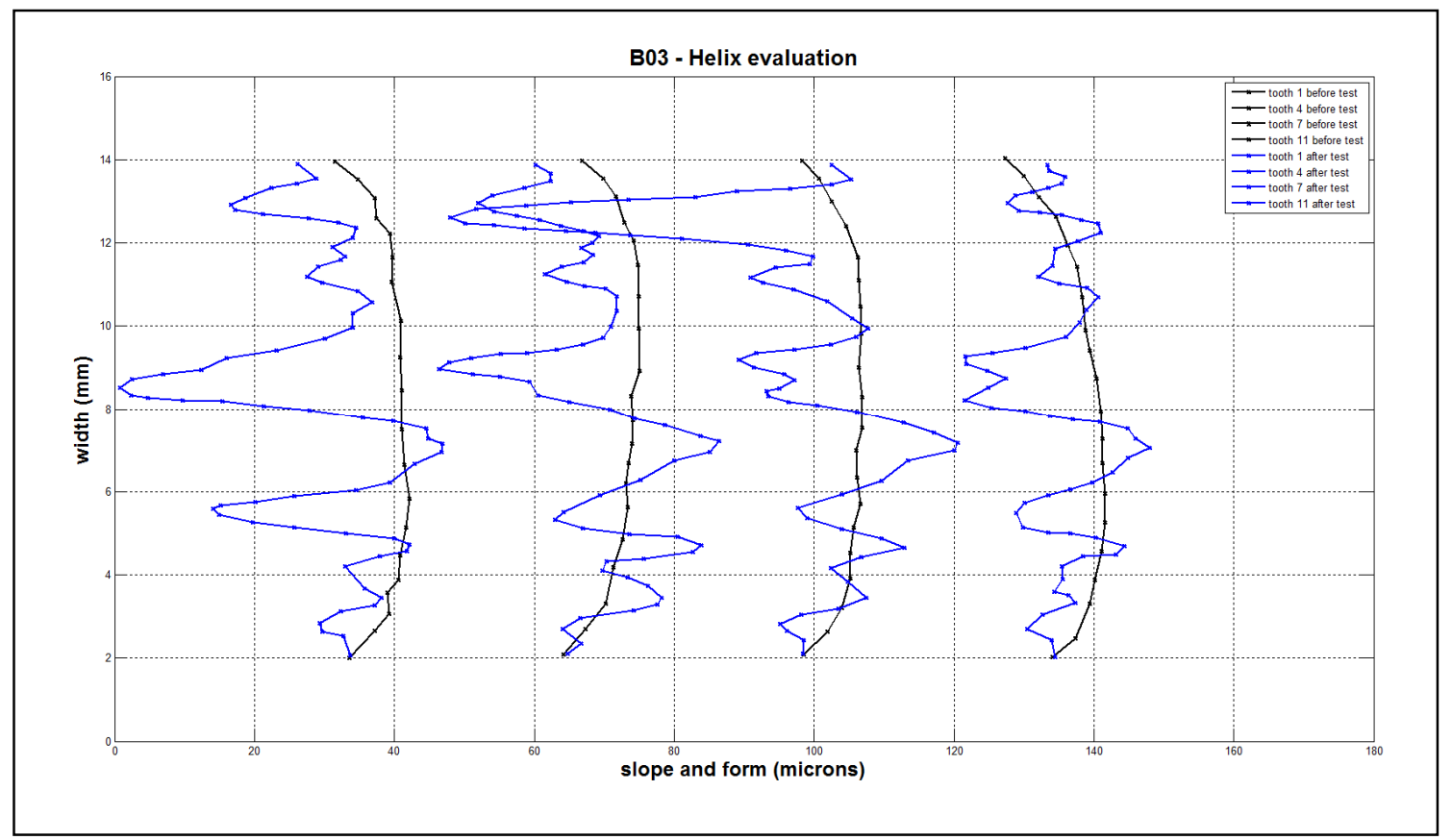


Figure H4 - B04 Gear Helix Evaluation based on WGT 350 measurements and plotted using MatLab routine.

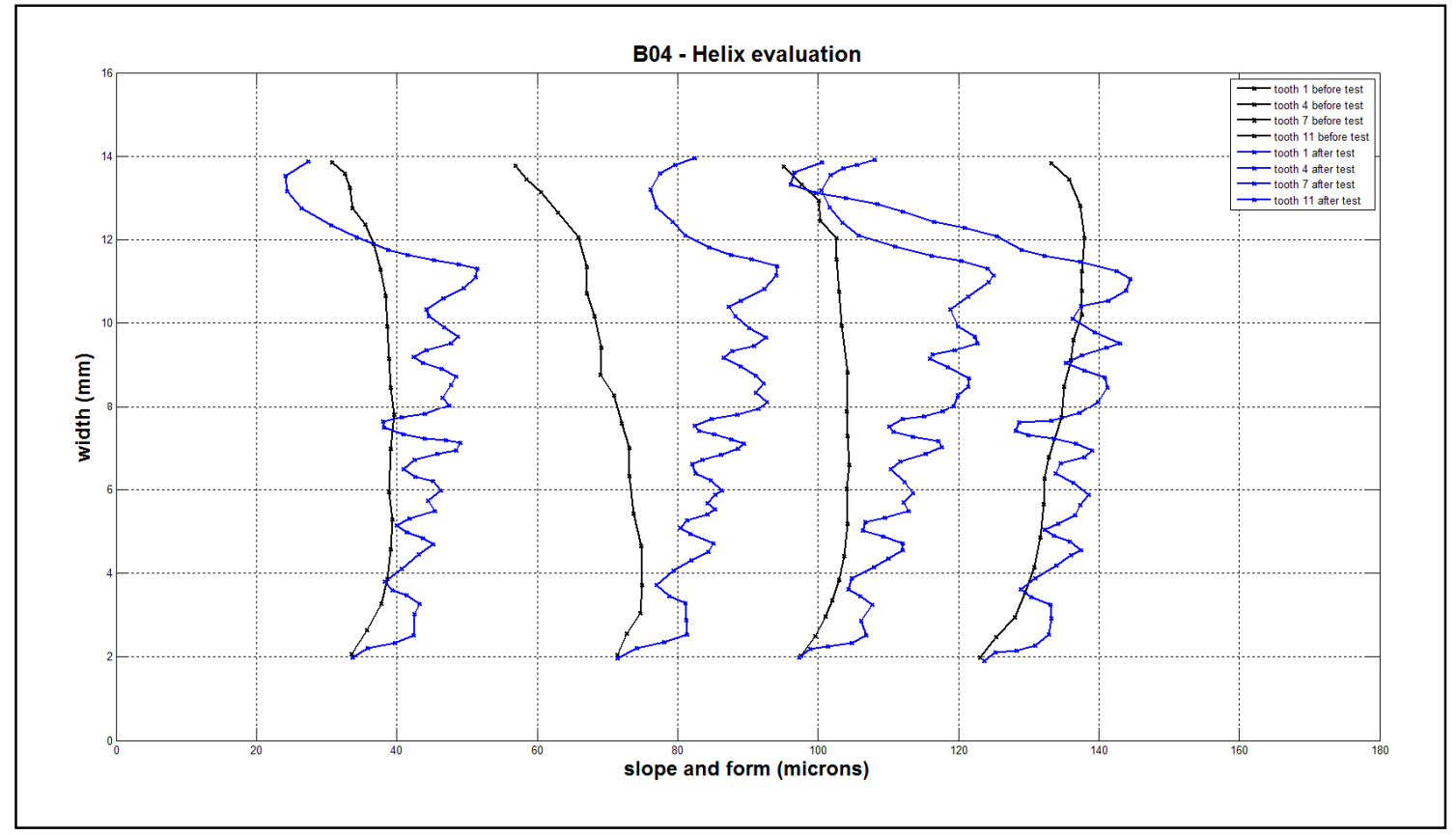

Figure H5 - B05 Gear Helix Evaluation based on WGT 350 measurements and plotted using MatLab routine.

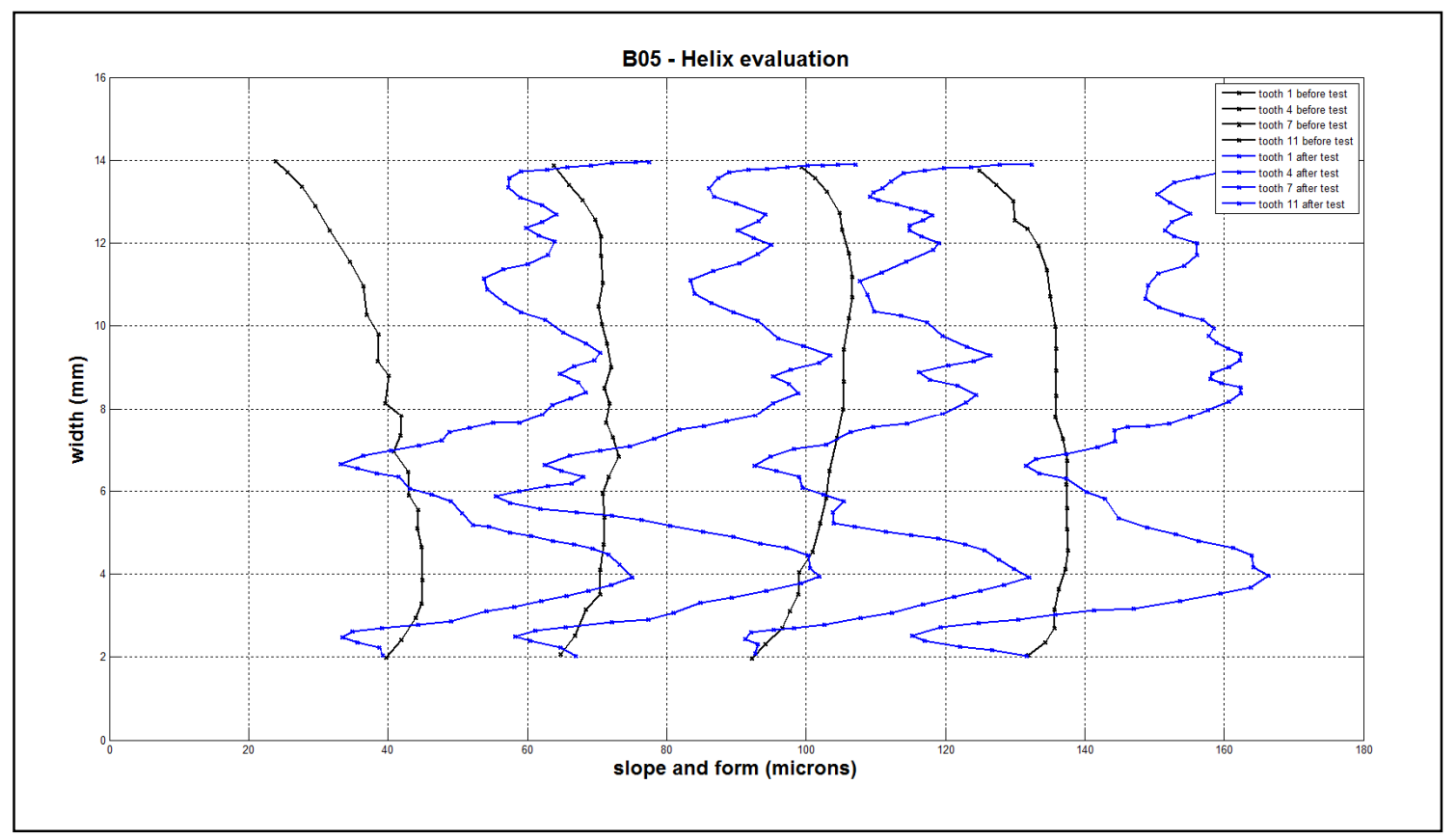




\section{APPENDIX I - FLANK HELIX MEASUREMENTS OF D01 TILL D05}

In this section, is shown Helix plots. On each one of those figures from 11 till 14 is seen teeth 1, 4,7 and 11 measurement done on WGT350 before and after fatigue test. Here, is shown measurements collected by a MatLab routine on WGT350 graph plots, another MatLab routine were required to plot the data as seen on this section figures.

To display an organized plot, all graph will follow the referred color here in set: (i) black lines referring to data before test and (ii) blue line referring to data after test. Also important to highlight that data curves references (lower width measurement) were changed and set to be equal, this modification objective is to have an organized plot, clean and allowing immediate slope and form values comparisons of blue or black lines. On the other hand, a value comparison in between black line values with blue line values are no longer accurate, since its reference has been adjusted to be plotted. Even tough, it's possible to compare the pattern between before and after test of each one of those tooth.

Figure I1 - D02 Gear Helix Evaluation based on WGT 350 measurements and plotted using MatLab routine.

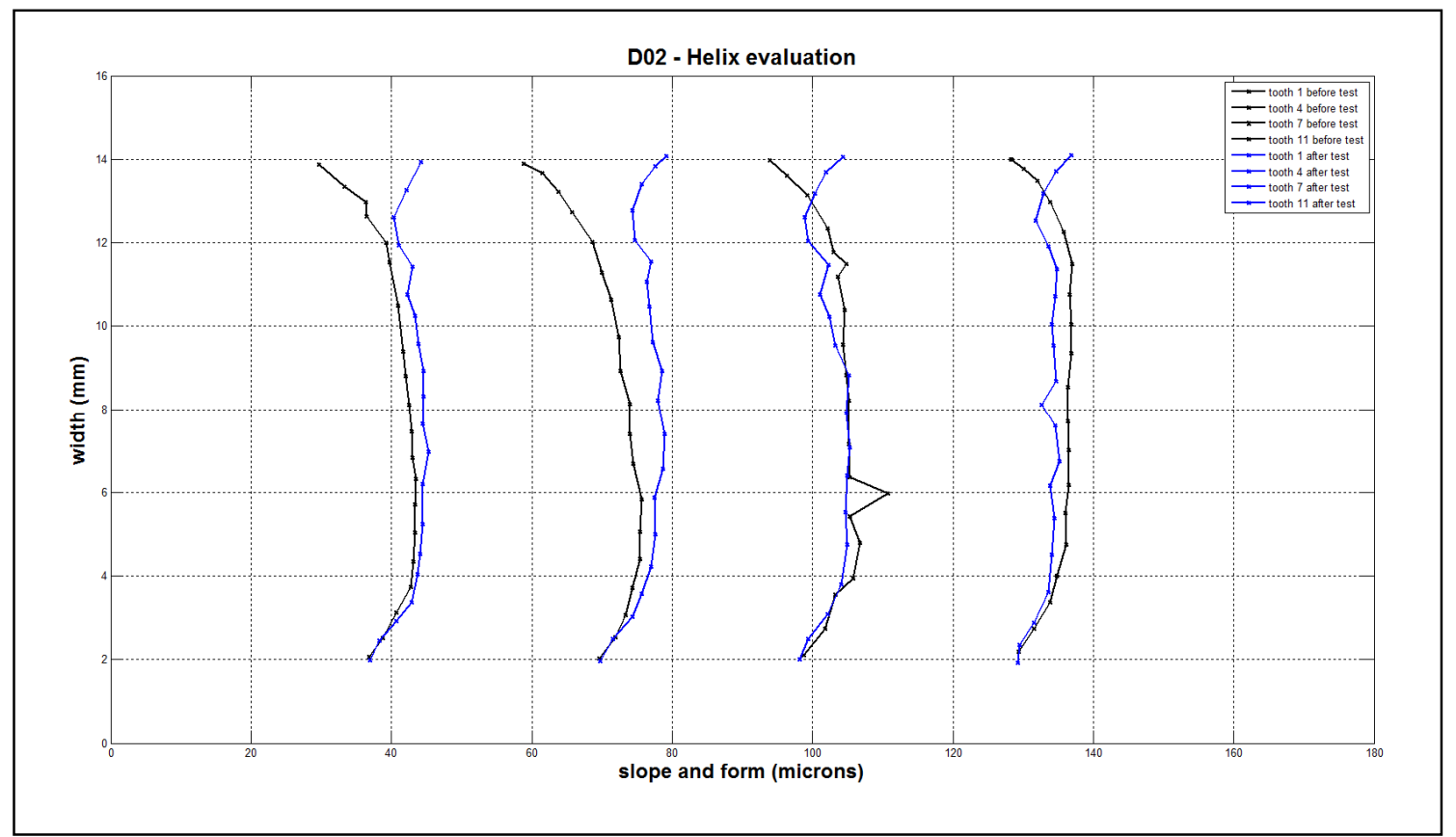


Figure I2 - D03 Gear Helix Evaluation based on WGT 350 measurements and plotted using MatLab routine.

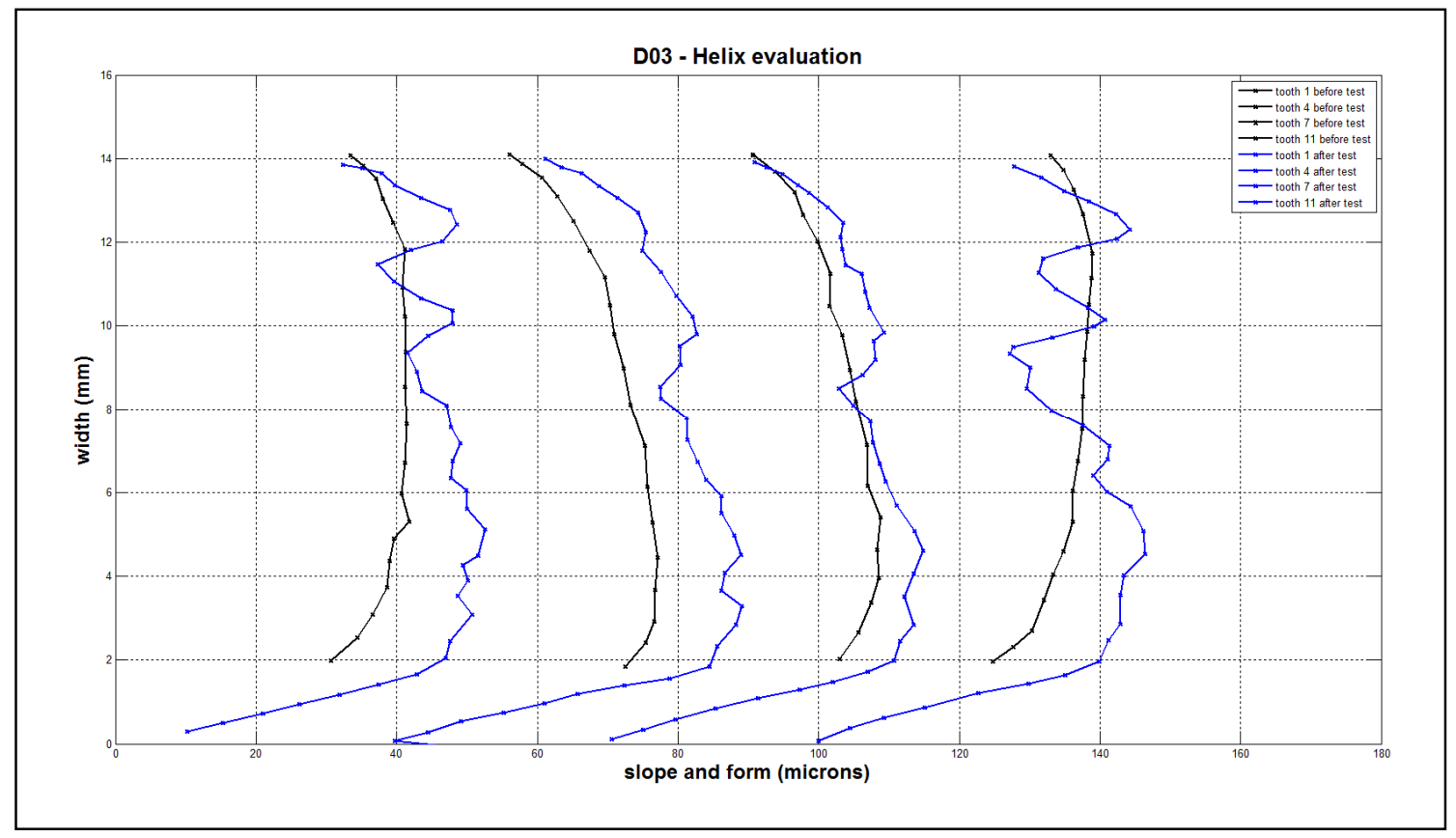

Figure I3 - D04 Gear Helix Evaluation based on WGT 350 measurements and plotted using MatLab routine.

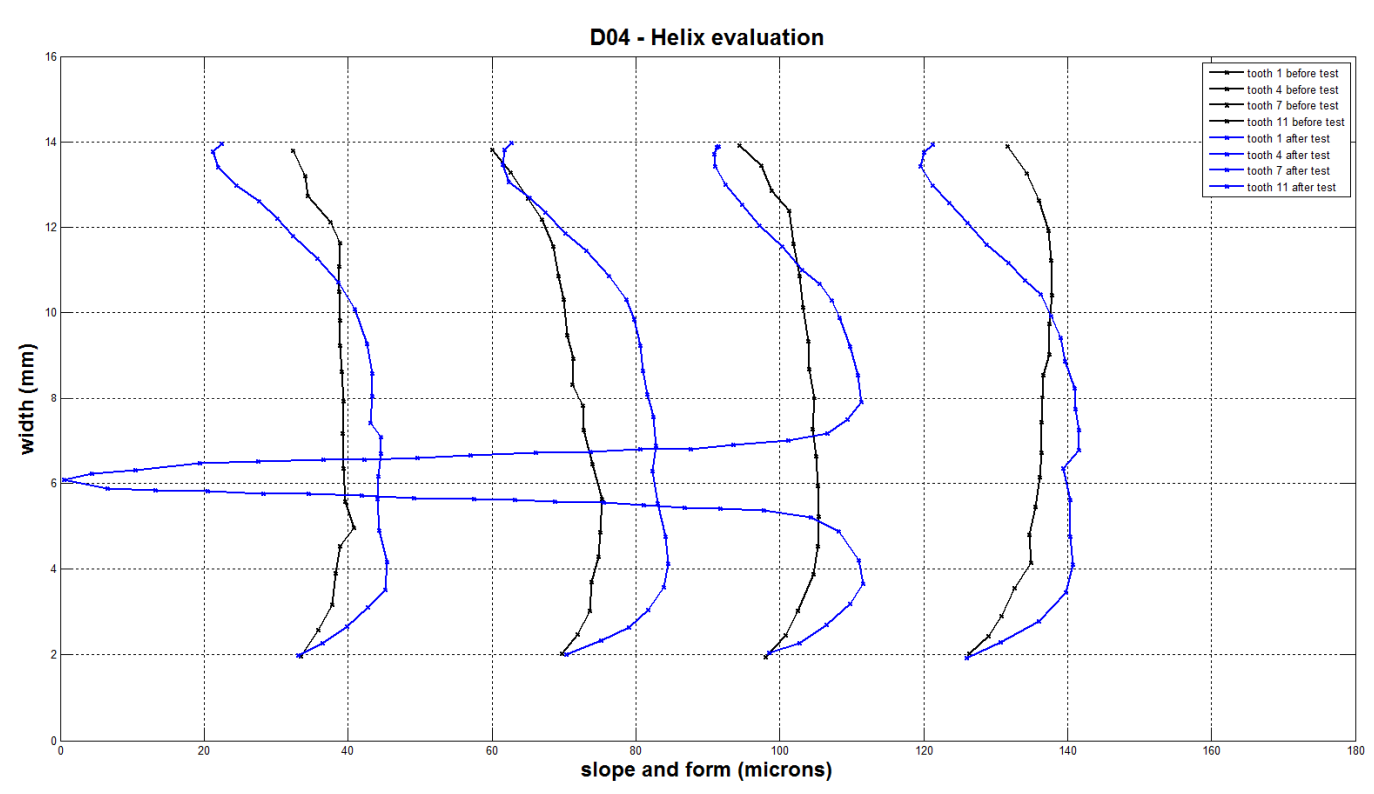


Figure 14 - D05 Gear Helix Evaluation based on WGT 350 measurements and plotted using MatLab routine.

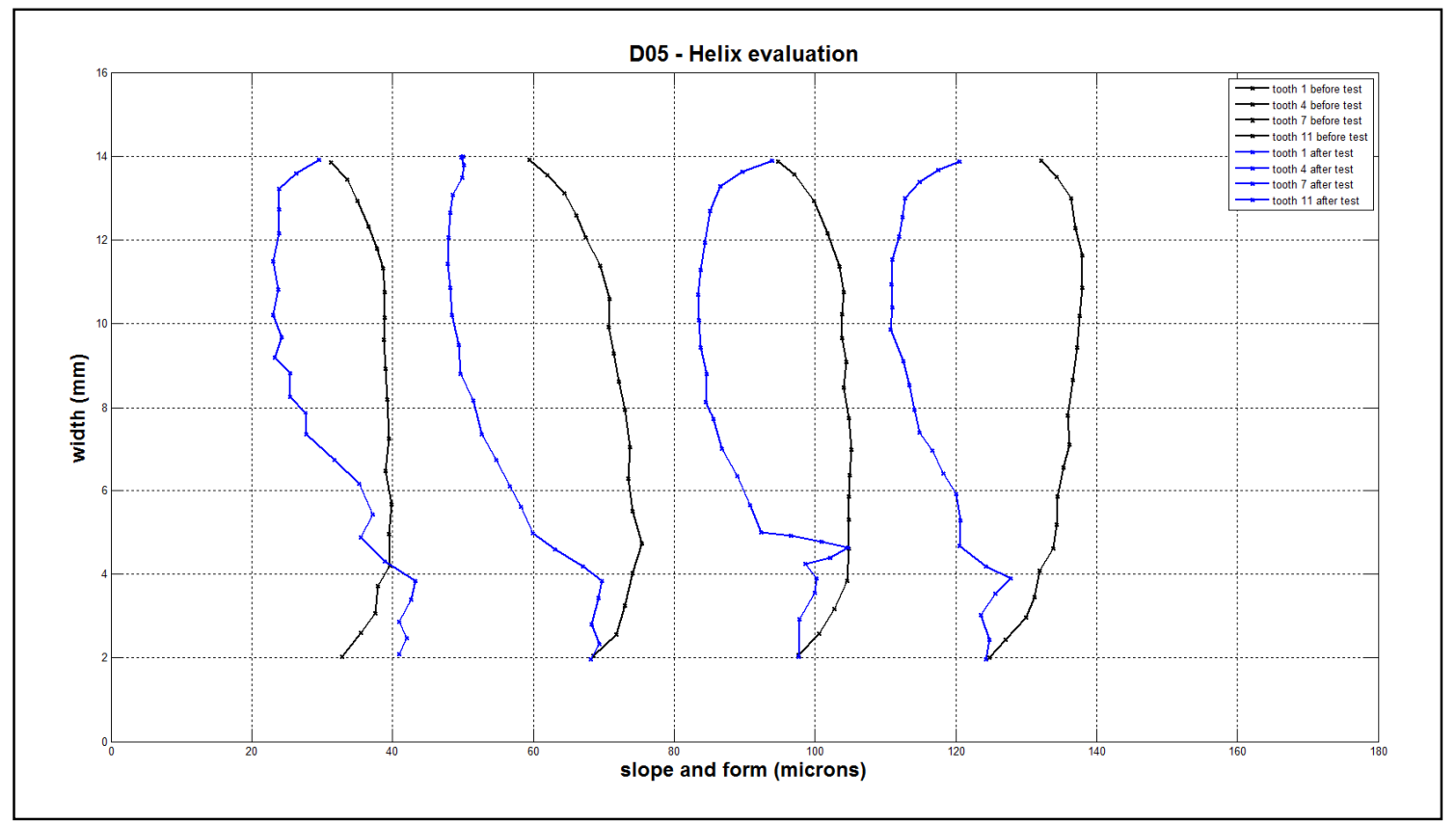

